\title{
HORSE BREEDING IN THEORY AND PRACTICE
}

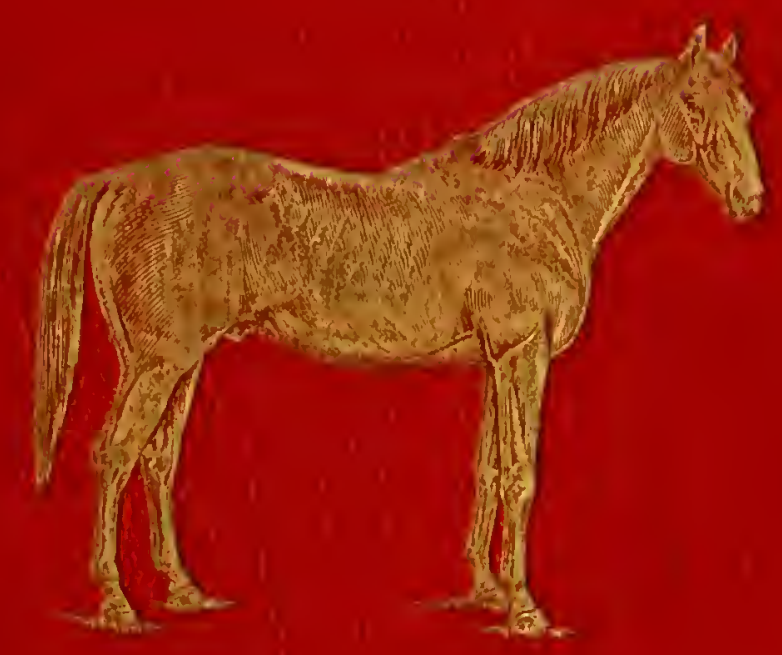

B. VON OETTINGEN 


\section{LIBRARY.}

UNIVERSITY : PENNSYLVANVA

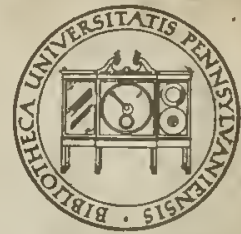

$$
\begin{aligned}
& V R 636.108 \\
& 0.88 .2
\end{aligned}
$$

FAIRMAN

ROGERS

COLLECTIION

$\mathrm{ON}$

HORSEMANSHIP

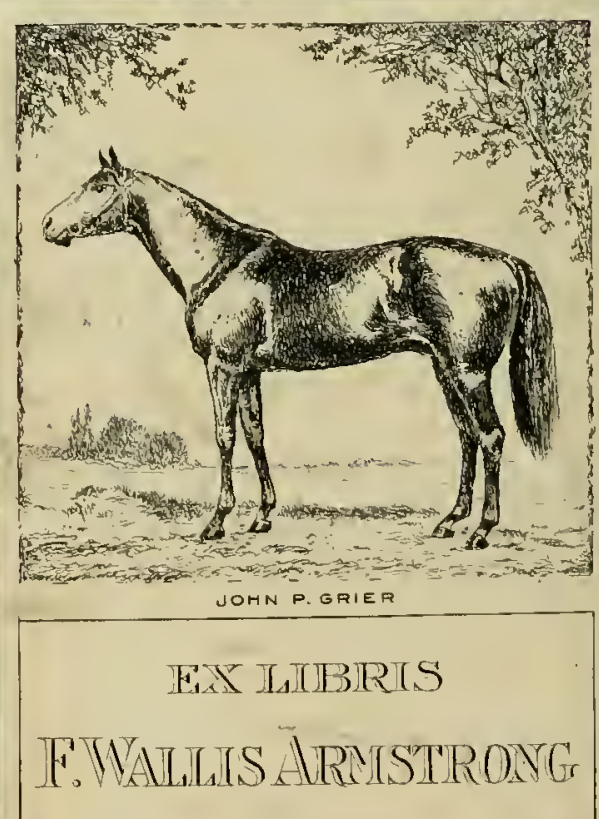




$4 c^{a} a^{a+2}$

\section{HORSE BREEDING}

IN THEORY AND PRACTICE

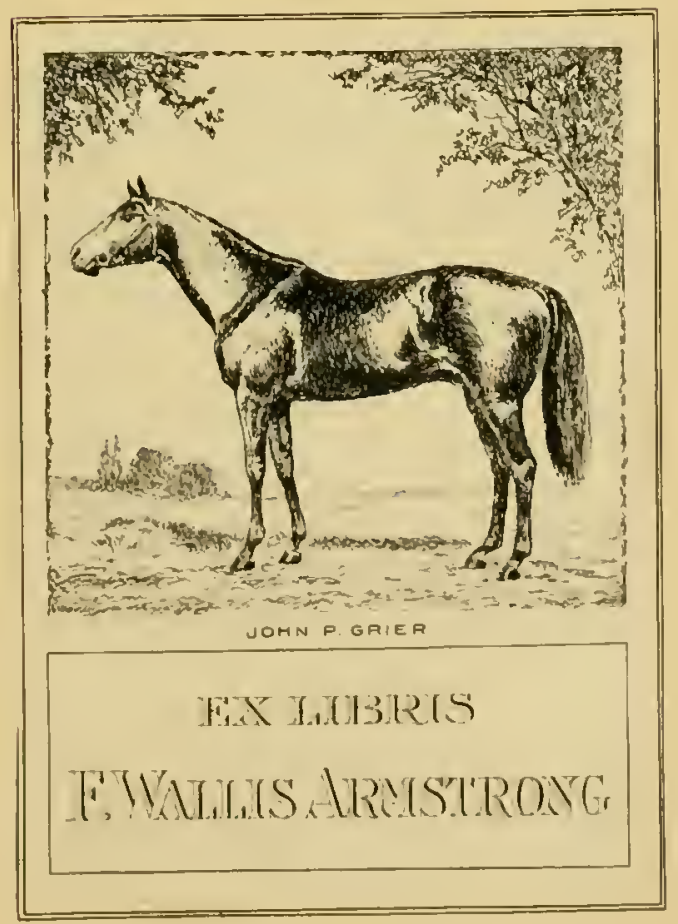


Digitized by the Internet Archive in 2009 with funding from

Lyrasis Members and Sloan Foundation

http://www.archive.org/details/horsebreedingi00oett 


\section{HORSE BREEDING IN THEORY AND PRACTICE}

\section{BY \\ BURCHARD VON OETTINGEN

\author{
I,ANDSTAIIMIEISTER AND DIRECTOR OF THF
} ROYAL STUD OF TRAKEHNEN}

LONDON :

SAMPSON LOW, MARSTON \& CO. 


$$
\begin{aligned}
& \checkmark K \text { NEW BCLTON } \\
& \begin{array}{l}
\text { h.6.106 } \\
\text { गes8. E }
\end{array}
\end{aligned}
$$




\section{PREFACE.}

$\mathrm{T}$

HE wish has often been expressed that practical men should malie known more generally their experiences in horse breding, but in desiring this it is easy to lose sight of the fact, that when a practical man commences to write he at once steps out of the brunds of the practical.

Joreover, it has to be taken into consideration that practical experience is only gained by long years of labour, whilst mere theorists may write their works when even quite young and practically without experience, but being gifted with critism, intellectual insight, and inspiration. 1 must also point out that my work at the "lrakehnen Stud has liept me always so actively employed that, unfortunately, I have not been able to spare the necessary time to go thoroughly through the large mass of material which I have accumulated in the course of many years, and to treat same in as "xhaustive a manner as an expert ought. In writing the last chapter on "The Establishment of Studs. "my time was very limited indeed, and I have had to be satisfied with simply griving an wutline.

The short historieal sketeh on the development of the lhoroughbred is the resuit of notes made from racing calendars and stud bowks, and the chapter on the alteration of weight differences is likewise the outcome of investigations made in the same quater. It was omly when l ame to the conclusion, from the present work, that there seemed to be a deterioration in the "looroughbred, eommencing from about the middle of the ninetexth century, that 1 again went through the Racing Calendar, and Stud bouk. in order to more closely investigate this apparent deterioration. In lhis waly originated the chapter comparing the capabilities (Leistungsfahiglicit) of the Thorughbred of to-day with that of an carlier period, and the deductions arising therefrom. The high regard which J have for the "Thorugghbred has not stopped, but rather stimulated me lo investigate and aspress candidly the ideats grot from studying the present state of the lhormenbred. I have taken nothing for gianted. The resules of these investigations have once again vindieated the nature of "Publie ()pinion" so well described by kanke. It has a true semse of what is needed, hut has men the slightent ideal 
of how to supply what is wanted. Is to whether the means which 1 propose for improving the Thoroughbred will be efficacious or not it is indeed difficult to prophesy. Experience alone will prove this.

The chapter on "Inbreeding" is a modest attempt to come to a logical conclusion in this interesting, but as yet unexplored, region of thought. Without doubt the study of this question is in its reriest infancy. Lp to the present time there las not been demonstrated the influence exerted by the bases of inbreeding, we do not ret know what is due to the own inbreeding of the base and what is due to its sex. Is it possible that the great prepotency of Melbourne, as shown in his female descendants, should be accounted for in the fact that the famous Termagant was the base of Melbourne's inbrecding? "The computation of "Inbreeding " has entailed much labour, as unfortunately the necessary material has neither been collected nor published anywhere, and 1 know very well that in this difficult work many errors have crept in.

In the chapter on lleredity, as well as in the practical part on llorse Breeding, much has been left out, as I take it for granted that only breeders and lovers of horses will read this work, who already know and are well acquainted with the elementary laws of horse breeding and sport racing. Iny man may make a mistalie, and I claim this indulgence, but it has been my earnest endearour to take from the practical what I have recognised as right in practice. 1 clo not liay any claims to completeness as regards the whole principles of breeding, such as is claimed in several books on this subject, as writers of these latter are often led into the error of copying ridiculous blunders of other, often injudiciously chosen, writers, and in this way spread incorrect teachings in this as in other branches.

For a period of twelve years in Trakehnen the long winter evenings were shortened and brightened by these hippological studies, the results of which 1 give to the public in the present work, with the sincere hope that it will promote the love of that noble creature, the horse, its breeding, and trial on the race course.

\section{BURCHARD VON OETTINGEN.}

Trakehnen, October 1st, 1907. 


\section{INDEX.}

\section{Part I. Trial of the Thoroughbred on the Racecourse and its value in the Breeding of Half-breds.}

CH.SPTER

1. Sources for Tracing the Development of the Thoroughbred and Race "lrials in England

2. Development of the Thoroughbred and Racing Trials in lingland. Most Important Events . . . . . . . . . . .

3. Origin and Clange in the Mreght Tables, and the Conclusions to be drawn therefrom . . . . . . . . . . . . 32

4. General Observations on the Value of the Thoroughbred for other Breeds . . . . . . . . . . . . . . . 50

5. I Comparison of what Thoroughbreds have done previously and what they are doing at the present time . . . . . . . .

6. Conclusions and Propositions as to the lmprovement and Breeding of Thoroughbreds . . . . . . . . . . .

\section{Part 1I. Heredity.}

1. General Observations on lleredity . . . . . . . . . . . ๖09

. The Transmission of Acquiled Characters . . . . . . . . 21.)

3. The Doctrine of Constancy and Individual Prepontency . . . 2.20)

4. Inbreeding . . . . . . . . . . . . . . . . . . .

5. Mercdiary Faults . . . . . . . . . . . . . . . 321

6. The Transmission of Coat Colour . . . . . . . . . . . 320?

7. The Art of Mating . . . . . . . . . . . . 33:3 


\section{Part III. The Practical Part of Horse Breeding.}

CHAPTER

1. Judging and Treating Breeding Material. . . . . . . . . 339

(a) Judging Covering Stallions . . . . . . . . . . 339

(b) Judging Brood Mares . . . . . . . . . . . . . . . 343

(c) Age and Treatment of Corering Stallions . . . . . . 345

(d) The Stallion whilst Covering . . . . . . . . . 347

(e) Age of Brood Mares . . . . . . . . . . . . . 350

(f) Twins . . . . . . . . . . . . . . 351

(g) Jirstlings . . . . . . . . . . . . . . . . . . 361

(h) The Treatment of Brond llares . . . . . . . . . 366

(i) Covering of Brood Mares . . . . . . . . . . . 367

(k) Fertilisation . . . . . . . . . . . . . . . 37.2

(l) The Time of Pregnancy of Brood llares . . . . . . 37t

(m) Abortion and Joint-illness . . . . . . . . . . . 376

(n) The Birth . . . . . . . . . . . . . . 380

(o) Treatment of the Browd Mare after the Birth . . . . . 381

2. The Judging and Treating of Foals . . . . . . . . . . . 383

(a) Judging Suckling Forals . . . . . . . . . . . 383

(b) Treatment of Suckling loals up to the time of ITeaning . 38.5

(c) Treatment of Weanlings . . . . . . . . . . . . 389

(d) Treatment of the Skin and Ilsots of W'eanlings . . . . 393

(e) The Treatment of Yearlings and Older Foals . . . . . 397

3. Training . . . . . . . . . . . . . . . . 399

(a) The Training Methods . . . . . . . . . . . . 400

(b) Stable Regulations and Instructions for Rubbing Down Horses . . . . . . . . . 406

(c) Fond whilst Training . . . . . . . . . . . 407

t. Establishment of Studs . . . . . . . . . . . . 409

j. Tables for Comparison of Various Measurements . . . . . . 422

6. Tables showing the Ancestors of Thoroughbreds in the Male Line 4:27 


\section{I. \\ Trial of the Thoroughbred on the Racecourse and its value in the breeding of Half-breds.}





\section{CHAPTER I.}

\section{Sources for tracing the development of the Thoroughbred and Race Trials in England.}

THERE is no breed in the world which places at the disposal of the investigator such an abundant and authenticated mass of material for the examination of the various problems on breeding as does the English Thoroughbred, but, unfortunately, up to the present time this material has been made very little use of.

lt is to be found chiefly - as far as I know-in the following :-

1. The General Stud Book by James Weatherby (Keeper of the Match Book, and Secretary of the Jockey Club, as successor of Tutting and Fawconer). Volume 1. appeared in 1793; the 5th Edition, very much improved and enlarged in 1891.

$$
\begin{aligned}
& \text { Volume 11..1821. th Edition, 189:. } \\
& \text { ", III. 1827. 4th ,, 18563. } \\
& \text {, IV. 1836. 4th ,, } 1899 . \\
& \text {.. V.1845. 3rd ,, } 1 \text { (s89. } \\
& \text {, VI. 1849. 3rd ,, } 1881 . \\
& \text {.. VJJ. 1853. 3rd ., 1894. } \\
& \because \text { VIII. 1857. 2nd ", } 1883 . \\
& \text { ". IN. 1861. End ", } 1903 . \\
& \text {. } \quad \text {.. } 1865 . \text { 2nd ., } 1893 . \\
& \text {, XI. } 1869 \text {. 2nd ", } 1904 . \\
& \text {. } \quad \text { N11. } 1873 . \\
& \text {.. } \quad \text { III. } 1877 . \\
& \text {.. IIV. } 1891 . \\
& \text {.. } \quad \mathrm{XV} .1885 \text {. } \\
& \text {. } \quad \text { XV1. } 1889 . \\
& \text {.. XVII. } 1893 . \\
& \text {.. XVll1. } 1897 . \\
& \text {. XIX. 1901. } \\
& \because \quad \text { X. } 1905 .
\end{aligned}
$$

2. An Introduction to a General Stud book by James Weatherby had already appeared in 1791, consisting of various collections of Pedigrees gathered from Racing Calendars and limspalpurs. 
3. The Turf Register and Sportsman and Breeder's Stud Book by William Pick.

$$
\begin{aligned}
& \text { I'olume I. appeared in York } 1803 . \\
& \text {, } 11 . \quad \text {. } \quad \text {,. } \quad \text {.. } 1805 \text {. } \\
& \text { " I11. " " " , " } 18 \text { ? } \quad \text { ? by R. Johnson. }
\end{aligned}
$$

In this Turf Register the pedigrees and racing performances of the most prominent stallions and mares are very clearly arranged. There are also many valuable notes added on Breeding.

4. The Ancestress of the English Thoroughbred, by Hermann Goos, 1885. 4th Edition extended by Dr. A. de Chapeaurouge, 1907.

5. Frentzel's Family Tables of English Thoroughbred Stock, 1889.

6. Modern Pedigrees by Antwerp and Lamplighter, New York, 1895.

7. Tables of Pedigrees of Thoroughbred Horses by Ch. Wackerow. Volume 1., 1900, from the earliest accounts to 1897 inclusive. Volume 11., 1904, from the earliest accounts to 1902 inclusive.

8. Historical List of all the Plates and Prizes run for on Clifton and Rawcliffe Ings, also since being moved to Knavesmire, near the city of York, etc., appeared in 1748 at York. It contains the Reports of the Races at York from 1709 to 1747.

9. The first Racing Calendar appeared in 1727, and was called: An Historical List of all Horse Matches run for in England and W'ales, by John Cheny, 24 Volumes covering the period from 1727 to 1750. From 1741 the Races in Ireland were also included.

10. After Cheny's death appeared the Continuation by Reginald Heber under the same title, 18 Volumes covering the period from 1751 to 1768.

11. After Heber's death there appeared the Continuation, under the title of: The Sporting Calendar, by William Tutting (Keeper of the Match Book at Newmarket), and Thomas Fawconer (Secretary of the Jockey Club), 4 Volumes, covering the perind from 1769 to 1772 . In the volumes for 1771 and 1772 the Races in Jamaica are included.

12. The Continuation appeared under the title of : Racing Calendar, edited by James WVeatherby (Keeper of the Match Book at Newmarket and Secretary of the Jockey Club), 135 Volumes, covering the period from 1773 to 1907.

Up to 1800 the Races in Ireland are included, but from 1801 there is simply an abstract from the Irish Racing Calendar given, and in 1903 and 1904 the Races in Ireland are left out altogether. Of Foreign Races are included: 1770, the Races in America (Annapolis, Phıladelphia and New York), 1776, 1776, 1777, the Races in Jamaica; 1776, the Races in France at Sablon and Fontaincbleau; and in 1792 and 1793, the Races at Moscow. Since $186+$ there has always been an Abstract of the important Races on the 
1. Sources for Tricins the Development of the "Thoroughbred, ete.

Continent included. Since the Autumn of 1867 the Hurdle Races have been left out, and since July, 1871, the National Hunt Flat Races have been omitted. Up to 1840 the Reports on Cock Fighting were included.

13. Irish Racing Calendar, 118 Volumes, covering the period from 1790 to 1907 .

14. Turf Innals of York and Doncaster, by John Orton, appeared 1843, and contains in une volume the Races at York and Doncaster from 1709 to 1843.

15. Bailey's Racing Register appeared in 1845 in three thick volumes, and gives in a very convenient and lucid manner an abstract of all the great Races in England and Ireland from 1709 to 1842.

16. The Racing Calendar Ibridged appeared in 1829, and contains in one volume the important Races in England and Ireland from 1709 to 1750 .

17. Pick's Racing Calendar, 49 Volumes, covering the period from 1709 to $18: 6$, containing all the Races in England and Ireland, appeared first under the title of: The Sportsman and Breeder's Vademecum, then as Racing Calendar. Since 1803 as The Annual Racing Calendar, since 1811 as Pick's (Annual) Racing Calendar. The first Editor, IV. Pick, died in 1816.

18. 'The Sporting Calendar, by John Pond, 18 Volumes, covering the period from 1751 to 1768 , containing the Races in England and Ireland. In the Appendix of the volume for 1751 all the Matches which were held at Newnarket from 1718 to 1751 are included.

19. The Pocket Racing Calendar for the Races in Great Britain from 1821 to $1840(?)$

20. An Historical List of Horse Natches, Plates and Prizes in Great Britain and Ireland, by B. Malker, 2 Volumes, covering the years 1769 to 1770.

21. The Sporting Magazine. First year of circulation, 1792. A competing paper appeared in 1839 under the title of "The Sporting Review," by Craven, and was amalgamated from 18.7 with the "Sporting Magazine." Also, the competing papers "The Sportsman" and "New Sporting Mlagazine," appeared in 18:31. Since 1855 the "Sporting Magazine" appeared with the addition: United with the Sportsman, sporting Review, and Sporting Magazine.

Bailey's Magazine, commencing 1870 (86 Volumes up 10 and including 1906), may be considered as a continuation of the Sporting Magazine (156 Volumes), which finished 1870.

22. The Steeplechase Calendar. I consecutive chronicle of the sport in Great britain from the yreat match over Leicestershire in 1826 to the cluse of 1814, to which is added the Irish Sport from the Autumn of 1812. Anonym, London, 1845.

23. Supplement to the Sterplechase Calendar. The Sport in Crrat 
Britain, Ireland and France, from January, 1845, to the close of the season 1846 , by Corbet.

The following appeared under the title of: The Steeplechase Calendar, etc. The next and last volume by Corbet appeared under the same title, hut did not include the Races in France.

24. The Steeplechase Calendar, by Joseph Osborne, 18 Volumes, covering the periods from 1848-49 to 1865-66.

25. The Racing Calendar Steeplechase Past, by Weatherby, 40 Volumes, covering the periods from 1866-67 to 1907.

26. The Olde New-Markitt Calendar of Matches, Results and Programmes, from 1619 to 1719 , by J. B. Muir, 1892.

27. Raciana, by J. B. Mluir, appeared 1890, and gave various interesting notes on the few Matches at Newmarket and York, as well as the rider's colours.

28. Gentleman's Magazine, 116 Volumes, covering the period 1731 to 1846.

29. The Sportsman's Dictionary, by experienced Gentlemen. London, 1778.

30. History and Delineation of the Horse in all his varieties, comprehending the appropriate uses, management and progressive improvement of each, with a particular investigation of the character of the Racehorse, and the business of the Turf, by John Lawrence, 1809. This ran through 14 Editions.

31. How to Chase, Ride, Train and Diet both Hunting Horses and Running Horses, by Jessis Markhanı. London, 1599.

32. The Complete Gamester, or Instructions how to play at Billiards, etc., together with all manner of usual and most gentle Games, either at Cards or Dice, to which is added the . Irts and Mysteries of Riding, Racing, Archery and Cock Fighting. 1680.

33. The Gentleman's New Jockey. Farrier's Approved Guide: containing the Exactest Rules and Methads for Breeding and Managing Horses in order to bring them up in the best manner for Profit, Pleasure, Service or Recreation, especially in what relates to Racing or Running, Coursing, Travel and War, etc. 3 Editions, 1696.

34. The Post and the Paddock, by The Druid. London, 1857.

35. Scott and Sebright, by The Druid. London, 1862.

36. Silk and Scarlet, by The Druid. London, 1862.

37. Field and Fern (South), by H. H. Dixon. London, 1865.

38. Field and Fern (North), by H. H. Dixon. 1865.

39. Saddle and Sirloin, by The Druid. London, 1870 .

40. Life and Times of The Druid (H. H. Dixon), by Hon. Francis Lawley. 2nd Edition, London, 1895. 
1. Sources for Tracing the Development of the Thoroughbred, etc.

41. A Treatise on the Care, Treatment and Training of the English Racehorse, by R. Darvill. London, 1828.

42. History of the British Turf from the Earliest Times to the l'resent Day, by J. Ch. Whyte. 2 Volumes, $18+0$.

43. Horse Racing: Its History, and Early Records of the Principal and Other Race Meetings. Anonym, 1863.

44. The History of Newmarket and Innals of the Turf, by J. P. Hore. 3 Volumes, 1886.

45. Portraits of Celebrated Race Ilorses of the Past and Present Centuries, bv Th. H. Taunton. 4 Volumes, 1887.

46. The Horse: How to Breed and Rear Him, by II. Day. London, 1888. 2nd Edition, 1890.

47. The Racehorse in Training, by 11 . Day. London, 1880. Tth Edition, 1892.

48. The Racehorse, by Marburton. London, 189. $189^{\circ}$.

49. Racing Life of Lord George Carendish Bentick, by John Kent.

50. Ashgill, or the Life and Times of John Osborne, by J. B. Radcliffe. 1900.

51. Breeding Racehorses by the Figure System, compiled by the late C. Bruce Lowe, by $W^{2}$. Allison. London, 1895. Translated into German, 1897, by $V$. Kirschy.

52. The British Thoroughbred Horse: His History and Breeding, together with an Exposition of the Figure System, by $W$. Mllison. London, 1901. 2nd Edition, 1908.

53. The Horse Breeder's Handbook, by Joseph Osborne. 1889.

54. Royal Ascot, its History and its Associations, by G. J. Cowthorne and R. S. Herod. $190 \%$. 1901.

5.). I'rom Gladiatenr to Persimmon, by Sydenham Dixon. London.

56. The English Turf, by Charles Richardson. London, $\$ 901$. 190 ร.

57. A History of the English Turf, by Th. A. Cook. 3 Volumes, about

58. The British Turf and the Men who have made it, compiled by the "Sporting Life." London, 1906.

59. On the Laws and Practice of Horse Racing, by Admiral Rous. Lumdon, 1852. and Issue, 1866.

60. Horse Racing in England, by Robert Black. London, 1893.

61. Kingsclere, by John Porter, edited by Byron Webber. 1.ondon, 1896 . 
62. Eclipse and O'Kelly. Being a Complete History, so far as is known, of that celebrated English Thoroughbred "Eclipse" (1764-1789), and of his breeder, the Duke of Cumberland, and of his subsequent owners, William Wildman, Denis O'Kelly and Andrew O'Kelly, now for the first time set forth from the original authorities and fanily memoranda, by Th. A. Cook. London, 1907.

63. Heroes and Heroines of the Grand National, by Finch Mason. London, 1907.

64. The Romance of the Derby, by Edward Moorhouse. 2 Volś., London, 1908. 


\section{CHAPTER 11}

\section{Development of the Thoroughbred and Racing Trials in England. Most Important Events.}

Gowg back to the time when England was ruled by the Romans, AngloSaxons and Normans, we find that Oriental blood was employed for the purpose of breeding Racehorses in this country. Shortly afterwarch the Crusades again gave the upportunity of introducing Oriental bluod, and as early as 1509-1547 Henry Vill. had special paddocks made in his stud at Hampton Court for the breeding of Racehorses. During the reign of James I. (1603-162.5) the importation of Oriental stallions increased very much, and during the reign of Charles II. (1660-1685) 30-40 Oriental mares, called the "Royal Mares," were brought over. IV have reason to suppose that up to 1750 about (i)-\$0 Oriental mares had been imported to England, whilst the number of stallions imported from the East was much greater. It seems that only a very fell of these Eastern stallions and mares were pure bred Irabs. The greater part were Berbers and Spaniards, whilst some were Turkish and others Persians. Also the home-bred English horse, so much praised by Cesar, and after him by many other writers on this subject, on account of its staying powers and speed, contributed very largely to the building up of the English Thoruughbred. These native-bred horses in England and I reland had the ehatater of the wild horses of the Steppes, and are mentioned as living in the forests as late as the seventeenth century, with the remark that they were better and more capable than any other breed in Europe: whilst nowhere was there to be found such good, nourishing grass, as in England and Ireland (sce the Sportsman's Dictionary, 1778). In the fourth part of the first volume of the "General Stud Book" there are 102 Eastern stallions given which have been used in the building up of the Thoroughbred, but hesides these there nere many uther liastern stallions imported and used hy breeders in England and Ireland (although the results, as far as the Thoroughbred is concerned, were probably very meagre), as can be gathered, for instance, from the publications in the old Racing Calendars. Of the 100 Oriental stallions which, according w the Stud book, were imported, about (i.) were imported before the famous Cookolphin Arabian. It is "orthy of note that, of the many Oriental stallions which were brought over to England 
after the famous Godolphin Arabian (probably a Berher), imported via Paris in 1730, none of them exercised any striking influence on the Thoroughbred of that time. Of the Oriental stallions imported from 1730 to the end of the eighteenth century (at least 40), probably the Cullen Arabian, imported in 1745, was the best, and even he did not leave behind any son of note, alluugh many of Cullen Arabian's children won races, amongst which wats Exutic, born 1756, up to the age of twelve inclusive, and although a son, called Camillus, born 1748, begot some race-winners, yet there has been nothing imperishable left behind. Of the many daughters of Cullen Arabian there are only two out of Lady Thigh in Fam. $12 \mathrm{a}$, one Foundation mare in Fam. 2 (born 1756), and one Foundation mare in Fam. 42 (born 1760) which can be said to have left anvthing lasting as breeding stock.

In the following list are given the 36 Oriental stallions which have played a very conspicuous part in the breeding of Thoroughbreds, and which occur very often in the pedigree of every Thoroughbred of our time.

We give below a list showing the probable year of their importation :-

1. 1635 Lord Fairfax's Morocco Barb.

2. 1660 Place's White Turk.

3. 1665 Dodsworth's (Mother imported in foal).

1. 1670 The Darcy Yellow Turk.

5. 1675 The Darcy White Turk, or Sedbury Turk.

6. 1680 The White-Legged Lowther Barb.

7. 1680 Shaftesbury Turk.

8. 1680 The Helmsley Turk.

9. 1687 The Stradling, or Lister Turk.

10. 1689 The Byerly Turk.

11. 1689 Oglethorpe Arabian.

12. 1690 Pulleine's Chestnut Arabian.

13. 1690 Leede's Arabian.

11. 1690 Fenwick Barb.

15. 1690 The Taffolet, or Morocco Barb.

16. 1695 The Marshall, or Selaby Turk.

17. 1700 Curwen's Bay Barb, or Pelham's Barb.

18. 1704 Holderness Turk.

19. 1706 Chillaby.

30. 1706 Darley's Irabian.

21. 1707 The St. Victor Barb.

2.2. 1708 Honywood's Arabian, or William's Turk.

23. 1708 The Akaster Turk.

24. 1709 Alcock's Arabian.

25. 1711 Bethel's Arabian.

26. 1712 The Strickland's Turk, or Carlisle's Turk or Barb.

27. 1713 Woodstock, or William's Arabian.

28. 1716 Bassett's Oxford Bloody-Shouldered Arabian. 
29. 1717 Wynn . Irabian.

30. 1718 Hall Arabian.

31. 1719 The Belgrade Turk.

32. 1720 Cyprus Arabian.

33. 1723 Lonsdale bay . Irabian.

31. 1723 ILutton's or Mulso Bay Turk.

3.5. 1725 Bloody Buttocks.

36. 1730 Godolphin Irabian.

Horse-racing as a popular amusement was indulged in even in the times of the Romans, and during the four years which King Severus passed at York (206-210) the Roman soldiers arranged races with Arabian horses at Wetherby, near York. King Athelstan (9:-910) was presented with racehorses, which had been bred in Germany, by his brother-in-law, Hugo Capet, Duke of Burgundy, later King of France.

The first race in England of which we have a reliable description took place in 1377. Unfortunately, the place is not stated.

This race was a match between the Prince of Wales (later Richard II.) and the Earl of Arundel. In all probability it took place at Newmarket. But already in 1309, and probably earlier, races (tournaments) had taken place at Newmarket (founded 1226, after the Plague had destroyed the old marliet). Further, according to reliable information, races took place at the following periods:-

1511 at Chester (the Silver Bell, since 1609 Silver (up).

1552 at Hattington (Scotland).

1574

1585

1587

1588

at Croydon in presence of the Queen.

1576 at Richmond.

1585 at Salishury. The Earl of Cumberland won the Golden bell in a three-mile race.

1590 at St. James' l'ark.

1590 at York.

1595 at Doncaster.

1599 at Carlisle (the Silver Be-1l).

1601 at Teviotdale.

1602 at Huntingdon.

1605 at Newmarket in presence of James 1. (1603-16:5).

1607 in November a hunting match or steeplechase took place at Huntingdon, between Lord Iladdington and Lord Shefield.

1617 at Woodham Moor, and at Lincoln races for the Cup took place in the presence of the King, James 1.

1620 at Paisley (Scotland), the Silver Bell.

1632. at Harleston (Silver Cup). 
1634 The establishment of the Gold Cup at Newmarket, the first winner of which, in 1719, was the Duke of Rutland's mare, Brown Betty, born 1713. by Basto (see liam. 5).

1661 at Epsom, formerly called Banstead Downs, in the presence of King Charles II. (1660-1685). Epsom was formerly famous as a health resort on account of the healing properties of its waters, and race meetings were held there even in the reign of James I. (1603-1625).

1672 at Liverpool.

Towards the end of the seventeenth century race meetings were held regularly at Newcastle, Northamptonshire, Winchester, and many other places, and somewhere about 1635 a match, described in detail, took place in Myde Park, London.

From 1654 to 1658 races were forbidden by Oliver Cromwell for political reasons, although he himself was a breeder and owner of racehorses.

There exists an exact register of the horses at the time when the Royal Tuttbury Stud in Staffordshire was handed over to the Parliament in 1649.

There was in the Royal Stud at that time:

23 Brood Mares with

23 Sucking Foals,

15 Brood Mares without Foals,

2.2 One-year-old Colts and Fillies,

17 Two-year-old Colts and Fillies,

16 Three-year-old Colts and Fillies,

23 Four-year and older horses.

After the Restoration, Charles II. (1660-168.5) established in 1665 the socalled King's Prizes, known as The King's (Queen's) or His Majesty's Plates-also called the Royal Plates. Run over the new round course at Newmarket, R. C.=3 miles, 6 furlongs, 93 yards (now R. C.=3 miles, 4 furlongs, 187 yards), with heats under 12 stone.

These King's Plates have played an important part in the testing of the breeding material, and they still exist, but without heats, and over shorter distances.

Under the reign of James 1I. (1685-1688), Gentlenten's Races took place in Newmarket, as, for instance, in 1688, under 12 stune, orer 4 miles, with 3 heats. Towards the end of the seventeenth century there were races with conditions for sale.

It can safely be taken for granted that as early as the beginning of the seventeenth century regular races were held at different places for the purpose of testing breeding material, whilst the races which took place before this time were more in the character of popular amusement.

The first breeding tests with which we are acquainted arose from matches, about which we have an account in the collection published by Muir in 1892. 
covering the period 1619 to 1719 , but, unfortunately, in many cases the names of the horses are not given.

The riders at that period were often Gentlemen, and sometimes even Kings. Charles II. and William III. ran and won many races. Of one race in 1674 the report reads as follows:-

" Vesterday His Majesty (Charles II.) rode himself three heates and a course, and won the Plate-all fower were hard and nęer ridden, and I do assure you the King wonn by good horsemanshipp" (see Olde New-Markitt Calendar, by Muir, page 19).

The distances were generally 4-6 miles, 1681 once 10 miles, 1708 once 12 miles. Most of the races had to be run and won twice, that is to say, the races were run with "heats." The age of the horses on the racecourse was generally over 6 years, the weight usually $8-12$ stone.

Newmarket was the chief racing place for all the best matches, and was famed as such, so that even Peter the Great expressed the wish to become acquainted with this sporting place.

In 1698 the Czar visited Newmarket in the company of William III., and participated in a race meeting at that place.

The following horses were winners of the races of that early period, and were used for the building up of the Thoroughbred :-

1. Old Careless, born about 1690, by Spanker and a Barb mare, ran and won 1698-1702 at Newmarket, over 5 and 6 miles.

$\because$ Honeycomb Punch, burn about 1692, by Taffolet or Morocco Barb, ran and won 1699 at Newmarket, over 4 miles.

3. Basto, born 170:- by Byerly Turk and the Bay Peg, ran and won several times 1708 and 1709 at Newmarket, orer 4 miles.

1. Duke of Bolton's Bay Bolton, horn 170., by Grey HautboyMakeless, rán and won 1712 and 1713 at Newmarket, оver 5 and 6 miles, having already won the Gold Cup over 4 miles at York in 1710.

5. Snail, born about 1710, by Whynot, ran several times at Newmarliet in 1718 , over 4 miles.

6. Flying Childers, born 1711, by Darley's . Irabian and lictty Leedes, won as a six, seven and eight-year-old, five matches at Newmarket, over 1 and 6 miles.

It was only after the yar 1728 that a fell races were arranged for fouryear-olds: at llambledon, 3 miles without heats, 10 stone, and at Scarborough, 2 miles with heats, 9 stone.

In 1732 Blacklegs won a match as a four-vear-old.

The first four-year-1)ld wimner of importance, as regards breeding, was Starling in 17:7, by bay Bolton (at llambleton).

A sweepstakes for four-l'ar-olds at Newmarliet took place in October, 1730 , and since $17: 31$ the same were arranged several vears in succession, 1 miles without heats, 8 stone, jo lbs. 
In order to give the small horses more chance in the races, in 1713. according to authority (but probably even earlier), the weights in special races were fixed according to the size of the horse. The races were called the "Give and Take Plates." Before 1750 the standard measurement for a horse was 12 hands high $=122 \mathrm{~cm}$.; normal weight 5 stone, and for each increase of 1 inch $\frac{1}{2}$ stone more. Also $7 \mathrm{lbs}$. was allowed for each year under seven years. In 1751 there were fifteen races for the King's Plates, and likewise fifteen for the Give and Take Plates. Whilst the races for the King's Plates are still in existence, the Give and Take Plates gradually disappeared in the first half of the nineteenth century. The qualifications for these latter were published for the last time in the Racing Calendar of 1858 . In the eighteenth century these races were important, in so far as several of the winners of the Give and Take Plates were the source from which came the material for the building up of the Thoroughbred. They were as follows:-

1. Grey Grantham, born about 1710, by Brownlock Turk, won 1717 at Newmarket.

2. Y. Cartouch, born 1731, by Cartouch, won four times as a five, seven and eight-year-old.

3. Squirt, born 1733, by Bartlet's Childers, won as a seven-year-old in Give and Take Plates with heats.

4. Inkaster Starling, born 1738, by. Old Starling, won as a nine-yearold twice, as a seven-year-old unplaced.

5. Dormouse, born 1738, by Godolphin Arabian, won as an eight to eleven-year-old, four races with heats.

6. Cub, born 1739 , by Fox, won as an eight-year-old once with heats.

7. Torismond, born 1739 , by Old Starling, won as a seven-year-old once.

8. Othello, born 1743, by Crab, won as an eight-ycalr-old.

The races for the King's Plates, established by Charles II. in 1665, formed, from about the beginning of the nineteenth century, the main trials of young Thoroughbreds, corresponding to our present so-called five classical races-Derby, Oaks, St. Leger, 1,000 and 2,000 Guineas Stakes. At first the King's Plates were only for six-year-olds under 12 stone, and over 4 miles with heats, but later both older and younger horses were admitted. Since 1750 a part of the King's Plates were also open for four and five-year-olds, over 2 miles with heats, under 9 and 10 stone respectively.

These King's Plates at first consisted of silver dishes of the value of $\mathcal{E} 100$, upon each of which was engraved the name and pedigree of the winner. It was not until the time of George I. (1714-1727) that, instead of these prizes, money prizes were given. This money was partly raised by gentlemen who themselves had no racehorses, with the express idea that this money should go to the Crown, for races over long distances with heats and heary weights, in order to improve the breed of Hunters, and not merely for breeding horses 
with beautiful forms and great speed. (See the Gentleman's Magazine, Ipril, 1739.)

The most prominent winners of the King's Plates up to 1850, as well as those which have played an important part for breeding purposes, were the following :-

1. Coneyskins 1712 by Lister Turk.

2. Cade 1734 by Godolphin Arabian.

3. Regulus 1739 by Godolphin Arabian.

4. Tartar 1743 by Herod.

5. Shakespeare 1745 by Hobgoblin.

6. Eclipse 1764 by Marske (or Shakespeare).

7. Conductor 1767 by Matchem.

3. Iloodpecker 1773 by Herod.

9. Highflyer 1774 by Herod.

10. Mercury 1778 by Eclipse.

11. IVaxy 1790 by PotSos.

12. Gohanna 1790 by Mereury.

13. Stamford 1794 by Sir Peter.

14. Sorcerer 1796 by Trumpator.

15. Dick Andrews 1797 by Joe Andrews.

10. Penelope 1790 by Trumpator.

17. Orville 1799 by Beningbrough.

18. Walton 1799 by Sir Peter.

19. Sir Paul 180.2 by Sir Paul.

20. Whalebone 1807 by Waxy.

21. Catton 1809 by Golumpus.

22. Master Henry 1815 by Orville.

23. Langar 1817 by Selim.

24. Guiccioli 1822 by Bob Booty.

25. Litlle Red Rover 1827 by Tramp.

26. Priam 18:8 by Emilius.

27. Birdcatcher 1833 by Sir Hercules.

28. Venison 1833 by Partisan

29. Bees Wing 1833 by Dr. Syntax.

30. Harkaway 1834 by Economist.

31. Lanercost $18: 35$ by Liserponl.

3:- Clarion 1836 by Sultan.

3:3. Nice Hawthorn $18: 38$ by Muley Molorb.

31. The Cure 1841 by Physician.

35. Ellerdale 1844 by Lanerost.

3ff. Canczou 1845 by Melbourne.

37. Flatcatcher 1845 by Touchstome.

38. Kingston 1849 by Yenisum.

39. Torment 1850 by . Tlarm.

10. Rataplan 1850 bie The Barom. 
The racing trials for horses in matches, which had been very costly for the owners, gradually became cheaper through the Sweepstakes, which were introduced at the beginning of the eighteenth century, and also by the increase of different Plates. In this way, and through the Handicaps, which were introduced in 1791, the participation in racing sport grew very considerably, less rich gentlemen now being able to participate in it. According to the statement of Idmiral Rous, there were for instance :-

\begin{tabular}{|c|c|c|c|}
\hline $176^{\circ}-$ & $\begin{array}{l}\text { Matches. } \\
\text {. . } \quad 19\end{array}$ & $\begin{array}{c}\text { Sweepstakes. } \\
: 38\end{array}$ & $\begin{array}{l}\text { Plates. } \\
.0 . j\end{array}$ \\
\hline 1807 & . . 189 & 26.3 & .969 \\
\hline 1843 & . $s 6 j$ & 897 & 191 \\
\hline
\end{tabular}

The further development of racing sport in England and Ireland can be seen from the following dates :-

1709. The first Racing Report of the Races at Clifton and Rawcliffe Ings, York (see Bailey's Racing Register). Gold Cup, 4 miles with heats, 12 stone-4 starters. Since 1731 the races take place at Knavesmire, York.

1710. Bay Bolton won the Gold ('up at York over 4 miles with heats, as a five-year-old, under 12 stone, the weight for six-year-olds.

1711. On the 11th of August, the opening of the new racecourse at Ascot in the presence of Queen Inn (1702-1714). A 6,50 Plate for six-year-olds, 12 stone, 4 miles with heats 7 starters. Ninner, Duke of St. Albans' chestnut colt, Doctor.

The racing reports gathered from newspapers and letters, covering the periods $1711,1712,1713,1720,1722,1724$ and 1726 . about the races held at Ascot, were first published in 1902 (see Royal Ascot, its History and Associations, by G. J. Cawthorne and R. S. Herod).

171\%. First race for five-year-old colts at York. The Ladies' Plate, 10 stone, 4 miles without heats. The proposition of this race, later called the Great Subscription, remained unchanged until 1758, that is to say, they remained unaltered for a period of 49 years. Since 1759 only the weight was reduced to 9 stone.

1715. First race for five-year-old mares at (Black) Hambleton. His Majesty's Gold Cup, 10 stone, 4 miles without heats - 15 starters. This race had always stronger fields than that for five-year-old colts at York. 1716,12 starters (winner, Brocklesby Betty, foundation dan of Fam. 23); 1717, 21 starters; 1718,19 starters; 1719,31 starters; 17.20 , 18 starters; 1721,20 starters; 1722, 2.2 starters; 1723, 19 starters; 1724, 26 starters. The proposition of this race remained unchanged, with the exception of the name, as long as the races were run at Hambleton, that is, up to 1775 . After 1776 , when these races took place at York, the racing propositions for three-years remained the same as for five-year-old mares: 10 stone, 4 miles-that is to say, they remained unaltered for a period of 64 years.

17I6. First racing report of the races which took place at Newmarket 
(apart from the matches from 1619 to 1719 , which have already been men. tioned above, and in the special collection by Muir).

1719. Bonny Black, foundation mare of the Fam. 39, won the Gold Cup at Hambleton as a four-year-old under 10 stone, at the weight for five-yearolds, over 4 miles, in a field of 31 starters. Bonny Black had already won a match as a three-year-old at Newnarket.

1725. First racing report of the races at Richmond.

1727. On the 12th of March, Tregonwell Frampton, Manager of the Royal Racing Stables under Willian 1II., Queen Ann, George I. and George II. died, at the age of 86 . He was called "The Father of the Turf," and was interred at Newmarket.

1727. Appeared the first Racing Calendar, under the title of : "An Historical List of all Horse Matches and of all the Plates and Prizes run for in England and in Wales (of the value of 6,10 or upwards) in 1727," by John Cheny. From this year there has appeared regularly up to the present time a Yearly Racing Calendar. In the first Racing Calendar were included the races run at 112 places, amongst which the following are well known to-day: Ascot Heath, Epsom, Hambleton, Liverpool, Nottingham, Richmond, Lewes, Lincoln, Ipswich, Stamford, Oxford, Peterborough, Winchester, York.

1728. First race for four-year-olds at Hambleton, Bishop Auckland, and Hunmanby, at Hambleton, 10 stone, 3 miles -15 starters; 1729, 13 starters; 1730, 17 starters; 1731, 15 starters; winner, Old Starling, born 17.27 by Bay Bolton, first four-year-old winner which played an important part in the breeding of the Thoroughbred, and shortly afterwards in the first years of these races for four-year-olds the following are noteworthy:-

Sedbury 1734 by Partner (won at Hambleton).

Fox 1735 by Partner (won at Malton).

Traveller 1735 by Partner (won at Hambleton).

1330. First race for four-vear-olds at Vewmarket, 8 stone, il lbs., 4 miles 4 starters.

First racing reports in the Raring (allendar of the races run at :

17.2S. Doncaster.

17:9. Chester.

1731. Canterbury.

1733. Manchester.

1741. Curragh of Kildare (Ireland).

From 1741 no races could be held with a prize of less than foro, exerpting the cases where the prizes were specially bequeathed (i.e., left as bequests).

At the same time the following weights were agreed upon for the "King's Plates ":-

For five-rear-olds 10 stone.

For six-vear-olds 11 stone.

lor seven-vear-old= 12 stone. 
1750. Jockey Club established at Newmarket.

1751. 15 King's Plates were run for in England as follows:-10 for six-year-olds, 12 stone, 4 miles with heats.

1 for five-year-olds, 10 stone, 2 miles with heats.

1 for four-year-olds, 9 stone, 2 miles with heats.

1 for four-year-olds and older, 4 miles with heats.

2 for five-year-old mares, 10 stone, 4 miles with heats.

15 Give and Take Plates.

In 1reland: 3 King's Plates, 4 miles with heats.

Mltogether there ran in England and 1reland 681 horses, of which winners were:-
21 four-year-olds.
34 five-year-olds.
26 six-year-olds.
36 older.
(i4 age unknown.

According to the Sporting Calendar by John Pond, only 490 horses ran, of which winners were:

18 four-year-olds.

33 five-year-olds.

24 six-year-olds.

45 older.

12 in Give and Take Plates.

175:. First known steeplechase match in I reland over $4 \frac{1}{2}$ miles, between Mr. O'Callagnan and Mr. Edmund Blake.

17.56. First race for three-year-nld colts, mares and geldings, on the 4 th of October at Newmarket-2 miles, 8 stone, $T \mathrm{lbs}$. During the next thirteen years this was the only race for three-year-olds, and there were always good fields.

There were run :

18 King's Plates in England.

.) Kring's Plates in Ireland.

There ran altogether, in England and 1reland, 924 horses -779 in England and 145 in Ireland - of which winners were:

1 three-year-old.

15 four-year-olds.

45 five-year-olds.

33 six-year-olds.

49 older.

73 age unknown.

1760. The establishment of Tattersall's in London by Richard Tattersall, who in 1779 bought Highflyer for $f:-500$, and thereby laid the foundation of his fortune. 
176:. Second October Meeting at Newmarket.

1763. First winner in the race for three-year-olds which played an important part for breeding purposes, viz., Virago (see Fam. 9, dam of Hollandaise 1..).

1764. On the lst of Ipril Eclipse was born in the Duke of Cumberland's stud at 11 indsor (horn 1721, died 1765), where already Crab, Marske and Herod had been born and lept as stallions.

176ii. First race for three-year-olds in Ireland; September, Curragh. 2 miles; 7 stone, $7 \mathrm{lbs}$.

1766. The first Classical (up-Race, which is still run at the present time: (Gold) Cup at Doncaster, at first for five-year-olds and older, 4 miles with heats: since $17 \%$. for four-year-olds and older, $t$ miles without heats: since 1786 for three-year-olds and older, 4 miles; since 1825, 2 miles, 5 furlongs; and since 1891 to the present time, 2 miles.

1731. July Houghton and Craven Meeting in Newmarket. For the first time the Craven (Trial) Stakes for three-year-olds and over, about 1? mileat very popular race, with very strong fields. For instance : 1775,29 starters; 1776,26 starters; 1777,29 starters; 1778,29 starters. Up to 181:2 no threerear-old was able to win the race. It the beginning there were many classical winners, viz: Pantaloon, Woodperker (three times). Pot8os, Buzzard (twice), llambletonian (twice), Selim, Rubens, and others. Sincr 1878 the race has been only for three-year-olds, over 1 mile.

1751 and 17\%2. Races in Jamaica were included in the Sporting Calendar.

1733. First volume of the Racing Calendar, by Weatherby, including also the races in Jamaica.

First race for two-year-olds and older at Newmariet in November, but in which no two-year-olds took part.

Two matches hetween two-year-olds and four-year-olds at Newmarket, of which the 1 wo-year-old colt baby won, over 4 miles.

Sir Ch. Bunbury (since 1768 Steward, the First Dictator of the Turf, burn 1740, died 1821), Lord Bolingbroke and Mr. J. Shafto were the Sicuards who had already sanctioned the races for two-yearolds in 1770, aboun which there were very different opinions.

19 King's Plates in England, of which were:

7 for six-year-olds: 12 stone; 1 miles with heats.

1 . six-year-olds; 1.2 stone: $3 \frac{1}{2}$ miles $w$ ith heats.

1 ,. live-rear-olds; 10 stone; 4 miles with heats.

1 ., five-year-olds: 10 stonc: 3 miles with heats.

1 , five-pear-olds: 9 stone: 3 miles with heats.

2 .. fiverearolds; 82 stonn'; 3 miles with heats.

1 .. four-year-olds; 9 stone; ; $2 \frac{1}{1}$ miles with heats.

1 .. four-year-olds and over: 1 miles with heats. 
1 for five-year-old mares; 10 stone; $3 \frac{1}{4}$ miles without heats.

1 ,, five-year-old mares; 9 stone ; 2 miles with heats.

1 , four-year-old mares; $8 \frac{1}{2}$ stone ; 2 miles with heats.

10 King's Plates in Ireland, of which were:

6 over 4 miles with heats.

2 without heats.

Altogether there ran 1,069 horses in England and 206 horses in Ireland.

1774. By special command of the King. from 1774 all King's Plates were to be run at York without heats.

1775. October, Newnarket, 1 mile match, one three-year-old, 8몰 stone, beat a two-year-old, Frontino, $6 \frac{1}{2}$ stone.

1776. In the Racing Calendar, the races in France (at Sablon and Fontainebleau), as well as those in Jamaica, were specified. heats.

From this time onward all races at Newmarliet were to be run without

First race for two-year-olds October, Newmarket: $\frac{1}{2}$ mile; 7 stone, 10 lbs.; 3 starters. Sweepstakes of $£ 50$ for each.

First St. Leger (convened as a sweepstakes) at Doncaster: 2 miles. colts, 8 stone; fillies, 7 stone, $12 \mathrm{lbs}$. The distances were later: Since 1806, 1 mile, 6 furlongs, 193 yards : since 1826,1 mile, 6 furlongs, 132 yards.

Veights since :

1790. 8 stone, $2 \mathrm{lbs}$, and 8 stone respectively.

1826. 8 stone, $6 \mathrm{lbs}$, and 8 stone, $3 \mathrm{lbs}$. respectively.

1839. 8 stone, 7 lbs., and 8 stone, 2 lbs.

1862. 8 stone, $10 \mathrm{lbs}$. , and 8 stone, $5 \mathrm{lbs}$.

188t. 9 stone, 0 lbs., and 8 stone, 11 lbs.

1787. The races in Jamaica were specified in the Racing Calendar.

The first winner in a race for three-year-olds which had an important bearing on breeding, viz., Highflyer.

1778. First St. Leger under this name at Doncaster -8 starters. Named after Lieut.-General Anthony St. Leger, of Park Hill, neighbour and friend of the Marquis of Rockingham, who becane Prime Minister in 1782, and owner of Alabaculia, which was the first winner of the St. Leger, 1776.

1779. First Oaks, on Friday, May 14th, at Epsom; 8 stone, 4 lbs.; $1 \frac{1}{2}$ miles-12 starters. Named after the estate belonging to Lord Derby, called the "Oaks," not far from Epson.

Distance since $187: 2: 1 \frac{1}{2}$ miles and 29 yards.

Weight since :

1787. 8 stone.

1808. 8 stone, 4 lbs.

1842. 8 stone, 7 lbs.

1862. 8 stone, 10 lbs.

189.2. 9 stone. 
1780. First Derby, on Mednesclay, the 4 th of May, at Epsom. Colts, 8 stone: fillies, 7 stone, $11 \mathrm{hhs}$ : 1 mile-9 starters.

The distances:

Since 1784. 13 miles.

Neights since:

, 187.2. 11 miles and 2!) yards.

1784. 8 stone, $3 \mathrm{lbs}$, and 8 stone respectively.

1801. S stone, $3 \mathrm{lbs}$., and 7 stone, 1:2 lbs. respectively.

1803. 8 stone, 5 lbs., and \& stone respectively.

1807. S stone, $7 \mathrm{hhs}$, and $S$ stone, 3 lbs. respectively.

1808. \& stone, 7 lbs., and \& stonc, 2) lbs. ,

1862. S stone, $10 \mathrm{lbs.,}$ and 8 stone, $5 \mathrm{lbs}$. ,

188t. 9 stone, and \& stone, 9 lbs., respectively.

The greater number of the races at Epsom were still run with heats.

October, Newmarket, three races for two-year-olds; one for fillies only, $7 \frac{1}{2}$ stone: and two for colts and fillies, distance 1 mile. Beside these, several other races for two-year-olds and over were held.

17S1. 19 King's Plates in England.

12 King's Plates in Ireland.

Only 8 of these were run without heats, and 1:3 over 4 miles with heats.

Altogether 1,069 horses ran in Ingland and 1 reland. The winners were:

9 two-year-olds.

77 three-year-olds.

100 four-year-olds.

58 five-year-olds.

38 six-year-olds.

39 older.

30 age not known.

Amongst the two-year-old winners was. Issassin, winner of the next year's Derby, and also Ceres, winner of next year's Oalis.

Afterwards (in 1785) Fidget won three races as at two-year-old, and later became sire of the nameless Derby winner of 1797. Besides this, ITren won five races as a two-year-old, and in 1795 became the dam of Bellisima, winner of the Oaks. At this time the races for two-year-olds became very popular.

1786. First classical race for two-year-olds: July Stakes at New marliet. Colts, 8 stone, 2 lbs; fillies, 8 stone; offspring of Eclipse or Highflyer, 3 lbs. extra. (This extra weight for Eclipse and Highflyer's offspring was customary in many other races, just as at an earlier period Herod's offspring had to carry 3 lbs. extra). Distance, 'T. Y. O. C.=i furlongs, 136 yards= $1,130 \mathrm{~m} .-7$ starters.

1790. The lirst races in Ireland for two and three-year-olds together. First volume of the Irish Racing Calendar. 
1791. First Handicap: The Oatlands Stakes, at Ascot; 2 miles-19 starters. Prize, 2,950 Guineas. Winner, Prince of Wales' six-year-old, Baronet, 8 stone, 4 lbs. The famous Escape, six-year-old, 9 stone, $10 \mathrm{lbs} .$, not placed. The three-year-old Vermin, 5 stone, 3 lbs., had the lightest weight, and started as favourite. The race was very popular, and there must have been about 40,000 people at Ascot. The following year this Handicap was removed to Newmarket, and in 1870 was won by the three-year-old, Adonis, 7 stone, 2 lbs., over the Cambridgeshire course. In 1804 it was won by Penelope, a six-year-old, 9 stone, $1 \mathrm{lb}$.

1791. Veterinary School founded in London by Charles Vial de Saintbel, a Frenchman, who became known in England through the dissection which he made of Eclipse in 1789, and the publicity caused by same. He was made first Professor of the Veterinary College of London.

1791. First race for two-year-olds in Ireland (at Ennis): $\frac{3}{1}$ mile. There was also a race for two-year-olds in the following year at Curragh.

First match with yearlings, October, Newmarket; 8 stone. Distance, Y. C. $=2$ furlongs, 147 yards $=524 \mathrm{~m}$. W'inner, the one-eyed grey mare of Mercury-Herod, which ran once again as a two-year-old not placed, then up to 14 years was used as a riding and carriage horse. After that gave birth to nine foals, amongst which were three foundation mares of the Fam. $5 \mathrm{~b}$. She was foundation dam of the Narigold, and when she was 27 bore her last foal.

The yearling, Cash (later Ariel), by Ready Rhino-Herod, won two matches in October at Newmarket against three-year-olds. Distance, Y. C.

The racing of yearlings was first officially forbidden in 1876 .

The two-year-old, Anthony, (1789) by Diomed and the Golden Rose, ran fourteen races and won in eleven (including one $w .0$.).

179.. Three races in Noscow, Russia, were included in the Racing Calendar.

First known steeplechase in Leicester, over 8 miles.

1793. First year's eirculation of the Sporting Magazine.

From this date the lengths of the different courses at Newmarket were stated.

First race for yearlings, sweepstakes, at Newnarket, October; 8 stone, 2 lbs. Distance, Y. C. -3 starters.

Besides this, two matches took place between yearlings.

Already in April a race liad taken place at Newmarket for two-year-olds, 7 stone, 4 lbs. Distance, $\frac{1}{2}$ mile- 1 starters.

22 King's Plates in England, and 13 in Ireland, of which 16 were over 4 miles with heats, and only 8 were over 2 to 4 miles without heats.

Altngether there ran in England and Ireland 923 horses. IVinners were:

3 one-year-olds.

28 two-year-olds. 


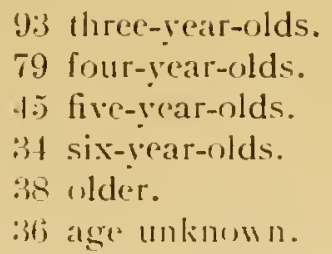

Mso, there were specified in the Racing Calendar three races which were run at Moscow (the eight-vear-old (irey Diomed, by Dinmed, won the Cup Prize, over 4 miles with heats).

First volume of the General Stud Book.

1795. One sweepstakes for yearlings at Newmarket, October; 8 stone. 2 lbs. Distance, I. C.

In consequence of the political unrest of the period, the breeding of Thoroughbreds and racing trials was neglected. It was not until after 1815 that it again commenced to recover.

On account of the Revolution, a part of the races in I reland did not take place. In the English Racing Calendar it is stated, erroneously, that no races were held, but some, as a matter of fact, did take place. In Ireland, the Racing Calendar for 1798 and 1799 appeared later ju one volume.

1799. On the 25th of March, at Newnarket, took place the famous match between Hambletonian, 8 stone, 3 lbs., and Diamond, 8 stone; distance, B. C $=1$ miles, 1 furlong, 138 yards. Both seren-ycar-olds. Hambletonian won in $7 \frac{1}{4}$ minutes.

1\$00. In Septrmber, at Duncaster, race for two-rear-old colts, 13 miles (later 1 mile); 8 stone.

1\$02. First Racing Report, about the three days' meeting at Gooduood.

Isot. The famous match for 1000 Guineas on the 25th of Iugust, at Sork. Over 4 miles, without regard to weight: Mr. lilint's Brown Phornville, seven-year-old, by lolunteer. Ridden by the ow nor.-Colonel Thornton's Vinagarella, about twenty-year-old, by Moodpecker. Rider, Mrs. Thronton. In the last mile the aged Vinagarella became lame, and therefore Brown Thornville won easily in 9 minutes, j9 seconds.

1S0.). On the 24th of . Iugust, at York, the famous match for 700

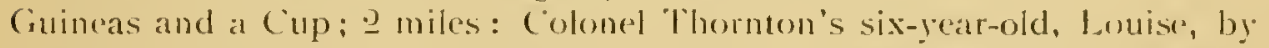
Pegasus, ! stone, fi lbs., rider, Mrs. Phornton, and Mr. Bhomfield's six-bearold, .llegro, by Pegatsus, 1:3 stone, 6 llos., rider, lirancis liuclele. Imidet the unequalled enthusiasm of a tremendous crowd of spectators, such as had never before been seen at lork, lhe famous lady rider beat lirancis Buckle, the most noted jockey of that period, after a very exciting struggle, by half a necls.

1607. Fitzwilliam Stakes, in September, at Doncaster; 13 miles, for two-year-olds and older. Since ligo Handicap.

For the lirst time the second classical race for two-rear-olds, the Haodeot 
Stakes, at Epsom, at Derby time: $\frac{3}{1}$ mile, for two-year-olds. Colts, 8 stone, 3 libs.; fillies, 8 stone.

For the first time: The Gold Cup at Ascot, for three-year-olds and older, $2 \frac{1}{2}$ miles. $1845-1853$ the prize was given by the Czar of Russia.

1809. For the first time: 2,000 Guineas Stakes at Newnarket, threeyear-olds. Colts, 8 stone, 3 lbs.; fillies, 8 stone. R. $M+=1$ mile- 8 starters. Distance the same up to the present time. Weight now: Colts, 9 stone; fillies, 8 stone, 9 lbs.

1513. For the first time: The Mrokingham Stakes (11andicap) at Ascot, $\frac{3}{4}$ mile.

1514. For the first time: 1.000 Guineas Stakes at Newmarket, threeyear-old fillies, 8 stone, 4 lbs. D. M.=7 furlongs, 178 yards. Distance since $1873 \mathrm{R}$. M. as in the 2,000 Guineas Stakes. Weight now 9 stone.

1516. For the first time: Gold Cup at Manchester, for three-year-olds and older.

18:3. For the first time: The third classical race for two-year-olds, The Prendergast Stakes, at Newmarket, October, T. Y. O.C.

18.4. For the first time: Chester Cup, for three-year-olds and older.

18.5. For the first time: Goodwood Gold Cup, for three-year-olds and older: 2 miles. Since 18337 lbs, allowed for horses whose dam or sire was Oriental (including Persian or Turkish). Since 1834, 14 or $28 \mathrm{lbs}$, allowed, according as one or both parents were Oriental. Since, the allowance was raised to 18 and 36 bs. respectively. In 1897 this allowance was done away with, as Orientals did not take part.

1S26. For the first time: The Clearwell Stakes, at Newmarket, October, for two-year-olds-11 starters.

On the 31st of March, 1826, Capt. Ross won the first steeplechase match which is recorded in the Racing Calendar, at Leicester, over 4 miles, with his famous IIunter, Clinker, by Clinker-Sancho-Fidget, and the Lily of the Valley, by Eclipse. A detailed description of this match is to be found in the Sporting Magazine, rolume 68, page 42.

18:7. 26 King's Plates in England, 16 King's Plates in Ireland-17 over 4 miles with heats, 16 without heats.

18:9. For the first time: The Criterion Stakes, for two-year-olds, at Newnarket, October.

First year's circulation of the Sportsman.

1830. First steeplechase, at St. Albans, for Cavalry Officers-16 starters.

1530-46. Lord George Bentick (born 1802 at $1 \mathrm{I}^{\top}$ elbeck, died 1848) had 100 brood mares, 60 racehorses, and 3 training establishments. He was the second Dictator of the Turf, and he cleared the racecuurse of defaulters.

1830. For the first time: The Lavant Stakes, at Goodwood, August; $\frac{1}{2}$ mile. 15th September, opening of the first railway in England, from Manchester to Liverpool, in the presence of the Duke of Ilellington. 
1s:31. The Racing Calendar recorded the famous bet of $M / r$. Osbaldiston (born 1787. He was a celebrated sportsman, and also well known on account (if his duel with 1.ord Bentick), who, on the jth of November, rode, at Neumarket, R. C., with 11 stone, 2 lbs., 200 miles $=322 \mathrm{~km}$., in 8 hours, 12 minutes, using 29 horses, which he changed every 4 miles.

1534. In the Spring Races at Newmarket the age of the horses was reckoned, as at the present time, from the 1st of January, instead of the 1st of Ilay. For all other racing places up to 1858 the following held good :-

"Horses take their ages from May Day, i.e., a horse foaled any time in the year 1830 will be deemed a year old on the 1st of May, 1831."

IN:36. On the 29th of February, first Liverpool Grand National Steplechase; 1.2 stone, 4 miles, gentlemen riders- 10 starters. Winner, Capt. Becher, after whom, later, the famous water leap was called, and over which Capt. Becher tumbted with Conrad. It was at first arranged as a Sweepstake with selling conditions, and was run 1836-1838 over a course at Maghull, and only since 1839 over the course at lintree, as at the present lime. Since 1843 as a Handicap.

1537. On the 25th of October, auction at Hampton Court of the Thoroughbreds (.) stallions, 4.3 brood mares, 13 colts, and 18 fillies, sold for 15,692 Guineas).

15.00, the stud of Thoroughbreds again established, and in 1894 disposed of for the second tine.

15i3s. For the first time: Gold Vase at Ascot.

1539. For the first time, the two famous Handicaps at Newmarket: "The Cesarewitch Stakes (the Russian Grand Duke . Mexander gave \&300), and The Cambridgeshire Stakes.

1540. For the first time: The Coronation Siakes at Ascot, three-year-old fillies, 8 stone, 7 lbs., 1 mile; and Stewards Cup (Handicap), $\frac{3}{4}$ mile.

The last reports on cock fighting in the Racing Calendar.

1842. At Epsom still five races with heats; at Goodwood three races with heats; at Doncaster two races with heats. At the smaller racing places most of the races with heats.

1843. For the first time: The Royal Hunt (up (Handicap) at Ascot, for three-year-ulds and older, 1 mile-2. 4 starters.

1S45-4\%. First volume of the Steplechase Calendar, by J. Osborne.

18.5I. For the first time: The (ity and Suburban Handicap at Epsom, $1 \frac{1}{4}$ miles -16 starters.

18.33. For the first time: The Lincolnshire Handicap, 1] miles, in March, at Lincoln. Still the first great Handicap of the year.

15.5.). Admiral Rous, "the third Dictator of the Turf," became Handicapper of the Jockey Club. The number of the two-yeatrold racing horses increase, and since 1856 outnumbers the three-year-olds.

1s.oti. In November, al Shrewsbury, races for yearlings. Colts, 7 stone. 
7 lbs.; fillies, 7 stone, 1 lbs. ; $\frac{1}{4}$ mile- 7 starters. Winner, lleroine, foundation mare in Fam. 2 b; 3rd, Saxony, foundation mare in Fam. 14: unplaced, Polly Peachum, foundation mare, Fam. 9.

1857. In November, at Shrewsbury, races for yearlings, as in $1856-4$ starters. NVinner, Tomboy, by ldleboy and Alexina. There were also several hurdle races with heats, of which the well-known half-bred mare, Heads or Tails (born 1854, by Sir Hercules, 27 years old), won a few.

15.58. In November, at Shrewsbury, races for vearlings, as 1856 -4 starters.

111 the King's Plates in England without heats, and only one over 4 miles.

15.)!. Lord Jersey died. He was the greatest opponent to races for two-vear-olds, and would not let lis own two-year-olds run. He won the Derby three tines: 1825 with Middleton, 1827 with Mameluck, 1836 with Bay Mliddleton. He won the Oaks once with Cobweb in 1824, and the 2,000 Guinea Stakes five times : 1831 with Riddlesworth, $183 \pm$ with Glencoe, 1835 with lbrahim, 1836 with Bay Middleton, 1837 with Achmet. 1le won the 1,000 Guinea Stakes twice: 1824 with Cobweb, 1830 with Charlotte II est.

18.9. In November, at Shrewsbury, races for yearlings: The Anglesey. Stakes, as in 1856-9 starters. Minner, Little Lady, foundation mare in Fam. .- c, dam of Camballo 2.

:33 King's Plates without heats in England, and 17 King's Plates in Ireland, of which one only was with heats.

Only at fifteen small racing places were races with heats still run.

1s(6). The proposal of Lord Redesdale to fix the minimum racing weight at 7 stone was rejected by the Jockey Club, and the minimum weight was fixed at 5 stone, 7 lbs., which was raised to 6 stone in 1889.

1563. The establishing of the National Hunt Committee.

1864. The Derby winner, Blair Athol, ran his second race in the Grand Prix at Paris, and was beaten with two lengths by Vermouth.

Since this year a small portion of the most important races on the Continent have been recorded.

1566-67. First volume of the Racing Calendar for Steeplechases, by Weatherby, as a continuation of that issued by $\mathrm{J}$. Osborne.

1866. For the first time: The Middle Park Plate (1,000 Sovereigns, given by Mr. W. Blenkiron) for two-year-olds, at Newnarket, October; 6 furlongs-15 starters.

1873. For the first time: Jockey Club Cup, at Newmarliet, Cesar. Course-6 starters. Winner, Flageolet.

157.2. Middle Park Stud was sold after the death of the Manager, Mr. II. Blenkiron; Blair Athol to the Cobham Stud Co., newly established. 
for I2.500 Guineas: Ciladiateur for T,000 Guineas to (apt. Ray; and Breadalbane for 6.000 Guineas to Germany.

1574. For the first time: The Brocklesby Stakes for two-yeatr-olds; 1․ mile, at Lincoln, in March-18 starters. First race in the year for twoyear-olds.

187.). For the first time: Dewhurst Plate for two-year-olds, at Newmarket: 7 furlongs-12 starters. Winner, Kisber.

1s;. First Race Neeting at Kempton Park (three meetings).

1879. For the lirst time: The Hartwicke Stakes for three-year-olds and older: $1 \frac{1}{2}$ miles, at Ascot.

Disposal of the Cobham Stud Co. which, after many a change, linally came into the possession of the International Horse. Igency and Exchange, 46a Pall Mall, London, S. 11 ., under the management of Mr. Mr. Mllison.

1ss(o. In England all the King's Plates were run over a two-mile course, but in Ireland there were still six over at three-mile course and two over a four-mile (as at present).

1856. The first $\mathscr{\&} 10,000$ race: Eclipse Stakes, for three and four-ycarolds, at Sandown Park; 1 1 miles-12 starters. Winner, Bendigo.

1557. The Royal Stud for Thoroughbreds was established at Sandringham.

15s5. Ormonde was sold for $L, 30,000$ to the Argentine, and afterwards to Mr. Macdonough, California, Menlo Stud Stock Farm, near Francisco, for $f: 31,000$, where he died.

1ss:9. For the first time: The Newnarket Stakes for three-rear-olds. Distance, $.1 . F .=1$ mile, :2 furlongs-17 starters. Winner, Donovan.

15:4. 'The second $\$, 10,000$ race: Princess of Wales', at Newmarket, for three and four-year-olds. Distance, B. M.=1 mile; July-7 starters. IVinner, lsinglass. Since 1901 for four-year-olds and older. Dislance since 1902 , Suff. St. $C=1 \frac{1}{2}$ miles.

159\%. Commencement of the American Jockey invasion.

In October Tod Sloan (born 1873) cance to England, rode in fifty-three races and won twenty. On the last racing day he rode five races, won four times, and once came in second. In the following year Sloan came to lingland in September, ran nincty-eight races and won forty-two, anong them the Middle Park Plate with Caman, beating Flying Fox. In 1899 Sloan ran 343 races and won 108, came second with Caiman in the 2,000 Guincas, and in the St. Leger. In the 1)erby the French grey colt. Holocauste, broke his fethck in struggling with Flying Fox.

In June, 1899, came the wo brothers. L. Reiff and J. Reiff (14-year-old); and in 1900 1). Maher (18-year-old), from America to England.

1899. Introduction of the . Iustralian starting machine for two-year-old races, and in the following year also for three-year-old races. 
1900. Of the ten most successful jockeys who had won more than 50 races five were Americans, of whom L. Reiff stood at the head with 553 mounts and 143 wins. The most famous English jockey up to this time, S. Loates, followed with 809 mounts and 137 wins; then J. Reiff, with 604 mounts and 124 wins. The American style of seat at race-riding was now generally accepted. In the following table are named the champion jockeys of England in the last 50 years.

\begin{tabular}{|c|c|c|c|}
\hline 18.59 & $\begin{array}{r}\text { Wins. } \\
\ldots \ldots \ldots . .109\end{array}$ & 1884 & $\begin{array}{l}\text { Wins. } \\
\ldots 241\end{array}$ \\
\hline 1860 & G. Fordham ..... & 1885 & F. Archer. \\
\hline 1861 & G. Fordham ................ 106 & 1886 & F. Archer \\
\hline 1862 & G. Fordham .............. 166 & 1887 & C. IVood. \\
\hline 1863 & G. Fordham .............. 73 & 1888 & F. Barrett \\
\hline 1864 & J. Grimshaw .............. 164 & 1889 & T. Loates \\
\hline 1865 & G. Fordham ... & 1890 & T. Loa \\
\hline 866 & Kenyon ........... & 1891 & M. Cannon \\
\hline 867 & G. Fordham .............. 145 & 1892 & M. Cannon \\
\hline 868 & G. Fordhan $\ldots . . . \ldots \ldots \ldots . . .121$ & 1893 & S. Loates .... \\
\hline 869 & G. Fordham ............... 95 & 1894 & M. Cannon ... \\
\hline 870 & II'. Gray .................. $7 \tau$ & 1895 & M. Cannon . \\
\hline 871 & G. Fordham .............. $s 8$ & 1896 & M. Cannon \\
\hline 872 & T. Cannon ................. \&8 & 1897 & M. Cannon \\
\hline 873 & H. Constable ............. 109 & 1898 & O. Madden ... \\
\hline 874 & F. Archer ................. 147 & 1899 & S. Loates ......... \\
\hline 875 & F. Archer ................. 172 & 1900 & L. Reiff ............ \\
\hline 1876 & F. Archer ............... & 1901 & O. Madden \\
\hline $187 \%$ & F. Archer .................. 218 & 1902 & W. Lane .... \\
\hline 1878 & F. Archer ................. 229 & 1903 & O. Madden ... \\
\hline 1879 & F. Archer ................. 197 & 1904 & O. Madden ............... 161 \\
\hline 980 & F. Archer ................. 120 & 1905 & E. Wheatley ...... \\
\hline 1881 & F. Archer .................. 220 & 1906 & IV. Higgs ................. 149 \\
\hline 1882 & F. Archer .................. 210 & 1907 & IV. Higgs ................. 145 \\
\hline 1883 & F. Archer .................. 232 & 1908 & D. Naher .................. 139 \\
\hline
\end{tabular}

1900. Twelve yearlings from Eaton were sold by auction for $£ 45,465$, amongst which were: Sceptre, for $\mathcal{E}_{10,000}$ to Mr. Sievier; Flying Fox, a four-year-old, for $\mathcal{L} 39,375=$ roundly, one million francs, to Mons. E. Blanc. 
Number of llorses which ran in England and Ireland.

\begin{tabular}{|c|c|c|c|c|c|c|c|c|c|c|c|c|c|}
\hline $\begin{array}{l}\text { In the } \\
\text { lear }\end{array}$ & 1 & $\begin{array}{l}2 \\
\text { Yea }\end{array}$ & 3 & der & 5 & $\begin{array}{c}\text { There } \\
\text { ran }\end{array}$ & $\begin{array}{l}\text { In the } \\
\text { Year }\end{array}$ & 1 & 2 & $s$ and & der & 5 & $\begin{array}{l}\text { There } \\
\text { ran }\end{array}$ \\
\hline & & "umbe & of $\mathrm{W}$ & iners & & & $\begin{array}{l}157 \% \\
1874\end{array}$ & 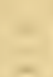 & $\begin{array}{l}691 \\
710\end{array}$ & $\begin{array}{l}(x) 22 \\
5-2\end{array}$ & $\begin{array}{l}324 i \\
3200\end{array}$ & $12 \overline{1}$ & $247 !$ \\
\hline 17.11 & - & - & - & - & - & 4117 & 1875 & 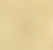 & 781 & (כ) & 34 & : & 2016] \\
\hline 17.51 & $\ldots$ & - & - & 21 & $I(i)$ & [isi & 1576 & 7 & 769 & ב2. & $: 310: 3$ & 300 & 201.21 \\
\hline 17.56 & - & - & 1 & 1.5 & - & 124 & 187 & & S) & (b) 1 & 318 & $: 330$ & 20157 \\
\hline 1757 & - & - & 1 & 51 & 1603 & (89;) & 1578 & - & 578 & 612 & $: 321$ & 291 & $20 \div 5$ \\
\hline l7is & - & - & 1 & 58 & 215 & $!: 31$ & 1879 & - & $s+1$ & 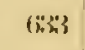 & 314 & :30.2 & $211: 3$ \\
\hline 1773 & $\ldots$ & 1 & $\cdots$ & - & - & $12 \pi$ & 1850 & - & $8: 20$ & 610 & 313 & $2 \times 3$ & $2(1) \div 6$ \\
\hline 1781 & - & 9 & $\pi$ & $I(k)$ & $16 i j$ & 10669 & 1881 & - & $s(x)$ & .587 & 30.4 & 279 & 1970 \\
\hline $17 ! 3$ & $: 3$ & 28 & 93 & 79 & 153 & 923 & 1892 & - & 840 & 553 & 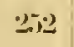 & 271 & 1916 \\
\hline 179.5 & 1 & 31 & 9) & 64 & $13: 2$ & 834 & 1883 & - & 859 & 1607 & $2(5) 2$ & 312 & 2070 \\
\hline & & ber of & & & & & 188.1 & - & 840 & 572 & 291 & 276 & $198^{2}$ \\
\hline & & iber of & Horse & that $r$ & & & 1885 & - & $85: 3$ & 578 & $27 !$ & 293 & 20134 \\
\hline 1797 & - & 67 & 191 & 142 & $31 \%$ & 712 & $1 S 86$ & - & s.80 & .93 & 思13 & 310 & 2076 \\
\hline 181$)^{2}$ & - & 39 & 142 & 123 & 340 & $(i, 50$ & 1857 & - & 908 & $5: 3$ & $2(x)$ & 286 & 21117 \\
\hline IS: 2 & - & 1 is & 390 & 0הבי & 570 & 1363 & $18 \mathrm{ss}$ & - & 919 & 566 & 287 & $3: 21$ & $21: 23$ \\
\hline 1819 & - & 204 & 119 & 24 & 378 & 1315 & $1854 !)$ & - & 1866 & 5,53 & $27 i$ & 316 & 2131 \\
\hline 18.56 & 7 & $5 \geq \tau$ & 457 & $2 \pi j$ & 376 & 1012 & 18901 & - & (13ij & 607 & 27.3 & ואיצ? & 2105 \\
\hline 18.57 & 4 & 518 & 500 & 251 & $: 3(i 1$ & $16^{\circ} 24$ & 1.801 & - & $10 i^{2} 2$ & 613 & (2)6 & 314 & 2315 \\
\hline $1 \sin$ & 4 & 601 & 436 & 280 & $31 \mathrm{~s}$ & $16: 341$ & $18(1)$ & - & 10.11 & 772 & 2336 & 365 & $2 x+4$ \\
\hline $18 ; 9$ & 9 & 576 & 496 & 240 & 324 & 1645 & $189: 3$ & - & 1118 & 727 & $: 399$ & $: 381$ & 2415 \\
\hline 1860 & - & 608 & $5: 1$ & $:(1) 2$ & 250 & 1717 & 1891 & - & 125 & 779 & 417 & 4.5 & 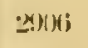 \\
\hline 1,601 & - & (itijl & 5.51 & 214 & 342 & 1767 & 1845 & - & $1: 37$ & 901 & 417 & tii & :3):! \\
\hline $1+602$ & - & $6 \div 6$ & 502 & 291 & 381 & 1830 & 1896 & - & 1276 & sis & 4.5) & 511 & 3111 \\
\hline $181 i 3$ & - & 643 & $511 \%$ & 291 & 393 & $1 \times 37$ & 1897 & - & $1: 369$ & 965 & $1 j 16$ & endi & :inisti \\
\hline 1861 & - & Gitit & 5.s & 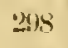 & $4: 35$ & 1918 & $1895^{\circ}$ & - & 1334 & $10: 3$ & 517 & $6 i 31$ & $: 2 \pi 71$ \\
\hline $1 \times(0 ; 5)$ & - & 659 & 572 & 364 & 47 & 2012 & 1899 & - & 1134 & $105 !$ & 5.85 & bi18 & 37.23 \\
\hline 1866 & - & 799 & 572 & 8.59 & $H ! 1$ & 21199 & 1900 & - & Sבית & 1110 & $53 ! 1$ & 658 & $: 3: 121$ \\
\hline 1.867 & - & 752 & 601 & 408 & 6337 & 2155 & $19) 1$ & - & 1115 & $117 !$ & 614 & $71 !$ & $: 39.5$ \\
\hline 1868 & - & sit & 631 & 118 & 617 & 2510 & 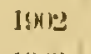 & - & $1: 3 \pi 1$ & 1070 & 617 & $7(62)$ & 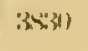 \\
\hline 18159 & - & 시른 & 673 & 402 & 617 & $2-234$ & 19M:3 & & \multirow{2}{*}{\multicolumn{5}{|c|}{ not given }} \\
\hline 1570 & - & $81) 7$ & 709 & 112 & 611 & 2,569 & $1 ! n 1$ & & & & & & \\
\hline $18 i 1$ & - & 7332 & 740 & 150 & $: \times 101$ & $24 \times 3$ & $194)$ & - & $1: 297$ & I(x)] & 576 & 677 & :35.51 \\
\hline 1872 & - & 699 & $6: 7$ & 240 & :3401 & 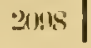 & I!หरi & - & I:ZIS & 981 & ; & 691 & :3ill \\
\hline
\end{tabular}

Since Iutumn, Is67, the Hurdle Races, and since July, 187, the Nalional Hunt fial Races, are mol given in above figures. 


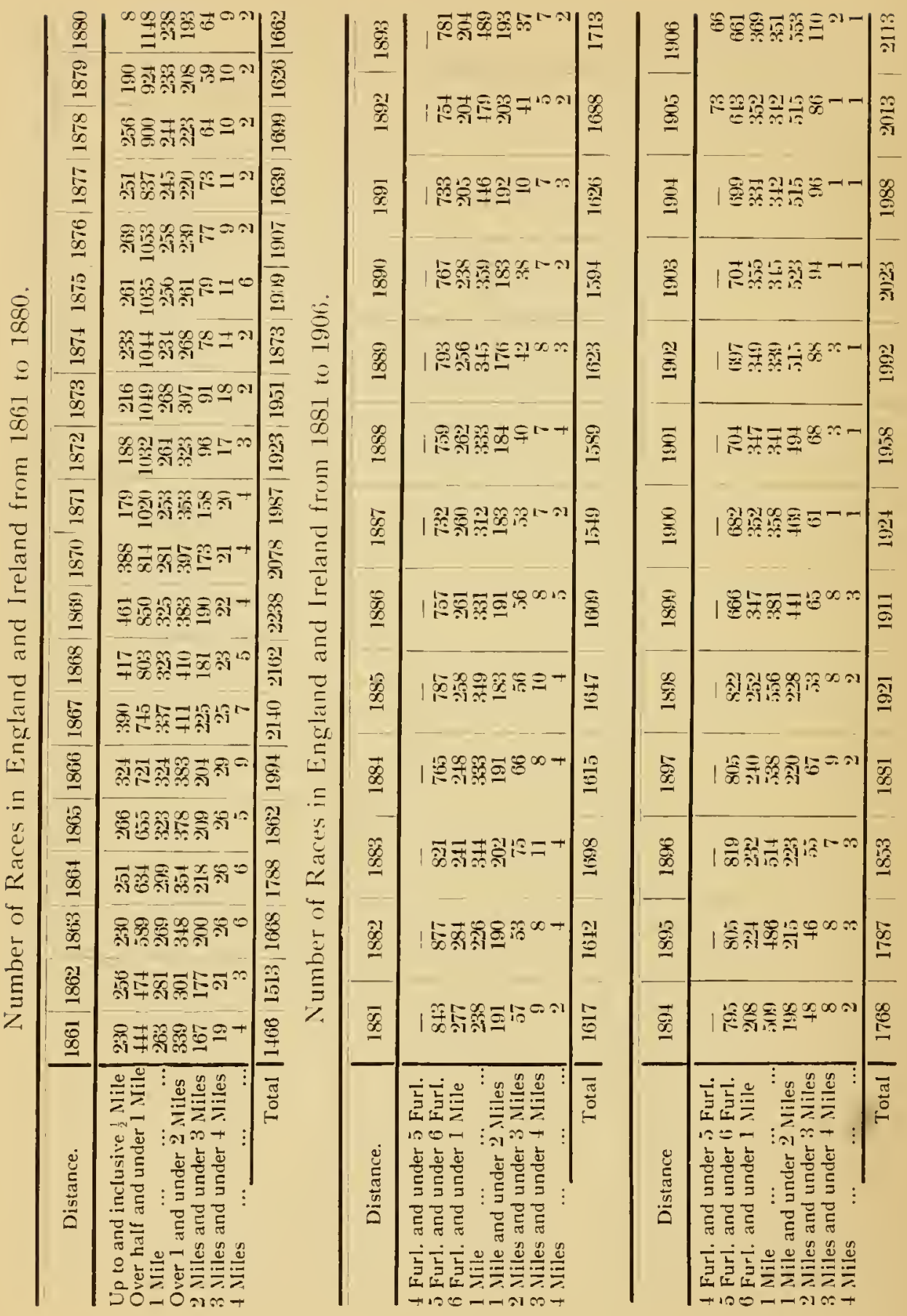


2. Development of the Thoroughbred, etc.

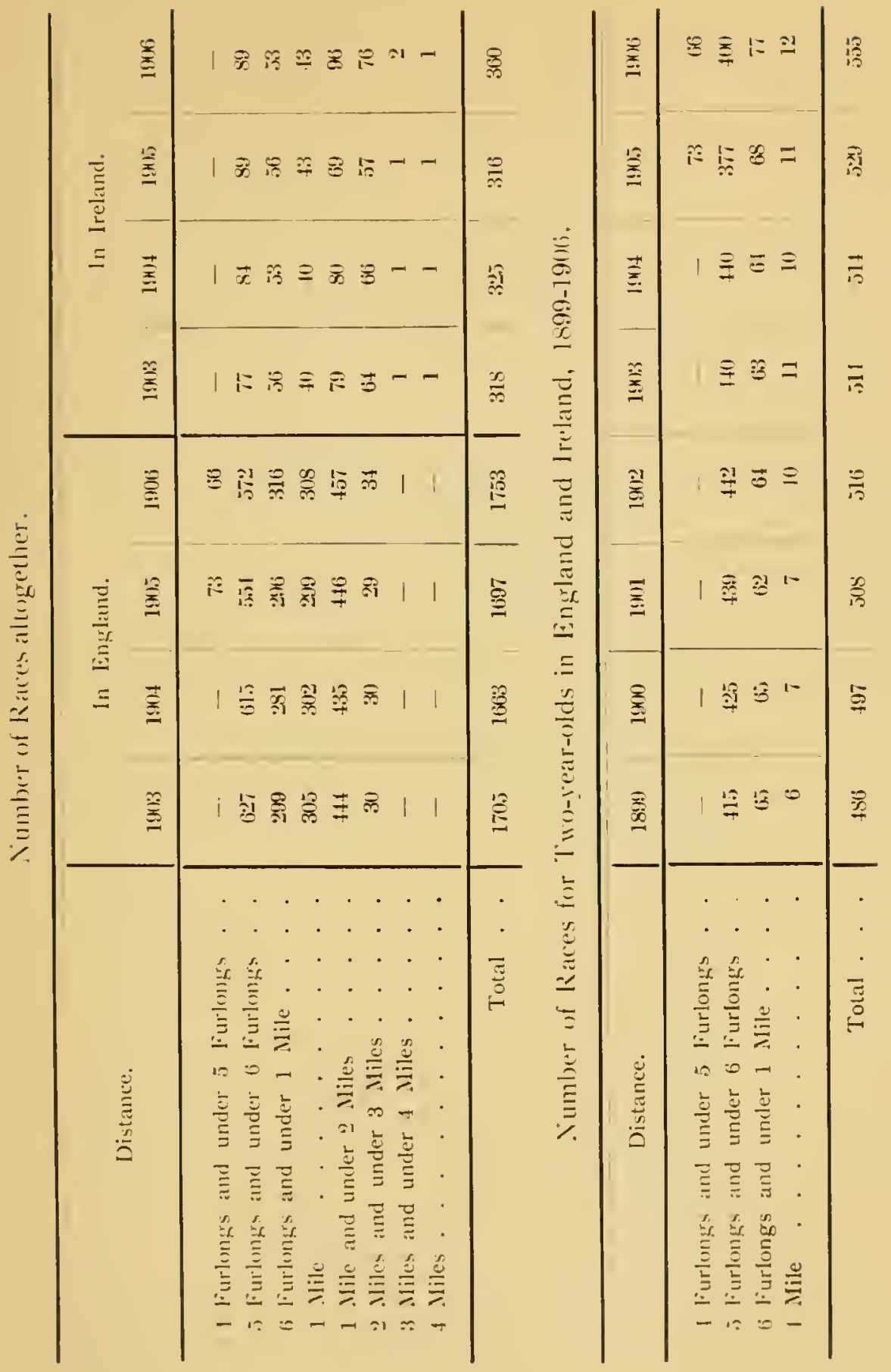




\section{CH.APTER III.}

Origin and Change in the Weight Tables, and the Conclusions to be drawn therefrom.

IN the beginning and middle of the eighteenth century, i.e., up to about 1760 , the difference in weight of horses of various ages was calculated generally at the rate of 1 stone $=14$ English lbs. per year. For instance :

$\begin{array}{lll}4 \text { year-olds } & 9 & \text { stone. } \\ 5 \text { year-olds } & 10 \quad \text { ", } \\ 6 \text { year-olds } & 11 & , \\ \text { Older } & 12 & ,\end{array}$

1759 to 1765 . October. Newmarket, so-called weight scale according to age for distance, B. C. $=$ about 4 miles.

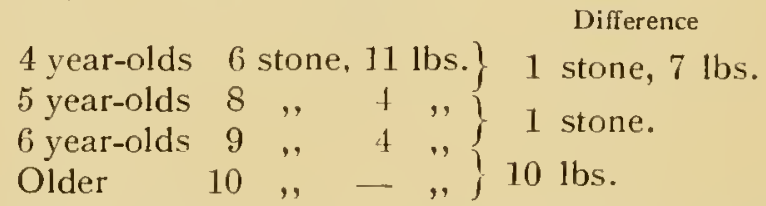

1766. So-called weight scale according to age at Newmarket. October. 1)istance, B. C. $=4$ miles.

\begin{tabular}{|c|c|c|c|c|}
\hline & & & & Difference \\
\hline $\begin{array}{l}4 \text { year-olds } \\
5 \text { year-olds }\end{array}$ & $\begin{array}{l}7 \\
8\end{array}$ & & 7 lbs. $\}$ & 1 stone, 2 lbs. \\
\hline olds & 9 & & & 12 lbs. \\
\hline Older & 10 & , & $-\quad, j$ & 7 lbs. \\
\hline
\end{tabular}

At the establishing of the Gold Cup in 1768 at Newmarket, to be run in October, over 4 miles, the weights were:

\begin{tabular}{|c|c|c|c|}
\hline 4 year-olds & & tone, - lbs. & Difference \\
\hline 5 year-olds & 8 & 5 & 1 stone, 5 lbs. \\
\hline 6 year-old & 9 & ", & 12 lbs. \\
\hline Older & 9 &,$\quad 10$ & 7 lbs. \\
\hline
\end{tabular}

At that time there was no universally accepted scale of weights, and the following examples show the average differences in weights in 1775 : 
3. Origin and Change in the Weight Tables, etc.

In July, at Newmarket, over 2 miles :

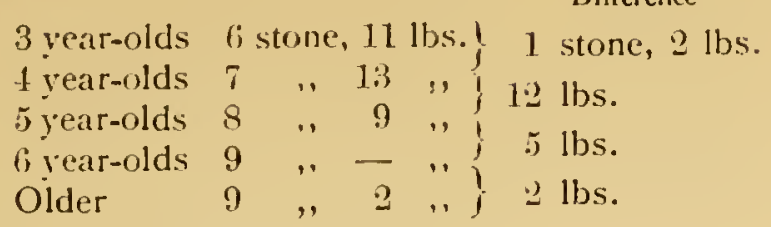

In August, at Newmarket, over 4 miles :

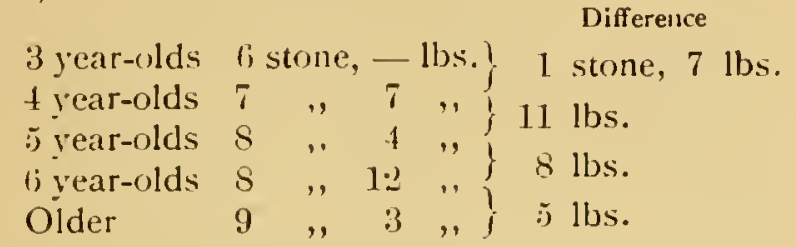

In October, at Newmarket, wer 4 miles :

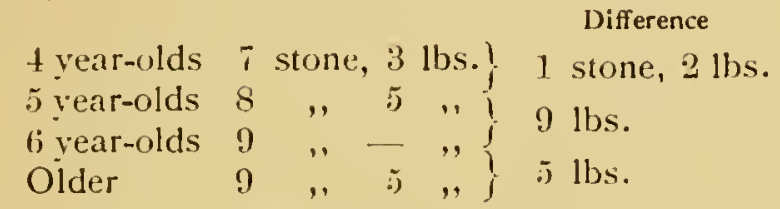

Hereafter the differences in weight were, on the average, about as follows :

1775 .

3 year-olds 4 year-olds 5 year-olds 6 year-olds Older

1 stone, 2 lbs. to 1 stone, 7 lbs.

10 lbs. to 1 stone, j) lbs.

.j lbs. to 1 stone, 2 lbs.

.2 lbs. to 8 lbs.

1800.

2 year-olds \} 1 stone, ].2 lbs. to 2 stone, $2 \mathrm{lbs}$.

3 year-olds

$t$ year-olds

j) year-olds

6 year-olds

Oider

1 stone to 2 stone.

7 lbs. to 1 stone, 2 lbs.

1 lbs. to $10 \mathrm{lbs}$.

? lbs. to $6 \mathrm{lbs}$.

1830.

2 year-olds $\} 1$ stone, $12 \mathrm{lbs}$. to 22 stone, $3 \mathrm{lbs}$.

3 year-olds

+4 year-olds

5 year-olds

6 year-olds

older

1 stone to 2 stone.

7 lbs. to 1 stone, $1 \mathrm{lb}$.

$2 \mathrm{lbs}$. to $9 \mathrm{lbs}$.

0 to 3 lbs. 
1850.

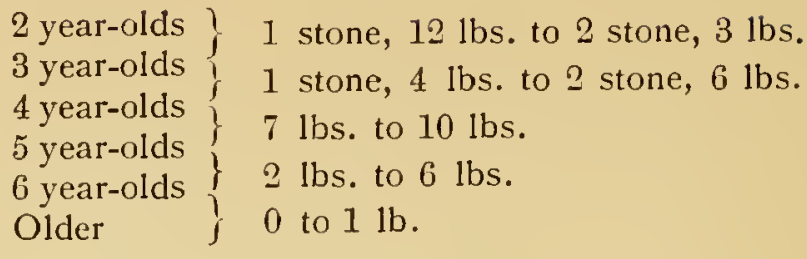

1900.

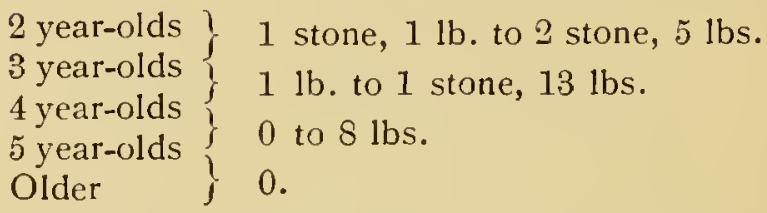

On page 35 is given the weight scale worked out by Admiral Rous in 1873. This scale has been frequently altered, and holds good at the present time. From 1832 the weights for the King's Plates were fixed for the different racing places in the Racing Calendar. At Newmarket alone were the weights for the King's Plates fixed by the Stewards. In the Racing Calendar for 1861 appeared for the first time the universally accepted weight scale for the King's Plates, which we give below. It was not until 1881 that the first copy of the weight scale worked out by Admiral Rous appeared in the Racing Calendar. A special table is given herewith for comparison of the weights from 1881 and of those from 1906. 
3. Origin and Change in the Weight Tables, etc.

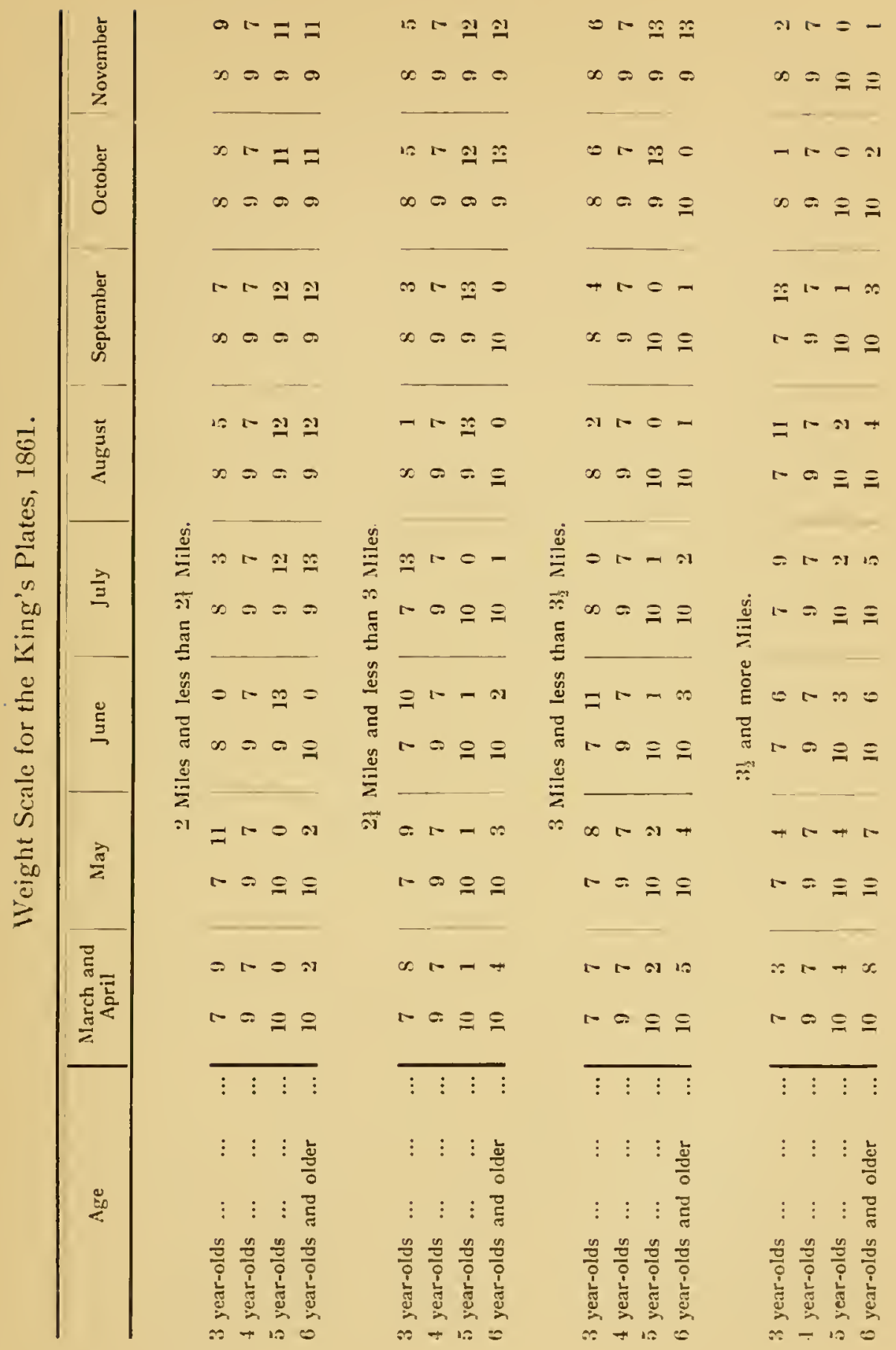


Trial of the Thoroughbred on the Racecourse, etc.

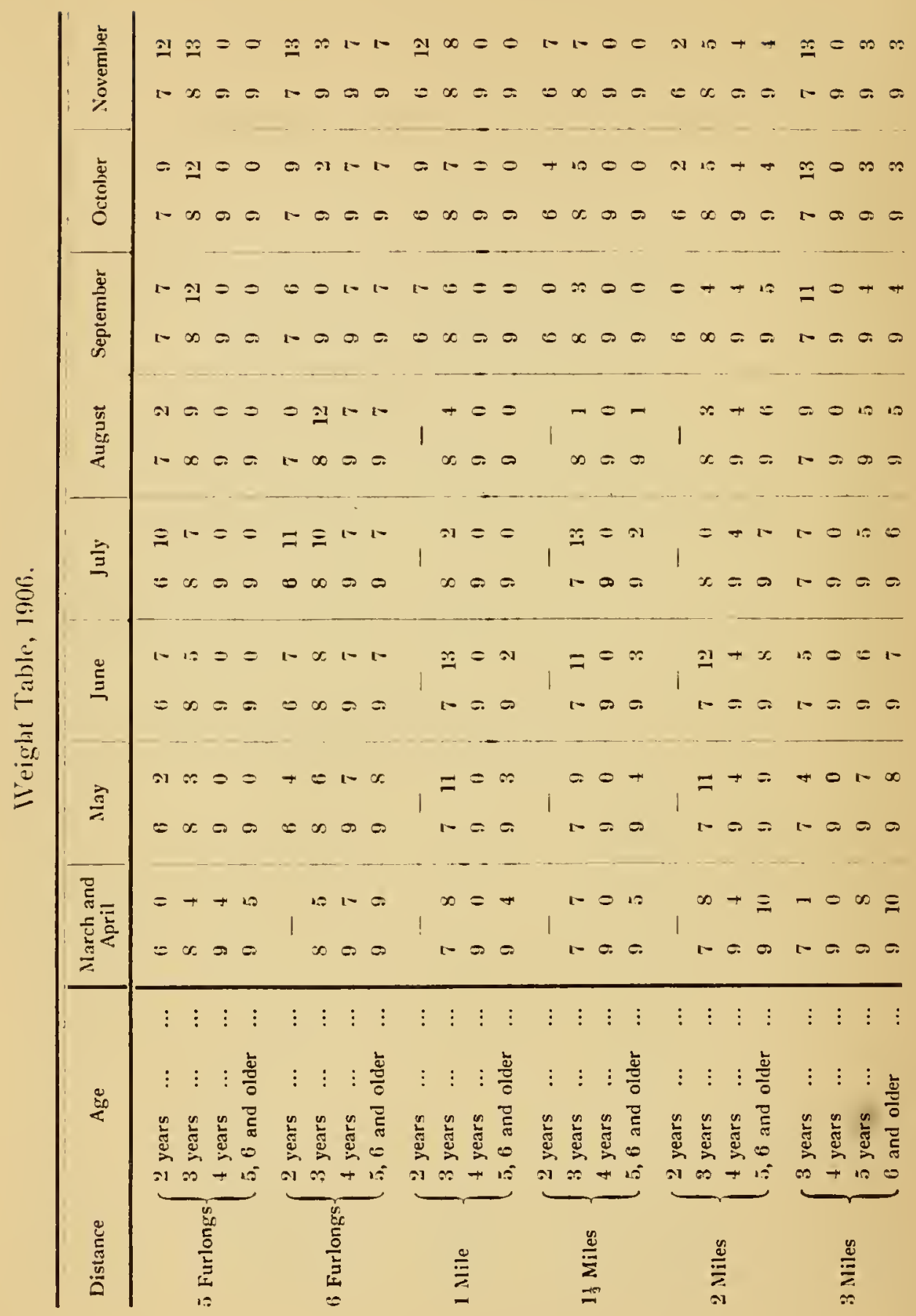


3. Origin and Change in the Weight Tables, etc.

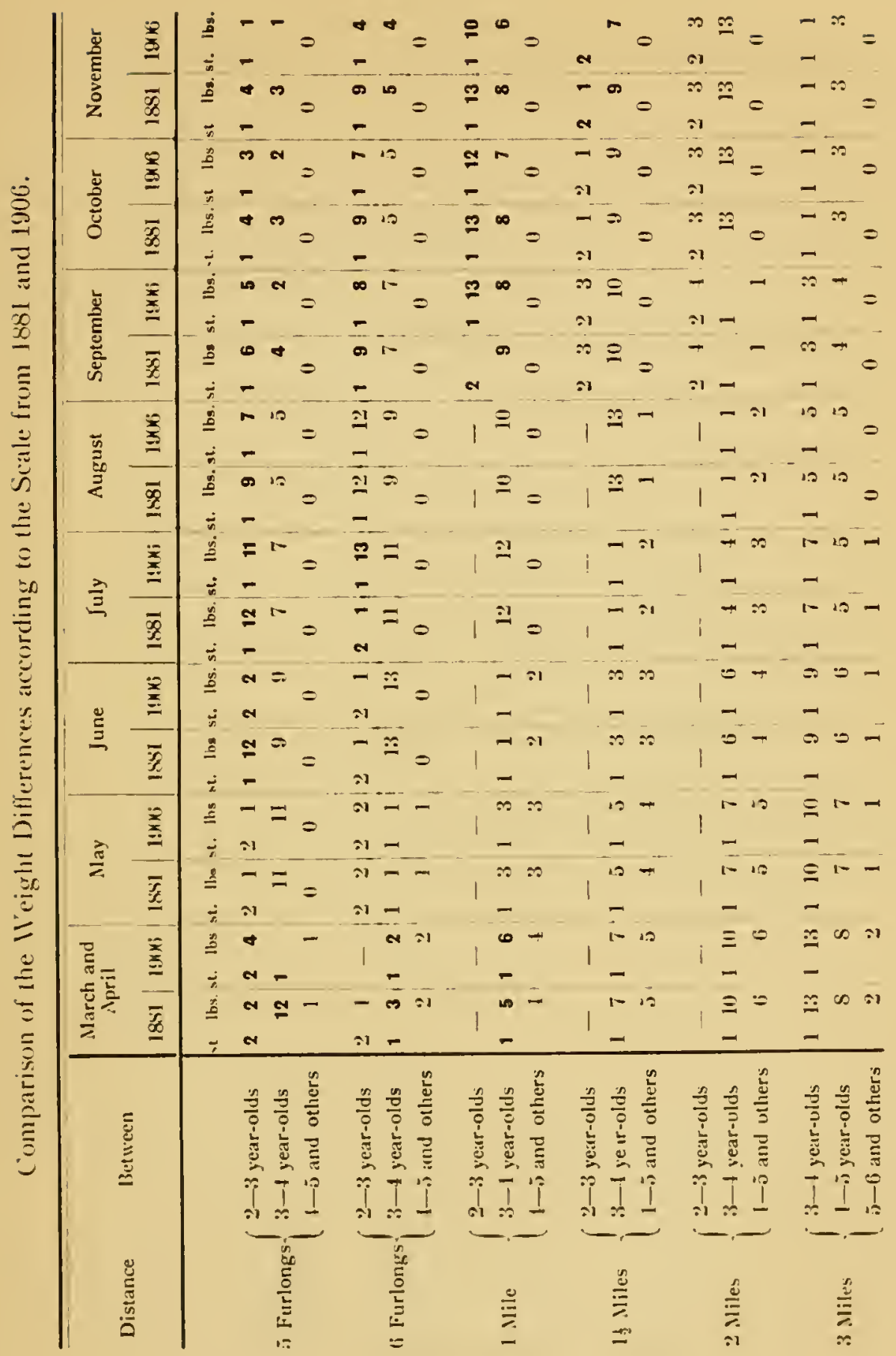


In order to arrive at a fair comparison of the weights carried formerly with those carried at the present time, hereafter will be given the weights for definite yearly periods, distances, seasons, and, of course, only for races without heats.

The first race for two-year-olds and older horses in November, 1773, at Newmarket, distance D. J.=about 2 miles, stipulated as weight for two-yearolds, 4 stone, $8 \mathrm{lbs}$., and for three-year-olds, 6 stone, $7 \mathrm{lbs}$, that is, a difference of 1 stone, $13 \mathrm{lbs}$. According to to-day's scale the difference would be $4 \mathrm{lbs}$. more, i.e., 2 stone, $3 \mathrm{lbs}$. It is very interesting to note how exactly, even at that time, the capabilities of the two-year-olds were valued against those of the three-rear-olds. For further comparison of the weights for twoyear-olds, as well as three-year-olds, the following examples will serve:-

17i7. October. Newmarket. Distance $\frac{3}{4}$ mile $A b . M .=6$ furlongs.

3 year-old, 8 stone (won). I Difference,

2 year-old, 6 stone, 8 lbs. $\int 1$ stone, $6 \mathrm{lbs}$.

According to the present day scale: 1 stone, $7 \mathrm{lbs}$.

1777. October. Newmarket. Distance Ab. M.=1 mile.

3 year-olds, 8 stone.

2 year-olds, 6 stone, 8 lbs. (paid forfeit). 1 stone, 6 lbs.

According to the present day scale: 1 stone, $12 \mathrm{lbs}$.

1781. October. Newmarket. Distance $\frac{1}{2}$ R. M. $=4$ furlongs.

a year-old, Assassin, 7 stone (won). $\quad$ Difference,

3 year-old, Puzzle, 8 stone, 3 lbs. $\left\{1\right.$ stone, $6 \mathrm{lbs}{ }^{1}$

According to present day scale : about 1 stone, $1 \mathrm{lb}$.

Assassin, winner of the next year's Derby, and the three-year-old, Puzzle, were racing horses of almost equal value, and Puzzle won directly afterwards the-at that time-classical Perram Stakes at Newmarket.

1800. October. Newmarket. Distance D. J.=about 2 miles.

Difference in weight between 2 and 3 year-olds, 1 stone, $12 \mathrm{lbs}$. , $\quad$, according to to-day's scale, 2 stone, $3 \mathrm{lbs}$.

$180 \%$. July. Newmarket. Distance 2 Y. O. C. $=5$ furlongs, 136 yards. Difference in weight between 2 and 3 year-olds, 2 stone, $2 \mathrm{lbs}$. , , according to to-day's scale, 1 stone, $13 \mathrm{lbs}$.

1800. October. Newmarket. Distance, 2 Y.O.C. $=5$ furlongs, 136 yds. Difference in weight between 2 and 3 year-olds, 1 stone, $7 \mathrm{lbs}$. , , $\quad$, according to to-day's scale, 1 stone, $7 \mathrm{lbs}$.

1800. October. Newmarket. Distance D. J.=about 2 miles.

Difference in weight between 2 and 3 year-olds, 1 stone, $12 \mathrm{lbs}$. " , according to to-day's scale, 2 stone, $3 \mathrm{lbs}$.

${ }^{1}$ Taking into consideration the usual 3-1bs. allowed for fillies. 
1830. July. Liverpool. Distance $2 \mathrm{Y} . \mathrm{O} . \mathrm{C} .=$ about 5 furlongs.

Difference in weight between 2 and 3 year-olds, 2 stone, $2 \mathrm{lbs}$. , , according to to-day's scale, 1 stone, $11 \mathrm{lbs}$.

1830. September. Doncaster. Distance $1 \frac{7}{2}$ miles.

Difference in weight between 2 and 3 rear-olds, 2 stone, 4 lbs. ," , according to to-day's scale, 2 stone, $3 \mathrm{lbs}$.

1850. July. Goodwood. Distance $2 \mathrm{Y}$. O. C. $=6$ furlongs.

Difference in weight between 2 and 3 year-olds, 2 stone, 2 lbs. , , $\quad$ according to to-day's scale, I stone, $13 \mathrm{lbs}$.

1850. July. Liverpool. Distance $2 \mathrm{Y}$. O. C.=about 5 furlongs.

Difference in weight between 2 and 3 year-olds, 2 stone.

," $\quad$, according to to-day's scale, 1 stone, $11 \mathrm{lbs}$.

1850. September. Doncaster. Dist. Red House $=5$ furlongs, 152 yds.

Difference in weight between 2 and 3 year-olds, 2 stone.

$$
\text { , } \quad \text {, according to to-day's scale, } 1 \text { stone, } 8 \text { lbs. }
$$

1850. October. Newmarlet. Distance 1 mile.

Difference in weight between 2 and 3 year-olds, 2 stone, 4 lbs. , $\quad$ ", according to to-day's scale, 1 stone, $12 \mathrm{lbs}$.

1850. October. Newnarket. Dist. 2 Y.O.C.=5 furlongs, 136 yards.

Difference in weight between 2 and 3 year-olds, 1 stone, 11 lbs. , , $\quad$ according to to-day's scale, 1 stone, $7 \mathrm{lbs}$.

18\%0. October. Newmarket. Distance $\frac{1}{2} \mathrm{Ab} . \mathrm{MI}=4$ furlongs.

Difference in weight between 2 and 3 year-olds, 1 stone, $10 \mathrm{lbs}$.

," , according to to-day's scale, I stone, $1 \mathrm{lb}$.

1850. October. Curragh. Distance Red Post $=1$ mile, 6 fur., 3 yds.

Difference in weight between 2 and 3 year-olds, 2 stone, $11 \mathrm{lbs}$. ," $\quad$, according to to-day's scale, 2 stone, $2 \mathrm{lbs}$.

For comparing three and four-year-olds the following examples will serve:-

1775. April. Newmarket. Craven Stakes. Distance 1 mile, 2 furlongs. $4 t$ yards.

Difference in weight between 3 and 4 year-olds, 1 stone, $7 \mathrm{lbs}$. ," according to to-day's scale, 1 stone, $7 \frac{7}{2}$ lbs.

1775. July. Newmarket. Distance 2 miles.

Difference in weight between 3 and 4 year-olds, 1 stone, 2 lbs. ,, $\quad$, according to to-day's scale, 1 stone, 4 lbs.

1775. August. Newmarket. Distance 4 miles.

Difference in weight between 3 and 4 year-olds, 1 stone, $7 \mathrm{lbs}$.

1800. ", according to to-day's scale, 1 stone, $9 \mathrm{lbs}$.

1800. April. Newnarket. Craren Stakes. Distance 1 mile, 2 furlongs, 41 yards.

Difference in weight between 3 and 4 year-olds, 2 stone. , , according to to-day's scale, 1 stone, $7 \frac{1}{2}$ lbs. 
1800. Nay. Newmarket. Distance 2 miles.

Difference in weight between 3 and 4 yearolds, 1 stone, $121 \mathrm{bs}$. according to to-day's scale, 1 stone, 7 lbs.

1800. July. Newmarket. Distance D. J.=about 2 miles.

Difference in weight between 3 and 4 year-olds, 1 stene, 5 lbs. ", " according to to-day's scale, 1 stone, 4 lbs.

1800. July. Stockbridge. Distance $=2$ miles.

Difference in W Weight between 3 and 4 year-olds, 1 stene, $3 \mathrm{Ibs}$. ," . according to to-day's scale, 1 stone, $4 \mathrm{lbs}$.

1800. July. Stamford. Gold Cup. Distance $=t$ miles.

Difference in weight between 3 and 4 year-olds, 1 stone, $7 \mathrm{lbs}$. ", ", according to to-day"s scale, 1 stone, $10 \mathrm{lbs}$.

1800. August. Ireland. Distance $=4$ miles.

Difference in weight between 3 and 4 year-olds, 1 stone, $3 \mathrm{lbs}$. , $\quad$, according to to-day's scale, 1 stone, 9 lbs.

1800. October. Newmarket. Distance $=2$ miles.

Difference in weight between 3 and 4 year-olds, 1 stone. ,, ., according to to-day's scale, $13 \mathrm{lbs}$.

1807. June. Ascot. Gold Cup. Distance $=2 \frac{1}{2}$ miles.

Difference in weight between 3 and 4 vear-olds, 1 stone, 4 Ibs. , , $\quad$, according to to-day's scale, 1 stone, $7 \frac{1}{2} \mathrm{lbs}$.

1830. April. Newmarket. Craven Stakes. Distance 1 mile, 2 furlongs, 44 yards.

Difference in weight between 3 and 4 year-olds, 2 stone, 4 lbs. , , , according to to-day's scale, 1 stone, $7 \frac{1}{2}$ lbs.

1830. May. Liverpool. Distance $=2$ miles.

Difference in weight between 3 and 4 year-olds, 1 stone, $6 \mathrm{Jbs}$. ," according to to-day's scale, 1 stone, $7 \mathrm{lbs}$.

1830. May. Epsom. Craven Stakes. Distance $=1 \frac{1}{4}$ miles.

Difference in weight between 3 and 4 year-olds, :2 stone.

, $\quad$, according to to-day's scale, 1 stone, $4 \mathrm{lbs}$.

1830. June. Ascot. Gold Cup. Distance $2 \frac{1}{2}$ miles.

Difference in weight between 3 and 4 year-olds, 1 stone, $6 \mathrm{lbs}$. according to to-day's scale, 1 stone, $7 \frac{1}{2}$ lbs.

1830. June. Manchester. Gold Cup. Distance 2 miles.

Difference in weight between 3 and 4 year-olds, 1 stone, 13 lbs. ," , according to to-day's scale, 1 stone, $6 \mathrm{lbs}$.

1830. July. Liverpool. Croxteth Stakes. Distance $1 \frac{1}{4}$ miles.

Difference in weight between 3 and 4 year-olds, 1 stone, 8 lbs. - , $\quad$, according to to-day's scale, 1 stone, $1 \mathrm{lb}$. 
3. Origin and Change in the Weight Tables, etc.

18:30. Iugust. Jorl. Distance 2 miles.

Difference in weight between 3 and 4 year-olds, 1 stone, 2 lbs. , $\quad$, according to to-day's seale, 1 stone, 1 lb.

18:30. September. Doncaster. Cup. Distance 2 miles, 5 furlongs.

Difierence in weight between 3 and 4 year-ulds, 1 stone, 3 lbs. , $\quad$. according to to-day"s scale, 1 stonc, $1 \frac{1}{2}$ lbs.

18:30. Octoher. Epsom. Metropolitan Stalies. Distance $1 \frac{1}{2}$ miles.

Difference in weight between 3 and 4 year-olds, 1 stone, 4 lbs.

$$
\text { ,. } \quad \text { aceording to to-day's scale, } 9 \text { lbs. }
$$

1850. April. Newmarket. Craven Stakes. Distance 1 mile, 2 furlongs, 44 yards.

Dificrence in weight between 3 and 4 year-ulds, 1 stone, $12 \mathrm{lbs}$. ", " according to to-day's scale, 1 stone, $7 \frac{1}{2}$ lbs.

1850. May. Epsom. Craven Stakes. Distance $1 \frac{1}{\frac{1}{4}}$ miles.

Difference in weight between 3 and 4 year-olds, "2 stone, 2 lbs. , ", according to to-day's scale, 1 stone, 4 lbs.

1850. June. Ascot. Gold Cup. Distance $2 \frac{1}{2}$ miles.

Difference in weight between 3 and 4 year-olds, 1 stone, 9 lbs.

," , according to to-day': scale, 1 stone, $7 \frac{1}{2}$ lbs.

1850. July. Liverpool. Croxteth Stakes. Distance $1 \frac{x}{4}$ miles.

Difference in weight between 3 and 4 year-olds, 1 stune, $8 \mathrm{lbs}$. ,. $\quad$, accurding to to-day's siale, 1 stone, $1 \mathrm{lb}$.

1850. September. Duncaster. (up. Distance 2 miles, j furlongs.

Difference in weight between 3 and 4 year-olds, 1 stone, jolbs. ," $\quad$, according to to-day's scale, 1 stone, $1 \frac{1}{2}$ lbs.

1850. October. Newmarket. Distance, T. Y. O. C.=.j furl., 136 y.ds.

Difference in weight between 3 and 4 year-olds, $7 \mathrm{lbs}$.

$$
\text { , } \quad \text { aceording to to-day's scale, } 4 \text { lbs. }
$$

"Those cxamples, which may serve as a comparison, are, especially in the rears before 1850 , often difficult to gather. as most races then were run with heats, and are therefore unsuitable as comparisons with the present time, and because only few races were set apart for horses of each age. The many Handicaps for horses of each age are very difficult to manipulate as comparisons. From abowe examples one easily can see, however, that the weight difference between two and threc-year-olds as well as between three and four-year-olds was, up to the year 1800, nearly acoording to the present sale of weights, and that they often were somewhat smaller fur longer distances. Förm ison to about 1850 the se weight differences increased somewhat, viz.: The differences between two and three-year-eslds less (about 2-7 lbs.), those between three and four-year-olds somewhat more (about $3-11$ lbs.). In other words, the proportion of weight carrying capability has been approximately the same up to about 1800 as it is to-day for two, threc and 
four-year-olds, whilst after about 1800 up to about 1850 the three-year-olds and the four-year-olds especially were expected to carry more, according to the table just quoted. It appears that the races for two-year-olds, which took place at that time in a reduced degree, and not too early in the year (which races became popular after Assassin's Derby victory, 1782), improved the capabilities (Leistungsfähigkeit) of the future three and four-year-olds, whilst after about 1850 the increase of races for two-year-olds, especially in the early part of the year, seems to have antulled gradually this favourable effect.

The changes of weight differences between four-year-olds and older horses are shown by the following examples, in their historic development:-

1775. April. Newnarket. Craven Stakes. Distance 1 mile, 2 furlongs, 44 yards.

Difference in weight:

According to present scale:

4 year-olds

5 year-olds

6 vear-olds

7 year-olds

Older

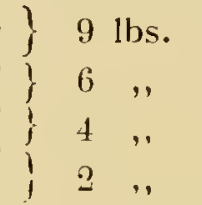

. $4 \frac{1}{2}$ lbs.

. . 0 ,

. 0 , 0

. 0 ,

1792. July. Newmarket. Distance 2 miles.

Difference in weight:

According to present scale:

4 year-olds

5 year-olds

6 year-olds

Older

$\left\{\begin{array}{cc}10 & \text { lbs. } \\ 5 & , \\ 2 & ,\end{array}\right.$

1792. October. Newnarket. Distance 2 miles.

Difference in weight:

According to present scale:

4 year-olds

5 year-olds

6 year-olds

Oider

$\left\{\begin{array}{cc}12 & \text { lbs. } \\ 6 & , " \\ 3 & ,\end{array}\right.$

1792. October. Newmarket. Gold Cup. Distance 4 miles.

Difference in weight :

According to present scale:

4 year-olds

5 year-olds

6 year-olds

Older

$\left\{\begin{array}{cc}11 & \mathrm{lbs} . \\ 5 & , \\ 1 & ,\end{array}\right.$

. . 6 lbs.

. . 0 ,

. 0 ,

1800. June. Ascot. Distance $2 \frac{1}{2}$ miles.

Difference in weight:

According to present scale :

4 year-olds

5 year-olds

6 year-olds

Older

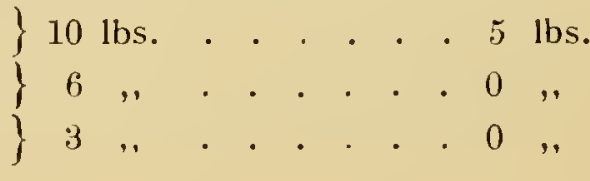


1800. July. Newnarket. Distance 2 miles.

Difference in weight:

According to present scale :

4 year-olds

5 year-olds

6 year-olds

Older

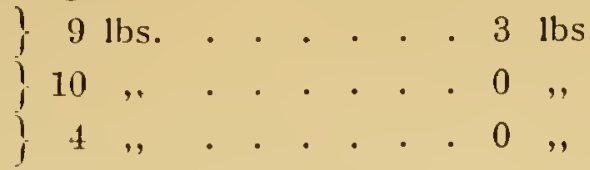

1800. October. Newmarket. Distance 2 miles.

Difference in weight :

Accurding to present scale :

4 year-olds ? 7 lbs.

5 year-olds

6 year-olds

Oider

$\left\{\begin{array}{llllllllll}7 & \text { lbs. } & \cdot & . & . & . & . & . & 0 & \text { lbs. } \\ 4 & . & . & . & . & . & . & . & 0 & , \\ 0 & . & . & . & . & . & . & . & 0 & ,\end{array}\right.$

1807. April. Newmarket. Craven Stakes. Distance 1 mile, 2 furlongs,

Difference in weight : 44 yards.

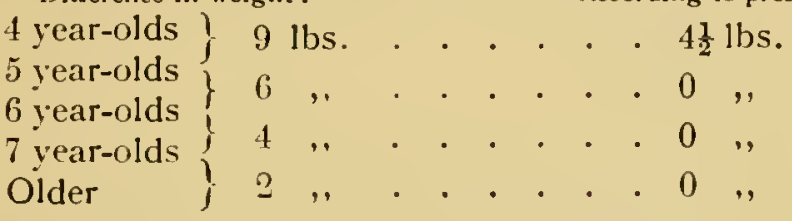

1807. June. Ascot. Gold Cup. Distance $2 \frac{1}{2}$ miles.

Difference in weight :

According to present scale :

4 year-olds

5 year-olds

6 year-olds

Oider

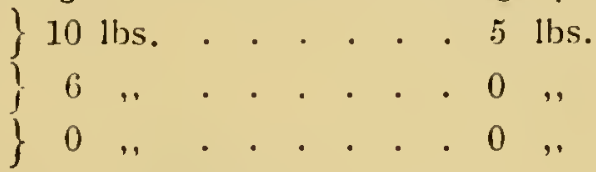

1830. April. Newmarket. Craven Stakes. Distance 1 mile, 2 furlongs, 44 yards.

Difference in weight:

According to present scale :

4 year-olds

5 vear-olds

6 year-olds

Older

\{

$9 \mathrm{lbs}$

$4 \frac{1}{2} \mathrm{lbs}$.

6 , . . . . . . . 0 . 0

4

1830. Nay. Epsom. Craven Stakes. Distance $1 \frac{1}{4}$ miles.

Difference in weight:

According 10 present scale :

4 year-olds

5 year-olds

6 vear-olds

Oider

$$
\begin{aligned}
& \text { ) } 9 \mathrm{lbs} . . .2 .3 \frac{\mathrm{ll}}{2} \mathrm{lbs} \text {. } \\
& \text { \} , . . . . } 0 \text {, } \\
& 3, . .00 \text {, . }
\end{aligned}
$$

1830. June. Ascot. Gold Cup. Distance $2 \frac{1}{2}$ miles. Difference in weight:

According to present scale :

4 year-olds

5 year-olds

6 year-olds

Older

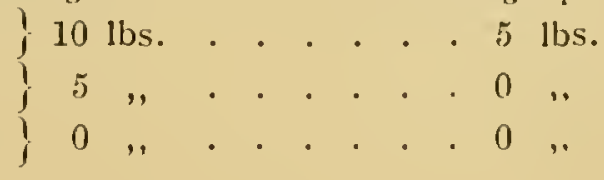


1830. June. Manchester. Gold Cup. Distance 2 miles.

Difference in weight:

According to present siak:

4 year-olds ; 8 lbs. . . . . 4 lbs.

5 year-olds

6 year-olds

Older

1830. August. York. Distance 2 miles.

Difference in weight:

According to present scale.

4 year-olds i 8 lbs. . . . . 2 lbs.

5 year-olds

6 year-olds

Oider

1830. September. Doncaster. Gold Cup

Difference in weight :

0 ,

0

4 year-olds

5 year-olds

6 year-olds

Oider

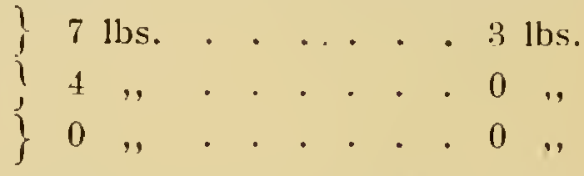

1830. October. Newmarliet. Distance 1 mile, 2 furlongs, 44 yards.

Difference in weight :

According to present scale :

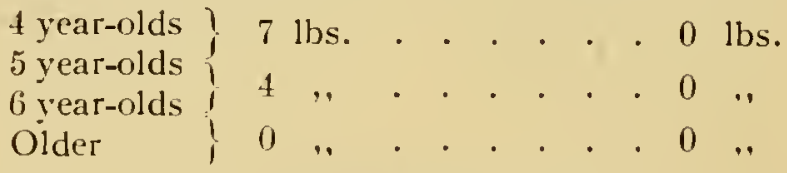

1850. April. Newmarket. Craven Stakes. Distance 1 mile, 2 furlongs, 14 yards.

Difference in weight:

According to present scale:

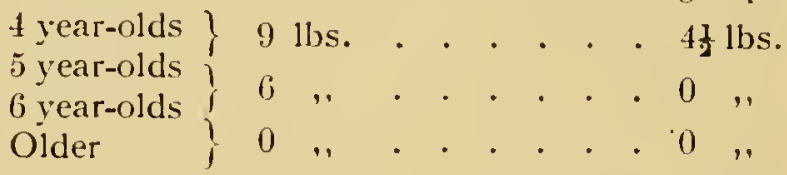

1850. May. Epsom. Craven Stakes. Distance 1妾 miles.

Difference in weight :

According to present scalc:

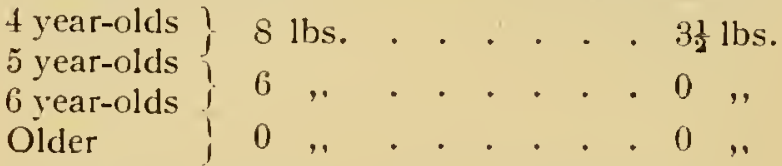

1850. June. Ascot. Gold Cup. Distance $2 \frac{1}{2}$ miles.

Difference in weight:

According to present scale:

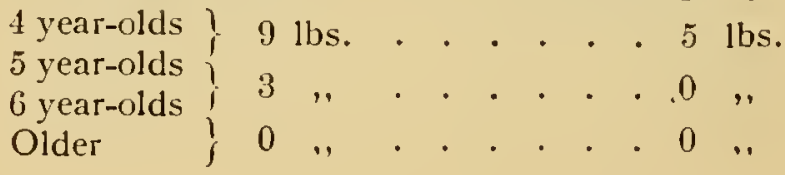


1850. September. Doncaster. Gold Cup. Distance 2 miles, 5 furlongs. Difference in weight:

According to present scale :

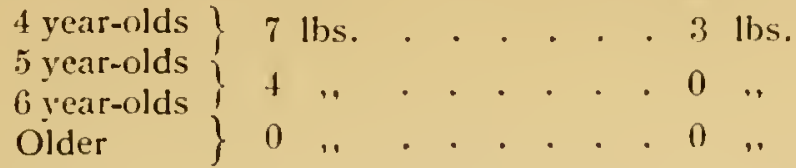

1850. October. Newmarket. Distance 1 mile, 2 furlongs, $4 t$ yards.

Difference in weight :

According to present scate:

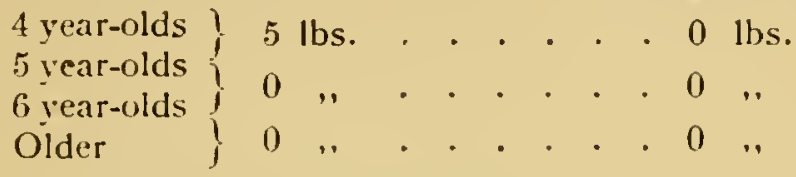

1851. May. York. Match. Distance 2 miles.

4 years Voltigeur, 8 st.
5 years Flying Dutchman, 8 st., $8 \frac{1}{2}$ lbs. (won)

Difference in According 10

weight:

$8 \frac{1}{2}$ lbs. . . 5 lbs.

It is difficult to compile examples of the King's Plates which can be of any use, as most of the King's Plates were formerly run with heats, and those without heats were only held for loorses of the same age. 'The few suitable examples which follow will suffice, however, for cur purpose :-

18:0. August. York. Distance 4 miles. Difference in 1)ifference in weight as per scale of
weight:

\begin{tabular}{|c|c|c|}
\hline $\begin{array}{l}4 \text { year-olds } \\
\text { ty year-olds }\end{array}$ & 1 stone, $: 2$ lbs. & $9 \mathrm{lbs}$. \\
\hline 6 year-olds & S,. & 2, \\
\hline Older & 2, & $0 \ldots$ \\
\hline
\end{tabular}

1832 to 1850. May and June, in Chester, Ascot and Manchester. Distance 3 miles.

Difference in Difference in weight as per scale of

3 year-olds weight: 1861 and 1881

4 year-olds

5 year-olds

6 year-olds and older

I 2 stone 10-13 lbs.

9-10 lbs.

$12 \mathrm{lbs}$.

$8-9 \quad$.

(i) -7 ,

5 ibs.

2

1850. August. York. Distance 2 miles.

Difference in weight:

1 stone, 3 lbs.

4 year-olds

5 year-olds

6 year-olds

Older
6 ,

3 ,,

2 ,
Difference in weight as per scate of 1801 and 1851

1 stone, 2 lbs. 1 stone, 1 lbs.

$\begin{array}{ll}5,, & 2,, \\ 0,, & 0, ", \\ 0,, & 0,,\end{array}$


From these weight differences it can be seen that the abilities of four, five and six-year-olds changed very little up to 1850. An improvement of horses, on the other hand, after six years old ceases gradually altogether. In other words, the influence caused by two-year-old races and mentioned on page 42 with the weight-differences between two, three and four-year-olds, has not affected the carrying capacity of four, five and six-year-olds, but has stopped the further improvement of six-year-olds. Accordingly horses at six years old reached the height of their capabilities (Leistungsfähigkeit).

The preservation of the capabilities of four, five and six-year-olds up to 1850 in the same proportion to each other (in spite or in consequence of the two-year-old races) is all the more remarkable, as in the same time (shown above) a progress in the development of three-year-olds, and still more of four-year-olds, must be assumed on account of the weight differences. From this we might suppose that the improved four-year-old would more closely approach the five-year-old and older horses, which ought to be seen by a diminution in the weight differences. As up to about 1850 this diminution did not take place, the continued improvement of five-vear-old and older horses must be all the more appreciated. After 1850, or even later, the frequent and very early two-year-old races seem also here to have impeded the favourable development of horses after their fourth year.

As a further example to confirm above conclusions, let me here mention the Thoroughbred Fitzwilliam Stakes, at Doncaster.

This race was founded in 1807, and set apart for two-year-olds and older horses, over a course of $1_{2}^{\frac{1}{2}}$ miles, with the following weights:

$\begin{array}{lllll}2 \text { year-olds } & 6 \text { stone. } & \text { Difference in weight: } \\ 3 \text { year-olds } & 8 & & & \\ 2 & \text { stone. }\end{array}$
According to present scale:

2 stone, $3 \mathrm{lbs}$.

$11 \mathrm{lbs}$.

0 ,

0,

0 ,

Two-year-olds in this proportionately long distance of $1 \frac{1}{2}$ miles had no chance (Oiseau was in 1811 the only two-year-old winner). Therefore the following changes were arranged in 1826 :-

\begin{tabular}{|c|c|c|c|c|}
\hline 2 year-olds & 5 & stone, & 10 lbs.? & $\begin{array}{l}\text { Difference in weight } \\
2 \text { stone, } 4 \text { Ibs. }\end{array}$ \\
\hline 3 year-olds & 8 & ," & - , , & 2 scone, 4 los. \\
\hline 4 year-olds & 9 & , & - & 1 " \\
\hline 5 year-olds & 9 & , & 6 & $6 \mathrm{lbs}$. \\
\hline 6 year-olds and older & 9 & ," & 10 & $4 \quad$, \\
\hline
\end{tabular}

The weight difference between two and three-year-olds is here only $1 \mathrm{lb}$. more than according to present scale. In this race from 1834 two-year-olds are left out. The weight difference of 1 stone (11 lbs. to-day) between three and four-year-olds remained stationary till the race became a Handicap in 
1850. The weight difference, however, between four and five-year-olds increased in 1834 to $6 \mathrm{lbs}$, in 1838 to eren $12 \mathrm{lbs}$., and decreased in 1839 to 7 lbs., whilst according to the present day scale the difference is nil. The weight difference between five and six-year-olds in 1834 came down to $2 \mathrm{lbs}$., and remained so to 1850 .

In the celebrated race between the three-year-old, Voltigeur, with 7 stone, $7 \mathrm{lbs}$. up, and the four-year-old, Flying Dutchman, carrying 8 stone, $12 \mathrm{lbs}$., over a course of 28 miles, in the Doncaster Cup, on the 20th of September, 1850 , there was a weight difference of 1 stone, $j \mathrm{lbs}$., against 1 stone, 1 졸 $1 \mathrm{bs}$. of the present scale. In the year later, in that well known match of the 13th of Nay, 1851, at York, over a distance of 2 miles, the four-year-old, Voltigeur, carried 8 stone, the five-year-old, Flying Dutchman, 8 stone, $8 \frac{1}{2}$ lbs. The weight difference was therefore $8_{2}^{1}$ lbs., against $5 \mathrm{lbs}$. according to present scale. There was each time a close finish. In the Doncaster Cup, Voltigeur won by half a length, and in the Match, Flying Dutchman won by one length. We may, therefore, assume that the then existing weight differences corresponded with the average capabilities of three, four and five-year-olds.

Therefrom follows that the present four and five-year-old racehorses are each $3 \frac{1}{2}$ lbs. worse than those in the middle of last century.

Against these conclusions one may, however, answer that it is just in consequence of the many and early two-year-old races, as well as in consequence of the progress made in the art of training in the second half of the nineteenth century, that the weight carrying capability of two and three-year-olds has been much improved, and that, therefore, three-year-olds have almost approximately reached that point of weight carrying capability which was formerly only held by five and six-year-olds.

The consequences, therefore, would of course be that the four-year-old and older horses could not improve in such proportion as to justify the greater weight differences which were formerly extant. Therefore, the weight differences between three, four and five-year-olds must become less. In other words, the zero point from which we count, i.e., the weight carrying capability of two and three-year-olds, would become much higher. If this were so, then, with the assumed quicker and better development of two and three-year-olds, the weight difference between these two ages ought also to have become larger. This, however, it is remarkable to have to say, is not so. In the gradual building of the weight scale, one point is very noticeable: that the weight differences between two and three-year-olds are almost the same to-day as they were at the end of the eighteenth century, when twoyear-old races were just beginning. On the other hand, the above-mentioned increase of weight differences of 2-7 lbs. between two and three-year-olds speaks for itself for the healthy development of two-year-olds in the years from about 1800 to 1850 . Furthermore, it is very doubtful whether the present two and three-year-olds are really better than they were in the middle 
of the nineteenth century. We have no proof of this. Such horses as Touchstone, Cotherstone, Orlando, The Baron, Surplice, Flying Dutchman, Voltigeur, Stockwell, West Australian, Fisherman, Blink Bonny, Thormanby, etc., are they really worse horses than our present day champions? All these said racchorses yet bclonged to those times in which there approximately existed for different ages that difference of weight which we have mentioned above for 18.50 .

Finally, we have only to deal with one point, namely, that two-year-olds themselves are much better than they were before. If this really be the case to such a great extent as to justify the above mentioned large weight differences, then the progress in the average records of time should be more remarliable than the comparisons show hereafter. As the weight difference between two and threc-ycar-olds as above-said are the same as they were one hundred years ago, the records of three-year-olds suffice for comparison. The increase of weight differences between two and three-year-olds after 1800 has been shown above to be a result of the improvement of three-yearolds, probably in consequence of the races for two-year-olds.

There is yet the possibility that the two-year-olds became worse. and that, therefore, the weight differences could rise. There is, however, no plausible reason for this possibility, whilst the assumed improvement of three-year-olds, through training and racing as two-year-olds, appears rery probable. It is, rather, to be assumed that as everything improves, twoyear-olds also improve in course of time. This, however, need not be at the expenses of three-ycar-olds.

In breeding. where only two-year-olds improve, one can hardly speali of a general progress. I seem, therefure, to be justified, for the salie of comparisons, in taking the capabilities of two-year-olds as a standard, although an improvement, however slow, in the course of time is clesirable, and also appears probable.

The weiglit differences in the scale for ling's Plates from the year 1861 are, as can be seen by comparison, considerably higher than to-day. The weight differences of the lirst weight scale of Idmiral Rous of 1881 , compared with those of the scale of 1906 , show, as can be seen from the figures of the table printed in larger type, that also in the last twenty-five years, a diminution of weight differences has taken place.

If one takes, therefore, the capabilities of two-year-olds as a standard, in comparing with the capabilities of other ages, one must consider the diminution of weight differences always as a sign of deterioration of stock.

According to above comparisons of weight differences in 1861, 1881 and 1906, from 1861 a gradual deterioration of stock appears probable if looked at from this standpoint.

Even if the pretended sreater capabilities of present two and three-rearolds were approximately right, there still remains a grave doubt whether the 
lessened development capability of four-year-olds and older horses has also produced a lesser resisting power, as we show later on, in the chapter on the capabilities of the Thoroughbred formerly and now.

Taken altogether, these observations justify us in assuming that, considered from the standpoint of the altered weight differences, the improvement of racehorses took place about up to the middle of the nineteenth century, and also specially, that a farourable development of four-year-old and older horses up to the sixth year existed. Furthermore, that simultaneously with the frequent occurrence of races for two-year-olds partly in the beginning of the year, and with the existence of the many short Handicaps, a retrogression of the development of racehorses after their third year began soon after the second half of the nineteenth century. The time in which the number of two-year-old racehorses began to exceed the number of threeyear-olds, denotes the turning point. 


\section{CHAPTER IV.}

\section{General Observations on the Value of the Thoroughbred for other breeds.}

THE prominent position occupied by the Thoroughbred in the breeding of half-bred horses ${ }^{1}$ has no equivalent in the breeding of any other animal. Breeding carried on for two hundred vears and for a set purpose, as it has been done in England and Ireland, where soil and climate constitute an environment admirably adapted 10 horse-breeding, has produced the Thoroughbred of the present day. Breeders of other animals have also done great things in the same time, but in the breeding of horses one very great factor has co-operated which is more or less absent in the breeding of other animals. This mighty factor was passion. Battle and sport are the foundations of horse breeding. These two have as if by magic produced a passionate love for this breeding. This love worlis with more power, wisdom, ingenuity and industry than all book wisdom of the wisest heads and all conscientiousness of the most industrious of men. To horse breeding, as well as to all that lives, the words of the Apostle Si. Paul apply: "The greatest of these is Love."

That this passion, otherwise so useful, may also lead to erroneous paths, is without doubt true in horse breeding. If this happens, and, unfortunately, it happens often, then wise counsel must step in; but this counsel must not kill the principal lever for the breeding of Thoroughbreds, namely, the justmentioned passionate love.

The facility with which the horse acclimatises itself everywhere has produced the spread of breeding of Thoroughbreds all over the world. All half-bred horses of the old and new world, even the Trotters, have, by mingling with the Thoroughbred, produced the required steel in their breeds, which otherwise could not have been produced as weil and as quickly. Even the breeds of the Steppes of Russia, America and

'In Germany all improved light breeds of horses, save the Thoroughbred, are called "edles Halbblut," which means "high quality Half-breds." All of these light breeds as well as the Thoroughbred itself are also called warm or hot-blooded horses, as contrasted with the cold-blooded heavy or draft breeds of horses, which are summarized in Germany under the general term " Kaltblut." 
Iustralia, have, with thr aid of Thoroughbred stallions (often, unfortunately, of very low quality), influenced the capabilities (1,eistungsfahigkeit) of their horses. When the ecelebrated Cossack officer, Ilotman Platoff (the celebrated stallion, H(tman Patoff, born 18:36, was called after him), about a hundred years ago, during the fight for liberty against Napoleon in the IVest of Europe", learned to know and to esteem the "lowroughbred, and other fine breods derived from same, he caused to be imported many thoroughbred stallions of groud, and of the best class, into the cossacli breeding stables near the Don, and the neighbouring Steppe breeding places. The consequenc: was that Russian cavalry soon after, in fact, up to the middle of the last century, were mounted on the best horses an army ever possessed. That was a time in which there were often matches betweren Cossack horses and Thoremghbreds. The most erebrated of these races took place on the the of Iugust, 18:5, near Sit. Petersburg, distance 75, versts, i.c., about sol kilometres. Two Cossack horses started against two Thoroughbreds. The Thoroughbred, Sharper, born 1819 in the stud of lord Egremont, by Octavious and Y. Imazon, won easily in 2 hours, to minutes, although he carried $10 \mathrm{lbs}$. more (:3 stone). The race took place on the high road, and the rider of Sharper broke a stirrup in the middle of the race. In England,

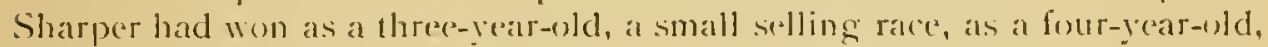
two small IIandicaps and a walk-ower, and as a five-year-old, three rakes, inclusive of two king 's Plates.

The good results which hate been obtained, eyperially in the Somb of France, by the mised Inglo-. Irabie breceling, seem lo lead to the conclusion that one could perhaps also improve the Oricntal horse, including the Irabs, in their own homes, by the intruduction of Thoroughbred stallioris. If the small mistalies made in brecding Thoroughbreds were aroided, it is more than probable that in future origrinal Oriontal breeds conld be improved by Thoroughbred stallions than vice iersa. Hattely, in England, they have tried to improwe Thoroughbreds by the infusion of Irabic bleod, but in rain. It is very remarliable, after the importation of Godolphin Irabian in 1730, that no Oriental stallion could produce any great influence on the breeding of Thoroughbreds, although in many races Oriental bloud was faroured by lighter weights, whilst, on the other hand, for example, the oflspring of Felipse and Highfleer had in many races to carry about 3 to j llss. more, according lo proposition. In the Goodwood Gold Cup Anglo- Irabic horses were favoured from lis:35 to 1 sig with $1.1 \mathrm{lbs}$. less weight, later with 18 los. less. Thereughbred Orientals, inclusive of Purks, Berbs, rte, had in this race at first $2 \mathrm{~h}$ lbs. less to carry, later 36 lbs., without having any success. These facts and figures (36) lls. less) are not suitable ('nough to rerommend larger importations of oriental blood for the breeding of light horses.

The most important spheres of action of the "Thoroughbred have been mentioned pret! "xhatsively above, namely, llalf-loreds, Trotters, Sirppo 
horses, and Oriental breeds. There still remains to be mentioned the coarser crosses of Thoroughbreds on some Coach Breeds and Heary Breeds. In these cases also the Thoroughbred has been successful, and the successes would probably have been more frequent if the said breed had not become spoiled and too heterogeneous through pampering without trials.

That the heary horse breeds have not amongst themselves produced a "Thoroughbred" drafter (sit venia verbo) as a representative of what they are capable of, is the regrettable consequence of the breeding which was built up for show without testing eapability. There has not been sought for prominent performances, but rather for zoological attributes. As long as the breeding of drafters is carried on in this one-sided way, one cannot expect any progress in the capability for employment (for use and breeding). The socalled early maturity of the cold-blooded stock, together with early decay, rests on its effeminating method of rearing. It is not quite the same as what is called in the breeding of other animals early maturity. Neither as regards its earlier capability nor as regards its earlier breeding capacity, is the drafter earlier mature than the Thoroughbred. The expression, "early maturity," ought, in my opinion, to be eliminated in the breeding of horses, for within every breed the so-called early maturity can be obtained in a short time by a corresponding practice, without at the same time causing the shortening of life which accompanies early maturity.

One often speaks of the better use of food with heavy lorses, but I think this is based on a wrong idea. Apart from the motion of exorbitant loads, as it is required, for example, in different industries and in the beetroot districts, the Half-bred, and also the Thoroughbred, perform the daily slow work of the drafter in agriculture, with considerably less food-a fact which I have noted at Beberbeck with teams composed of drafters and Halfbreds. With quicker work, however, in trotting, the draft horse falls still more behind. He requires still more food, and finally gets off his feed. Tu make good use of food when doing nothing, or working at a comparatively slow rate, does not mean much. That the drafter of to-day, in spite of his irrational breeding, can, on account of its heavy weight, carry a larger load than a Thoroughbred, is unquestionable.

There is also no doubt that the Thoroughbred will bear a load corresponding to its weight, viz., 20 cwts. (which, considering the usual weights of practice, is a large load, a good Belgian horse carrying about 80 cwts.) quicker, at longer distances, and over worse roads. In a Thoroughbred, through many generations, more muscle power, energı, etc., has been amassed than in the present day drafter. Of course, it is to be expected that a reasonable breeding of cold-blooded stock, based on performance, will also here beat the Thoroughbred. The great popularity and spread of the heavy breeds is based principally on their easy temperament, which has been bred into them by pampering them. That a drafter bred on performance will be just as easy in temperament as the actual draft horse is highly improbable, because 
it is very difficult to combine two qualities which are so different and so contradictory as is the case with powerful energy and comfort. This ease, clemanded only by easy workmen, will always influence the good quality of the material. The great mistake made in comparing different breeds of horses is always the consequence of unjust parallels, as, for example, want and use of food, quict and restless temperament of light horses at quick work, and of heary horses at slow work. The same unjust comparison is often made with the so-called Thoroughbred knacker and a picked prominent llalf-bred. II ithin the light breeds the same difference in the temperament of horses is observable. The most pleasant and most reasonable temperament as a riding borse is in most cases the Thoroughbred when it has left the track, and the most difficult, violent and nervous temperament is found in Half-breds when they are taken seriously into training. The less highly bred the horse is, the more the above symptoms show themselves. If the work which is required from a horse in training often reaches the limits of its capacity, or even exceeds it, it is natural that a certain fear to do the work shows itself. Thereby nervousness ensues, which in Thoroughbreds shows itself most with the worst of them. I have seen Kinscem, Ormonde, Flying Fox, Ard Patrick and others, as quiet and reasonals]e before a race as after one.

Of all Half-bred horses which so far have been trained for races in Trakehnen, I have only observed three which remained absolutely quiet and reasonable up to the last quick gallop, namely, Morgenstrahl, born 1896 , by Blue Blood and Noba, winner of the Fanfarro Race, 1900, in Insterburs; and the two Cadiz fillies, Jagdkönigin and liastanie, both winners of the Breeders' Races in Königsberg. These three horses were so superior to their comrades that the limits were reacbed neither in racing nor in training.

In consequence of its hard training, the Thoroughbred acclimatises itself very easily in different districts, whilst the softly raised draft horse is most sensitive in this regard. Pure-bred Percherons, which 1 saw in the year 1887 in the magnificent Steppes of the Russian Royal stud at Derkul, Government Charkow, soon lost their type, and had already in the second generation an Arabic appearance, thereby reminding one of their ancestors. In the form of croup one could still distinguish the Percheron; in its noble bearing, in the vivacity of its temperament, one seemed to recognise the Arabian cross-bred. The pure-bred Suffolls and Clydesdales reared there were soon stunted in their growth, and could not stand the rocky meadows and strong sun. The long marches, which are necessitated by the life on the Stcppes, from the meadows to the distant watering-places, did not offer them the comfort which they were accustomed to, and which is necessary to their srowth. Drafters bred on performance would very likely, if repuired, stand the life of the Steppes, just as the Thoroughbred can be raised in a low, watery district. Of course, neither the draft horse on the Steppes nor the Thorouglibred on the marsh would prosper.

$\triangle$ real breed of heary horses founded on performance tests would not 
produce the powerful dimensions which are, unfortunately, to-day still demanded, and even rewarded. The judging of horses only by measurements expressed in ligures, whether it be for weight or dimensions of certain parts of the body, is not sufficient, and often dangerous. For many years the measuring of cannon bones has played the leading part, which, as a matter of fact, is often very useful in helping the eye when judging of the strength of the fundament within the same breed. The comparing of cannon measurements of different breeds of horses has become a modern sport.

Often incommensurable values are compared. The fore foot of a drafter which has become thick through doing nothing or only light work, is most probably (without science showing it definitely) of a softer texture, therefore less capable of resistance than that of the Thoroughbred or Halfbred, grown hard through hard work. The examinations of different cannons by Professor Krämer, in Bern, seem to confirm the above supposition. I here observe that comparative examinations on this subject lose in value if the bones are not bones of well-known horses. One must know genealogy, age, as wall as the feeding and training of horses, if the bones are to be used for experiments in pressing and bending, as well as for showing specific gravity. One only may compare results if they are taken from horses of the same breed, same standard quality, same age, same state of health, especially as probably small differences may have a great importance in these experiments. If the quality of the cannons is of equal value, one must first answer the question how advantageously the weight of the body is supported, i.e., how much weight rests on a definite square measurement of supporting surface. This question is answered by putting the square of the cannon girth in proportion to the body weight which it supports. If, however, the question is put how easily the supporting column (in this case the cannon) can break or bend, the cube of the eannon girth must be put in proportion to the weight.

Apart from the different quality of the mass of the bones in different breeds, of the larger marrow cavity of the cannon in drafters, ete., I believe that these measures often lead to mistaken conclusions. Practical use and experience will often lead to other and more correct results. In any case, the present horses of draft blood are the most unsuitable breeds to create in warm-blooded 1 Ialf-breds, strong, good and firm fore legs. In the breeding of drafters, as well as in that of many IIalf-breds, one may observe that a soft method of rearing with little movement, and then only at a slow rate, is eminently suitable to produce strong (i.e., big) fore legs.

Against all these errors and dangers there is only one remedy, namely, performance tests. The isolated attempts to introduce at exhibitions and shows performance tests of heary horses will become general, if only the general public rightly honours same and requires them. I'ulgus vult decipi, erşo decipiatur-till it is undeceived.

In the year 1867, at the last Napoleonic Exhibition in Paris, several 
prizes were given for horses which eould arry the largast load in proportion to their wn weight. I very excellent idea. The priqe (I betiene the first) was not won, however, by a clraft horse, but by the colt 11 apsikas, bred in Porgel (Estland), 60 degrees latitude, later for a long lime loe Royal stud stallion in Torgel. Purely listande, i.e., of light breed -a small, wallproportioned horse, similar to a small bast Prussian. This Imexpected result, however, has, so it appears, prevented a repetition of this proposition.

The knowledge that sham is easier and cheaper te produce than the real thing, and the fact that there are still people enough who are deceived by sham, has just as much a deteriorating influence in the preducing of wine and tobaceo and other things as in the breeding of horses. The claims of the consumers on the capabilities, and the interest of the producer to produce as cheaply ats pessible, will always be difficult to meet. The cratuness of the breeder to produce showy stock rery cheaply has often made greater progress than the expert knowledge of the consumer. Experts of cattle breeding complain about the same thing. Finally, one would think that the real thing, Truth, would prevail in the end. The breeding of Thoroughbreds and Trotters bred for performance is genuine, as wel] as, for example, the unfortunately dying out breeding of the Russian Steppes: the breeding of horses for the Russian soldiers, formerly in the Lkrane, num at the Manitseh river, was very much based on it. Since the cultivation of land, and with it the breeding of cattle and the use of the plough, have driven away the breeding of the Steppes from the magnificent Uleraine to the Manitsch river, and from thence still further and further into the unsuitablo salt-containing Eastern Steppes; even Russia, so rich in horses, is forced to establish a modern system of horse-breeding in order to produce horses for its soldiers. The hope to get thereby just as nicc-looking cavalry horses as the other great nations is a sop to vanity, and suppresses the knowledge that horses lose thereby in capability. The difliculty of the organisation of a successful breeding of horses, like we have in liast Prussia, is perhaps thereby under-estimated, and the quality of the brexd of horses produced with a beatiful sham appearance is over-estimated.

That one bas to light against this production of the beatuful sham, aren in the breeding of Thoroughbreds, one can see in many linglish studs which breed for the yearlings' marliet. In this respect, in the course of the last fifty years, things have gone very much to the bad in England, as now more than half-aceording to some authors ren nine-tenths-of all phoroughbred yearlings are brought up for the yoarlings' market. Wthough this showy stock very seldom becomes also breeding stock, on account of the race tests in any case, not breeding stock which has an improtant influence on the breeding of "Thoroughbreds-it is a pity" that through human sin much gond material is wasted which was destincd by Divine Nature to be chosen material.

Ipart from the performances obtained by no other breed than the "Thornughbred, over an! distance and under any weight, the Thoroughbred 
is also superior to all other breeds in various breeding performances. This is an eloquent proof that the tests to which the Thoroughbred is subject have also had a good influence on its fertility and longevity (as will be shown hereafter by many examples). The number of dams which, according to the statement of the General Stud Book, have prodticed sixteen living foals, is such a large one that for want of space they have been left out of the following lists. We only mention brood mares which have produced seventeen and more living foals. Is the first volume of the General Stud Book only appeared in 1793, the foals born in the eighteenth century are only given in so far as they were known throtigh the Racing Calendar, the chief basis of the Stud Book. It is clear, therefore, that in the first book many living foals are not mentioned, as they have not appeared on the racecourse. Therefore, the number of brood mares which in the eighteenth century produced seventeen and more living foals is less in the following list :-

Register of Thoroughbred Mares in the General Stud Book which have had not less than seventeen ]iving foals.

\begin{tabular}{|c|c|c|c|c|c|}
\hline No. & Names of the Mares. & 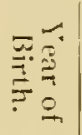 & Sires of the Mares. & 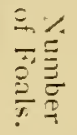 & Remarlis \\
\hline 1 & Sister to Sampson & $172: 3$ & Greyhound & $21)$ & $\begin{array}{l}\text { Dam of the } \\
\text { Fam. 12 a }\end{array}$ \\
\hline 2 & Pratt's Old Mare & 1750 & Squirt & 17 & $\begin{array}{c}\text { Dam of the } \\
\text { Fam. } 24\end{array}$ \\
\hline 3 & Mare & 1777 & Natchem & 18 & Fam. 15 \\
\hline 4 & Mare & 1780 & Y. Marske & 18 & $\begin{array}{c}\text { Dam of the } \\
\text { Fam. } 34\end{array}$ \\
\hline 5 & Nimble & 1784 & Florizel & 17 & $\begin{array}{c}\text { Dam of the } \\
\text { Fam. } 32\end{array}$ \\
\hline 6 & Heiress & 1786 & Paymaster & 17 & Fam. 12 \\
\hline 7 & Nare & 1788 & Adamant & 17 & Fam. 3 \\
\hline 8 & $\begin{array}{l}\text { Amelia (Grand- } \\
\text { daughter of No. 2) }\end{array}$ & 1788 & Highflyer & 17 & Fam. 24 \\
\hline 9 & Beatrice & 1791 & Sir Peter & 17 & Fam. 7 \\
\hline 10 & Mare & 1791 & Y. Marske & 18 & Fam. 9 \\
\hline 11 & Platina 0. & 1792 & Mercury & 19 & Fam. jb \\
\hline 12 & Bay Javelin & 1793 & Javelin & 17 & $\begin{array}{l}4 \text { year-old first foal } \\
\text { Fam. } 3 \text { a }\end{array}$ \\
\hline 13 & Mare & 1795 & Skyscraper & 17 & Fam. $2 \mathrm{e}$ \\
\hline 14 & Miss Gunpowder & 1797 & Gunpowder & 20 & $\begin{array}{l}3 \text { year-old first foal } \\
\text { Fam. } 34\end{array}$ \\
\hline 15 & Rosamond & 1798 & Buzzard & 17 & Fam. $5 a$ \\
\hline 16 & $\begin{array}{l}\text { Carthage (Firstling) } \\
\text { sister to Hannibal D. }\end{array}$ & 1798 & Driver & 18 & Fam. 3 \\
\hline 17 & Grey Duchess & 1799 & Pot $8 n s$ & 19 & $\begin{array}{l}4 \text { year-old first foal } \\
\text { Fam. } 6\end{array}$ \\
\hline
\end{tabular}


Ni. Names of the Mares.

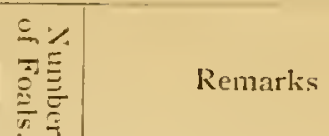

\begin{tabular}{|c|c|c|c|c|c|}
\hline 18 & Miss Haworth & 18182 & Spadille & 17 & $\begin{array}{l}3 \text { year-old first foal } \\
\text { Fam. } 29\end{array}$ \\
\hline $1 !)$ & Mare & 1802 & Precipitate & 18 & $\begin{array}{l}4 \text { year-old first foal } \\
\text { Fam. } 5\end{array}$ \\
\hline 20 & $\begin{array}{l}\text { Bronze O. } \\
\text { sister to Castrel }\end{array}$ & 1803 & Buzzard & 17 & Fam. $2 \mathrm{~d}$ \\
\hline 2$]$ & Mare & $180: 3$ & Star & 17 & Fan. 23 \\
\hline$\cdot 2 \cdot 2$ & Mare & 1,504 & llambletonian & $\begin{array}{l}18 \\
\text { or } \\
19\end{array}$ & Fam. 18 \\
\hline 23 & $\begin{array}{l}\text { Minniac (Firstling) in } \\
\text { 19 years }\end{array}$ & 1sisk; & Shuttle & 19 & Fam. $4 \mathrm{~b}$ \\
\hline 24 & Mare & 18017 & Shuttle & 18 & $\begin{array}{l}\text { Not in the } \\
\text { Goosichen Table } \\
\text { Frentzel Fam. 4t }\end{array}$ \\
\hline 25 & $\begin{array}{l}\text { Barrosit (Grand- } \\
\text { daughter uf No. 5) }\end{array}$ & $1 s 0 s$ & $\begin{array}{l}\text { Vermin } \\
\text { out of Nike } O .\end{array}$ & 17 & Fam. 32 \\
\hline 26 & Lady of 'he lake & 1809 & Sorcerer & 17 & Fam. 43 \\
\hline 27 & Mare & 1810 & Sancho & 1!) & $\begin{array}{l}3 \text { year-old first foal } \\
\text { ram. } 18\end{array}$ \\
\hline 23 & Wire & 1811 & IVaxy & 17 & Fam. 1 \\
\hline 29 & Miss Cantley & 1812 & $\begin{array}{l}\text { Stamford } \\
\text { out of Sister to Silver }\end{array}$ & 17 & Fam. $5 b$ \\
\hline 30 & $\begin{array}{l}\text { Mare (Firstling) } \\
\text { (Daughter of No. 24) }\end{array}$ & 1812 & Dick Andrews & 17 & $\begin{array}{l}3 \text { year-old first foal } \\
\text { Not in Goosschen } \\
\text { Table } \\
\text { Frentzel Fam. } 46 \\
\text { Fan. } 8\end{array}$ \\
\hline 32 & Mare & 1812 & Shuttle & 20 & $\begin{array}{l}4 \text { year-old first foal } \\
\text { Fam. 15 }\end{array}$ \\
\hline$: x 3$ & Sister to Corduroy & 1812 & Shuttle & 2) & $\begin{array}{l}4 \text { year-old first foal } \\
\text { Fam. } 5\end{array}$ \\
\hline 34 & Nancy & 1813 & Dick Andrews & 17 & Fanı. 9 \\
\hline 35 & Coquette & 1814 & Dick Andrews & 18 & $\begin{array}{l}4 \text { year-old first foal } \\
\text { fram. } 12\end{array}$ \\
\hline 36 & Filagree & $181 j$ & Sonthsayer & 18 & $\begin{array}{l}4 \text { year-old first foal } \\
\text { Fam. } 1 \text { b }\end{array}$ \\
\hline 37 & Nare & 1815 & Election & 17 & Fam. 2 a \\
\hline 38 & Mare & 1815 & Orville & 18 & $\begin{array}{l}4 \text { year-old first foai } \\
\text { Fam. } 12\end{array}$ \\
\hline ; & Velocipede's dam & ISIT & Juniper & 18 & $\begin{array}{l}4 \text { year-old first foal } \\
\text { Fam. 3c }\end{array}$ \\
\hline 40) & Marion & 1810 & Tranp & 17 & Fam. 5a \\
\hline 41 & Snowball & 1819 & Prime Minister & 18 & $\begin{array}{l}4 \text { year-old first foal } \\
\text { Fam. } 2 \text { a }\end{array}$ \\
\hline
\end{tabular}




\begin{tabular}{|c|c|c|c|c|c|}
\hline No. & Names of the Mares. & 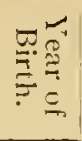 & Sires of the Mares. & 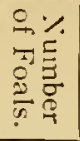 & Remarks \\
\hline 42 & Irmida & $1 s 1 !$ & Rinaldo & 17 & Fam. 15 \\
\hline $4: 3$ & Bequest & 1819 & Election & 17 & Fam. 3 a \\
\hline 44 & Emmat & 1819 & Orville & 18 & Fam. 3 a \\
\hline 45 & Mare & 1819 & Rubens & 17 & Fam. 27 \\
\hline 46 & Adeline & 1821 & Sootheayer & 17 & $\begin{array}{l}4 \text { year-old first foal } \\
\text { Fam. } 11\end{array}$ \\
\hline 47 & Katherine & $18: 21$ & Southsayer & 18 & Fam. 22 \\
\hline 48 & Monimia & 1821 & Muley & 17 & Fam. $12 \mathrm{a}$ \\
\hline 49 & Mairṣ & 1823 & Friday & 19 & F:I17. $5:$ \\
\hline 50 & Mare & 1823 & Merlin & 17 & F:am. 10 \\
\hline 51 & Miss Thomasina & 1823 & l'elbeck & 17 & Fam. 15 \\
\hline 52 & Mare & 1824 & Cervantes & 17 & Fiım. 1 \\
\hline 53 & Emma & 1824 & I'hislier & 17 & Fam. T \\
\hline 54 & Mare & 1825 & Phintom & 17 & Fam. 1 \\
\hline 5 & Banter & 1826 & Master Henry & 17 & Fam. 14 \\
\hline 56 & $\begin{array}{l}\text { Margellina } \\
\text { sister to Memnon L. }\end{array}$ & 1826 & $\begin{array}{c}\text { Whisler out of } \\
\text { Manuella O. }\end{array}$ & 19 & Fam. 11 \\
\hline 57 & Europa & $18 \not 29$ & Reveller & 17 & $\begin{array}{l}4 \text { year-old first foal } \\
\text { Fam. } 2 \mathrm{e}\end{array}$ \\
\hline $5 S$ & Medea & 1831 & Whislier & 18 & Fam. 8 a \\
\hline 59 & Mare & 1832 & Whislier & 19 & Fam. 1 \\
\hline 60 & Lat Belle & 1833 & Voltaire & 17 & $\begin{array}{l}4 \text { year-old first foal } \\
\text { Fam. } 17\end{array}$ \\
\hline 61 & Manilla & 1833 & Mulatto & 17 & $\begin{array}{l}4 \text { year-old first foal } \\
\text { Fam. } 26\end{array}$ \\
\hline (i2) & Valentine & 1833 & Voltaire & 17 & Fam. 12 a \\
\hline 63 & Annette & $18: 35$ & Prian & 19 & $\begin{array}{l}4 \text { year-olel first foal } \\
\text { Fam. } 16\end{array}$ \\
\hline 64 & Parade & 1835 & Pantaloon & 18 & $\begin{array}{l}3 \text { year-old first foal } \\
\text { Fanm. } 2 \mathrm{e}\end{array}$ \\
\hline 65 & Executrix & $18: 36$ & I.iverpool & 18 & $\begin{array}{l}4 \text { year-old first foal } \\
\text { Fam. } 4\end{array}$ \\
\hline 66 & Mare & 1536 & Muley & 18 & $\begin{array}{l}3 \text { year-old first foal } \\
\text { Fam. } 8\end{array}$ \\
\hline 67 & Lapwing & 1837 & Bustard & 17 & $\begin{array}{l}3 \text { year-old first foal } \\
\text { Fam. } 19\end{array}$ \\
\hline 68 & Nartha Lynn & 1837 & Mulat to & 18 & Fam. $2 \mathrm{c}$ \\
\hline 69 & Mare & 1837 & Pantisloon & 18 & Fam. 14 \\
\hline 70 & Palmỵra & 1839 & Sultan & 17 & Fam. 12 a \\
\hline 71 & Iretbusa & 1839 & Elis & 18 & Fam. 20 \\
\hline 72 & Bohemienne & 1839 & Confederate & 18 & $\begin{array}{l}4 \text { year-old first foal } \\
\text { Fam. } 8\end{array}$ \\
\hline 73 & Equation & 1839 & Emilius & 18 & Fam. 7 \\
\hline
\end{tabular}




\begin{tabular}{|c|c|c|c|c|c|}
\hline No. & Names of the Mares. & 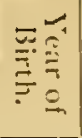 & Sires of the Mares. & 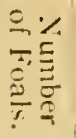 & Remarlis \\
\hline 74 & Flurence & 1839 & Velocipurere & 20 & latm. 2a \\
\hline 7i) & Mare & $1 \leqslant 39$ & Sir llereules & 19 & $\begin{array}{l}4 \text { year-eld lirst fo:l } \\
\text { Fam. } 2\end{array}$ \\
\hline 76 & Treacherous & 1839) & Pantiloun & 17 & Fam. உ2 \\
\hline 77 & Mare & $18: 59$ & l'lenipotcontiary & 17 & $\begin{array}{l}4 \text { year-old first foal } \\
\text { liam. } 10\end{array}$ \\
\hline 78 & Barbarinal & 1810 & Plenipotentiary & is & Fann. $2: 3$ \\
\hline 79 & Titania & 1840 & Emilius & 17 & Fatı. $3 c$ \\
\hline Si) & Boardingr-School-.Miss & $18+1$ & Plenipotentiary & 17 & liam. $3 \mathrm{~b}$ \\
\hline si & Emerald & 1841 & Defence & 17 & Fam. $3 a$ \\
\hline 8.2 & Phỵsalis & 1841 & Bay Middleton & 17 & Fam. $8: 1$ \\
\hline 83 & Mare & 1841 & Plenipotentiary & 18 & $\begin{array}{l}4 \text { year-rid first foal } \\
\text { Fam. } 1 \text { at }\end{array}$ \\
\hline 84 & $\begin{array}{l}\text { Black Bess (Grand- } \\
\text { daughter of No. 45) }\end{array}$ & 1812 & Sheet Inchor & 17 & $\begin{array}{l}3 \text { year-old first foal } \\
\text { Fan. } 27\end{array}$ \\
\hline 85 & Stamp & 1842 & Emilius & 17 & Fant. 1 \\
\hline sio & $\begin{array}{l}\text { The Duchess of } \\
\text { Lorraine }\end{array}$ & 1842 & Pantaloon & Is & $\begin{array}{l}4 \text { year-old first foal } \\
\text { Fam. ? }\end{array}$ \\
\hline 87 & Little Fineh & 1812 & Hornsea & 17 & $\begin{array}{l}4 \text { year-old first foal } \\
\text { Fam. } 29\end{array}$ \\
\hline Ss & $\begin{array}{l}\text { Eulogy } \\
\text { (Daughter of No.68) }\end{array}$ & 1843 & Euclid & 19 & Fam. 2c \\
\hline 89 & $\begin{array}{l}\text { Jocose } \\
\quad(\text { Daughter of No. } 55)\end{array}$ & $181: 3$ & Pátitalon & 17 & Fam. 14 \\
\hline ו) & Queen Mary (Firstlingr) & 1813 & Gladiator & 19 & $\begin{array}{l}4 \text { year-old first foal } \\
\text { Fam. } 10\end{array}$ \\
\hline$! 1$ & Tour de liorce & 1843 & Sir llercules & 17 & Fam. 22: \\
\hline trz & 1)efenceless & 1844 & Defence & 1!) & $\begin{array}{l}\text { 4ycar-old first foal } \\
\text { Fam. } 6 \text { a }\end{array}$ \\
\hline 93 & Sister to 1,eaconfield & $18 H$ & Birdcatcher & $1 s$ & $\begin{array}{l}3 \text { year-old first foal } \\
\text { Not in Goosschen } \\
\text { Table } \\
\text { lirent\%ll lam. } 72 \\
\text { Fam. 2a }\end{array}$ \\
\hline $9 ; 5$ & Makeless & 1814 & St. Martin & 17 & $\begin{array}{l}4 \text { year-old first foal } \\
\text { Fam. } 2 \mathrm{~d}\end{array}$ \\
\hline 96 & The Prairie Bird & 1811 & Touchstone & 211 & $\begin{array}{l}4 \text { year-old first foal } \\
\text { Fam. } 1 \text { a }\end{array}$ \\
\hline 97 & Sultana & 1811 & Hetman Platoff & 17 & Fam. 6 a \\
\hline 98 & Themis & 184 & Touchstone & 17 & $\begin{array}{l}4 \text { year-old first foal } \\
\text { Fam. } 9\end{array}$ \\
\hline 9 & Contraction & Istis & Esmilius & 17 & $\begin{array}{l}4 \text { year-old first foal } \\
\text { Fam. } 43\end{array}$ \\
\hline I00 & Nourmahal & 181.5 & 1.anercost & 17 & $\begin{array}{l}4 \text { year-old first fonl } \\
\text { Fam. 2e }\end{array}$ \\
\hline
\end{tabular}




\begin{tabular}{|c|c|c|c|c|c|}
\hline No. & Names of the Mares. & 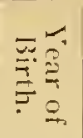 & Sires of the Mares. & 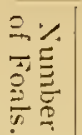 & Remarks \\
\hline 101 & Troica & 1845 & 1.anercost & 17 & Fam. 3 a \\
\hline 102 & Midia & 1840 & Scutari & 17 & fam. 3 a \\
\hline 108 & Sacrifice & 1847 & Voltaire & 17 & Fan. $1 \mathrm{~d}$ \\
\hline 101 & Sunflower & 1847 & Bay Middleton & 18 & $\begin{array}{l}4 \text { year-old first foal } \\
\text { Fam. 1 c }\end{array}$ \\
\hline 1015 & Agnes & 1848 & Pantaloon & 17 & Fam. $2 S$ \\
\hline 106 & Plush & $181 \mathrm{sin}$ & Plenipotentiary & 19) & Fam. 1 a \\
\hline 107 & Bay Rosalind & 184!) & Orlando & 20 & Fam. 11 \\
\hline 108 & Butterfly & 184!) & Knight of the Whistle & 18 & Fam. 20 \\
\hline 109 & Gossamer & $184 !)$ & Birdcatcher & 17 & Fam. 19 \\
\hline 110 & Niss Conyngham & $181: 1$ & Slane & 18 & $\begin{array}{l}4 \text { year-old lirst foitl } \\
\text { Fam. } 1\end{array}$ \\
\hline 111 & Amazon (in 18 years) & 1850 & Touchstone & 18 & $\begin{array}{l}4 \text { year-old first foal } \\
\text { Fam. } 4\end{array}$ \\
\hline 112 & Latona & 1850 & Lanercost & 17 & $\begin{array}{l}4 \text { year-old first foal } \\
\text { Fam. } 3\end{array}$ \\
\hline 113 & Georgiana & 18.51 & Touchstune & 17 & Fan!. 7 \\
\hline 114 & Black Cotton & 1852 & liaugh-a-Ballagh & 17 & $\begin{array}{l}4 \text { year-old first foal } \\
\text { Fam. } 6\end{array}$ \\
\hline 115 & Jeu d'Esprit & 1552 & Wlatcatcher & 17 & Fam. 7 \\
\hline 116 & Mare & $18 \% 2$ & Jon & 19 & $\begin{array}{l}4 \text { year-old first foal } \\
\text { Fam. } S \text { a }\end{array}$ \\
\hline 117 & Defamation & $18 \% 3$ & Jagro & 17 & $\begin{array}{l}4 \text { year-old first foal } \\
\text { Fam. } 14\end{array}$ \\
\hline 118 & Stuff and Nonsense & 1853 & The Libel & 18 & $\begin{array}{l}4 \text { year-old first foal } \\
\text { Fam. } 1\end{array}$ \\
\hline 119 & All's IVell & 1854 & The Cure & 17 & Fam. 5 \\
\hline 120 & Christabelle & 18.54 & Fermhill & 17 & Fam. 3c \\
\hline 121 & Countess & 1854 & Slane & 18 & $\begin{array}{l}3 \text { year-old first foal } \\
\text { Fam. } 8\end{array}$ \\
\hline $1 * 2$ & $\begin{array}{l}\text { Countess of } \\
\text { IVestmorland }\end{array}$ & 1854 & Nelbourne & 1!) & $\begin{array}{l}4 \text { year-old first foal } \\
\text { Fam. } 3\end{array}$ \\
\hline 123 & Daisy & 1854 & Touchstone & 18 & Fam. 12 \\
\hline 124 & Mledia Noce & 1854 & Weatherbit & is & Fam. 5b \\
\hline 120 年 & Electra & 1855 & Touchstone & 17 & $\begin{array}{l}4 \text { year-old first foal } \\
\text { Fam. } 2 \mathrm{e}\end{array}$ \\
\hline $126 i$ & Mare & 1855 & Melbourne & 18 & Fam. $5 n$ \\
\hline 127 & Emotion & 1856 & Alarm & 17 & Fam. 1 a \\
\hline 128 & Amethyst & 1857 & Touchstone & 18 & Fan. 11 \\
\hline 129 & Codicil & 1857 & The Cossack & 19 & Fam. $6 a$ \\
\hline 130 & Emm: & 1857 & Storm & 18 & $\begin{array}{l}3 \text { year-old first foal } \\
\text { Fam. } 19\end{array}$ \\
\hline 131 & Lambda & 1857 & I'mbriel & 17 & Fanı. 19 \\
\hline 132 & Spring Blossom & 18.57 & Touchstone & 17 & $\begin{array}{l}4 \text { year-old first foal } \\
\text { Fam. } 14\end{array}$ \\
\hline
\end{tabular}




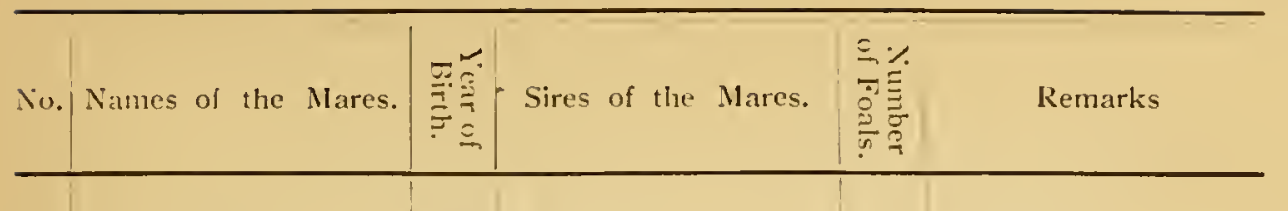

\begin{tabular}{|c|c|c|c|c|c|}
\hline 133 & Charmione & $18 \bar{s}$ & Oriando & 17 & Fam. $2 \mathrm{c}$ \\
\hline 134 & 1.ady Blanche & $14: 58$ & Voltigeur & 17 & Fam. $2 \mathrm{~d}$ \\
\hline 135 & Miss lanny & 1858 & Vanderdeclien & 19 & Fant. 8 \\
\hline 136 & Tartlet & $1 \sin$ & Birdcatcher & 19 & Fan. 21 \\
\hline 137 & Vergiss . Mein niclut & 18.53 & Flying Dutchman & 17 & Fan. 9 \\
\hline 138 & 1)ewdrop & 1859 & Mildew & 19 & $\begin{array}{l}4 \text { year-old first loal } \\
\text { Fan?. } 8 \text { a }\end{array}$ \\
\hline 139 & Entremet & 1859 & Sweetmeat & 17 & Fam. $2 \mathrm{e}$ \\
\hline 140 & . Nare & 1159 & Lambton & 18 & $\begin{array}{l}4 \text { year-old first foal } \\
\text { Not in Goosschen } \\
\text { Table } \\
\text { Frentzel Fam. } 83 \\
\text { Fam. 2a }\end{array}$ \\
\hline 142 & Pompadour & 18,59 & Stockwell & 18 & $\begin{array}{l}4 \text { year-old first foal } \\
\text { Fam. } 2 \mathrm{e}\end{array}$ \\
\hline 143 & Mare & $185 ; !$ & Y. Meibourne & 17 & Fam. 9 \\
\hline 14 & Imaranth & 1860 & Newninster & 17 & $\begin{array}{l}3 \text { year-old first foal } \\
\text { Fam. } 20\end{array}$ \\
\hline 145 & Bonny Bell & ISti) & Voltigeur & 17 & Fam. 10 \\
\hline 146 & Crucifixion & 1860 & Pelion & 17 & Fam. 27 \\
\hline 147 & Last l.tive & 1830 & Innandale & 18 & $\begin{array}{l}4 \text { year-old first foal } \\
\text { Fam. } 4\end{array}$ \\
\hline 148 & Novara & $1860)$ & Weatherbit & 19 & Fam. ㄴ \\
\hline 149 & Old Orange Girl & 1860 & kingston & 17 & Fam. 7 \\
\hline 150 & Pimpernel & 1860 & Sweetmeat & 17 & Fam. $2 \mathrm{c}$ \\
\hline 151 & The Doe & 1861 & Turnus & 18 & Fanı. 8 \\
\hline 152 & Mystery & 1861 & .lugur & 17 & Fan. 2e \\
\hline 153 & Tomfoolery & 1861 & King Tom & 17 & Fam. 2 \\
\hline 154 & Nare & 1861 & Wild Dayrell & 17 & $\begin{array}{l}4 \text { year-old first toal } \\
\text { Fam. } 3 \mathrm{~b}\end{array}$ \\
\hline 15 & Agile & 1862 & Gibraltar & 17 & $\begin{array}{l}4 \text { year-old first loal } \\
\text { Fam. } 3\end{array}$ \\
\hline 156 & Lad! Charlotte & 1862 & Fiandango & 17 & $\begin{array}{l}4 \text { year-old first foal } \\
\text { Fam. } 8\end{array}$ \\
\hline 157 & Money Spinner & $1 \times 5$ & Marsyas & 17 & $\begin{array}{l}4 \text { year-old first foal } \\
\text { Fam. } 16\end{array}$ \\
\hline 155 & Danish Rose & $18(i 3)$ & Surplice & 17 & $\begin{array}{l}4 \text { year-old first foal } \\
\text { Fam. } 12\end{array}$ \\
\hline 1.59 & Delilalı & 1864 & Thormanby & 18 & $\begin{array}{l}4 \text { year-old first foal } \\
\text { Fam. 1c }\end{array}$ \\
\hline 160 & Laur:t & 1861 & J.ambton & 18 & Fam. 17 \\
\hline 161 & Lina & 1861 & Stockwell & 18 & $\begin{array}{l}4 \text { year-old first foal } \\
\text { Fam. } 7\end{array}$ \\
\hline 162 & Murcia & 18601 & T.ord of the Isles & In & $\begin{array}{l}4 \text { year-old first foal } \\
\text { Fam. } 6\end{array}$ \\
\hline
\end{tabular}




\begin{tabular}{|c|c|c|c|c|c|}
\hline $\mathrm{N}_{11}$. & Names of the Mares. & 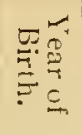 & Sires of the Mares. & 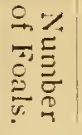 & Remarlis \\
\hline 1163 & Ninna & $186 t$ & Buccaneer & 18 & $\begin{array}{l}3 \text { year-old first foal } \\
\text { Fam. } 11\end{array}$ \\
\hline 164 & Queen Esther & 1864 & Warlock & 22 & $\begin{array}{l}4 \text { year-old first foal } \\
\text { fam. } 4 c\end{array}$ \\
\hline 165 & Perea & 1865 & Voltigeur & 17 & Fam. 3 \\
\hline 1608 & Siluria & 1865 & Caracticus & 17 & Fam. 1 a \\
\hline 167 & The Splyynx & 1865 & Newminster & 18 & Fam. 2 a \\
\hline 168 & Thrift & 1865 & Stockwell & 17 & $\begin{array}{l}4 \text { year-old first foal } \\
\text { Fam. } 10\end{array}$ \\
\hline J69 & Jenny Diver & 18606 & Buceaneer & 20 & $\begin{array}{l}4 \text { year-old first foal } \\
\text { Fam. } 20\end{array}$ \\
\hline 170 & Night jar & 1860 & Wild 1)ayrell & $19 ?$ & Fam. 15 \\
\hline 171 & Cestus & 1867 & Newminster & 17 & Fam. $3 \mathrm{~b}$ \\
\hline 172 & l'erformer & 1867 & Idventurer & 17 & $\begin{array}{l}4 \text { year-old first foal } \\
\text { Fam. I c }\end{array}$ \\
\hline 173 & Canonical & $186 \mathrm{~s}$ & Cathedral & 20 & $\begin{array}{l}4 \text { year-old first foal } \\
\text { lam. } 11\end{array}$ \\
\hline 174 & Furiusa & 1868 & Orlando & 17 & Fam. 45 \\
\hline 175 & Lady Mortimer & 1865 & Kettledrum & 18 & $\begin{array}{l}4 \text { year-old first foal } \\
\text { liam. } 10\end{array}$ \\
\hline 170 & Claretto & 1869 & Claret & 19 & $\begin{array}{l}4 \text { year-old first foal } \\
\text { Fam. } 25\end{array}$ \\
\hline 177 & liva & 1869 & Breadalbane & 17 & $\begin{array}{l}4 \text { year-old first foal } \\
\text { Fam. } 2 \text { e }\end{array}$ \\
\hline 178 & Wee Lassie & 1869 & Scottish Chief & 18 & Fam. 2 a \\
\hline 179 & True Blue & 1570 & Oxford & 20) & l⿳am. 32 \\
\hline 180 & Cherry Duchess & 1571 & The Duke & 18 & Fam. 27 \\
\hline 181 & Mirror & 1571 & Speculum & 17 & Fam. 49 \\
\hline 182 & Pillage & 1871 & Cambuscan & 19 & $\begin{array}{l}4 \text { year-old first foal } \\
\text { Fam. } 8\end{array}$ \\
\hline 183 & Pomona & 1871 & Slirmisher & 18 & lFam. 19 \\
\hline 184 & Turn of the Tide & 1871 & Mandrake & 17 & Fam. 18 \\
\hline 185 & $\begin{array}{l}\text { Enigma } \\
\text { (Daughter of No. 167) }\end{array}$ & 1872 & The Rake & 17 & Fam. 2 a \\
\hline 186 & Katrine & 1872 & Blair Ithol & 17 & $\begin{array}{l}4 \text { year-old first foal } \\
\text { Fam. } 5 \mathrm{~b}\end{array}$ \\
\hline 187 & Katrine & 1873 & 13linkhoolie & 17 & $\begin{array}{l}4 \text { year-old first foal } \\
\text { Fam. } 9\end{array}$ \\
\hline 185 & Orchestra & 1573 & Trumpeter & 17 & $\begin{array}{l}4 \text { year-old first foal } \\
\text { Fam. } 12\end{array}$ \\
\hline 189 & St. Editha & 1873 & Kingley Vale & 18 & $\begin{array}{l}4 \text { year-old first foal } \\
\text { Fam. } 16\end{array}$ \\
\hline 190 & Stone Chat & 1873 & Adventurer & 17 & $\begin{array}{l}4 \text { year-old first foal } \\
\text { Fan. } 16\end{array}$ \\
\hline
\end{tabular}




\begin{tabular}{|c|c|c|c|c|c|}
\hline No. & Names of the Mlares. & 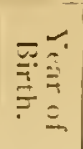 & Sires of the Mares. & 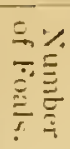 & Remarlss \\
\hline 191 & Miss Manmeringr & 1871 & Blair Ithol & 19 & $\begin{array}{l}\text { 4yeartold lirst loitl } \\
\text { liam. } 43\end{array}$ \\
\hline 192 & p'ilunflower & 1874 & The l'almer & 17 & J:am. 20 \\
\hline 193 & Shatemuc & 1871 & Oxford or The Dulse & is & $\begin{array}{l}4 \text { year-old first toul } \\
\text { Fam. } 2 \mathrm{~d}\end{array}$ \\
\hline 194 & leluni & 1870 & Rosicrucian & 18 & Fan. 4 \\
\hline 195 & Mrs. Knight & 1576 & Knight of the Garter & 17 & $\begin{array}{l}3 \text { yeatr-old first foal } \\
\text { Fam. } 4 \mathrm{~b}\end{array}$ \\
\hline $19 ;$ & Osimunda & $1570^{\circ}$ & Sterling & 17 & Jinm. 2 ล \\
\hline 1918 & Festival & 157 & Sterling & 17 & $\begin{array}{l}4 \text { year-old first foal } \\
\text { Fam. } 1 \mathrm{~b}\end{array}$ \\
\hline 198 & Jlluminata & 1877 & Rosicrucian & IS & $\begin{array}{l}4 \text { year-old first foal } \\
\text { liam. I c }\end{array}$ \\
\hline $19 n$ & Miss Mabel & ]87 & Finght of the Garter & 17 & Fom. 11 \\
\hline 2010 & l'ink Thorn & 1577 & Cathetral & 18 & $\begin{array}{l}\text { 4year-old first foal } \\
\text { Fam. In a }\end{array}$ \\
\hline 201 & Soplyy & 1575 & St. Mbans & 18 & $\begin{array}{l}4 \text { year-culd first foal } \\
\text { Fram. 12 }\end{array}$ \\
\hline 2() 2 & Celandine & 1578 & King Tom or Macaroni & 17 & liam. I c \\
\hline 203 & Eugrenie & 1878 & Pero Gomez & 17 & Fam. 16 \\
\hline 204 & Loving Cup & 1588 & Brown Bread & 17 & $\begin{array}{l}4 \text { year-uld first foal } \\
\text { Fanm. } 23\end{array}$ \\
\hline 205 & Rent Day & Ists & Macgrugor & 17 & $\begin{array}{l}4 \text { year-old first foill } \\
\text { Fam. } 3 \text { b }\end{array}$ \\
\hline $2(k)$ & True Love & 1878 & Sterlingr & Is & Fan1, 3c \\
\hline 207 & Bonny Rose & 1579 & Rosicrucian & is & f:am, 31 \\
\hline 2015 & Biserta & 1850 & Lord tyon & 17 & Finn. 22 \\
\hline 20,9 & Fisher Lass & Is'I & Exminster & 18 & $\begin{array}{l}4 \text { yertr-c)ld first foal } \\
\text { f:un. }\end{array}$ \\
\hline 210 & Hall Mark & 1850 & Standard & 17 & Fimll \\
\hline 211 & $\begin{array}{l}\text { Jennie Winkle } \\
\text { (I)aughter of No. 169) }\end{array}$ & 1880 & Mr. Winkle & 17 & $\begin{array}{l}4 \text { year-(1)d first foal } \\
\text { futm. } 20\end{array}$ \\
\hline 212 & 1) nn:mola & 1483 & Doncaster & 18 & $\begin{array}{l}\text { 4year-old lirst foal } \\
\text { Fam. } 6 \text { al }\end{array}$ \\
\hline 213 & Red Clove & $185: 3$ & Sterling & 17 & $\begin{array}{l}4 \text { year-old first foal } \\
\text { Fam. } 3 \mathrm{~b}\end{array}$ \\
\hline
\end{tabular}

I linow of no horse breed which can show, even approximately, stoh breeding merits as the English breed of Thoroughbreds, alcording to the above list. In Trakehnen only wo case's have taken place, in more than 100 years, in which one horse has given birth to 19 living foals. To the following list I have to add that wo Thoroughbred dams with 18 foals each are net mentioned. 
List of brood Mares in Trakehnen laving produced 17 and more living foals.

\begin{tabular}{|c|c|c|c|c|}
\hline No. & Names of the Dams. & Lorn. & Sires of the Dams. & $\begin{array}{l}\text { Number } \\
\text { of Foals. }\end{array}$ \\
\hline 1 & 1.enma & 1810 & Oronocco I & 17 \\
\hline 2 & 1)uellona & 1814 & Rodrich & 17 \\
\hline 3 & Destemona & 1815 & Caril & 17 \\
\hline 4 & Junia & 181: & Rodrich & 17 \\
\hline 5 & Bazilia & 1817 & Caril & 18 \\
\hline 6 & Crab & 1818 & Oronocco 1 & 18 \\
\hline$\tau$ & Czarina & 1818 & Oronocco 11 & 17 \\
\hline 8 & Zoraide & 1818 & Rodrich & 18 \\
\hline 9 & Anta & 1819 & Pretender & 19 \\
\hline 10 & Arcade & 1819 & Pretender & 18 \\
\hline 11 & Persienne & 1819 & Teheran ox & 19 \\
\hline 12 & Ingloise & 1820 & Scrapall $\mathrm{xx}$ & 17 \\
\hline 13 & Litima & 1820 & Blackamoor $\mathrm{xx}$ & 17 \\
\hline $1 t$ & Victory & $18 \div 0$ & Blackamoor $\mathrm{xx}$ & 18 \\
\hline 15 & Doris & 1821 & Scrapall $x x$ & 17 \\
\hline 16 & Nasora & $18 * 2$ & Eminlik ox & 18 \\
\hline 17 & Olympia & 1823 & Blackamoor $\mathrm{xx}$ & 17 \\
\hline 18 & Collina & 1820 & Trafalgar & 17 \\
\hline 19 & Alzire & 1828 & The Cryer $\mathrm{xx}$ & 17 \\
\hline 21 & Hipponome & 1834 & Acarnas & 17 \\
\hline 21 & Jupine & $18: 34$ & Sterling $1 \mathrm{~V}$ & 17 \\
\hline x) & Medina & $18: 38$ & Big Ben $x x$ & 17 \\
\hline 3 & Vecordia & $18: 39$ & Black Hambleton xx & 17 \\
\hline 24 & Intha & $185 \%$ & Paragone & 17 \\
\hline 25 & Perina & 1561 & Promoter & 18 \\
\hline 26 & Injurie & 1863 & Venerato & 17 \\
\hline 27 & Lava & 1863 & Grezano & 17 \\
\hline 28 & Herz & 1866 & Duplicat & 17 \\
\hline 29 & Harmonica & 1874 & Journey & 17 \\
\hline 30 & Atella & 1883 & Flügel & 17 \\
\hline
\end{tabular}

In order to fully appreciate the work of Thoroughbreds not attained also in Trakehnen, as regards the living born foals of one dam, I add a list of brood mares, of Half-breds having produced 17 and more living foals.

I. Beberbeck. Stud Book, Volume 1. 312 brood mares.

1. Lolly 1862 by Hipparch . . . . . 17 foals.

2. Custozza 1867 by Thanatos . . . . . 17 ",

3. Gurly 1868 by J. Harlequin . . . . 18 ,"

II. Ostpreussen. Stud Book, Volume IV. 4.060 brood mares.

1. Kunzine 1881 by Mercos No. 4868 . . . 17 foals.

2. Granate 1882 by Dativ No. 2763 . . . . 17 ,

3. Viva 1883 by Vivat No. 2073 . . . . . 17 ,

Besides these only 11 dams which had 16 foals. 
[I1. IIannover. Stud Book, Voltume III. 3,5.27 brood mares.

1. Godelinde 1872 by Goldoni No. 2399 . . 18 foals.

2. Numana 1873 by Nord No. 107 . . . 18 ,,

The superiority of the English Thoroughbred over all Half-breds and draft breeds is perhaps shown still more clearly from its special work at a great age, or under difficult circumstances, as can be seen from the following "xamples. I have to add that there are numerous cases in which Eaglish Thoroughbred mares 22 to 29 years old have still brought forth living foals. In the following list only such cases are specially menlioned where the offspring of these aged mares have shown themselves to be really good horses, either on the racecumse or in the stud.

Special Breeding Performances of Old Thoroughbred Nares in England.

No. Name and Pedigree. Born. Breeding Performances.

1 Brown Farwell by. Matheless-Brimmer.

2 Sister to Sampson by Greyhound - Curwen Bay Barb

3 Little Ilartles Mare by: Bartlett's Childers out of Flying Whigg.

1 Look at Me Lads by Grasshopper - Newton's Bay Arabian.

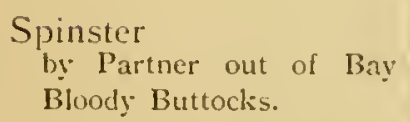

1710 16 foats, 7 of which were Pedigree Mares in Fann. 4. One born in the 20th year (had 16 foals); one in its 21st year (dam of Sweepstakes); one in the 22nd rear (dam of Dormouse); and one in the 25th year (dam of Matchem).

1723 20 foals. In its 26th year Sally, a good racer, 4 to 6 years, and a good Brood Mare (11 foals).

13 foals, of which were: Tortoise, Janus, IBlank, Old England, Trimmer, Shakespeare (according to Lawrence sire of Eclipse). In its $241 \mathrm{~h}$ ve:tr Miss Meredith, Foundation Mare in Fam. 15, and in its 25th year, a mare with frood progeny.

Is a 6 year-old, at Newmarket, King's Plates, ran, not placed. Had first foal when 20) years of age. 12 foals, amongst which were: in its 21 st year, Lottery by Blank; at 24 it matre by . Incastor Sterling. Foundation Mare of Fom. 14; at 30, Whisper by Blank, a grood racer; at 32, Amazon by Blank, a good racer and broed mare; at 33 its last foal.

Won the King's Plates as a 5 and 6 yearold at Newmarket. First foal at 8 years of age; Spinster (Foundation Mare in Fam. 4), 11 fouts. At 2.5, Golden Grove, liuundation Mare of Fam. id. 


\begin{tabular}{|c|c|}
\hline No. & Name and Pedigree. \\
\hline 6 & $\begin{array}{l}\text { Sister to Regulus } \\
\text { by Godolphin Arabian out } \\
\text { of Grey Robinson. }\end{array}$ \\
\hline 7 & $\begin{array}{l}\text { Miss WVillinson } \\
\text { By Regulus out of Miss } \\
\text { Layton. }\end{array}$ \\
\hline $\mathrm{s}$ & $\begin{array}{l}\text { Duchess } \\
\text { by Whitenose out of } 19 \\
\text { year-old Miss Slamerkin. }\end{array}$ \\
\hline 9 & $\begin{array}{l}\text { Spiletta } \\
\text { by Regulus out of Mother } \\
\text { Western. }\end{array}$ \\
\hline 10 & $\begin{array}{l}\text { Cypron } \\
\text { by Blaze out of Salome. }\end{array}$ \\
\hline 11 & $\begin{array}{l}\text { Pratt's Old Mare } \\
\text { by Squirt-Mogul. }\end{array}$ \\
\hline
\end{tabular}

12 Mare by Rib out of Mother IVestern.

Polly by Black and All Black out of Fanny.

Mare (chestnut) by Tartar-Mogul.

Calliope by Sloutch out of Lass of the Mill.
Breeding Performances. racer. Foundation Mare in Fam. 43.

16 foals, of which were: at 23, Grey Mare by Snap, Foundation Nare in Fam. 11 (Birdcatcher); and at 27, Mussulman, a famous

Won a race as a 4 yeár-old. First foal when 8 years old. 10 foals. At 25, Mare by Syphon,

Ran 1-7 year-old 16 times and won 12 times. First foal at 9 years old. At 23 , Pyrrha, Foundation llate in Fan. 7 , which had Beatrice when 20 years old, dam of 17 foals.

Not run. First foal at 10 years old. At 23 , Garrick (own brother to Eclipse), and at 25 , Briseis.

Not run. First foal at 5 years old. 16 foals without a break, amongst which was Herod.

Not run. 17 foals, amongst which were 12 good racers. At 24 Purity (by the 25 year-old (Iatchem), dam of Rockingham and 12 other foals. Lived to 27 years old. Fam. 24.

Not run. Five grey foals, amongst which, at 24 , was the Foundation Mare of Marsyas, Kingston, Archibald, Hermit, etc. Fam. 12.

3 year-old ran once. First foal at 8 years old. 14 foals, amongst which were: King Fergus, at 20; Fanny, Foundation Mare in Fanı. 6; and at 25, Cast-off (a good racer).

Did not run. First foal at 6 years old. 16 foals, amongst which were: 10 chesnuts by Eclipse; at 21, Mercury; at 23, Volunteer ; at 28, Queen Mab, a famous Foundation Mare in Fann. 9, with 16 foals, amongst which were Remembrancer L., and at 20, Remembrance, Foundation Mare in Fam. 9.

4-6 year-old ran six times, won twice. First foal at 7 years old. 10 foals, of which were : at 18 , Omphale L.; at 20 , Sir John Falstaff, a grood racer; and at 25, a Mare by Phonomenon, that gave birth to living twins, amongst which a Foundation Mare in Fam. "2 


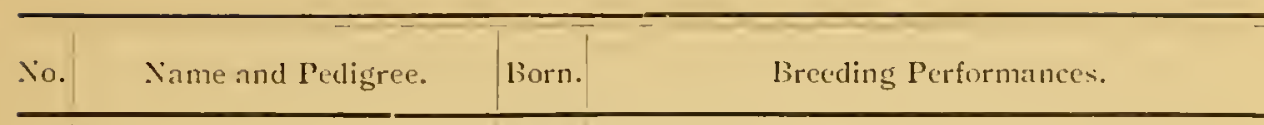

16

Jö

by Spectator-Blank.

17. Mother Brown

by Trunnion-Old England.
18

Crpher

by Squirrel out of 23 yearold Mlare by Regulus.

19) Termagant

by Tantrum out of Cantatrice.

20

Mare

by Siphon out of 25 yearold Miss Willinson.

21 Calash by Herad out of Teresa.

20

Cälia

by Herod out of Proserpine (own sister to Eclipse).

23 Miss Ilervey by Eclipse out of Clio.

21 Fanny by Eclipse out of 20 yearold Polly.
1767 Ran as a 3 and 4 year-old four times, won once. First foal at 11 years old. 10 foals. .It 20 , Patriot, a very fine racer, winning 28 year-old.

$17 \pi 1$ 4-12 year-old ran 39 races and won 20. Is 8 year-old, and in foal, won il race wer 4 miles. As a 9 vear-old, after foaling, ran again four times and won four times (amongst which was one King's Plates). Is a 10 yearold ran six times, winning five times (one Fing's Plates). As an 11 year-old ran six times, winning twice. Is a 12 year-old ran once, not placed. Had 6 foals. .It 21 , Toby, a sood racer, in Ireland; at 2j, Jerry Sneak, a splendid racer in Ireland, won as a 13 yearold, and was the sire of many crood racehorses: at 26. Mare by Bagrot, grandmother of Nabocklish, Fam. 4. Was about 30 years old when it had last foal.

4 rear-old ran once. 12 foals. 3 Foundation Mares in Fam. 3 a. At 24, Nfred by John Bull.

First foal at 12 years old (Edmund and in L). 11 foals by 10 different stallions. It 19 , Evelina, dam of Orville, Paulowitz, and Cervantes; at 27, Sparrow Hawk (3rd in 1)).

Did not run. First foal at 10 years old. 16 foals without a break, last foal in itw 2uth year. loundation . Mare of Fam. 43.

4 year-old ran six times, winning fwe times. löirst foal at 8 years old. 11 foals, amonngst which as Firstling Paragon I, at 1.4, Whislicy; and at 24, Lady Charlotte, dam of Cwrw 2.

1775

3 year-old ran once. 14 foals. it 23 , lighland Fling by Spadille.

3 and 4 year-old ran eight times, won twice. First fonl at 8 years old. 13 foals. It 22. Haphazard by Sir Peter, and at 23, a Mare by Sir l'eter lioundation Mare of liam. 3.j.

Not run. First fonl at 11 years old. 14 foals, of which was Volante O., l'om. 6. 
No. Nanme and Pedigree.

25 . Ilare

bỹ Natchem (28 year-old) -Siphon.

36 Horatia

by Eclipse out of 28 yearold Countess.

27 Camilla

by Trentliam out of

Coquette.

28 Expectation

by Herod out of 21 yearold Mare by Slim.

Faith

by. Pacolet out of

Atalanta.

30

\section{Luna}

by. Herod out of

Proserpine.

3 Maria

by Telemachus out of 20 year-old A-la-Grecque.

:32. Nimble

by Florizel out of Rantipole.

:3 Pewet L.

by Tandem out of Termasant (No. 19)

34 Prunella

by Highflyer out of

Promise.
1787

3 rear-old ran seren times, won once. 18 foals, of which were: Eager D, Fidget, Bustler; at 24, Professor (ran twice as a 3 year-old); at 25. Sinbad (later Rainbow, ran as a 3 and 4 year-old four times, won twice); and at $2 \overline{7}$, Frn (ran once as a 3 year-old, and had $\&$ foals). At 32 last foal.

3-5year-old ran nine times, won three times. First foal at $\&$ rears old. 15 foals. At 19. Archduke D, and at 25, Paris D.

3-6 year-old ran seventeen times, won six times. First foal at 9 years old : Y. Camilla, a famous Foundation Mare in Fam. 11. 12 foals, at 25. Jerboa, Foundation Mlare of Venison, The Little Wonder D, St. Simon, Orme, etc.

5 year-old ran twice. Fïst foal at 8 years old. Spinster, Foundation Mare in Beberbecls. 13 foals. At 23, Anticipation, Foundation Mare in Fanı. 4 (Executrix, Vanessa, Manganese, Alice Hawthorn, etc.).

1779 Ran and won $3-7$ year-old, ran as 8 yearold, not winning. First foal at 11 years old. 9 foals. At 18, Nlarcia ; at 22, Vesta, Foundation Nares in Fam. 2 a at 24 . Camillus.

Not run. First foal at $\&$ (?) years old. 14 foals. It 24, Cardinal Puff by Cardinal.

3 and 4 year-old ran eight times, won twice. First foal at 8 years old. 10 foals. At 15 , Marianne, dam of Octavius D and Caroline $\mathrm{O}$; at 22. Narybella, lioundation Nare in Fam. 23 ; and at 24, Breslaw, a famous racer.

2-6 year-old ran 36 times, won 19 times. First foal at 7 years old. 17 foals, of which were once twins, Nike $O$, and at 23, Donna Clara, Foundation Nlare for Fan. 32.

$3-6$ year-old ran thirteen times, won four times. First foal at 8 years old. 9 foals. At 16. Sir Paul; at 18, Pauline L; and at 26, Clinkerina (dam of II umphrey Clinker).

3-6 year-old ran eleven times, won three times. First foal at 8 years old. 12 foals, of which 9 were very fine. At 23, Prudence, Foundation Mare in Fam. 1 a. 


\begin{tabular}{|c|c|c|c|}
\hline No. & Xime and Pedigree. & Born. & Breeding l'erfurmance. \\
\hline 35 & $\begin{array}{l}\text { Sister to Silver } \\
\text { by Mercury-Herod. }\end{array}$ & 1790 & 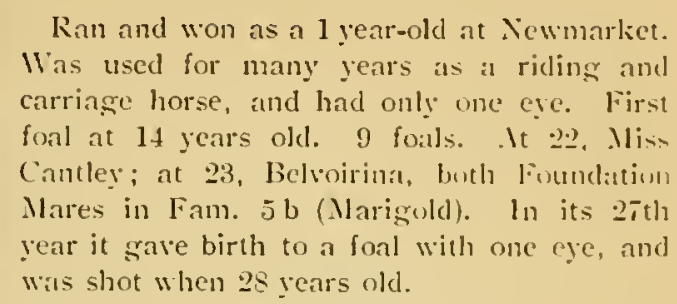 \\
\hline 36 & $\begin{array}{l}\text { Evelina } \\
\text { by Highnyer out of Ter- } \\
\text { magant (19 year-old). }\end{array}$ & 1791 & $\begin{array}{l}\text { 3-jyear-old ran eight times, won threw } \\
\text { times. First foal at } 7 \text { years old. 12 foals. . It } \\
\text { 22, Paulowitz by Sil Patul. }\end{array}$ \\
\hline 37 & $\begin{array}{l}\text { Platina } 0 \text {. } \\
\text { by Mercury-Herod (own } \\
\text { sister to } .0 .33 \text { ). }\end{array}$ & 1792 & $\begin{array}{l}\text { Ran once as a } 3 \text { and once as it } 4 \text { year-uld, } 0 \text {. } \\
\text { First foal at } 5 \text { years old. } 19 \text { foils. It } 2 J \text {. } \\
\text { Adclicia (ran as a } 2 \text { year-old and became a } \\
\text { good brood mare); at } 27 \text {, Aliarius (a very rood } \\
\text { racer } 2-4 \text { year-old, won (wice as a year-old, } \\
\text { was not placed in the } 5 \text {. Leger), and lived to } \\
28 \text { years of age. }\end{array}$ \\
\hline 38 & $\begin{array}{l}\text { Mare } \\
\text { by Potsos out of Editha. }\end{array}$ & 1794 & $\begin{array}{l}\text { Not run. First foal at } 7 \text { years old. } 12 \text { foals, } \\
\text { once dead twins. At } 24 \text {, the srandmother of } \\
\text { Neville, Fam. } 3 \text { c. }\end{array}$ \\
\hline $3 ! 1$ & $\begin{array}{l}\text { Mare } \\
\text { by Slivscaper out of } \\
\text { Isabel. }\end{array}$ & 1795 & $\begin{array}{l}\text { Not run. } 17 \text { foals. It } 25 \text {. Wisentere, a good } \\
\text { racer. }\end{array}$ \\
\hline 40 & $\begin{array}{l}\text { Mare } \\
\text { by Precipitate - Mood. } \\
\text { pecker. }\end{array}$ & 1796 & $\begin{array}{l}\text { Not run. First foal at } 8 \text { years old. } 10 \text { foals. } \\
\text { It } 25 \text {. Monimia, a good lateer and Foundation } \\
\text { Mare in Fam. } 12 \text { a; at } 27 \text {, The ('aptain, a } \\
\text { grood racer. }\end{array}$ \\
\hline 41 & $\begin{array}{l}\text { Mare } \\
\text { by Ruler out of Tree- } \\
\text { creeper. }\end{array}$ & 1797 & $\begin{array}{l}\text { Ran three times as a } 3 \text { year-old. First foal } \\
\text { at } 12 \text { years old, Oiseau by Camillus. } 13 \text { foals. } \\
\text { At } 25, \text { Miss Crachami, a good racer, and } \\
\text { Foundation Mare in Fam, } 42 \text {. }\end{array}$ \\
\hline 42 & $\begin{array}{l}\text { Y. Lalage } \\
\text { by Cloncolate out of } 21 \\
\text { year-old Lalage, which } \\
4-12 \text { year-old won races. }\end{array}$ & 1797 & $\begin{array}{l}\text { Ran four times as a } 2 \text { and } 3 \text { yeatrold, won } \\
\text { once. } 11 \text { foals. It } 2-1 \text {, l) Vere by feramor } \\
\text { a famous racehorse in lreland. }\end{array}$ \\
\hline 43 & $\begin{array}{l}\text { Marcia } \\
\text { by Coriander out of liaith } \\
(3-i) \text {. }\end{array}$ & 1797 & 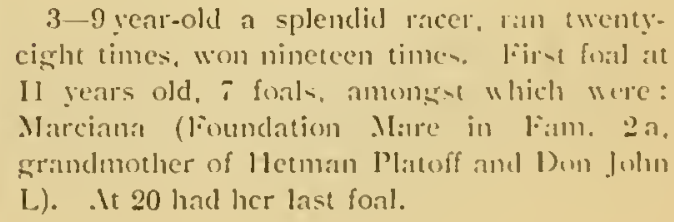 \\
\hline
\end{tabular}




\begin{tabular}{|c|c|c|c|}
\hline No. & Name and Pedigree. & Born. & Breeding Performances. \\
\hline$H$ & $\begin{array}{l}\text { Rosamond } \\
\text { by Buzzard out of Rose- } \\
\text { berry. }\end{array}$ & 1798 & $\begin{array}{l}3 \text { and } 4 \text { year-old a good racer, ran nine } \\
\text { times, won three times. First foal at } 6 \text { years } \\
\text { old. } 17 \text { foals. At } 22 \text {, Barefoot } L \text {, and } 26 \text {, a } \\
\text { Mlare by Tramp, Foundation Mare in Fann. } 5 \text { a. } \\
\text { lived to } 31 \text { years of age. }\end{array}$ \\
\hline 4.5 & $\begin{array}{l}\text { Mandane } \\
\text { by Potsos nut of } \mathrm{Y} \text {. } \\
\text { Camilla. }\end{array}$ & 1800 & $\begin{array}{l}\text { Ran six times as a } 2 \text { and } 3 \text { year-old, won } \\
\text { I wice. First foal at } 4 \text { years old. } 13 \text { foals, } \\
\text { amongst which were: Mannella } O \text {, Altisidora } \\
\text { L; at } 20 \text {, Lottery by Tramp; at } 21 \text {, Brutan- } \\
\text { dorf by Blacklock; and at } 22 \text {, a Mare by } \\
\text { W'hisker, dam of Liverpool. Lived to } 26 \text { years } \\
\text { of age. }\end{array}$ \\
\hline 46 & $\begin{array}{l}\text { Parasol } \\
\text { by Potsos out of Prun- } \\
\text { clin (No. 34) }\end{array}$ & 1800 & $\begin{array}{l}\text { Ran and won } 3-8 \text { year-old, at } 8 \text { years in } \\
\text { foal, ran four times and won twice. First foal } \\
\text { at } 9 \text { years old. } 12 \text { foals, amongst which were: } \\
\text { Partisan, Pindarri } 2 \text {; at } 19 \text {. Pastille } 2,0 \text {; and } \\
\text { at } 24 \text { her last foal. }\end{array}$ \\
\hline 47 & $\begin{array}{l}\text { Mare } \\
\text { by Delpini out of Tipple } \\
\text { Cyder. }\end{array}$ & $180^{\circ 2}$ & $\begin{array}{l}\text { Not run. lirst foal at } 6 \text { years old. } 15 \text { foals. } \\
\text { At } 20 \text {, Wildwood, and at } 21 \text {, Sir Calton, both } \\
\text { good racers; at } 23 \text {, The Colonel L, and at } 24 \text {. } \\
\text { The Captain, a famous racer. }\end{array}$ \\
\hline 48 & $\begin{array}{l}\text { Thomasina } \\
\text { by Timothy out of Violet. }\end{array}$ & 1804 & $\begin{array}{l}\text { A very good racer } 2-5 \text { year-old. First foal } \\
\text { at } 8 \text { pears old. } 16 \text { foals without a break. }\end{array}$ \\
\hline 49 & $\begin{array}{l}\text { Remembrance } \\
\text { by Sir Solomon out of } \\
20 \text { ycar-old Queen Mab. }\end{array}$ & 1805 & $\begin{array}{l}\text { A very good racer } 3-5 \text { ycar-old. First foal } \\
\text { at } 8 \text { years old. } 13 \text { foals. At } 26 \text {, Oblivion, dam } \\
\text { of Springy Jack and grand-dam of Daniel } \\
\text { O'Rourlse D, Foundation Mare in lim. } 9 \text {. }\end{array}$ \\
\hline .0 & $\begin{array}{l}\text { Liftle Fully } \\
\text { by llighland Fling out of } \\
\text { Harriet. }\end{array}$ & I 800 & $\begin{array}{l}\text { Ran three times as a } 3 \text { year-old, won once. } \\
\text { First foal at } 6 \text { years old. } 13 \text { foals. At } 24 \text {, } \\
\text { Folly, foundation Mare in Fam. I (Miami, } \\
\text { Rosicrucian, etc.). }\end{array}$ \\
\hline 51 & $\begin{array}{l}\text { Liselfe } \\
\text { by Hanbletonian out of } \\
\text { Constantia. }\end{array}$ & 1806 & $\begin{array}{l}\text { A grood racer } 3 \text { and } 4 \text { year-old. lirst foal at } \\
7 \text { years old. } 14 \text { foals. At } 24 \text {, Clearwell } 2 \text {. }\end{array}$ \\
\hline 52 & $\begin{array}{l}\text { Snowdrop } \\
\text { by Ilighland Fling out of } \\
\text { Daisy. }\end{array}$ & 1806 & $\begin{array}{l}\text { A good racer } 2-4 \text { year-old. lirst foal at } i \\
\text { years old. } 11 \text { foals. At } 22 \text {, Galantine } 1 \text {. }\end{array}$ \\
\hline 53 & $\begin{array}{l}\text { Miss IVasp } \\
\text { by IIay out of Trum- } \\
\text { petta. }\end{array}$ & 1807 & $\begin{array}{l}\text { Won as a } 2 \text { year-old. First foal at } 7 \text { years } \\
\text { old. At } 21 \text {, Y. Muley, and at } 23 \text {, Vespa } O \text {. }\end{array}$ \\
\hline 54 & $\begin{array}{l}\text { Mare } \\
\text { by Walton out of } 19 \text { year- } \\
\text { old Y. Noisette. }\end{array}$ & 1808 & $\begin{array}{l}\text { Not run. First foal at } 10 \text { year-old. } 11 \text { foals. } \\
\text { At } 23 \text {, Black Agnes, Foundation Mare in Fam. } \\
\text { 23. }\end{array}$ \\
\hline
\end{tabular}




\begin{tabular}{|c|c|c|c|}
\hline No. & Dame and Pedigree. & | Born. & Breeding Performances. \\
\hline jij & $\begin{array}{l}\text { Manuclla } O \text {. } \\
\text { by Dick Andrews out of } \\
\text { Mlandane (No. 45). }\end{array}$ & $1 S 09$ & $\begin{array}{l}\text { 3-5year-old min thirteen tines, won three } \\
\text { times. First foal at } 8 \text { years old. } 15 \text { foals with- } \\
\text { out a break, amongst which were: Memnon } \\
\text { L, Belzoni, Margelina (had } 19 \text { foals); at } 20 \text {, } \\
\text { Nitocris, loundation Mare in linm. } 11 \text { (1\%isher- } \\
\text { man, Strathcona, etc.); at } 21 \text { and } 22 \text {, two good } \\
\text { racers, Belshazzal and Belluno. }\end{array}$ \\
\hline 56 & $\begin{array}{l}\text { Lady of the Lake } \\
\text { by Sorcerer out of } 20 \\
\text { year-old Mare by Salt- } \\
\text { ram. }\end{array}$ & 1809 & $\begin{array}{l}3-4 \text { year-old a good racer. First foal at } 6 \\
\text { rears old. } 17 \text { foals. At } 22 \text {, Ophelia, Founda- } \\
\text { tion Nare in Fam. } 43 \text {, and last foal at } 27 \text { years } \\
\text { old. }\end{array}$ \\
\hline ji & $\begin{array}{l}\text { Bella } \\
\text { by Beningbrough out of } \\
\text { Peterea. }\end{array}$ & 1811 & $\begin{array}{l}\text { Ran twice as a } 6 \text { year-old, won once. First } \\
\text { foal at } 8 \text { rears old. } 13 \text { foals. At } 24 \text {, Bellona, } \\
\text { Foundation Mare of the liam. } 4 \text {; at } 25 \text {, last } \\
\text { foat, ran in race. }\end{array}$ \\
\hline is & $\begin{array}{l}\text { Wire (sister to Thale- } \\
\text { bone) } \\
\text { by llaxy out of Penelope. }\end{array}$ & 1811 & $\begin{array}{l}3 \text { and } 4 \text { year-old a good racer. lirist foal at } \\
8 \text { years old. } 17 \text { foals. At } 22 \text {, Verulam; at } 26 \text {, } \\
\text { her last foal. Lived to } 28 \text { years of age. }\end{array}$ \\
\hline 59 & $\begin{array}{l}\text { Mare } \\
\text { by Orville out of Miss } \\
\text { Grimstone. }\end{array}$ & 1812 & $\begin{array}{l}\text { Not run. } 14 \text { foals, amongst which were: } \\
\text { Tranby by Blacklock; at } 22 \text {, Miss Bowe, and } \\
\text { at } 25 \text {, a Mare by Priam or Zinganee, the two } \\
\text { chief Foundation Mares in Fam. } 21 \text {. }\end{array}$ \\
\hline 60 & $\begin{array}{l}\text { Sister to Corduroy } \\
\text { by Shuttle out of } 18 \text { year- } \\
\text { old landy Sarah. }\end{array}$ & 1812 & $\begin{array}{l}\text { Not run. First foal at } 4 \text { years old. } 20 \text { foals, } \\
\text { the first } 19 \text { without a breali, at } 24 \text {, the last foal } \\
\text { by } 1 \text { Waverley (ran as a } 2 \text { year-old, had } 11 \text { foals, } \\
\text { and became Foundation Mare in Fam. 5), and } \\
\text { lived to } 26 \text { years old. }\end{array}$ \\
\hline 61 & $\begin{array}{l}\text { Octariana } \\
\text { bỹ Octarian_-Shuttle. }\end{array}$ & 1815 & $\begin{array}{l}2 \text { and } 3 \text { year-old a good racer. First foal at } \\
5 \text { years old. } 15 \text { foals. At } 22 \text {. Crucifix O. 2. } 1 \text {, } \\
\text { dam of Surplice D. L. }\end{array}$ \\
\hline $6: 2$ & $\begin{array}{l}\text { Silvertail } \\
\text { by Y. Gohanna-Orville. }\end{array}$ & 1815 & $\begin{array}{l}3-4 \text { year-old a good racer. First foal at } 6 \\
\text { years old. } 15 \text { foals. At } 24 \text {, Bonney Bonnet, } \\
\text { Foundation } 2 \text { lare in lam. } 2 \text { d. }\end{array}$ \\
\hline 63 & $\begin{array}{l}\text { Mare } \\
\text { by Comus-Delpini. }\end{array}$ & 1816 & $\begin{array}{l}\text { Not run. Jirist foal at } 14 \text { years old. } 8 \text { fonls. } \\
\text { At } 22 \text {. Miss Lydia, Foundation Mare in Fan. } \\
9 \text {; at } 24 \text {, Nutwith L. }\end{array}$ \\
\hline 61 & $\begin{array}{l}\text { Lacerta } \\
\text { by Zodiac out of Jerboa. }\end{array}$ & 1816 & $\begin{array}{l}3-4 \text { yen-old moderate racer, then was used } \\
\text { as a riding horse. First foal at lo years old. } \\
15 \text { foals. At } 21 \text {, Little Wonder D, ind at } 25 \text {, } \\
\text { little liary, grand-dam of St. Angela, St. } \\
\text { Simon's dam, Foundation Mare in Fam. 11. }\end{array}$ \\
\hline
\end{tabular}




\begin{tabular}{l|l|}
\hline No. & Name and Pedigree. \\
\hline 65 & $\begin{array}{c}\text { Mare (Velocipede's } \\
\text { dam) } \\
\text { by Juniper-Sorcerer. }\end{array}$ \\
$\begin{array}{l}\text { Electress } \\
\text { by Election-Stamford. }\end{array}$ \\
67 & $\begin{array}{l}\text { Mare } \\
\text { by Rubens out of Tippity- } \\
\text { wichet. }\end{array}$ \\
$\begin{array}{l}\text { by Sultan out of Dulcinea. } \\
\text { Clari } \\
\text { by Smolenslio-- } \\
\text { Precipitate. } \\
\text { Guiccioli } \\
\text { by Bob Booty out of } \\
\text { Flight. }\end{array}$ \\
\end{tabular}

liorn. Breeding Performances.

1817 Not run. First foal at 4 years old. 18 foals, of which 7 were Foundation Mares in Fam. 3 c. It 24, Nerope, grand-dam of Galopin D, Moslem 2, and Knight of the Crescent.

1819 2-4 year-old a grood racer, once slipped twins. First foal at 9 years old. 11 foals. At 16 , Vauban; at 19, Miss Twiclienham (dam of Teddington); at 20, Nare by Sir Hercules (Foundation Mare in Fans. 2 and had 20 foals); at 22, Splitrote (grand-dam of St. Albans L, Savernake and Gang Forward 2); last foal at 24 years old.

18192 and 3 year-old ran twice, was second in $O$. First foal at 6 years old. 17 foals, amongst which were: Phosphorous D, May Day 1; at 20, Firebrand 1 ; and at 23, Ratafia, Foundation Mare in lian. 27.

18232 - 6 year-old a very good racer. Ran as a 6 year-old, after foaling and again in foal (produced a healthy foal), six times, won twice, in the ling's Plates, over 4 miles with heats, in September, in Curragh (6 starters), and in Northumberland Handicap in October, at Curragh (11 starters, amongst which were such good horses as Sliylarls, a 3 year-old, and Napoleon, a 5 year-old). First foal at 6 years old. 13 foals, amongst which were: Birdcatcher and Faugh-a-Ballagh; at 21 , living twins, one of which was Gramachree, Foundation Mare in Fam. 11; and at 24, St. John (later Carlow), a good racer $2-i$ year-old.

Not run. First foal at 8 years old. 17 foals without a breals. Foundation Nare in Fam. 1.

Not run. First foal at 5 years old: Clara, 2-5 year-old a good racer, and Foundation Mare in Fam. 6 a. The next $i$ foals were used in Ireland as riding horses. 13-18 she was a riding horse, and afterwards, $19-27$, she had $i$ more foals, the greater part of which were good racers. Altogether she had 10 foals, and lived to 29 years of age.

1826 2-4 year-old a very good racer. First foal at 8 years old. 13 foals. At 24 , Kernel, Foundation Mare in Fans. 6 a. 


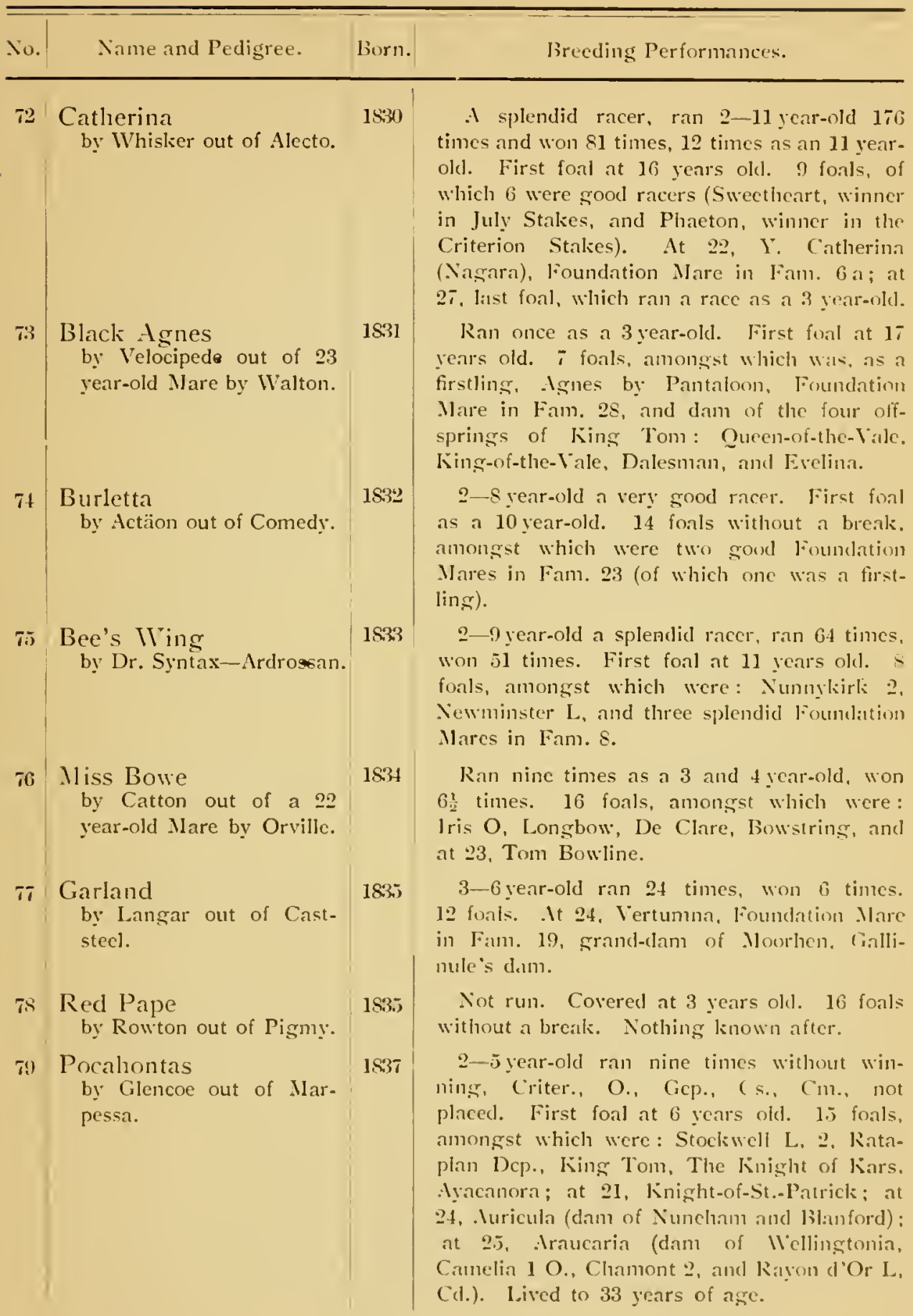




\begin{tabular}{|c|c|c|c|}
\hline No. & Name and Pedigree. & Born. & Breeding Performances. \\
\hline 80 & $\begin{array}{l}\text { Lapwing } \\
\text { by Bustard-Muley. }\end{array}$ & 1837 & $\begin{array}{l}\text { Nit run. Covered at } 2 \text { years old, had first } \\
10 \text { loals without a break. Altogether } 17 \text { foals, } \\
\text { the last in her } 29 \text { th year: Sir Niel. }\end{array}$ \\
\hline 81 & $\begin{array}{l}\text { Alice Hawthorn } \\
\text { by Muley Moloch out of } \\
\text { Rebecca. }\end{array}$ & 1838 & $\begin{array}{l}\text { 3-7 year-old a splendid racer, ran } 71 \text { times } \\
\text { and won } 50 \frac{1}{2} \text { times, which included } 17 \text { King's } \\
\text { Plates, Gcp and Dcp twice. First foal } \\
\text { at } 11 \text { years old, and had } 7 \text { loals without a } \\
\text { breals. Afterwards she slipped and had } 3 \text { more } \\
\text { foals, that is, } 10 \text { loals altogether, } 3 \text { splendid } \\
\text { Foundation Mares in Fam. 4c, of which were } \\
\text { Terrona (grand-dam of Queen Esther, which } \\
\text { had } 22 \text { foals); afterwards, after she had } \\
\text { slipped, at 19. Thom } \\
\text { Fauconberg. Oulston and Findon. and also Lord }\end{array}$ \\
\hline \$2 & $\begin{array}{l}\text { Mare } \\
\text { by Beiran out of Addy. }\end{array}$ & 1838 & $\begin{array}{l}\text { Not run. First foal at } 13 \text { years old. } 12 \\
\text { foals, amongst which was Christabelle, grand- } \\
\text { dam of Aspirant. Lived to } 27 \text { years of age. }\end{array}$ \\
\hline 83 & $\begin{array}{l}\text { Florence } \\
\text { by Velocipede out of Mar- } \\
\text { garette. }\end{array}$ & 1839 & $\begin{array}{l}2 \text { - } 3 \text { year-old ran ten times, won three times. } \\
\text { first loal at } 5 \text { years old. } 20 \text { foals without a } \\
\text { breals. }\end{array}$ \\
\hline 84 & $\begin{array}{l}\text { Revival } \\
\text { by Pantaloon out of } \\
\text { Linda. }\end{array}$ & 1839 & $\begin{array}{l}\text { Not run. Covered at } 2 \text { years old. } 8 \text { foals } \\
\text { without a break. Altogether } 16 \text { foals, in spite } \\
\text { of having made a journey to France and back. }\end{array}$ \\
\hline 85 & $\begin{array}{l}\text { Boarding-School-Miss } \\
\text { by Plenipotentiary out of } \\
\text { Marpessa. }\end{array}$ & $18+1$ & $\begin{array}{l}\text { A grood racer } 3 \text { to } 5 \text { year-old, ran } 22 \text { times, } \\
\text { won } 6 \text { times. First foal at } 7 \text { years old. } 17 \\
\text { loals without a break, amongst which were } 6 \\
\text { Foundation Mares in Fam. } 3 \text { b, then lilled. }\end{array}$ \\
\hline$\$ 6$ & $\begin{array}{l}\text { Physalis } \\
\text { by Bay Middleton out of } \\
\text { Baleine. }\end{array}$ & $18+1$ & $\begin{array}{l}2-5 \text { year-old ran } 22 \text { times, won } 6 \text { times. } \\
\text { First foal at } 7 \text { years old. } 17 \text { foals. At } 23 \text {, } \\
\text { Legacy, Foundation Mare in Fam. } 8 \text { a. }\end{array}$ \\
\hline 87 & $\begin{array}{l}\text { Mare } \\
\text { by Little Red Rover out } \\
\text { of Eclat. }\end{array}$ & 1811 & $\begin{array}{l}\text { Not rum. First foal at } 8 \text { years old. } 15 \\
\text { foals, amongst which were : Buccancer; at } 22 \text {, } \\
\text { Lady Audley (Touchet's dams, and at } 24 \text {, } \\
\text { Stars-and-Stripes, Foundation Mare in Fam. } 14 .\end{array}$ \\
\hline 8s & $\begin{array}{l}\text { Jocose } \\
\text { by Pantaloon out of } \\
\text { Banter. }\end{array}$ & 1843 & $\begin{array}{l}\text { Ran four times as a } 4 \text { year-old, won twice. } \\
\text { First foal at } 6 \text { years old. } 17 \text { foals, amongst } \\
\text { which were: Macaroni } 2 \text { D, Dcp., and at } 24 \text {. } \\
\text { Flippant, loundation Mare in Fam. } 14 \text {. }\end{array}$ \\
\hline 89 & $\begin{array}{l}\text { Queen Mary (Firstling) } \\
\text { by Gladiator out of a } 3 \\
\text { year-old Mare by Pleni- } \\
\text { potentiary. }\end{array}$ & 1813 & $\begin{array}{l}\text { Not run. First foal at } 4 \text { years old. } 19 \text { foals } \\
\text { (and one dead foal), amongst which were: } \\
\text { Blink Bonny D.O., at } 21 \text {, Blinlshoolie, and at } \\
27 \text {, Bonnie Doon, Foundation Mare in Fam. } 10 . \\
\text { Lived to } 29 \text { years of age. }\end{array}$ \\
\hline
\end{tabular}




\begin{tabular}{|c|c|}
\hline No. & Came and Pedigree. \\
\hline 90 & $\begin{array}{l}\text { Ferina } \\
\text { by Venison out of } \\
\text { Partiality. }\end{array}$ \\
\hline 91 & $\begin{array}{l}\text { Maid of Masham } \\
\text { by Don John out of Miss } \\
\text { Lydia. }\end{array}$ \\
\hline 92 & $\begin{array}{l}\text { Iaricot (Firstling) } \\
\text { by Mango or I Lanercost } \\
\text { out of the } 4 \text { year-old } \\
\text { Quecn Mary. }\end{array}$ \\
\hline 93 & $\begin{array}{l}\text { Mare } \\
\text { By- Birdcatcher-Hetman } \\
\text { Platoff. }\end{array}$ \\
\hline 94 & $\begin{array}{l}\text { Catharina Hayes } \mathrm{O} \text {. } \\
\text { by Lanercost out of Con- } \\
\text { stance. }\end{array}$ \\
\hline 95 & $\begin{array}{l}\text { Gertrude } \\
\text { by Hautboy out of Middle. }\end{array}$ \\
\hline 96 & $\begin{array}{l}\text { Doiselte } \\
\text { by Nutwith out of Mar- } \\
\text { mora. }\end{array}$ \\
\hline 97 & $\begin{array}{l}\text { Miss Ignes } \\
\text { by Birdcatcher out of } \\
\text { ignes. }\end{array}$ \\
\hline 18 & $\begin{array}{l}\text { Torment } \\
\text { bỵ Alarm-Glencoe. }\end{array}$ \\
\hline
\end{tabular}

Breeding lerformancen.

1844 Not run. First foal at 5 years uld. 16 foals. .It 2:- Pretender $2 \mathrm{D}$.

1815) 3-6 year-old a very good racer. First foal at 8 vears old. 14 foals. It 21 . Faraway; at 22, Lady Masham, both Foundation Mares in liam. 9, the latter also dam of the two own brothers, leter Hrd, and "limothy. Icp.

1S47 3-7 year-old a very grood racer. First foal at 8 years old. 13 fonls, anongst which were: Caller Ou 1.; at 21, Lady Langden (dam of Sir Berys D and Hampton Gep. Dep.); at 24. Ware by lietledrum, Foundation Nare in lam. 10; at 25, lentil (ran four times as a 2 year-old).

1850 Not run. 10 foals, amongret which was Solon. At 22, Xenophon.

1850) 2-4 year-old a splendid racer. First fonl at 6 years old. 13 foals. At 20 , Narquise de Caux, Foundation Mare in Fann. 22: last foal at 2.5. Lired to 27 years of itge.

1850 Dot rum. First foal at 9 years old. 12 foals, amongst which were two foundation Mares in Fam. 3. At 22, Carnation; last foal at 2.5. Reflex (ran as a 2 and 3 ycar-old. Won once).

1850 2-9 year-old ran 91 times, won 17 times. Won once ns an syear-old. Is a 9 yenr-old, in foal, ran four times without winning. lirst foal at 10 years old. 9 foals, amongst which was . Icceptance, Foundation llare in fianl. 2. Last fonl at 21.

1850 Kan 2-4 year-old, won once. First foal at 6 years old. 16 foals, amongst which were six prominent loundation Wares. It 23, Innes sorel, linumdition Nare in liam. I6. Jived to 27 years of nge.

18;n .Is a 2 and 3 reatr-old a very good racer. First foal at 5 years old. 14 fonls, including living dwins, 13 lilat and F Sharp, $;$ Foundation Vares in Fom. J0, Tormentor $O$, and at 22. Peine de ('our (dam of 1)espair and Caris. sima). 


\begin{tabular}{|c|c|c|}
\hline No. & Name and Pedigree. & Born. \\
\hline 99 & $\begin{array}{l}\text { Blue Bell } \\
\text { by Heron-Zinmerman. }\end{array}$ & 1851 \\
\hline 100 & $\begin{array}{l}\text { Nelly Hill } \\
\text { by Springy Jack out of } \\
\text { Anne Page. }\end{array}$ & 1851 \\
\hline 101 & $\begin{array}{l}\text { Katherine Logie } \\
\text { by Flying Dutchman out } \\
\text { of Phryne. }\end{array}$ & 1853 \\
\hline 102 & $\begin{array}{l}\text { Christabelle } \\
\text { by Fernhill-Beiram. }\end{array}$ & 1854 \\
\hline 103 & $\begin{array}{l}\text { Bathilde } \mathrm{Cm} \text {. } \\
\text { by Stockwell out of } \\
\text { Babette. }\end{array}$ & 1858 \\
\hline 104 & $\begin{array}{l}\text { Anonyma } \\
\text { by Stockwell out of Miss } \\
\text { Sarah. }\end{array}$ & 1859 \\
\hline 105 & $\begin{array}{l}\text { Lady Sefton } \\
\text { by West-Australian out of } \\
\text { Clarissa. }\end{array}$ & 1861 \\
\hline 106 & $\begin{array}{l}\text { Palmflower } \\
\text { by The Palmer out of } \\
\text { Jenny Driver (had } 20 \\
\text { foals). }\end{array}$ & 1874 \\
\hline
\end{tabular}

Breeding Performances.

Not run. First foal at 5 years old. 14 foals. 3 Foundation Mares in Fam. 25. At 25, Aura (dam of Orcan). Lived to 34 years of age.

Ran ?-4 year-old and won as a 3 year-old four times. First foal at 6 years old. 14 foals. At 23, Jessie, Foundation Ware in Fam. 17.

Ran 3-6year-old 61 times and won 11 times. First foal at 8 years old. 13 foals, anong which were: Bothwell 2, King o' Scots, and at 23, Pompeja, Foundation Mare in Fann. 3.

Not run. First foal at 5 years old. 17 foals, amongst which were three Foundation Mares in Fam. $3 \mathrm{c}$. At 23, Sorcery (dam of Aspirant OD, and See Me OO).

Ran 2-6 year-old 24 times, won 5 times. First foal at 8 years old. 14 foals. At 20, Lowland Chief, and at 24, Queen Bathilde, Foundation Mare in Fam. 23.

Ran 2-5 year-old 14 times, Won 4 times. First foal at 7 years old. 15 foals, amongst which were four Foundation Mares in Fam. 21. It 23, Lonely $O$, and at 24, Hawlistone.

Ran once as a 2 and once as a 3 year-old. First foal at 5 years old. 14 foals, amongst which were: Sefton D, two Foundation Mares in Fam. 25, and at 24, Quid Pro Quo by Isonomy.

Ran 2-4 year-old 13 times, and won 4 times as a 2 year-old. First foal at 7 years old. 17 foals without a break, amongst which were: E1 Dorado, St. Florian, three Foundation Mares in Fam. 20; at 22, Nusa $O$, and at 23, Palmaro (ran and won as a 2 and 3 year-old).

Special Breeding Performances of Old Thoroughbred Mares from abroad.

\begin{tabular}{|c|c|c|c|}
\hline No. & Name and Pediggree. & 13orn. & Breeding Performances. \\
\hline
\end{tabular}

1 Vittoria

by Milton out of Geane, Meudon Stud in France, owned by L. Napoleon.
1823 First foal at 7 years old. 18 foals, amongst which were: Nautilus Cd. three times, and Romulus F.D., at 23, Vergogne F.O., at 25, last foal. 


\begin{tabular}{|c|c|c|c|}
\hline No. & Name and Pedigree. & Born. & Breeding Performances. \\
\hline 2 & $\begin{array}{l}\text { Miss Furey } \\
\text { by Whalebone - Sooth- } \\
\text { sayer. }\end{array}$ & 1824 & $\begin{array}{l}\text {. It } 25 \text {, Dunna Sol (dam of Negresse), Fam. } \\
\text { 18. }\end{array}$ \\
\hline 3 & $\begin{array}{l}\text { Maid of Ionor } \\
\text { by Champion out of } \\
\text { Etiquette. }\end{array}$ & $18: 99$ & $\begin{array}{l}\text { It } 2.2, \text { Kohinoor U., at } 23 \text {, Darinoor, Fam. } \\
14 \text {. }\end{array}$ \\
\hline 4 & $\begin{array}{l}\text { Mermaid } \\
\text { by Whalebone unt of Miss } \\
\text { Emma. }\end{array}$ & $18: 29$ & It 23 , Seahorse Hr., Fam. 12. \\
\hline 5 & $\begin{array}{l}\text { Irory } \\
\text { by Humphrey Clinker out } \\
\text { of Ildegarda. }\end{array}$ & 1832 & It 29 , Collingwood 1I. U. \\
\hline li & $\begin{array}{l}\text { Jessy } \\
\text { by Emancipation out of } \\
\text { Eliza. }\end{array}$ & 1835 & It 24, Belle Etoile, Fam. 25 . \\
\hline$i$ & $\begin{array}{l}\text { Mlice Carneal } \\
\text { by Sarpedon out of } \\
\text { kuwena. }\end{array}$ & 1836 & $\begin{array}{l}12 \text { foals, amongst which were Lexington; } \\
\text { at } 21 \text {, Lmpire, and at } 23 \text {, Annette. Fam. } 12 \text { a. }\end{array}$ \\
\hline 8 & $\begin{array}{l}\text { Dolphin } \\
\text { by Priam out of Mermaid. }\end{array}$ & 1836 & $\begin{array}{l}\text { It } 20 \text {, Shark } \mathrm{H}_{11}, \text { and at } 22 \text {, Chingachgook. } \\
\text { Fam. 1.2. }\end{array}$ \\
\hline 9 & $\begin{array}{l}\text { Victoria } \\
\text { by Elizondo-Saracen. }\end{array}$ & 1841 & $\begin{array}{l}\text { It } 22, \text { Vera Crux, and at } 23 \text {, Victorieuse } \\
\text { F.O. Fam. 1 c. }\end{array}$ \\
\hline 10 & $\begin{array}{l}\text { Bathilde } \\
\text { by I. Emilius out of } \\
\text { Odine. }\end{array}$ & 1842 & $\begin{array}{l}\text { It 24, Orpheline (dam of Fra Diasolo, F.L.). } \\
\text { Lam. } 5 \text { a. }\end{array}$ \\
\hline 11 & $\begin{array}{l}\text { Ennui } \\
\text { by Bay Middleton out of } \\
\text { Blue Devils. }\end{array}$ & $18+3$ & $\begin{array}{l}14 \text { foals, amongst which were Saunterer and } \\
\text { Loiterer; at } 24 \text {, Constance. Fan. } 11 . \text {. }\end{array}$ \\
\hline 12 & $\begin{array}{l}\text { Belle de Nuit } \\
\text { by Y. Enilius out of } \\
\text { Odine. }\end{array}$ & $1 S 4$ & $\begin{array}{l}\text { It } 23 \text {, Good-Night (dam of Pourquoi Gi.). } \\
\text { Fam. } 5 \text { a. }\end{array}$ \\
\hline 13 & $\begin{array}{l}\text { Officiotts } \\
\text { by Pantaloon out of } \\
\text { Baleine. }\end{array}$ & 1847 & $\begin{array}{l}\text {.It 2I, Dami B.B., at } 22,11 \text {. Nüstro B.B. } \\
\text { Fam. } 8 \text { a. }\end{array}$ \\
\hline 14 & $\begin{array}{l}\text { Sunrise } \\
\text { by Enilius out of Sunset. }\end{array}$ & 1848 & It 23, Succes. Fam. 3. \\
\hline 15 & $\begin{array}{l}\text { Partlet } \\
\text { by 13irdcatcher out of } \\
\text { Gipsy. }\end{array}$ & 1819 & It 25 , Patriarche. Fam. 19. \\
\hline 16 & $\begin{array}{l}\text { Vermeille } \\
\text { by The Baron out of Fair } \\
\text { Ilelm. }\end{array}$ & 1853 & $\begin{array}{l}13 \text { foals, amongst which Were: Vermouth } \\
\text { G.P., B.B., and Vertugadin Gl., B.B., at } 24 \text {, } \\
\text { Extra (dam of Excuse Cd.). lived to } 29 \text { years } \\
\text { of age. Fam. } 3 \text { b. }\end{array}$ \\
\hline
\end{tabular}




\begin{tabular}{l|l|l|l|l}
\hline Nu. Name and Pedigree. & Born. & Breeding Performances. \\
\hline 17 & $\begin{array}{c}\text { Thrift } \\
\text { by Stockwell out of } \\
\text { Braxey. }\end{array}$ & 1865 & $\begin{array}{c}\text { Covered 3 years old. } 17 \text { foals and once } \\
\text { twins; at 24, Avoir. Fann. 10. }\end{array}$ \\
$\begin{array}{l}\text { Clotho RO. } \\
\text { by Bois Roussel out of }\end{array}$ & 1866 & $\begin{array}{c}\text { At 20, Cleodore Prd., at 25, Clôture. Fam. } \\
\text { sa. }\end{array}$
\end{tabular}

The Thoroughbred also seems to have the superiority over the Half-bred as regards longevity, as can be seen from the three following lists.

Of the Half-breds known to me. the Royal Country Stallions in Celle have reacled by far the greatest age, but even these are inferior to the Thoroughbred regarding their maximum performances, etc, as the last of the three tables following shows.

Register of the Thoroughbred Stallions born in England which have attained the age of 25 years and more.

\begin{tabular}{|c|c|c|c|c|}
\hline No. & Names of Stallions. & born. & Sires of Stallions. & $\begin{array}{l}\text { Age } \\
\text { reached. } \\
\text { lears. }\end{array}$ \\
\hline 1 & The Lister Turk & abt. 1680 & $\ldots$ & abt. 32 \\
\hline 2 & Jigg & abt. 1702 & Byerly Turk & abt. 28 \\
\hline 3 & Bay Bolton & 1705 & Grey Hautboy & 31 \\
\hline 4 & The Belgrade Turk & abt. 1710 & - & abt. 30 \\
\hline$\dot{j}$ & Flying Childers & 1715 & Darley Arabian & 26 \\
\hline 6 & Partner & 1715 & Jigg & 29 \\
\hline 7 & Crab & 1722 & Alcocli's Arabian & 28 \\
\hline 8 & The Godolphin Arabian & 1721 & - & 29 \\
\hline 9 & Monkey & 1725 & Lonsdale Bay Arabian & $29 \mathrm{~A}$. \\
\hline 10) & The Bolton Starling & 1727 & Bay Bolton & 29 \\
\hline 11 & Young Cartouch & 1731 & Cartouch & 28 \\
\hline 12 & Sedbury & 1734 & Partner & 25 \\
\hline 13 & The Ancaster Starling & 1738 & Starling & 26 \\
\hline 14 & Regulus & 1739 & Godolphin Arabian & 26 \\
\hline 15 & Blank & 1710 & Godolphin Arabian & 29 \\
\hline 16 & Jolly Roger & $17+1$ & Roundhead & 31 A. \\
\hline 17 & Sampson & 1745 & Blaze & 32 \\
\hline 18 & Janus & 1746 & Janus & $34 \mathrm{~A}$. \\
\hline 19 & Matchem & $171 \mathrm{~s}$ & Cade & 33 \\
\hline 20 & Marske & 17.50 & Squirt & 29 \\
\hline 21 & Snap & 1750 & Snip & 27 \\
\hline 2.2 & Syphon & 1750 & Squirt & least 25 \\
\hline 23 & The Godolphin Colt & 1754 & Godolphin Arabian & 27 \\
\hline 24 & Matchless & 1754 & Godolphin Irabian & abt. $32 \mathrm{~A}$. \\
\hline
\end{tabular}




\begin{tabular}{|c|c|c|c|c|}
\hline No. & Nimes of Stallions. & Born. & Sires of Stallions. & $\begin{array}{l}\text {. Ige } \\
\text { reached. } \\
\text { lears. }\end{array}$ \\
\hline 2.5 & Squirrel & $175 t$ & Traveller & 26 \\
\hline 20 & Engineer & 1750 & Sampson & 26 \\
\hline 27 & Centinel & 1758 & Blank & $26 \wedge$. \\
\hline 28 & Jalap & 1758 & Regulus & 29 \\
\hline 29 & Bay Malton & 1760 & Sampson & 26 \\
\hline 30 & Tantrum & 1760 & Cripple & least 26 \\
\hline 31 & Coriolanus & 1762 & Whistlejacliet & least 29 \\
\hline 32 & Morwick Ball & 1762 & Regulus & 25 \\
\hline $3: 3$ & Pilgrim & 1762 & Sampson & 26 \\
\hline 31 & Chrysolite & 1763 & Blank & 25 \\
\hline $3 ;$ & P'acolet & 1763 & Blank & 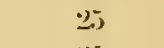 \\
\hline 30 & Ranthos & 1763 & Matchem & 31 \\
\hline 37 & Eclipse & 1764 & Marslie & 25 \\
\hline 38 & Goldfinder & 1764 & Snap & 25 \\
\hline 39 & Phlegron & 1765 & Matchem & 25 \\
\hline 40 & Trentham & 1766 & Sweepstalies & abt. 20 \\
\hline 41 & Fagsergill & 1766 & Snap & 25 \\
\hline 42 & Paymaster & 1766 & Blank & 25 \\
\hline 43 & Solon & 1766 & Sampson & 27 \\
\hline 4 & Sharls & 1771 & Marslie & $2.5 \mathrm{~A}$ \\
\hline 55 & Young Marslse & 1771 & Marsise & 29 \\
\hline 46 & Potsos & 1773 & Eclipse & 27 \\
\hline 17 & IVoodpecker & 1733 & Herod & $\underline{5}$ \\
\hline is & Jupiter & $17 \pi t$ & Eclipse & 28 \\
\hline 49 & King Fergus & 1775 & Eclipse & $\pm i$ \\
\hline 50 & IVeasel & 1776 & llerod & 25 \\
\hline .31 & Dioned & 1767 & Florizel & 31 A. \\
\hline 52 & Dronc & 1777 & Herod & 20) $\mathrm{A}$. \\
\hline 33 & Ruler & 1777 & I. Marslie & 30 \\
\hline 54 & Dungantion & 1780 & Eclipse & $2 \mathrm{~S}$ \\
\hline 5 & Messenger & 1780 & Nambrino & 251. \\
\hline 50 & Delpini & 1781 & Ilighflyer & 27 \\
\hline 57 & - llexander & 1782 & Eclipse & $2 !$ \\
\hline is & Trumpator & $178: 2$ & Conductor & 26 \\
\hline 59 & Driver & 1783 & Trentham & 28 \\
\hline 60 & Meteor & 1783 & Eclipse & 28 \\
\hline 61 & Windlestone & 1783 & Magnet & 吕) \\
\hline 62 & Sir Peter & 1784 & Highfler & 27 \\
\hline $6: 3$ & Star & 1785 & Highnyer & $26 \ldots$ \\
\hline$(i 1$ & Traveller & 1785 & Highflyer & 25 \\
\hline (ii) & Competitor & 1780 & Eclipse & (3) \\
\hline 60 & Cocker & 17sij & Trentham & $21 ;$ \\
\hline $6 \overline{1}$ & Grog & 1756 & Tanden & 27 \\
\hline 68 & Star & 1786 & Highflyer & $25 x$ \\
\hline (i) & Dragon & 1757 & Woodpecker & 25.1 \\
\hline
\end{tabular}


Trial of the Thoroughbred on the Racecourse, etc.

\begin{tabular}{|c|c|c|c|c|}
\hline No. & Names of Stallions. & Born. & Sires of Stallions. & $\begin{array}{l}\text { Xge } \\
\text { reached. } \\
\text { Years. }\end{array}$ \\
\hline 70 & Gohanna & 1790 & Mercury & 25 \\
\hline 71 & Waxy & 1790 & Pot8os & 28 \\
\hline $7 \underline{2}$ & $\begin{array}{c}\text { Parrot (ran } 2-5 \text { years } 28 \\
\text { times and won } 9 \text { times) }\end{array}$ & 1791 & Dungannon & 36 \\
\hline 73 & Diamond & $179^{2}$ & Highflyer & $27 \mathrm{~F}$ \\
\hline 74 & Hambletonian & 1722 & King Fergus & 26 \\
\hline 75 & King Bladud & 1792 & Fortunio & 27 \\
\hline 76 & Stamford & 1794 & Sir Peter & 26 \\
\hline$\pi$ & Whip & 1794 & Saltram & $31 \mathrm{~A}$. \\
\hline 78 & Bobtail & 1795 & Precipitate & 27 \\
\hline 79 & Eagle & 1796 & Volunteer & $30 \mathrm{~A}$. \\
\hline 80 & Sorceser & 1796 & Trumpator & 25 \\
\hline$\$ 1$ & Quiz & 1798 & Buzzard & 28 \\
\hline $8 \cdot 2$ & Orrlando & 1749 & IVhisliey & 25 \\
\hline 83 & Orville & 1709 & Beningbrough & 27 \\
\hline 84 & Walton & 1799 & Sir Peter & 26 \\
\hline 85 & Remembrancer & 1800 & Pipator & 29 \\
\hline 86 & Sir Oliver & 1800 & Sir Peter & 29 \\
\hline 87 & Castrel & 1801 & Buzzard & 26 \\
\hline $8 s$ & Sir David & 1801 & Trumpator & $27 \mathrm{k}$. \\
\hline 89 & Sir Walter Raleigh & 1801 & Waxy & 30 \\
\hline 90 & Grimaldi & 1802 & Delpini & 28 \\
\hline 91 & Fyldener & 1803 & Sir Peter & 26 \\
\hline ! 2 & Hollyhock & 1804 & Master Bagot & 25 \\
\hline 93 & IVaxy Pope & 1806 & Waxy & 25 \\
\hline 94 & Ad Libitum & 1807 & Whisliey & $30 \mathrm{~F}$. \\
\hline 9.5 & Bluster & 1808 & Orlando & $26 \mathrm{~A}$. \\
\hline 96 & Grimalkin & 1808 & Chance & $26 \mathrm{D}$ \\
\hline 97 & Hanulet & 1808 & Hambletonian & $27 \mathrm{~F}$ \\
\hline 98 & Phantom & 1808 & Walton & $26 \mathrm{D}$. \\
\hline 99 & Rainbow & 1808 & IValton & $26 \mathrm{~F}$. \\
\hline 100 & Comus & 1809 & Sorcerer & 28 \\
\hline 101 & Muley & 1810 & Orville & 27 \\
\hline 102 & Tramp & 1810 & Dicli Andrews & 25 \\
\hline 103 & Bijou & 1811 & Orville & $25 \mathrm{~F}$ \\
\hline 104 & Dr. Syntax & 1811 & Paynator & 27 \\
\hline 105 & Captain Candid & 1813 & Cerberus & $25 \mathrm{~F}$. \\
\hline 106 & Elector & 1813 & Election & $25 \mathrm{D}$. \\
\hline 107 & The Cryer & 1814 & Sorcerer & $28 \mathrm{D}$. \\
\hline 108 & Gaudy & 1814 & Peruvian & $27 \mathrm{D}$ \\
\hline 109 & Talma & 1814 & Sorcerer & $28 \mathrm{r}$. \\
\hline 110 & Allegro & 1815 & Orville & $29 \mathrm{r}$. \\
\hline 111 & Oracle & 1815 & Sorcerer & $27 \mathrm{n}$. \\
\hline 112 & Spectre & 1815 & Phantom & $26 \mathrm{~F}$. \\
\hline 113 & Dr Eady & 1816 & Rubens & 26 \\
\hline
\end{tabular}




\begin{tabular}{|c|c|c|c|c|}
\hline No. & Names of Stallions. & Born. & Sires of Stallions. & $\begin{array}{l}\text { Age } \\
\text { reached. } \\
\text { Vears. }\end{array}$ \\
\hline 114 & Tandem & 1816 & Rubens & $25 \mathrm{~F}$ \\
\hline 115 & Cavalier & 1817 & Limbton & $27 \mathrm{D}$ \\
\hline 116 & Parchement or Tring & 1817 & Thumderbolt & $25 \mathrm{~F}$. \\
\hline 117 & St. Patrick & 1817 & W'alton & 26 \\
\hline 118 & Trance & 1817 & Phantom & $29 \mathrm{~F}$ \\
\hline 119 & Minister & 1818 & Prime Minister & $26 \mathrm{~F}$. \\
\hline $1: 20$ & Belmout & 1819 & Thunderbolt & $29 \mathrm{~F}$. \\
\hline 121 & Marcellus & 1819 & Selin & $25 \mathrm{~F}$. \\
\hline 122 & Abrou & I 820 & Whisker & $25 \mathrm{~J}$ \\
\hline 123 & Emilius & 1820 & Orville & 27 \\
\hline 121 & General Mina & $18 * 20$ & Camillus & $26 \mathrm{~F}$ \\
\hline 125 & Lottery & $18: 20$ & Tramp & $25 \mathrm{~F}$ \\
\hline $1 \pm 6$ & Middleton & 1822 & Phantom & $25 \mathrm{R}$. \\
\hline 127 & Sir Geolfrey Peveril & 1823 & IVlialebone & $25 \mathrm{D}$. \\
\hline 128 & lioyal Onk & 1823 & Catton & $26 \mathrm{~F}$. \\
\hline $1: 29$ & Mamelulie & 1824 & Jartisan & $2 \pi \mathrm{F}$. \\
\hline 130 & Pantaloon & 1824 & Castrel & 26 \\
\hline 131 & Terror & 1805 & Magistrate & $25 \mathrm{~F}$ \\
\hline 132 & Velocipede & 1825 & Blacklock & 25 \\
\hline 133 & Iaron & 1826 & Moses & $26 \mathrm{AU}$. \\
\hline 134 & Lawnsleeves & $18 \div 6$ & Dr. Syntax & $25 \mathrm{Ir}$ \\
\hline 135 & Sir Hercules & $18: 6$ & Whalebone & 29 \\
\hline 130 & Birmingham & 1827 & Filho da Puta & $27 \mathrm{lr}$. \\
\hline 137 & Giovanni & $18: 28$ & Filho da Puta & 26 \\
\hline $1: 35$ & Minster & $18 \cdot 9$ & Catton & $26 \mathrm{~F}$. \\
\hline $1: 39$ & Trustee & $15: 0$ & Catton & $27 \wedge$ \\
\hline 140) & limigrane & 1831 & "lramp & 25.10. \\
\hline 141 & General Chasse & $18: 31$ & Ictaeon & 25 к. \\
\hline 142 & Glencoe & 1831 & Sultan & $26 \mathrm{~A}$ \\
\hline 143 & Touchstone & 1831 & Camel & 30 \\
\hline 144 & Safeguard & $18: 32$ & Defence & 26 \\
\hline 145 & Sheet Anchor & 1.832 & Lottery & 311. \\
\hline 146 & Birdeatcher & 1833 & Sir llercules & 27 \\
\hline 1.17 & 1.angford & 1833 & Sir Hercules & 2511. \\
\hline 148 & Slane & 1833 & Royal Oali & 25 \\
\hline 149 & Y. Confederate & $18: 31$ & Confederate & $25 \mathrm{I}$. \\
\hline $1: 0)$ & llarkaway & $1 \times 31$ & Econonnist & 25 \\
\hline 1.51 & Melbourne & $18: 34$ & Humphrey Clinker & 25 \\
\hline $1: 0^{2}$ & Mickle Fell & $1 \leqslant 3$. & Catton & $28 \mathrm{Im}$ \\
\hline $1 .: 3$ & Yorkshire & 1634 & St. Nicolas & 25. \\
\hline 151 & Sportsman & $1 \times 3 ;$ & Flexible & 2511. \\
\hline 15ij & Bloomsbury & $1 \leqslant 360$ & Mulatto & $2 ; 11$. \\
\hline lini & Oroonocko & $1 \times 36$ & Camel & $36 \mathrm{1}$ \\
\hline 157 & P'olydorus & 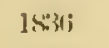 & Priam & a) 11. \\
\hline 158 & Sampson & 1 1ホ行 & Cetus & 0. \\
\hline
\end{tabular}


Trial of the Thoroughbred on the Racecourse, etc.

\begin{tabular}{|c|c|c|c|c|}
\hline No. & Names of Stallions. & Forn. & Sires of Stallions. & $\begin{array}{l}\text { Age } \\
\text { reached. } \\
\text { Years. }\end{array}$ \\
\hline 159 & Sovereign & 1836 & Emilius & $26 \mathrm{~A}$ \\
\hline 160 & St. Swithin & 1837 & Velocipede or St. Nicholas & $27 \mathrm{n}$. \\
\hline 161 & Alonzo & 1837 & Alpheus & 25 \\
\hline 162 & Satirist & 1835 & Pantaloon & $26 \mathrm{D}$ \\
\hline 163 & Consternation & 1811 & Confederate & $25 \mathrm{~A}$. \\
\hline 164 & The Cure & $18+1$ & Physician & 25 \\
\hline 165 & Orlando & $18+1$ & Touchstone & 27 \\
\hline 166 & IVeatherbit & 1842 & Sheet Anchor & 26 \\
\hline 167 & King of Naples & $184 t$ & Slane & $28 \mathrm{n}$ \\
\hline 168 & Springy Jack & 1845 & Hetman Platoff & 2) $\mathrm{D}$. \\
\hline 169 & Surplice & 184.5 & Touchstone & 26 \\
\hline 170 & Voltigeur & 1817 & Voltaire & 27 \\
\hline 171 & Harpsichord & 1818 & T'ouchstone & $26 \mathrm{I}$ \\
\hline 172 & Stilton & 1849 & Cotherstone & $26 \mathrm{D}$ \\
\hline 173 & Captain Cornish & 1850 & Jago & $25 \mathrm{D}$. \\
\hline 174 & Sittingbourne & 1850 & Chatham & $27 \mathrm{D}$. \\
\hline 175 & Arthur IVellesley & 1851 & Melbourne & 25 \\
\hline 176 & Epaminondas & 1851 & Epirus & $25 \mathrm{D}$ \\
\hline 177 & King Tom & 1531 & Harkaway & 27 \\
\hline 178 & Marsyas & 1851 & Orlando & 25 \\
\hline 179 & Professor Airey & 1852 & Mathematician & 27 \\
\hline 180 & Bonnie Scotland & $18: 33$ & Jago & $27 \mathrm{~A}$ \\
\hline $1 S 1$ & Cotswold & 1853 & Newcourt & 260 \\
\hline 182 & Forbidden Fruit & 1853 & Birdcatcher & 200. \\
\hline 183 & Kentucky & 18.3 & Micliey Free & $31 \mathrm{D}$. \\
\hline 184 & Leamington & 1853 & Faugh-a-Ballagh & $25 \mathrm{~A}$ \\
\hline 185 & Amsterdam & 18.54 & The Flying Dutchman & 25 \\
\hline 186 & The Attorney General & 1854 & Melbourne & $20 \mathrm{D}$ \\
\hline 187 & Gunboat & $18.5 t$ & Sir Hercules & 29 \\
\hline 188 & WVardermarslie & 1554 & Birdcatcher & $26 \mathrm{D}$. \\
\hline 189 & Kelpie & 1855 & Weatherbit & $27 \mathrm{AU}$. \\
\hline 190 & Flash in the Pan & 1856 & Pontifex & 25 \\
\hline 191 & Gaspard & 1856 & Daniel O'Rourke & $26 \mathrm{D}$. \\
\hline 192 & The Spealier & 1556 & Filbert & 25 \\
\hline 193 & Athos & 1857 & Prime Minister & $25 \mathrm{AU}$. \\
\hline 194 & Buccaneer & 18.57 & IVild Dayrell & $30 \mathrm{o}$. \\
\hline 195 & Mainstone & 1857 & King Tom & 28 \\
\hline 196 & Thunderbolt & 1857 & Stockwell & 31 \\
\hline 197 & Carbineer & 1858 & Rifleman & 25 \\
\hline 198 & Don John & 1858 & Wild Daỹrell & 25 \\
\hline 199 & Kettledrum & 1858 & Rataplan & 270 \\
\hline 200 & Drummer Boy & 1859 & Rataplan & $28 \mathrm{n}$ \\
\hline 201 & The Marquin & 1859 & Stockwell & 27 AU. \\
\hline 202 & Victor & 1859 & Vindex & 27 \\
\hline 203 & Conrad & 1860 & Kingston & $25 \mathrm{AU}$. \\
\hline 204 & Grimston & 1860 & Stockwell & $26 \mathrm{D}$. \\
\hline 205 & Macaroni & 1860 & Sweetmeat & 27 \\
\hline
\end{tabular}


4. Value of the Thoresughbred for Other Breeds.

\begin{tabular}{|c|c|c|c|c|}
\hline$x i$. & Sames of stallions. & liorn. & Sires of Stallions. & $\begin{array}{l}\text { Jge } \\
\text { reached. } \\
\text { lears. }\end{array}$ \\
\hline 206 & Mail Train & 1861 & Girmenor & $27 \mathrm{AU}$ \\
\hline 207 & The Dart & $186: 3$ & Lord Fauconberer & 2 \\
\hline 208 & Lectures & 1863 & Colsterdile & 2 \\
\hline 209 & Vespasian & $186 ; 3$ & Newminster & 27. \\
\hline 210 & $\mathrm{Ni}$ & $196: 3$ & General Miiliams & $20 i$ \\
\hline 211 & Friponnier & 1864 & Chevalier d'Industrie & 2511 \\
\hline 212 & Hermit* & 1564 & Newminster & 26 \\
\hline 213 & Pathfinder & 1864 & Thormanby & 2911 \\
\hline 214 & Tibthorpe & 1864 & Voltigeur & 26 \\
\hline 21.5 & Tynedale & 1864 & Wartocls & 26 \\
\hline 216 & BoId Inỵrell & $186 \%$ & Wild Dayrell & 26 \\
\hline 217 & Rosicrucian & 186.5 & Beadsmาaห & 26 \\
\hline 218 & Tregeagle & 3865 & Wild Diyrell & 24.16 \\
\hline 219 & The Drummer & 1866 & Rataplan & $26 \mathrm{AC}$ \\
\hline $2 \cdot 211$ & Dutch Skitter & listiti & The Flying Dutchman & 25 \\
\hline 221 & Goldfinsch & 1866 & Audubon & 20 \\
\hline $2 \cdot 2 \cdot 2$ & The Bobby & 1867 & Loiterer & $\underline{2}$ \\
\hline 2.3 & Jing Cole & 1867 & King Tum & $24 i .11 \%$ \\
\hline $2 \geq 1$ & Lord Glascrow & 1867 & Rapid Rhone & 20 \\
\hline 5 & Syrian & $1 \times 67$ & Mentmore & $24 ;$ \\
\hline $2 \cdot 2 i$ & Grindmaster & 1815 & Giladiateur & $31 \mathrm{Al}$. \\
\hline 29 & Rapture & 1869 & Dioplantus. & 2.5. \\
\hline 2.28 & Interos & 150 & Loiterer & $2 ; \mathrm{AC}$. \\
\hline $2 \cdot 24$ & Gang Forward & 1870 & Stockwell & $2 \mathrm{Al}$ \\
\hline 230 & iscetic & 1571 & Hermit & 24 \\
\hline 231 & Controvers! & 1871 & Lambton or The Miner & $2 ;$ \\
\hline 232 & George lirederick & 1871 & Marsyas & $2 ; 1$ \\
\hline 233 & Friar Tuck & 1872 & Hermit & $27 \mathrm{~A}$ \\
\hline 234 & Galopin & 1872 & Vedette & 27 \\
\hline 235 & Hampton & 1572 & Lord Clifden & 25 \\
\hline 23 & The Iluleteer & 1873 & Mogador & 20 \\
\hline 237 & Spendthrift & 1573 & Blair Ithol & 27 \\
\hline 238 & Springfield & 1573 & St. .Ilb:tns & 2.5 \\
\hline 39 & Empire & 1973 & Tom kingr & $20 ;$ \\
\hline 210 & Midlothian & 1854 & Rataplan or Strathcona & 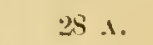 \\
\hline 241 & Beauclerc & 1575 & Rosicrucian & تיט \\
\hline 210 & T"hurio & 1875 & Tibthorpe or Cremorne & 27 \\
\hline 213 & Charaxus & 1876 & Distin & $24 ;$ \\
\hline 211 & Fend Or & $18 \pi$ & Donciaster & 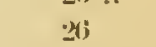 \\
\hline 21.5 & Prestompans & 187 & Prince Charlie & $2 \pi$ \\
\hline 216 & Leamington & 158 & Faugh-a-Ballagh & ai \\
\hline 217 & Despair & $187 !$ & See Sall & 25 \\
\hline 218 & St. Simon & lssI & Galopin & 27 \\
\hline 219 & kendal & ISS3 & liend $\mathrm{Or}$ & 小 \\
\hline & $\begin{array}{l}A \text { Died in America. } \\
O \text { Died in Austria. }\end{array}$ & $\begin{array}{l}\text { D-Died i } \\
R \text { Died it }\end{array}$ & $\begin{array}{l}\text { I. Died in } \mathrm{F} \\
\text { AL Died in }\end{array}$ & ralia. \\
\hline
\end{tabular}

"In General Stud look, Vol. .IVIl., page 938 , the age of llermit is wrongly given as an years. 
Register of the Thoroughbred Stallions born in America which have attained the age of 25 years and more.

1. Celler 1776 by Janus

2. Jolly Friar 1783 by Janus

3. Old Friendship 1783 by Apollo

4. Centinel 1800 by Diomed

5. Peacemaker 1800 by Diomed

6. Sir Archy 180:5 by Diomed

was 28 years old.

7. Eclipse 1814 by Duroc

, $2.5,,$,

8. Manalopan 18 . 8 by Johnson's Medley

9. Wagner 1834 by Sir Charles

10. Grey Eagle 183.5 by Wundpecker ," 28

11. Y. Langford 1840 by Langford ", 35

12. Revenue 1843 by Trustee

13. Star Davis 1849 by Glencoe

14. Jexington 1850 by Boston

1.). Wild I rishman 1850 by Glencoe

16. Brown Dick 1851 by Margrave

17. Rifleman 1855 by Glencoe

18. Isteroid 1861 by Lexington

19. Norfolk 1861 by Lexington

20. Ansel 1862 by Lexington

21. Baltimure 1862 by Revenue

2.2. Morris 18603 by lexington

23. Pat Malloy 186:) by Lexington

24. Vauxhall 1865 by i.exington

25. Exchange 1866 by Endorser

26. Glenelg $1866 \mathrm{by}$ Citadel

27. Enquirer 1867 by Leamington

28. Longfellow 1867 by Leamington

29. Lyttleton 1867 by Leamington

30. Regent 1867 by Bonnie Seotland

, 25

$, 2,27$

.25

,

, 26

, .28

,$\quad 28$

(. $\quad 25$

, $\quad 29$

,, 29

,$\quad 26$

,,$\quad 20$

,$\quad 25$

, $\quad 2.5$

(

(1) $\quad 25$

,, 31

, 20

.. 26

,. $2($;

31. Bigarom 1868 by Bonnie Scotland

32. Eolos 1868 by Leamington

(.).

,$\quad 2.5$

,,$\quad 29$

33. Nathan Oaks 1868 by Bonnie Scotland „, 26

34. Spindrift 1868 by Bonnie Scotland ., 2.5

35. Joe Daniels 1869 by Australian , 27

36. Springbok 1870 by Australian

37. Grinstead 1871 by Gilroy

38. Big Sandy 1872 by Australian

39. Fiddlesticks 1874 by Lexington

40. Himyar 1875 by Mlarm

41. Falsetto 1876 by Enquirer

,$\quad .97$

, 2.5

,

, 20

, 30

, 28 
42. The Jacobite 1876 hy Prince Charlie was 29 years old.

43. Irish Kingr 1876 br Iongfellow

44. Foxhall lists by ling Ilfonso

45. Onondaga 1879 by Leaminglon

$\begin{array}{llll}, & 25 & , & , \\ , & 26 & , & , \\ , & 25 & , & ,\end{array}$

Register of the Royal Country Stallions in Celle which allained the age of 25 years and more.

\begin{tabular}{|c|c|c|c|c|}
\hline Ni). & Name of Stallion & [Burn. & sire. & $\begin{array}{l}\text { lge } \\
\text { reached. } \\
\text { years. }\end{array}$ \\
\hline 1 & Prevenant & 1755 & unknown & 27 \\
\hline 2 & Inique & 1798 & Inique & 26 \\
\hline 3 & 1. Ambush & 1811 & limbusli II. & 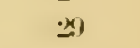 \\
\hline 4 & Faustinus & 18I.) & Kitylichen & 31 \\
\hline$\dot{j}$ & Torador & 1817 & Grosstenor $\mathrm{xx}$ & 26 \\
\hline 6 & Nil & $18: 40$ & Gilfi & 32 \\
\hline$i$ & Tancred 11. & $18 \div 21$ & Herodot $x x$ & $2 i$ \\
\hline 8 & Diomed & 1820 & Stallion in Ivenacls & 25 \\
\hline 9 & Bundler & $18: 4$ & Coriander $\mathrm{xx}$ & 26 \\
\hline 10 & Dorado & $18 \div$ & Aly̆ & 28 \\
\hline 11 & Gomul & 1521 & Intonius & 27 \\
\hline 12 & Whisker & 1824 & Gilfi, Araber & 27 \\
\hline 13 & Brigadier & 1:25 & Romano & 26 \\
\hline 1.1 & Gallas & 15 & Giallits & 26 \\
\hline 15 & Goblet & 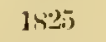 & Y. Bobtail & 25 \\
\hline 16 & Hotspur & 1525 & Oberon & 27 \\
\hline 17 & Paroli & 195; & 1. Sebastian & 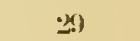 \\
\hline 18 & Malcolm & $15 \% 3$ & Imbaldo xx & 27 \\
\hline $1 !$ & Trijan & $16 \div 3$ & Inthras & 27 \\
\hline 20 & Ibelard & $18 \div 7$ & Imbaldo $x x$ & 525 \\
\hline$\geq 1$ & Britannicus & 1827 & Idrast xix & ; \\
\hline wey & Bucephalus & 1827 & Imbaldo $\mathrm{xx}$ & 50) \\
\hline $2: 3$ & Ilelote & 1827 & Robin Hood xx & 23 \\
\hline 21 & Phönix & 1827 & Robin Hood xx & 3i) \\
\hline 25 & Mambrino Il. & 1828 & Rubello & 26 \\
\hline 26 & Radical & 1824 & 1. Sebastian & 25 \\
\hline 27 & lieiram & $18: 4)$ & Hercules & 28 \\
\hline 28 & Mameluck & 1 1 & Inthrax & 26 \\
\hline 里) & Montrose & $13: 31$ & Hanibal & $2 i$ \\
\hline 30) & Robuste & $18: 31$ & Morisen & $2 \pi$ \\
\hline 31 & Claret & Is:33 & Cavalier xx & היה \\
\hline$: x^{2}$ & Ï̈iesco 1. & 1503 & I:anhoe & $2 \cdot 3$ \\
\hline 33 & Muff & $1 \times 31$ & Brutindorf $x x$ & 26 \\
\hline $3 !$ & Musquito & $1 \times 331$ & Babram $x x$ & 26 \\
\hline 35 & Negrociator & $1 \times 31$ & C:avalier xx & 3 \\
\hline 36 & Hector 11. & $1 \times 3$ & Tancred & 27 \\
\hline 37 & Heron & IS:35; & Cavalier & ;20 \\
\hline
\end{tabular}


No. Name of Stallion

born.

Sire.

inge

reached.

Years.

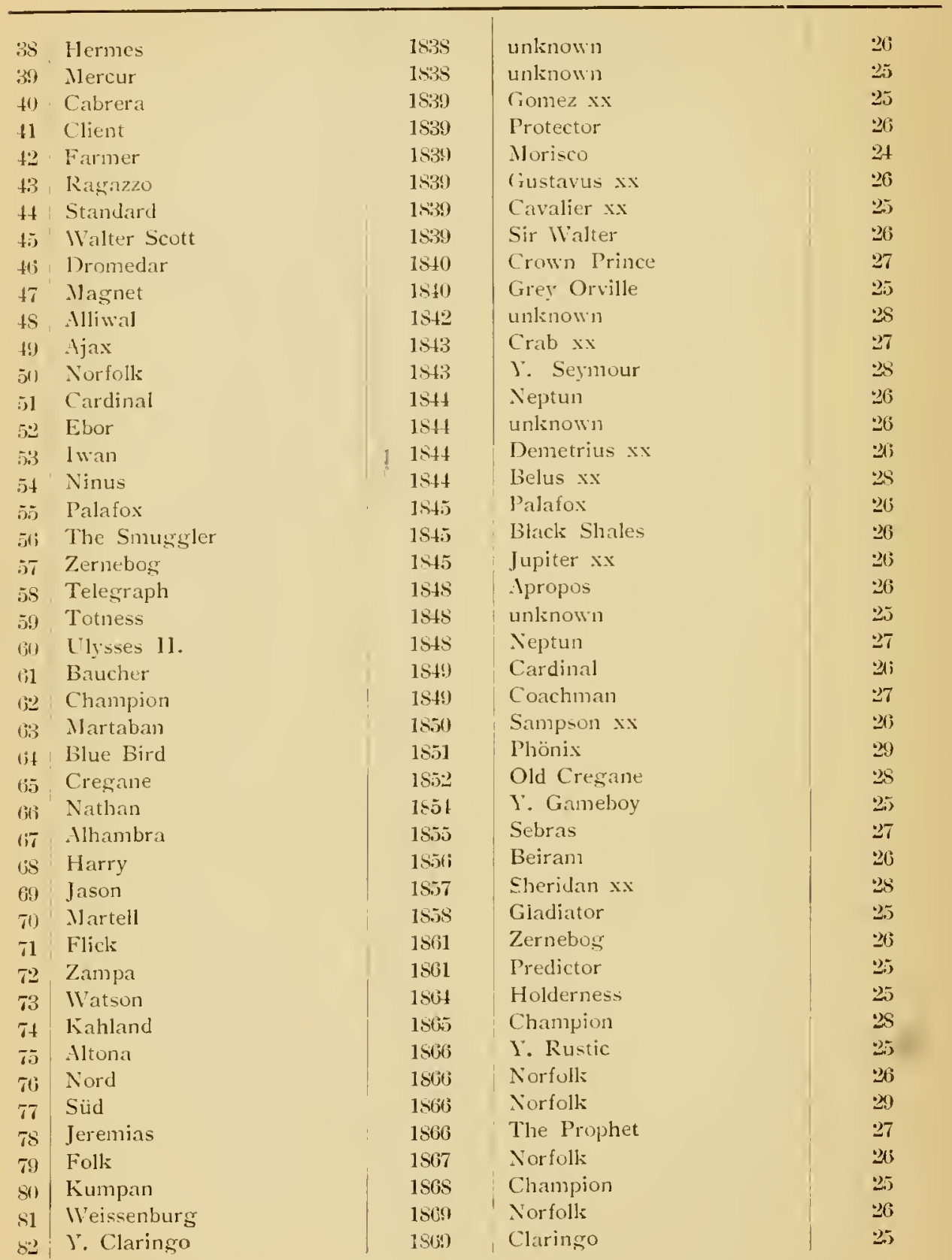




\begin{tabular}{|c|c|c|c|c|}
\hline Nu. & Name of Stallion. & Born. & sire. & $\begin{array}{l}\text { lgge } \\
\text { reached. } \\
\text { l'ears. }\end{array}$ \\
\hline 83 & Averbers & 1870 & Norfolk & 26 \\
\hline 84 & Nadock & 1870 & Nurfolli & 27 \\
\hline 85 & Tellus & 1871 & Duplicat & 25 \\
\hline 86 & Y. Norfolk & 1871 & Norfolls & 20 \\
\hline 87 & Norman & $18 \pi 1$ & Norfoll: & 20 \\
\hline 88 & Nornicls & 1871 & Norfolk & 25 \\
\hline s!) & Nordlicht & 1872 & Norfolk & 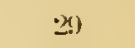 \\
\hline 10 & Norval & $187^{\circ} \cdot 2$ & Norfoll: & 26 \\
\hline 91 & Figaro & 1573 & Flick & .27 \\
\hline$(12)$ & Granada & 1573 & Alhambra & 28 \\
\hline 43 & Derwisch & 1874 & Lord Derby & 20 \\
\hline$\$ 4$ & Nording & 1575 & Nord & 23 \\
\hline 15 & Jessick & 1870 & Jeremias & 25 \\
\hline 96 & Juli & 1977 & Julius & 27 \\
\hline 9 & Augur Il. & 1578 & Belfort xx & 27 \\
\hline 18 & Jaspis & 1878 & Jason & 26 \\
\hline 99 & Julianus & 1878 & Julius & 20 \\
\hline
\end{tabular}

The following instances of the performances of -0 year-old and older Thoruughbred stallions in the production of first-class racing and breeding stock show very clearly that a great age in Thoroughbreds is no detriment in a grond heredity power. In this respect other light breeds, and more particularly heavy breeds, are a long way behind Thoroughbreds.

\begin{tabular}{|c|c|c|c|c|}
\hline 1 & Bverly Turk & 1680 & 21 & Jisgr and Basto \\
\hline 2 & Flying Childers & 171.5 & $\begin{array}{l}30 \\
-20\end{array}$ & $\begin{array}{l}\text { Snip } \\
\text { Brown Basto, Fam. 9.6 }\end{array}$ \\
\hline 3 & Partner & 1718 & $\begin{array}{l}21 \\
21 \\
202 \\
23 \\
23 \\
23 \\
24 \\
20 \\
20 \\
27 \\
20 \\
29\end{array}$ & 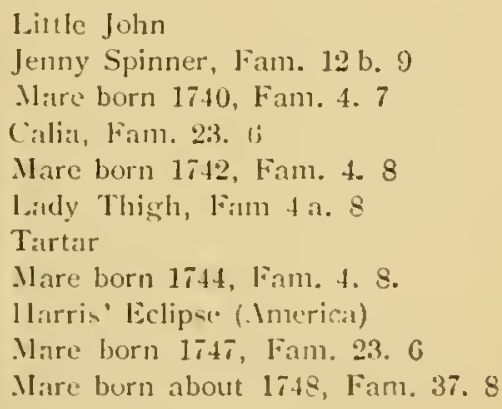 \\
\hline 4 & IJobgoblin & 1724 & 20) & Shakespeare \\
\hline
\end{tabular}


No. Name of the Stallions. Born. Age.

Produced the following progeny.

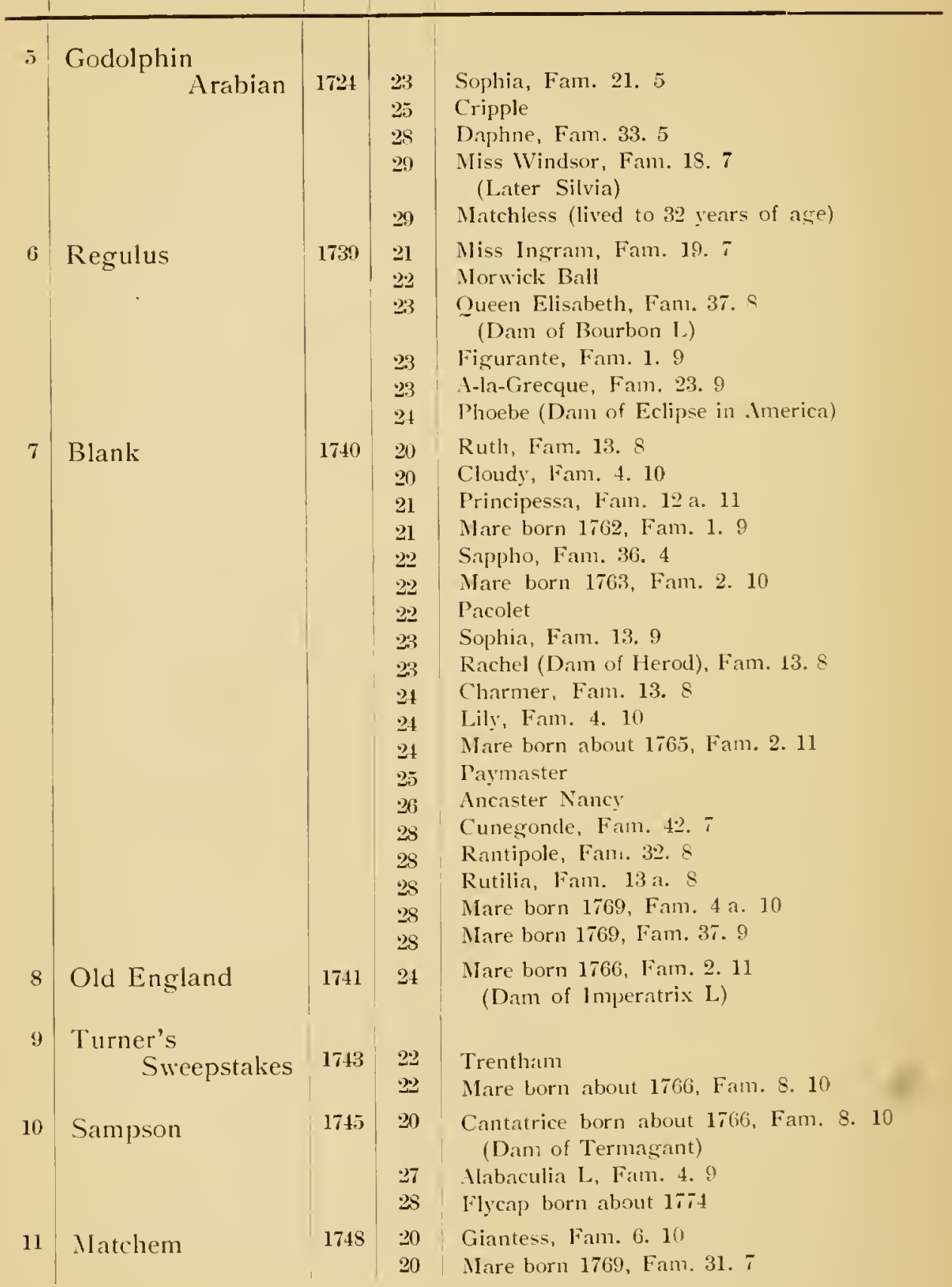


4. Value of the Thoroushbred for Other Breeds.

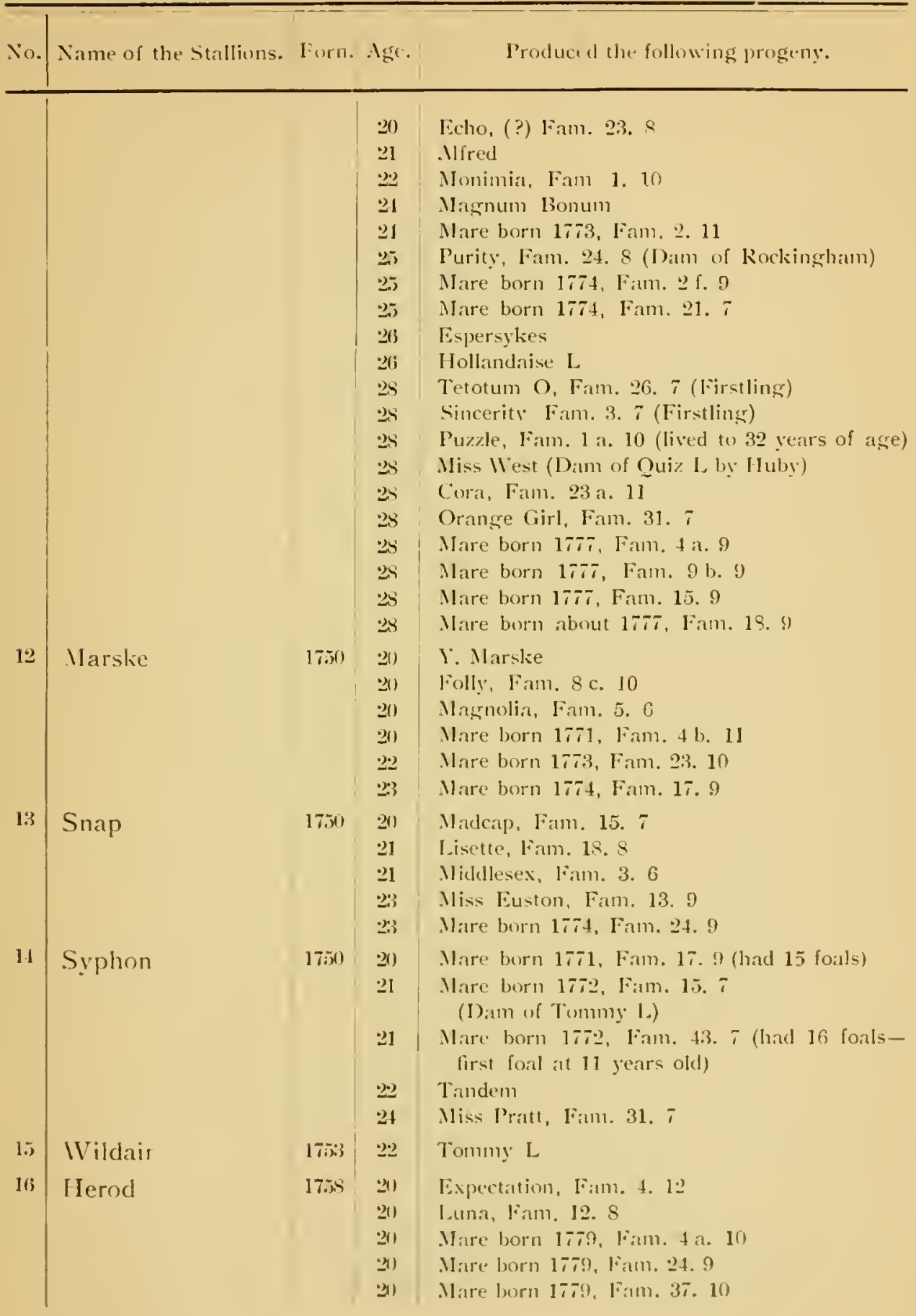


No. Name of the Stallions. Born. Age.

Produced the following progeny.

\begin{tabular}{|c|c|c|c|c|}
\hline & & $\beta$ & $\begin{array}{l}21 \\
21 \\
21 \\
21 \\
21 \\
21 \\
21 \\
21 \\
22\end{array}$ & $\begin{array}{l}\text { Ploenomenon L } \\
\text { Bagot } \\
\text { Maid of the Oalss O, Fan. } 3 \text { b. } 7 \\
\text { Macaria, Fam. t. } 9 \\
\text { Mare born 1780, Fam. 5a. } 6 \\
\text { Mare born 1780, Fam. 5a. } 7 \\
\text { Nare born 1780, Fam. 5c. } 6 \\
\text { Nare born 1780. Fam. 7. } 6 \\
\text { Editha, Fam. 3. } 6\end{array}$ \\
\hline 17 & Eclipse & 1764 & $\begin{array}{l}30 \\
20 \\
21 \\
21\end{array}$ & $\begin{array}{l}\text { Queen Mab, Fam. } 9 \text { b. } 9 \text { (out of the 28-year- } \\
\text { old Tartar Mare) } \\
\text { Violot, Fam. 13. 11 } \\
\text { Bobtail, Fam. } 3 \text { b. } 7 \\
\text { Serpent }\end{array}$ \\
\hline 18 & Florizel & 1768 & $\begin{array}{l}20 \\
20 \\
21\end{array}$ & $\begin{array}{l}\text { Tartar L } \\
\text { Lucy, Fam. 2. } 13 \\
\text { Ninety-Three L }\end{array}$ \\
\hline 19 & Y. Marstie & 1771 & $\begin{array}{l}21 \\
202 \\
22 \\
23\end{array}$ & $\begin{array}{l}\text { Shuttle } \\
\text { Mare born 1794, Fam. 2. 1:? } \\
\text { Mare born 1794, Fam. } 113 \\
\text { Mare born } 1795, \text { Fan. } 8 \mathrm{~d} .12\end{array}$ \\
\hline 2() & Putsos & 1773 & $\begin{array}{l}20 \\
20 \\
22 \\
23 \\
24 \\
25 \\
25\end{array}$ & $\begin{array}{l}\text { Mare born 1794, Fam. } 1 \text { 1.2 } \\
\text { Mare born 1794, Fam. } 3 \mathrm{e} .7 \\
\text { Mare born 1796. Fan. 17. } 11 \\
\text { Champion D and L } \\
\text { Dabschick, Fam. 12. } 10 \\
\text { Tyrant D } \\
\text { Grey Duchess, Fam. } 6.11 \text { (had } 19 \text { foals, and } \\
\text { lived to be } 28 \text { years old) }\end{array}$ \\
\hline $2 \mathrm{I}$ & IT'oodpecker & 1773 & $\begin{array}{l}20 \\
20 \\
21 \\
23 \\
24 \\
24 \\
24\end{array}$ & $\begin{array}{l}\text { Chesnut Slim, Fam. } 5 \text { d. } 7 \\
\text { Mare born 1794, Fam. 11.9 } \\
\text { Catherine, Fam. } 11 \text { b. } 9 \text { (Dam of Golumpus by } \\
\text { Gohanna) } \\
\text { Ephemera O, Fam. 3 b. } 18 \\
\text { Mare born 1798, Fam. 6. 12 } \\
\text { Mare born 1798, Fam. 12 a. } 13 \\
\text { Mare born 1798, Fam. 24. } 10\end{array}$ \\
\hline $2-2$ & Diomed & $177 \pi$ & $\begin{array}{l}23 \\
27 \\
28\end{array}$ & $\begin{array}{l}\text { Ball's Florizel } \\
\text { Sir Archy } \\
\text { Duroe }\end{array}$ \\
\hline 23 & Saltram & 1780 & 24 & Mare born 1805 (Dam of Timuleon) \\
\hline 24 & Alevander & 1782 & $\begin{array}{l}201 \\
202\end{array}$ & $\begin{array}{l}\text { Ptrepolis, Fam. } 12.10 \\
\text { Berenice, Fam. } 3 \text { b. } 9\end{array}$ \\
\hline
\end{tabular}




\begin{tabular}{|c|c|c|c|c|}
\hline$\therefore i$. & Xitume of the Stallions. & born. & Isse. & Produced the following prosgeny. \\
\hline 35 & Trumpator & 1782 & $\begin{array}{l}22 \\
24 \\
20 \\
27 \\
20 \\
21 \\
20 \\
2 \pi \\
20\end{array}$ & $\begin{array}{l}\text { Berenice, Fam. 14. } 10 \\
\text { Boadicea, Famt. 14. } 10 \\
\text { Thalestris, Fam. 2 c. 1.5 } \\
\text { Deita, Fam. 2f. } 14 \\
\text { Mare born 1803, Fam. 9. } 10 \\
\text { Woodnymph, Fam. 4 b. } 14 \\
\text { Mirth, Fam. 26. } 9 \\
\text { Pawn, Fam. } 1 \text { d. } 11 \\
\text { Prue, Fam. 11. } 10\end{array}$ \\
\hline $21 ;$ & Dun Ouisote & 1751 & $\begin{array}{l}21 \\
21 \\
21 \\
202\end{array}$ & $\begin{array}{l}\text { Cervantes } \\
\text { Pea Blossom, Fam. 1:2 a. } 16 \\
\text { Zoraida, Fam. 25. } 10 \\
\text { Imadis }\end{array}$ \\
\hline 27 & Sir Peter & 1784 & $\begin{array}{l}201 \\
201 \\
20 \\
20 \\
21 \\
21 \\
21 \\
21 \\
21\end{array}$ & $\begin{array}{l}\text { Petronius L. } \\
\text { Clinlier (2nd in 1..) } \\
\text { las, Fam. } 2 \text { f. } 13 \\
\text { Pluver, Fam. 27. } 10 \\
\text { leruvian } \\
\text { Fadladinida, Fam. 13. } 11 \\
\text { dametta, Fam. 1. 14 } \\
\text { Opal, Fam. } 3 \text { b. } 7 \\
\text { Zaida, Fam. 25. } 9\end{array}$ \\
\hline $2 i$ & IIaxy & $17(m)$ & $\begin{array}{l}21 \\
211 \\
20 \\
21 \\
21 \\
24 \\
21 \\
21 \\
21 \\
21 \\
21 \\
21 \\
213 \\
27\end{array}$ & 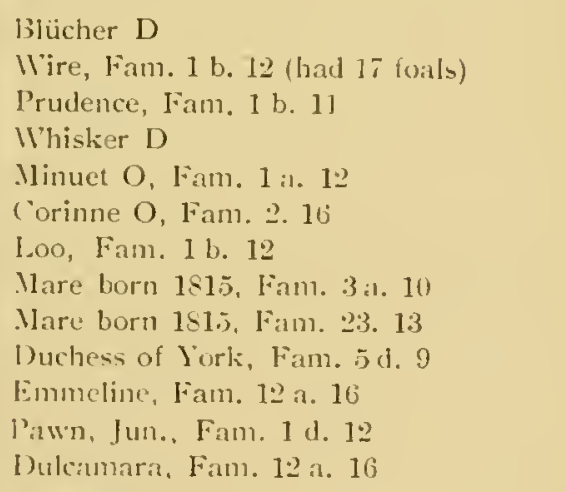 \\
\hline 39$)$ & Gulnanna & $17(n)$ & $\begin{array}{l}21 \\
.23 \\
203\end{array}$ & 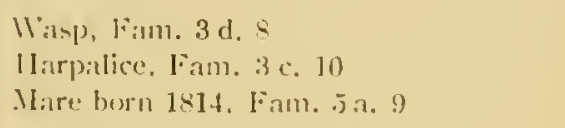 \\
\hline$: 31$ & Hambletonian & $17 \div 2$ & $\begin{array}{l}211 \\
2 ! 3\end{array}$ & $\begin{array}{l}\text { Ware born 1813, Fẻm. \&c. 1.j } \\
\text { (incrub, Fam. 16. } 6\end{array}$ \\
\hline 31 & Stamford & $17 ! 4$ & $\begin{array}{l}211 \\
2.3 \\
35\end{array}$ & 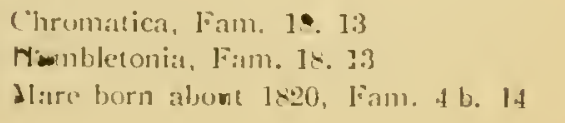 \\
\hline
\end{tabular}


No. Name of the Stallions. Born. Age. Produced the following progeny.

\begin{tabular}{|c|c|c|c|c|}
\hline 32 & Sorcerer & 1796 & 2) & Mare born 1817, Fam. 19. 1' \\
\hline 33 & Haphazard & 1797 & 20 & $\begin{array}{l}\text { Hazardess, Fam. } 10 \mathrm{~h} .7 \\
\text { Figaro }\end{array}$ \\
\hline 31 & Quiz & 1798 & $\begin{array}{l}20 \\
20 \\
202 \\
2.5\end{array}$ & $\begin{array}{l}\text { Irbis, Fam. 12. } 11 \\
\text { The Odd Trick, Fam 6. } 12 \\
\text { Globe, Fam. } 5 \text { c. } 9 \\
\text { Irethissa, Fam. 12. } 11\end{array}$ \\
\hline 35 & Orville & 17964 & $\begin{array}{l}20 \\
20 \\
30 \\
21 \\
24 \\
24 \\
25 \\
25 \\
20 \\
27 \\
27\end{array}$ & $\begin{array}{l}\text { Bizarre } \\
\text { Emilius D } \\
\text { Etiquette, Fam. } 1411 \\
\text { Georgina, Fam. } 32.12 \\
\text { Suuvenir, Fam. } 12 \text { a. } 17 \\
\text { Edmund } \\
\text { Manille, Fam. } 1 \text { b. } 13 \\
\text { Zcre, Fam. } 11.12 \\
\text { Mare born } 1826 \text {, Fam. 19. 12 } \\
\text { Nare born } 1827 \text {, Fam. } 11 \text { b. } 11 \\
\text { Mare born 1827, Fam. } 19 \text { a. } 11\end{array}$ \\
\hline 36 & Walton & 1799 & $\begin{array}{l}20 \\
21 \\
21 \\
2: 3 \\
23 \\
23 \\
23 \\
23 \\
23 \\
24 \\
25\end{array}$ & $\begin{array}{l}\text { Miss Fanny, Fam. 12. 12 } \\
\text { The Twinkle, Fam. 19.12 } \\
\text { Mare born 1821, Fam. 25. } 11 \\
\text { Galena, Fam. 23. } 14 \\
\text { Miss Patrick, Fam. } 4 \text { b. } 16 \\
\text { Mrs. Fry, Fam. 15. } 11 \\
\text { Mare born 1823, Fam. 19. } 11 \\
\text { Mare born 1823, Fam. } 9 \text { b. } 12 \\
\text { Miss Emma, Fan. } 12.12 \\
\text { Rosalia, Fam. } 19 \text { a. } 11\end{array}$ \\
\hline 37 & Castrel & 1801 & $\begin{array}{l}21 \\
202\end{array}$ & $\begin{array}{l}\text { Castrellina, Fam, } 3 \text { a. } 11 \\
\text { Pantaloon }\end{array}$ \\
\hline 38 & Selim & 1812 & $\begin{array}{l}21) \\
21 \\
2 \cdot 2\end{array}$ & $\begin{array}{l}\text { Nanine, Fam. } 3 \text { a. } 10 \text { (1)am of (ilaucus) } \\
\text { Mlare born } 1824 \text {, Fam. } 9 \text { b. } 11 \\
\text { Turquoise O, Fam. } 1 \text { b. } 12\end{array}$ \\
\hline 39 & Whalebone & 1007 & $\begin{array}{l}21 \\
211 \\
211 \\
21 \\
21 \\
21 \\
29 \\
22 \\
23 \\
23\end{array}$ & $\begin{array}{l}\text { Spaniel D } \\
\text { Mins Petworth, Fam. } 3 \text { c. } 11 \\
\text { Naind, Fam. 9.12 } \\
\text { Dryad, Fam. 3c. } 11 \\
\text { Fretna Green, Fam. } 3 \text { c. } 11 \\
\text { Mermaid, Fam. 12. } 13 \\
\text { Myrrha, Fam. 10.8 } \\
\text { Baleine, Fam. 8c. } 16 \\
\text { Bodice, Fam. 8c. } 16 \\
\text { Mare born 1831, Fam. } 10 \text { b. } 8\end{array}$ \\
\hline
\end{tabular}


No. Name of the Stallions. Born. Ige.

Produced the following progeny.

\begin{tabular}{|c|c|c|c|c|}
\hline 411 & Catton & 1819 & $\begin{array}{l}20 \\
202 \\
24 \\
24\end{array}$ & $\begin{array}{l}\text { Inne, Fam. 6. } 14 \\
\text { Mündis D } \\
\text {. Dalia, Fam. 42. 12 } \\
\text { Miss [Bewe, Fam. 2]. 12 (lived to } 29 \text { years of } \\
\text { age) }\end{array}$ \\
\hline 41 & Conmus & 18199 & $\begin{array}{l}21 \\
21 \\
21 \\
24 \\
25 \\
25 \\
25 \\
25 \\
25 \\
26 \\
20 \\
210\end{array}$ & $\begin{array}{l}\text { Mirdlime, Fam. 2f. 12 } \\
\text { Mare born 1831, Fam. 12. } 13 \\
\text { Mare born 1834, Fam. 2 a. } 16 \\
\text { Mare born 1834, Fam. 2 a. } 15 \\
\text { (irey Momus } \\
\text { Shire Oaks, Fam. 6. 14 } \\
\text { Mare born 1835, Fam. } 8 \text { b. } 17 \\
\text { Mare born 1835, Fam. 17. } 13 \\
\text { Mare born 1836, Fam. 8b. } 17 \\
\text { Mare born 1836, Fam. 5b. } 11 \\
\text { Mare born 1836, Fam. } 47.7\end{array}$ \\
\hline+2 & Ifuley & $18] 11$ & $\begin{array}{l}201 \\
23 \\
25 \\
26 \\
26\end{array}$ & $\begin{array}{l}\text { Muliana, Fam. } 9 \text { b. } 14 \\
\text { Rachel, Fann. } 2 \text { b. } 17 \\
\text { Mare born } 1836, \text { Fam. } 8 \text { b. } 15 \\
\text { l.jttle Wonder D } \\
\text { Mare born } 1838 \text {, Fam. } 19 \text { a. } 12\end{array}$ \\
\hline 43 & Tramp & 1810 & $\begin{array}{l}21 \\
21 \\
23 \\
21 \\
21 \\
21\end{array}$ & 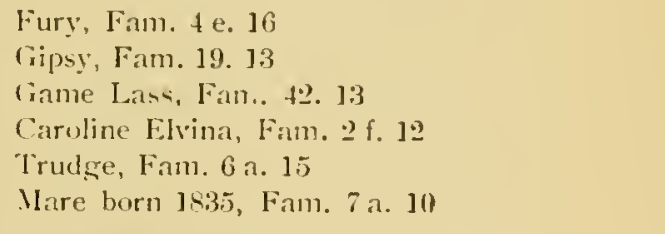 \\
\hline+1 & Dr. Syntax & 1811 & $\begin{array}{l}21 \\
206 \\
26 ; \\
26 i \\
27 \\
27\end{array}$ & $\begin{array}{l}\text { Bee's Wing, Fam. } 8 \text { a. } 16 \\
\text { bee's Wingr, Fam. 1 d. } 14 \\
\text { Syntaxina, Fam. Jc. } 11 \\
\text { Mare born 1838, Fam. 1 c. } 13 \\
\text { The Doctur } \\
\text { Dorothy, Fam. 6.1. } 15\end{array}$ \\
\hline 4i) & Parlisan & $1 \leqslant 11$ & $\begin{array}{l}01 \\
21 \\
21 \\
21 \\
203 \\
23 \\
23\end{array}$ & $\begin{array}{l}\text { Venison } \\
\text { Ciladiator } \\
\text { Cyprian O, Fam. 23. 15 } \\
\text { Mare burn 1834, Fam. 6. } 14 \\
\text { Constance, Fam, 22. 10 } \\
\text { Mare born 1835, Fam. 11 a. } 14\end{array}$ \\
\hline 10 & Imerioan Eclipse & 1811 & $\stackrel{21}{21}$ & Biriwner's lielipue \\
\hline 17 & Pantaloon & $18: 1$ & $\begin{array}{l}211 \\
.11 \\
.11 \\
.11\end{array}$ & 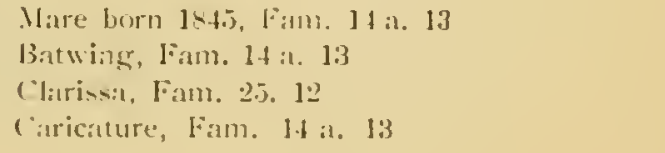 \\
\hline
\end{tabular}


No. Name of the Stallions. Born. Ige.

Produced the following progeny.

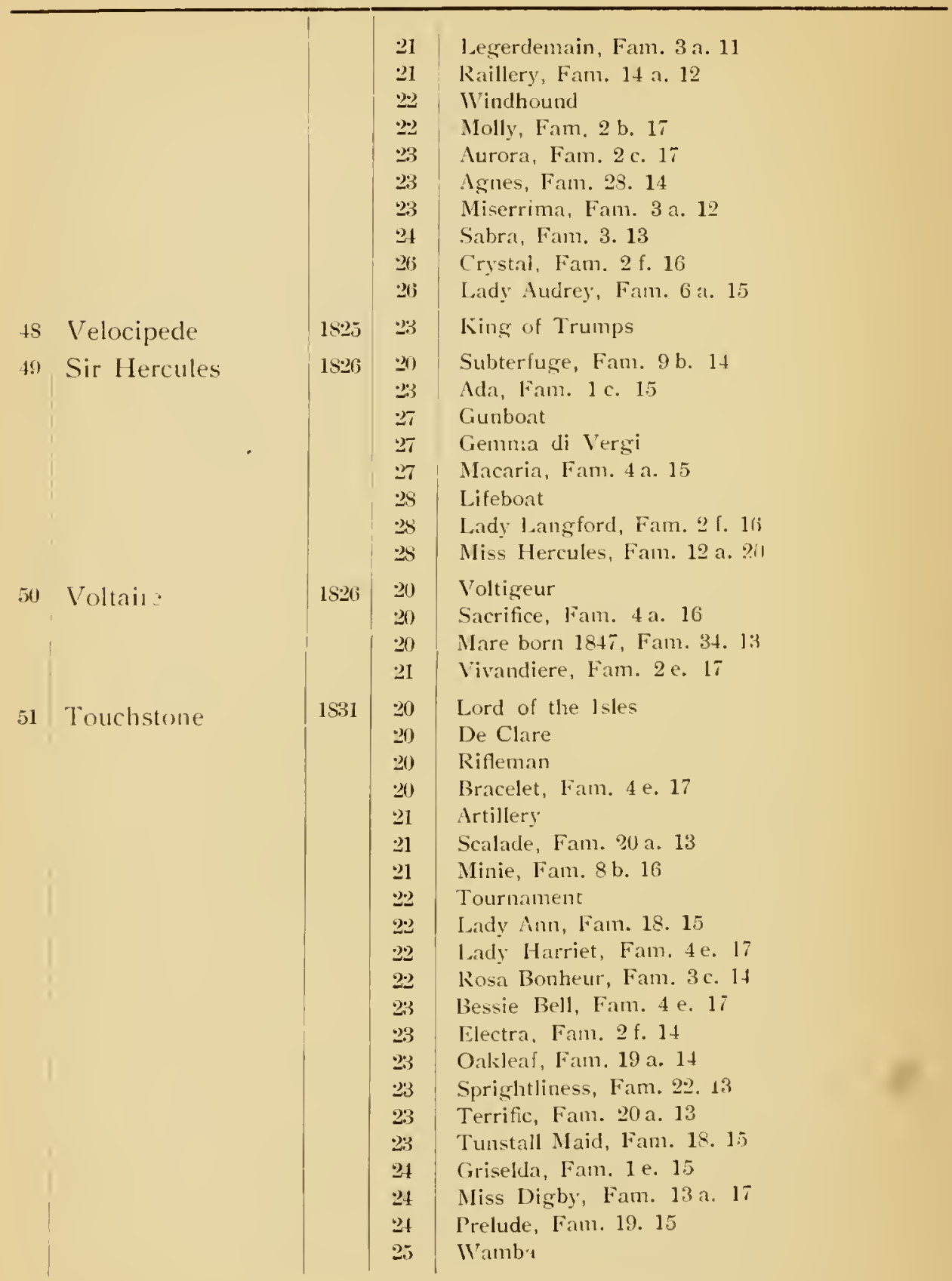




\begin{tabular}{|c|c|c|c|c|}
\hline No. & Name of the Stallions. & Born. & Ige. & Produced the following progeny. \\
\hline 52 & Birdcátcher & 1833 & $\begin{array}{l}25 \\
26 \\
26 \\
26 \\
26 \\
27 \\
27 \\
27 \\
23 \\
23 \\
20 \\
20 \\
20 \\
20 \\
20 \\
20 \\
20 \\
20 \\
21 \\
21 \\
21 \\
223 \\
23 \\
23 \\
23 \\
24 \\
21 \\
21 \\
23\end{array}$ & 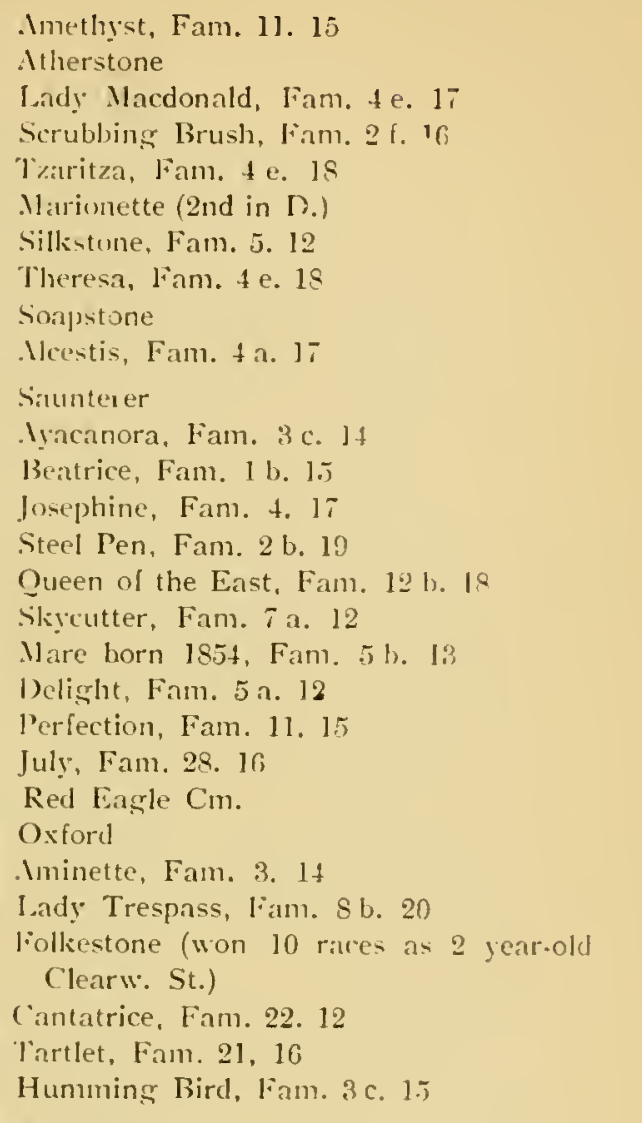 \\
\hline 53 & Gladiator & 1833 & 21 & Ventre St. Gris \\
\hline it & Caravan & J $18: 34$ & 21 & Souvenir \\
\hline 5i, & Melbourne & 1834 & $\begin{array}{l}201 \\
20 \\
201 \\
20 \\
20 \\
20 \\
20 \\
20 \\
21\end{array}$ & 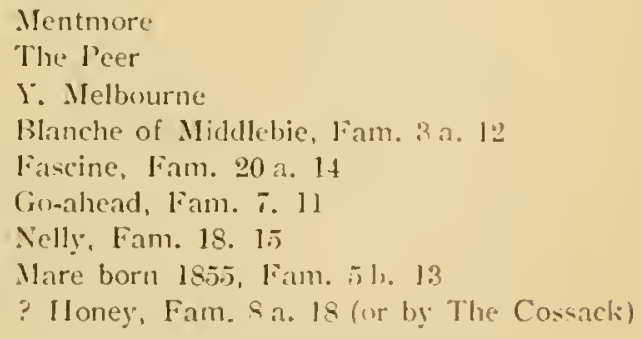 \\
\hline 30 & Newcourt & 1840 & 22 & Cecrops \\
\hline 57 & Orlando & 1811 & 20 & $\begin{array}{l}\text { Chattanooga } \\
\text { Fuiryland, Fam. 3r. } 12\end{array}$ \\
\hline
\end{tabular}


Nu. Name of the Stallions. Born. Age. $\quad$ Produced the following progeny.

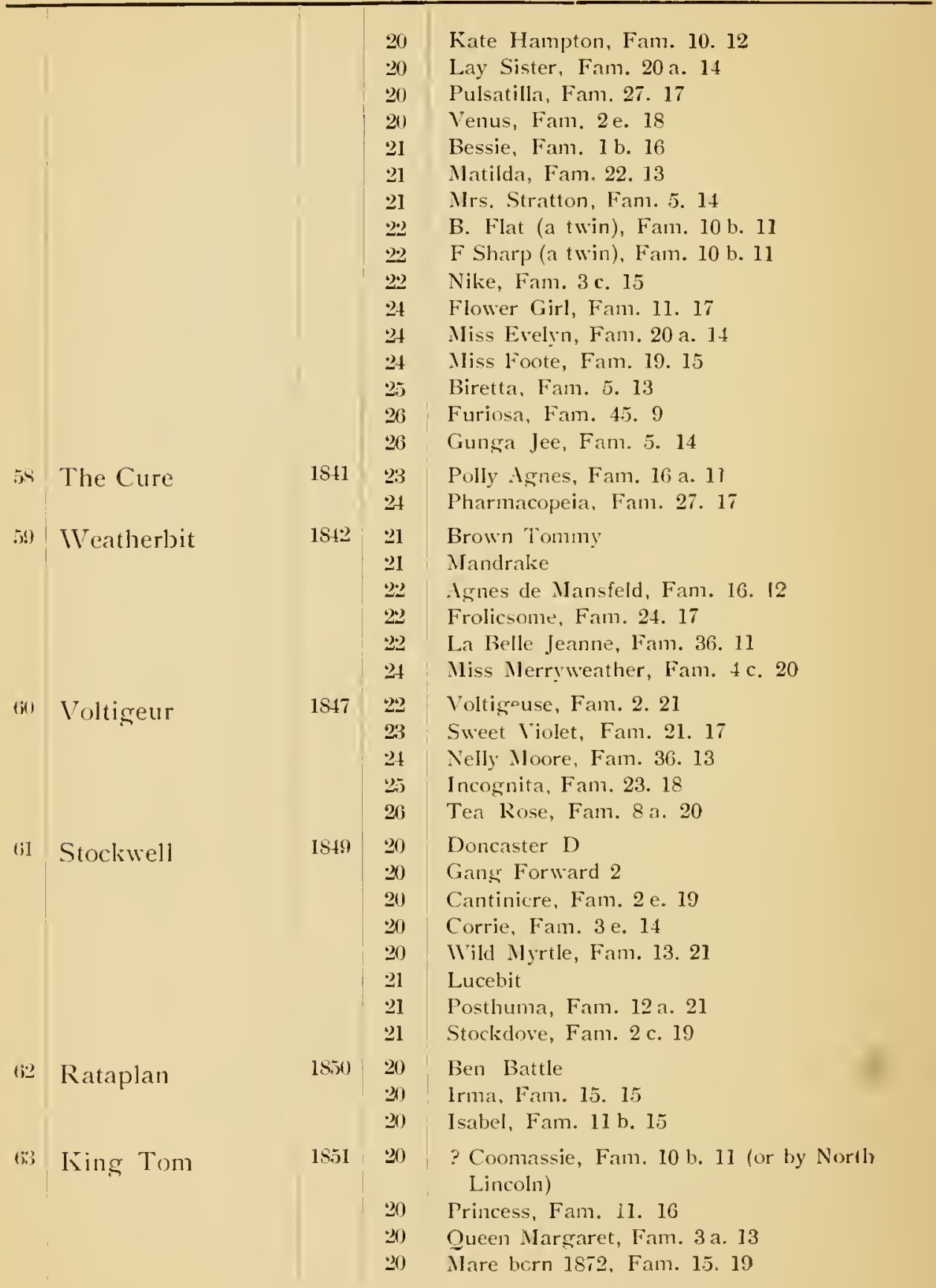


No. Nime of the Stallions. Born. Ige.

Produced the following prongeny.

\begin{tabular}{|c|c|c|c|c|}
\hline & & & $\begin{array}{l}21 \\
21 \\
21 \\
21 \\
21 \\
21 \\
22 \\
22 \\
22 \\
22 \\
23 \\
24 \\
24 \\
25 \\
26 \\
26\end{array}$ & 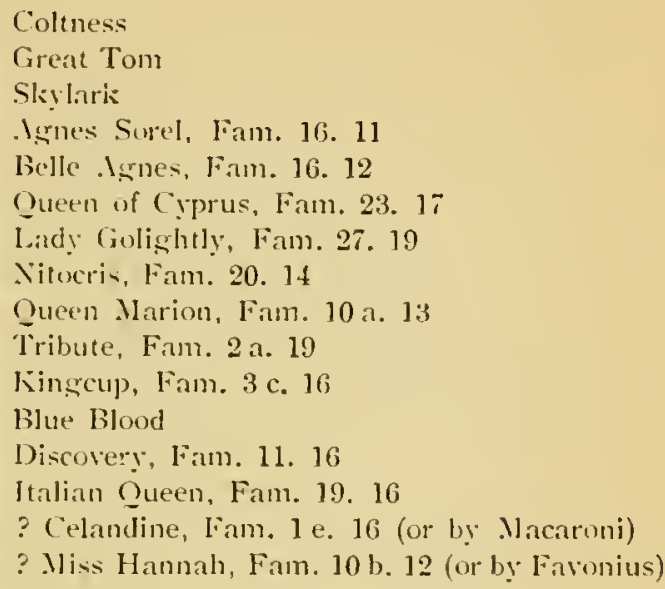 \\
\hline (6) & Bonnie Scotland & $185 ; 3$ & 21 & Bramble \\
\hline$(\mathrm{ij})$ & Leamington & 1853 & $\begin{array}{l}21 \\
21 \\
23 \\
24 \\
24 \\
24 \\
25\end{array}$ & $\begin{array}{l}\text { Jaconct, Fam. } 4 \text { a. } 20 \\
\text { Perfection, Fam. } 4 \text { a. } 20 \\
\text { Sensation } \\
\text { Iroquois D } \\
\text { Spinaway, Fam. } 11 \text { b. } 16 \\
\text { IYandlote, Fam. } 4 \text { a. } 22 \\
\text { Francesca (which had at } 25 \text { year-old Imos } \\
\text { Fudd by IJowland) } \\
\text { Onondaga }\end{array}$ \\
\hline 166 & Gunboat & 1854 & $\begin{array}{l}21 \\
23\end{array}$ & $\begin{array}{l}\text { Torpedo } \\
\text { Frisate, Fam. 20. } 16\end{array}$ \\
\hline 67 & Saunterer & 1854 & $2 \cdot 2$ & Elegrance, Frm. 12 b. 19 \\
\hline lis & Toxophilite & $185 \overline{5}$ & $\begin{array}{l}20 \\
20 \\
21 \\
21 \\
24 \\
24 \\
24\end{array}$ & 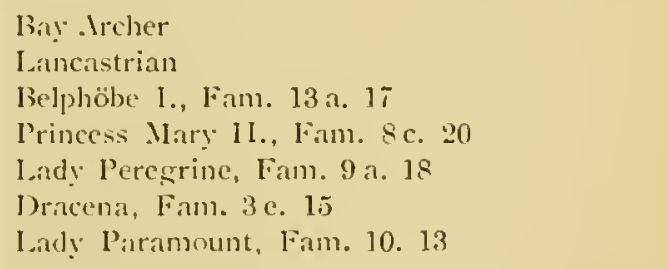 \\
\hline (39) & Buccaneer & 1857 & $\begin{array}{l}231 \\
20 \\
23 \\
23 \\
27 \\
25 \\
27 \\
27 \\
25 \\
201\end{array}$ & $\begin{array}{l}\text { Balvany } \\
\text { Vederemo } \\
\text { Vinea } \\
\text { Budagyongye N.I. } \\
\text { lencll O.D.U. } \\
\text { Ollyan-nincs P.L. } \\
\text { Talpra Magyar } \\
\text { lins-or } \\
\text { Kincs }\end{array}$ \\
\hline
\end{tabular}


No. Name of the Stallions. Born. Age.

Produced the following progeny.

71 Adventurer

72 Dollar

73 Bláir Athol

1857

20

20

$20 \quad$ Mare born 1878, Fam. 19. 16

21 Damages, Fam. 3 d. 13

22 Dart, Fam. 3. 15

$23 \mid 6$ (2 year-old winner in England)

25. Thunderstorm

20 Krakatoa

27

$1859 \quad 20$

21

21 Hirondelle, Fam. 10. 13

21 Pinta, Fam. 2. 22

21 Linda, Fam. 11.17

21 Planchette, Fam. 7. 13

22 Black .lgnes, Fam. 37. 19

2.) Wheatsheaf, Fam. 1 d. 17

1860

20 Lpas

24 Brocage

24 Dauphin

186120 Child of the Mist

20 Chiming Bells, Fam. 8 b. 19

20 Chiquilta, Fann. 20. 15

20 Glenrosa, Fan. 8 d. 19.

20 Insignia, Fam. 9 b. 16

21 Cyclopaedia, Fam. 10 b. 13

7 Norfolk

75 Savernake

76 Hermit
$1861 \quad 24$

$1863 \quad 23$

23

1864
Emperor of Norfolk

Bandit

Nickel

20 . Istrologer

20 Friar Balsam

20 Hazlehatch

20 Bella Donna, Fam. 10 a. 12

20 Devote, Fam. 4 a. 19

20 Fleur de Marie, Fam. 8c. 21

20 Hermia, Fam, 19. 17

20 Seraphine, Fam. 10 b. 13

20 Sly, Fan. 11 a. 18

21 Melanion

2l . Iustralia, Fanı. 1 c. 19

21 Seclusion, Fam. 2 c. 19

21 Single Barrel, Fam. 9 e. 18

21 Spoleta, Fam. 2. 20

22 Heaume 


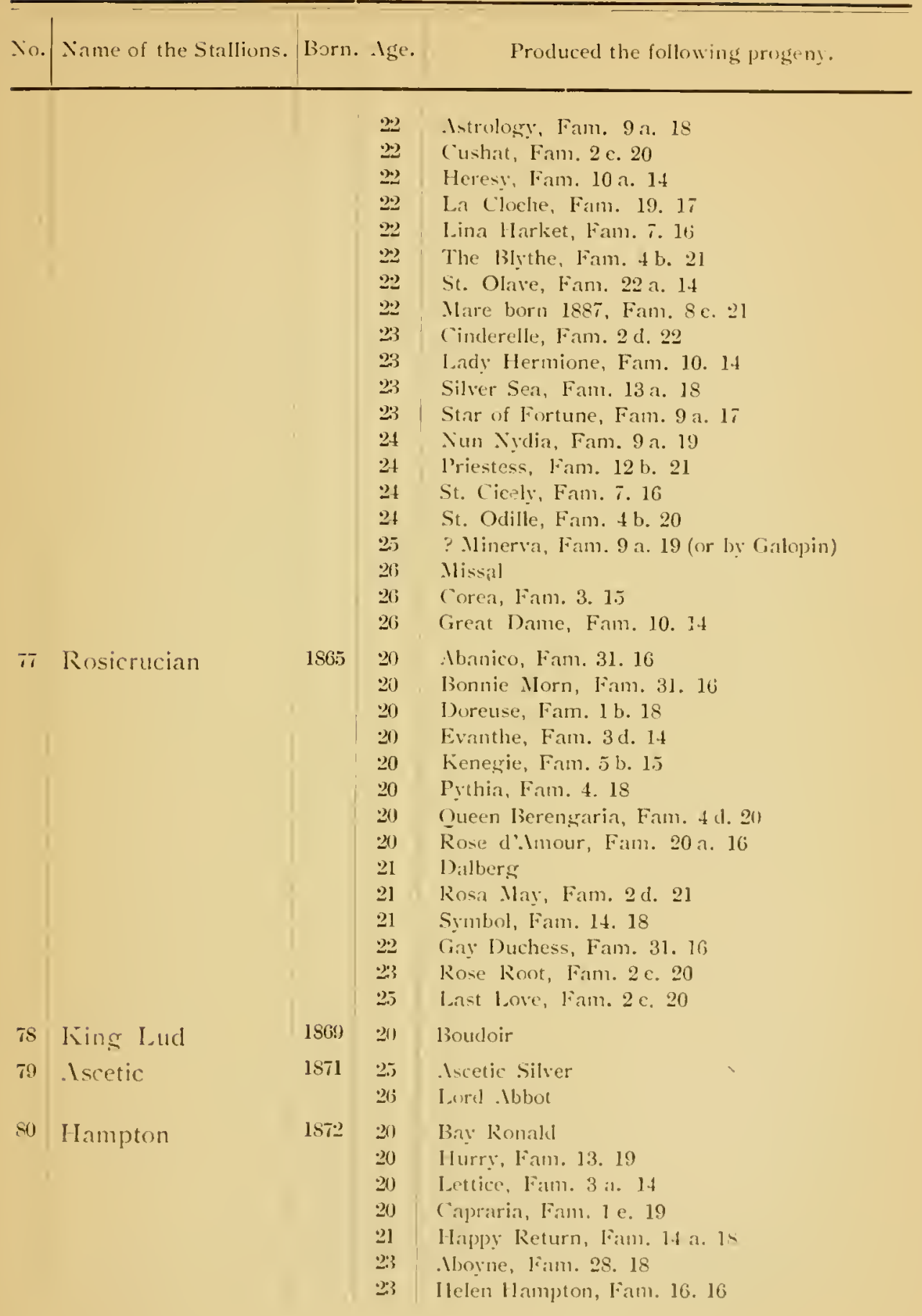


Xo. Mitme of the Stallions. Burn. Ige. Produced the following progeny.

81 Galopin

82 II aisenknabe

83 Kisber

st Springfield

85 Chamant

si Master Kildare
Stub-liampton, Fam. 10 b. 13

Thames Valley, Fam. 4 b. 21

Gadfly, Fam. 22. 16

lnquisitive, Fam. 3 e. 17

1872 29 Gilopin Lad

Ganache

20 Galeazzo

2) Gilata, Fam, 13 a. 19

(2) Pindi, Fam. 4 b. 20

20 Flitters, Fam. 1 b. 17

2l Grafton

21 Guerrier

21 Briseis, Fam. 2 d. 23

21 Galatia, Fam. 13 a. 19

2l Gelatine, Fam, 10 b. 13

21 ioletta, Fam, 22 a. 16

21 The Message, Fan, 20. 19

21 Gialopade 11., Fam. 8 c. 22

21 Miss Primrose, Fam. 1 b. 18

22 Brio

2:2) Disraeli

2.2 Canter, Fan. 16 a. 13

22 Daints, Fam, 20 a. 16

2.) Gathinthia, Fam, 7 a. 15

23 Galopin Lassie, Fam. 14. 17

23 Gallandra, Fam, 20 a. 17

21 Itbarra, Fam. 3 b. 18

24 Merry Gal, Fam. 5. 16

24 Nattie, Fam. 3. 16

25. . Vida, Fam. 9 a. 19

2.) Galicia, Fam, 10 a. 16

26 ? Nphabet, Fam. 14. 19 (or by Friar's Balsam)

1872 21 Bogdany

187321 Sperbers Bruder

1873 2.2 Cromlix, Fanı. 11 b. 15

20 - 25 (only 5 unimportant winners)

$1874 \quad 20$ Habenichts

$20 \quad$ Vollmond

22: Pomp

21 : Letzter Mohikaner

187523, Ostende Express 


\section{CHAPTER V.}

\section{A comparison of what Thoroughbreds have done previously and what they are doing at the present time.}

THE inportance of a solid and lasting formation of legs in the breeding of Half-breds, and the confession of Thoroughbred breeders that an improvement of this fundament by the infusion of Thoroughbred is very seldom if at all possible, raises the question as to $w \mathrm{hy}$ in this respect, which is so important, the Thoroughbred mar not be improsed, or, in other words, how one can breed Thoroughbreds which are able to give the necessiry stamina for the fundament. The answer to this question leads, lirst of all, to that well-known query, whether our present day Thoroughbred has not become worse during the last century, especially in respect to fundament.

We have not got enough exart and reliable statements of time of the races in former years, to compare them with the performances of to-day, and, therefore, cannot answer this question from records impartially. The records of Filying Childers at Newmarket over the Round Course and the Beacon Course were until now incorrectly said to be the best, and eren unsurpassed records of Thoroughbreds on a racecourse. This erronemus opinion probably originated by a wrong division, which William Pick makes in his Turf Register. Vol. 1., p. 12, where he reckons wat the recurd of Flying Childers to be nearly a mile a minute, that is to say $5.0 \frac{\pi}{2}$ feet = about ${ }^{2}$ metres, in a second, whereas from a correct caleulation follows a record of only lis metres in one sereond. Busdes, eren an exatet and reliable measure of time would not be a safe guide as to the differeme capalcity, because the distances for which horses were trained have changed so much, and formerly the tests nearly alwats louk place in several heats. It is $u$ be assumed that the present day Thureughbred for a cistance of 1 (u) $1 \frac{1}{2}$ finglish miles is quicker than the Thoroughbred of a hundred rears age. This aleme. however, would not be a sufficient prost of the improvement of the Thoroughbred in general, especially as regards its adaptability to improwe Half-breds, as we have no records to go through ats to hou quickly the former Thoroughbred could cover distances of 1 (1) $1 \frac{1}{2}$ linglish miles if 
trained by the method in rogue to-day, which is without doubt an improved one, especially if it had been trained for races for that distance, and not for t-mile heats.

Also, the measurements of time which we have for classic races for about the last fiftr vears, are insufficient to go on. Of the Derby and Oalis at Epsom we have the time fron the rear 18t6. Of the Doncaster St. Leger from the vear 1810. The tables given on the next page are characteristic.

Is the measurement of time can only give a correct measurement of proven performances for races of steady motion (as I have already shown mathematically in 1899 in my treatise entitled, " Das Vollblutpferd in seiner Bedeutung fur die 1 Jabbblutzucht"), it is not at all surprising that the above measurements are an insufficient criterion for the capacity respecting the yuality of horses. For example: acourding to these measurements, the very moderate (Cicero weuld be a by far better horse than either Ormonde, Persimmon, Flying Fox, Ard Patrick; and of the Oaks winners, Cherry Lass better than I. F Fleche, Sceptre, and Pretty Polly, and the celebrated ITheeliff-Fortune would be the worst. The times given for the St. Lecger would make us beliere that Troutbeck was the best of all, and that Challacombe was as goud as Pretty Polly, and much better than Ormonde, Galtce More, Persimmon, etc.

According to the Derby time, Ormonde ought to be worse than his predecessors, Melton and Kettledrum. Furthermore, Sir Tatton Sykes, born 1813. ought to be much better than Ormende, Persimmon and Caltee More. Is a matter of fact, from these messurements we can see that the Derby and Oals times have become considerably shorter than those for the longer distance St. Leger. The reason probably is, that with the invasion of the Imerican juckeys, the quicker setting off made more difference in the shorter races than in the longer St. lecger, or perhaps the speed of the present day Thoroughbred has not improved so much for longer distances as for slowrter ones. Or, again, it is possible that the changing of the starts for the Derby and the Oaks, which took place in the rear 187:- has effected an improrment of the time, as this alteration caused the first incline to be less difficult. Of course, one must not lose sight of the fact here that since 1872 the Derby distance has been lengthened by 23 metres, and the St. leger clistance (in 1826) has been shortened by 56 metres.

To draw a definite conclusion as to capabilitr, even from the latest, reliable measurements of time seem to be out of question, especially when these measurements have to be compared with those of a hundred years ago.

Most measurements of olden times are given in Orton's Turf Annuals, esperially for the lork races. I great deal of these measurements has, unfortunately, been given for races in which insignificant horses ran. The following riamples (from the earliest times to 1832) give the best record performances 1 have found: 
5. The Thoroughbred in the Past and Present.

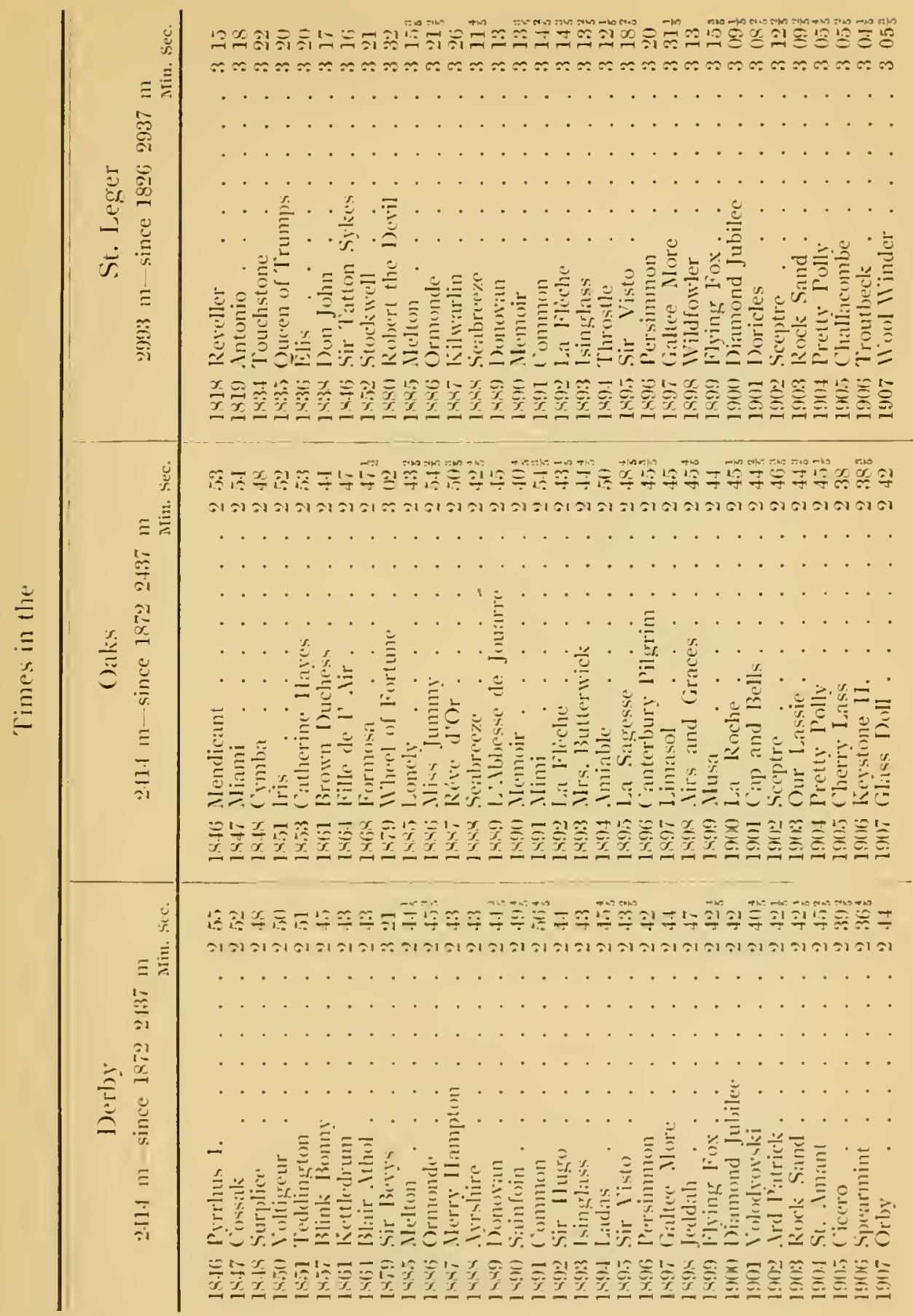




\section{Distance 4 miles $=6437.2 \mathrm{~m}$.}

1759. Cade 5 vear-old by Cade, 9 stone ..... Min. Sec

1759. Silvio 5 year-old by Cade, 10 stone . . . . . . . . . 8 . 8

1759. Careless 9 year-old by Regulus, 9 stone . . . . . . . 88

1764. Beaufrement 6 year-old by Tartar, 8 stone, 7 lbs. . . . . 751

1766. Bay Malton 6 year-old by Sampson, 9 stone . . . . . $743 \frac{1}{2}$

1793. Huby 5 year-old by Phoenomenon, 8 stone, 7 lbs. . . . $730 \frac{7}{2}$

1801. Sir Solomon 5year-old by Sir Peter, 8 stone, 7 lbs. . . . $710 \frac{1}{2}$

1802. Alonzo 4 year-old by Pegasus, 8 stone, 7 lbs. . . . . 78

1803. Haphazard 6 year-old by Sir Peter, 8 stone, 10 lbs. . . 732

180j. Sasoni ó year-old by Delpini, 8 stone, 4 lbs. . . . . 728

1809. Petronius 4 year-old bỵ Sir Peter, 7 stone, 9 lbs. . . 725

1810. Theresa 5 year-old by Jambletonian, 8 stone, 5 lbs. . 730

2. Distance 2 miles $=3218.6 \mathrm{~m}$.

Min. Sec.

1804. Witchcraft 3 year-old by Sir Peter, 8 stone, 2 lbs. . . . 340

1815. Catton 6 year-old by Golumpus, 8 stone, 2 lbs. . . . . 333

1824. Fair Charlotte 6 year-old by Catton, 8 stone, 10 lbs. . 328

1827. Mulatto 4 year-old by Catton, 8 stone, $3 \mathrm{lbs}$. . . . . . 334

1829. Velocipede 4 year-old by Blacklock, 7 stone, 12 Ibs, . 333

1830. Fortitude 4 year-old by Whislier, 7 stone, 8 lbs. . . . 329

\section{Distance $1 \frac{3}{4}$ miles $=2816.3 \mathrm{~m}$.}

Min. Sec.

1809. Sheba's Queen 3 year-old by Sir Solomon, 8 stone . . . . 320

1824. Sandbeck 6 year-old by Catton, 8 stone, 3 lbs. . . . 313

1826. M'Adan 3 year-old by 'Tramp, s stone, 5 lbs. . . . . . 317

1826. Belzoni 3 year-old by Blacklock, 8 stone, 5 lbs. . . . . 321

1827. Medoro 3 year-old by Cervantes, s stone, 5lbs. . . . . 312

1827. Maleck 3 year-old bỹ Blacklock, 8 stone, 5 lbs. . . . . 317

1828. Velocipede 3 year-old by Blacklock, 8 stone, 5 lbs. . . . 320

1829. Sir Hercules 3 jear-old by 11 halebone, 8 stone, 5 lbs. . . 315

1830. Chancellor 3 year-old by Catton, 8 stone, 5lbs. . . . . 318

\section{Distance $1 \frac{1}{2}$ miles $=2414 \mathrm{~m}$.}

Min. Sec.

1804. Quid 3 year-old by Star, 8 stone . . . . . . . 254

1804. Lady Brough 3 year-old by Stride, 8 stone . . . . . . 247

1824. Abron 4 year-old by IThisker, 8 stone, 6 lbs. . . . . . 251

1826. Helenus 5ear-old by Soothsayer, 9 stone, 6 lbs. . . . . 249

1827. Mauto 3 year-old by Tiresias, 8 stone, 3 lbs. . . . . . 250

1832. Retainer 3 year-old by Jerry, 8 stone, 7 lbs. . . . . . 247

I These two races were run at Doncaster, all the others at York. 
5. Distance $1 \frac{1}{4}$ miles $=2011.6 \mathrm{~m}$.

Min. Sec.

18:8. Laurel 4 veatold by Blacklock, \& stone . . . . . . . 2 15

1832. Mare 3 year-old by Figaro, 8 stone, $\geq$ lbs. . . . . . . 2 1:

\section{Distance $1 \mathrm{mile}=1609.3 \mathrm{~m}$.}

Min. Sec.

1811. Bethlem Gaber 3 year-old by Surcerer, 8 stone, - 1bs. . . 140

1826. Brother to Miss Fanny 3 year-old by 11 alton, 8 stone, 6 lbs. 1 4:3

1827. Tom Jones 3 year-old by Ibjer, s stone, jlbs. . . . . I 45

1829. Voltaire 3 year-old by Blacklock, os stone, j lbs. . . . . 14

7. Distance Redhouse at (Doncaster) $=5$ furlongs, 152 yards $=1145 \mathrm{~m}$.

IIin. Sec.

1s:6. Moonshine ? year-uld by Grey Middleham, S stone, j lbs. 1 I:

8. Distance T. Y. C. $=5$ furlongs, 44 yards $=1046.2 \mathrm{~m}$.

1806 Min. Sec.

180s. Middlethorpe ? year-old by Shuttle, s stone, 22 lhs. . . . 120

1824. Indrogeus 2 year-old by Ilinos, 8 stone, j̆lbs. . . . . 1 15

1827. Velocipede 2 year-old by blacklock, 8 stone, 5 1br. . . . 19

1827. Slut 2 year-old by "Tramp. 7 stone, 11 lbs. . . . . . . 114

18:8. Mare 2 year-old by Jack Spigot, 8 stone, 2 1bs. . . . . 114

1830. Chorister 2 year-oid by lottery, 8 stone, jlbs. . . . . 18

1830. Clarence 2 year-old by Comus, 8 stone, j lbs. . . . . . 111

besides the above, the following interesting performances are well linumn :-

1. Distance $6120 \mathrm{~m}$. at Newmarket, Round Course $=3$ rmiles, 6 furlongs, 93 yards.

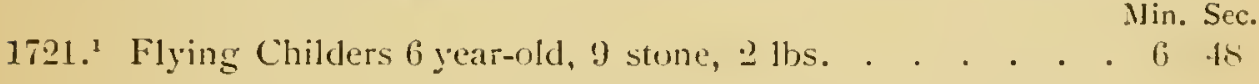

2. Distance $6764.6 \mathrm{~m}$. at Newmarket, B. C. $=4$ miles, 1 furlong, 138 yards.

1721.2 Flying Childers, (j year-c)ld . . . . . . . . . 730

175\%." Matchem 7 year-old, 8 stune, 7 lbs. . . . . . . . . 7 20

1792." Hambletonian 7 year-t)ld, \& stume, :3 1bs. . . . . . . 7 j.j

. It Eclipse's time several horses . . . . . . . . 8 -

3. Distance $1610 \mathrm{~m}$. at Newmarket, R. $M 1=1$ mile, 1 yard.

177:3.5 liretail trear-uld by Squirrel, s stone. . . . . Min. Sec.

'Sporting Calendar by J. Pond, Vol. 1., 17.51, p. 203.

= Turf Register by Pick, Vol. 1., p. 12.

${ }^{3}$ Turf Register by Pick, Vol. 1., p. J36.

- Raciana by .lluir, p. 162.

s Racing Calcndar 1763, p. 14; Turf Regrister by lick, Vol. 11., p. 327; Morning Post and Daily. Idvertiser, 19h .Ipril, 1773; and Sporting .Magazine, Vol. 1V., p. 325. 
The time measurement in the race of Firetail at Newmarket over a mile shows a record performance of almost 2.5 $\mathrm{m}$. in the second, and appears to me to be incorrect. Possibly a printer's error crept in the "Morning Post," and the others took the report from the newspaper. The remaining time measurements show the following maximum performances:-

\begin{tabular}{|c|c|c|c|c|c|c|}
\hline $\begin{array}{c}\text { Distance } \\
\text { Meter. }\end{array}$ & lear. & Place. & lame of Horse. & $\begin{array}{l}\text { Age } \\
\text { lear. }\end{array}$ & $\begin{array}{l}\text { Weight. } \\
\text { st. lb. }\end{array}$ & $\begin{array}{l}\text { Meter in } \\
1 \text { Second. }\end{array}$ \\
\hline 6764,6 & 1792 & Newmarlset & Hambletonian & 7 & 83 & 15,55 \\
\hline 6137,2 & $180^{\circ}$ & I)oncáster & . 1 lonzo & 4 & 87 & 15,04 \\
\hline 6120 & 1721 & Newmarket & Flyogng Childers & 6 & $9 \stackrel{2}{2}$ & 15,00 \\
\hline 3218,6 & $18: 4$ & Yorle & Fair Charlotte & 5 & S 10 & 15,41 \\
\hline 2993 & 1818 & Doncaster St. Leg. & Reveller & 3 & 82 & 15,35 \\
\hline 2816,3 & 1827 & York & Medoro & 3 & 85 & 14,67 \\
\hline 2414 & 1804 & York & Ladỵ Brough & 3 & 80 & 14,45 \\
\hline 2011,6 & 1832 & Yorls & Mare by ligaro & 3 & $s 2$ & 15,21 \\
\hline 1609,3 & 1811 & York & Bethlem Gaber & 3 & 82 & 16,09 \\
\hline 1145 & 1820 & Doncaster & Moonshine & 2 & 85 & 15,90 \\
\hline 1046,2 & 1830 & York & Chorister & 2 & 85 & 15,38 \\
\hline
\end{tabular}

The best known reeord performances in England during the last twentyyears are as follows:-

\begin{tabular}{|c|c|c|c|c|c|c|}
\hline $\begin{array}{l}\text { Distance } \\
\text { Neter. }\end{array}$ & Vear. & l'lace. & Nimle of Horse. & $\begin{array}{l}\text { lge } \\
\text { lear. }\end{array}$ & $\begin{array}{l}\text { Weight. } \\
\text { st. Ibs. }\end{array}$ & $\begin{array}{l}\text { Meter in } \\
1 \text { Sccond. }\end{array}$ \\
\hline$+2 \div 24,6$ & 1880 & Doncister. Cup. & Dresden China & 4 & & 17,24 \\
\hline 3646,6 & 1881 & Newmarlset. Ces. & Fuxhall & 3 & 712 & 15,12 \\
\hline 3646,6 & 1880 & Newmarket. Ces. & Robert the Devil & 3 & 86 & 14,05 \\
\hline $29: 37$ & 1904 & Doncaster. St. Leg. & Pretty Polly & 3 & S 11 & $15, \mathrm{SS}^{\prime}$ \\
\hline 2414 & 190.5 & Epsom. Derby & Cicero & 3 & $9-$ & $15,18^{2}$ \\
\hline 2114 & 1905 & Eipsom. Oaks & Cherry Lass & 3 & $9-$ & $15,28^{2}$ \\
\hline 1609,3 & 1885 & Lincoln. Hdep. & Bendigo & 5 & $S \quad 5$ & 16,62 \\
\hline 1407,4 & 188: & Manchester. Hdcp. & Tuastmaster & 5 & 96 & 17,28 \\
\hline 1206.9 & $188^{\circ}$ & Epsom. Moodc. St. & Beau Brummel & 2 & $8 \quad 12$ & 15,91 \\
\hline 1006 & $188^{2}$ & Manchester & Chislehurst & 2 & $8 \quad 10$ & 15,16 \\
\hline
\end{tabular}

The proportionately little differences in time in the classic races show that these times are the most reliable, and, therefore, the most suitable for

Best time in the St. Leger.

Best time in the Derby.

${ }^{3}$ Best time in the Oalis. 
making comparisons. A little more than 15 metres to the second seems to be about the distance which the better kind of racehorses of former, as well as of the present time can do. Just about the middle of this period lived the celebrated 17 est I ustralian, born 1850, winner of the Derby, St. Leger, and 2,000 Guineas. Mlso its record for the St. lecger was 15.41 metres, for the Iscot Gold Cup (distance 3,98:- metres) 15.11 metres. In the Iscot Gold Cup of 186:3, Buckstone's record, as a four-year-old, with 8 stone, 7 lbs., after a dead leat, was 16.10 metres, and in the year 1897 , Persimmon's record, as a four-year-old, 9 stone, was 11.018 metres.

The compilation of the best reourds of ancient and modern times, after taking into consideration all acressories, show therefore, neither a progress nor a retrogression in the capability of the Thoroughbred. ()nly within the last few years the American methed of riding, with its quick getting off, and its quick pace all along. serems to have affected a little the shortening of the time, and to prevent greater differences of time as happened previously.

Inwever, the English race calendars show that there exists a marked difference in the endurance of older horses. The difficulties of training racelyorses, and especially the diffeulty of bringing so-called lirst-class racehorses after their fourth or fifth year again on the track, are well linown, and are caused by the insulficient resisting capacity of the fundament of the present day Thoroughbred.

Every sportsman will acknowledge this regrettable fact, i.e., that the galloping capability of the present day "Thoroughbred has been more developed than the less developed fundament ran stand.

In order to get an actual basis to compare the performances of older Thoroughbreds of former times with those of the present time on the flat, I have compiled, in the following tables, begioning with the most ancient times up to the time of lie celebrated lisherman (therefore about the middle of the last contury), the most prominemt performanees of older horses on the llat. Is a rule, I have only taken such horeses that have won flat races as eight-year-nlds and abose that age (ientlemen's llat races are not mentioned, but the more celebrated steepleweldasers I have noted. I have compiled on the basio of the male asernt. Of the ligures given under each horse, the one wn the left shows the are at which the horse first started racing, and the one on the right shews the age at which it won last on the flat. Sires of horses which have won races all a creat age have also their own perfermances mentioned in the same manner as a emparison.

To) judse of the influence of the early and frepuent twe-pear-old races, 1 have given, with the sires as well as with the dams of the respective racehorses, in which month they first ran as woryear-olds, and how ofter altogether as two-year-olds. Ithere the dam did not run at all as a two-yearold 1 have not mentioned her at all. 


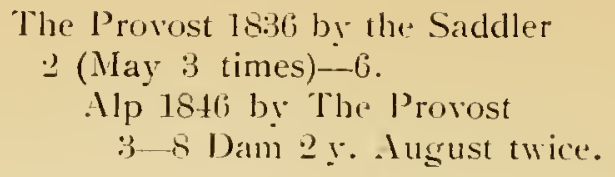

means, The Provost ran as a two-year-old first in May, and altogether as a two-year-old three times, and won at last as a six-year-old. Alp ran first as a three-year-old. Its last win was as an eight-year-old. Its dam ran first as a two-year-old in August, and altogether twice as a two-year-old.

\section{Darley's Arabian born 1702.}

1. BRISk 1711 by Darley"s . Irabian.

5.

Foxhunter (Cole's) 1727 by Brisk.

(Half-brother to Cones slins.)

6-9.

MOSLS 1746 by foxhunter (cile's).

4.

Otho 1760 by Moses.

\pm-7 .

1. COXCOMB 17i1 by Otho.

$3-4$, then up to 18 year-old used as a hunter, at 17 year-old won a Match over 2 miles with heats at St. Ives.

Blackcock 17 \&2 by Coxcomb. 3 - 8 .

2. ILEPI'() 1711 by Darley's Mrabian.

5.

Hobyololin 1724 by . .leppo.

$5-9$.

SIIAKLSPLARE: 1745 by Hubgoblin.

$4-8$.

l'incher 1765 by Shakespeare.

4-9. (Half-Brother to King Fergus).

3. FIIIY(: C'HILI)ERS 1715 by Darley's . Irabian.

$6-8$.

1. Second 1732 by Flying Childers.

5-11.

2. Blaze 1733 by lilying Chikers.

$5-7$ (up to 10 year-old without winninge).

1. SAMPSOX 1745 by Blaze.

$5-7$.

1. Engine(er 175.5 by Sampson.

$5-10$.

MAMBRINO 1768 by Engineer (s. to America).

5-8 (up to 11 vear-old without winning).

2. Bay Malfon 1760 by Sampsun.

$4-8$.

3. Pilgrim 1762 by Sampson.

4-12. 
5. The Thoroughbred in the P'ast and Present.

1. Sulun 1766 by Sampson.

$1-10$.

2. SCRUI 1751 by Blaze.

$4-10$.

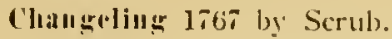

$4-10$.

3. Ninip 1736 by Flyingr Childers.

$\overline{5}$

S.AP 1750 by Snip.

$6-7$.

1. Iathom S Suap 7059 by Snap.

$4-8$.

ST.1RTIMG T(O./ 17\%.2 by Lathom's Snap.

$4-10$.

2. Melaplysician 176:3 by Snap.

$4-8$.

3. (ioldfuder 1764 by Snap.

$1-6$.

1. КNIGIT ERR.NT 17Tt by Goldfinder.

is 8 .

‥ DOCTOR 17is by foldfinder.

3-9.

4. Prize 1767 by Snstp.

j) -9.

5. Mexican 1775 by Snap) (s. to Virginia).

3. 8 .

4. B.IR'IIA'T'X (or Bleeding) Childers about 1716 by Darley srabian.

(Not run).

1. Syuirt 1732 bỵ Bartlet's Childers.

i) 8 (up to 11 year-old without wimning, also in Give and Take Pl.).

1. STPlller 1750 by Squirt.

4.

1. Sweer William 1i68 by Syphon.

48 .

2. Siweeluriar 1869 by syphon.

10.

1. CHOCOL.1TE $17 \%$ by sweetbriar. $3-5$.

Jerry Sneak 1796 by (hocolite wut of 25 year-old Mother

2 (October, once)-13. [irown. 4-11.]

1. Fitzjerry 180- by Jerry Sneak.

$2-9$.

2. IInIIymount 181ㄹ by Jerry Sneak.

$4-10$.

2. E.ICISEM.1.N 17, h Swectbriar.

8-12.

2. M.IRSKl: 1750 by Squirt.

4 (5) and o year-old without winning).

1. Eclipse 1704 by Marslie.

$5-6$. 
1. JA I'ELIN 1772 by Eclipse.

4.

1. Chance 1780 by Javelin (s. to Russia).

$4-8$.

2. Helmet 1788 by Javelin.

$3-10$.

3. I'. Jaźclin (later Antrim) 1795 by Javelin.

$2-8$.

2. POTSOS $17-33$ by Eclipse.

4- 10 .

1. Coriander 1786 by lot 8 os.

3-8.

N arcia 1797 by Coriander.

$3-9$.

2. Telescope 1786 by Pot8os.

3 - 8 .

3. Alderman 17st by Potsos (s. to Tirginia).

3-9.

4. Druid 1790 by Pot 8 os.

3 -s.

5. W'axy 1790 by Pot8os.

3-6. D.

1. Waxy Pope 1806 by Maxy.

3-6. D.

1. Noble 1816 by IVaxy Pope. $5-9$.

2. Gossoon 1818 by Waxy Pope.

4-9 (Half-bred).

3. Starch 1819 by Waxy Pope.

3-8.

Confusion 1832 by Starch.

7-10.

4. Sliylark 1826 by Waxy Pope (1836 s.

3-8. to America).

St. Lawrence 1837 by Sliylarli (or 2-10. Lapwing).

(10 year-old Chester Cup).

2. Whalebone 1807 by Waxy.

3-6. D.

1. Waverley 1817 by Whalebone.

$3-5$.

1. The Saddler 1828 by Waverley.

2 (Sept., twice)-6. Dep.

1. The Prorost 1836 by The

2 (Mlay, 3 times) -6. [Saddler. Alp 1846 by The P'rovost.

3-8. Dam 2y. Aug., twice.

2. Shadow 1836 by The Saddler. 2-11. Dam 2 y. April once.

3. Inheritress 1840 by The Saddler. 2-10. 
4. Radulphus 1843 by The Saddler. $2-14$.

5. Sir Peter Laurie 1873 by The Saddler. 3. I famous steepler.

(1852 3ri in Liverpoul Grand National.) Hall Court 1859 by Sir Peter Laurice. . famous steepler. 1865 and 1869 2nd in Liverpool Grand Viational.

6. Vesta 1843 by The Saddler. $2-9$.

2. The Bard 1833 by IIaverley (s. to Russia).

2 (June, twice)-4. (Own brother to The Saddler).

Iliss Burns 1840 by The Barl. $5-9$.

3. Sambo Sutton 1834 by Waverley:

6-10. Dan 2… April, once.

2. Camel 18:20 by Whalebone. $3-5$.

1. Ibbas .lirza 1831 by Camel.

3.

The 11 idow 1839 by . Ibbas . Mirza.

7-9 (10) year-old without winning, s rear-old ('m.)

2. Touchstone 1831 by Camel.

2 (Sept., twice)-6 L. Icp. $2 \times$ Dep. $2 \times$

1. Cotherstone 1840 by Touchstone.

2 (Oct., twice)-3, 1), 2.

Bordeaux 1847 by Cotherstone. 2-9. Dam 2 1. Sept., once.

2. Orlando 1841 by Touchstone. 2 (Juni, 5 times) - 5. D.

1. Octavia 1849 by Orlando. $3-8$.

2. Fractious 1853 by Orlando. 2-8.

3. Ithuriel 1841 by Touchstone.

3.

Sir Rowland Trenchard 1549 by Ithuriel. 28 .

3. Sir lsaac 1 \&31 by Camel.

(Not run).

Yardley 1,840 by Sir Isanc.

3-8. Dam 2y. July, twice.

4. Caravan 18.3.1 by Camel.

3-7. (2nd 1).) . Icp.

5. Camelino 1\$30 by Camel.

2-8. Dam $2 y$. July, twice.

6. Queen of the Gipsies 18.10 ly Camel.

2-9. Dam 2 9 . June, once.

3. Busk 1824 by IIhalebone.

ㄴ. 
4. Sir Hercules 1826 by II'halebone.

2 (Oct., once and 1 w. o.) -4 . (Sire of many guod steeplechasers).

1. Birdeatcher 1833 by Sir Hercules. 2 (Oct., once) - 4 .

1. The Baron 1842 by Birdcatcher.

3. L. Cs.

Zouate 1855 by The Baron.

3.

The Lamb 186:2 by Zouave.

1 famous steepler.

1868 and 1870 winner in Liverp.

Gr. Nation.

2. Kingfisher 1842 by Birdcatcher.

$6-8$.

3. Blarney 1847 (or 48) by Birdcatcher. $7-12$.

4. Kinight of St. George 1851 by Birdcateher. 2(Sept., 4 times) - 3. L.

1)an O'Connell 1858 by linight of St. (Not run).

The Liberator 1\$69 by Dan

[Georse.

3 -5. 6-15y. steeplechase.

[O Connell.

1879 winner in Liverpool Grand

National. Ran as 17 year-old in

Liverpool Grand National.

(Dam 2 y. Sept., twice).

2. Masnum Bonum (later Discount) 1835 by Sir 4. Famous steepler.

1844 winner in Liverpool Frand Natimal.

is. Merman 1826 by Whalebone.

3-9.

6. The Sea 1830 by Whalebone.

3. Later in Steeplechases.

1840 also in Liverpool Grand National.

Freetrader 1849 by The Sea.

느.

1850 winner in Liverpool Grand National.

7. Bodice $1831 \mathrm{by}$ Whalebone.

3 -s.

3. Wh islier 1812 by Waxy.

3-6. D.

1. Memnon 1822 by Whisker (s. to Russia). 2 (Sept., twice) -5. L.

1. Seventr-four 1833 by Memnon.

3. A famous steepler, twice 2nd Liverpool

Grand National.

2. King Cole 1833 by Memnon.

$2-9$. 
5. The Thoroughbred in the Past and Present.

2. Catterick 15:2 by Whisker.

3.

1. Jerry abosut 1830 by Catterick.

1 famuss steepler. (Half-bred).

1940 winner in liverpool Grand Vational.

2. Roderick kandom about 1832 by Catterick.

.I famous steepler. (Ilalf-bred).

3. Catherina 1430 by Whisker.

2-11.

6. Doricles 1790 by Pot

4 -8.

7. Olizer 1791 by Putous.

3-8. Dam 2! July, 3 times.

s. Thereabouts 1792 by Potsos.

$4-9$.

9. Triffle 1795 by Potsos.

$2-8$.

10. P'arasol 1800 by Potsos.

$3-8$.

3. SATELLITE $17 T 4$ by Eclipse.

3-6 (up to 9 year-old w ithout 1 inning).

Kiren-Happuch 17s9 by Satellite.

3-8. (Half-sister to Waxy).

1. JPITER 1TTt by Eclipse.

3-4. (Own brother (n) Volunteer and Nercury).

1. Carlock 1785 by Jupiter.

2-9.

$\because$ Ilılkin 1 ¿̇ by Jupiter.

3 -8.

4. KING FERGIS 17T by Eclipse.

$3-6$.

1. Beningbrough 1791 by King liergus.

3 -6. L.

1. Orville 1 iga by Beningbrough.

2 (. Iugust, twice) - s. I.

1. Muley 1910 by Orville.

5.

1. Morinen $1 \leqslant 19$ by Muler.

3 -

Taurus 14o6 by Morisco (or Phántom)

(1:39) ‥ to (icrmany).

3 (and up to 7 year-old without winning).

1. John "Gaunt 18:3s by Tourus.

$3-7$.

Hungerford 18.19 by John o Gaunt.

2-s. Dam a year-old. June, mere

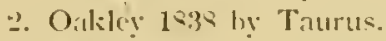

$3-7$.

2. Mlas 1426 by Muley.

1-?. 
3. Muley Moloch 1830 by Muley. 2 (May, twice) -5 .

1. Nice Nawthorn 1838 by Muley Moloch. 3-7. Gep. Dep. $2 \times$. Mother, Grani mother, and Great-grandmother not rum.

2. Morpeth 1841 by Wuley Moloch. 2 - 8 .

ł. Dulcimer 1836 by Muley.

3.

Dulcet 1842 by Dulcimer. 3-10.

2. Fitz Orville 1812 by Orville.

$3-6$.

Gondolier 1.926 by Fitz. Orville.

$$
3-8 \text {. }
$$

3. Master Henry 1815 by Orville. ¿-7 (7 year-old, 6 races, won).

Dam $2 y$. July, twice.

4. Indrew 1816 by Orville.

$3-4$.

Cadland 1825 by Andrew.

3-6. D. 2.

Miss Kitty Cockle 1834 by Cadland. 4-9. Dam $2 y$. Sept., once.

5. Hizalre 1820 by Orvillp.

$5-6$.

1. Nike 1830 by Bizarre. $3-8$.

2. Mus 1833 by Bimarre.

$3-8$.

3. Bellissima 1835 by Bizarre. 3-9.

6. Emilius 1820 by Orville.

3-4. D.

1. St. Nicholas 1827 by Emilius 2 (Sept., twice) $-3 . \quad$ (1840 s. to Germany).

1. St. Lawrence 1833 by St. Nicholas.

2 (Aug, twice.)

Grillade 1852 by St. Lawrence. $1-9$.

2. California 1833 by St. Nicholats. (Not run).

Romeo 1850 by Califomia. 2-10. $12 \mathrm{y} .3 \mathrm{rd}$ in Liverp. Cr. Nat.

2. Priam 1827 by Emilius.

3 -5. D. Gcp. $2 \times$.

1. Y. Priam (later Wild llero) 1836 by P'rianm.

2 (Oct., 3 times.)

'lom Tough 1840 by Y. l'riam.

3-8. (IIalf-bred). 
2. Zoroaster 1836 by Priam.

ב-8. Dam $2 y$. June, twier

2. Sc ud 1804 by Beningrometigh.

$3-4$.

. Ietion 18:2 by Scud.

3-6.

Aimwell 1535 by Actäion.

2-9. Dam 2y. Ipril, once.

‥ Hambletonian 1792 by king lergus.

3-8. L.

1. IV hitelock 1803 by llambletonian.

4.

Blacklock $181+$ by lWhiteleck.

2 (lug., 3 times) - -

1. Brutanderf 1821 by Backlocls.

$3-6$.

1. Hetman l'dituff 1826 Ly birutandorf.

$3-4$.

1. The Cossack 1844 by Hetman Patolf.

2 (July, once) -3. D). (up) to 8 year-old

without wituning, but very good).

- Mcibiade 1860 by 'The Cossack.

3. 1865 winner in Liverp. (ir. Nilt.

2. "Timotheus 18ts by lletman Plitoff.

2-9. J)am 2 y. June, once.

2. Plyysician 1899) by Birutand(mf.

2 (Oet., once) $-\tilde{z}$.

Aristotle 1839 by Physiciall.

$3-8$.

3. Iretic 183:3 by 13rutanderf?

4-10. (Half-bred.)

4. Cay land ábout 1833 by Brotandor.

1 famous steepler (Hajf-bred).

1842 winner in liverponl Grand National.

$\because$ Brownlock 19.2.2 by Blitelilock.

$3-9$.

3. Velocipede 1505 hy Blatelock.

2 (.Ipril, 4 timess) - 4 .

1. Valculissino 1832 by Velocipede. $2-8$.

2. Hornsea 1832 by Vincipede.

3 4. Gicp.

Catarnct 1sfo by IIornasia.

$3-5$.

W'aterfall INIR Wy c:ataract.

$\because-8$.

3. Maid of Tean Valley 18fis by Velecipede.

2 o. Jam 2 y. April, unce.

1. Y. BHaclilock 152.j by Baclilock.

3. 
Maxpie 1834 by Y. Blacklock.

$?$ (Sept. 3 times, and 1 w.o.) - 5 .

1.ough Bawn 1848 by Magpie. $3-10$.

5. Tranby 1 \&og by Blacklock (s. to America).

$1-\mathrm{g}$ ( $\mathrm{ran}$ as it jyear-old $\mathrm{in}$ the famous bet of Mr.

Osbaldeston).

I-am-not-aware 1835 by Tranby:

-9. Dam $2 \mathrm{y}$. June, 6 times.

i. Flacrow 1826 by Blacklock.

3. Famous steepler.

7. Voltaire 1826 by Blacklock.

2) (April, twice) -3 .

Charles XII. 1836 by Voltaire.

$3-7$. L.

little Charley 1848 by Charles Xil.

I famous steepler.

1858 winner in Liverp. Gir. Nat.

2. ('amillus 1803 br Hambletunian and Faith.

3.

1. Oisenu 1809 by Camillus.

$3-7$.

2 (.Ipril, 5 times) -7 .

Revolution 1827 by Oiseau.

3 - s.

?. Nisgistrate 1814 by Camillus (s. to Russia).

3-5. (Falf-brother to Fleur de Lis.)

1. Coroncr 1825 by Magistrate. $3-9$.

‥ Terror 1825 by Magistrate. $3-9$.

3. Garus 1812 by Hambletonian.

$3-9$.

4. Anticipation 1812 by Hambletonian.

3-7. Dam 2 y. May, once. (Half-brother to Clinker).

Presentiment 1821 by Anticipation.

2-8.

3. Hippona $179+$ bo ling lergus.

4-8.

4. Ilarter 17of by ling Fergus.

$3-8$.

¡. Queensberry (later Picadilly) 1794 by King Fergus.

5 .

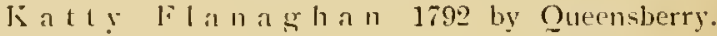

$4-9$.

6. THFFIN 1735 by Eclipse.

48.

7. Fl.1MER 17T6 by Eclipse.

$5-9$.

Q. MERCIRY $17-8$ by Eclipce.

3-6. (Own brother to Jupiler and Volunteer). 
1. Ohd ciold 178; by Mercury. $3-8$.

2. P'rccipilate late by Mercury (1-0:2 s. lo Virginia).

3-5. (Own brother to Goliama).

1. B o b t a i I 1795 by l'recipitate. 3-10. Dam 2! ? July, 4 times.

2. M a t r a n $3-8$.

3. R oll a 1797 by l'recipitalte. $2-8$.

4. La n $210 n$ 18(1) by Precipitale. $3-10$.

3. Gohanna 1790 by Nercury.

3 - 10. (Own brother to Precipitate).

1. Gol u $\mathrm{mp}$ p 4 - 1802 by Gohimn:1. (Not russ).

Catton d 809 by Ciolumpus. $3-8$.

1. Mulitto 182is by ciltum. 3-6. Dep.

1. Discord 1837 by Mulitlu. 5-8. Dan $2 y$. Junc, 1 wice.

2. African 1839 by . Mulitto. $3-8$.

2 Corence 1825 ly (atton (18:37 - th Virginiat). 3-11. Dam 2y. May, twice.

3. Cistercian 1826 by Callon. 3-10. (Ow's brother to 'The Nun).

4. The Poet 18?6 by (atton.

A famous steepler.

5. The Chancellon 18:- by cattor. $3-8$.

6. Diana 1S2S by Callon. 2-8.

7. The Nun 1829 by Calton.

7-9. Later a fammus stcepler up (1) 11 veatsokl. Twice leant. St. Ch. Won.

$\therefore$ David 1529 by Catton.

3-5. (Half-Drother to Despot). (riblos 14t4 by David. $3-9$.

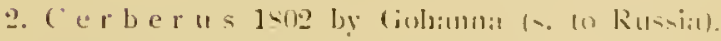

3-9. (Sire of many" womel racers).

3. Election lelt by Golannm.

3- 万. D. Dam 2y. Jume. 4 times.

1. Ixish $1>1+$ hy Election. $3-9$.

2. Manfred 1814 by Election. 3 .5.

Bilbery 1520 by Minfred on by Medley (bs 49. 
4. Zu le ik a 1810 by Gohanna. 4-8. (Dam of Helenus).

1. Brigadier 1792 by Mercury. $4-10$.

5. Shaver (earlier Little Wirley) 1ig? by Mercury, 6-10.

9. JOE AMDREUS (eartier Dennis O!) 1TR by Eelipse 4.9.

Dick Andrea's 1797 by Joe Andrews. 36.

1. C $w \mathrm{r} w 1809$ by Dick Andrews. 3-7. 2.

‥ Tr a m p 1810 by Dicli .Indrews. 3-4.

1. Lottery (tiatiew linker) 1820 by liamp (1833 s. to 3-6. Dep.

[France).

(.) large progeny by Lotlery winning up to 7 year-old, hatf-brother to Altisidora and Brutandorf).

1. Lottery $1 \$ 29$ by Lottery.

.r-16. Steepler. (Not in the Stud book, but ought 10 be a Thoroughbred).

1839. II'inner in l.xp. Gr. Natiun.

2. Zohrab 1831 by Lottery. 3-10.

3. Sheet Anchur 1832 by lottery

3- $4 . \quad$ (s. 10 (iemany).

1. Collingwood 1843 by Sheet Anehor. $2-7$.

2. Weatherbit $1 \& 42$ by Slieet Anchor. 3-4.

Weathercoel 1851 by Weatherbit.

I famous steepler. 1857 and 1858 and in Liverpool Grand National.

2. Litlle Red Rorer 1827 by Tramp.

$3-i$.

3. Trateller 1828 by Tramp.

2-9.

4. Vagrant 1828 by Tramp.

$3-10$.

5. Liverpool 18:8 by 'Tramp.

2 (Sept., onee) - 5 .

1. Lanerenst 1835 by Liverpoul.

3-7. Cm. Dam 2y. Oet., once.

Van Tromp 184t by Lanereost (s. to Russia).

2 (July, 3 times and 1 w. o.) -5. L. Gep.

Prince of Orange 1853 hy Van Tromp. $2-8$.

2 . Xiluoth 1837 hy Liverpool.

$2-9$. 
5. The Thoroughbed in the P'ast and Present.

3. I Eritials leoman 1840 by l.iverpool. 2. (.lay, b times).

Bourgeois 1850 by . I British Veomnn. $3-11$.

4. Birkenhead 1\&43 by liverpoul.

(Not run).

New Brighton 1850 by Birkenhead. $4-10$.

10. SALTRAM 1780 by Eclipse (1793 s. I0 Russia).

3-5. D.

11hiskey 1789 by Saltram.

3-4.

1. El e a nor 1708 by Whiskey.

3-7. D. O. (Half-sister to Sorcerer and dam of Muley).

2. Whir ligig 1798 by Whislicy.

$4-8$.

3. Ru m b o 1800 by Whiskey.

2-11.

4. S p 1803 by 11 hisker.

3-10. Daur 2 .. July, 4 times.

11. IOLUNTEER (later Cornet) 1780 by Eclipse.

3-5. (Own brother to Mercury and Jupiter).

1. Vagic 1794 by loolunteer.

$4-5$.

II o u ut a i ne e 1802 by Magic.

$3-11$.

2. Eagle 1796 by Volunteer (s. to Virginia).

3-9. (Own brother to Spread Eagle D., half-brother to

Diclelot D.)

As mode us 1807 by Eagle.

$3-9$.

12. ERAS.MUS 1781 by liclipse.

3. (Own brother to Nexancler and Don Quixote).

Esher 179.5 by Erasmus.

6-8. (Half-brother to J'ecrasus).

13. MLEXAVDER 1782 by liclipse.

3-7. (Own brother to Erasmus and Don Quixote).

Bucephalus 1sire by . Mexander (s. to Russia).

$3-8$.

14. METEOR 17S3 by F.clipse.

$3-7$.

1. Meteora 1802 by Meteor.

3-8. O. 1)am 2 y July, L icc.

2. Imbo 1809 by lletere (or by Diamond).

2 (Oct, once) -6 .

I. iston I\&2I by .Imbo.

$3-13$.

15. GLYPOIVDER liRt by Eclipes (n. 10 Russia).

3-9. (Own brother to Soldier 1779 by Eclipse).

$3-7$. 
Trial of the Thorvughbred on the Racecourse, etc.

16. PEGASUS liot by Eclipse. $4-7$.

1. King Edward (earlier Shum Sheer Jung) 1795 by Pegasus. $6-8$.

2. Novice $179 \mathrm{j}$ by Pegasus.

?-S.

3. Parnassus 1797 by Pegasus.

$5-11$.

4. Laura 1800 by Pegisus.

2-8. (Dam of Dr. Eady' by Rubens).

5. Hippomenes 1802 by Pegasus.

$2-8$.

17. DON QUI.IOTE 1784 by Eclipse.

3-8. (Own brother to Erasmus and Mexander).

1. Sancho 1801 by Don Quisote.

3-4. L.

C a 17 n 0 n-b a I 1810 by Sancho.

3-9. (Half-brother to Grimaldi and sire of many good hunters and steeplers).

Counsellor 15:2 by Cannon-ball (or by (hilde Harold). $5-8$. (Half-bred).

2. Artichoke 180: by Don Quixote.

$3-8$.

3. Amadis $180 \overline{\mathrm{b}}$ by Don Quixote.

4.

1. P a 1 m e r i n 1816 by Amadis (s. to Russia).

2 (May, once) -4 .

Aggravator 1832 by Palmerin.

5 -12. (Half-bred).

2. 11 a $g$ i c about 1820 by . Imadis.

1 famous steepler. (Half-bred).

18. SERPENT 1 TS6 by Eclipse.

$3-10$.

2. Stripling 1765 by Marslic.

4-8.

3. Y. Marslie 1771 by Marske.

4.

1. RULER $17 \%$ by I. Marslie.

3-5. L. (Haif-brother to Rattler and Magnum Bonum).

High Eagle 1790 by Ruler.

$3(?)-9$.

2. TRIMMER IRES by Y. Marske.

$3-8$.

3. SHLTTLE 1793 by Y'. Marslie.

$3-5$.

1. Cambric 1807 by Shuttle.

2-S. (Ran and frequently won several times in al day).

Dam ㄴ. July, twice.

2. Engrazer 180 - by Shuttle.

3-6. (Ran and frequently won several times in a day). 
4. Hephestion 1771 by Marske.

$4-9$.

2. MISs l'k0CTOR (or Smalliropes) 1733 by Bartlet's Childers.

5-11. (Lp to 13 year-old without winning).

\section{Byerly Turk born about 1680 .}

1. B.IS'TO 1702 by Byerly Turk. 6-8. (Sire of Old Ebony, Fundiation Mase in Fam. 5).

‥ JIAli about 170? by Byerly Turk.

(Not run).

P'artuel (Mr. (roft's) 1718 by Jigg.

z- 8 . (Lp to loyear-old without winnings).

1. PARTXIR (Grisewood"s) 1730 by Partner.

5-14. (15 year-old without winning).

(Sire of Miss Elliot, Grimcrack's Dam, Foundation Mare in Fam. 23).

9. SEUBURI 1734 by Partner.

$4-10$.

Tantiry 1749 by Sedbury.

$4-11$.

3. OLI) TRAYELLLR li3i by l'artner.

$4-7$.

1. Skim 1748 by Old Traveller.

4 - 8 .

2. Daiuty Dary 1752 by Odd Traveller.

4-11.

D.A I'TD 1766 by Dainty Dary.

4-12 (also in Give and Take Plates).

3. Squirrel lijst by Old Traveller.

$4-7$.

1. 11EASEL 1760 by Squirel.

$5-11$.

2. FURIBAND 176; by Squirrel.

3-10 (aiso in Give and Take Plates).

3. SLIII 1771 by Squirrel.

3-10.

4. LITTLL JOLI 1740 by Partner.

4-8. (Lp to 10 veatrold without wiming).

5. TARTAR (earlier Partner) 17.43 by l'artner.

$5-7$.

(King) Ilerol 17.58 by Tarlar.

5) -9.

1. FLORIZEL 1768 by llerod.

4-6.

1. Dioned liti by litorizel (s. 10 . Imeric:a).

3-6. D.

1. Cirey i) i o m e d 1785 by Dimned. $3-8$. 
2. C 1 a u c us 1786 h Dioned.

$3-10$.

3. Ig a m e m 170 n 1790 by Diomed.

$3-12$.

2. Ulysses 17T bỹ lilurizes (s. to Virginia).

$3-10$.

Play or Pay 1791 by ['ỵsses (s. tu lirunia). 3-9.

3. Fortunio 1779 bั lilorizel.

$3-10$.

Sir Ferd in a $11 \mathrm{~d}$ (later ling Bladud) 1792 by Fortunio. $2-12$.

4. Bustler 1784 by Florizel.

$2-9$.

.). Prizefighter 1784 by lilorizel.

$2-9$.

S w o r d s m i 11 1796 by Prizefigliter.

$4-7$.

1. Fencer 1Sth by Swordsman.

4.

Vivian about 1824 br fiencer.

1834-1843 al famous steepler.

2. Spartacus 180 by Swordsman. $3-9$.

2. MAGNET 17\%0 by Herod.

$4-9$.

1. Noodle 1781 by Mignet.

$3-8$.

2. 1]indlestone 1783 by Magnel.

$3-8$.

3. Shazel 1785 by Mitguet.

$4-9$.

3. BACCHL's 1771 by Herol.

(Not run).

1. Rutland 1783 by bacchus. 4-9.

2. Kitty 1785 by Baccluus.

$3-9$.

3. Bacchus 1788 bỵ Bacchus. $3 \mathrm{~S}$.

4. HOODPECKER 1773 by llerud.

$1-7$.

1. Seagull 1786 hy Hondpeclier.

$2-8$.

2. Chanticleer 17.5 by Wnodpeclier.

2 (April, 6 times) - 5.

1. Travelle 1797 by Chanticleer.

2-9.

2. B o b $B$ ont $y$ 1s04 by Chanticleer.

2 (Sept., twice) -4.

Napoleon 1824 మ. Bob Booty (1833 s. to France). $2-9$. 


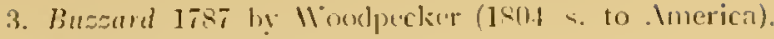

2 (Oct., twice) -i.

1. $\mathrm{Q}$ แ $\mathrm{i} \% 1798$ by Bu\%ard.

$3-9$. I.

1. Roller 1414 by Quiz.

3-11. (Sire of many good steeplers).

‥ Euphates 1810 by ()ui\%.

$6-13$.

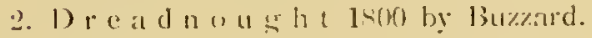

3 - 8 .

3. 13 1 a i n 11 , 1 $m$ 1 141 by Bu\%ard.

$2-8$.

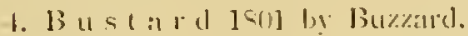

$2-10$.

5. Castrel job] by Bumard.

34.

1. Bustard 1413 by ('istrel.

$3-5$.

Remmant 1-33 by Buatard.

$4-10$.

2. Nerlin 1:1, by ('astrel.

2 (.lug., 3 timess) -4.

lancastrian leos by Merlin.

$\because$ (Oc., (wice, ] w. o.) -8.

Jis: Mus luay about l\&43 by Lancastrian.

(Half-bred) lose wimes in liverp. Cir. Nation.

b. $S$ e $l$ i m $18(1)$ by Buzzand.

$4-6$.

1. Champion $1=12$ by Selim.

3.

1)onninglon l-20 by (hampion.

3-10.

2. Sultan 14]6 by Siclim.

2 (fuls, unce) …

1. De:sunt 1-31, by Sultan.

2-4. (Ilalf-broller to David by (atlom).

2. Npheur 1430 hy Sultan.

3.

1. Jahn Dor 1846 by Apheus.

$3-9$.

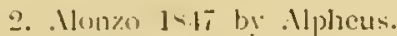

30.

3. Islmated 1-31 he Sultan.

3 year-old in racing stable of lord Jersey, but not

Sire of mint sood steeplers. [run.

1. Mbd-(cl-kider about 1 s.40 by Ishmael.

1 fimusus s(appler. (Ilalf-bred).

14.51 and $1-, j]$ winner in liverp. (ir. Nation.

$\therefore$ The su itcher ]\&4? by Ishmael.

.1 fanmous steepler. 
Trial of the Thoroughbred on the Racecourse, etc.

4. Caliph 1832 by Sultan.

$2-10$.

万. Hampton 1833 by Sultan.

3.

Lady Florit 1838 by Hampton. $3-11$.

6. Adrian 1834 by Sultan.

3-13.

7. Jereed 1834 by Sultun (1846 ․ 10 Russia).

2. (.lug., twice).

I. Greatheart 1840 by Jereed.

3.

Gaylad 1846 by Greatheart.

3-10. (Half-bred, and half-brother to

Gaffer Green by Obadiah).

2. Bishop of Romford's Cob 1840 by Jereed. 3-10.

3. Thurgarton 1841 by Jereed.

5-10. (Half-bred) and in Steeplechases.

3. Langar 1817 by Selim.

2 (Oct., (wice) -9.

1. Philip 1. 1828 by Langar.

2. (Sept., (wice, and I w, o.).

Montague 1846 by Philip J.

$3-8$.

2. Potentite 1830 by Langar.

$2-12$.

3. Leander 1932 by Langar.

3.

The Marchioness 1846 by Leander.

3-9. (Half-bred).

7. R ub e ns 180.5 by lizzard.

3- $\tilde{5}$.

1. Gainsboroush 1813 by Rubens.

$3-7$. Ditm 2 ycar-old Oct., once.

2. Wourematus $181 j$ by Rubens.

4-8. Dan 2 year-old June, twice.

3. Dr. Endy 1816 by Rubens and Laura $2-8$

6-12. Dam 2year-old June, twice.

4. Hampden 1819) by Rubens.

$3-5$.

Guildford 1826 by Hampden. $2-9$.

5. Vandyke 1s:os by Rubens.

5--10. (Ilalf-bred, also in hurdle races).

11 ye:ur-ind in sleeplechises.

4. Olive 1787 by IVoodpecles.

$2-10$.

5. I. II'oodpcitier 1794 by II'oodpectier.

(Not run). 
j. The Thoroughbred in the l'ast and Present.

1. 11 o o d ma $12180 \%$ by 1 . 11 oodpecker. 38.

Clarily 1530 by 11 oodınan.

5-?) (1lalf-bred). It se:al-old, steeplechases. I famous I-41 uinnes in 1,iverpl. Mr. Nation. [sterpler.

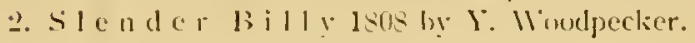

$$
3-9 .
$$

6. Brother to lizaldi lign by lloodpectier.

4 8. (Sire uf many aumel humters).

5. FITZHEROJ 17:3 by Herad.

(Not ruir).

Wufti 1783 by liitherud.

$3-9$.

i. II: HIO 17\%t by Herod.

$3-\mathrm{j}$.

Siorpion 1785 by 11 'mio.

$3-12$.

i. J1:T/E 17T+ by Herud.

$3-4$.

1. Trifle 17s- by Justice.

$3-9$.

2. Rasper (Iater Douglas) 17:2 by Justice.

$3-9$.

3. Wentor list by Iustice.

3-11. (Half-brother to siwectbriar).

1. Midnight (later Mhip) 17.9 Wy Justice.

$2-10$.

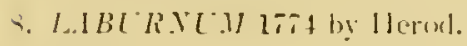

$+-9$.

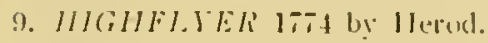

35.

1. Rockinsham 17s lug Hishlofer.

1 i. Dep. (fi sear-ald. IT rices non).

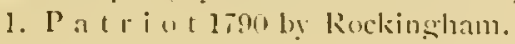

$2-8$.

2. B

$3-8$.

2. Delpini (earlier Hackucod) 17-1 by Highllyer.

$3-\tilde{j}$.

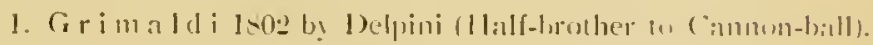
$\bar{i}-\mathrm{S}$.

1. Frimaldi ivezo by Gimaldi.

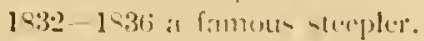

2. l'antmuime $1 \leq 20$ log Cirimaldi 1 - 16 in Hemer Sililies.

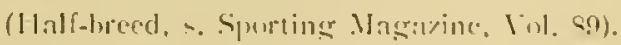

$\because$ B $u \leqslant(1$ e r lat by 1)elpini.

$4-6$.

The Major 1 oon his Bustler. $3-11$.

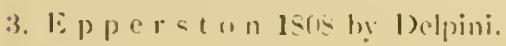
$4-8$. 
3. Harlot (earlier Comnilass) 1783 by Highntyer. $4-13$.

4. Sir Peter 17st by Hightiyer.

3 . . D. D.

1. In brosio $\mathbf{1 7 9 3}$ by Sir Peter. $3-6$. L.

Huntingdon 1806 by Ambrosio.

4-9. (Ran only in unimportant races atgatinst ver! inferim horses). Dan 2 vearold War, 10 times.

2. Mr. T eazle 1793 by Sir Peter.

(Notrun).

Y. Vestris $18 n$ by Mr. Teazle.

3.

Mathew 1838 by Y. Vestris.

6-9. .I famous steepler.

1847 winner in Liverp. (ir. Nation.

3. Sir Solomon (earlier Tankersley) 170k by Sir l'eter. $3-6$.

Cambrian 1804 by Sir Solomon.

$3-11$.

4. Haphazar $\$ 1797$ by Sir Peter. $3-9$.

1. Don Cossack 1810 by Haphazard. $3-5$.

1. Prosody $1 \$ 18$ by Don Cossack. 3-10. (Often many times in a day).

2. The Tarta 18?1 by Don Cossack. 6-8. (Not in Stud Book).

3. Reform 1829 by Don Cossack. 3-9. (Often many times in a day).

(Not in Stud Book).

?. Filho da Puta 1812 by Haphazard.

" (Sept., once, and $1 \mathrm{w}, \mathrm{o}$.) -4 . L.

1. Troy 1820 by Filho da Puta.

3.

Pumplsin 1829 by Troy. 3-9.

2. Conductor 1890 by Filho da Putil.

$3-4$.

Oswald 1832 by Conductor. $3-10$.

3. Forester Lass 1g2l hy liilho da l'ut: 6-s.

4. Haji Baba 1821 by Fillo da Puta. 3-9.

5. Orthodox 1821 by Filho da Puta. 3-11. Dam 2 year-old May, twice.

6. Dr. Faustus 1822 by Filho da Puta. $3-9$.

1. Jesuit 1.834 by Dr. Faustus.

1 famous steepler. (Half-bred).

Ran and won at 24 year-old? 
2. Ohadiah 1834 by 1)r. Fanstus.

2 (Sept., once, and 1 w. o.) - 3 .

Satilor (later Galfer fireen) 1-39) by: Obadiah.

(i-1.1. (llalf-bred, aud half-brother to

Ginglad by Gircathenrt).

(Often many times in at clis)

3. Jupslay 1837 by Dr. Faustus.

3 -4.

Huntsman 18.53 by Tupsley.

$3-4$.

1862 winner in Liverp. Gr. Natiun.

4. The (handler 1836 by Dr. Fatustus.

I famous steepler. (Half-bred). 1848 winner in Liverp. Gr. Nation.

7. Joclios 1823 by loilho da Puta.

3-9.

S. (hico 18:5) by Fillo da Puta.

3-11.

(). Independence 18:6 by fillio dia Putil or by silu"l. $2-9$.

| wood 1800) by Filhu) dit ['ut:1

10. Giovanni 1828 by Fillan da Putit.

3-7. (8 year-old 3 times very well run).

(Half-brother to Potentate by lantratr:

3. Victorine 1816 by Haplha\%and.

$4-9$.

4. Figaru 1819 by Haphazard (18:31 ๖. (10 Mocklenburs). $3-6$.

Isitite $18: 31$ by lijgato.

2 14. (Lp to 15 year-old, also in hurelle races)

$\therefore 11$ a I t o n 1799 dy Sir l'eter.

$: 3-6$.

1. ['hantom Isos by 11 altom (1830) $\rightarrow$, (1) Mecklenburin).

3. I. D.

1. Spectre 181.j by I'hintom.

3. .5.

Ciranby 1-23 by Spectre. $3-8$.

2. Vinnish 1825 by Pliantom (1536- to linst l'russial)

2 (June, 4 times) - -

Sunbeam 1833 by Vianislo.

2 - 8 .

2. Partisan 1:11 by Waltom.

35.

1. Mimmeluke 15:3t hy l'atisint.

3-5. ().

\%cuhus 14:31 by Mimelulic.

310.

‥ I'atron 1826 by Partican.

3. 2 . 
Peter Simple about 1839 by Patron.

A famous steepler. (Half-bred).

1849 and 1853 winner in Liverp. Gr. Nation.

3. Venison 1833 by Partisan.

2 (July, once) -4 .

1. New Forestdeer 1841 by Venison.

3-10. (Half-bredd).

?. Herbert 1846 by Venison.

$2-8$.

4. Gladiator 1833 by Partisan.

3. (2nd D).

Napier 1840 by Gladiator.

2 (Sept., 3 times) -3.

Indian Warrior 1849 by Napier. 2-S.

3. Waterloo 1814 by Walton.

3.

1. Conquest 1822 by IV'aterloo.

3-5. (Ran and won often several times in a day).

2. Caleb 1828 by I'aterloo.

2-8.

4. St. Patrick 1817 by IValton.

3-4. L.

1. Daniel 1832 by St. Patticls.

3-9.

2. St. Francis 1835 by St. Patricli.

$2-8$.

3. Garry Owen 1837 by St. Patrick.

$2-10$.

6. D i t t o 1800 by Sir Peter.

$3-7$. D.

Luzborough 1820 by Ditto.

4-8.

7. Sir Pa u I 1802 by Sir Peter.

$3-5$.

Paulowit\% 1813 by Sir Palul.

$3-7$.

1. Cain 1822 by Paulowitz.

$3-5$.

1. Ion 1835 by Cain.

2 (July, 4 times) -4 .

Poodle 1849 by Ion.

2-9. Dam 2 year-old Oct., once.

2. Barney Bodlsin 1830 by Cain.

$2-8$.

2. Little Boy-blue 1825 by Patilowitz.

2-9.

3. Changeling 18gs by l'nulowitz (or Swap).

3-9. Dam 2 vear-old Sept., once.

\&. C; a d inal York 1804 by Sir Peter.

$3-5$. 
Idvance 181.5 by Cardinal York.

$3-5$.

1. Vanguard $18: 35$ by Advance.

4. A famous steepler. (Half-bred). 1843 winner in Liverp. Gr. Nation.

2. I'ioneer 1840 by Advance.

I famous steepler. (Hall-bred). 1846 winner in Liverp. Gr. Nation.

9. P o u I t o n 180.5 by Sir Peter.

$3-8$.

10. Clinker 1805 by sir Peter.

3-4. (Half-brother to Inticipation).

Clinker 1806 by Clinker.

I famous steepler.

5. Star 178,5 by llighflyer (1811 s. to .Imerica).

2 (Oct., once) -6 .

N r. G u n d y 1804 by Star.

$3-9$.

6. Slyseraper 1786 by Hightlyer.

3 - 8. D.

7. Il'alnut 1786 by Highflyer.

$3-5$.

Lign um Vit a e 1797 by Walnut.

3-8. (Sire of many good racers).

8. Phacton 1787 by Highflyer.

$3-11$.

9. Sit. George 1789 by Highflyer.

$3-10$.

Topsy-Turvey 180.5 by St. George.

$7-10$. (Died at 16 year-old through physic in training for Welter Stakes in Bibury).

Nimrod 1820 by Topsy-Turvey.

$6-10$.

10. Sling 1789 by Highnyer.

$3-11$.

11. Tidy 7889 by Highfyer.

3-8.

12. Grouse 1790 by Higliflyer

3. ( 4 and jyear-old without winning).

First Fruits 1797 by Grouse.

2-9.

13. Diamond 1792 by Jighflyer (s. to France).

$3-8$.

1. Queen of Diam ond s 18u19 by Diamond.

$3-7$. (Own sister to king of Diamonds).

2. King of Diamonds $1 \leqslant 10$ by Diamond.

3-8.

King of Trumps 1827 by king of Dianonds. $3-8$.

14 Lark 1792 by Highflyce.

$3-8$. 
Trial of the Thoroughbred on the Racecourse, etc.

15. Louisa 1792 by Highflyer.

4-9.

16. Delamere 1793 by Highflyer. 3-s.

10. BOURDEAUX 1774 by Herod.

4-6. (Own brother to Florizel).

Higllander 1783 by Bourdeaux. 3-9.

11. GLILDFORD 1775 by: Herod. $4-9$.

12. BOIER 1776 by Herod. $3-8$.

13. ROIER (later Tom Tug or 'Tug) 17i- by Herod.

$3-9$.

Cornet 1792 by Rover.

3-9.

14. GOLDEN DUN 1777 by Herod.

3-11.

15. DRONE 1777 by Herod (s. to America).

$3-10$.

16. ANIIL $17 \% 7$ by Herod.

$4-9$.

17. FORTITUDE 1777 by Herod.

$4-7$.

John Bull 1789 by Fortitude.

3. D.

A du ira 1 Nels on 1795 by John Bull ( $\therefore$. to America). 3-S. Dam 2 year-old July, 3 times.

18. PHÖNOMENON 1780 by Herod (1803s. to America).

$3-5$. L.

Huby 1788 by Phönomenon s. to Rlisia).

$3-8$.

Driver 1798 by Huby.

$3-9$.

19. BAGOT 1780 by Herod.

4.

1. Master Bagot 1787 by Bagrot.

$3-5$.

1. D a w d l e 1795 by Master Bagot. 2-S. (Half-bred).

2. Holly hock 1804 by Master Bagot.

$4-8$.

Hesperus 1820 by Hollyhock.

4-10.

2. Loyal 1796 by Bagot.

3-s. (Half-bred).

2. Soldier 1758 by Tartar.

4-9. 


\section{Godolphin Arabian born 1 i24.}

1. CIIIE 1734 by Godolphin Mrabian.

6. (i to 11 year-old without winning).

1. Trumlion 174 by Cade.

$1-10$.

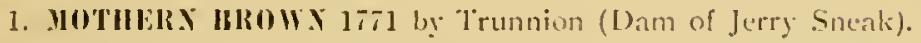
$1-11$.

2. TITIXIA 17it by Trunnion. 4-11.

2. Y. ('ille 1747 by Cade.

(Not run).

1. HACHlidoR tiog by Y. Cade.

$4-9$.

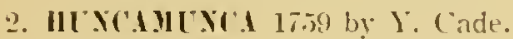
$7-11$.

3. IIIIEL 17i;- by Y. Cade. $4-10$.

4. CIRABISElin 170.5 by Y. Cade. 4-10. (Half-brother to Morwick Gall by Regulus).

3. Changeling liti by (ade.

(i. (Own brother to Matchem).

LE sATli 1759 by changreling.

$4-8$.

1. Siampstom Ciale 17ti by Cade.

5 - 6.

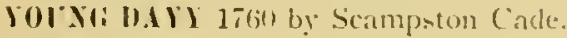

4-10.

5. IItchrin lite by Code.

$5-10$.

1. TIRF lö60 by Matchem.

4-6.

Inetor lo-:3 by 'Turf.

$3-5$.

BLFFER list by Mentor.

$4-10$.

2. B.ISkl:R 1761 by Matchem.

3-9.

Tyeress 1700 by Banker.

$=11$.

3. Cositilly 1702 by Matchem.

4 S. (16 year-old without winning).

Thelfurd 1772 by Conundrum.

$+9$.

4. R.IITHOS lo63 by Matchem.

4-9.

5. CHIMST 176n by Matchem.

$4-6$. 
Uruegist 173.5 by (hymist.

4 -8. 7 year-old ciold Cup in Chester and 6 other races.

6. PANTALOON 1767 by Matchem.

$4-6$.

1. Merry Indrews 17s3 by Pantaloon.

$2-9$.

2. llufier 1784 by l'antaloon.

$4-8$.

7. G01.loFIXCll 1767 by Matchem.

$3-10$.

Q. (OSIIC(TOR 1765 by Mlatchem.

4- 6.

1. Imperator 1766 by Conductor.

$3-8$.

PIP.ITOR I786 by Imperator.

2 (July, once) -7 .

Romembrancer 1800 by Pipator.

3-4. L.

Recordon 1807 by Remembrancer.

$3-5$.

Welcone 1819 by Recordon.

(Not run).

Sir William about 1830 by W'elcome.

A famous steepler. (Half-bred).

1838 winner in Liverp. Gr. Nation.

2. Trumpator 1782 by Conductor.

$3-4$.

1. MIMATOR 1790 by Trumpator (s. to Kussia). 3-8. (Half-brother to timwell D).

2. PAYNATOR 1791 by Trumpator (s. to Russia). 2 (July, 4 times) -9.

1. Offa's Dyke (earlier Occator) $18 n$ by Paynator. $5-9$.

2. Marksman 1808 by Paynator. $5-18$.

3. Dr. Syntax 1811 by Paynator. $3-12$.

1. Be e's $\mathbb{W}$ i n $g$ 1 1833 by- Dr. Syntax. 2 - 9. Dep.

2. The Doctor 1834 by Dr. Syntax. $2-9$.

3. A j a x 1838 by Dr. Syntax. $3-8$.

4. F a m a 1838 by Dr. Syntax. $3-8$.

i). Sy nt ax in a 1838 by 1)r. Syntax. $2-8$.

6. II o pe about 1838 by Dr. Syntax. 1 famous steepler. (Half-bred). 
3. REPEATOR 1791 by Trumpator. 3-9.

4. SORCERER 1796 by Trumpator. $3-5$.

1. Soothsayer $18(18$ by Sorceter (1 203 s. (1 Rusias). 3 -5. L.

1. Wel beck 1815 by Suthsityer.

(Not rusi).

Bedlanite 18:23 by Welbeck.

3-4.

Saul 1835 by Bedlamite. $3-10$.

․ H elenus legl by Sindhilyer (1835 to Germany) $3-8$. ind Zuleikia +8

2. Comms 1809 by Sorcerer.

$3-4$.

1. Rev c I I e 1815 by Comun.

3-8. L.

1. Oberon 1827 by Reveller.

$3-11$.

$\because$ Busplorus 1436 by Reveller.

$3-8$.

2. Corint hi a n 1 \& 19 by Comus.

$3-5$. (Half-brother to Jerry).

Russel 182 (j by Corinthian.

$3-8$.

3. II u m phrey CI inker 180.2 by comus. $3-5$.

Melburne 1834 by If Imphrey Clinlier.

3- 5. (6 year-uld very grood but did not win).

Sir "latton sives 1843 by Melbourne.

3-4. L.. :-

Mr. Silien 18is) by Sir Tatton Sylies.

2 (1). (lallf-bred).

3. Smolenstio 1410 by Sincerer.

3-4. D). 2. (Sire of matng racers which frequently ran several race in une daly, as, for instance, Thorngrose, $15 \div-2 \pi)$.

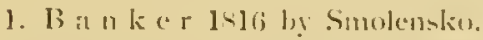

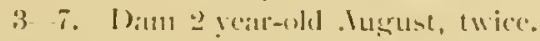

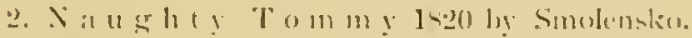
(j-11.

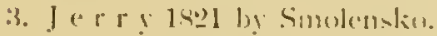

3. L. (Sire of many ? ?

Tomboy 10.en by Jerry.

$3-\pi$.

Vutwith l-10 ly lomber.

2 (Iune, 3 times) -3. I..

1. Noinetse 1-5) by Xiumith.

$2-?$ 
2. Knight of Kars 1855 by Nutwith.

3.

1. The Colonel 1863 by Finight of Kars. I fanous steepler. (Half-bred). 1869 and 1870 winner in liverp. Gr. Nation. (s. to Germany).

2. New Oswestry 1864 by Knight of (Half-bred).

[Kars.

A famous steepler, and sire of many good steeplers, amongst which were:

1. Zoedone 18it by New Oswestry. 1883 winner in liverp. Gr. Nat.

2. Savoyard 1878 by New Oswestry. 1887 and in Liverp. Gr. Nat.

1. Bourbon 1811 by Sorcerer (s. to Kussia).

$3-6$.

1. Com te d'Arto is $18 \geq 0$ by Rourbon.

$3-8$.

2. Il d e r m a 17 182* by Bourbon (s. to Russial).

2-7. (nd L).

3. Fle ur d e l, is 1822 by Bourbon.

3 - \&. Dep. Gcp. $2 \times$ ( 8 pear-old Gcp.).

5. IERNATOR 1796 by Trumpator.

$3-5$.

homeo 1>04 by Vernator.

$4-9$.

6. REBEL, 1796 hy Trumpator.

3-9. Dam 2 year-old Oct., once.

7. CHIIPENHAII 1790 by Trumpator.

$3-8$.

S. SIR D.]l'1D 1801 by Trumpator (s. to Russia). $2-9$.

9. AJ.FRII) 1770 by Matchem.

4. (Own brother to (onductor).

Tickle Tohy 17\&6 by Alfred.

t-9.

10. ('RlTU lïl by Matchem.

3-11.

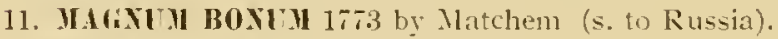

$4-i$.

Ritler 1784 by Masnum Bonum.

4-9. (Half-brother to Ruler by Y. Marske).

12. ESPERSYKRS 1775 by Matchem.

(Not run).

1. Conquerur 17ig by Espersylies.

4-11. (9 year-old Gold Cup. Chester).

2. Sir l'uter Pellet 17s0 by Espersylies.

$3-8$.

6. Sportsillan 1753 by Cade.

$4-8$. 
7. Hero (earlier Slape) 1753 by Cade.

5- 13.

1. FIRIAR 1768 by llero.

+11 . (Sire of many good racers).

2. Allisis 1720 by llero.

$5-11$.

3. IALACiL 1776 by Hero.

4 12. (Dam of Y. Lalage 1797, s. p. 69).

8. Northumberlaud 1754 by Cade.

$4-5$.

BCFF $1 ; 66$ by Northumberland.

$4-12$.

9. Nilvio $175+$ by Cade.

$4-11$.

10. Noringhtly 1754 by Cade.

$1-9$.

11. Flylax 1756 by Cade.

6--11.

12. Fmilius about 1750 by Cade.

?

I.AYUK Kl: 176.5 by Enilius.

7-13. (.11so in Give and Take Plates).

2. WUR IUISEE 1738 by Godolphin Irabian.

7-11. (.1so in Give and Take Plates).

1. Villiant 1755 by Dormouse.

4-10.

2. Morimont $175 \mathrm{~s}$ by Dormouse.

4-10. (Sire of many good racers).

3. RElil ILN 1739 by Godolphin Arabian.

ij- $i$.

1. Citto 1ifí by Regulus.

t-11.

2. 'Irajan 1748 by Regulus.

$5-9$.

3. (Alloless 1751 by Regulus.

$1-9$.

1. IIougsius 1752 by Regulus.

$1-9$.

HORLSTER 176. by Dionsius.

$4-12$.

5. Ipollo 175.5 by Resulus.

$4-10$

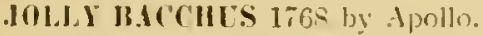
3-9.

6. Nullan 1757 by Regulus.

$6-9$.

7. Ilolwirk Jall 1762 by Regulus.

4-7. (llalf-brother in Carabineer by 1: Cade). 
1. CAVEMIISI (earlier Quintus) 1784 by: Morwick Ball.

3- 8 .

2. GLSTAYS 1785 by Morwick Ball.

$4-8$.

8. Sejanus 1764 by Regulus.

4. (Half-brother to Herod).

CIIRROKEE 1780 by Sejanus.

$4-10$.

9. Bucephalus 1764 by Regulus.

4-9.

10. Demular 1764 by Regulus.

$4-8$.

4. 'THE (A)WER STIISIOIX 1740 by Godolphin Arabian.

(Not ruis).

Sweepstakes 1749 by The Gower Stallion.

$5-10$.

5. BIRR IHIM $\mathbf{1 7 4 0}$ by Godolphin . Trabian.

6-9.

1. Aleides 1753 by Babraham.

$5-7$.

TONZIR 1768 by . Alcides.

$4-9$.

2. Bosplorus 1754 by Babraham.

$4-9$.

3. 'Traplill 1754 by Babraham.

$4-9$.

4. Allericus 1755 by Babraham.

$4-11$.

5. Cardiual I'ufí 1760 by" Babraham.

4--9.

6. Fop ]760 by Babraham.

4-11.

6. IB.J IVE'T 1740 by Godolphin Arabian

$5-10$.

Siplim 1760 by Bajazet.

$5-9$.

7. BLAXK 1740 by Godolphin Arabian.

$5-10$.

1. Iottely 1752 by Blank.

$5-11$.

2. Coutest 1753 by Blank.

$5-9$.

3. IInlatills 1756 by Blank.

$4-9$.

4. Intinolls 1759 by Blank.

$4-9$.

5. Pancake 1759 by Blank.

4-10.

6. Clatswortll 1762 by Blank.

$4-9$. 
7. Jarolet 1763 by Blank.

4-7. (Also in Give and Take l'lates).

Y. PACOLET 1780 by Pacolet.

3 -4.

('ilizen 1785 by l. l'acolet.

4-9. (s. 10 India)

S. Paymastre (earlier Jesmond) lifit; by lilank.

4 -8. (Sire of many good racers).

9. Intouster lites by Blank.

5-10.

5. (III) FX(iLIXI) 1741 by Godolphin Irabian.

$5-8$.

Imlaranthus lito by Old Engrand.

$4-9$.

!). II)(iLl, 1741 by Godolphin Arabian.

Whistlejacket 1749 by Mogul.

$4-10$.

CORIOLIXIN 1762 by Whistlejacliet.

$4-7$.

Coquelte 1783 by Coriolanus.

$5-8$.

10. WHITESOSE 174? by Godolphin . Irahian.

8.

11. YIRZA 1749 by Godolphin .Irabian.

(i-9.

12. ('RII'PI. 1750 by Godolphin . Irabian.

6. ?

1. Gimurack 1760 by Cripple.

$4-11$.

2. 'T'antrum 1760 by Cripple.

$8-10$. (Sire of Termagane 177-?).

(OPPliRBOTTOMl lizg by Tantrum.

$3-10$.

13. I.(1F'l 1753 by Codolphin Arabian.

$4-6$.

sliut 1762 by lofty.

$1-11$.

Alcock's Arabian born albout 1703.

1. ('R.Il 172:2 by .llcock's . Irabian.

$5-$.

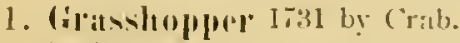
i) 9.

2). ('rall (kouth's) 17:36 by ('rab.

.5 9. ((p) to 12 year-old withuse winningr).

3. Ril, 1736 by Crab.

(3-9.

1. Mustand litl by Crab,

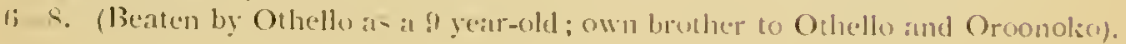


1. GAMAIIOE about 1758 by Bustard.

1. Noble 1767 by Gamahoe.

$4-11$.

2. Ilippolitus 1767 by Gamahoe. $4-13$.

3. Olympns $176 \pi$ by Gamahoe. $4-8$.

4. Cromahoo $177+$ by Gamahoe. $4-6$.

1. DUCHESS $17 \pi 5$ by Cromaboo. 3-8.

2. SH.4MROCK 1789 by Cromaboo. 3-11.

5. 0lympia 1757 by Gamahoe. 6-11.

6. Watcmau 1779 by Gamahoe. 6-10.

7. Farmer 1759 by Gamahoe. 6 -8.

8. Ramuculus 1782 by Gamahoe. $4-10$.

2. LEXYOX 1766 by Bustard. j- 7 .

1. Tom Turf 1781 by Lemnox. 4-7. (Lp to 9 year-rlal without winning).

2. P'epina Tou 1782 by Lennox. $3-9$.

5. (Mlıcllo (or Black and All Black) 1743 by Crab. j-9. (Also in Give and Take Platec).

(i). Why Not 174 by Crab. $5-8$.

7. Crab 1744 by Crab. $4-12$.

1. MILKSOP 1760 by Crab. 5-13.

2. CIIAMI'AIGNE 1764 by Crab. $4-10$.

8. Orounoke 1745 by Crab. $5-6$.

FLISIINA MOLLY 1750 by Oronnoko. $8-11$.

9. Slepplerel's ('ral) 1745 by Crab. (Not run).

SI'Y 1759) by Shepherd's Crab. 4 - 8.

10. Suectatol 1749 by Crab. $5-9$.

1. SCLPIICR 1762 by Spectator. 4-9. 
5. The Thorumbred in the Past and Present.

2. MARK AXTIONY 176 by Spectitor.

3-9. (Half-brother to Hightlyer).

1. Neorge 1780 by Vark Anthony. 3 - 8 .

2. Mark-1ı0 17\&3 by Marls Anthony. $3-8$.

3. NIMIVER 1769 by Spectator. $4-10$.

11. Brilliant 1750 by Crib.

46.

1. XIB(1B $17(j 2)$ by brilliant.

4. 9.

2. Blid.AR10 1763 by Brilliant.

3-8.

3. RItIIMONI) 1763 by Brilliant.

4 8. (Sire of many grood racers in Treland).

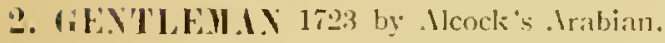

; 11 .

The Darcy White Turk born about 1650.

(or Sedbury lurli).

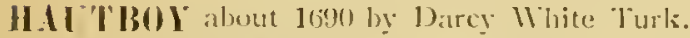

1. Arry IIatluoy about liag by Hatulboy.

1. 13AY 1301.TOX 170.j by Grey Hauboy.

.j.

1. (Civduphin's) Whitefunt 1719 by Biy liolton.

.5. 9.

M()LOTT() 173t5 by llitefoot.

j-8.

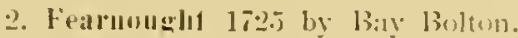

$5-4$.

3. Old Natrling 17:-7 by Bay Bulton.

$1-7$.

1. INC.STEh STARLMG 173 by Old Starlingr.

; 9. (.1ko in Give and Take Plates).

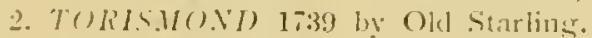

5-7. (1lso in Give and lake l'lates).

3. $K 1 . M 1746$ by Old Starling.

16.

Tinn 17.50 by skint. $4-13$.

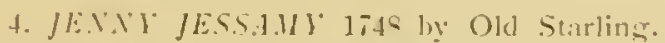
$5-9$.

3. HERSELS 17.5 by Od, Stirling. $5-9$.

1. I.woly (Bulton) lide by liay Rulton.

$5-12$.

2. I. IMPRl: 1715 by Grey Hatbor.

7--10. (Lp to 10 year-ild without winging. Own brother to Bay

[Bolcon.) 
2. CInmsey about 1700 by Hautbog.

1. Olil) lox 1714 by Clumsey:

5.9.

1. (iuliah 1730 by Old liox.

(j.

1. CH.HMPON 1739 by Goliah. 5- 10.

2. HLNT'S JIGG 1741 by Goliah (Nare with-1 free $4-10.4$ [generation.) Jigg of Jiggs 1745 by Hunt's Jigg (and the dam $4-7$.

‥ Yerry Andrew 1730 by Old lox.

5-11. ( $\mathrm{U}_{\mathrm{p}}$ to 13 year-old without winning).

FRIBBLE 1746 by Merry Indrew.

6-S.

3. ('ub 1739 by Old Fux.

is S. (Also in Give and Take Plates). CLERICLS 1763 by Cub.

$4-11$.

$\because$ Fox CLB 1714 by Clumser.

S.

Dunkirk 1725 by Fox Cub.

$6-11$.

3. Old Windham 1719 by Hautboy.

5-11.

1. CIXXAMOX 17.22 by Old Windham.

6-7. (Up to 9 year-old without winning)

1. II-mal 1735 by Cinnamon.

5-s. (Lp to 10 year-old without winnity).

?. Brisk 1737 by Cinnamon.

$5-8$.

2. GREYLEGS 1725 by Old Windham.

5) 6. (Lp to 10 vear-old without winning).

Belgrade Turk born about 1710 .

V. BEI(IRII)E about 1729 by Belgrade Turk.

1. Voluntar 1735 by Y. Belgrade.

6-8.

2. OMl Stamdand 1736 by Y. Belgrade-

$5-6$.

IASOX 1749 by Old Standard.

4-11. (Sire of many sood racers). 
5. The Thoroughbred in the Past and Present.

\section{Lister Turk burn :lbut 16:0.}

1. (I.IN'IER) NI.IKE about 1705 ly L Lister "Turli.

l)river (Burers) 1732 by lister Snidie.

$.5-8$.

I.ITTLE JIRIVLR 1743 hy Driver.

5- 12. (Give and Take Plates).

1. Y. Mriver 175 by litele Driver.

t-i. (.llo in Give and Talie l'lates).

?. ('icero 1765 by l.ittle 1)river.

$4-10$

2. (ONEYSIIXS 1512 by lister Turk.

5-7. (S year-s)l without winning).

Holderness Turk born :bout 1698.

IIIR'TLEYS BLISI) IIURSE about 17:0 by Holderness Turk.

Forrester (('poft's) 1736 by Hartley's Blind Horse.

6.

1. Rilstilts 17.5 by Forester. .

511 .

‥ RIPOXY 1749 by Formter.

$5-11$.

Oxford Bloody-Shouldered Arabian bern abuut 1 irin.

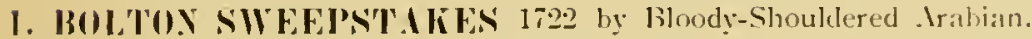

if 6 . (U'p to 11 year-old without winning).

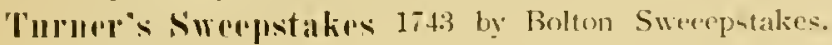

(Not run).

TRETTIAM 1766 ly: Turner's Sweepsakes.

3-10.

Invirer 1793 by Trentham.

$4-8$.

2. BRISK 172.5 hy Bilondly-Shouldered Iraldian.

j. (lip to 9 year-old without winnings).

If in above schedule all horses born before lonol and all sleeplechasers are left oild, there remains 21 t horses which have won al seven years and older. 13:- of them have parents which did not run as wor-year-olds. 53 come from sires which ran as twe-yeareolds (only of of them before June), al come from dams which ran as awe-year-e)ds (only of of them before June), and only in

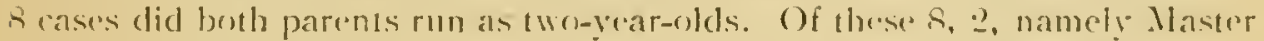
Henry and lanerost, won races up to the age of only seven inclusior. 
Further, the sires and dams ran on an average less than twice as two-year-olds. Horses which only won up to the seventh year inclusive are only mentioned in exceptional cases, i.e., . Mice Hawthorn, whose sire, Muley Noloch, ran as a two-year-old in May, but the dam, granddam and greatgranddam of Mlice Hawthorn did not run at all. Such and similar compensations may often be found in the cases mentioned above.

1 do not claim that the above tables are complete, and mistakes may have, here and there, crept in. Everyone who has studied old horse-race calendars will know how difficult and wearying such tables are, and that mistalies are very difficult to avoid. Taking it altogether, however, we may safely draw the following conclusions from the above-named dates:-

1. Performances on the flat at a great age are more certainly inherited from sires and dams who did not run themselves as two-year-olds than from those who did. The running as two-year-olds in more than two consecutive generations seems to deprive the parents of the suitability to produce racehorses who run for long with success.

2. The using of horses on the racecourse up to a great age seems to strengthen their breeding power in producing first-class racehorses and prominent stud stallions. The most significant examples of this are the following champions of Thoroughbreds which appear almost in every pedigree of our present day Thoroughbred horses several times, namely, Regulus, Noodpecker, Buzzard, Pipator, Election, Paulowitz, Master Henry, Little Red Rover, Lanercost and Collinguoud, won up to their serenth rear inclusive. Imperator, Chanticleer, Ilambletonian, Orville, Catton, Reveller and Sultan, won up to their eighth year inclusive. Herod, Mark Intony, Joe Indrews, Paynator, I Iaphazard, Quiz, Cerberus and Langar, won up to their ninth year inclusive. Matchem, PotSos and Gohanna, won up to their tenth year inclusive. Squirt, grandsire of Eclipse, and Mambrino, foundation stallion of the Imerican trotter, ran up to their eleventh year inclusive, although they only won up to their eighth year inclusive. Dr. Syntax, sire of Bee's Wing, which as a nine-year-old won four races, won again as a twelve-year-old three good races. Out of the great number of the chief founders of Thoroughbreds which won as sis-yearolds, let me here mention only the following eight: Eclipse, Waxy, Whalebone, IVhisker, King Fergus, Beningbrough and Touchstone.

3. The use of stallions with race performances at a great age for breeding purposes decreases more and more during the first half of the nincteenth century. In spite of the increase of Thoroughbred breeding, the examples of prominent performers at a great age already diminish before the middle of the nineteenth century (see tables, pages 108-141).

4. The most famous steeplechasers in the first half of the nineteenth century are descended, with few exceptions, from parents which did not run as two-year-olds.

5. Most stallions and mares which ran as two-year-olds, and yet pro- 
duced horses which could run for long successfully, ran as mo-year-olds late in the year, and seldom more than once or twice.

As in our time nearly everything which is healthy runs as a wo-year-old, it is remarliable that in examining the question from this point of riew, there are still actually brought out points which speak against two-year-old races, especially against the early and frequent ones. In the last ten years, up to and inclusive of 1906,63 horses born in England or 1reland still won flat races as eight-year-olds and older. The number 63 is a very small one considering that the breeding is to-day five to ten times more extensive. Therefore a retrogression in this respect is distinctly recognisable. Of these 63 horses, 27 have dams which never ran as two-year-olds. Of the remaining 36 dams, only 14 ran before the $]$ st of June, generally only one to three times; 3 ran seven times; 2 eiglnt times, and 1 ten times. The above 63 horses have 50 different sires, of which 9 did not run as two-year-olds. Of the remaining 41 sires, only 19 ran before the 1 st of June as tro-year-nlds. Osbeck ran and won longest-up to its twelfth year. He was born in 189. by Common, who did not run as a two-year-old, and his dam . llibcel ran once as a two-year-old in September.

Also, several American and Australian horses won in England as eightyear-olds and older. Imongst the latter is especially to be mentioned Australian Star 1896, by Australian Peer and Colours, who won as an eightyear-old a Handicap in Alexandra Park, $1 \frac{1}{1}$ miles, 9 stone, $12 \mathrm{lbs}$, against five four-year-olds, with 8 stone to 9 stone. Further, Merman 189. by Grand Flaneur out of Seaweed, who, as an eight-year-old, won the Gold Vase at Ascot, 21 miles, carrying 9 stone, 1 lbs., against the four-rear-old Scintillant, and Perth (under 9 stone), and three others. Iustralian Star. as well as Merman, come from dams which did not run as two-year-olds. Also, Australian Peer, the sire of Iustralian Star, did not run as a two-yearold, and Grand lilaneur, a mariel on the Iustralian raceeourse, and sire of Merman, ran and won as a two-year-old only once, in January, which month, for the salie of comparison, corresponds to our July.

To further show the hardness and stamina of the Thoroughbred up to about the middle of the nimetecntl century, the following examples of excellent performances on the flat may serve. Of course, there are some horsus given which. from the point of view of the regular racecourse freguenter, belong to an inferior class. 
No. 1. Partner 1730 by Partner.

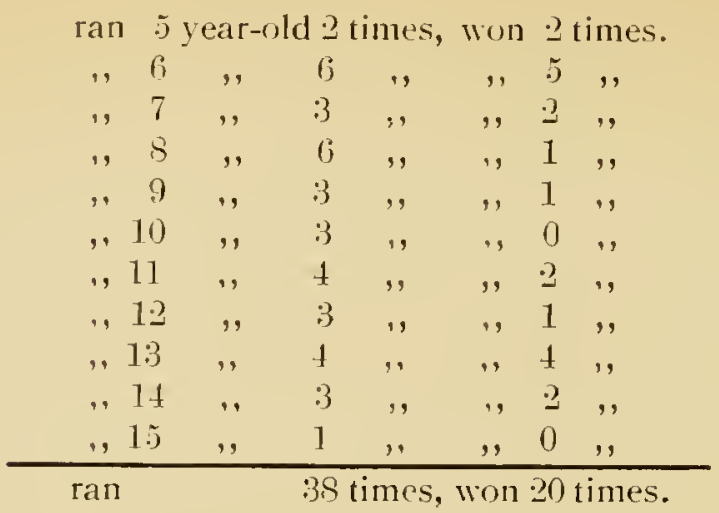

No. 2. Black Chance 1732 by. Hutton's Bay Barb. ran 5 year-old 2 times, won 2 times.

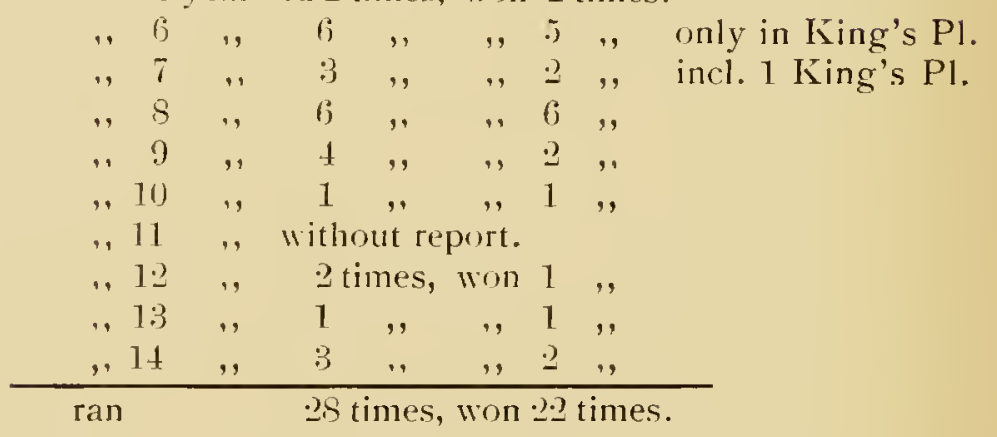

No. 3. Sedbury 1734 by Partner.

$$
\begin{aligned}
& \text { ran } t \text {-yearold } 2 \text { times, won } 1 \text { time. } \\
& \text {,. . , , } 3 \quad,, \quad, 3 \text { times. }
\end{aligned}
$$

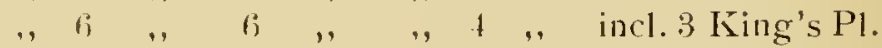

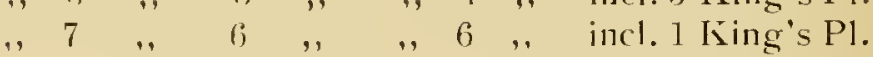

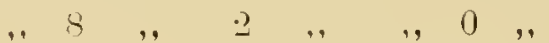

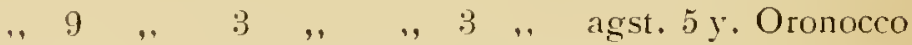

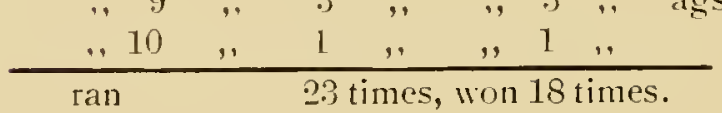

No. 4. 1744 Nlatch at Lincoln 14 miles $=22,530 \mathrm{~m}$.

Mr. Southcote Parker's if year-old horse won with one length in 39 minutes, against 11 r. Gilbert Colecut's 21 year-old horse. See "IIestminster Journal," the $23 / 6 / 1744$. 
No. 5. Gustavus 174.) by ('roft's Forrester.

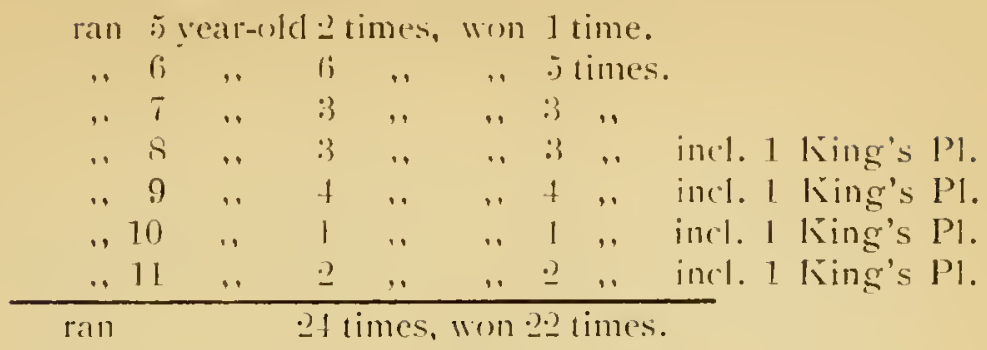

No. li. (albbage 1757 by South.

ran f year-old : 3 times, won 11 times.

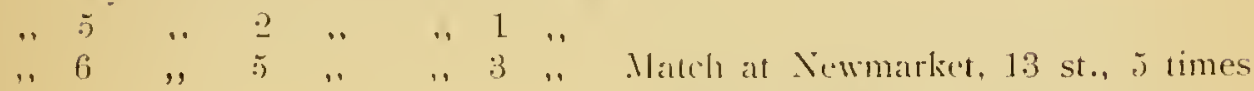

$$
\begin{aligned}
& \text { R. } \mathrm{C}^{\circ}=30,50,596 \mathrm{~m} \text {., in 5.) minutes, } 30
\end{aligned}
$$

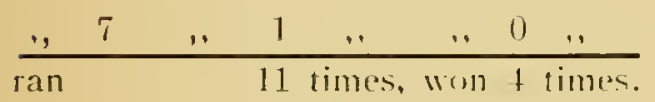

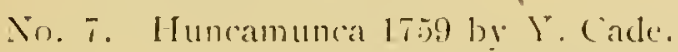

ran 7 year-old 1 time, won 1 time. King's Pl.

. s .. 3times, .. - times incl. 1 King's Pl.

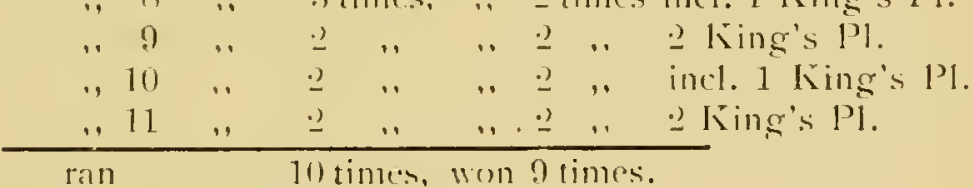

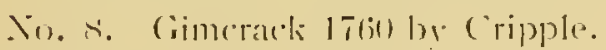

ran tyear-old 7 times. won $T$ times.s.

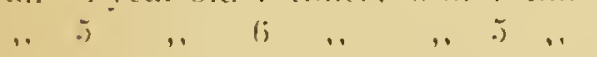

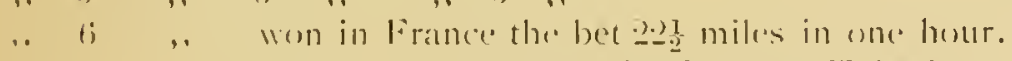

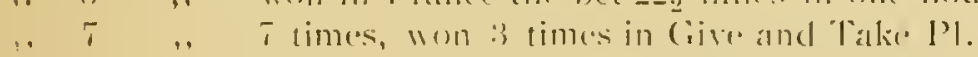

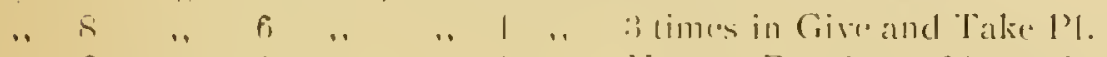

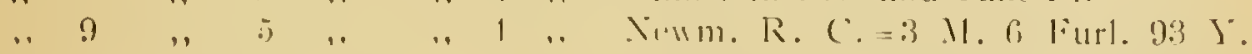

9 st against 9 herses of a grod dass.

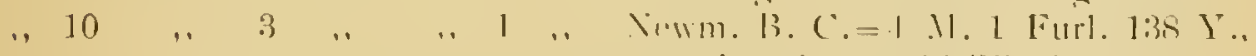

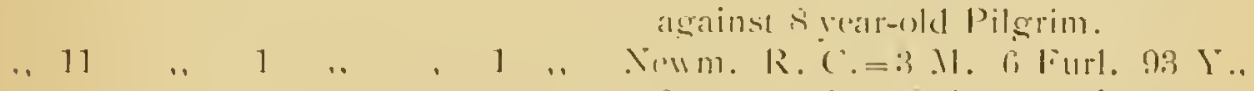

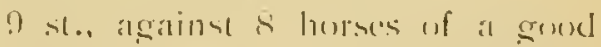
(latses amenger which were Bellariw syear-rold, Sportsman iprar-edel.

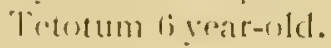


No. 9. Trentham 1766 by Sweepstakes.

ran 4 year-old 6 times, won 6 times.

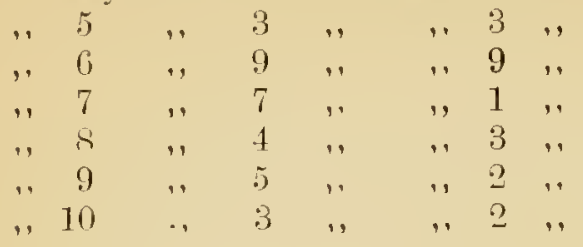

and twice as second.

incl. Jockey Club Pl. Newm. B. C.= t Mi. 1 Furl. 138 Y. 4 behind.

ran

37 times, won 26 times.

No. 10. Nother Brown 1771 by Trunnion.

ran 4 vear-old 2 times, won 0 times.

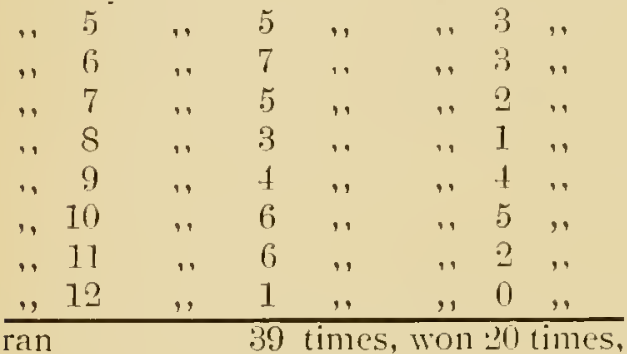

was in foal.

incl. 1 King's Pl. after fualing.

incl. 1 King's P1.

and 4 times as second.

ran 39 times, won 20 times, and had 6 good foals, amongst which at 2.; years Jerry Sneak, s. No. 24.

No. 11. Pot8os 1773 by Eclipse.

ran 4 year-old 5 times, won 0 times, twice second in big races.

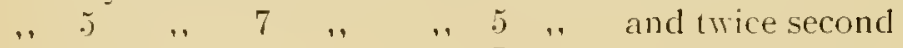

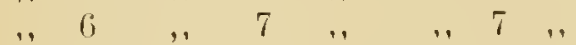

$, 7,11,, \quad, 8 \frac{1}{2},, \quad$ and unce second.

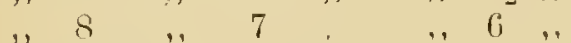

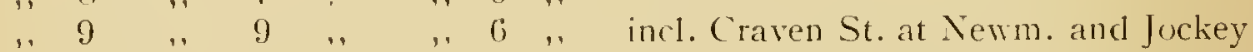

Club Pl. at Newm., and 3 times second.

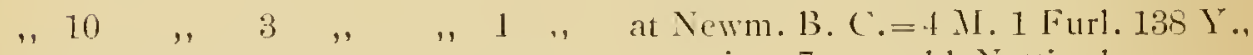

ran 49 times, won $33 \frac{1}{2}$ times. against 7 year-old Nottingham.

No. 12. Titania 1774 by Trunnion.

ran 4 year-old 5 times, won 3 times, and once second.

, 5 , 8 , , , 7, , and once second.

" $6 \quad, \quad+, ., \quad, \quad 1$, and 3 times second.

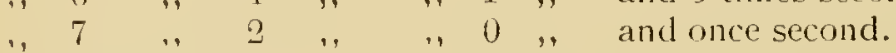

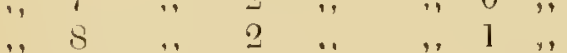

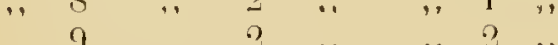


ran 10 year-old : times, won "2 tines. Kingr's Pl.

, $11 \quad$ " $2 \quad$, 1 , 4 miles with heats, 3 behind, incl. one 6 vear-old.

ran

27 times, won 17 times.

No. 1:3. I.alage 1770 by Hero.

ran 4 year-old 7 times, won 6 times, incl. 1 King's Pl, and oncre second.

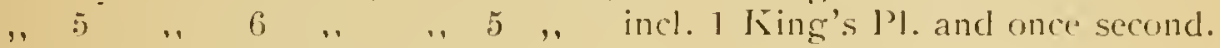

,, $\quad$ ( $\quad 2 \quad 2 \quad, \quad, \quad 2 \quad$,

, 7 ., without report.

, \& ., 2 times, won 1 time, and once second.

" $9 \quad 9 \quad 5 \quad$, , , 5 times, incl. 1 King's Pl.

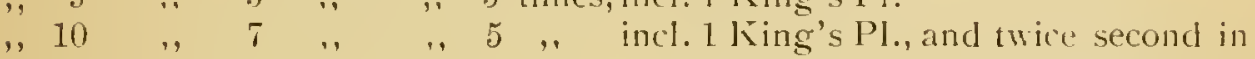
good company. fi year-old Cherokee beaten twice.

, $11,, \quad 3 \quad,, \quad, 1$, King's Pl. and once second.

, 12, , $6 \quad, \quad 4$, , incl. 1 King's Pl. in good company, and once second.

ran 35 times, won 29 times.

No. 14. Quibbler 1780 by Minor.

ran 4 year-old : times, won 1 time.

, 5 , , $3 \quad$, , , 0 ,

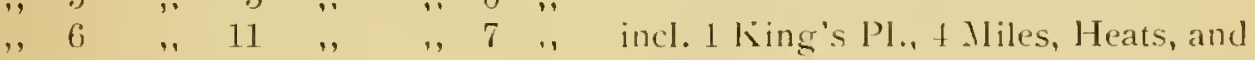

Match at Newmarket, $\$$ st. 7 lbs., 23

Miles (=37 lim.) in .57 min., $10 \mathrm{sec}$.

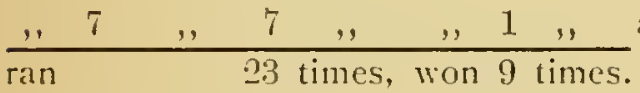

at Lewmarliet.

ํ. 15. Exciseman 1781 by Sweetbriar.

ran Syear-old j times, won 3 times. 1 miles with heats, against, 4, jand

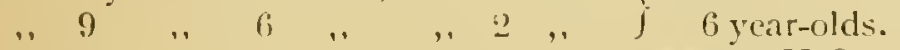

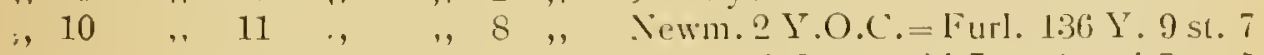
atgst. -3 vear-old 7 st. 4 and 7 st. ij

34 year-old 7 st. $9-7$ st. 11

25 year-old 8 st. 2 and 8 st. 9

(Serpent by Eclipse)

1 ulder 9 st. 7

against 8 horses.

$, 11,30, \ldots \quad 4,$, on the 12 th 11 ay, Newm. $2 l^{\circ} .() .($.

$5 \mathrm{I} .136 \mathrm{Y}$., S st. 4, against 1 yearold II leetor, 8 st.

on the 2.2nd May, Epsom, 4 M., I l'ats,

3 Heats second and 1 I Ieat first. 
Newm. 2 Y. O. C.=5 Furl. 136 Y. 8 st., against jyear-old Stallion by lispersykes, 7 st. 8.

Newm. 2 Y. O. (.$=.5$ Furl. $136 \mathrm{Y}$. 8 st. 12, against 3 vear-1)ld Mare by Jupiter, 7 st. 10.

ran $1 \%$ year-old $1 \%$ times, won $i$ times. Newm. 1 Mile, 8 st. 1?, against 4 yearwld Peggr. sit. $t$.

Newm. 3 Miles, sst. 12 agst. 34 r.-o. 7 st. $11-8$ st. $t$.

25 y.-o. 7 st. 11 and 8 st. 13. (Eager D).

On the following dav, $\because$ Y. O. C. = F Furl. 136 Y., 9 st., against 3 y.-o. 7 st. 2 (Brother to Sir John).

. It Warwick, 1 Mile, Heats, against 6,3 and 4 year-olds and one older.

On the following day,

+ Miles, Heats, 8 st. 9, against

26 year-old, 8 st. 7 and 8 st. 12 , and ij times second.

$\overline{\text { ran } \quad 4 \text { times, won } 24 \text { times. }}$

No. 16. Mlentor 1784 by Justice.

ran 3 year-old 5 times, won 1 time, in Derby not placed.

, $4 \quad, \quad 10 \quad, \quad, 4$ times.

$\because, \quad, \quad 8 \quad, \quad, 4,, \quad$ incl. 1 king's P1.

$\begin{array}{llllllllllll} & , & 6 & , & 9 & , & & , & 5 & ,\end{array}$

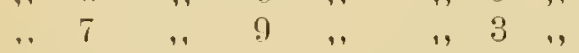

"

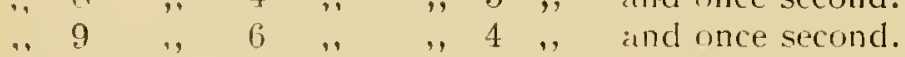

. $10 \quad, \quad 4 \quad ., \quad, \quad 2$," and twice second.

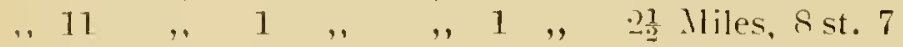

against 13 year-old, 6 st.

15 vear-old, 8 st. 4 .

2 older 8 st. 12.

$\overline{\text { ran } \quad 56 \text { times, won } 27 \text { times. }}$

No. 17. Huby 1788 by Phönomenon.

ran 3 vear-old "2 times, won 0 times, second in the St. Leger.

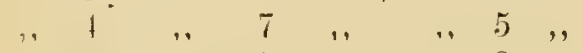

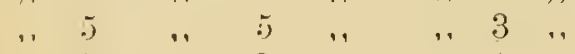

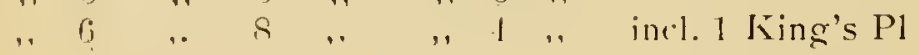


ran $\tilde{i}$ year-old + times, won 1 time.

" $8 \quad . \quad 7 \quad, \quad, 3 \frac{3}{,}, \quad$ in the best company, of $3,4,5$ and li year-olds. Dead heat, 8 st. 3, at Iewes, with the jear-old I Herminnc O., s st. 1, which won j rares in the same year, and wice second.

ran 33 times, won $16 \frac{1}{2}$ times.

o. lis. St. Gerge 1789 by Jighflyer.

ran 3 gear-old fines, won 1 time, in Derby mut placed.

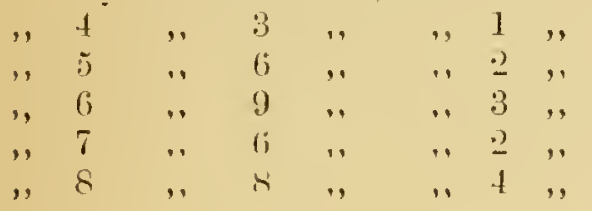

incl. 1 King gis l.

Jockey Club Pl. at Newm. B. C:= 4 M. 1 Furl. 138 Y., Bst. 11

against $1+$ yeár-()ld, 7 st. 2

15 year-old, sst. 3

2 older ist. 11

ahd 3 times secund.

, 9 , 9 , , , 5 , against 3, 4 and jear-olds, further against 7 year-old Paynatur and 8 rear-old . limator

and 3 times second.

"10 $10 \quad 6 \quad, \quad, 1$, and once second, 4, jand ti year-olds behind.

$\overline{\operatorname{ran}} \overline{5: 2}$ times, won $19 \overline{\text { times. }}$

So. 1!). . limator 1790 by Trumpator.

ran 3 year-old 4 times, won 0 times.

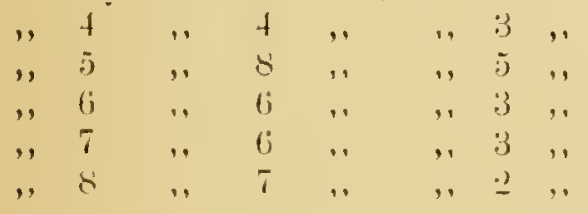

Craten St. at Vewm. against li gearold Spread Eagle 1)' and against? 3,4 and jowir-olds and ulder and wice serond.

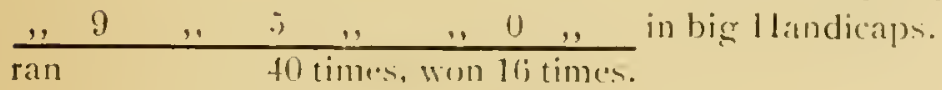

Vu. 20. Colanna 1790 by Mereury.

ran 3 year-old \& times, won (i times, second in Derby.

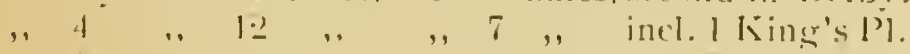

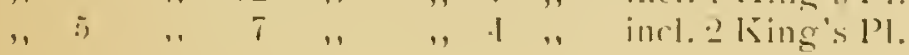


ran 6 year-old 5 times, won 3 times, incl. 2 King's Pl.

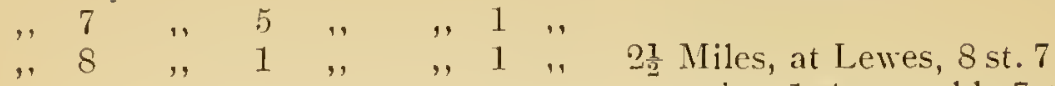

$$
\begin{aligned}
& \text { against } 14 \text { year-old, 7st. } 11 \\
& 26 \text { year-old, } 8 \text { st. } 2 \\
& 1 \text { older, } 8 \text { st. } 7
\end{aligned}
$$

$$
\begin{aligned}
& \text {, } 9 \quad, \quad 7 \quad, \quad, \quad 3 \quad, \quad 1 \text { llile, } 9 \text { st. } \\
& \text { against } 24 \text { year-olds, } 7 \text { st. } 8 \\
& 15 \text { year-old, } 8 \text { st. } 6 \\
& 16 \text { year-old, } 8 \text { st. } 6
\end{aligned}
$$
and twice second.

, $10 \quad, \quad 3 \quad, \quad, \quad 1 \quad, \quad 2 \frac{1}{2}$ Mliles, at Lewes, 8 st. 7 against 25 year-olds, 7 st. 12 and 8 st. 4

$\overline{\text { ran } \quad 48 \text { times, won } 26 \text { times. }}$

No. 21. Hambletonian 1792 by King Fergus. ran 3 year-old 6 times, won 6 times, L. Dcp.

, $4 \quad, \quad 4 \quad, \quad, \quad, 4 \quad, \quad$ Dcp.

$,, 5 \quad, \quad 7 \quad, \quad, \quad, \quad 7 \quad$,

., $6 \quad$, not

" 7 " 2 times, won 2 times, Donc. St.

, $8 \quad, \quad 1 \quad, \quad, \quad 1$, 4 Miles, at York, 9 st. against 5 year-old Dion and 6 year-old Timothy, 8 st. 10 , both first-class racehorses which won good races in the same year.

$\overline{\text { ran } 20 \text { times, won } 20 \text { times. }}$

No. 2.2. Bubtail 1795 by Precipitate.

ran 3 year-old 3 times, won 2 times, in the Derby not placed.

$\begin{array}{llllllll}, & 4 & , & 7 & , & , & 3 & , \\ . & 5 & , & .2 & , & , & 1 & , \\ , & 6 & , & 7 & , & , & 7 & , \\ , & 7 & , & 5 & , & , & 3 & , \\ , & 8 & , & 7 & , & , " & 4 & ,\end{array}$

Newm. 2 Y. O. C.=5 Furl. 136 Y., 8 st.7, against 5 year-old Muley Moloch, 8 st. 4

Newm. 1 Mile, 8 st. 4 against 6 yeár-old Surprise, 8 st.

Newm. Hdc., 1 M. 2 Furl. 44 Y., 9 st., agst. 4 y.-o. Northampton, 7 st. 3

5 y.-o. Eleanor D. O., 8 st. 12

jy -o. Flambeau, 6 st. 12

6 y.-o. Georgiana, 8 st. 10 
ran !) year-old 7 times, won + times. Newm. D. J.= 2 M. 97 Y.. 8 st. 10, agst. 13 y.-o. 7 st. 4

6 y.-o. Dick Andrews 9 st. 4

6 y.-o. Surprise 7 st. 13.

Newm. 1 M. 2 Furl. 44 Y., 8 st.9, agst. 7 y.-0. Lignum Vitae 8 st.

Newm. 1 II. 2 liurl. 14 Y., Sst., agst. 6 y.-o. Penelope 8 st.

ran

38 times, won 24 times.

No. 23. Eagle 1796 by Volunteer.

ran 3 year-old 5 times, won 3 times, third in the Derby.

, 1 , , 4 ,

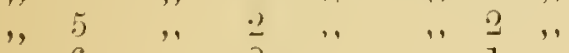

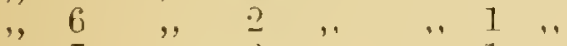

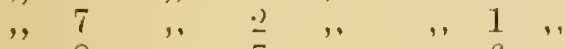

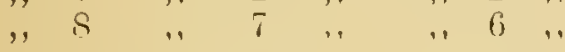

" $9 \quad$ " 4 , $4 \quad$, 3 " Nerm. 1 Mile, 9 st. 7 , agst. 1 y.-o. Dreadnought 5 st. 8.

Newm. 1 Mlile, 9 st. 4 , agst. 6 y.-o. Marianne 7 st. 6 .

$\overline{\text { ran }} \quad 26$ times, won 17 times.

No. 24. Jerry Sneak 1796 by Chocolate.

(As a racing horse also called Toby and Y. Toby).

ran ? year-old 1 time, won 0 times, in October.

$\because 3$, 7 times, . 2 ..

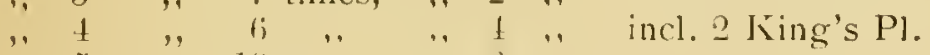

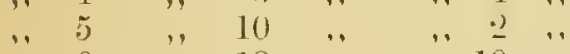

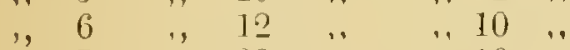

"7 7 "

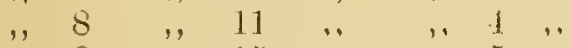

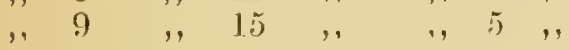

:) Miles, 9 st. 7 , agst. 5y.-o. 8 st. 10 \& 4 y.-o. 7 st. 8, 1.) June, at (urragh, 4 Miles with Heats, second to 6 y.-o.. Blacklegs, Traveller behind, and won on the same day Match, 1 Miles, $18 \mathrm{st} .=$ $11.4 .3 \mathrm{lig}$. against a Hunter 18 st., in 9 min. 27 sec. ( 7 sec. quicker than the Hunter). Covered 180556 mares, of which only 3 were barren.

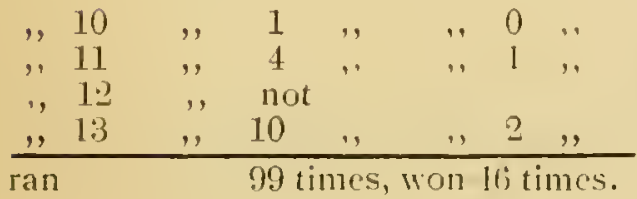

1 Miles, agst. $69-0$. Midas.

1 Miles, agst. 8 y.-1, Miclas. 
No. 25. Marcia 1797 by Coriander.

ran 3 year-uld 1 time, won 0 times.

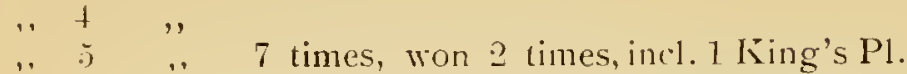

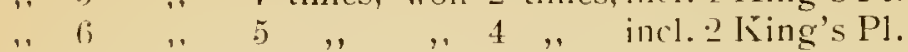

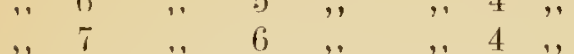

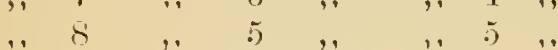

Gold Cup at Newcastle, Gold Cup York, Doncaster St., 4 M., 8 st. 10, agst 33 v.o. 6 st. (amongst which was Sir Paul), and 14 y.-o. 7 st. 7 . , 9 " 4 . , , 4 " àlways against young horses incl. 1 King's Pl.. 8 st. 9, agst. 3 y.-o. 6 st. 7 and $4 y,-0.7$ st. $T$.

ran

28 times, won 19 times.

No. 26. Rumbo 1800 by Whisker.

ran 2 year-old $\cong$ times, won 1 time.

, $3 \quad$, $5 \quad$ " . , 2 times, incl. 1 King's Pl.

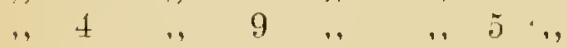

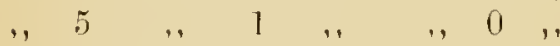

," $6 \quad$,

$, 1,7$,

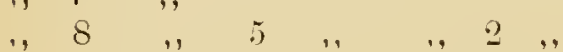

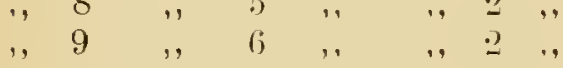

$2 \frac{1}{2}$ Miles at Ascot, 9 st., agst. 24 y.-o. 7 st. 9 and $+5 y-0.8$ st. 5 .

, $10 \quad$ " $11 \quad, \quad$, 7 , Gold Cup at Newm., 1 M. 2 F. 44 Y., 8 st., agst. 13 y.-o. 6 st. 11

$74 \mathrm{y} \cdot-0.7$ st. to 9 st. 7

35 y.-o. 7 st. 12 to 10 st., amongst which many good racers. , 11 , 2 , , , 1 , $2 \frac{1}{2}$ Miles at Ascot. Selling race,

ran 41 times, won 20 times. agst. $26 y$ y.o. and 1 older.

No. 27. Sir Darid 1801 by Trumpator.

ran - year-old 2 times, won 1 time.

, $3 \quad, \quad 5 \quad 7, \quad, \quad 5$ times.

$\begin{array}{lllllllll}, . & 4 & , & 9 & \text {, } & & , & 6 & ,,\end{array}$

$\begin{array}{lllllllll}1, & 5 & 4 & 4 & 9 & , & , & 9 & ,\end{array}$

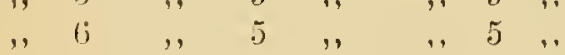

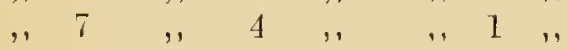

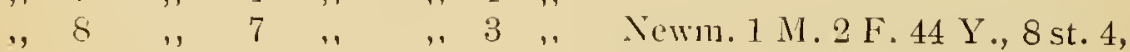
agst. 5 y.-o. Eaton 8 st. 8 . 
5. The Thoroughbred in the Past and Present.

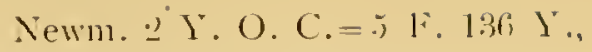
8st. 10 . agst. 4 y.-o. Morel O. S st. ?2.

ran 9 year-old? times, won $1 \frac{1}{2}$ times. Newm. 3 .lliles, 8 st. 7 , acrst. i) y-o. Vandylie 8 st.

Newn. 1 il. 2 F. 44 Y.. S st. T, at 7 y.o. Deceiver \& st. 4 ,

Dead lieat.

ran 45 times, won $31 \frac{1}{2}$ times.

No. 28. I.angton LA(1)-2 by l'reripitate.

ran 3 year-old 4 times, won 3 times.

\begin{tabular}{|c|c|c|c|c|}
\hline 4 &, & 12 & " & " \\
\hline 5 & ", & 8 & , & , \\
\hline 6 & , & i & " & , \\
\hline 7 & ,. & 10 & , & , \\
\hline 8 & . & 6 & , & . \\
\hline
\end{tabular}

Oatl. St. át Jewm. ? Miles, 9 st. 11, arst. 9 young horses $3-6$ y.-o.,

and $\delta y-o$. Meteora O. S st.

Gold Cup at Niwm. 1 Mile, 9 st. 7. agst. 13 y.-0.j st. 12

$1+\mathrm{y} .0 .7 \mathrm{st} .10$

45 y.o.sist.j jo st. 10

$16 \mathrm{r}-0.9 \mathrm{st}$.

1 older S st. 12,

agrst. \& horses.

Newm. 1 Mile, 9 st. 6, agst. (i younger horses, and on the same day a race, 4 Miles, third, ? behind, started as favourite.

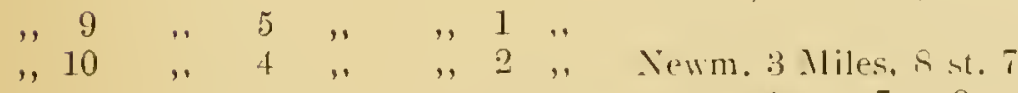

agst. 4 y.r. 7 st. 9 and $5 y,-0.8$ st. 3

ran

5.) times, won 27 times.

No. 29. Cambric 1807 b\% Shuttle.

ran 2 year-old 5 times, won 2 times, first race, July St. at Newm. against 7 liorse's.

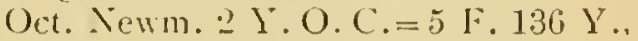
second to Vulture,

and on the same day won over

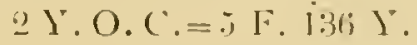

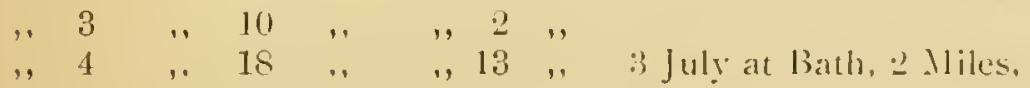
agst. $23 y-1)$ and $2 ?+1$ y.o. 
1 July, 2 Miles, Heats, agst. 13 y.-o.,

and immediately afterwards 4 Miles, Heats (3 times), agst. $24 \mathrm{y}$.-o. and $16 y$.o.

16 July at $\mathrm{V}$ inchester, 1 Mile, second, 3 behind.

17 July, 2 Miles, Heats, 8 st. 11, agst. $1+y \cdot-0.8$ st. 8 .

6 August, Oxford, 4 Miles, second, $1+$ y -o. behind.

8 . Iugust, 2 Miles, Heats, not placed.

5) September, 2 Miles, agst. $13 \mathrm{y}$-o., $14 \mathrm{y}$-o. and 1 older, and immediately after 3 Miles, Heats, 8 st. 9 , agst. $13 \mathrm{y},-\mathrm{o} .6$ st. 12.

18 September, 3 Miles, Heats, agst. $1+\mathrm{r}$.-o. and $16 \mathrm{v}$.-o.

$\because 4$ September, 4 Miles, lleats, agst. 13 y.-o.

2 October, 3 Miles, Heats, agst. $1+$ y.-o.

ran 5 year-old 1 time, won 1 time. Oatlands St. at Newm., 2 Miles,

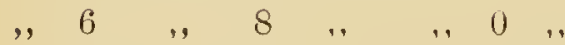
agst. \& good racers.

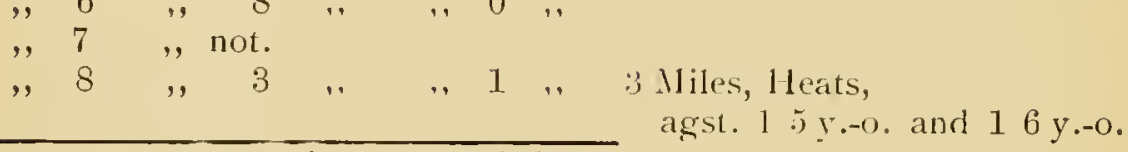

ran 45 times, won 19 times.

No. 30. Marksman 1808 by Paynator.

ran 5 year-old 4 times, won 1 time.

, $6 \quad 6 \quad, \quad 3 \quad,, \quad$, 1 , 1 ,

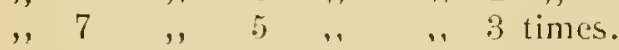

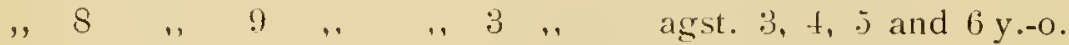

, 9 , 9 \& $\quad, \quad, \quad, \quad 5 \quad, \quad$ incl. 1 King's Pl.

agst. 4 and $6 \mathrm{y} .-0$.

, $10 \quad, \quad 9 \quad 9 \quad, \quad, \quad 5 \quad, \quad 4-3$ Miles.

,, $11 \quad,, 3 \quad, \quad, \quad, \quad 0 \quad$,

agst. $3,4,5$ and 6 y.-o.

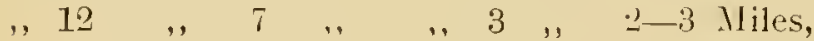

, $13,, 8,,, 2,, \quad$ agst. 3,4 and 5 y.-o. and 1 times second. 
ran 14 year-old $t$ times, Won 1 time, at Basingstoke, 8 st. 10. Selling race agst. $+3 y,-0.7$ st. 1 to 7 st. 4 $1+y-0.8$ st. 3 25 i. - o. S st. 9 and 8 st. 1$]$

agst. 7 horses.

, $15 \quad,, \quad 5 \quad, \quad, \quad 3 \quad$, at l.ewes, 9 st. 4 , arst. 3 y.-o. 6 st. 11

ty-0.8st. at Basingstoke, 9 st. 1 agst. $23 \mathrm{y} \cdot-0.7$ st. 1

$1+y-0.7$ st. 12

1 . y.-o. 8 st. 6

$16 \%-0.9$ st. $t$

1 oider 9 st. 1

agsst. 6 horses.

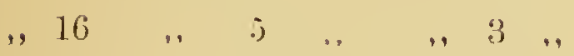

2 Selling Races at Lewes.

at (anterbury, - Miles, with Heats, 9 st. s,

agst. $2+y-0$. 8 st. 6

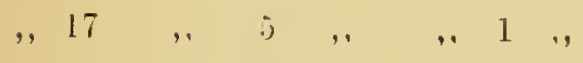

The Trin Pl. to.j0 at Tunbridge

IVells, :3 $\mathrm{Ml}$., with Heats, 9 st. 11, agst. $1: 3$ y.-0.T st. 4

1 .j $y-0.9$ st. 11

and twice second.

, 18 , 3 , , 1 , The Vimmen's l', at Ihford, 650 , or Miles, witl Heats, agrst. 1 :3 y.o. (Ioneysuckle by IThalebone)

$1 \pm y-0$. (Jack Bunce by $Y$. (inhanna)

1 i y.-u. (Roderich Randow bi Regrent)

agst. :3 horses, which had won races in the same year, and twice second,

latst race 1 t Sept., at Hastings, the

Town Pl., M., with Heats, 650

:3 y.-(). Honeysuckle 7 st.6.. 1. 18 y.-(). Marksman 9 st.8. . 2. (i y.-o. Barbary st. $13 \ldots 3$. 
No. 31. Cannon-ball 1810 by Sancho.

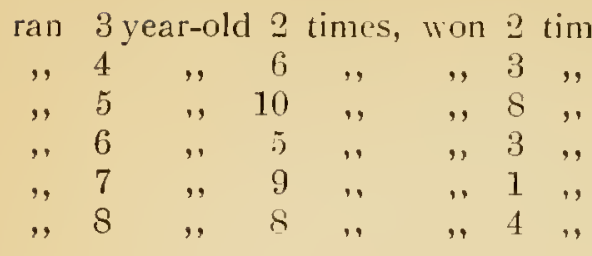

beat Catton, Langold, Viscount, Wanderer, Slim, and King of Diamonds.

Oatl. St. at Newm., 2 Miles, 9 st. 3 agrst. $14 y-0.7$ st. 5 to 7 st. 12

15 y.-o. 8 st. 6 -2) 6 y.-0. 8 st. 8

agst. 7 horses (amongst which were the $t y-0$. IVaterloo and $6 y \cdot-o$. Anticipation).

Jockey Club Pl. at Newm. B. C.= 4 M. 1 F. 138 I., 8 st. 11 agst. $2+y .-0.7$ st. 2

$15 \mathrm{y} .-0.8$ st. 3

agst. 3 good racers.

King's P1. at Newn., agst. $4 y_{-0}-0$. Waterloo.

IIdc. at Newnı., 2 Miles, 9 st. 4 agst. 33 y.-0. 7 st.

$1+y-0.8$ st. 7

agst. 4 horses,

and twice second at Newmarket.

, $9 \quad,, 8 \quad 8 \quad, \quad,, 3 \quad$,

Crav. St. at Newm., 1 M. 2 F. 44 Y., 9 st. 7

agst. 73 y.-o. 5 st. 10 (incl. Wouvermans).

$5+y-0.8 \mathrm{st}$.

4 .) V.-o. 8 st. 9

agst. 16 good racers.

ling's P]. at Newm.

agst. $6 y .-0$. Skim and $7 y,-0$. Anticipation.

Jockey Club Pl. at Newm. B. C.= 4 M. 1 F. 138 Y.., 8 st. 11

agst. 24 y.-0.7 st. 2 (Sam. D) and

7 st. 5 (IVouvermans). and twice second at Newmarliet. 
5. The Thoroughbred in the Past and Present.

No. 32. Dr. Sỵntax 1811 ḅ̣ Paynator.

ran 3 yearold 8 times, won 5 times.

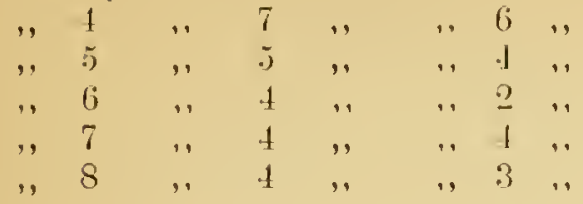

Gold ( up at Lancaster, 3 11., 8 st. 12 agst. 6 y.-o. Paulowitz 8 st. 12

Fold ('up at Preston, 3 M.. \& st. 12 agst. : ? y.-u. 6 st. 6

ince second Gold ('up at Richmond 4 young horses behind.

, $9 \quad, \quad 4 \quad, \quad, 4,4 \quad$ Gold Cup at Lancaster, 3 11., 8 st. 12 agst. $24 y-0.8$ st.

(iold Cup at Preston, 3 MI., 8 st. 12 agst. $2+y .-0.8$ st.

Gold Cup at Richmond, 4 M., 8 st. 10 agst. 3 3 y.-0.6 st. 10

$1+\mathrm{ro}-0.8 \mathrm{st}$.

$15 \mathrm{y} \cdot-0.8 \mathrm{st} . \mathrm{t}$

asst. 8 good racers.

$\begin{array}{lllllll}\text {, } & 10 & \text {. } & 4 & , & , & 3\end{array}$

Cold (up at Preston, 3 M., 8 st. 12 agst. 1 \& y.-0. 8 st.

and $1 \mathrm{y}$ y.-o. Reveller L. 8 st. 12

Gold Cup at Richmond, 4 M., 8 st. 10 agst. 5 y..-0. 8 st. 7

second in the Gold Cup at Lancaster to ti y.-o. Reveller L.

, $11 \quad, \quad 4 \quad, . \quad, 2 \quad 2 \quad$,

Gold cup at Richmond, \pm M.. s st. 10 agst. 4 y.-o. 8 st.

Gold Cup at Northallerston, $t$ M., D. st. 1

agst. 23 y y,-0.tist. 7 and 6 st. 1.2

$15 \mathrm{v.-(1.85t.12}$

1 older 9 st. 1

atgst. 4 horses,

and $t$ ice second in the Gold cup at Preston

and in the forld Cup at lancaster.

$\frac{,, 12}{\operatorname{ran}} \frac{5 \quad, \quad, \quad 3 \quad . .}{49 \text { times, wion } 36 \text { times. }}$

Nowcastle, Dlites, ? st.

agst. 13 y.-(). (i st. 10

and $1+\mathrm{i} \cdot-\mathrm{cos} .8 \mathrm{st} .3$

Gold (up at Pontefract, . . . . . 9 st. 1 agst. I r.o. Sst. 
Gold Cup at Richmond, 4 M., 9 st. agst. 23 y.-o. 6 st. 10

$1.4 \mathrm{y} \cdot-0.8 \mathrm{st}$.

15 y.-0. 8 st. 9

agst. 4 horses.

In this last race Dr. Syntax fell down immediately after winning, but without damaging itself.

No. 33. Euphrates 1816 by Quiz.

ran 3 year-old 5 times, won 1 time, D. and 1 . not placed 2 , third.

, $4 \quad, \quad 3 \quad, . \quad$.. 2 times.

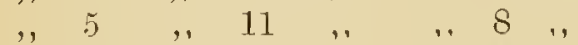

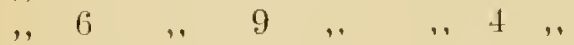

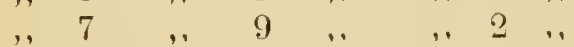

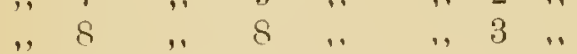

Gold Cup at Cheltenham, 3 M., 9 st. 4 agst. 14 v.-o. 8 st.

and 25 y.-0. 8 st. 11 , amongst

which were Sharper by Octavius, who won the race in St. Petersburg over 75 versts.

Oxfordshire St., 2 Miles, 8 st. 12

agst. 13 y. -0.6 st. 8

34 y.-0. 7 st. 11 to 8 st. 1

16 y.-o. 9 st. $3=$ Escape

agst. 5 horses.

Gold Cup at Lichfield, 3 M., 8 st. 12 agst. 4 y.-o. 8 st.

$\begin{array}{lllllll}. & 9 & \text {, } & 13 & \text {, } & & \end{array}$

Gold Cup at Newton, 3 Miles, 8 st. 12 agst. $14 \mathrm{y}$.-0. 8 st.

35 y.-o. 8 st. 8

agst. 4 horses.

Gold Cup at Worcester, 4 MI., 9 st. 4 agst. $24 \mathrm{y},-0.8 \mathrm{st}$.

16 y.-0. 9 st. 1

agst. 3 horses.

Cup at Molverhampton, 3 Miles, 9 st. agst. 14 y.-o. 8 st. 2

25 y.-0. 8 st. 10

agst. 3 horses.

Gold Cup at Lichfield, 3 Miles, 8 st. 6 agst. 13 y.-o. 6 st. 6 34 y.-0. 7 st. 10 to 7 st. 12

agst. 4 horses. 
Gold Cup at Oswestry, $3 \frac{1}{2}$ MI., 9 st. 2 agst. 4 y.-o. Buttler 8 st. and 5 y.-0. Hesperus 8 st. 9

ran 10 year-old 11 times, won 5 times. King's Pl. at Chester agst. $5+y \cdot-0$.

Gold Cup at Lichfield, 3 M., 8 st. 12 agst. 1 y.-O. Cain 7 st. 12 and $; y-0$. Longwaist $8 \mathrm{st} .6$

Gold Cup at Oswestry, $3 \frac{1}{2}$ M., 9 st. 2 agst. $2+y \cdot-0 . S$ st.

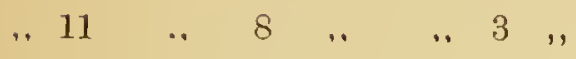

King's P1. at Lichfield, 4 M.., Heats, agst. $14 \mathrm{Y} .-\mathrm{O}$. $16 \mathrm{v} \cdot \mathrm{-O}$. 1 older

agst. 3 horses.

Guld Cup at Oswestry, 3 Miles agst. I y.-o. Mayfly.

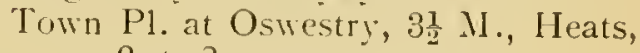
9 st. 2 agst. 4 r.-o. Mayfly 7 st. 10 - and 5 y.-o. Cymbeline 7 st. 12

and 3 times second in good races with large fields.

$\begin{array}{llllllllll} & 12 & & 10 & \ldots & & & 5 & 5 & \end{array}$

King's P1. at Chester 9 st. 10 agst. $14 \mathrm{v} .-0.8 \mathrm{st} .2$ and 1 ; y.-0.9 st. 6

Gold Cup at Ludlow, 3 . Vliles, 8 st. 12 agst. 3 y.-o. Alcaston 6 st. 6 and $8 \mathrm{y} .0$. Hesperus $9 \mathrm{st}$.

Gold Cup at Worcester, 4 .I., 9 st. 1 agst. $1+y .-0.8$ st. and $16 y-1) .8$ st. 12

Gold Cup at 11 rexham, 9 st. 7 agst. 1 +f y.-(1. Sst. ; and 25 j. -0 st. 9 and 9 st.

and twice second.

$\begin{array}{llllll}. & 13 \quad, & 9 & , & & \end{array}$

Gold ('up at L.udluw, 3 Miles, S st. 12 agst. $3 y-0$. Melody ti st. j) and $5 y$ y. -o. Samprion 8 st. 10

(up at línlierhampion, 3 .lliles, 9 st. agst. 4 y.-o. Mufti 8 st. 2 and 6 y. -0 . Euxton 9 st.

King's Pl. at Lichficld, \& .1., Heats, 1.2. st. agst. 7 y.-0. . Mderman 1.) st. and it times secund. 
No. :34. Prosody 1818 by Don Consack.

ran 3 year-old fi times, won 2 times, at Goudw. 2 Miles, second, and immediately after 2 Miles with Heats, also second.

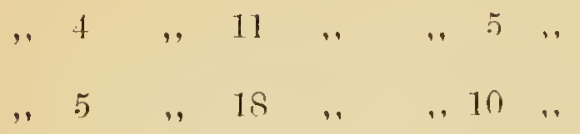

1 times winner two dars one after the other ower 1 and 2 Miles with Heats. and $t$ times second.

1 July, about 2 Miles with Heats, second,

? July, 3 Miles, first,

and won the same day

about 2 ? liles with [leats, first.

6 . Jugust, 2 Miles with Heats, first.

7 . Iugust, Miles with Heats, first, and immediately after

2 Miles with Heats, first.

13. Iugust, 2. Miles with Heats, third, and on the same dav 1 Mile, first.

27 August, 2 Mliles with Heats (three times), first.

28 . Iugust, 4 Miles with Heats (three times, nnce dead heat, twice first), first.

17 Sept., $:-1$ Miles with Heats, first.

18 Sept., 2 Miles with Heats, first.

$\begin{array}{llllllll}. & 6 & \quad, & 11 \quad, & , & 4 & \end{array}$

and 5 times second.

Won the 3 first races with Heats, then 25 August, - Miles, second, and 2 Miles with $[$ [eats, third.

26 . Jugust, about 2 Miles with Heats, second,

and immediately after

about 2 Hiles with Heats, second.

1 Sept., 끌 Miles with Heats, second.

1.5 Sept., 2 Miles with Heats (3 times), second.

4 October, $2 \frac{1}{4}$ Miles with [Ieats, not placed.

:) October, $2 \frac{1}{4}$ Miles with Heats, first.

$, \quad 7 \quad, \quad 11 \quad, \quad,, \quad 7 \frac{1}{2}$, and once second.

7 July, $7 \frac{1}{2}$ Miles with Heats, first.

13 July, $2 \frac{1}{4}$ Miles, second,

and immediately after

$1 \frac{1}{1}$ Mides with Ileats (3 times), twice dead heat. 
3 . August, 4 Miles with Heats, first. 2.) August, 2 Miles with Heats, first.

7 Sept., about 2 Miles with Heats, first, and on the same day about 2 Miles with Heats, first.

1.) Sept., $2 \frac{1}{4}$ Miles with Heats, first (Gold Cup at Exeter, 4 behind).

16 Sept., about 2 Miles with Heats, first.

ran 8 year-old 13 times, won 8 times, and twice second, always against younger horses.

23 August, 2 Miles, second, and immediately after

21 Miles with Heats, not placed.

24 August, about 2 Miles with Heats (3 times), first against $15 \mathrm{y} .-0$. and 14 y.-o. on the same day.

1 Mile with Heats, not placed.

5) Sept., 3 Miles with Heats (3 times), second.

6 Sept., 2 Miles with Heats, first, agst. $15 \mathrm{y},-0$. and $14 \mathrm{y},-u$.

12 Sept., 2 Miles with Heats, first.

13 Sept., 214 Miles with Heats, first, agst. 15 y.-o. and 4 y.-o. Conquest. , $9 \quad$, $6 \quad$,, , 1 , 15 August, $2 x$ Miles, third.

16 August, $2 \frac{1}{4}$ Miles, second.

28 August, $2 \frac{1}{4}$ Miles with Heats, first, 9 st. 4 agst. 23 y.-o. under 7 st. 1 and 7 st. 7

29 August, $2 \frac{1}{4}$ Miles, second.

12 Sept., about 2 Miles, third.

13 Sept., 21 Miles with Heats, second.

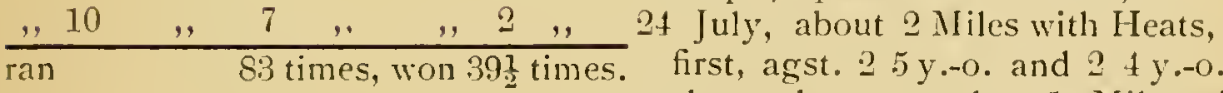
and on the same day 1 Mile with Heats $(4$ times, once dead heat, once first), second, $15 \mathrm{Y} .-\mathrm{o}$. and 2 $4 y$ - - o. behind.

6 Nugust, 21 Miles, second,

and on the same day about 3 Miles with Heats (3) times), second 1 $6 y,-0$. and $25 y$ y.o. behind.

21 Iugust. 3 . Miles with Heats, first, S st. 11 
agst. $15 \mathrm{y} \cdot-0.8$ st. 5 , and

$13 \mathrm{y},-0.6$ st. 7

3 Sept., 2 Miles with Heats, third.

4 Sept., 2 Miles with Heats, second.

Was covered at 11 years old, and had 6 foals, of which several won good races.

No. 35. Hesperus 1820 by Hollyhock.

ran 4 year-old 3 times, won 0 times.

$\begin{array}{rrrrrrrr}, & 5 & , & 12 & , & , & 6 & \text {, } \\ , & 6 & , & 16 & , & , & 8 & , \\ , & 7 & , & 14 & , & , & 4 & , \\ , & 8 & , & 9 & , & , & 2 & , \\ , & 9 & , & 10 & , & ,, & 6 & , \\ & & & & & & & \\ , & 10 & , & 13 & , & , & 7 & ,\end{array}$

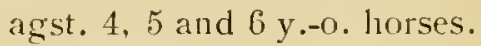

under a high weight against younger horses.

City Pl. at Chester, 9 st. 2 agst. 4 y.-o. Grimbald 8 st.

Gift of Earl Grostenor at Chester, 2 Miles, 9 st. ?

agst. $25 \mathrm{y} .-0.8$ st. 12

Gloucestershire St. at Cheltenliam, 8 st. 7 agst. $54 \mathrm{y} \cdot-0.7$ to $8 \mathrm{st}$. and $35 \mathrm{y} \cdot-0.7 \mathrm{st}$. to $8 \mathrm{st} .8$

agst. 8 horses.

Gold Cup at IVorcester, 4 Miles, 9 st. 2 agst. 34 r.-0. 7 st. 12 to 8 st. 2 Handicap at ITrexham, 2 Miles, IIeats, 9 st. 5 agst. $33 \mathrm{y} \cdot-0.7 \mathrm{st}$. to $7 \mathrm{st} .3$ 14 y. -0.8 st. 6 $16 \mathrm{y} .0 .8$ st. 12

agst. 5 horses, and 6 times second in good races.

ran 77 times, won 33 times.

No. 36. Liston 1821 by Imbo.

ran 3 year-old 1 time, won 0 times.

\begin{tabular}{|c|c|c|c|c|}
\hline , 4 & ," & 4 & times, & won 3 \\
\hline 5 & ,, & 8 & , & ,", \\
\hline 6 & ,, & 14 & ," & \\
\hline 7 & ," & 16 & ,' &, 10 \\
\hline, 8 & ," & 11 & , , & , \\
\hline
\end{tabular}

Oxfordshire St., 2 Miles, 9 st. 5 agst. $23 y \cdot-0.6$ st. 5 and 6 st. 12 and $24 y \cdot-0.7$ st. 7 and 8 st. 2

agst. 4 horses. 
5. The Thoroushbred in the Past and Present.

Gold Cup at Warwick, 4 Miles, 9 st. 5) agst. 34 y.-0. 8 st. 3

Cup at Abingdon, 3 Miles, 9 st. 2 agst. (i y.-o. Jocko 9 st.

and 3 times second.

ran 9 yearold 6 times, won 3 times. Selling Race at $\mathbf{A b i n g d o n , ~} 3$ Miles, 9 st. 2

agst. $34 \mathrm{y} .-0.8 \mathrm{st}$.

and $2.5 y .-0.8$ st. 9

agst. 5 horses,

and once second.

, $10 \quad, \quad 7 \quad, \quad, \quad 3 \quad$,

Cup at Newport, 3 Miles, 8 st. 13

agst. 4 y.-o. Paradox \& st. 7

Selling Race at Mbingdon, 2 Miles, agst. $13 \mathrm{y} .-0$. and $34 \mathrm{y}-()$.

and 3 times second.

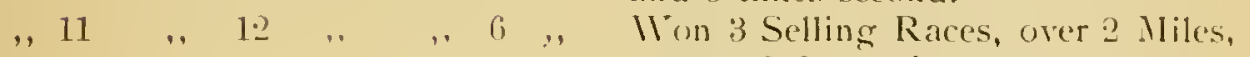
aest. $36 \mathrm{y},-()$. hurses.

Salperton Si. at Cheltonham, 2 Miles, . 9 st. 7 agsit. $1: 3 \mathrm{y} \cdot-0.7 \mathrm{st}$.

and 1 it y-o. 8 st. $T$

and on the same day

Cup, 3 Miles, 9 st. 1

agst. 5 y.-o. Thorngrove \& st. 11

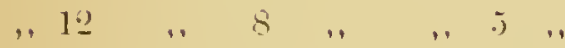

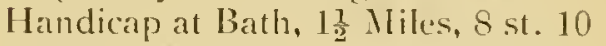
arst. 35 y.-0. 7 st. 10 to 8 st. 1

(Sinbad)

and 26 y.-o. 8 st. 2 and 9 st.? (Bryan)

agst. 5 horses.

2 Selling Races, $1 \frac{1}{4}$ and 2 Miles. agst. young horses.

Beaufort St. at Ciloucester, about ? Miles, Heats, agst. $13 y \cdot-0$. and $16 y .-0$., and on the following day City Member's Pl., 2 Miles, ILeats. agst. $6 y$ y. (). I larry.

, 13 , 7 , , 1 , Selling Race at Bath, 3 Miles, 9 st. ." agst. 1 y.-(). 7 st. 13

and second in Gold Cup at Dewport.

$\frac{, 14 \quad, \quad 2 \quad, \quad, \quad, \quad 0 \quad, ",}{96 \text { times, won } 50 \text { times. }}$ 
No. 37. Fleur de lis 182.2 by Bourbon.

ran 3 year-old 4 times, won 3 times, 1 . not placed.

$$
\begin{aligned}
& \text {, } 4 \text {, } 7 \text {, , } 7, \text { Dср. } \\
& \text {, } 5 \quad,, \quad 6 \quad,, \quad,, 4 \quad,
\end{aligned}
$$

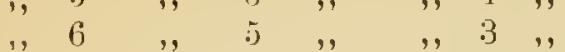

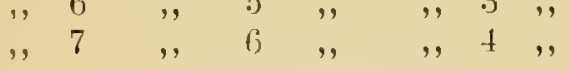

$$
\begin{aligned}
& \text {, } 8 \quad, 2 \quad 2 \quad,, \quad, 1 \text {, } \\
& \text {, } 9 \quad,, \quad 1 \quad,, \quad,, \quad 0 \quad,
\end{aligned}
$$

second in the Gold Cup at Goodw., 9 st. 11 agst. 4 y.-o. Priam D. 9 st. 5 behind $4 \mathrm{y} \cdot-0$. Variation 8 st. 11 which had won 4 good races in the same year.

ran 31 times, won 22 times.

No. 35. Conquest 18:2 by W'aterloo. ran 3 year-old 3 times, won 2 times.

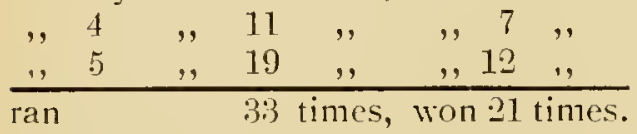

Altogether Races with Heats, 2 - $2 \frac{1}{2}$ Miles.

ran and won on August 9 at Salisbury in 3 races, one after the other without a pause, with fresh opponents, viz.:--

1. Sweepstakes, 2 Miles, 8 st. 7

2. The City Member's Pl., 2 Miles with Heats.

3. The City Bowl, 21 lliles with Ieats.

Ran on the 16th August at Blandford the following 3 races:

1. Dorsetshire St., 2 Miles, not placed.

2. Selling Races, $2 \frac{1}{4}$ Miles, won, and inmediately after

3. Nember's Pl., $2 \frac{1}{4}$ Nliles, second.

IVon on the 23rd August at Taunton, $2 \frac{1}{4}$ Miles with Heats, against 3 good racehorses.

IVon on the 24th August in the Town Pl., $2 \frac{1}{4}$ Miles with Heats.

Non on the 28th August in Bridgwater Ladies' P1., $2 \frac{1}{4}$ Miles with lleats, 
and on the 29th August at the same place Member's Pl., 23 Miles with Ileats (3 times).

and on the 12th Sept. at Exeter Member's Pl., 217 Miles with Heats.

Ran on the 13th Sept. at the same place IIandicap P1., $2 \frac{1}{4}$. Miles with Heats, not placed,

and was victorious immediately afterwards in the City Nember's P1., 21 Miles with Heats.

Ran on the 206th Sept. at Dorchester Tradesmen's Pl., about 2 Miles with Heats, not placed.

and won on the same day in the Ladies' Pl., about 2 Miles with Heats (3 times), agrainst 4 good racehorses,

and ran on the following day at the - same place Yeoman's Pl., 2 Miles with Heats, against 3 good racehorses, won the first Heat, but fell in the second Heat, injuring itself inwardly, and had to be killed.

No. 39. Tranby 1826 by Blacklock.

November 1831. The famous bet of Mr. Osbaldiston to ride 200 miles in 10 hours, an number of horses. 1 on in 8 hours, 12 minutes, with 89 horses, amongst which was the 5year-old Tranby by Blacklock, which had to go four times, $f$ miles each time, with about one hour's pause each time. Tranlyy took for the :-

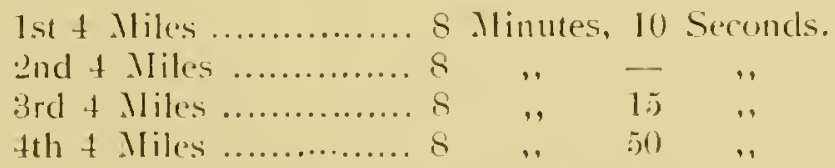

In the next year Tranby ran and won wice at Newmarket in a well contested rare, then became Sire of "I am not aware," who won races 2-9 yearold, and was then sold to Imerica, where he got renowned as sire of Vandal's dam.

No. 10. Catherina 18:30 by Whisker.

ran 2 rear-old "2 times, won 0 times, twicre serond.

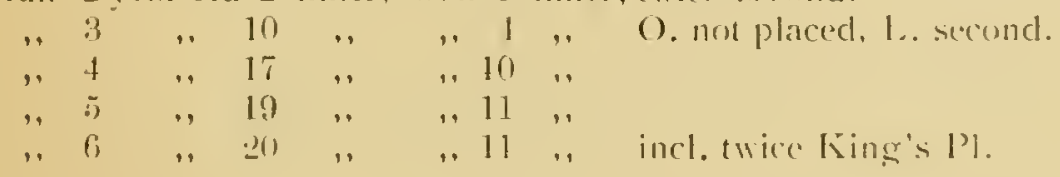


ran 7 year-old 18 times, won 6 times, incl. once King's Pl.

, 8 , 12 , . , 5 ., The Tradesmen's Cup at Manchester, $2 \frac{1}{4}$ Miles, 8 st. 7

agst. 24 y.-o. 7 st. and 7 st. 12

$15 \mathrm{y},-0.7 \mathrm{st}$.

1 older 8 st. 5

agst. 4 horses.

1 Ixing's Pl. agst. - 4 y.-o.

and $t$ times second.

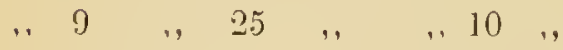

The Tradesmen's Cup at Burnley, 201 Miles, 8 st. 11

agst. 34 y.-o. 8 st. 2 1 older 9 st.

agst. 4 horses.

.. $10 \quad, 25 \quad, \quad, 1.2 \quad \ldots$

ladies' Purse at Chester, about 2 Miles with IIeats

agst. $33 \mathrm{y} \cdot-\mathrm{o}$.

$1+y_{0}-0$.

$15 \mathrm{~V} \cdot-\mathrm{O}$.

agst. 5 horses.

L.ancashire St. at Burnley, about 3 Miles, Heats

agst. $4 y_{.-0}$. The Shadow and 5 y.-o.

St. Leonard

and immediately after

I free P1., about 3 Mliles, Heats agst. $2+y_{0}-0$. and 1 is y.o.

Swerpst. at Chesterfield, 2 Miles

acst. $f+y .-0$. and $15 y_{0}-0$.

and on the following day

Sweepst., about 2 Miles

agst. $1: 3 y_{\cdot-0}$. and $1+y_{\cdot}-0$.

and immediately after

The Town Pl., ? Miles, Heats (3 times)

agst. 23 y.-o.

$1+\mathrm{y} .-0$.

$15 \mathrm{y} \cdot-\mathrm{o}$.

agst. 4 horses.

The Tally-lio St. at IVelshpool, ? Miles, Heats (:3 times), 11 st. 5

aust. $13 y \cdot-0.8$ st. 7

$2+\frac{1}{2}-0.9$ st. 5

15 y.-o. 10 st. 10

1 older 11 st. .j

acst. 5 horses, 
and on the following day

The Innkeeper's Purse, $1 \frac{2}{2}$ Mliles, I Jeats ( 3 times)

agst. $13 \mathrm{y} \cdot-0$. and $25 \mathrm{y} \cdot-\mathrm{o}$.

ran 11 year-old:28 times, won 1: times. The All-aged St. at Tenbury, 2 Miles, I. Teats ( 4 times), 8 st. 11

arst. 25 y.-o. 8 st. 7 and 8 st. 10

3 older 8 st. 11 to 9 st. 3

agst. 5 horses.

Swerpst. at Bridgnorth, about 2 Miles, Jeats (3 times)

arst. $14 \mathrm{y} \cdot-0 ., 15 \mathrm{y} .-0$. and 1 older, and on the following day

I Gold Cup, about 2 Miles agst. $1+y_{\cdot}-0 ., 15 y_{0}-0$. and 1 older.

On the $30 t h$ August second in Race oret $1 \frac{1}{4}$ Mliles with Heats.

On the following day third in a Race over 2 Mliles, and on the same day

- second in a Race over $1 \frac{1}{1}$ Miles with lleats.

On the :sth Sept. second in a Race urer about 3 Mliles,

and immediately after winner in

Pottery St., about 2 Miles, Heats

agst. $24 \mathrm{y} .-0$. ,

and on the following day winner in

Town St., about 2- Miles

agst. $23 \mathrm{y} \cdot-\mathrm{n}$.

()n the 6th October Sweepst. at Knutsford, ? Miles agst. $1+y \cdot-0 .$,

immediately after not placed in Race over 20.4 Miles,

and on the following day second, 18

lliles with I leats (3 times), 3 behind.

On the 1 th Octuber third in a Race uver 2 Miles with l Ieats.

and on the same day

Tally-lu St. at Welshpoul, 2 Miles,

IIcats, 11 st. 5

agst. 4 y.-(). Hautboy 9 st. 11

and on the following day

Innkeeper's Purse, $1 \frac{7}{2}$ Miles, Heats (I (imes), 9 st. 8 


$$
\begin{aligned}
& \text { agst. } 13 \text { y.-o. } 7 \text { st. } 3 \\
& 2 \text { older } 9 \text { st. } 8 \text { and } 9 \text { st. } 13 \\
& \text { (amongst them Kitty Cockle) } \\
& \text { 18th October Moorland St. at Leek, } \\
& \text { about } 2 \text { Miles, Heats ( } 3 \text { times) } \\
& \text { agst. } 23 \text { y.-o. and } 1 \text { older. }
\end{aligned}
$$

ran 176 times, won 81 times, and had 9 good foals.

No. 41. 1saac 1831 by Figaro.

ran 3 year-old 2 times, won 1 time.

$$
\begin{aligned}
& \text {, } 4 \text {, } 3 \text {, , , } 2 \text { times. } \\
& \text {, } 5 \text {, } 8 \text {, } 8 \text {, , } 1 \text { time, King's Pl. }
\end{aligned}
$$

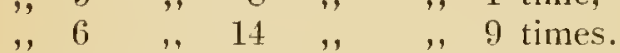

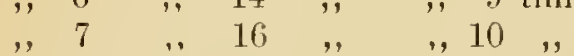

$$
\begin{aligned}
& \text {, } 8 \quad, \quad 23 \quad, \quad, 18 \text {,, }
\end{aligned}
$$

Handicap at Coventry, $1 \frac{1}{2}$ Niles, 9 st. agst. 13 y. -0.6 st. 1

14 y.-0. 7 st. 4

25 y.-0. 8 st. and 8 st. 6

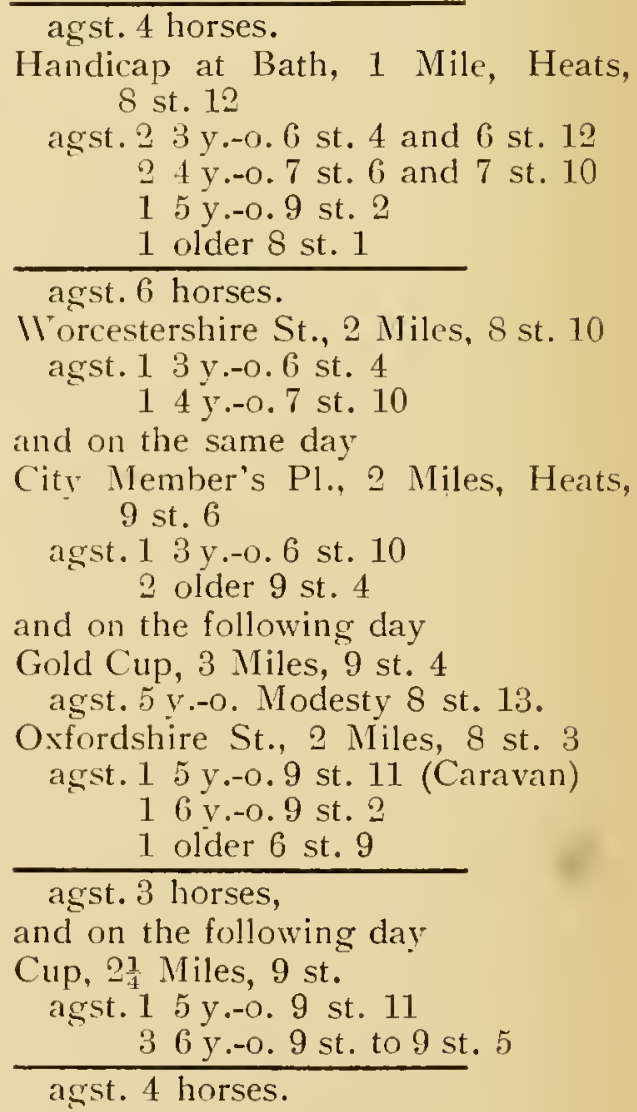


Leamington St. at Warwick, 2 Miles, 8 st. 3 agst. $24 y \cdot-0.6$ st. 7 and 7 st. 7

15 y.-0. 7 st. 13

$26 \mathrm{v}-0.8 \mathrm{st} .5$ and 8 st. 10 (King Cole)

agst. 5 horses.

On the following day second in the Warwick Cup, over 4 Miles, 5 y.-o. Caravan behind.

On the following day

Stand St., 2 Miles, 8 st. 10 agst. $23 \mathrm{y} \cdot-0.6 \mathrm{st}$. and $6 \mathrm{st} .5$, and immediately after third in King's Pl., 2 Miles, Heats.

King's P1. at Leicester agst. $13 y$.-o. and $14 y$-o., and on the following day

Fold Cup, about 3 Mliles, 9 st. 4 agst. $13 \mathrm{y} .-0$. and $15 \mathrm{y} \cdot-0.6$ st. 7 and 9 st. 1.

Gold Cup at Shrewsbury, 3 Miles agst. 5 y.-o. Caravan, and on the following day

King's Pl., 3 Miles agst. 13 y.-o. and 25 y.-o.

Cup at Oswestry, $1 \frac{1}{2}$ Miles, not placed, 5 starters.

Immediately after

w. o., $1 \frac{1}{2}$ Miles

and

Member's St., $1 \frac{1}{2}$ Miles, Heats (3 times), 8 st. 13 agst. 13 y.-o. 7 st.

and $14 y$.-o. 7 st. 11

ran !) year-old 15 times, won 5 times. Worcestershire St., 2 Miles, 9 st. .) agst. 24 y.o.- 7 st. 4 and 7 st. 10

Cup at Oxford, $2 \frac{1}{4}$ Miles, 9 st. agst. $24 \mathrm{y},-0.7$ st. 13 and 8 st. 2 16 y.-0. 9 st. 5 (Caravan)

agst. 3 horses.

16ith Sept. at Shrewsbury, 2 Mliles. not placed.

17 th Sept. second Gold Cup, 3 .IIles.

18th Sept. King's Pl., :3 Miles agst. 5 good horses, 
and on the same day second in Member's Pl, about $2 \frac{1}{2}$ Miles, Heats.

Gold Cup at IIrexham, about 3 Miles, 9 st. 1

agst. 13 y.-0. 7 st.

$1+y_{0}-0.8$ st. 1

$16 y .-0,9$ st.

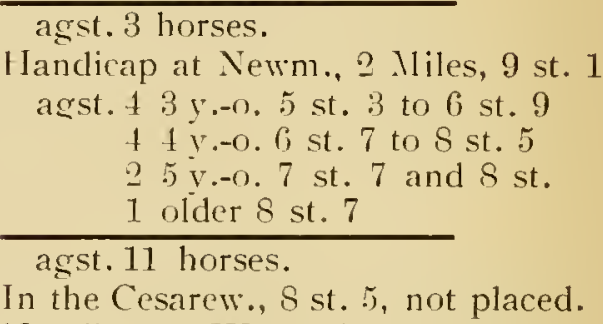

ran 10 year-old 13 times, won 1 time. Handicap at Warwick, 2 Miles, 9 st. 4 agst. 64 y.-o. 6 st. 12 to 8 st. 4 $35 \mathrm{v},-0.7$ st. 8 to 8 st. 10 1 older 7 st. 7

asst. 10 horses.

On the same dar in Handicap second. Ran further in many big Handicaps. " 11 , 5 , , 2 , 5 in small and large Ilandicaps,

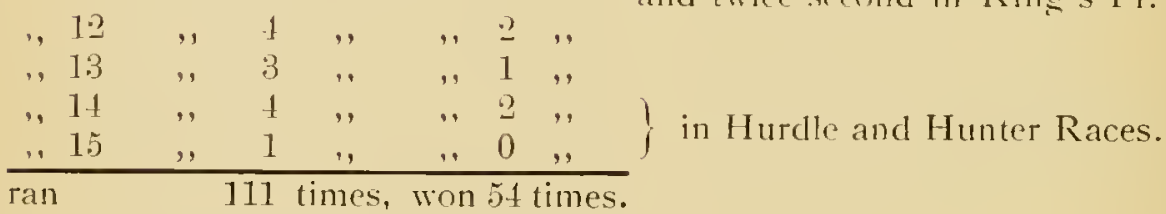

No. 42. Zohrab 1831 by Lottery.

ran 3 year-old 6 times, ron 3 times.

\begin{tabular}{|c|c|c|c|c|}
\hline , 1 & ," & 8 & , & , \\
\hline 5 & , & 6 & , & , \\
\hline 6 & ," & 10 & ,' & 3 \\
\hline 7 & , & 7 & , , & ", 1 \\
\hline S & , , & 17 & ,, & , 11 \\
\hline
\end{tabular}

1 King's Pl.

,

,

,

Victoria St. at Cheltenham, 2 Miles, 9 st. 7 agst. 13 y.-0. 6 st. 12

and 25 y. -0.7 st. 12 and 9 st. 2

Ladies' Pl. at Kelso, $2 \frac{1}{2}$ Miles, 9 st. 2 agst. $13 y+-0.7$ st. 7

$1+y,-0.7$ st. 7

$15 \mathrm{y},-0.7 \mathrm{st} .8$

$16 \mathrm{y} \cdot-0.8 \mathrm{st} .3$

acst. 1 horses, 
often ran and won twice on the same dily.

ran 9 year-old 11 times, won : times. Gold Cup in Egrlinton Park, 21 Miles, 12 st. 4

$$
\begin{array}{r}
\text { agst. } 26 \text { y.- } 6.12 \text { st. } 4 \\
\text { (Cardinal Puff) }
\end{array}
$$

1 older 12 st. 4

uften ran twice on the same day.

, $10 \quad$, $18 \quad, \quad, \quad 6 \quad$,

often won twice on the same day, mostly in Races with lleats. last Race at Kelso, $1 \frac{1}{2}$ Wiles, 9 st. 9 agst. $2+y \cdot-0.7$ st. 13

1 5) y.o. 9 st. 6

1 olcler 9 st. 3

rall

S:3 times, won 36 times.

agst. $t$ horses.

N(1. 4:3. The Potentate 18:30) by Langar.

ran 2 year-old 3 times, won 0 times.

. 3 , 7 , , , 1 , and twice second (Derby at York and St. leger at Liverpool).

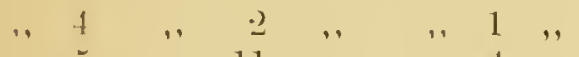

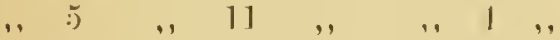

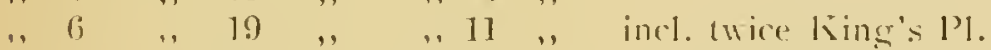

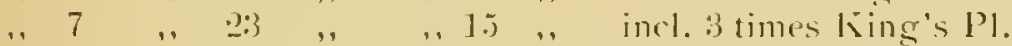

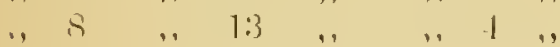

Goodwoud Cup, áganst 19 horses of a goud class (of which were Epirus, Euclid, Retricver, Verulam).

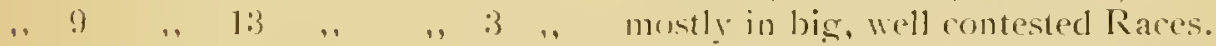
The Glasgow Cup at Paisley, 2 Miles, S st. B

acsit. 5) y.-1). Rejected 7 st. 12

last Race, 1 free Ilandicap, 1 . Mile, lleats (3 times), 8 st. 9

agst. 20 y. -0.7 st. 3 and 9 st. 2

ran 91 times, 1 non 39 times.

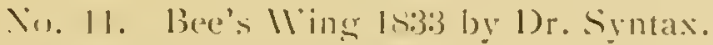

ran : gear-old :3 times, won 2 times.

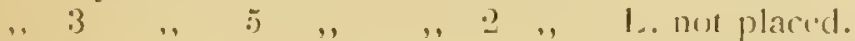

, 1 , 1 , , (; ., incl. 1 king's P.

, 5 " $\quad 9$,

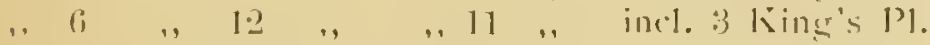

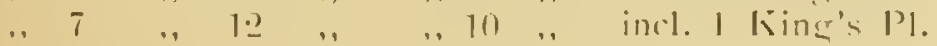


ran 8 year-old 10 times, won " times. Trial St. at Chester aggst. $23 \mathrm{y} \cdot-\mathrm{O}$.

15 y.-O.

1 older.

agst. 1 horses.

Gold Cup at Newcastle, 2 Miles, 9 st. 3 agst. 4-y.-o. Calypso 8 st. 5 and 6 y.-o. Lanercost Acp. 9 st. 3

Gold Cup at Stockton, 9 st. 8 agst. 13 y.-o. 6 st. 11

$14 \mathrm{v},-0.7$ st. 7

15 y.-0.9 st. 5

agst. 3 horses.

Cup at Doncaster agst. 5 y.-o. Shadow.

, 9 , , $5 \quad, \quad, \quad 4$, , Gold Cup at Ascot, $2 \frac{1}{2}$ Miles agst. 24 y.-o.

and 2 older (of which were Lanercost Acp.)

\section{agst. 4 horses. \\ Gold Cup at Newcastle, 2 Miles agst. 6 y.-o. Charles XII. L. \\ Gold Cup at Doncaster agst. 3 y.-o. Attila \\ 6 y.-0. Charles XII. L. and $6 y,-0$. Shadow}

agst. 3 horses.

ran

64 times, won 51 times.

No. 45. Adrian 1834 by Sultan.

ran 2 year-old 10 times, won 1 time.

\begin{tabular}{|c|c|c|c|c|c|c|}
\hline , 4 & ," & 9 & ", & ", & ", & \\
\hline , 5 & , , & 12 & ", & ," & ". & \\
\hline, 6 & , & 3 & , & , & $0 \quad$, & \\
\hline , 7 & , & 14 & ", & , & 5 times. & Ran chiefly in Races over $1 \frac{1}{2}$ to $2 \frac{1}{2}$ \\
\hline $\begin{array}{l}1,8 \\
9\end{array}$ & ," & $\begin{array}{l}18 \\
90\end{array}$ & , & , & ", & Miles with Heats, and beat many \\
\hline , 10 & "' & 14 & ", & , & ," & younger horses, often in Selling \\
\hline , 11 & , , & 7 & ,, & ,", & , , & Kaces. \\
\hline , 12 & "' & 4 & , & , & , & also in Hurdle Races. \\
\hline „13 & , & 3 & ," &, & $\because$ & वाल० \\
\hline
\end{tabular}


No. th. The Shadew 1836 by the Saddler.

ran 2 year-old 4 times, won 2 times.

\begin{tabular}{|c|c|c|c|c|}
\hline , 3 & & 4 & ", & 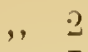 \\
\hline 4 & , & 10 & ", & \\
\hline 5 & ," & 19 & ," & ,, 13 \\
\hline 6 & , & 21 & ., & , 11 \\
\hline 7 & ," & 17 & ," &, 12 \\
\hline 5 & ,", & 10 & ," & $10 \quad 1$ \\
\hline
\end{tabular}

O. not placed.

incl. twice King's Pl.

incl. 3 times King's Pl.

incl. once King's PI.

Gold Cup at Wrestern Meeting, 2. .1., S st. 13

agst. 13 y.0. 7 st. .5)

and $1+y .-0.8$ st. 5

and on the following day

A Plate for all ages, 2 Miles, Heats.

1 Plate of $\$, 50$ at Perth, 2 Niles, Heats

agst. 14 y.o-o. and $15 y,-0$.

and on the same day

Member's Pi., about 2 Miles, 10 st. 2 agst. 4 y.-o. Armytage 9 st. 9

. 9 , $18 \quad, \quad, \quad 9$,

Cleveland Cup at Volverhampton, 3 Miles, 9 st.

agst. 13 y.-o. Coer de Lion 7 st. 2

$1+\mathrm{y} \cdot-0.7 \mathrm{st} .6$

$15 y,-0.9$ st. 7

16 y.-0.9 st. 11

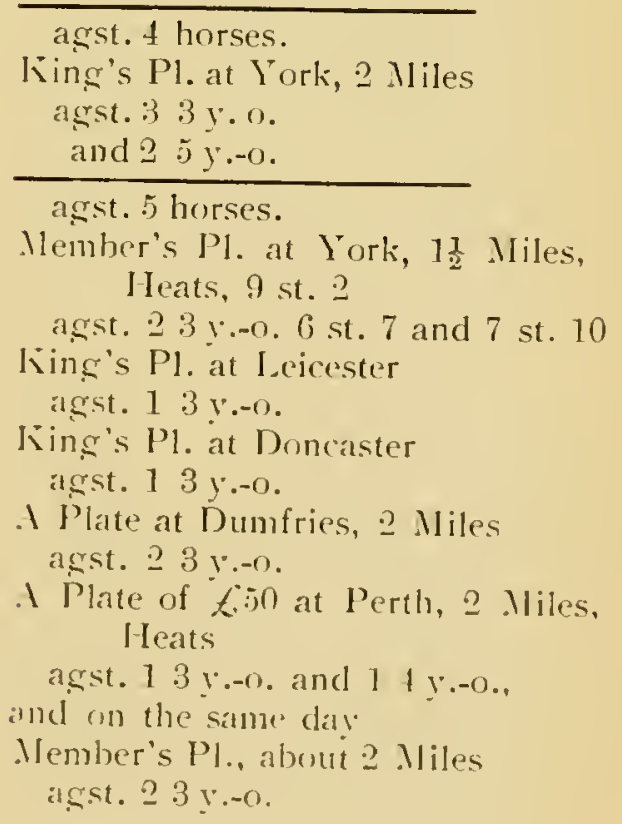




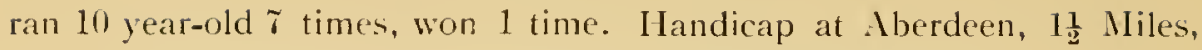
9 st. 5

agst. $16 \mathrm{y}$-o. 8 st. 1

and 1 older 8 st. 4

, $11 \quad$ " $3 \quad$, " 1 , Welter St., Gentleman Rider, 1 Mile agst. 3 older.

$\overline{\text { ran }} 113$ times, won 64 times.

No. 47. St. l.awrence 1837 by Skylark or Lapwing.

ran 2 year-old 6 times, won 2 times.

, 3 , 6 , , , 2 , ,

$\begin{array}{lllllllll}, & 4 & 4 & 0 & 16 & , & , & 9 & ,\end{array}$

,

",

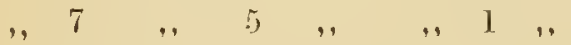

, $8 \quad, \quad 4 \quad, \quad, \quad, \quad 0, ., \quad$ in the best company.

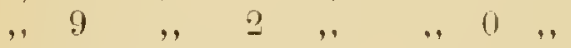

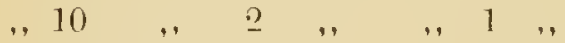

Tradesmen PI. IIandicap at Chester, 7 st. 10

agst. 29 horses of a good class, amongst which were: Mendicant 4 y. - 8 st. (second), Newcourta 7 st. 10 (third), Annandale $5 \mathrm{y}$-0. 7 st. 13.

In the Cesarew. 8 st. 2, not placed.

$\overline{\text { ran }} 62$ times, won 29 times.

No. 48. Lady Flora 1838 by Hampton.

ran 3 year-old 4 times, won 2 times.

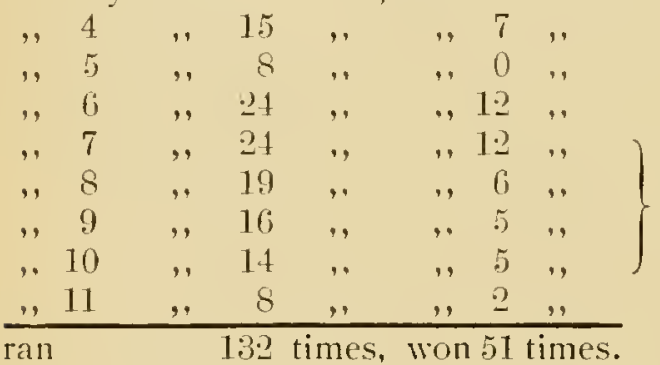

Almost all in Races with lleats $1 \frac{1}{2}$ to 3 Miles, against younger horses.

Also ran in Hurdle Races.

No. 49. The Sailor (later Gaffer Green) $1839 \mathrm{by}$ Obadiah.

(Not in Stud Book, a Thorouglibred nevertheless).

ran 6 year-old 6 times, won 4 times.

, $7 \quad, \quad 11 \quad, \quad,, 4$,

,, $8 \quad, \quad 24 \quad,, \quad, \quad 7 \quad$, 
ran 9 year-old 15 times, won 4 times, and 5 times second.

(hester Handicap, $\frac{3}{4}$ Mile, 5 st. 12 agst. 3 3y.-o. 4 st. 12 to 5 st. 3 $74 y,-0.5$ st. 11 to 6 st. 13 1 older 7 st. 8

agst. 11 horses.

Shrewsbury Handicap, $1 \frac{1}{2}$ Miles, 6 st. 12

agst. $15 \mathrm{y} \cdot-0.7$ st. 6 $36 y-0.7$ st. 2 to 7 st. 12 1 older 8 st. 10

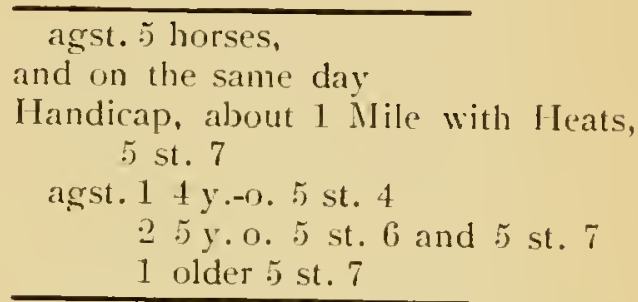

, $10 \quad, \quad 15 \quad,, \quad, 44$, agst. 4 horses.

Chester Grand Stand Cup, about 1늘 Miles, 8 st. 2 agst. 53 y.-0. 4 st. 12 to 6 st. 10 $2 \pm y .0 .6$ st. 11 and 7 st. 10 25 y.-0. 7 st. 6 and 8 st. 2 16 y.o. \& st. 10 1 older 8 st. 6

agst. 11 horses.

Shrewsbury Ser. St., 1 Mile with Heats, 7 st. 7

agst. $25 y+-0.5$ st. 11 and 6 st. Newport IJandicap, 1 Mile, 8 st. 7 agst. $23 y--(1.6$ st. 6 $15 y \cdot-0.7$ st. 13

agst. 3 horses.

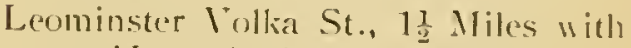
lleats ( 1 times), 8 st. 12 agst. $4+y,-0.6$ st. 10 to 7 st. 10 $16 y-0.7$ st. 13

$\begin{array}{lllllll}. & 11 \quad, \quad & 20 \quad, & & 1\end{array}$ agst. 5 horses.

Ratn in good Races and in good company.

Wrexham Iandicap, 11 Miles with Heats ( 4 times), 7 st. 9 
agst. 13 y.-0. 7 st.

$3+y \cdot-0.6$ st. 13 to 7 st. 11

26 y.-o. 8 st. 1 and 8 st. 7

agst. 6 horses.

ran 1:2 year-old 10 times, won 0 times. Ran in good Races and in good company.

, 13, , not run.

" 14 , 8 , , 1 , in several big Handicaps in good company.

IValsall Member's St., about $1 \frac{1}{2} \mathrm{M}$. with Heats (4 times), 8 st. 11

agst. $13 \mathrm{y} \cdot-0.7$ st. 1 and $2+y \cdot-o .7$ st. 13

$\overline{\text { ran }} 109$ times, won 25 times.

No. j). Inheritress (Foundation Mare of Veilchen) 1840 by The Saddler.

ran 2 year-old 4 times, won 1 time.

, $3 \quad, 2$, , , 0 ,

, 4 , 10 , , 4 times.

, 5 , 24 , , 14 ,, incl. 4 King's Pl., 2 Miles, Heats.

, $6 \quad$, 17 , , 7 , incl. 2 King's Pl., 2 Miles, Heats.

" 7 , 15 , , , , 9 ," incl. 3 King's Pl., 2 Miles, Heats, and

3 Miles.

Liverpool Cup, 2 Miles

agst. 16 horses.

, 8 , 18 , , 11 , second in the Gr. Metrop. St. at Epsom, 22 horses behind.

The Cheshire St. at Chester, 1 Mile 3 Furl., 8 st. 9

agst. $13 \mathrm{y} \cdot-0.5$ st. 2

$14 y$-o. 7 st. 2 (The Swallow)

15 y.-0.8 st. 10 (Pyrrhus I D.)

1 older 7 st.

\footnotetext{
agst. 4 horses.

Handicap at Nottingham, 2 Miles, S st. 9

agst. $23 y$.-o. 5 st. 4 and 5 st. 7

$1+\mathrm{y}$.-o. 6 st. 9

1 older 7 st. 11 (Yardley)

agst. 4 horses,

and on the following day

The Chesterfield Handicap, $1 \frac{1}{2}$ Miles, S st. 10

agst. 23 y.-o. 4 st. 12 and 5 st. 6
} 
j. The Thoroughbred in the P'int and l'resent.

and on the same day

$$
\begin{aligned}
& \text { King's P1., } 2 \text { Miles, } 1 \text { Ieats, } 10 \text { st. } \\
& \text { acst. } 13 \text { y.-0.8 st. - } \\
& \text { t. }+y,-0.9 \text { st. } t \\
& \text { li i v".-o. } 10 \text { st. }
\end{aligned}
$$

algst. :3 horses.

Sicond in the Volverhampton Si., $2 \frac{1}{2}$.liles, 3 behind, and on the following day

The Cleveland Cup at IV istrerhampton, :3 Mliles, 9 st. (i)

ayst. 1.3 y.-1). 6 st. 5

$1+\because 0-0.8$ st. 2

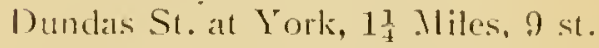
asst. $33 y$ y.ot). 6 st. 8

$\frac{1+y-0.8 \text { st. }-2}{\text { acrst. } t \text { horses. }}$

Calconian Handicap, : Miles, s. st. $1: 3$

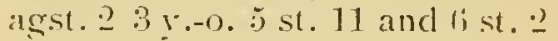
1. li i.t). Ast. (Plaudit)

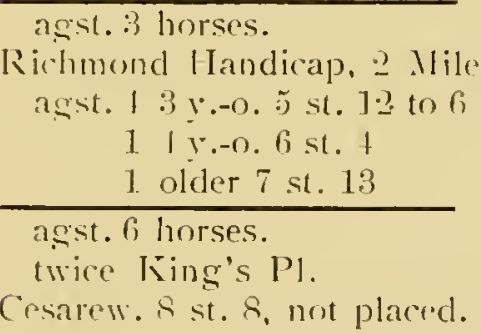

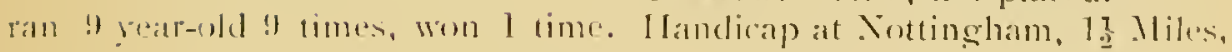

$$
\text { S st. } 7
$$

agst. $1+y \cdot-1) .7$ st.

I times second and

in 3 bic 1 Jandicaps not placed.

. In , $\quad 5 \quad, \quad, \quad 1$, Mandicap) at Molverhampton, $1 \frac{1}{2} .11$. T st. i)

agst. 1 :3 y.-0. o st. 6

and 1 fi y.-o. 6 st. 4

and in l big Handicaps nut plared.

ran 104 times, won tif times.

i. ol. 1)uket 18foby Dukimer.

ran 3 year-old 6 times, won 1 time.

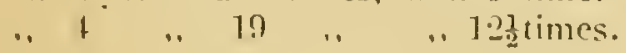

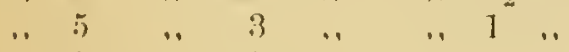

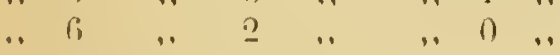


ran 7 year-old 5 times, won 0 times.

$$
\begin{aligned}
& \text { " } 8 \text {, } 7 \text {, , } 4 \text {, Handicap Newn. } 2 \text { Y. O. C. }=5 \text { Furl. } \\
& 136 \text { Yds., } 7 \text { st. } 8 \\
& \text { agst. } 43 \mathrm{y} \cdot-0.6 \text { st. } 4 \text { to } 7 \text { st. } 7 \\
& 14 \text { y.-0. } 8 \text { st. } 5 \\
& 16 \text { y.-o. } 8 \text { st. } 9 \\
& \text { agst. } 6 \text { horses. } \\
& \text {, } 9 \quad, \quad 7 \quad, \quad 1 \quad,
\end{aligned}
$$

agst. 7 horses

twice second in big Handicaps.

Cesarew. and Cambr. not placed.

, $10 \quad$, $8 \quad, \quad$, , 2 ,

Berlishire St. at Reading, 2 Miles, 6 st. 13

agst. $13 \mathrm{y} \cdot-0.6$ st. 3

44 y.-o. 6 st. 10 to 8 st.

$15 \mathrm{y} \cdot-0.8$ st. 1

agst. 6 horses.

Handicap at Newm., 1 Mile, 6 st. 5

agst. $23 \mathrm{y} \cdot-0.6$ st. 12 and 7 st.

14 y.-o. 7 st. 12

$25 \mathrm{y} \cdot-0.7 \mathrm{st}$. and 8 st. 8

(Russborough) second

$16 \mathrm{y} \cdot-0.7$ st. 7

agst. 6 horses.

Once second and once third in big Handicaps.

In the Tradesmen's Pl., 43 Starters, not placed.

, 11 , 1 , , , 0 ,, In the Royal Hunt Cup at Ascot, 30 Starters, not placed.

$\overline{\text { ran }} 58$ times, won $21_{\frac{1}{2}}^{\frac{1}{2} \text { times. }}$

No. 52. Radulphus 1843 by The Saddler.

ran 2 year-old 1 time, won 0 times.

, 3 ,, 6 times, won 0 ," twice not placed.

, 4 , 5 , , , 3 ,

, . , , 4 , , 4 , 1 ,

"

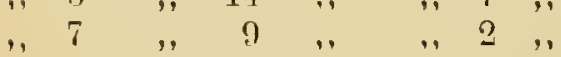


ran 8 year-old 7 times, won 4 times. Handicap at York, $1 \frac{1}{4}$ Miles, 8 st. 12 agst. 43 y.-o. j) st. 2 to 5 st. 12 and $4+y-0.7$ st. 2 to 8 st. agst. 8 horses.

Fitzwilliam Iandicap at Doncaster, 1 Mile, 8 st. 12 agst. 53 y.-o. 5 st. 9 to 7 st. 6 and $35 y-0.6$ st. 5 to 8 st.

agst. 8 horses.

Handicap at Richmond, $\frac{5}{8}$ Mile, 9 st. .j agst. $42 y \cdot-0$. j st. to 6 st. 10 and $15 \mathrm{y} .-0.7$ st. 10

$\begin{array}{llllllll}, & 9 & , & 8 & , & , & 2 & \end{array}$

agst. 5 horses.

Craven St. at York, 1 Mile, 9 st. :3 agst. 3 y.-o. Lady . Ignes 6 st. 10 and $4 y-0$. Iris 8 st. 4

, $10 \quad, 11 \quad, \quad, \quad 2$,

Trial St. at Airdrie, $1 \frac{1}{2}$ Miles, 9 st. i) agst. $23 \mathrm{y},-0.7 \mathrm{st} .4$

$14 y,-0.8$ st. $t$

$25 \mathrm{y} \cdot-0.8$ st. 10 to 9 st. 1

1 older 8 st. 9

agst. 6 horses.

Sweepst. at Paisley, $1 \frac{1}{4}$ Miles, 9 st. 7 agst. 23 y.-0. 7 st. 4

, $11 \quad$, $9 \quad,, \quad,, 4 \quad 4$,

Member's Pl. at Paisley, ? Miles, 9 st. 5 agst. $1+\mathrm{y}-0.0$ st. 9 and $16 \mathrm{y} .-0.9$ st. 2

Trial St. at Lanark, $1 \frac{1}{4}$ Miles, 9 st. 2 agst. 23 y.-o. 7 st. 4 and 7 st. 9 and 1 older 9 st. 2

, $12 \quad, \quad 10 \quad, \quad, \quad, 4 \quad$,

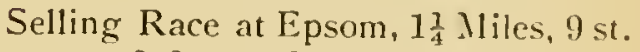
agst. $23 y$ y.-o. 6 st. 13 to 7 st. 5

$15 \mathrm{y} .-0.9$ st. 2

1 older 9 st. 3

agst. 4 horses,

and on the same day second in

Railway Pl., $\frac{3}{t}$ Mile, 53 - and 5 y.-0. behind.

Handicap at Marwick, 5 Furl., \& st. :agst. $13 y \cdot-0.6$ st. 9

14 y.-o. 7 st. 6

1 older 8 st. 7

, $13 \quad$, $9 \quad, \quad \quad, \quad 0 \quad$, , $\quad$ in big Handicaps. 
ran 14 year-old 12 times, won 4 times. Selling Race at Ripon, about 1 Mile. 7 st. 11

$$
\text { agst. } 13 \text { y.-0. } 5 \text { st. } 9
$$

and 16 y.o. -0.5 st. 11

agst. 2 horses,

and won 2 Gentlemen's Races at Newmarket over $\frac{1}{2}$ Mile.

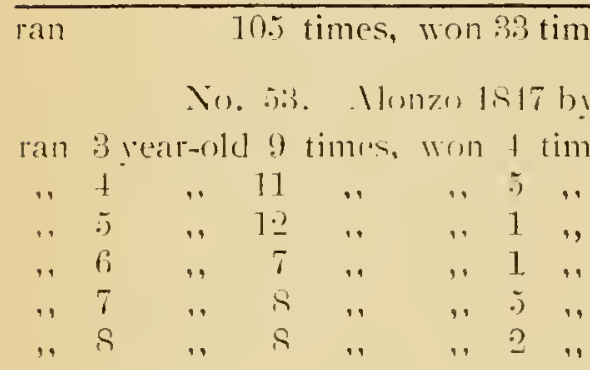

St. Liz. Ilandicap at Northempton, $1 \frac{1}{4}$ Miles, 8 st. 4 arsist. 13 y.-o. 5 st. 6 $5+\%$-o. 5 st. 10 to 7 st. 3 16 y.-o. 8 st.

agst. 7 horses.

llandicap at Ludlow, $1 \frac{1}{4}$ Miles, 8 st. 3 agst. $3: 3$ y.-o. 6 st. 6 to 6 st. 12

35 y.-0. 6 st. 12 to 8 st. 1 $26 y,-0.7$ st. 3 and 7 st. 10

agst. 8 horses,

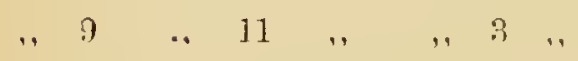
and 3 times third in good Handicaps. St. Liz. Handicap at Northampton, $1 \frac{1}{4}$. Miles, 8 st. ? asst. $33 \mathrm{y} .-0.4 \mathrm{st} .10$ to $6 \mathrm{st}$.

$74 \mathrm{v} .-0$. j) st. 12 to 7 st. 7

$15 \mathrm{v} .-0.6$ st. 10

1 older 7 st. 5$)$

agst. 12 horses.

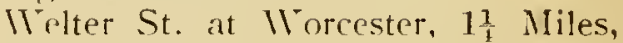
11 st. 3

agst. 13 y.-०. 8 st. 6

$14 \mathrm{y},-0.8 \mathrm{st} .6$

$15 \mathrm{y},-0.9$ st. 11

agst. 3 horses.

L ancaster Cup, $1 \frac{1}{2}$ Miles, 8 st. 7 agst. 33 y.-o. 5 st. 7 to 6 st. 5 and $2+\mathrm{y} .-0.7 \mathrm{st}$. and 7 st. 12

:3 times second.

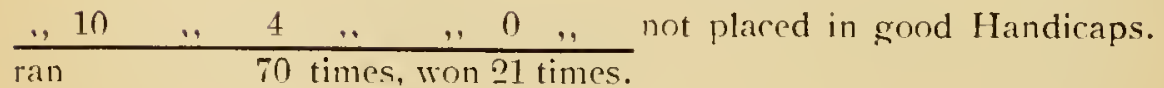


No. 5t. Poodle 1849 by Ion.

ran 2 year-old 3 times, won 2 times.

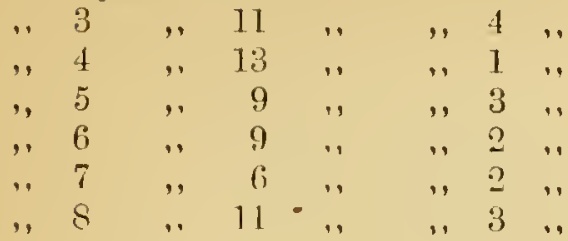

Gr. Metrop. St. at Epsom, $2 \frac{1}{4}$ Miles, 8 st.

agst. $8391 .-1)$; st. 20 to 6 st. 8

$2+1 .-0.5 \mathrm{st} .4$ and $9 \mathrm{st}$.

(Fisherman)

I (iv-0.8 st. \& (Winlefield)

1 older 7 st. 1:3

agst. 12 horses.

Handicap at Newm., 1 Mile, 9 st. 'agst. 4 \& $y-0 .(0$ st. !) to 7 st. 9

1 ; $; i .-1,8$ st. 3

1 wdeler st. 7

agst. 6 horses.

Ling's Pl. at Newmarket

agst. $2+(\because,-0$. and 1 i) y.-0).

and in the Cesarew.., st. s, not placed.

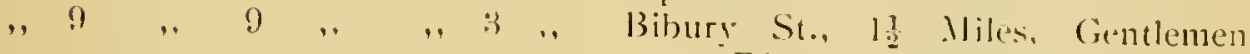
Riders, 10 st. 8 agst. $\doteq+y,-1), s$ st. $1: 3$ and 1 ; y.-0. 11 st. in the Cesaren., 7 st., not placed.

\begin{tabular}{|c|c|c|c|c|c|}
\hline \multirow[t]{2}{*}{$\operatorname{ran}$} & \multicolumn{5}{|c|}{71 times, won 20 times. } \\
\hline & & No. 5 & ). $/ 1 r$. & Sylkes & (Ilalf - - \\
\hline ," & 3 & 11 & times, & won? & $\begin{array}{l}1 \text { time. } \\
2 \text { times. }\end{array}$ \\
\hline ,. & 4 &., & ". & ". & 0 \\
\hline , & 5 & 11 & ,. & , &.. \\
\hline , & 6 & 11 & ., & & ,, \\
\hline , & 7 & , , & ,. & & , , \\
\hline ,. & 8 & ., & .. & ., & 1 \\
\hline
\end{tabular}

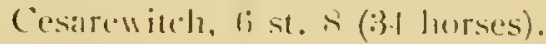

King's Pl.

Roxburgh llandicap, 1] Miles, 7 st. di agst. :3 : $3(1 .-1) .1$ st. (10) (1) .) st. 13

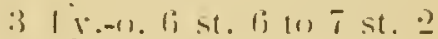

$+j i \cdot-1), j$ st. 11 to 7 st. 10

1 older 7 : 1.13

agst. 11 lonses.

in the Cesarew., 7 st. \&, and in the Cambr.. s st., not placed. 
ran 9 year-old 13 times, won 2 times. City Handicap at Lincoln, $1 \frac{1}{4}$ Miles, 7 st. 9 agst. 83 y.-o. 4 st. 10 to 5 st. 9

34 y.-o. 5 st. 12 to 7 st. 6

15 y.-o. 6 st. 10

1 older 7 st. 2

, $10 \quad$, $\quad 3 \quad$, $\quad$, $0 \quad, \quad$ in good Handicaps.

ran

65 times, won 10 times.

The most imposing performances of the above 55 examples from 1730 to 1850 begin with Marksman, born 1808, and slowly decrease after Inheritress, born 1840. In spite of the enormous increase of Thoroughbred breeding after 1850, we do not find any examples in the second half of the nineteenth century which can show better performances in this respect, and only a few with anything like these performances. According to the above examples, the best time seems to have been 1830 to 1840 , as in this period the following horses, which accomplished extraordinary performances, were born, namely: Catherina, 1saac, Zohrab, Potentate, Bee's WVing, The Shadow, St. Lawrence, The Sailor, and Inheritress.

All these facts go to prove that the hardiness and endurance of the Thoroughbred, and especially the resisting capacity of its foundation, decreased about the second half of the nineteenth century. Whether at the same time the speed of the Thoroughbred, for distances up to $1 \frac{1}{2} \mathrm{English}$ miles, increased, cannot be clearly shown, but it is probable. Height and appearance have been improved, or, as the classical defender of the present day racing sistem, Admiral Rous, says, "Te have bred more for size and strength." Yes, we have obtained that in our highly improved light breeds, but also at the same time a retrogression in the performing capacity which is required outside the racecourse. The celebrated Gimcrack, born 1760, No. 8 in above list, to which Admiral Rous points somewhat contemptuously as a "generally small horse," is supposed to have been only. 14 hands; according to other statements, 13 hands, $3 \frac{1}{2}$ inches, equalling 141 $\mathrm{cm}$. Idmiral Rous contends that in 1700 the average height was 13 hands, 3 inches, and that since then this average height has risen every twenty-five years by 1 inch, and ought therefore to be now 15 hands, 3 inches, equalling $160 \mathrm{~cm}$. 
The following list gives the sizes of the most important Thoroughbred stallions which I have been able to obtain.

\begin{tabular}{|c|c|c|c|c|c|c|}
\hline No. & Names of Stallions. & Born. & Sire. & Hands, & $\begin{array}{c}\text { Size in } \\
\text { Inches. }\end{array}$ & cin. \\
\hline 1 & Dariey .Irabian & 1702 & $\longrightarrow$ & 15 & - . & 152,4 \\
\hline$\underline{2}$ & Mixbury & 1704 & The Curven Bay Barb & 13 & 2 & 137,2 \\
\hline 3 & Godolphin Irabian & 1724 & … & 15 & - & 152,4 \\
\hline 4 & Sampson & 1745 & Blaze & 15 & $\underline{2}$ & 157,5 \\
\hline j & Matchem & $17+8$ & Cade & 15 & 1 & 154,9 \\
\hline (i) & Herod & 1758 & Tartar & 15 & 3 & 160,0 \\
\hline$\tau$ & Fclipse & 1764 & Marslie & 15 & $\underline{2}$ & 157,5 \\
\hline 8 & Sir Peter & 1784 & Highflyer & 16 & - & 162,6 \\
\hline 9 & Gohanna & $17(x)$ & Mercury & 15 & 1 & 154,9 \\
\hline 10 & Sorcerer & 1796 & Trumpator & 16 & $1 \frac{1}{2}$ & 166,3 \\
\hline 11 & Orville & $17(9)$ & Beningbrough & 16 & - & 162,6 \\
\hline 12 & Castrel & 1801 & Buzzard & 16 & - & 162,6 \\
\hline 13 & Whalebone & 1807 & IVaxy & 15 & $\frac{1}{2}$ & 153,7 \\
\hline 14 & Partisan & 1811 & IValton & 15 & 2 & 157,5 \\
\hline 15 & Dr. Syntax & 1811 & Paynator & 15 & - & 152,4 \\
\hline 16 & Filho da Puta & 1812 & Haphazard & 16 & - & 162,6 \\
\hline 17 & Lottery & 18210 & Tramp & 16 & - & 162,6 \\
\hline 18 & Camei & 1822 & Whalebone & 15 & 2 & 157,5 \\
\hline 19 & Iulatto & $18 \div 3$ & Catton & 15 & 2 & 157,5 \\
\hline 311 & L.ittle Red Rover & $18 \div 7$ & Tramp & 14 & 3 & $14 !, 0$ \\
\hline 21 & Plenipotentiary & 1831 & Emilius & 15 & 2 & $157, \overline{5}$ \\
\hline 2.2 & Touchstone & $18: 31$ & Camel & 15 & 2 & 157.5 \\
\hline 23 & Mundig & 1832 & Catton & 15 & 3 & 160,0 \\
\hline $2-4$ & Sheet Inchor & 1832 & L.otter! & 16 & 1 & 165,1 \\
\hline 25 & Birdcalcher & $18 \times 3$ & Sir Hercules & 15 & 3 & $10), 0$ \\
\hline $26 ;$ & Harkaway & 1834 & liconomist & 16 & 2 & 167,6 \\
\hline$\underline{-1}$ & 1)on John & 1 linis & Tramp or llaverley & 15 & 3 & $1(x), 0$ \\
\hline 2 & I3loomsbury & 1836 & Mulatto & 15 & 3 & 160,0 \\
\hline$: 3$ & Nutwith & 1510 & Tomboy" & 15 & $-\$$ & 158,2 \\
\hline 3! & Newminster & 1842 & Touchstone & 15 & 2 & $1: 37,5$ \\
\hline 31 & Chanticleer & $18+3$ & Birdcatcher & 16 & - & $162,6 \mathrm{i}$ \\
\hline$: 32$ & The Cossack & $184 t$ & Iletman Platoff & 15 & $\ddot{2}$ & 157,0 \\
\hline 333 & Daniel O'Rourke & 184!! & Birdeatcher & 15 & $\check{2}$ & $1: \pi$ \\
\hline 31 & Stockwell & 1849 & The Baron & 16 & - & $1 t^{i 2}, 6$ \\
\hline 35 & 1.exingrton & $1 \sin 0$ & Boston & 15) & 3 & $1(x), 0$ \\
\hline 36 & Saunterer & 18 is & Birdcatcher & 15 & $: 3$ & 181,0 \\
\hline 37 & Buccanecr & 1857 & Wild Daỵrell & 1.5 & 3 & 101.0 \\
\hline : $\mathrm{xs}$ & Macaroni & $18(0)$ & Swcetmeat & 15 & 3 & 160,0 \\
\hline (⿻) & Blair Ithol & 1801 & Stockivell & 16 & - & 160,6 \\
\hline 41 & 1.clio & $180^{\circ}$ & II est . Iustralian & 15 & 2 & 1.i, \\
\hline 41 & Rosicrucian & 1865 & Beadsman & 16 & - & $16 \div .19$ \\
\hline 42 & Prince Charlic & 1869 & Blair Ithol & 17 & - & $17 \cdot 2.7$ \\
\hline
\end{tabular}




\begin{tabular}{|c|c|c|c|c|c|c|}
\hline \multirow{2}{*}{$\mathrm{N} n}$. & \multirow{2}{*}{ Nimes of Stallions. } & \multirow{2}{*}{ Liorn. } & \multirow[b]{2}{*}{ Sire. } & \multicolumn{3}{|c|}{ size in } \\
\hline & & & & IIands. & lnches. & $\mathrm{cm}$. \\
\hline 43 & Ilector & 1872 & Virgrilius & 15 & $\ddot{2}$ & 157,5 \\
\hline 4 & Petrarch & 1873 & Lurd Clifelen & 15 & 3 & 160,11 \\
\hline 45 & Springfield & 1873 & St. Mibans & 16 & - & $16^{2}, 6$ \\
\hline $4 i$ & Chamant & 1 1st & . Mortemer & 16 & - & 162,6 \\
\hline 47 & Beauclerc & 1875 & Rusicruci:un & 15 & 3 & 160,0 \\
\hline 48 & 1sonomy & 187.5 & Sterlin:r & 16 & -. & $162,(i$ \\
\hline 49 & Charibert & 1876 & Thormanby & 15 & $: 3$ & 160,11 \\
\hline 50 & Bend Or & 1877 & Doncaster & 16 & 1 & 105,1 \\
\hline .31 & Despair & $187 ! !$ & Sec Saw & 15 & $\underline{2}$ & 157,5 \\
\hline $5: 2$ & St. Simon & $1 \mathrm{~s}+1$ & Cialopin & 16 & $\frac{1}{2}$ & $16 i 3, y$ \\
\hline 53 & Esterlin: & 1882 & Sterlingr & 16 & - & $162,0^{\circ}$ \\
\hline 54 & Melton & 189. & Manter Kildare & 15 & 3 & 160,0 \\
\hline 35 & lughrim & 1893 & Xenophon & 16 & $\ldots$ & 162,6 \\
\hline$\ddot{0}$ & Kiencial & $184: 3$ & Bend Or & 16 & 1 & 165,1 \\
\hline $5 \overline{1}$ & Minting & 1853 & Lared Ifyon & 16 & $\frac{1}{1}$ & 163,2 \\
\hline 58 & Ormonde & 1853 & Bend Or & 16 & 1 & 165,1 \\
\hline 59 & Gallinule & 1881 & Isonomy & 16 & - & 162,6 \\
\hline 60 & Iyrshire & 1885 & Hampton & 16 & - & 162,6 \\
\hline 61 & Carbine & 1885 & Musket & 15 & $3 \frac{1}{2}$ & 161,3 \\
\hline 62 & Winlsfield & 1855 & Barcaldine & 15 & 3 & 160,0 \\
\hline (3) & Imphion & 1856 & Roseber! & 15 & 3 & 160,0 \\
\hline 61 & Donot:a1] & 1886 & Galopin & 16 & 1 & 165,1 \\
\hline 65 & Enthusiast & $18 s 6$ & Sterlingr & 16 & - & 162,6 \\
\hline ikj & Melanion & 1886 & Hermit & 16 & - & 162,6 \\
\hline 137 & Hackler & 1897 & Petrarch & 16 & 1 & 165,1 \\
\hline 65 & Martagon & 1887 & Bend $\mathrm{Or}$ & 16 & - & 162,6 \\
\hline $6 !$ & Srinfoin & 1857 & Springtielıl & 15 & 3 & 160,0 \\
\hline 70 & St. Serf & 1857 & St. Simon & 16 & 3 & 170,2 \\
\hline$\pi 1$ & Common & 1888 & 1 sonumy & 16 & 1 & $16 i 5,1$ \\
\hline 72 & Orme & $188 !$ & Ormonde & 16 & 1 & $1(6,1$ \\
\hline 73 & Suspender & $188 !$ & Muncaster & 16 & 2 & 167,6 \\
\hline 74 & Red Prince & 1889 & Kendal & 15 & 3 & 160,0 \\
\hline 75 & Childwick & 180 & St. Simon & 16 & - & 162,6 \\
\hline $7 i$ & Isinglass & 1890 & Isonomy & 16 & 1 & 165,1 \\
\hline 77 & Grey I.en & 1891 & Pepper and Salt & 15 & 2 & 157,5 \\
\hline 78 & Ladas & 1891 & Hampton & 16 & -- & 162,6 \\
\hline $7 ! 1$ & Sir Visto & 1892 & Barcaldine & 16 & 1 & 165,1 \\
\hline 80 & Persimmon & 1893 & St. Simon & 16 & 2 & 167,6 \\
\hline 81 & Galtee Mnre & 1894 & Kendal & 15 & $33^{3}$ & 162,0 \\
\hline 82 & Velasquez & 1894 & Donovan & 15 & 3 & 160,0 \\
\hline 83 & Flying Fox & 1896 & Orme & 16 & $\frac{1}{2}$ & 163,9 \\
\hline 84 & Ird Patrick & $18 \%$ & St. Florian & 16 & - & 162,6 \\
\hline 85 & Maintenon & $19 K 3$ & Le Sitgittaire & 16 & $3 \frac{1}{2}$ & 171,5 \\
\hline
\end{tabular}


In order to arrive at a fair comparisom, it is necessary to take sis of the most important stallions from the above table for three different periods, and we then gret the follow ing interesting restults:

1.st l'eriud-Second Ilalf of the lighternth cintury.

\begin{tabular}{|c|c|c|c|c|c|c|}
\hline Matchem & born & 17.48 & 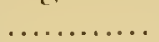 & $1.5+. ! 1$ & (וIוI. & $\sin$ \\
\hline Ilered & . & 17.58 & & 160.0 & . & . \\
\hline Eclipse & & $176 \mathrm{t}$ & & 1.57 .5 & " & \\
\hline Sir Petro & $\because$ & 1784 & & $1(i-2 . t i$ & ". & \\
\hline Gohannal & n & 1790 & & 1.54 .9 & ". & \\
\hline Orville & $\because$ & 1790 & n........... & $1(i=. .1 j$ & . & \\
\hline
\end{tabular}

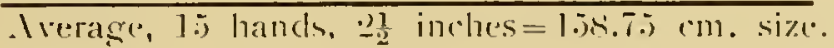

End l'eriod-Middle of the Nincteenth century.

louchstune born $\mid x^{3} 31$

$157.5)$ (m) size.

Birdcatcher , 1 16:33

160.1 .. .,

Newminster ., 184.)

Steckwell ., 1 int?

$\begin{array}{lll}\text { Buccaneer } & \text {, } & 18.5 \\ \text { Macaroni } & \text {. } & 1800\end{array}$

1.57 .5

$162.0 \%$

$16(1) .1)$

,

,

Irerasce, 1.5 hands, 3 inches $=1.59 .6 \mathrm{~cm}$.

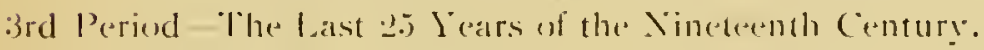

Isonomy born 187.j ......... 16.2. (m) size.

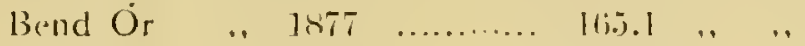

St. Simen .

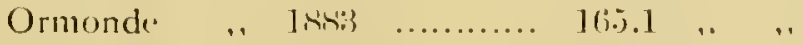

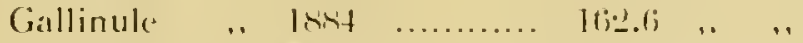

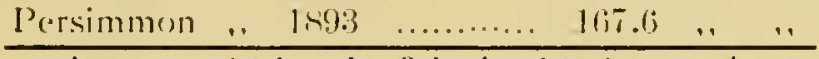

Irerage, lfi hands, $\frac{3}{2} \mathrm{inch}=16$ t.j cme size.

From abose it can be seen that the height of the Thoroughbred in lingland has increased somewhat quiclier than . Imiral Rous supposed. The desire 4 increase the height serms $t 0$ mo tu be dangerous, and will probably rery suon only be possible at the expense of the fundament. The present day situation of racing and breeding secoms to be approathing nolens a'olens a further increase of height.

To judgre rightly of the retrogressive mesement in the brerding of Thorouglibreds, as regards their general capabilities (1,eistungstihiglecit). one must take into consideration that the races of to-day are rum over shorter distances, and 1 ithout heats, and on an average at a considerably quicker pace that was the case loo years ago, and also that the increased pace malies greater demands an the fundament. One must, therefure, expeet that the resisting strength of the fundament will be more quickly used up. "o the

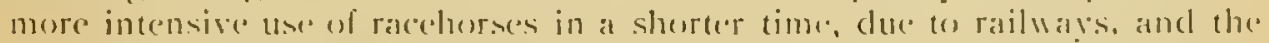
numerous raceenurses, as well as to the many short races, which repuire a quick getting off, must be attributed the fact that the nerves and health are more quickly destroged. The question as to whether the Thoroughbreds of 100 years ane would not also have deteriorated quicker if they had had 
to undergo present day methods, and as to whether the present day Thoroughbreds, on the other hand, would not have held out longer under the conditions of 100 years ago, may be answered as follows: Both cases can only happen slowly, i.e., if the supposed causes have lasted and had an influence for several generations. On the basis of physiological observations and practical experiences, we must assume that the present over-exertion, especially of the younger Thoroughbred stock, for many consecutive generations, has an unfavourable influence on the constitution. These unfavourable effects, which are an especial consequence of the many early races of twoyear-olds, are distinctly recognisable, also outside the course, by manyretrogressive signs. The most conspicuous of these consists in the fairly regular decrease of the percentage of pregnant mares, from about 80 per cent. in the year 1851 to 70 per cent. in recent years.

The following list, the figures of which are taken from the General Stud Book, gives an exact summary of this retrogressive movement :-

\begin{tabular}{|c|c|c|c|c|c|c|c|c|}
\hline \multirow{2}{*}{ Year } & \multicolumn{3}{|c|}{ Foals Born } & \multirow{2}{*}{ Aborted } & \multirow{2}{*}{ Barren } & \multirow{2}{*}{$\begin{array}{l}\text { Total } \\
\text { Covered }\end{array}$} & \multirow{2}{*}{$\begin{array}{c}\text { Pregnant } \\
o \\
o\end{array}$} & \multirow{2}{*}{$\begin{array}{c}\text { Aborted } \\
\%\end{array}$} \\
\hline & Colts & Fillies & Total & & & & & \\
\hline 1851 & 538 & 597 & 1135 & 43 & 302 & 1480 & 79,59 & 3,65 \\
\hline 1852 & 536 & 538 & 1074 & 71 & 334 & 1479 & 77,42 & 6,61 \\
\hline 1853 & 574 & 558 & 1132 & 42 & 369 & $15+3$ & 76,09 & 3,71 \\
\hline 1854 & 619 & 582 & 1201 & 35 & 40 & 1676 & 73,75 & 2,83 \\
\hline $18 \tilde{5}$ & 610 & $6 \cap 9$ & 1219 & 73 & 480 & $177 z$ & 72,91 & 5,65 \\
\hline 1856 & 705 & 6.50 & 1355 & 61 & 48 & 1861 & 75,97 & 4,31 \\
\hline 1857 & 691 & 679 & 1370 & 58 & 4.52 & 1880 & 75,96 & 4,106 \\
\hline 1858 & 756 & 736 & 1492 & 64 & 424 & 1980 & 78,59 & 4,11 \\
\hline 1859 & 745 & 722 & $1+67$ & 77 & 484 & 2028 & 76,13 & 4,99 \\
\hline 1860 & 738 & 220 & 1464 & 85 & 495 & 2047 & 75,67 & 5,49 \\
\hline 1861 & 750 & 749 & 1499 & 73 & 5:3 & 2097 & 74,96 & 4,64 \\
\hline 1862 & 718 & 763 & 1481 & 68 & $56: 2$ & 2111 & 73,38 & 4,39 \\
\hline 1863 & 783 & 757 & 150 & 89 & 556 & 2184 & 74,54 & 5,41 \\
\hline 1864 & 756 & 811 & 1567 & 95 & 237 & 2199 & 75,59 & 5,72 \\
\hline $186 \mathrm{j}$ & 786 & 799 & 158.5 & 136 & 550 & 2277 & 75,55 & 7.90 \\
\hline 1866 & 8501 & 850 & 1706 & 96 & 686 & 2488 & 72,43 & 5,33 \\
\hline 1867 & 893 & 964 & $185 \overline{7}$ & 84 & 645 & 2586 & 75,06 & 4,33 \\
\hline 1868 & 972 & 949 & 1921 & 127 & 719 & 2767 & 74,02 & 6,20 \\
\hline 1869 & 1009 & 1041 & 2050 & 113 & 669 & 2832 & 76,38 & $5,2 * 2$ \\
\hline 1870 & 983 & 965 & 1948 & 125 & 727 & 2800 & 74,04 & 6,03 \\
\hline 1871 & 928 & 968 & 1896 & 147 & 6993 & 2736 & 74,67 & 7,20 \\
\hline 1872 & 881 & 888 & 1769 & 94 & 694 & 2557 & 72,86 & $\mathbf{5}, 04$ \\
\hline 1873 & 862 & 858 & 1720 & 74 & 621 & 2415 & 74,29 & 4,12 \\
\hline 1874 & 857 & 871) & 1727 & 72 & 657 & 2456 & 73,25 & $4,(x)$ \\
\hline
\end{tabular}


5. The Thoroughbred in the Past and Present.

\begin{tabular}{|c|c|c|c|c|c|c|c|c|}
\hline \multirow{2}{*}{ Year } & \multicolumn{3}{|c|}{ Foals Born } & \multirow{2}{*}{ Aborted } & \multirow{2}{*}{ Barren } & \multirow{2}{*}{$\begin{array}{c}\text { lotal } \\
\text { Covered }\end{array}$} & \multirow{2}{*}{ Pregnant } & \multirow{2}{*}{ Aburted } \\
\hline & Colts & Fillies & Total & & & & & \\
\hline 1875 & 824 & 910 & 1734 & 86 & 634 & 2454 & 74,16 & 4,73 \\
\hline 1876 & 879 & 803 & 1742 & 121 & 636 & 2499 & 74,55 & 6,55 \\
\hline 1877 & 941 & 915 & $18 \pi 6$ & 105 & 722 & 26683 & 73,09 & 5,37 \\
\hline 1878 & 926 & 969 & 1895 & 168 & 723 & 2786 & 74,$0 ;$ & 8,14 \\
\hline 1879 & 1002 & 952 & 1954 & 108 & 798 & 2380 & 72,10 & 5,19 \\
\hline 18त्रा & 969 & 054 & 1923 & 161 & 801 & 2885 & 72,24 & 7,73 \\
\hline 1881 & 928 & 1063 & 1991 & 110 & 520 & 2927 & $71,7 \mathrm{~S}$ & 5,24 \\
\hline 1882 & 920 & 9963 & 1916 & 140 & 818 & 2874 & 71.54 & 6,81 \\
\hline 1883 & 1006 & 958 & 1964 & 121 & $T 48$ & 2833 & 73,60 & 5,80 \\
\hline 1884 & $1(x) 1$ & 956 & 1957 & 127 & 801 & 2885 & 72,23 & 6,09 \\
\hline 1885 & 1019 & 1061 & 3081 & $1: 29$ & 7.33 & 29162 & 74,58 & 5,84 \\
\hline 1866 & 1100 & 1086 & 2166 & $1: 39$ & 791 & 3096 & 74,45 & 6,03 \\
\hline 1887 & 1063 & 1069 & $21: 32$ & $13 \pi$ & 861 & 3128 & 72.47 & 5,96 \\
\hline 1888 & 1069 & 1141 & 2210 & $1: 21$ & $8: 39$ & 3178 & 73,60 & 5.50 \\
\hline 1859 & $13(3) 1$ & 1159 & 23601 & 146 & 940 & 3446 & 72.72 & 5,83 \\
\hline 1890 & 1213 & 1290 & 203 & 144 & 1002 & 3649 & 72,54 & 5,44 \\
\hline 1891 & 1305 & 1419 & 2787 & 192 & 1120 & $4(009$ & 72,68 & $(3,45$ \\
\hline 1502 & 1517 & 1523 & 3040 & 2.28 & 1206 & 4.504 & 73,24 & 7,82 \\
\hline 189.3 & $166^{\circ 2}$ & 1613 & 3275 & 156 & 1412 & $484: 3$ & $7(1,81$ & 4,55 \\
\hline 1894 & 16.51 & 1562 & 3213 & 184 & 1400 & 4797 & $70, S^{2}$ & $5,4 \pm$ \\
\hline 189.; & 1674 & 1557 & 3231 & 124 & 1324 & 4682 & 71,60 & 3.70 \\
\hline 1846 & 16.52 & 1652 & $: 304$ & 144 & 1257 & 4735 & 72,82 & 4.15 \\
\hline 1897 & 1785 & 1753 & 3535 & 150 & $13 x$ & 5021 & 73,45 & 4.17 \\
\hline 1998 & 1774 & 1762 & 3536 & 149 & 14.00 & 5135 & 71,76 & 4,114 \\
\hline 1899 & 1706 & 1808 & 3514 & 147 & 1405 & .0060 & דיב.20 & 4.112 \\
\hline 1900 & 1753 & 1674 & $31: 27$ & 169 & 1310 & tex) & 73,301 & 4.711 \\
\hline 1901 & 1743 & 1697 & 3440 & $1: 35$ & $1+16$ & 4094 & $71,(6 j)$ & 3,86 \\
\hline 1902 & 1797 & 1651 & 3448 & $1: 38$ & 1380 & 4966 & $7: 2.21$ & 3,85 \\
\hline 1913 & 1629 & 1564 & 31193 & 123 & 1364 & 4681 & $7(1,85)$ & 3,71 \\
\hline 194 & 1585 & 1512 & 3107 & 132 & 1272 & 4511 & $\pi 1,80$ & 4,108 \\
\hline 1905 & 1312 & 1461 & 2773 & 121 & 1091 & :2068 & (22,7:2 & 4, 4, \\
\hline$I ! x) 6$ & 1418 & 13381 & 2799 & 117 & 1153 & $4(1) 69$ & $7(1) .17$ & 4.111 \\
\hline
\end{tabular}

This fairly uniform decrease in the perontage of pregnant mares begins just at the time at which, lesked at from the standpoint of changed weight differences, the decrease of the capabilities (Leistungsfähigkeit) of the Thoroughbred seems to commence. In the chapter relating to the changed weight differences (looked at from this one-sided point of view), a slow improvement of the Thoroughbred stock is supposed to have wisted in the first half of the nineteenth century. Corresponding with this, the percentage 
of foaling mares seens also to have increased in the first half of the nineteenth century. Aecording to some superficial tests, I have found that the perrentage of foaling mares varies in the years 1800 to $1810 \mathrm{from} 74$ to 75 per cent. The statistics in the General Stud book begin only with the year 1846 , and are as follows:-

\begin{tabular}{|c|c|c|c|c|c|c|c|c|}
\hline \multirow{2}{*}{ lear } & \multicolumn{3}{|c|}{ Funds born. } & \multirow{2}{*}{ Aburted } & \multirow{2}{*}{ Barren } & \multirow{2}{*}{ Cuvered } & \multirow{2}{*}{ Pregnant } & \multirow{2}{*}{ Aborted } \\
\hline & Stallions & Mares & Total & & & & & \\
\hline 1846 & $4 ! 1$ & $48 I$ & 972 & 41 & 310 & 1323 & 76,57 & 4,05 \\
\hline 1847 & 486 & 491 & 977 & 47 & 303 & 1327 & $7 \pi, 17$ & 4,59 \\
\hline 1845 & $48: 3$ & 493 & 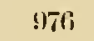 & 57 & 304 & $13: 37$ & 77,$20 ;$ & 5,52 \\
\hline $181 !$ & - & - & - & - . & -- & - & - & - \\
\hline $1 \sin 11$ & 504 & 537 & 1041 & jl & 282 & 1374 & 79,48 & 4,67 \\
\hline
\end{tabular}

The year lis.) therefore, sems to be the sulminating point in this respect.

The list of Thoroughbred mares in England which have produced 17 and more living foals, shows from 18.j) a distinct retrogression. In spite. of the double to fourfold number of brood mares in the second half of the nineteenth century, the breeding performances have decreased in this respect, with the exception of the estrardinars performanes of Oueen listher, born listit, which produced o.2 foals. The number of mares with 17 and more foals is far from haring incroased proportionately, and the highest performanee of en foals becomes more scarer. In the last thirteen years,

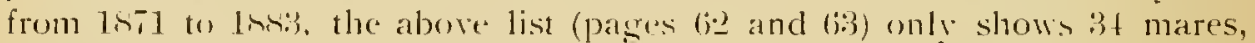
none of which have produced 20) foals, and only :- (Pillage, borm 1471, and Miss Mannering, born 1874) 19 foak-mhilst previumsly in the thirteen years from $18: 3.2$ to $18+4$ (in which tinte there were only a quarter of the brood mares existing as compared with lsil w 1883), 40 mares are mentioned, of which :2 mares (Florence, born 1839), and Prairie Bird, born 1844) have preduced :0 foals, and 6 mares (Whiskermare, born 1s:3. Annette,

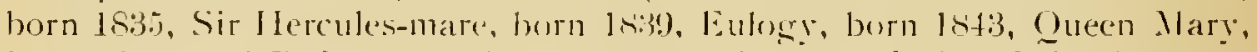
born 1843, and Dofeneeless, born 1itt) produced 19 foals. It is also noteworthy that from about 18.00 , in the cases of most mares with 17 and more foals, the mares had been mated when three years old oftener than before 1850 .

1lso, the performaners of old mares under difficult conditions, as previously indicated (pages (i.j-7(i), slow from about 1850 a clecrease both in the number of extraordinars performanees and in the importance of same. Ifter Pocahontas, born 18:37. Nice Hawthorn, born 1838, Queen Mary, born 1843, llaricot, born 1847, we do not find in the subsequent years any breceding performanees to equal them. 
The decreased performances of hroud mares ats regards the number of

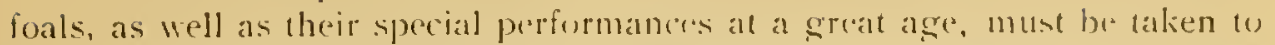
verify the fate that the longevity of the mares in the seound half of the nineteenth exentury has somerhat decteased. I nfortunately, I am not able to give a sufticiently long list of the longereity of the mares as a proste but one mar well assume that the length of the lives of the mares has dereased just as much as that of the stallions, and this fact is proved by the list given

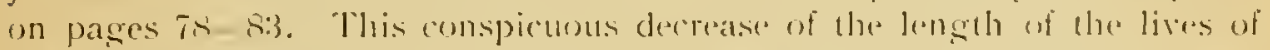

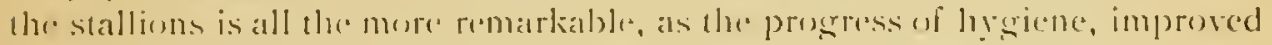
stabling, and other conditions. ats well as the more reasonable training of

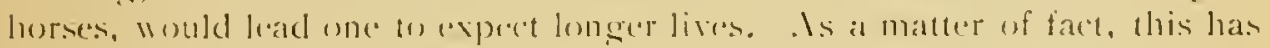
taken plare in the human rate. I changere in the length of the lives of English born stallions in the lirst half of the nimetenth exntury is not recrgnisable from the above list. In the middle of the nineternth century. however, these long lives serm onee again to ocetur, as shown by the

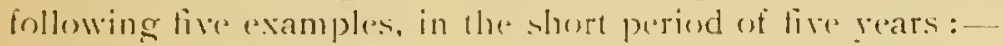

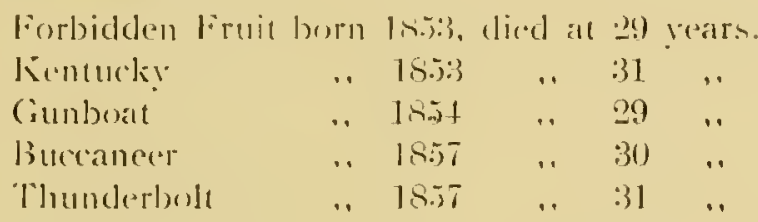

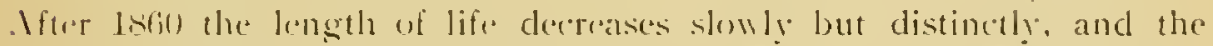
number of stallions which have altained 2.5 years is much tou small in proportion to the remarkably increased breding. The number of stallions which hate attained 2.5 yeats and more has been in no decade sreater than in that of 18.jo to 18.j9, namely, :30. Mlready in the following two decades a

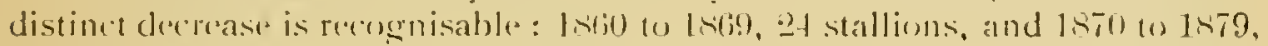
20 stallions.

firom the list of Imerican burn thoremghbred stallions (pagre st), we can also gather that the lenghth of their lives has decreased. (One (an distinetly" recognime this decrease about ten years later than in lingland, hut it is more sudden and more apparent. (2)perially if the enomoms inerease in Theroughbred brerding is taken inter comsiderations.

firom a fumber consideration of the table (pages st 10 100) We can glean

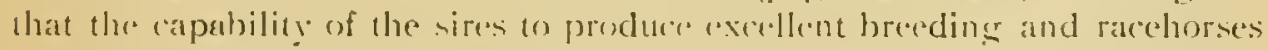
has also decreased from aboun throt twentireh vear, since the middle of the last century, and that considerably. The middle of the nimetenth rentury" shows also in this respect a distinet rulminating print. Of the stallions which have excelled after 1800 through prominent production, I can only mention $t$ in the abov"table, namely, Rosicrucian, Mermit, lamptom, and Calopin, and here I may make the following remarle: (1) Rosicrurian is a som of 
Beadsman, which only ran twice as a two-year-old, the first time in July. All his ancestors in the male line never ran as two-year-olds. (2) Hermit is a son of Newminster, which did not run as a two-year-old. (3) Hampton is a son of Lord Clifton (by Newminster), who only ran three times as a twoyear-old, the first time in June. (4) Galopin is a son of Vedette, who only. ran twice as a two-year-old, first time in August.

Of the remaining stallions which lived a long time and produced prominent stock at a great age, one may mention, after 1860 , the following horses in England: Springfield, Beauclerc, Bend Or, and St. Simon. Springfield, born 1873, attained 25 years, but produced in the last six years only 5 unimportant winners. Bcauclerc, born 1875, also attained 25 years, and in the last six years produced only 1 unimportant winner. Even St. Simon, who reached 27 years, seems after his twentieth year to have deteriorated with regard to his former very prominent breeding power, as in the last eight years he could not produce any winner of importance. In the case of Bend Or, born 1877, 26 years old, the remarkable phenomenon blood of Old Stockwell seems to have had some effect still, as at the age of 24 he could still produce two such good horses as Golden Gleam and Rouge Croix, and as a 25-year-old, Radium, and Gold Rioch.

Not only in theory, as I mentioned in the chapter about weight-differences, but also various statistics (as already given), point repeatedly to the middle of the nineteenth century as a period from which the retrograde movement in the development of Thoroughbred breeding in England seems to have begun. It is worthy of note that it is just at the time when the almighty Third Dictator of the Turf, Admiral Rous, who first of all became celebrated by working out the scale of weights, became Handicapper of the Jockey Club (1855). The number of short races (1 mile and under 1 mile) begins to be more than half the races run, and the number of two-year-old racehorses (see page 29) begins to exceed the number of three-year-olds (1856). The impatience of many racing stables anxious to get money, and the power of the boolimaker, are finally the causes of the races for yearlings being held for four consecutive years (1856 to 1859). The most energetic opponent of the races for one and two-year-olds, Lord Jersey, died in 1859. Buccaneer, born 1857 (who still could produce as a 24-year-old Budagyongye N.D., as a 25-year-old Feneck OD. U., and Ollyannincs PL., and as a 27 -year-old Talpra Magyar), ran as a five-year-old his last race at Salisbury with 9 stone, 5 lbs. up, the mile in 1 minute, 38 seconds $(=16.42$ $\mathrm{m}$. per second), and therefore beating any mile performance before or since. Certainly Bendigo ran in 1885 a mile in 1 sccond quicker $(=16.62 \mathrm{~m}$. per second), but he carried, also as a five-year-old, 1 stone less). Abd-el-Liader won in 1850 the Liverpool Grand National, in the largest field $(32 \mathrm{com}-$ petitors), and as long as the race was run, on the then existing track, in the shortest time (9 minutes, 57! seconds). In 1849 Stockwell was born, the 
champion of the whole English Thoroughbred breeding. ${ }^{\text {' Leamington. }}$ born 1853, is most probably the last Thoroughbred stallion born in England which as a 2t-year-old could produce an Epsom Derby winner, namely, Iroquois. The number of pregnant mares reached the never before attained number of about 80 per cent. (1851). The number of foals born exceeds 1850 , the first thousand (1,041 foals) and in 1859 the second thousand $(2,028$ foals). English Thoroughbred breeding was in every respect in the zenith of its performances, and England herself had become the first world power after the Crimean war! In this decade were produced such a great number of racecourse champions and never-to-be-forgotten pillars of Thoroughbred breeding as has never occurred before or since in such a short period. The following examples demonstrate this :-

1. The lilying Dutchman D. u. L. 1849.

$\therefore$ Voltigeur D. U. L. 1850.

3. Teddington D. 1851 .

4. Newminster L. 18.51 .

5. Stockwell 2 t. L. 1852.

6. West Australian 2, D. u. L. 1853.

7. Rataplan Gold Vase Asc. 1853, Dcp. 185. u. 18 King's Plates.

8. Wild Dayrell D. 1855.

9. Blink Bonny D. u. O. 1857.

10. Vedette 2 แ. Dcp. 1857.

11. Beadsman D. 1858 .

12. Saunterer Gcp. 1858.

13. Fisherman Acp. 1858 and 1859.

14. Thormanby D. 1860.

15. St. Albans L. 1860.

16. The Vizard 2. 1860.

. Imongst these 16 champions I have also numbered Fisherman, whe never attained classical honours, but who has become famous because

Stocliwell's progeny has won 17 times classical races in England (amongst these the Derby 3 times, and the St. Leger 6 times) and 16 times ran as seconds. The 3 first places were occupied by Stockwell's progreny 1862 in the 2,000 Guineas, and 1866 in the Derby: Even at 20 years of age, Stockwcll had a breeding power so as to produce in one vear the winners of the Derby and the 2,000 Guinens, as well as the seconds in the Derby, St. Leger, and the Oaks. Amongst these was one of the greatest heroes, namely, Doncaster. Isinglass, St. Gatien, I roquois and Doncaster were the last Derby winners who could still win big races as five-year-olds. Doncnster did not run as a two-year-old: Stockwell is in modern times most nearly approached as a sire by St. Simon. The St. Simon progeny has also won up to now 17 classical races, amongst them twice the Derby and 4 times the St. Leger, but were only 10 times seconds. One must, howerer. consider that St. Simon reached $2 \pi$ years, while Stockwell, unfortunately, dicl six years earlier, that is, as a 21 year-old (5th of May, 1870). Stockwell, furthermore, has produced 209 winners, who have won 1.147 races. St. Simon, on the other hand, only 160 winner (up 101907 inclusive), who have won 506 races. In their best years Stockwell's progeny. won 132 races, and St. Simon's progeny 47 races in one ycar. 
between the age of two and six he ran in 119 races and won fig. He is perhaps the last representative of that hardiness and toughness which are. unfortunately no longer in the same degree, the attribute of our present das Thoroughbreds. ()ld Tom Parr used to say very characteristically of Fishorman: "H( would never begin to gut until he had run two miles, and then he would wear the heart sut of anything on four legs; how he could be trained on a turmpike road, and raced esery day, and never leave a grain in the manger, theugh he ate more solid com than any other horse."

It is worthy of note that also in . Imerican Thoroughbred breeding the ling of all Thoroughbred stallions, ourresponding to our Stockwell, was borll at almust the same time, namely, Lexington (18.j) by Buston and Alice. (arneal), sire of about (fon horses, amongst which 236 winners. The Imerieans, whe are beliexers in time performances, were especially. astonished at Yew Orleans, in 18.5.5, ty the hitherto unattained performance of Lexington in the mated against time ( 4 miles in 7 minutes, 193 seconds. (arrying $103 \mathrm{lbs.}$.) 1 must here remark that Lexington berame blind as a six-year-old, very likely in conseguence of an inflammation of the eyes, first in the right eye, and then in both. During his 21 years activity at the stud this defect was not inherited by his progeny, but they vero plainly inherited his longevity. Mlow the trotter leing in Ineriog was born at the same time, namely. Ilambletonian 1 is 49 by . Ibdallah.

Sorm after this high tide mark of English lhoroughbred brecting, more and more notice was taken of ideas which tended to a deterioration of the Thoroughbred. The classical and very chever advocate of Thorughbred breeding, Admiral Rous, published in 18.5, in the preface to his bouk, "On the laws and practice of horse racing," and 1800 in Bailey"s Magazine. volume 1R, "Racing Past and Future," a defence of the present racing sy'stem with its short distances, light wrights, and many bets. He admits the numerous and early breakdowns of Thuroughbreds, but attributes them to the too frequent starts caused by railways. Ite further points out the greater pace, which has incrased rery much in consecfuenes of the shorter distances, and of the larger fields. Finally, the Admiral points to the same champions of Thoroughbred breeding as mentioned above, and concludes therefoum that the Thoroughbred has never been as good as now. Idmiral Rous (born 179.5, died 187i) passed his best years just at the height of Thoroughbred breeding, which had greater attractions than the starting point of the retrograde movement could have.

Against the opinion of Idmiral Rous we have the interesting judgment of a practical man, who also lived in the times preceding and succeeding the above-mentioned zenith of Thoroughbred breeding. 1 am speaking of the well-known jocker. John Osborne, born 1833, who rode his first race in 1846 and his last race in 189\%. Osborne says (vide . Ishgill \& Radcliffe, page 4.8):-." "The method of training horses in the present day is quite different to what it was when I hegan. Jorses are very much lighter now; they 
have neither the bone nor the substance that Thoroughbreds liad fifty years ago. Of course, in the old times they were sweated a good deal. I Ieary cloths were put on them, and they were gralloped three and four miles in them. That plan has been discontinued for many years. I don't know that sweating is weakening to a horse. Old John Scott was a great believer in sweating and bleeding. I am certain that the constitution of horses of the present day would not stand such work; the modern breed is neither so robust nur so strong. liormerly it was quite a common thing for horses to run threr and four mile heats. If they were subjected to that now they wouldn't be able to come sut of the stable for a month after. I have had some experience myself of riding horses in heats-two mile heats; and I have ridden in four mile races but never in four mile heats. I am fully convinced the constitution of horses of the present day is not as strong as it was forty or fifty years ago. It puzzles me to account for the degenerat tion in the stoutness of the modern racehorse; really, I cannot point to the" rause of it."

The well-known Malton trainer, Charles Lund, speaking about this (purstion in the year 1\$99, expresses himself as follows (1) 1shgill, page 430): "Racehorses in the olden dats were, on the average, 2 stone heavier than those of the present day. Now, horses like Touchstone and West Australian were, if one might say so, twice the size in substance and power in comparison with the present day racehorse, most of which are light of bone and substance, and comparatively weedy in appearance. Present day trainers have gone in for speed too much, with the result that the great majority of the horses are tor finely built."

The following points show that Thoroughbred horses in England hase deteriorated from the middle of the nineteenth century as far as capabilities (I.eistungsfähigkeit), hardiness, and the whole constitution are concerned:-

(1) Diminution of weight differences between weights for horses of different ages.

(2) Decreased capability of the older racehorses on the flat.

(3) The decreased percentage of pregnant mares.

(t) The decreased number of foals.

(.) The decreased breeding performances of the brood mares at a great age and under diflicult circumstances.

(i) 'The shorter lives of 'Thoroughbreds and the decreased prepotency' at a great age. 


\section{CHAPTER VI.}

\section{Conclusions and Propositions as to the Improvement and Breeding of Thoroughbreds.}

TiIE great and important duties which the Thoroughbred accomplishes in the breeding of other light horses, justified, and even also obliged, those people who stood outside the domain of Thoroughbred breeding, to criticise the basis of all its performances, and breedings, on which it is built up, and on which it continues. Our present day Thoroughbred is the outcome of race propositions, and of the manner of breeding and training, which those propositions entail. These propositions are made on human understanding, and are influenced by human misunderstanding, and what is much worse, by many side interests. Only by clever and purpose-answering race propositions, as well as by reasonable breeding and rational training, shall we be able to still more improve our Thoroughbreds. Ifter the observations made in the previous chapters, there seems to be no doubt that our present day Thoroughbred needs improving, and its room for improvement is just as certain as agreeable.

The chief attacks which have been made up to now against the Thoroughbred may be summed up as follows:-

1. Faulty conformation.

ฉ. Want of endurance for long distances.

3. Wrant of cleverness.

4. A too weedy and light fundament.

5. I want of capacity to carry heary weights.

6. Nervousness, difficult temperament, and the bad use of food.

1.-Faulty conformation. This oldest reproach against the Thoroughbred, rests partly on the fact that we over-estimate our knowledge of this matter, and partly on our inexperience in the judging of horses in training. I call to mind the opinion of the Landstallmeister von Burgsdorf, expressed in 1817 in a special brochure. He went on to say that most English Thoroughbreds had sparin, and that the English Thoroughbred must shortly disappear. We must not forget that from chipping cone chips, and that of course every lind of breeding must produce a certain percentage of faulty 
individuals. Training and racing, moreover, show up many little fátults in form which would not have been noticed when merely looking at a horse as, for example, at shows. Nevertheless, it is interesting and instructive to see that the Thoroughbred, even with such great faults, is still capable of performing very astonishing things, whilst a non-Thoroughbred with the same faults in most cases could not do anything. If with fauly conformation it is still capable of doing well, the substance used must be very good indeed.

The su-called Biedenweg's instructions for judging horses competing for State prizes, which are still in vogue in the Prussian State, but probably very rarely followed, can, in my opinion, be dispensed with. One cannot lay down laws to judge the conformation of a horse to suit all cases. '1he views as to what form a horse should have differ, and are, as history teaches, also changeable. There have been times in which long-legged horses were preferred to short-legged ones, and in which long shin bones were considered an advantage. I also believe that many so-called faults in a lorse of 100 years ago were more dangerous than they are to the present day horse, and rice versa. In short, our doctrine as to the conformation of a horse will always have its limits, and will often change in the future, according to the experiences which we gather from the racecourse and from the other uses to which the horses are put. Races and other tests of performances will in themselves destroy what is useless. If, however, Biedenweg's instructions exclude a priori certain conformation, we rob those horses which are supposed to br better of the opportunity of showing that they can perform better things.

The better form has not been proclaimed as such by the racillating opinions of judges, but acquired for itself the right to be judged as such by beating competitors. The history of Thoroughbred breeding teaches that even without such police rules as Biedenweg's instructions are, faults are finally eradicated automatically. The best example of an effective elimination of faults by racing is perhaps the walk of the Thoroughbred. I know of no breed which produces so many horses which walk so correctly as the English Thoroughbred. Where is the half-bred stallion in Germany which without freshness walks as correctly, gracefully and beautifully as . Ird Patrick, who, besides, won the Epsom Derby, and beat the four-year-old Rock Sand, and Sceptre? The eradication of horses with irregular walk is not done in England, as in the breeding of Half-breds, or as they do when buying hnrses for military mounts, by picking out the regular walkers for the stud or military service, but by training and the struggle on the racecoursc. Military conmissioners can be as strict as they like in refusing to bu! irregular walkers, but they will never be able to judge as keenly and as correctly as the winning post. Anybody can prove this statement if he will only examinc the same horses two years later at the troop after mancuvres. He will find there are more irregular walkers than in the racing stables. Thr regular walk of horsis that have not worlied, but are well led in, disappears very often with worling as buter melts in the sun. 
Race propositions must therefore offer sufficient opportunity to eradicate these different fauls, and not protect, for example, the roarers, not leave too much to chance, and not damage the temperament of young horses by too many short races. Those handicaps, so much loved by the bookmakers, for horses of all ages, with the light weights, for distances under 1 mile, have also mo value for breeding, and should be much more restricted than is at present, unfortunately, the case in England. The making of racecourses. for example, over uneven ground (as more detailed in No. 3), may also have a favourable effect on the form of Thoroughbreds by eradicating every. unharmonious conformation of racehorses.

2.-- Want of endurance for long distances. This reproach is the most common, and not to use a stronger expression, a very ill-considered one. We have proved that no other race of horses has attained the endurance of the Thoroughbred in many long distance races. Generally the above reproach is applied to the former long races over 4 miles with heats, as they took place in the time of Eclipse. We must, howerer, emphasise that the removal of these long races with heats was the first step towards progress. The errors made must be found somewhere else, and as we shall soon see, rne did not go far enough in the shortening of distances.

Not only representatives of Half-bred breeders, but also breeders and admirers of Trotters and Thoroughbreds, have always rightly aimed at the production of a horse with the greatest possible endurance as the goal of their breeding efforts. Is history shows us, however, they were wrong in demanding performances over too great distances in order to reach this goal. They forgot that races should not onlr prove which is the best, but that the object of this test, and of the training for this test, should be to improve the stock. I flat race, for example, over 20 kilometres or more (in Moscow there exists one for 20 versts) does not improve the stock but ruins it. The breeders of Trotters in America tried in the middle of the last century to get 100 miles covered in 10 hours. Ifter that they wanted 20 miles doing in 1 hour. Ther also attained this result. They, however, observed at the same time that the health of the horses suffered, that is to say, that horses did not improve, but rather their value as breeding stock depreciated. The practical Americans soon found that the right distance for testing breeding stock is the one on which one can train horses, i.e., prepare them without damaging their health, and they found-and I think they are right- that this right distance is 1 English mite. Russian Trotters kept to their long distances. The result is, firstly, the American Trotter has improved its record in 1818 of 3 minutes per mile to 2 minutes to-day: secondly, the American Trotter to-day shows more endurance for every distance than the Russian. The improved record of Russian Trotters since 1860 (there are no reliable statements for former years) is mainly due to the establishment of good racecourses (hippodromes instead of roads), and to the introduction of stlkies instead of the four-wheeled droskies, which 
weighed two and more puds heavier. In 1860 the six-year-uld Welisar from Chrenowoi, ran in a four-wheeled drosky 2 versts in 3 minutes, 27 seconds, and in 1896 the best record was in a sulky, 2 rersts in 3 minutes, $13 \frac{1}{2}$ seconds. Since 1893, the year in which sulkies were generally introduced, th record performances of Russian 'Trotters have made very little proseress indeced: for example, in 1896 the best record for $1 \frac{1}{2}$ rersts was 2 minutes, b.j $\frac{1}{2}$ seconds, and in 1907 the best record for the same distance ? minutes 14 seconds. "Trotting races for distances over more than 1 mile are justified and uscoful as a test for an individual horse in use, just the same as all hinds of long distance rides for "Thoroughbreds and Half-breds. Is a matter of principle. one ought to distinguish tests for breeding stock from tests for atock in use. The former have for object the improving of the production of capable. breeding stock, whilst the latter serve to show what the maximum performance is, and how it may possibly be best attained, if need be, without considering whother the stock is hereby damaged or not.

The question, what distance, looked at from the abose point of riew, is the right one for flat races, still requires solving. At the time of Eilipse the chicf races "ere run over distances of 4 miles. The classical Derby is now run in all countriesuver about 2,400 metres. The Grand Prix de Paris uver 3,000 metres. The Doncaster St. Leger 2,937 metres. The longest races are in France, in the Prix Gladiateur, 6,200 metres. In England there are now mo races over 3 miles. In Ireland there is still one over 4 miles, and two over 3 miles, and in Germany, Second Class Iutumn Meeting in Hoppegarten, 1, sul) metres. In the course of time the art of training has, of course, made much progress, and trainers have learned that gallops for more that $-2,000$ (1) $2,+100$ metres, also in preparation for the berby and longer distances, are bad. The gallops which were still in foree twenty years agu, and which were often run likw a race over the Derby distance, have almost totally ceased. Mlost traners incline to the opinion that such long gallops do not improse the condition of the horse, but rather the reverse. If that is so, it is a mintake to have flat races for longer distances than about 2,400 metres. It is a mistake to have races wer distances which do not improve the rightly trained sturk, but rather do it harm. To fix the right limit with certainty is very diffirult, but the Derby seems to be the utmost limit for a useful race distance. $\mathrm{My}$ own opinion is that for three-year-olds 2,000 metres, and for two-yearolds 1,200 metres is the correct and most useful test distance, and 1 should comsider it a sign of progress if all so-called classical racen, i.c., such as serve for breeding stock, were raced over these distances, as is the ease with the Imerican Jrotters. Fraining would not only be facilitated thereby, but the horses would also prosper more. To train horses for different distances at the same time, and especially for very shore distancess of s(o) (1) 1.400 metres, as is now demanded, is of no use whaterer for breeding stock. Ratces held for long and short distances, in order us use stock which is not used for breeding. may be useful and instructive, as long as the number 
of these races is kept in moderate bounds and suitable to the requirements. For the same reasons there should not be too many selling races and handicaps.

It is wrong to believe that races of 2,000 metres for three-year-olds and 1,200 metres for two-year-olds would give little chance to so-called stayers, and would thus endanger the aim and end, namely, the breeding of horses for endurance. WThat is called generally a horse with plenty of endurance, and therefore one specially suitable for long distances, is not the same as what is, called a stayer on the racecourse, as opposed to a flyer. I really believe that flyers are often more suited for long distance rides and other feats of endurance, as well as for steeplechases, than stayers.

The quickest pace in which a horse can gallop a certain given distance without endangering the speed necessary for the finish, I would like to call its special pace. This special pace diminishes in the case of every horse with the growing distance. In the case of horses which we call stayers, this diminishing of special pace is less than in the case of those which we call flyers. At the same time the former have to put in a less speed than the latter. The scale at which this decrease takes place, and the amount of reserved speed, show in which degree the horse is a stayer or a flyer. Here 1 would like to further remark that the stayer can increase its speed only very little or not at all after a pace which is under its special pace.

The special pace $t$ of the stayer is greater than the special pace $t_{1}$ of the flver. The staver, therefore, gains on the way per metre a distance equal to i. On the whole distance $d$ the advantage of the stayer amounts to $d$ M. If s equals the speed of the stayer, and $s_{1}$ equals the speed of the flyer, the question is whether

$$
\text { d. } \mathrm{MI} .>\mathrm{s}_{1}-\mathrm{s} \text { or d. M. }<\mathrm{s}_{1}-\mathrm{s}
$$

i.c., whether the flyer on a given distance can, through its superior speed, gain the advantage which the stayer has obtained on the way (about to the distance) on account of its greater special pace. If this be not the case, the flyer will have to increase somewhat its special pace, but only in so far as $\mathrm{s}^{1} \longrightarrow$ s becomes larger than d.M.

The pace in steeplechases is limited by obstacles and the ground. The stayer therefore in steeplechases is prevented from fully developing its chief force. The flyer, on the other hand, can make use of the pace necessitated by the ground, which perhaps just corresponds to its special pace, and put on a better speed at the finish. The less the obstacles, the more even the ground, the more advantage there is for the stayer.

In races of such great distances as in the above-mentioned 20 rerst race at Muscow, the racing galloping ceases more or less, and the special pace of the stayer as well as of the flyer (ceteris paribus, viz., in horses of approximately ecual (lass) becomes pretty equal. For this reason, in case of such unraceable distances, the flyer will very often have the advantage.

The above comparisons apply in general only to borses of approxinately 
equal class. A horse of the first class, for example, which is at the same time a stayer, may have a greater speed than a horse of the third class which is a typical flver. In a race between the two, therefore, the latter will not have need to put on his better special pace. In the case of such champions of the course as Gladiateur, Kinesem, Ormonde, Plaisanterie, Isinglass, etc., it will be very difficult, for want of a reliable standard, to clecide whether they are stavers or flyers. The best proof as to whether two horses belong to different classes will be found in the fact that one of them can beat the other with the tactics of the stayer as well as those of the flyer. Is a rule, I would say that racehorses between whom there is more than a stone bolong to ww different classes.

3.-Hant of clezerness. This reproach has a certain justification, as it very likely is possible to still further increase the cleverness of the Thoroughhred by a different method of rearing and other tests. In consecpuence of their peculiar rearing up, for example, the Steppe horses, or the horses brought up in the Ilauerpark of Sababourg (Beberbeck), are certainly superior to the Thoroughbreds in cleverness. It is reasonable to expect and also probable, that the race tests for many generations only on flat, level tracks, may catuse neglect of several useful and important qualities of the horse in use (riding liorses, school horses, hunters and military horses). To these qualities belong chicfly cleverness, which is best cultivated and tested in steeplechases. But also the shape of the flat racing track may have a farourable influence in cultivating and rewarding cleverness. Quite level and flat tracks, as, for example, those of Newmarket, are not suitable. Of the classical courses which 1 know, the Derby Epsom course is the nost suitable for testing and rewarding the ability to go up and down hills and to pass such sharp bends as are found on very few steeplechase courses. The Epsom Derby course is known as a hard and very reliable test. Its chief ralue consists in the following:

1. The very strong incline, about half a mile from the start, demands so much from the horse, that roarers, unfit horses, and those of inferior quality and without sufficient stamina, drop out rery early or hatre finished with the last incline before the winning post.

?. The uneren and often rarving ground, with its several sharp bends, requires great efturts in the cleverness of the horses.

3. I one-sided capacity does not avail. Such a one-sided capacity might exist, for example, in the special cleverness to climb up a hill well; it is possible over-built horses with strong hind quarters might excel here. Or, again, such at one-sidedness might enable them to go better down hill, which show's a better developed fore part than hind part. linally, this -at Epsom unavailing - one-sidedness might be a tou long galloping-stride (Caloppsprung). It is possible that this too long galloping-stride results from a conformation that has not the necessary symmetry, which enables the linerse to adapt in time the pace to an uneven sround and to sharp corners. This 
lack of symmetry would be less troubling on a level and straight course like Vewmarliet; perhaps it should even be of some use.

In the breeding of Ialf-breds, Steeplechasers have often been used with good results. In Beberbeck, The Colonel, twice winner at Liverpool, has produced well, and from him are derived the good and strong limbs which, through Optimus and Obelisk (the dams of which were daughters of Colonel) one often finds in Trackehnen. The number of Steeplechasers which have been suceessful in English 'Thorughbred breeding is indeed rery small. The best known example is Touchstone's grand-dam, Boadicea (Banter's dam), who, between its serenth and twelfth year proved itself an execllent hunter, and as a 19-year-oid produced Touchstone's dam. I great mistake in all propositions for steeplechases both here and abroad is that geldings have to carry 3 to 5 lbs. less weight, instead of having to carry is lbs. more. Such classical steeplechase races as the great Liverpool National should only be for colts and fillies, weight for age. Then also steeplechases would supply more useful sires. Everybody who has seen the Grand National steeplechase at Liverpool, run over a distance of 7,200 metres, with its 32 jumps, of which each is a great performance, and who could admire the 11-year-old Manifesto coming in as a victor for the second time among 2. , and 19 starters, carrying 1.2 stone, $7 \mathrm{lbs}$., will at once admit that such a performance is just as imposing as a Derby victory, and the only thing to be regretted is that Manifesto, like many other steeplechasers, was a gelding. The mare Empress by Roval Bloud, who ended her career as a steeplechaser as a winner of the Grand National Steeplechase in 1880, produced still 9 good foals, among them in 1889 Red Prince by Kendal, winner of the Lancashire Handicap Steeplechase, one of the best known sires in Irish 11 unter breeding, and an ideal mating stallion for llalf-bred mares.

The value of steeplechases as a breeding test depends on the kind of track. Steeplechase tracks like Iuteuil are less suitable for testing breeding stock, because the kind of obstacles and the crround there admit of nearly the same pace as in flat races, besides, the distance, acourding to the observations just made, is ton long to be a useful test for hat racing. Steeplechase courses like. Iuteuil ruin the material more than diliticult tracks like Liverpool, for example. Perhaps this accounts for the fact that the same horse (Peter Simple, Abd-el-Kader, The Culonel, The Lamb, Manifesto) has won and obtained places more than once in the liverponl Grand National, Whilst only one horse (Wild Monarch, won 1sis and 1879) has done the same feat in the Auteuil great steeplechase. In ohstacle course which has to be used also for the test of breeding material, ought to consist of difficult obstacles, and varying, if at all possible, uneven fields with sharp corners. The distance ought to be in proportion to the topugraphy and soil, about 6,000 metres, with a run in of at most j00 metres. Obstacles as well as the ground should prevent an uninterrupted flat race pace, and admit at most only of a good hunting gallop up to the distance (the finish), which, 
of course, must admit of a real struggle in the best pace, just as in a flat race. Such tracks would, of course, demand a much more careful preparation for riding and jumping than is the case at .Itueuil. Whoever burs the winner of the great . Iuteuil steeplechase hoping to get a good hunter "ill very likely be deceived. Horses who have elanced to win on such tracks as I have just described, must be so far prepared that they are alwats well in hand and always willing to suit their pace to the ground. They must be very carefully jumped, so that they may be able to get over large and various obstacles safely at the required pace and without giving tom much awaly. Finally, after having surmounted the last obstacle, they must still have chough speed left for the final struggle. A tust over such a cours. in, naturally, different to a test on the flat. The latter will certainly measur. more correctly the galloping capacity, whilst the former malses more demand in the cleverness, doeility, temperament, stecessful training, and, finally. 'nt the speed of the horse. All these pualities have great inflence in the practical breeding of horses, especially of military horses. 1 consider it an advantage that on sucl courses so-called flyers have a greater chance of unning than stavers, especially as the flyers are more symmetrically built. and have more energy than the stayers. It is very noteworthy and interesting that the development of al sreat speed over short distances reguires a horse to be symmetrically built. One will hind more often amongst stayers high-legred and narron horses with upright shoulders and straight pastern than anongst flyers. Noreover, we do not here speak about flyers which are inly very quick over 500 metres. but about such which, carrying 80 kilos, after a gallop of 6.000 and more metres, and wer about 30 difierent jumps, still retain enough energy, force and breath to be able to put on enough rpeed tu suceed in the final struggre. These are not only performances worthy of recommendation, but they are also suarantee for characters, which are especially valuable for all noble laalf-breds.

Enfortunately, such steeplechases, so important as tests of breeding strek, are becoming more and more satree. especially where bookmalsers "xrercise much influence on racing propositions, like they do in England, "wen for that racing propositions (many handicaps and short selling races). It is very clear that the special tritiners are afraid of the work and preparation newessary for horses for such steeplechases, especially as very fell of them are sullieiently conversant with it. This worle belonges partirularly to gentlemen riders, and here Germany is probably on top. This work will alse cultivate the love and knowledge which are essential to the future breeder of Thoroughbreds and Half-breds, and, finally, this work will produce and animate the love of sport and the daring which soon disappears in long perinds of peace, and without which every peesple would soon decay. The further spread and reserve of steeplechases. especially for enentlemen riders ur ufticers, might also prevent a modern return of the times of ancient Greece and kome, in which saves did the fighting and daneinge whilst the Grand Seigneurs losked on and applatuded. 
4.-Faulty and ton light fundament. This reproach, unfortunately justified, is the weals point in the whole Thoroughbred breeding. Every Half-bred breeder knows how difficult it is to improve by the infusion of Thoroughbreds the important fore-legs, and any rider who linows the Thoroughbred outside the racecourse will confirm that the galloping capacity of the same is a greater one than the fundament will stand. Whoever has ridden Steppe horses will know what good fore-legs can stand. In this respect, next to the Steppe horse come the Irish Half-breds. We have seen in Germany several such Irish Half-breds on our steeplechase courses. Certainly they broke down now and again, but they were put right and won races again, and they did performances on three legs which a classical Thoroughbred can very seldom perform. As typical examples of these kinds of performances, let me mention the following Thoroughbreds and Halfbreds :- Red Nob (Half-bred) 1866 by Neville, Et Cetera 18st by Town Moor, Teviot 1886 by Marden or Lucebit, Gardenia 1888 by Reveller, Handy Andy (Half-bred), Sixpence 1889 by Man-of-TVar, Balrath (Half-bred) 1893 by Alban, Sportsman (1 Half-bred) 1894 by the Dethroned, Scotch Moor 189.j by Town Moor.

It is fairly clear from the observations made in the chapter on weight differences, as well as from the list of horses whose fundament admitted of their successful use on the racecourse up to their eighth year, and even beyond that, that the two-year-old races, and especially those which are run early in the year, may be considered the chief cause of a general retrogression in the capabilities (Leistungsfähigkeit) of the Thoroughbred, as well as of the special deterioration of the fundament. A sequence of the early. two-rear-old races, as well as of the many early entry closings, is the breaking in of the rearlings, which often takes place in July, and the dangerous trials of same in autumn. The rearlings lose through these causes the benefits of grazing, which really cannot be over-estimated. How much the long-extended grazing influences the production of good fore-legs can best be seen with the Steppe horses and IIalf-breds in Ireland. It is clear that the morement connected with life on the Steppes, on hard meadows (in summer day and night), produces better, i.e., stronger, dryer and firmer fore-legs than the 2 hours' training and the 22 hours' rest in the stable of the yearlings. Besides, the American training method, to keep them in motion twice daily, and to give them plenty of walking exercise, seems in this respect to be rery effective, especially as it aroids the effeminate treatment which is resorted to in Europe without any advantage whatever. In America in 1893 I thought that the American Thoroughbreds possessed better fore-legs and a more regular walk than our horses, perhaps since then, however, even there the steady increase of races for two-year-olds in the early. part of the year (even beginning in January), may also have had a bad influence in this respect. The law which has been in force in France since 1867 that two-year-olds must not be run before the 1st of August is rery 
reasonable. Unfortunately, this law has been extended since 1907 , so that in July also, races may be run for two-year-olds (at most two on each race day), for a prize up to 5,000 francs, for a distance not over 1,000 metres. In Germany, since 1905, the 2nd of June has been fixed as the earliest date for two-year-old races. The above particulars on changes in weight differences seem to prove that the few two-year-old races not run too early in the year have had a favourable effect on Thoroughbred breeding. Yet $I$ believe that the complete elimination of two-year-old races would be the hest means to improve Thoroughbred breeding, and especially to improve the fundament. As long, however, as the brokmakers and their following are so powerful as ther have been hitherto, a change will scarcely be pussible in England in this respect. One may expect the unfeeling energy necessary to carry out such a trenchant rule, together with prudence and careful work, onl in France and Germans. In Germany, first of all, one might attain an improvement by forbidding two-rear-old races before the 1st of September, and also to offer the best prizes for four-year-olds and older horses, for distances of 2,000 or 2,400 metres.

If one, however, objects to this, the fact that most classical winners of about the last fifty years are derived from dams who ran and won as tworear-olds, the question seems to be justified, as to whether these clams have produced so well, in spite of, or in consequence of, the two-year-old tests. Those fillies as well as crilts which can stand the two-year-old race tests are, especially if they come out as winners, without doubt by nature the best and hardest horses, and can therefore produce well in spite of wo-year-old races. That the two-rear-old races are a good thermometer as to hardness, health, and quality of roung horses can be taken for granted, just as surely as we can accept their directly injurious influence. Besides, these young horses, through their early training, luse benefits which nothing can replace, such as the one year's grazing, which is especially useful for the whole constitution, and more particularly the fundament. Morenver, alsainst the fact that most classical winners are derived from dams which have also run as two-year-olds, we have the other fact, which I have discussed above, that horses with prominent performances at creat ages, as well ats the champions of steeplechases, are derived in the majority from stallions and dams which did not run as two-year-olds. If two-year-old races are altogether abolished, I would recommend, and especially for countries in which grazing is interrupted by a long winter, to break in the vearlings late in the autumn, to work them weil through the winter, and to send then again as two-year-olds in the summer to grass.

5. - Il ant of cupacity for carrying heary aieights. I du not consider this reproach justified. The statement so often heard that horses carried heavier weights formerly is only cortect in so far as the so-ralled hing's Plates are concerned, in which five and six-rear-olds and older horses mere tested under great weights up (1) 1.2 stone, mostly for 1 miles with heats. 
Besides, in the 11 King's Plates, the never beaten Eclipse carried, for example, as a five and sir-year-old, in its remaining 8 races, only 8 or 9 stone. In the second half of the nineteenth century 8 stone, $7 \mathrm{lbs}$. Was the usual weight for four-year-olds. The weights of the Derby, of the St. Leger, and the Oaks, have been increased by 1 stone since their inauguration. In the first races for two-year-olds (1780) the weight for colts was 7 stone, 9 lbs., and in the first classical races for two-year-olds (1786), 8 stone, 2 lbs., against 9 stone of to-day. In Germany the Derby reight is the highest, namely, 58 litos. Then follows England with 57.15 kilos. France and Austria with 56, America and Russia with 54.9. A greater weight than 58 kilos for three-year-olds in classical tests does not seem to be desirable, especially as the danger to the legs would thereby be increased without obtaining any advantages. A great mistake, however, are the feather weights, which are still usual in England in many handieaps and selling races. English earnest hippologists have often tried to do away with this, but the power of the bookmaker has alwar's prevented it.

6.-Meruousness, difficult temperament, and bad use of food. I only mention this altogether unjustified reproach on account of completeness. If a chronometer must go as well as it ought to, you must handle it in a proper manner, and the sane thing applies to the Thoroughbred. The worse and more unreasonable the rider, the less is he suited to handle a capable Thoroughbred. The Thoroughbred demands only a reasonable, not a tender treatment, and no gond Thoroughbred can bear, for example, to stand a whole day or longer in the stable. In right hands, the Thoroughbred is neither nervous nor dificult, and uses his food better than any other race of horses, especially when it works hard. The above reproach is a characteristic judgment of people who do not know the Thoroughbred thoroughly, and who cannot distinguish the racehorse in training from the Thoroughbred in use.

On account of the above observations, I make the following proposals:-

1. Forbid two-yearold races before the 1st of September, also under 1,000 metres.

2. Regulate distances for threc-year-olds and older in breeding races to $1,600-2,400$ metres.

3. Establish well-endowed races for four-year-olds and older for 2,0002,400 metres.

4. Lay out flat races over uneven ground with sharper turnings than is usual in Germany up to now, after the model of Epsom.

5. Arrange steeplechases as breeding races for four-year-olcls and older colts and fillies on courses with great obstacles, varying and uneven ground and sharp turnings, for 4,000 - i,000 metres, with age weights for gentlemen riders.

6. Abolish all races under $1,(100$ metres, all handicaps for two and threeyear-olds, all hurdle races, and the so-called Biedenweg rules. 
7.-- Ibolish the early entry clusings, as they lead to too carly trials, put heary taxes on breeders, and are the cause that good horses often rannot run in important races.

8. Increase breeders' rewards, so that more breeders may participate in the earnings of races, and irrerease the interest in the breeding of hardy. yearlings.

If, however, the representatives of Thoroughbred breding will decline to reorganise the race trials in the above-described or a similar manner, and will persist in going on as they have done up to now, and if, further, as is unfortunately the case in England, the influence of bonkmakers and other husiness people should increase in the framing of racing propositions, the proud words, "pro republica est dum ludere videmur," will soon be no longer true as regardi race tests. Half-hred breders will then be forced 1 , apply to their own breeding the care and expense which has been the cause of the origin of the Thoroughbred, and they will be forced to demand from their own breeding stock those tests of performances which have made the Thoroughbred sn capable. In other words, I Ialf-bred breeders will themselves have to produce a sort of Thoroughbred. Of course, that is a long way off, and several generations will have to work before that standard cin he reached to which the Thornughbred of in-day has attained. 

II.

Heredity. 



\section{CH.HPI:R I.}

\section{General Observations on Heredity.}

THE most important and difficult werk of the breeder is the mating of brood mares. It is acknowledged that the breeding of horses is more difficult than the breeding of our other clomestic animals. Darwin contends that only few people hase an idea what an amount of matural capacity, and how many. years' practice are neecsiary, to become an expert breeder of pigcons. IIow much more diflicult, more complicated, and above all, how much mere wearisome, is horst breeding : Judging from the results of mating. not only many bears' practice, but alse a sharp eye and an impartial consideration are essential. besides the breeding material and the soil which atre at the disposal of the breeler, mating and the manner of rearing are the fundamental factors in horse breding. Finn if we have to aclinowledge that the lirst work, mamely, mating. is the more dilticult part, Iet, on the other hand. the mistakt which most frepuently oceurs, not only in the breeding of horses, but also uf eur othere comestic animals, is that one expects everything from the sire and its mating, and omits to give that care to the rearing without which. (sern with the wisest mating and the best brededng material, mothingerseat is produced. The breeder's are must be able to distinguish what is due to the mating and what is due to the reatring with respect to the soil.

We do not know any gremeral rules or laws indicating which characters are generally ur mestly tramsmitted by the sire and which by the dam. The. former dexerine that the sire ustally iransmits the forequarters and the dam the hindequarters is met rorrect. liven il there are sires who mearly alwals transmit certain parts of the body or intellectual characters, there are, on the wher hand, dams who gremerally transmit the same characters. There does not exist a lised regularity of transmission, aither on the part of the dam or on that of the sire. Cenerally, other things being equal, both dam and sire hatre the same proneness to transmit the peruliar characters uf their bod! and temperanent down to the minutest particular. .11so, they may transmit. more or less, qualities which they do not themselves possess, hut which (as barwin eontends) one of their ancestors as far bak as lle twelfth gemeration josse'sised. 
Transmission works like the memory - often far distant, unimportant matters are recalled, often nearer and more important ones are passed over. 11 physiological psychology they speak of a muscular memory, even of a memory of matter. The supposition that transmission is also somewhat determined by the memory of the germ plasm is in the same manner justified. In contrasting, for example, different growths of the real and grafted picea, pungens, argentea, one can distinctly see how memory acts in transmission, as the ingrafted branch, remembering too much its position and duty on the mother tree, does not therefore attain that regular straightness which distinguishes its mother type, and which only can be produced by grafting with the top shoots.

Even if both parents generally take equal shares in transmission, yet in every instance different combinations of the mutual heritages will arise. This is the consequence of the results of the fights which are fought out between mutual heritages in the segmentation nucleus (fertilised egg). And these results cannot always be foreseen, and often change. The innumerable combinations which are thus possible are the cause of the different fine shades of individuality and, finally, also of variations.

In the Goos tables we find many examples of dams which had conspicuously cleverer sons than daughters, and rice-versa. There have also been many stallions whose transmission differed in the same way, for example, Stockwell and Newminster were distinguished principally on account of many good sons, while Melbourne and King Tom produced more good daughters. This peculiarity in transmission often continues through many generations, as, for example, through Melbourne to Wizard and Odoardo, down to the Half-hred stallion Optimus, which excelled rery conspicuously through having better daughters than sons. In Trakehnen, the Journey-mares excelled in their splendid produce, whilst the daughters of V'enezuela succeeded rery seldom. On the other hand, Venezuela's sires were much better than the sons of Journey.

Sexual characters are also transmitted in the same equal manner by the sire and dam. Stallions which fertilize badly, for example, also produce dams which conceive badly, and bad covering stallions also produce dams which come badly and seldom in heat. Even the qualities to produce twins are not transmitted by the dams only, but also by sires descending from families where twins have often been born. Sunflower, born 1813, for example, descended on its mother's side from a family in which there had never been twins up to its foundation mare, Lavton, Barb Nare (fam. 4). But her sire, Castrel, was own brother to Bronce, winner of the Oaks 1806 . who produced twins four times, and transmitted this quality several times, down to Rigolboche, sire of the Derby winner Cremorne, and even later. Sunflower also produced twins, and transmitted this quality to four conseculive generations, and perhaps still further. In this case we must suppose that her sire, Castrel, transmitted this quality. It was the same case with 
Crystal (born 1851 by Pantaloon), who was inbred to Castrel, and Selin, her dam, to Rubens and Selim, produced twins twice, again transmitting this quality, yet no ancestor on her mother's side ever had twins.

If a dam .11. has produced by a sire B. a prominemt sire product MI.I., and with several other sires less good dam products have been produced, the retention of the blood of the dam $\mathrm{X}$. by her son $\mathrm{Xl}$.I. is 10 be reermmended, as his daughters, if well done, are taken as brood mares to the stud. They will transmit the best part of the blond of dam M. improved by B. it is therefore not absolutely necessary, in order to preserve the blood of a wellproven dam, to breed always from her direct daughters. The same purpose is attained-a generation later-by breeding from the daughters of her son. The correctness of this procedure stands and falls, of course, with the generally acknowledged doctrine that sire and dam, all things being equal, take equal share in the transmission.

The equal value of sires and dams in transmission is contrary to the doctrine of Bruce Lowe, i.e., that certain breeding characters (Running and Sire families) are for several generations always transmitted by the dams. It can be seen already from this that the breeding theory of Bruce Lowe, from the Figure system, is untenable. In any case, Bruce I.no wrerestimates the effective power of transmission which a gond brood mare can maintain for following generations, notwithstanding the conseguent influencr. of other blood. If the Bruce Lowe theory that there are special Sire and special Running families were true, the transmission power of dams must be totally different to that of sires, and that is not the case. Besides, Bruce Lowe has not figured out the Sire-families in the same way as the Runningfamilies, but has estimated them without figures. In the book published in 1901 by $\mathrm{II}^{*}$. Allison, "The British Thoroughbred Horse," he gives on page 101 the number of stallions which have been produced by each family as sires of classical winners. The families according to that are as follows:-

Family 3 with 72 stallions.

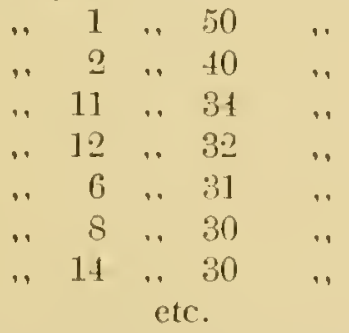

It is therefore quite evident that the Bruce Lowe Sire-families $(3,8,11$, 12. 14) are totally different to those ascertained by figures. Besides, the aborr table shows that the great difference affirmed by Bruce Lowe does not exist between Sire and Running-families. The three best Rumning-families, 1 , 3 and 3 (which according to $A l l i s o n, 1901$, range $1,3,2$ ), are, according in 
the above table, also the three best Sire-families, and the two Runningfamilies, 1 and $\cdot$, are, as regards the production of sires, far superior to Bruce Lowe's Sire-families, $8,11,12$ and 14. Even the outside fanily 6 , is just as good as the celebrated Sire-family 12, and better than the Sirefamilies 8 and 14. Thereby the aflirmed difference between Running and Sire-families is wanting. Moreover, this fact gives, if looked at from a special point of view, an interesting figure proof of the above contention that both parents, sire and dam, have an equal influence on their progeny. If the Bruce Low doctrine were true, then in the case of the present day racehorses of about the s.jth generation, the one foundation mare ought to have a greater influence than the other about $17,000,000$ ancestors, and she ought to be able to seal her Sire or Running-eharacter on her family. Very interesting-if it were true!

In the figure estimate of the different families as regards the production of classical winners in Running-families, as well as of sires of classical winners in Sire-families. we must take into consideration that a classical winner can only be counted once, or at most three times, acording as it has won one or three classical races. A sire of classical winners, however, can be counted one to about serenteen times, according as he has produced one or seventeen classical winners. Stockwell, for example, is sire of three Derby winners, six St. Legers, one Oaks, four 2,000 Guineas, and three 1,000 Guineas winners-altogether seventeen classical winners. Is a winner in the $: 2,000$ Guineas Stakes and in the St. Leger, Stockwell is marked with two numbers in the running quality of family 3 , and with seventeen numbers in the sire quality of the same family. I classical winner can only obtain number three for the running quality of his family, but for the sire quality he can, like Stockwell, obtain seventeen or even a higher number. This show: distinctly that the value of the figures which determine the quality of Running or Sire-families cannot be talien as a standard. Statistical comparisons of the figures which represent the elassical winners and of those which represent the sires of elassical winners, are, therefore. not justified, and lead to wrong conclusions. The followers of the Bruce Lowe doctrine, when judging of the pedigree of a prominent racehorse whose sire does not come from a Sire-family, surmount this obstacle by saying that perhaps the best sire blond was brought by the sire of the mother or by the sire's sire. This does not, however, reconcile itself with the fundamental idea of the contended transmission of sire or running qualities only through the dan's side. The proportion of running, sire, and outside families changes in the pedigree of a horse with every generation so much that the value of a horse differs according to the generation which is taken as a basis. According to one's wish, one could, from this point of view, judge a horse favourably or unfarourably. According to Chamant, for example, the proportion of sire and outside families changes as follows:- 
1. Ciencral Obnersillions on Heredily.

\begin{tabular}{|c|c|c|c|c|c|c|}
\hline \multirow{2}{*}{\multicolumn{2}{|c|}{ II. Generation, }} & \multicolumn{2}{|c|}{$\begin{array}{l}\text { Rumning- } \\
\text { family: }\end{array}$} & \multirow{2}{*}{$\begin{array}{l}\text { Sire- } \\
\text { family. }\end{array}$} & \multirow{2}{*}{$\begin{array}{c}\text { Jumily } \\
3 . \\
1\end{array}$} & \multirow{2}{*}{$\begin{array}{l}\text { Outside } \\
\text { f:umily }\end{array}$} \\
\hline & & 2 Incestors. & 1 & & & \\
\hline III. & .. & $4 \quad 1$ & $\because$ & — & 1 & I \\
\hline $1 \mathrm{~V}$ & , & . & 3 & 2 & 1 & $\ddot{2}$ \\
\hline $\mathrm{V}$ & ., & 16 & 1 & 3 & 1 & 8 \\
\hline VI. & ., & 32 & 9 & (i) & .1 & $1: 3$ \\
\hline VII. & , & 64 & 19 & 12 & 5 & 23 \\
\hline XVII. & $, .05,5$ & 530 & $7: 37$ & $1,-1339)$ & + & (i;i),:36i:3 \\
\hline
\end{tabular}

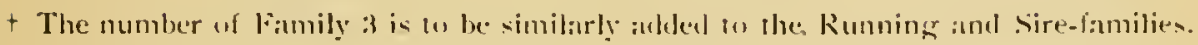

If Chamant had produced hadly. one could have made the exenes that he had, in the seeond and third generation, more running bleod than sire blesd. If he produces well, it is evident from the seventeenth greneration (or avell earlier) that he has more sire blood than running blond. These different ways of judging horses are indeed aluass possible for our present day horses, but not for the three foundation sires of the linglish Thoroughbred. for Eclipse, a marvel on the racecourse, had, just like IIerod, who between the age of 5 to 9 years won in (i races, no rumning bleed whatever in his pedigree, whilst liatehem, who between the age of 5 to 10 geatrs wom in 10 races, can show very little running bleod (only family 1) and no sire blook at all in his pedigree. All this ought to be sufficient to show how untenable Bruce Lowe's breeding theory with the number system is. Even if cleverness in the grouping of figures and cliverse statistical tables oftron seem to suppert the Bruce lowe theory, one only needs to be reminded that tigures prove anything, and ofterl give very remarkable answers to fochlish or unjustified questions. II $i$ th the same justification we could alse divide the blend of the sires into Running and Sire-families. Bruce Lowe's breeding theory, with its figure system, belongs to those errors which are fairly harmless, with this advantage even, that breeders now trouble themselves more with the pedigree of the breeding material than formerly. (Charles Richardson ("The English Turi," page 2:-27) rightly settles the whole Bruce lowe dectrince by saying: "I had some thought of criticising this figure sy'stem theory, but, never seriously taken up by breeders, it is alread! a dead letter, succumbing through sheer inanition."

The influence of sires and dams on their progeny varies in reality from the fact that the sires are, on the average, more impertant representatives of their race than the mueh more numerous dams can cree be. It is, therefore, to be assumed that the sires will oftener imprint their individuality than the dans. From this point of view can be explained the justitication and the especial value of the tables of breeding sires in the male line, ats I have shem in the tables which follow. The recognised Goos tables in the fomale line up to the respective foundation dams are pre-eminently suitable for the correct judgment of Thoroughbred dams. The breeding tables of broud mare's, com- 
piled according to the families on the dam's side, in the same manner as the Thoroughbred dams in the Goos tables, are used to serve this purpose in the stud books of the Roval studs at Trakehnen and Beberbeck. In these tables all dams which have produced nothing great are left out. The classical winners in the Thoroughbred tables correspond to the Royal stud and country stallions. Is in all breeding of animals, by far more female than male animals are necessary, the stallions will on an average excel the brood mares in quality. The chief thought expressed in the Goos tables, as well as in the tables just mentioned in the stud books of Trakehnen and Beberbeck, is therefore based on the claim that a division of the blood on the mother's side may also take place. Through it the practical breeder will obtain an easy means to judge rightly and quickly the chances of a good heredity in every single instance, and the investigator obtains an easy survey and insight in transmission affairs. It is a mistalie, therefore, to conclude that the dam has a greater influence on the progeny than the sire, whilst from the above, it only follows that a good or prominent mare has more, i.e., more favourable influence, than a mare of less value, and that it is therefore important to use as good a dam as possible. It is taken as a matter of course that the stallion is good, especially as he can be more easily obtained, i.c., for a single covering, than a good mare. 


\section{CHAPTER II.}

\section{The Transmission of Acquired Characters.}

Tue ingenious Darwin theory of natural selection in breeding has latterly been much attacked. Critics, however, have not ret substituted it by anything better. The development of the genus horse is a proof of the Darwin theory. The destruction of the unfit is, according to Darwin's theory, casy. to prove. The creation of the fit, which he speaks of, is explainable by the transmission of acquired characters, without it, it remains an enigma. The controversy as to the transmission of acquired characters seems to me to have become a war of words, just like the controversies about pure breeding. constancy and individual prepotency. The opponents of the doctrine of the transmission of acquired character admit that those acquired characters of the body, which when acquired influence also the germ cells, or, to speal: more correctly, the corresponding so-called "determinants" of the germ cells, can be transmitted. The question therefore arises. Which of the characters acquired by the body do not influence at the tine when they are acpuired corresponding these germ cells? The above opponents admit also the effects of climate and food, for example, on the body and on the germ cells and the transmission of characters thus acquired. The transmission of immunities has also been proved by 'Tizzoni, for example, in the case of raby-proof rabbits and tetanus-proof mice, and by Ehrlich, in the case of rizin and abrin-proof mice. Mares which have suffered from severe glanders and got over them scem, as far as mo observations at Beberbeck and Trakehnen go, to transmit a certain immunity. Of course, it is possible that this transmission of immunities by the dam is deceptive, and has only been transmitted to their progeny by the milk of the dam. I have never been able to prove that sires transmit such immunities.

.Il characters acfuired by the body exercise an irritation through the acquirement. $11 \mathrm{c}$ must, therefore, assume that the germ plasm nourished by the body, and thus connected with the same, is subject to every such irritation, even if only in a very small degree. The cutting off the tail, as in the case of dogs, very likely produces an irritation of the gern cells, which is, however, not strong enough, and not of such a lind that transmission 
without a tail follows. The cutting off the tail in the case of dogs belongs, as a matter of fact, just as little to acoutired characters as the circumcision of lews: both are changes made from the outside, not acquired characters. Only irritations which for a long time and regularly. especially in early years, have led to the acquirement of certain characters, as, for cxample, the trottingr training of Trotters, which commences when they are weaned foals, will be able to inflence the cerresponding "determinants" of the germ cells in such a manner that a fatrourable aptitude for the charaters acquired from the parents is more or less transmitted. Thus is explained the transmission of changes produced by the continuous influence of sround. The best example for this which I personally know is the previously mentioned change of the Percherons in the Russian Government Stud, Derkul (Government ("harkow), which was caused by the Steppes, and transmitted. Darwin has especially emphasised the great influenee which ground, climate, food and manner of living exereise on the complete organism of animals in every lind of breeding. Important changes in the breeding steck may be effected by changes in the mode of living, which brecelers can bring about. Practical horse breeding is based on these important and fundamental truths, and reckons with the transmission of many acquired charaters. To these belong first of all, health, power, hardiness, habits, action, and the construction of the sleletom, combined with the action and changed by cortain exercises. For example, the firmly established and early begun galloping exercises of the Thoroughbred have transformed the previous more curved hind legs into more straight ones, and the less developed withers into prominent ones. On the other hand, the trotting exercises have, in the course uf sereral generations, produced straighter pasterns, longer middle, and flatter withers. likewise the more upright shoulders of the draft horse, su suitable for dratling heavy loads, have been produced by degrees and transmitfed in consequence of constant practice in drawing heary loads through many consecutive generations. The deformation of the slefeton. which the Frenchman, De Gasté, takes to be the result of exorbitant trotting exercises, consists for the most part in a prolongation of the ilium, a stunting of the ischium, and the diminution of the shoulder angle.

Foals from parents which have been harnessed in their vouth are more easily brolien to harness than foals from parents which have never heen harnessed. It is more difficult to break in for riding foals the parents of which have nest been ridden or very little than those from parents which have been riclden for a long time. This is most noticeable in the case of breaking in young horses for jumping. In Trakehnen the brood mare Pirna, probably in conseguence of an accidental opportunity, had accustomed berself not to be fastenced up, or if she were fastened up, to get louse by cunning or foree. Pirna transmitted this quality to several of her progeny. In the celebrated stud Palo. Alto (California), October, 1893, l noticed as a very remarkable thing in the meadows that the foals of Trotters, unlike foals of 
Thoroughbreds, nearly alwaly trotted, if they were urged on hy at handierchicf or whip. There was only one foal which galloped more than trotted, and that foal was the product of a Tretser statlion out of the Thoroughbred mare Satanella. Is is well known, the Imericans liked until recently to infuse Thoroughbred blood into their Trotters. The staltion Pale. Mleo and mare Maud S. are the best examples of this experiment. Briefly, the transmission of habits and faculties is in horse breeding a fact provable by many Namples, and the most impurtant means for the improvement of the breeds.

. Is Darwin has prosed by many examples, however, bot only hahits and reverness are transmitted, but all those characters which are acquired by continueus exercise of the same and acoording to the effect of the atcquirement they may either injure or benefit the breed. In the following are given a fer examples showing that the explanation of progress or retrogression in breeding by variation and selection alone seems to be insufficient.

In . Iugust, 1906, in Trakchnen, anong the tho-year-old fillies which were destined w become brood mares, the following ten fillies. by reason of their ancestry and conformation, were put on training lor the IIalf-bred breeding races 1907 in liönigsberg:- -

1. Meleedrosscel by Pomp and Achtbare by Optinrus.

2. Lantenspielerin by Geheimrat and latute by lehnsherr.

3. Leibeigene by Pomp and Leibrente by Optimus.

4. Pocsic by Gehcimrat and leststrasse by lechnsherr.

5. Irt by Pomp and . Irgolis by linin.

6. Jutizäatin by Geheimrat and Justicia by Passian.

7. Julisenne by Greif and Julia by Oreus.

r. Hohkönigsibers by lomp and Iohke by Apis.

9. Lore by Pomp and Louisa by liffenbein.

10. IIeimathe by Pomp and Hloidenelfe by Elfenbein.

If we consider the three different Thoroughbred sires (Pomp. Geheimrat. and (ireif) of the above te:n fillies of egual value, we should have, first of all, to judge the sires of the dams of these ten fillies in order to rightly estimate, by reason of the ancestry of the mares, their racing chances. If we helieve in the transmission of acquired characters, we must assume that the two IIalfbred sires, (Optimus and lechnsherr, both of which were brought up in the Mauer l'ark of Sababure, near Beberbeck, had there acequirrod hetter faculties for groing, hardiness, and stronger heart and lungs, and all things being ecpual, ought also to have transmitted these characters better than the stallions Orcus, Apis, l:lwin and lifenhein, which were brought up in Trakehnen, and therefore hat less chance of exereise and galleping from their earliese youth. loxeled at from this standpoint, the merit of the ten fillies in the last autumn trial. 1906. Was about in the same order as shuwn b! the above table. The last four were soen after talien awal from rare traning. In the summer, 1907, Cos. jand fi were the slowest. Finally there nnly remained the four 
grand-children of Optimus and Lehnsherr. On account of influenza in Trakehnen, in July, 1907, we were unable to send any horses for racing to Lïnigsberg. The race took place, therefore, on the 20th July, 1907, at Trakehnen, and there the four fillies passed the post in the same order as above list.

The well-known . Imerican Trotter, Mambrino Chief (II.) by Mambrino Paymaster, had already been used as a stallion for breeding of Trotters hefore he was trained for trotting races. He produced badly, however, and his progeny were not able to win any trotting races. Nambrino Chief was then trained several vears for trotting, and afterwards transmitted so well the faculty for trotting that he became one of the most favourite trotting sires in Kentucky.

We can find many similar examples of this in horse breeding, and it would be somewhat artificial to explain this striking fact by variation and selection alone. Above all, time necessary for variation and selection is wanting. It is also unjustifiable to explain by variation and selection alone the retrogressive movement in Thoroughbred breeding-as illustrated above - as a consequence of the many and early two-year-old races. The deterioration of the fundament of the Thoroughbreds, which increases slowly from generation to generation, remains a puzzle without the transmission of acquired characters. Selection will, for all that, always try to avoid horses with bad fundament as much as possible, and a bad fundament can scarcely be considered as a necessary accompaniment of variation, which produces at the same time racing capability in early youth. The fact that racehorses possessing such a good fundament that they still win flat races as eightyear-olds and older are mostly derived from parents which did not run as two-year-olds can, without a transmission of acquired characters, only be explained if a good fundament were an obstacle for successful racing as twovear-olds. On the contrary, we find in the above tables (pages 108-141) many examples proving that horses which did not run as two-vear-olds, but up to their eighth year and older, successfully produced progeny which distinguished themselves as two-year-olds on the racecourse. Dr. Syntax, for example, ran and won between the age of 3 and 12 vears, and produced from several mares which had not run as two-vear-olds, two-year-old winners, amongst them the celebrated Bee's W'ing. How is it, then, that the reverse happens so much more seldom? All these and similar occurrences can be much more easily and more naturaliy explained if we accept the theory of transmission of acquired characters.

In the history of Thoroughbred breeding, one may give as an example for the transmission of acquired characters, the fact that many Orientals which were imported into England after Godolphin Arabian, i.e., after 1730 . did not inherit the capability for running in the same measure as the children and grand-children of Godolphin Arabian (Cade and Matchem), or as the descendants of the already earlier imported Byerly Turk, Darley Arabian. 
and many others. In spite of the less weights which were accorded to the offspring of Oriental parents in several races (in the Goodwood Gold cup. for example, $36 \mathrm{lbs}$.), the later imported Orientals could not compete any longer against the progeny of the earlier imported ones. On the other hand, the offspring of Herod, Highfyer and Eclipse were given in many races extra weights of about 3 to $5 \mathrm{lbs}$., as a contrast to the offspring of Matchem and Conductor, as descendants of Godolphin Arabian, who was later imported than Byerly Turk (foundation sire of Herod and Highfler) and Darley. Arabian (foundation sire of Eclipse). In consequence of the transmission of acquired characters, the heritage, with respect to racing capability, has slowly increased. The other explanation of this fact, i.e., by variation and selection, is, if we consider the few generations and the not too numerous individuals coming into consideration for selection in which the difference of transmission was already distinctly recognisable, somewhat artificial, unless we suppose that the variation of the germ plasm has been at least guided by the acquired characters of the body. Finally, the followers of Weissmann's theory may say that it is not the acquired characters of the body which have guided the formation or variation of the germ plasm, but that the activity necessary to acquire new characters had a direct influence on the germ plasm. In my opinion, the whole controversy about the transmission of acquired characters seems to terminate on this or that wrangling.

The belief in the transmission of acquired characters is for the breeder the chief stimulant to diligent and well-considered work. Without this belief there would be a great danger of breeding material being neglected. The breeder would have much less pleasure in his work once the most interesting part of stud work disappeared. 


\section{CHAPTER 111.}

The Doctrine of Constancy and Individual Prepotency.

THERE has been much dispute over the question as to how surely the characters of breeding stock, and especially the just-mentioned changed characters of same, are inherited. With the exception of a few extravagant, short-lived cloctrines (as, for instance, that of Buffon), the unimportant deriations of the different doctrines from one another are just as remarkable as the great disagreements of the doctrinaires. Even in ancient Greece, about 300 years B.C.. Aristotle tanght in his celebrated work, Historiu Animalium, if somewhat less thoroughly than it is taught to-day, the same theory of constancy and even individual prepotency, just as later the professor of the Vienna veterinary school, Johann Gottleb Wollstein (born 1737), and his eminent disciple the Imperial Stud Inspector, Johann (hristoply Justinus (died 182.4), did. The doctrines of the old Justinus (Allg. Grundsätze zur Vervollkommung der Pferdezucht, Wien 181.5), which are considered classics still at the present time, have been later partly rearranged more precisely but at the same time have been impaired by won Weckherlin. H. v. Nathusius and Settegast. There are often only wordy wars, and doctrines expressed carelessly and regardless of correctness, causing a long book war. It is difficult to decide who was the original author of the doctrine of individual prepotency. Of course, the teachers of the present time hate the advantage of regarding these questions from the pedestal of the latest biological researches, and they are therefore enaloled to give to these questions more precise and more explicit answers, which, above all, are scientifically better founded.

Heredity in horse breeding is all the more sure, i.e., all the more corresponding to the purpose of horse breeding: in short, is more constant the longer the line of ancestors which has been trained in serviceable management to acquire the intended performance, and which has been farourably tested. Of course, the word "constant " admits progress in the intended capabilities, and only designates the constant direction of the breeding. II. V. Nathusius calls the products of capable parents "highly bred." According to this the 
above sentence could be summed up as follows: The heredity in hores breeding is all the more constant the more highly bred ancestors are found in the pedigree of both parents. In so far as in so-called pure breeds a guarantere is given that their ane estors are highly bred, the albere senteme could also be read: The heredity is all the more constant the purer the breed is bred. Purity of breed is, of course, not meant in the zoulogical sense, as H. l'. Vathusius requires. Acourding on this we rould not call the Mlerinos, with their obscure origin, and not eren the present day Thoroughbred, pure bred. The purity of breed coming here into consideration does not only require at pure pedigree, as recorded in the stud book, but also the prouf of a rational rearing, as well as of sufficient performances of their respective ancestors. For example, Thoroughbred breeding would lose erery prospect of success if breeding material were used which latd been pampered without training and tests, especially if it is continued for several generations. Such breeding stock would not transmit constantly in the sense of their breed, but hy transmitting their own acquired characters, consisting in effeminacy and lack of capabilities, they would transmit a character opposed to the object of breeding, i.e., they would not transmit constantly in the sense of their race. In natural breeds, as, for example. Steppe horses and drabs in the?r own homes, purity of breed, as regards pedigree and capabilities, is guaranteed more or less by local conditions and espectally hy natural selection in the fight for life. The surer this guarantee is the more can one rely on a constant heredity of these natural breeds. In covery breed where the method of management is irrational, at first the intended capabilities disappear, afterwards constant heredity, and finally, after several generations, more or less

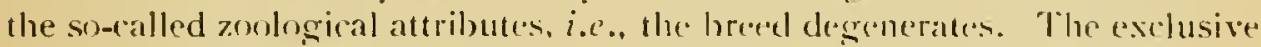
attention paid to a recorded pure pedigree (and as long as it is possible of the just-mentioned rowlogical attributes, without taking into consideration the performanee. which really is the base of all) hats, with the aid of unreasonable shows, already ruined many formerly capable breeds, as, for example, the Suffolls, the Cleveland bays, atrd in recent times perhaps alon a part of the Clydesdales.

livery sensible breeder of Thoroughbreds or Tronters can whtain from the stud book and race calendar the mecessary information for the eorrect judgment of the breeding stoxki from which he may expeet it constant heredity, i.e. one which serves the purpone. In the breeding of laalf-breds judging is, of course, someu hat mere ditheult, as the ate ate ner rate calendars. but it is just as necessary, and will generally be based on the manner of rearing and the condition of the soil. In recent times some stud books of Half-breds give some information, as they record esperially the brood mares which have received prizes at shows. These hurses which have been rewarded at shows cannot generally be recommended when breeding for performances. There are horses which, for example, arcording to their 
pedigree, are pure Trakehners, but having been irrationally reared (perhaps they have not been taken to grass, or had not sufficient exercise), they cannot be taken as pure-bred Trakehners. That breeding with such stallions in Trakehnen cannot lead to good results, therefore cannot have a constancy, in spite of so-called purity of breed, is a priori probable, and I have experienced it myself at Trakehnen. The performances and other characters required in every kind of horse breeding are much surer of being transmitted the longer the line of ancestors which have been bred on favourable soil in connection with a rational method of raising, as well as of the selection of the most capable stock for breeding. By improving the soil and other things, the intended characters of the products can also be improved. The heritage, which in this way becomes more valuable, is the cause of progress in all kinds of breeding. Neither Justinus nor the other old teachers of the doctrine of constancy have ever believed in uncliangeable breeds in the sense of the progress of their performances.

The chances of success in crossing different breeds depend on the compatibility of the most important characters which the two breeds possess, and on the possibilities of realising the new breeding form aimed at by crossing and its performances to be produced. Unreasonable wishes will remain wishes here, as everywhere else. Cross breeding, even if it otherwise miscarries, acts on fertility and good constitution just as favourably as fresh blood. The best example of a successful cross breeding in the history of horse breeding is the mixing of the American Trotter and Thoroughbred. The chief characters of Trotters and Thoroughbreds which are required to be combined are hard sinews and bones, well developed lungs, strong heart, action, and healthy nerves. Coarse crosses of either English or Oriental Thoroughbreds on Draft breeds may be prominent products well adapted to certain practical uses, but not to breeding. Darwin writes about cross breeding and its success as follows: "The possibility to form different breeds by cross brecding has been very much exaggerated. Certainly many cases are known which prove that a breed can be modified by an occasional crossing of certain individuals carefully chosen, and which possess an intended character. It would, however, be very difficult to produce a new breed which would represent a good average of two different breeds or kinds. Sir J. Sebright has made special experiments in this respect, but without success. The progeny from the first crossing of two pure breeds is, as I have found in the case of pigeons, fairly, and sometimes extraordinarily, alike in character, and everything scems to be simple. If one, however, pairs these crossbreds for a few consecutive generations, scarcely two of their progeny will be alike, and one gets a clear idea of the great difficulty of success."

There are breeds which are not related to each other, but which pursue the same purpose and have attained approximately the same results. According to what I have just said, there would be no objection to the mixing 
of such breeds. On the contrary, such a mixing, especially with a breed which ranks higher, on account of performance, would act farourably as an infusion of new blood, i.e., it would tend to farourably influence constitution, energy, vigour and fecundity. Such mixings between the Thoroughbred and the different noble breeds in all parts of the world have taken place with good results. The use of Thoroughbred sires in the Steppe breedings of Russia has led to very good results. Arabian and Persian stallions have produced well in the East Prussian Military. Studs. In the same way also, the mixing of a military horse bred perhaps in Australia, and possessing the neessiry characters, with the East Prussian horse breed, would probably have good results. On the other hand, however, it seems to me that a mixing of the Russian and American Trotters would be very precarious, not on account of the various blood which predominates in these two breeds, but on account of their very different training. caused by different race propositions. The manner of rearing these two Trotting breeds is just as different as their conformation. The characters of the American Trotter, trained for the mile, cannot be so easily mised with the characters of the Russian Trotter, trained for longer clistances. These two breeds are not of equal value regarding the kind of their performances, they possess different forms, and cannot therefore be classed as of wholly equal breed. The mixing of the same would not be an infusion of fresh blood, but almost a cross, with all its dangers, but yet not without prospects for a final success after long and systematical breeding.

In every breeding, may it be ever so pure, and may the rearing of its progeny be ever so rational, one will always find inclividuals which transmit the desired breed characters particularly well, and, on the other hand, individuals which transmit them badly. The ability of breeding stock to transmit the characters desired in the case of every breed in a prominent manner is called individual prepotency. Sires as well as mares may have a special individual prepotency, even when (if only exceptionally) they do not themselves possess in a prominent manner the character which they. prominenty transmit. On the other hand, there are sires and dams which, although themselves possessing in a high degree the desired breed characters, transmit the same very seldom or not at all. There har bern Thorouglibreds which belonged to the best on the course, as, for eximple. the wo champions of the racecourse. Cotherstone and Ciladiateur, and yet their progeny performed litte or nothing at all. I have linow n horses with revellent hocks, and again others with a splendid action, nevertheless, their progeny was noted for bad hocks, or a bad action. Of course, this lack of transmission is not the rule.

()ne of the most important tasks of the breeder will be to get prominent sires with special individual prepoteney. I'hether those individuals which excel by new formations of nature (according to Darwin's single vatration) 
are-as Settegast says - endowed with a special individual prepotency, is a question which, as far as horse breeding is concerned, cannot as yet be definitely answered. In any case, male products in horse breeding are more prone to take on individual variations than female products. The aggregate of filly yearlings is therefore always more equal than that of colt yearlings. Of course, one will naturally prefer to use stallions with individual variations or moditications which appear favourable for the purpose of breeding. and to establish and make the greatest possible use of these characters by inbreeding. It is possible that generally such stallions are specially endowed with individual prepotency. 


\section{CHAPTER IV.}

\section{Inbreeding.}

THE investigations of scientists in biology and transmission have made, nut conly in Germany, but also in other civilised countries, especially in England and Imerica, most considerable progress. Numerous regularly appearing periodicals have lately been created regarding this subject, amongst which is one that is especially worthy of notice appearing in England since 1901 under the name of "Biometrica" (Professor Pearson), in which-as the name already indicates-all mathematically tangible phenomena of biology and transmission are discussed. Laws of inbreeding are, however, to my linowledge, neither formulated in our country, nor in the just-mentioned "Biometrica." 1t appears to me the reason is that the material for such investigations in animal breeding is available to a sufficient extent only for the English Thoroughbred breeding, and this field is, unfortunately, up to the present not wholly familiar to the scientists. Even the book by Davenport ("Principles of Breeding"), published in America, 1907, which gives many very interesting insights into the mathematically-expressed laws of transmission, leaves somewhat to be desired in its chapter on inbreeding. From the Thoroughbred of to-day are already known 20 to 25 generations of their genealogy. We could therefore make their pedigrees, with 1 to 17 millions of ancestors, whose abilities and capabilities are to be seen in the racing calendars since 200 years. It shows how enormous is the material we possess in the Thoroughbred, and that it is without equal in any other breed!

Common ancestors constitute the basis of inbreeding. 13y "free generations" is meant the number of generations between the common ancestors and the sire on the one side, and between the common ancestors and the dam on the other side. In the following example of Stockwell, the common ancestor, Orville, is removed from the dam. Pocahontas, by two generations, and from the sire, The Baron, by three generations. Therefore the common blood of the basis, Orville, must altogether run five generations before entering the blood of Pocahontas and The Baron. From this is derived the 
term "five free generations." It can also be seen from the same example that Waxy and Penelope form the basis of a different inbreeding with six free generations, three on each side. We further can notice that The Baron himself has four free generations to $\mathrm{IT}^{\mathrm{T}} \mathrm{xy}$ and four free generations to Penelope, and accordingly supports or increases the inbreeding of his son Stockwell on the same basis.

Stockwell

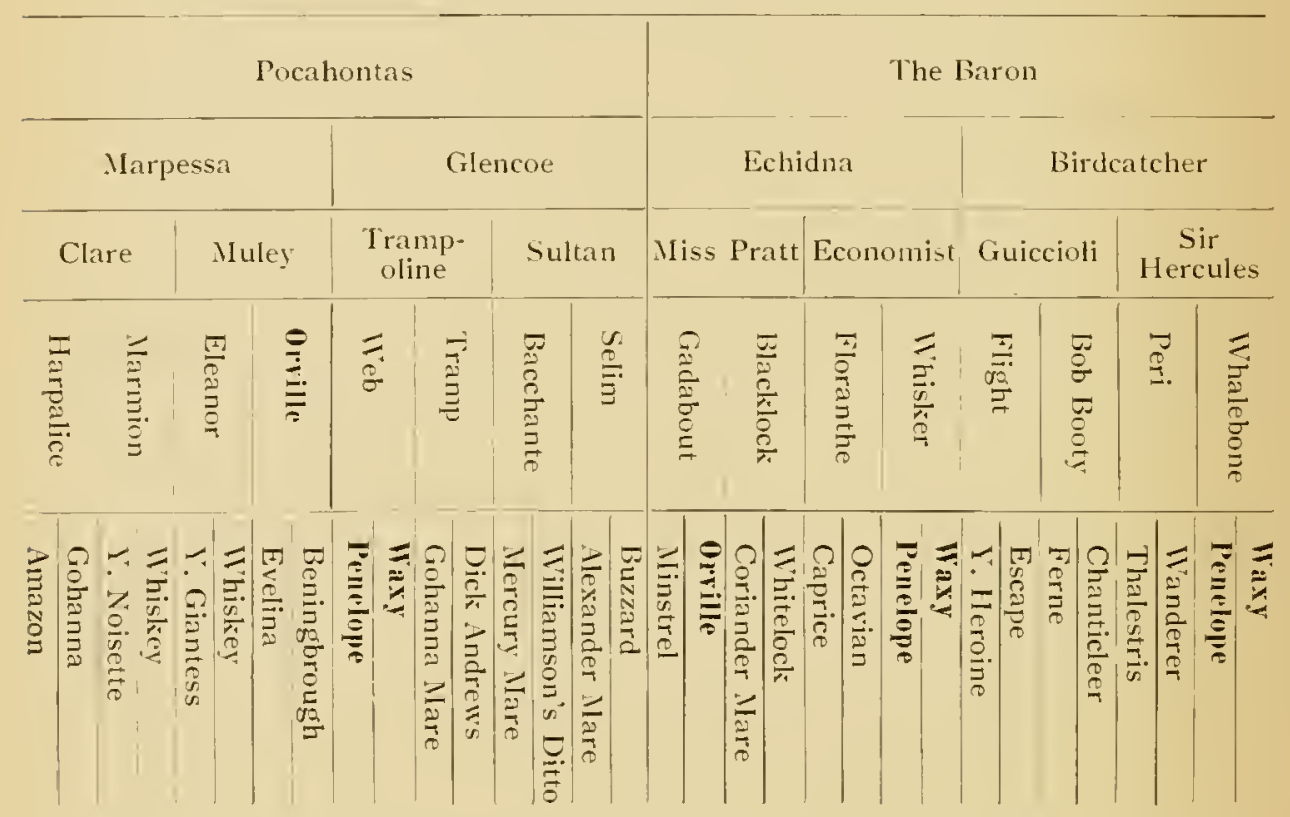

If sire and dam come from the same stallion (mating of brother and sister), that is an inbreeding with 0 free generation, and when it happens that a stallion is sire and grand-sire on the dam's side, that is to say, when he produced a foal out of his own daughter, that is an inbreeding with -1 free generation. In fixing the number of free generations, no notice is taken to which side they are allotted. On the other hand, in giving the number of removes, it is seen at once how many free generations are on the dam's side and how many on the sire's side. For instance, Stockwell is inbred to Orville in the fourth and fifth remove. It is usual in this designation to count both parents, commencing with the dam. It seems to me, however, improbable that finer shades in the laws of inbreeding can be detected by the help of removes than by that of free generations. I personally have not succeeded. In making these attempts, one is apt to run into the danger of getting together certain interesting geometrical groups, and to shape out fanciful and imaginary things, and in this way lose sight of the real facts. 
I myself know of no law of breeding which can be settled or confirmed by the sistem of removes.

Both parents, as well as all ancestors, have heredity shares on the offspring. The question now arises how great is the share of each of them. The second generation consists of two parents, both of which possess equally great shares. To each of the parents may be therefore ascribed half. Now the question arises how much of this half each parent individually transmits, and how much of it he transmits as mediator of his ancestors. The heredity share coming from each parent must therefore be considered to consist of two parts which-as is shown at once-must be equally great. It first a still unknown fraction e is supposed to be the individual heredity share of one parent. Both parents therefore individually possiess 2 e shares, and figure as mediators of the remaining part $1-2 \mathrm{e}$, which is ascribed to the wal of the ancestors. Thereby follows the demand that the fraction e is also ralid for all former generations. Accordingly the four grand parents have the share $2 \mathrm{e} .2 \mathrm{e}=4 \mathrm{e}^{2}$, the eight great grand parents $2 \mathrm{e} .2 \mathrm{e} .2 \mathrm{e}=$ $8 \mathrm{e}^{3}$ ete. The total heritage will thus be represented by:

$$
2 e+4 e^{2}+8 e^{3}+16 e^{1}-. . . .=1 .
$$

in which the 1 figures as the entire heritage. The value of this series quickly decreases until they soon become inlinitesimal. From the above equation 1) one can eliminate -2 and write instead:

$$
\left.2 \mathrm{e}\left(1+2 \mathrm{e}+4 \mathrm{e}^{2}+8 \mathrm{e}^{3}-. . . \quad .\right)=1 \text { - } .\right)
$$

As the value contained in brackets near the 1 is equal to the series in equation 1), it follows by substitution 2 e $(1+1)=1$, therefore e $=1$ (4_-3.)

Thereupon follows that each parent individually transmits only the half of its total heritage, while the other half, also $=14$, is transmitted on the offspring by each parent as mediator of all his ancestors. This is known in England as the so-called Gaiton's law of ancestral heredity. But whether and in what manner Galton has furnished a mathematical proof of it is unknown to me.

Table 1. gives a scheduled classification of the heredity shares for 10 generations, and Table 11 . the same in a lucid pedigree form. One sees that not quite I-millionth represents the heredity share of an ancestor in the tenth generation. As it is inconvenient to count with fractions, one can multiply all numbers by $26: 114$. Thereby the heredity unity is the share of an ancestor in the tenth generation, accordingly $=1=2$ "and the heritage of the offspring equals $2621+4=2{ }^{18}$. The last column in Table 1 . shows the heredity share of the various generations. One sees that each higher generation has the half of the share of the preceding one. Below is shown the total of all generations up to and including the tenth. If one adds the remaining heritage of the still further removed generations of 512 the whole heritage of the offispring $=2^{18}$ is obtained. 
TABLE I.

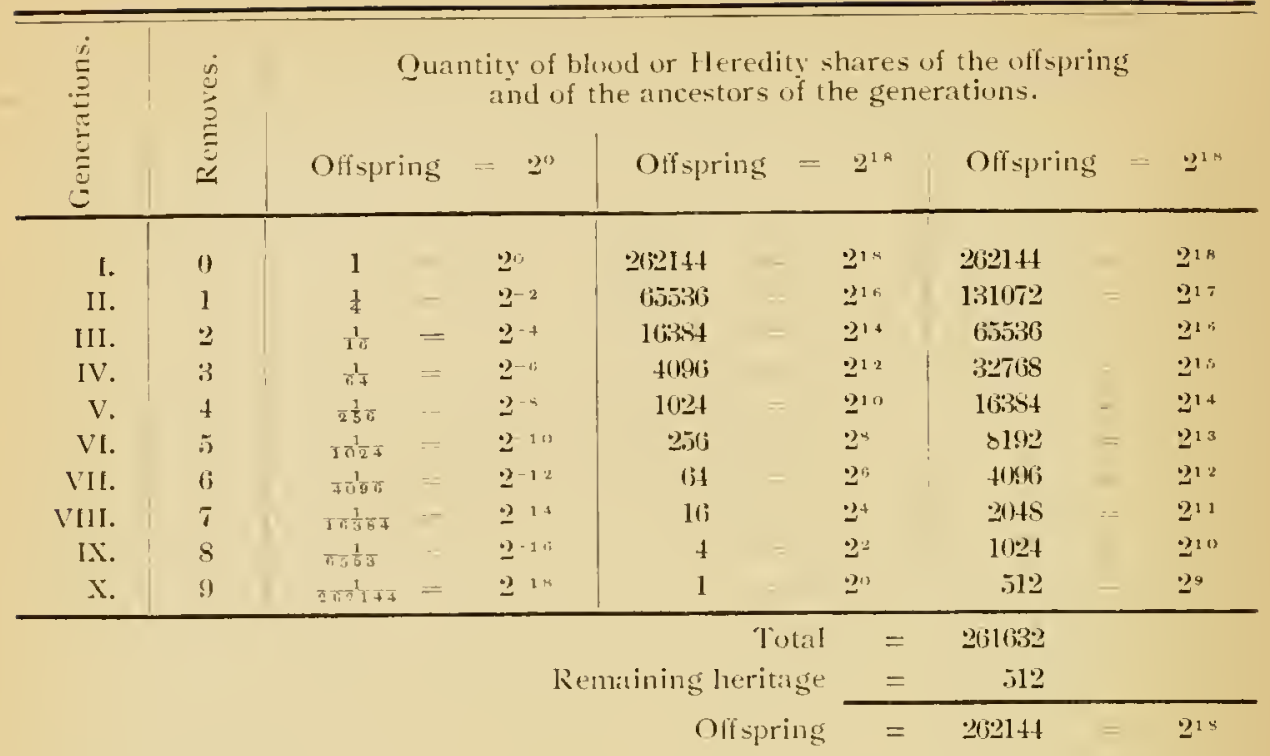

The question is now to find for the different inbreedings a measure for their effect in transmission, i.e., the inbreeding amount. From a simple mathematical consideration it follows-as is shown below-that the inbreeding amount cannot be in any case a total, i.e., also not equal to the transmitted quantity of blood, or what is the same, equal to the total of heredity shares of the basis of inbreeding. These quantities of blood are not at all the same for the same number of free generations, but change very much according as the removes are allotted to the two sides of the pedigree. From Table III. one sees, for instance, that in the case of 6 free generations = totalling 10 removes, the quantities of blood transmitted by the basis of inbreeding increase $\mathrm{from} 512$ to 65,537 , while as may be here mentioned in anticipation - the product of the heredity shares remains equally great, i.e., $2{ }^{16} \cdot 20=$ $2^{14} \cdot 2^{2}=2^{12} \cdot 2^{4}=2^{10} \cdot 2^{6}=2^{8} \cdot 2^{8}=2{ }^{16}$, therefore independent of the different removes in the case of immutahle free generations (for information see Table II.).

If $a^{1}$ and il ${ }^{11}$ represent the heredity shares of the basis of inbreeding, and $\mathrm{J}_{1,2}$ the corresponding inbreeding-anount, it follows that

$$
\left.\mathrm{J}_{1,2}=\mathrm{F}\left(\mathrm{a}^{1}, \mathrm{a}^{11}\right)-4 .\right)
$$

If $a^{1}$, or a ${ }^{11}$ becomes infinitesimal-i.e., in the case of far removed inbreeding - it follows that $\mathrm{J}_{1,2}$ also becomes infinitesimal or 0 , although the quantity of blood as such remains existing but not operating as inbreeding. It is therefore certain that

is no equation.

$$
\mathrm{l}^{7}\left(a^{1}, a^{11}\right)=a^{1}+a^{11}(-5,)
$$


4. Inbreeding

\begin{tabular}{|c|c|c|c|c|c|c|c|c|c|}
\hline & & & 晕 & 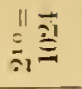 & $\stackrel{x}{31}$ & 莎 & 기 & 51 & $\hat{\text { S1 }}$ \\
\hline & & 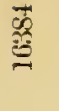 & $\begin{array}{l}\| \\
\stackrel{2}{2}\end{array}$ & 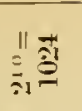 & $\stackrel{m}{: 1}$ & $\because$ & 今1 & تี & 文 \\
\hline & 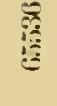 & " & $\stackrel{亏}{\xi}$ & $\stackrel{1 !}{3}$ & $\stackrel{x}{2}$ & $\bar{\nu}$ & 䓜 & $\ddot{\therefore}$ & 引1 \\
\hline & $\begin{array}{l}\| \\
=\end{array}$ & 51 & $\begin{array}{l}\| \\
\approx \\
\approx\end{array}$ & $\underbrace{\| \vec{\jmath}}_{\Delta=1}$ & $\stackrel{x}{3}$ & 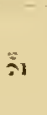 & 31 & דี & $\bar{\vdots}$ \\
\hline & 11 & & $\stackrel{g}{=}$ & 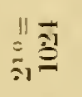 & 累 & $\stackrel{x}{\circ 1}$ & 莎 & โ1 & $\because$ \\
\hline$\frac{\dot{1}}{3}$ & 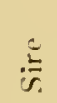 & $\stackrel{0}{=}$ & ज1 & 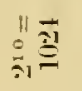 & $\stackrel{x}{3}$ & $\stackrel{x}{3}$ & 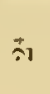 & ธิ & 引 \\
\hline II & & + & 拳 & 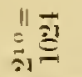 & $\therefore 1$ & 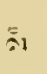 & 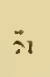 & ถิ & כו \\
\hline$\frac{x}{31}$ & & & $\stackrel{\square}{\partial 1}$ & !l & $\stackrel{x}{=1}$ & 今̀ & $\overrightarrow{B 1}$ & 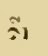 & 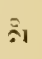 \\
\hline & & & 善 & 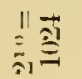 & $\stackrel{x}{=1}$ & 1. & 1) & $\stackrel{\vdots}{\circ}$ & 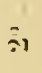 \\
\hline$\frac{5}{5}$ & & 荄 & $\stackrel{\Delta}{\overrightarrow{3}}$ & $\begin{array}{c}\| \overrightarrow{\mathrm{I}} \\
\vdots \\
\vdots \\
\vdots\end{array}$ & ○. & 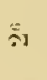 & כ) & $\tilde{\vdots 1}$ & (1) \\
\hline & 施 & & 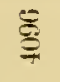 & $\frac{\| \overrightarrow{3}}{\overrightarrow{3}}$ & $\stackrel{x}{\because 1}$ & ถั & 莐 & $\therefore 1$ & 三 \\
\hline & II & & $\tilde{\partial}$ & $\sum_{01}^{1+\frac{3}{\triangleq}}$ & $\stackrel{1}{\circ}$ & 5 & נה & 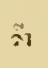 & $\therefore$ \\
\hline & $\|$ & & 竎 & ${ }_{0}^{\|}$ & ถึ & 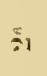 & 51 & 31 & 5 \\
\hline & $\Xi$ & & 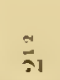 & $\sum_{: 1}^{11}$ & $\stackrel{5}{3}$ & วิ & 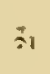 & $\stackrel{01}{01}$ & 今̀ \\
\hline & & & 㡎 & 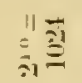 & $\therefore 1$ & 今. & $\therefore$ & $\therefore 1$ & $\hat{i 1}$ \\
\hline & & & 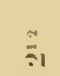 & 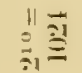 & $\therefore$ & 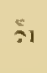 & $\stackrel{\square}{3}$ & ऽ1 & 가 \\
\hline
\end{tabular}




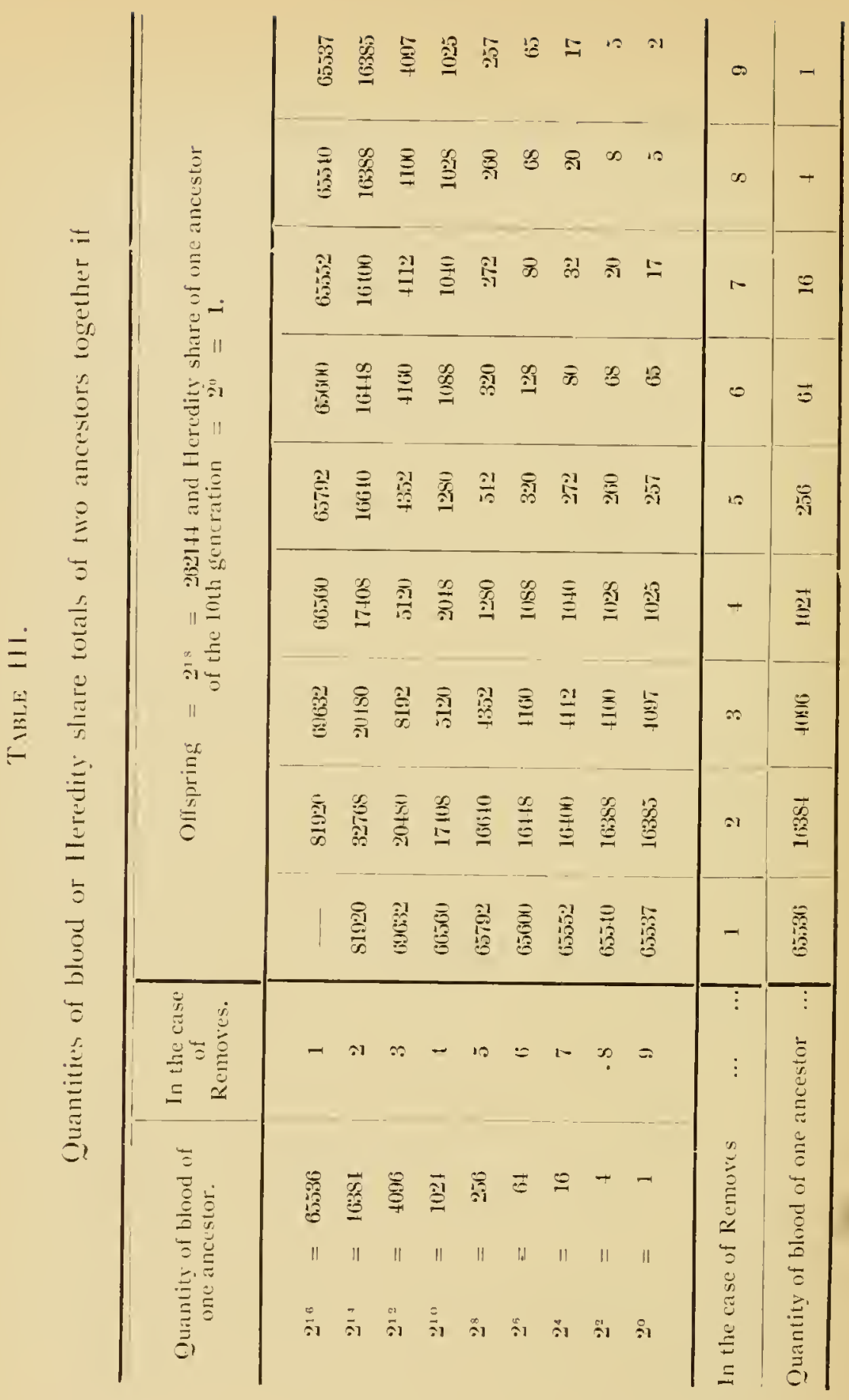


The simplest function which fulfils the condition that $J_{1,2}$ becomes 0 if a ${ }^{1}$ or $a^{11}=0$ reads as follows:

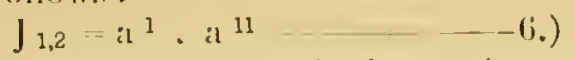

in words: The inbreeding amount equals the product of the hredity shares of the basis. Acourdingly Table $\mathrm{IV}$. is constructed. The unity to be chosen is an arbitrary one, as a unity to be absolutely described is unconceivable. The heredity shares are powers to 2 , therefore their product also indicates in powers to 2 the inbreeding amount. Accordingly, not the quantity of blood, but the inbreeding amount as settled by the free gencrations, is the effective agency which asserts the influence of the common ancestor in memory and in transmission with the power of the inbreeding amount. From the number of free generations, one can calculate at once the inbreeding amount $F$ as

$$
F=22(81-f)
$$

in which $f 1=$ that number of free generations to which the inbreeding amount is supposed $=1$. One can convince oneself of the exactness by regarding the values in Table IV., for instance according to column iii., therefore $f \mathrm{i}=6$. It follows in case of

$\because$ free generations: $\mathrm{F}=22(6-2)-28=256$

(; free generations: $\mathrm{I}: 2=22^{2(6-6)}=20=1$

Accordingly the free generations are a practical and simple means for the calculation of the inbreeding amounts.

TABLE IV.

\begin{tabular}{|c|c|c|c|c|c|c|}
\hline 昱 & 焉 & \multicolumn{2}{|c|}{ 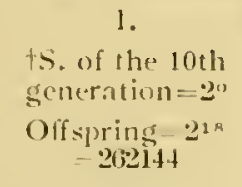 } & 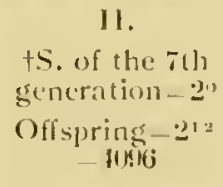 & 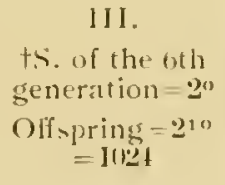 & $\begin{array}{c}1 \mathrm{l} . \\
+5 . \text { of the } 10 \text { th } \\
\text { seneration }=2^{-19} \\
\text { Offspring }=2 \\
=1\end{array}$ \\
\hline-1 & 3 & $2^{30}$ & 11773711821 & $21 \times \quad 262111$ & $2^{24}=110384$ & $2-6$ \\
\hline 11 & 4 & $2^{2 *}$ & 20 istimisto & $24=60530$ & $2^{12}-10 ! 16 ;$ & $2 \cdot A=\quad \frac{1}{36}$ \\
\hline 1 & j & 221 & 67108861 & $2^{14} \quad 16 ; 381$ & $210-\quad 11024$ & $210=x^{3} 53$ \\
\hline 2 & 6 & $2: 4$ & 16767216 & $212 \quad 41996$ & $24=20$ & $2^{11}=$ कर्षण \\
\hline$: 3$ & 7 & $2: 2$ & $1191: 30.1$ & $1(1) \geq 4$ & $2 n-$ & $212 \quad 1818 \div$ \\
\hline 4 & 8 & $2^{211}$ & 1018536 & 24 & $24=$ & $2-11=\quad \cos \frac{1}{3} 30$ \\
\hline 5 & 9 & 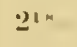 & $20 i 214$ & 2 & $2^{2}=$ & 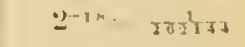 \\
\hline i; & I1) & $2+1 i$ & 6ij.i36; & 2 & 2 & 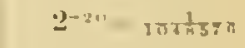 \\
\hline$T$ & 11 & $\underline{y_{1}+}$ & $16 i 381$ & 22 & $2^{2}=$ & $2^{-22}=\pi 18150 \pi$ \\
\hline$s$ & 12 & 212 & 4096 & $23=$ & $2-6$ & $2-3 \quad$ 16. \\
\hline
\end{tabular}

Inbreeding amount in the case of

tS. = Ileredily share of one anciestor (see Tables 1 , and II.). 
There is yet a broad and uncultivated field before us for further mathematical considerations. I will here only point out that if the basis of inbreeding occurs oftener than twice in the pedigree the inbreeding amount cannot be equal to $a^{1}, a^{11}$, a ${ }^{111} \ldots$ but is equal to $a^{1} \cdot a^{11}+a^{1}, a^{111}+a^{11}$ . $\mathrm{a}^{111}+\ldots \ldots \ldots$ for which we can also write :

$J_{1,2,3}=J_{1,2}+a^{111} \cdot B_{1,2}$, where $B_{1,2}=$ quantity of blood $=a^{1}+a^{11}$.

Thereupon we could still establish special values for the inbreeding supports. Another consideration would be necessary for the inbreedings with more than one basis.

In every kind of animal breeding, one has sought and attained improvement and progress by inbreeding to prominent ancestors as a basis. It is evident that the merits of a prominent ancestor have more chance of being transmitted, if this prominent ancestor appears as a basis of an inbreeding, in the pedigree of the product, for the breeding of which the mating is made. If we construct the case theoretically that two animals are mated with each other, which neither in themselves nor in mating with each other, can show any inbreeding whatever, we must ascribe to each separate ancestor of a generation, for example, therefore, to each of the 128 ancestors in the seventh generation, the same possibility and chance of the thorough heredity of its characters. As amongst these 128 ancestors there are very probably several of inferior value, there is just as much probability that the inferior ancestor thoroughly transmits as well as the prominent ancestor. Only on the basis of inbreeding the prominent ancestors is a better chance of transmission to be expected. If all ancestors were faultless and equally prominent, every inbreeding would be clispensable. The inbreeding of any developing breed must necessarily be very close at first. The question now arises how close useful inbreeding shall be to-day, and how often it shall be repeated. Furthermore, on which ancestors as a basis the inbreeding must be founded. Too much inbreeding in Thoroughbreds as well as in Half-breds has often ruined good tribes. In Thoroughbred breeding 1 recall the too frequent inbreeding undertaken by Lord Derby (9 times with 0,6 times with 1 , and 9 times with 2 free generations) with the daughters of Papillon, dam of the Derby winner Sir Peter. The best part of the female progeny of the celebrated Papillon was ruined by it. Much of the blood of Eclipse has been spoiled by inbreeding too, and we have lost at Trakehnen in the same way, as will be shown later on, a great part of the blood of Thunderclap. Whilst the inbreeding mania of Lord Derby was a distinct fiasco, the breeding of the Duke of Grafton, undertaken almost at the same time, in the reasonable limits of at least 2 free generations, has produced fron the valuable blood of Prunella, and her daughter Penelope, the most celebrated family of all Thoroughbreds.

As further examples for the failure of inbreeding with 0 free generation the progeny of following mares will serve :- 
1. Sister to Regulus 1743 by Godol. Arabian, with 16 foals, amongst which 6 with 0 free generation.

2. Maid of all Work 1786 by Hightyer, own sister to Cowslip L. and dam of Mleteora $\mathrm{O}$.

3. Sister to Parrot 1812 by Waltom, with 8 foals, amongst which 4 with 0 free generation.

The progeny of the products with 0 free generation is omitted in Goos' tables. There are ret many other examples in which this exaggerated inbreeding would have had a favourable opportunity to manifest its eventual merit. Its failure is the most plainly recognised through their progeny-ats well as in the case of the children of Papillon-not having plaved the rote to bre assured of a place of honour in the Goos' tables.

There has been also much damaged by exaggerated inbreeding in the American Thoroughbred breeding. Among the many instances of 0 free generation I have not found a single one in the American Stud Bowk which has been of importance to breeding. I have only found one good racelorse in the Imerican breeding, and that is Henry 1819 by Sir Archy-Diomed (i.e., 1) Diomed), celehrated by his famous match with the American Lelipse in New York, 1823. The sire of American Eclipse-equally prominent on the racecourse as at the stud-was Duroc 1806 by Diomed-Grey Diomed. As Grey Diomed is a noted son of Diomed, Duroc atso appears to be bred to Diomed with 0 free generation. The excellent performance of Duroc on the racecourse and at the stud made me sceptical, and exact investigation in the American Stud Book proved to me, therefore, that the aforesaid Crey. Diomed is not descended from Diomed, but from Medley, a son of Cimcrack.

A sire as well as a mare may be the basis of inbreeding, but as the stallion will, of course, have a more numerous progeny, his good characters can be recognised much more easily and quickly than those of a mare, especially if she has only produced well by one stallion. Generally, therefore, in mating one will aim at inbreeding $\mathrm{t}$ a a stallion. As, morener, in most cases good stallions have produced several good offsprings. Whilst most good mares have produced a few good offsprings, it often happens that grood mares give place to the nearest good son as a basis for the inbreeding. Foond mares will only be alble to serve as a basis for an inbreeding in those cases where, as for example, with Penelope, Termagant, Banter, Pocahontas, ete., they, with two different prominent products on each side of the pedigree, determine themselves the closest linit of the inbrecling. The few mares which serve as basis of inbreeding belong to the most prominent. Velocipede's dam, for example, the basis of St. Simon's inbrexding, must be specially mentioned as the mother of 18 foals, of which 9 are mentioned in Goos' tables, a fine performance indeed! But, as wilt he scen from the abore, in most cases the stallion forms the basis of inbreeding. 
In order to correctly estimate the most successful inbreedings in Thoroughbred breeding, we have classified the following examples of the best stallions, according to the degree of their inbreeding. We find them :-

With 1 free generation 11 stallions (only 3 or 4 successful)

, 2 free generations 23 stallions

\begin{tabular}{|c|c|c|c|c|}
\hline 3 & & & & \\
\hline 4 & & $"$ & -0 & " \\
\hline 5 & ," & ", & 10 & ," \\
\hline 6 & " & ", & 01 & "' \\
\hline 7 & "' & " & (.) & "' \\
\hline 8 & " & " & 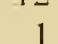 & " \\
\hline & & & & \\
\hline
\end{tabular}

The figures of the free generations and the basis of same are given for every stallion indicated and also for its parents. In every case, not only the closest inbreedings, but also the more distant ones, up to 5 free generations, are added. Six and more free generations are only mentioned where no closer inbreedings exist, or where the same appear necessary to give a complete and correct idea of the whole inbreeding.

A clear table of the inbreedings of the best sires and their parents seems to me to be the best means for arriving by nearer critical considerations at practical and useful conclusions. In a few exceptional cases I have added the inbreedings of grand-parents. A more comprehensive consideration of the pedigree of the best stallions, especially of the so-called basis and its inbreeding, may be interesting and also instructive in the case of some horses. I do not believe, however, this way to be suitable for arriving at useful statistical results. I fully appreciate the difficulties of finding the right examples from the whole Thoroughbred breeding. It is certain that 1 have often overlooked a better stallion and added a worse one instead. Furthermore, as 1 have also taken into consideration American and Australian Thoroughbred breedings, and have also mentioned the most important representatives of olden times up to Eclipse, a correct selection of stallions has been all the more difficult. I am quite aware that 1 have mentioned amongst stallions with 1 free generation several less successful, but this was necessary, as otherwise their number would have been too small from which to draw statistical conclusions.

As an interesting example of incest breeding with -1 free generation, I may mention, besides Y. Paragone 1852 by Paragone and Lanterne by Paragone, produced in Germany, and which ran as a three-year-old twice unplaced, also Jigg-of-Jiggs of English Thoroughbred breeding. He was born 17.5 by the three-year-old Hunt's Jigg (ran four to ten years of age) out of a mare by Heneage's Jigg, who was the dam of Hunt's Jigg, and was herself bred to a Jigg mare with -1 free generation. This Jigg-of-Jiggs, so closely 
inbred, was a good racehorse, ran and won between the age of four and seven, ran as an eight-year-old without winning, and was then used as a stallion for Half-bred breeding. IIs own brother Heel-and-Toe ran between the age of four and seven without winning.

I have yet found in the General Stud Book Regulus, born 1764, by Regulus out of Sappho by Reguhus, with - l free generation, a moderate racehorse, of whom is asserted that he knocked his hip when in embryo, and that had this not happened he would probably have been much better than his less closely inbred brothers and sisters (see Family 4:3). In any case, he has been of no importance in Thoroughbred breeding. He was set apart as covering stallion in Richmond in Yorkshire for 3 gruineas. Is I never found recorded a foal by him, the supposition is that he was infertile.

Imong the stallions with 0 free generation the following 13 (up to now I found no more in the General Stud Book) are wortlyy of mention as crood or moderate racehorses: -

1. Silverieg 174:3 by Cartouch-Old Cartouch.

o) Olu Cartouch.

$13 \mathrm{~h} .3+$ inclese $=140,3 \mathrm{~cm}$. of height. Ran and won between the age of four and nine, also in Give and Take Plates, and beat Little Driver.

‥ Trap 1759 by Blank-Godol. Mrabian.

0 God. . Irabian.

3. Sharper 1700 by bajazet out of Sister q Regulus.

0 Gud. Irabian.

1. Filch 176 by bajazet out of Sister to Regulus.

0 Cod. Arabian.

5. George 179:3 by Dungannon out of Sister (o Soldier.
0 Eclipse.
Sire of 5 unimportant winners.

i. Lucan 1796 by Sir Peter ont of Brown Charlotte.

0 Hightlyer.

7. Robin Radbreast 1790 by Sir Jeter out of IVren.

0 I'apition.

Sold as statlion to Americi and worthy of mention there as grand-sire in the female line of sumpter, 1818 , by sir . Irchy.

ฉ. Igonistes 1797 by Sir Peter out of 11 ren.
() Paipillon.
sire of 2 unimporlant winners.

9. Cocoatree 1797 by Miodpreker-Herod.

0 llerind

10. Hedley 1stre by Sir Peter out of Maria.
1) llightlyer. (Not to be confused with lledley by Gohanna born
le033).

11. Bromn Stout 1804 by Sir Peter out of Brown Charlotte.

0 llighllyer.

1.). Ivanhore 1 sit by Phantom osut of Sister a Parrot.

o 11 alon. 
13. Cedric D. 1821 by Phantom out of Sister to Parrot. 0 IVaiton.

As a three year old remarkable racehorse, ran nine times, amongst which the Derby. As a five year old was set apart as stud stallion in Lambton Grange, 1) urham. Have never found recorded a foal by him.

Of the stallions with 0 free generation I only know the following four which have had any influence on Thoroughbred breeding :-

1. Turner's Sweepstakes 1743 hy Sweepstakes.

0 Mare br Basto.

He was sire of : 1, Trentham, born 1766; 2, Nare, born 1760. Foundation mare in Fam. 8 and dam of Sharper 1788 by Ranthos: 3, Lady, born 17.58, who had 11 foals.

2. Omar 175.2 by Gridol. Arabian.

0 Godol. Arabian.

He was sire of : 1, Miss Spindleshanks, Fam. 9; 2, Nisa, who had 9 foals; 3, Marplot's dam; 4, Confidence's dam; 5. Blemish, who ran and won between the age of three and nine.

3. Bahraham Blank 1758 by Babraham.

0 Godol. . Irabian.

He is also worthy of note as being the sire of Carbuncle.

4. Milo 1802 by Sir Peter.

0 Papillon.

He was half-brother to the winner of the Oaks, Bellissima, ran and won as a three and four-year-old, and was the sire of many good racers, among which Rosalla, grand-dam of Ithuriel (Fam. 2 d), and Eryx born 1816.

Stallions witl 1 Free Generation.

\begin{tabular}{|c|c|c|c|c|}
\hline No. & Names of Stallions & Born & Sire & Dam \\
\hline 1 & $\begin{array}{l}\text { Barcaldine } \\
1 \text { Darling's dam (by } \\
\text { Birdcatcher) } \\
5 \text { 'Touchstone }\end{array}$ & 1578 & $\begin{array}{l}\text { Solon } \\
5 \text { Whalebone (Grand- } \\
\text { sire of Birdcatcher } \\
\text { and Touchstone) } \\
6 \text { W'asy } \\
6 \text { l'enelope }\end{array}$ & $\begin{array}{l}\text { Ballyroe } \\
\text { 3 Birdcatcher }\end{array}$ \\
\hline 2 & $\begin{array}{l}\text { Delpini } \\
1 \text { Blank } \\
7 \text { Darley's Arabian }\end{array}$ & 1781 & $\begin{array}{l}\text { Highflyer } \\
5 \text { Sister to Mixbury } \\
6 \text { Darley's Irabian }\end{array}$ & $\begin{array}{l}\text { Countess } \\
\text { ? }\end{array}$ \\
\hline 3 & $\begin{array}{l}\text { Flying Fox } \\
1 \text { Galopin (by Vecl- } \\
\text { ette) } \\
6 \text { Stockwell } \\
7 \text { Pocahontas }\end{array}$ & $1 S 96$ & $\begin{array}{l}\text { Orme } \\
5 \text { Pocahontas } \\
6 \text { Birdcatcher (Grand- } \\
\text { sire of Stockw.) }\end{array}$ & $\begin{array}{l}\text { Vampire } \\
2 \text { Vedette } \\
6 \text { Birdcatcher }\end{array}$ \\
\hline
\end{tabular}


4. Inbreceding.

\begin{tabular}{|c|c|c|c|c|}
\hline No. & Names of Stallions & hiorn & Sire & Dam \\
\hline 1 & $\begin{array}{l}\text { Friponnier } \\
1 \text { Orlando } \\
7 \text { Orville } \\
7 \text { Walton }\end{array}$ & 1Stit & $\begin{array}{l}\text { Chevalies } \\
\text { d'Industrie } \\
5 \text { Orville } \\
6 \text { Waxy }\end{array}$ & $\begin{array}{l}\text { Tension } \\
\text { j llation } \\
\text { 6 llbatebone }\end{array}$ \\
\hline i) & $\begin{array}{l}\text { Jighland Fling } \\
1 \text { Herod }\end{array}$ & 1798 & $\begin{array}{l}\text { Spadille } \\
+ \text { Parlner } \\
+ \text { Resulus }\end{array}$ & $\begin{array}{l}\text { Calia } \\
\text { (j Darley's Arabian } \\
6 \text { Betty leedes }\end{array}$ \\
\hline i) & $\begin{array}{l}\text { Lnight of St. } \\
\text { Ceurge } \\
1 \text { Sir Iflercules } \\
6 \text { Potsos (by liclipuse) }\end{array}$ & 18.11 & $\begin{array}{l}\text { Birdcatcher } \\
5 \text { If ixdpeclier } \\
6 \text { Eclipse }\end{array}$ & $\begin{array}{l}\text { Maltese } \\
\text { \& Surcerer } \\
\text { ¿ Potsos }\end{array}$ \\
\hline $\bar{\imath}$ & $\begin{array}{l}\text { The Niner } \\
1 \text { Birdeatcher } \\
7 \text { Orville } \\
\text { i Waxy } \\
\text { - Penclope }\end{array}$ & $181 ; 1$ & $\begin{array}{l}\text { Rattaplan } \\
\text { j Orvilte } \\
\text { f Wiaxy } \\
\text { i l'encelope }\end{array}$ & $\begin{array}{l}\text { Manganese } \\
\text { F Sir Peter } \\
\text { 7 Trumpator }\end{array}$ \\
\hline \multirow[t]{2}{*}{$s$} & $\begin{array}{l}\text { Ninety Three } \\
1 \text { Herod } \\
6 \text { Flying Childers }\end{array}$ & 1790 & $\begin{array}{l}\text { Florizel } \\
\text { \& Flying Childers }\end{array}$ & $\begin{array}{l}\text { Inseray } \\
3 \text { Snap } \\
6 \text { Bartl. Childers }\end{array}$ \\
\hline & $\begin{array}{l}\text { Orest } \\
1 \text { Touchstone }\end{array}$ & 18.5 & $\begin{array}{l}\text { Orestus } \\
\text { t Setim }\end{array}$ & $\begin{array}{l}\text { Lady Louisa } \\
\text { + llaxy } \\
\text { \& Penclope }\end{array}$ \\
\hline 10 & $\begin{array}{l}\text { Paulowitz } \\
1 \text { Hightyer } \\
1 \text { Termasant } \\
\text { (3 Godol. Irabian) } \\
\text { 6 Regulus }\end{array}$ & 1813 & $\begin{array}{l}\text { Sir Paul } \\
+ \text { Resrulus } \\
\text { (by (ind. Mrab.) } \\
\text { is Sipip }\end{array}$ & $\begin{array}{l}\text { Evelina } \\
+ \text { Regulus } \\
+ \text { Godol. Irabian } \\
+ \text { Blaze }\end{array}$ \\
\hline 11 & $\begin{array}{l}\text { IV ellingtoniat } \\
1 \text { Pocalontas } \\
2 \text { Touchome } \\
\end{array}$ & $1,4(6)$ & $\begin{array}{l}\text { Chattanimgara } \\
\text { I Whalebone }\end{array}$ & $\begin{array}{l}\text { Araucaria } \\
\text { j Selim } \\
\text { j Orville } \\
\text { is llaxy } \\
\text { 6 Penelope }\end{array}$ \\
\hline
\end{tabular}

Stallions with ? Free Generattions.

1

Implicon

2 . Newminster

2 18s; Roseberv

3 Touchistone

180.1

Chanticleer

5 Codol. . Irabian

f $1 \%$. Childers

6 \{ birt. Childers
Suicide

- IIhalebone

lerne

f F. Chitders

Ibars. Childes 


\begin{tabular}{|c|c|c|c|}
\hline No. & Names of Stallions & Born & Sire \\
\hline 3 & $\begin{array}{l}\text { Boston } \\
2 \text { Diomed } \\
5 \text { Eclipse } \\
6 \text { Marslie (by Squirt) }\end{array}$ & $\mid \begin{array}{c}1833 \\
\text { in } \\
\text { Antericy }\end{array}$ & $\begin{array}{l}\text { Timoleon } \\
5 \text { Trentham } \\
7 \text { Squirt }\end{array}$ \\
\hline 4 & $\begin{array}{l}\text { Brutandorf } \\
2 \text { Potsos } \\
5 \text { Herod }\end{array}$ & 1821 & $\begin{array}{l}\text { Blaclilock } \\
3 \text { Highflyer } \\
4 \text { Herod } \\
4 \text { Eclipse }\end{array}$ \\
\hline 5 & $\begin{array}{l}\text { Cap à Pie } \\
2 \text { IVaxy }\end{array}$ & 1837 & $\begin{array}{l}\text { The Colonel } \\
3 \text { Highfirer } \\
4 \text { Eclipse } \\
4 \text { Herod }\end{array}$ \\
\hline 6 & $\begin{array}{l}\text { Cardinal York } \\
2 \text { Herod } \\
5 \text { Regulus }\end{array}$ & 1804 & $\begin{array}{l}\text { Sir Peter } \\
3 \text { Regulus } \\
4 \text { Godol. Arab. } \\
5 \text { Flying Childers } \\
5 \text { Fox }\end{array}$ \\
\hline 7 & $\begin{array}{l}\text { Gohanna } \\
2 \text { Tartar (by Partner) } \\
4 \text { Syuirt } \\
5 \text { Mogul (by Godol. } \\
\text { Arabian) } \\
6 \text { Godol. Arabian } \\
6\left\{\begin{array}{l}\text { Bart Childers } \\
\text { F1. Childers }\end{array}\right.\end{array}$ & 1790 & $\begin{array}{l}\text { Mercury } \\
\pm \text { Godol. Arabian }\end{array}$ \\
\hline 8 & $\begin{array}{l}\text { Galopin } \\
2 \text { Vultaire (by Black- } \\
\text { Jock, and grand- } \\
\text { son of Phantom) }\end{array}$ & 1872 & $\begin{array}{l}\text { Vedette } \\
3 \text { Blacliocls } \\
6 \text { Wratton }\end{array}$ \\
\hline 9 & $\begin{array}{l}\text { Golumpus } \\
2 \text { Herod (by Tartar) } \\
5 \text { Cade (by Godol. } \\
\text { Arabian) }\end{array}$ & $181)^{2}$ & $\begin{array}{l}\text { Gohanna } \\
2 \text { Tartar (by Partner) } \\
4 \text { Squirt } \\
5 \text { Mogu] } \\
6 \text { Godol. Arabian }\end{array}$ \\
\hline 10 & $\begin{array}{l}\text { Hannover } \\
2 \text { Vandal } \\
7 \text { Emilius (by Orville) }\end{array}$ & 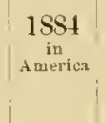 & $\begin{array}{l}\text { Hindon } \\
6 \text { Emilius }\end{array}$ \\
\hline 11 & $\begin{array}{l}\text { Humphrey Clinker } \\
2 \text { Sir Peter (by High- } \\
\text { fyer and out of a } \\
\text { Nare by Snap) } \\
4 \text { Trumpator } \\
7 \text { Eclipse }\end{array}$ & 1822 & $\begin{array}{l}\text { Comus } \\
5 \text { Herod } \\
5 \text { Snap (by Snip) } \\
6 \text { Cade } \\
6 \text { Eclipse }\end{array}$ \\
\hline
\end{tabular}

\section{Mare by Ball's Florizel \\ 5 Marske}

Mandane

$\therefore$ Cade

\section{Sister to Cactus 5 Eclipse}

\section{Charmer}

1 Jerod

Mare by Herod

3 Partner

$4\left\{\begin{array}{l}\text { Bart. Childers } \\ \text { lil. Childers }\end{array}\right.$

Flying Duchess

4 Plantom (by:

IVaton)

6 Potsos

Catherine

4 Godol. Arabian

5 Partner

Bourbon Belle

7 Orville

\section{Clinkerina \\ 5 Regulus \\ 6 Snip}




\begin{tabular}{|c|c|c|c|c|}
\hline No. & Names of Stallions & Born & Sire & Dam \\
\hline 12 & $\begin{array}{l}\text { Janissary } \\
2 \text { Stockiwell } \\
5 \text { Touchstone } \\
5 \text { Nelbourne }\end{array}$ & 1857 & $\begin{array}{l}\text { Isconomy } \\
3 \text { Birdcatcher }\end{array}$ & $\begin{array}{l}\text { Janetle } \\
3 \text { Touchstone } \\
6 \text { Bee's IIing's dam }\end{array}$ \\
\hline 13 & $\begin{array}{l}\text { Muley Moloch } \\
2 \text { Beningbrough (by } \\
\text { King lergus by } \\
\text { Eclipse) } \\
4 \text { Highflyer } \\
5 \text { Eclipse }\end{array}$ & IS30) & $\begin{array}{l}\text { Muley } \\
4 \text { Echlipse } \\
5 \text { Iferod }\end{array}$ & $\begin{array}{l}\text { Nancy } \\
3 \text { Eclipse } \\
4 \text { Herod }\end{array}$ \\
\hline 11 & $\begin{array}{l}\text { Orville } \\
2 \text { Herod (by lartar) } \\
6 \text { Regulus } \\
6 \text { Ruby .Iare }\end{array}$ & 1799 & $\begin{array}{l}\text { Beningbrough } \\
3 \text { Tartar } \\
4 \text { Miss Slamerlin } \\
6 \text { Godol. Arabian }\end{array}$ & $\begin{array}{l}\text { Evelina } \\
4 \text { Blaze } \\
4 \text { Regulus } \\
4 \text { Godol. Irabian }\end{array}$ \\
\hline 1.5 & $\begin{array}{l}\text { Partisan } \\
2 \text { Hightlyer (by } \\
\text { Herod) } \\
3 \text { Eclipse } \\
4 \text { Snap } \\
6 \text { Regulus }\end{array}$ & 1811 & $\begin{array}{l}11^{\top} \text { alton } \\
3 \text { Herod } \\
3 \text { Snap } \\
4 \text { Regulus } \\
6 \text { Partner }\end{array}$ & $\begin{array}{l}\text { Parasol } \\
5 \text { Regrulus } \\
6 \text { Godol. Arabias }\end{array}$ \\
\hline 16 & $\begin{array}{l}\text { Petrarch } \\
2 \text { Touchstone } \\
i \text { Selim }\end{array}$ & 1873 & $\begin{array}{l}\text { Lord Clifden } \\
6 \text { Paynator }\end{array}$ & $\begin{array}{l}\text { Laura } \\
4 \text { Whalebone (Grand- } \\
\text { sire of Touchstome) } \\
5 \text { Selim }\end{array}$ \\
\hline 17 & $\begin{array}{l}\text { Priam } \\
2 \text { Whisliey } \\
5 \text { Herod } \\
5 \text { Eclipse }\end{array}$ & 1027 & $\begin{array}{l}\text { Emilius } \\
3 \text { Hishfyer } \\
4 \text { Eclipse }\end{array}$ & $\begin{array}{l}\text { Cressida } \\
3 \text { Herod } \\
3 \text { Matchem }\end{array}$ \\
\hline 18 & $\begin{array}{l}\text { The Saddler } \\
2 \text { Waxy } \\
4 \text { Sir Peter } \\
5 \text { Hiwhflyer }\end{array}$ & 1829 & $\begin{array}{l}\text { Maverley } \\
3 \text { Highflyer } \\
5 \text { Matehen }\end{array}$ & $\begin{array}{l}\text { Castrellina } \\
4 \text { Herod } \\
4 \text { Eelipse } \\
5 \text { llighflyer } \\
\text { Grand-sire : } 3 \text { Herod } \\
\text { Grand-dan : 4 Herud }\end{array}$ \\
\hline 19 & $\begin{array}{l}\text { Sainfoin } \\
2 \text { Sicuckell } \\
6 \text { Touclistone }\end{array}$ & 1887 & $\begin{array}{l}\text { Springfield } \\
5 \text { Sultan (Grand-sire } \\
\text { of Pocahontas) } \\
6 \text { Camel ) Parents of } \\
6 \text { Banter) Touchstone }\end{array}$ & $\begin{array}{l}\text { Sanda } \\
3 \text { The Baron Parents } \\
3 \text { I'ocithontas) Stockwell }\end{array}$ \\
\hline 20 & $\begin{array}{l}\text { Le Sagittaice } \\
2 \text { Strathoonan (by } \\
\text { Newninster) } \\
6 \text { Melbuurne }\end{array}$ & 1892 & $\begin{array}{l}\text { Le Sancy } \\
3 \text { Windhound } \\
3 \text { Alice Hawthorn } \\
5 \text { Touclistone }\end{array}$ & $\begin{array}{l}\text { La Dauphine } \\
5 \text { Touchstone } \\
5 \text { Melbourne }\end{array}$ \\
\hline
\end{tabular}




\begin{tabular}{|c|c|c|c|c|}
\hline No. & Names of Stallions & Born & Sire & Dam \\
\hline 21 & $\begin{array}{l}\text { Saltram } \\
2 \text { Regulus } \\
+\left\{\begin{array}{l}\text { Bart. Childers } \\
\text { F1. Childers }\end{array}\right. \\
5 \text { Sister to Old } \\
\text { Country IVench } \\
6 \text { Bay Bolton }\end{array}$ & 1780 & $\begin{array}{l}\text { Eclipse } \\
3 \text { Sister to Old } \\
\text { Country Wench } \\
+ \text { Snake }\end{array}$ & $\begin{array}{l}\text { Tirago } \\
3 \text { Mlare by Basto }\end{array}$ \\
\hline 20 & $\begin{array}{l}\text { Sorcerer } \\
2 \text { Nlatchem } \\
6 \text { Partmer }\end{array}$ & 1790 & $\begin{array}{l}\text { Trumpator } \\
4 \text { Godol. Arabian } \\
4 \text { Partner }\end{array}$ & $\begin{array}{l}Y^{\prime} . \text { Giantess } \\
j \text { God. Arab.) } \\
\text { j Partner } \begin{array}{c}\text { Grand } \\
\text { sire ol } \\
\text { Matchem }\end{array}\end{array}$ \\
\hline 23 & $\begin{array}{l}\text { WVisdom } \\
2 \text { The Baron } \\
2 \text { Pocahontas } \\
5 \text { Emilius (by Orville) }\end{array}$ & 1873 & $\begin{array}{l}\text { Blinkhoolie } \\
5 \text { Whalebone } \\
6 \text { Orville }\end{array}$ & $\begin{array}{l}\text { Mine } \\
\text { j Orville } \\
5 \text { Whisker } \\
6 \text { Whalebone }\end{array}$ \\
\hline
\end{tabular}

Stallions with 3 Iiree Generations.

\begin{tabular}{|c|c|c|c|}
\hline 1 & $\begin{array}{l}\text { Iustralian } \\
3 \text { Whislier (by Maxy } \\
\text { and Pedelope) }\end{array}$ & 18.58 & $\begin{array}{l}\text { Wrest-A tustralian } \\
6 \text { Trumpator }\end{array}$ \\
\hline & $\begin{array}{l}\text { Beadsman } \\
3 \text { Tramp } \\
5 \text { Orville } \\
7 \text { Buzzard } \\
8 \text { Trumpator } \\
8 \text { Eclipse }\end{array}$ & 1855 & $\begin{array}{l}\text { W eatherbit } \\
3 \text { Orville } \\
5 \text { Y. Giantess } \\
6 \text { IVoodpecker } \\
6 \text { Trumpator }\end{array}$ \\
\hline 3 & $\begin{array}{l}\text { Beningbrotigh } \\
3 \text { Tartar (by Partner) } \\
4 \text { viss Slamerkin } \\
6 \text { Godol. Arabian } \\
6 \text { \{li. Childers } \\
\text { Bart. Childers }\end{array}$ & 1791 & $\begin{array}{l}\text { King Fergus } \\
5\left\{\begin{array}{l}\text { Fi. Childers } \\
\text { Bart. Childers }\end{array}\right. \\
6 \text { Bay Bolton }\end{array}$ \\
\hline 4 & $\begin{array}{l}\text { Blacklock } \\
3 \text { Highflyer } \\
4 \text { Herod } \\
4 \text { Eclipse }\end{array}$ & 1814 & $\begin{array}{l}\text { IVhitelock } \\
3 \text { Herod } \\
3 \text { Eclipse } \\
\text { ? Matchem }\end{array}$ \\
\hline 5 & $\begin{array}{l}\text { Buccaneer } \\
2 \text { Edmund (by Orville) } \\
5 \text { Paynator }\end{array}$ & 1857 & $\begin{array}{l}\text { Wild Dayrell } \\
4 \text { Selim }\end{array}$ \\
\hline & $\begin{array}{l}\text { Buzzard } \\
3 \text { Cade (by Godol. } \\
\text { Arabian) } \\
5 \text { Partner } \\
6 \text { Flying Childers } \\
7 \text { Bald Galloway }\end{array}$ & 1787 & $\begin{array}{l}\text { IVoodpeclier } \\
6 \text { Dar]. Arabian }\end{array}$ \\
\hline
\end{tabular}

\author{
Emilia \\ 4 IVaxy \\ 4 Penelope (by \\ Trumpator)

\section{Mendicant} \\ 6 Eclipse \\ 6 . Alexander \\ 6 Sir Petel- \\ 6 Buzzard \\ 6 Mare by Mlexander \\ Mare by Herod \\ 3 Partner \\ j J1. Childers \\ 5 Confederate Filly
}
Mare by Coriander
1 Potsos (by Eclipse)
2 Herod
5 Snap
Mare by Little Red
Rover

4 Beningbrough (Sire of Orville)

Missfortune

4 Godol. Arabian

6 Bald Galloway 


\begin{tabular}{|c|c|c|c|c|}
\hline No. & Names of Stallions & Lorn & Sire & Dam \\
\hline 7 & $\begin{array}{l}\text { Cain } \\
3 \text { Hightyer } \\
6 \text { blank }\end{array}$ & $182 \cdot 2$ & $\begin{array}{l}\text { Paulowitz } \\
\text { I Ilimhflyer } \\
\text { I Termagant } \\
\text { c Resulus (by Godol. } \\
\text {.Irabian) }\end{array}$ & $\begin{array}{l}\text { Mare by Paynator } \\
4 \text { Rachel (I)an of } \\
\text { Highflyer) } \\
5 \text { Blamk (by Godol. } \\
\text {. Irabian) }\end{array}$ \\
\hline 8 & $\begin{array}{l}\text { Carbine } \\
3 \text { Browl Bess (by } \\
\text { Camel) } \\
\text { 5 Touchstone }\end{array}$ & $\begin{array}{c}185 ; \\
\text { In: } \\
\text { Autral. }\end{array}$ & $\begin{array}{l}\text { Musliet } \\
+ \text { Tomchstone } \\
+ \text { Camel }\end{array}$ & $\begin{array}{l}\text { The Nersey } \\
3 \text { Touchstone } \\
4 \text { Camel }\end{array}$ \\
\hline$!$ & $\begin{array}{l}\text { The Colonel } \\
3 \text { Hishnyer } \\
4 \text { Herod (by Tartar) } \\
4 \text { Eclipse } \\
7 \text { Blank (by Codol. } \\
\text { Irabian) }\end{array}$ & 1825 & $\begin{array}{l}\text { II hisker } \\
3 \text { llerod } \\
4 \text { Snap } \\
6 \text { ('ide (by Godol. } \\
\text { Irabian) }\end{array}$ & $\begin{array}{l}\text { Mare by Delpini } \\
5 \text { Tarlar } \\
5 \text { Blank } \\
6 \text { Resulus }\end{array}$ \\
\hline 10 & $\begin{array}{l}\text { Conductor } \\
3 \text { Partner (by Jigg) }\end{array}$ & 1767 & $\begin{array}{l}\text { Matchem } \\
\text { J Mare by Spanker }\end{array}$ & $\begin{array}{l}\text { Mare by Snap } \\
4 \text { Partier's dam } \\
6 \text { Byerly Turl (Sire } \\
\text { by Jimer) }\end{array}$ \\
\hline 11 & $\begin{array}{l}\text { Domino } \\
3 \text { Lexinerton (by } \\
\text { Boston) } \\
7 \text { Cilencue }\end{array}$ & $\begin{array}{l}1891 \\
\text { in } \\
\text { Amerilest }\end{array}$ & $\begin{array}{l}\text { Himvar } \\
7 \text { liuley }\end{array}$ & $\begin{array}{l}\text { Mannie Grey } \\
2 \text { Lexington } \\
\text { Grand-dam } \\
1 \text { Reel (by } \\
\text { Glencoe) } \\
2 \text { Boston }\end{array}$ \\
\hline 1:2 & 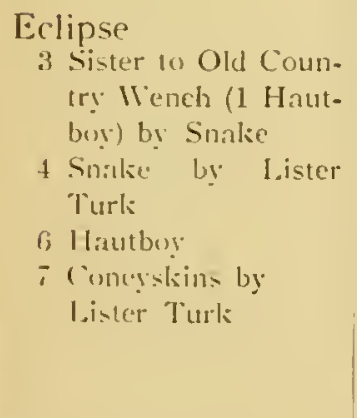 & $173 t$ & $\begin{array}{l}\text { Marske } \\
5 \text { Hautboy (Grand-sire } \\
\text { of Old Country } \\
\text { llench and Snake) } \\
5 \text { Lister Turls }\end{array}$ & $\begin{array}{l}\text { Grimcl-dam (Mother } \\
\text { Western) } \\
3 \text { Hautboy } \\
\text { j Brimmer }\end{array}$ \\
\hline $1: 3$ & $\begin{array}{l}\text { Emilius } \\
3 \text { llighllyer (by } \\
\text { llerod) } \\
4 \text { liclipse } \\
6 \text { Blank } \\
\text { (by Goclol. Irabian) }\end{array}$ & 1820 & $\begin{array}{l}\text { Orville } \\
2 \text { llerod } \\
\text { fi leeculus (by Godol. } \\
\text {. Irabian) }\end{array}$ & $\begin{array}{l}\text { Emily } \\
\text { 3 Eclipse } \\
+ \text { Ilerod } \\
+ \text { Blank }\end{array}$ \\
\hline
\end{tabular}


Heredity.

\begin{tabular}{|c|c|c|c|c|}
\hline No. & Names of Stallions & Born & Sire & Dam \\
\hline 14 & $\begin{array}{l}\text { Emperor of Norfolk } \\
3 \text { Glencoe } \\
6 \text { Emilius } \\
6 \text { Tramp } \\
7 \text { Sir Archy }\end{array}$ & $\begin{array}{c}1885 \\
\text { in } \\
\text { America }\end{array}$ & $\begin{array}{l}\text { Norfolli } \\
5 \text { Sir Archy } \\
5 \text { Sumpter (by Sir } \\
\text { Archy) }\end{array}$ & $\begin{array}{l}\text { Marian } \\
5 \text { Emilius }\end{array}$ \\
\hline 15 & $\begin{array}{l}\text { Epirus } \\
3 \text { Sir Peter } \\
\text { (by Hightlyer) } \\
4 \text { Diomed } \\
5 \text { Herod } \\
5 \text { Eclipse }\end{array}$ & 1834 & $\begin{array}{l}\text { Langar } \\
4 \text { Highfyer }\end{array}$ & $\begin{array}{l}\text { Olympia } \\
3 \text { Herod }\end{array}$ \\
\hline 16 & $\begin{array}{l}\text { Fitz James } \\
3 \text { Touchstone } \\
5 \text { Pantaloon } \\
\text { (by Castrel) }\end{array}$ & 1875 & $\begin{array}{l}\text { Scottish Chief } \\
5 \text { Orville } \\
6 \text { Selim (by Buzzard, } \\
\text { Sire of Castrel) }\end{array}$ & $\begin{array}{l}\text { Hawthorn Bloom } \\
6 \text { Muley } \\
7 \text { Buzzard }\end{array}$ \\
\hline 17 & $\begin{array}{l}\text { Flying Dutchman } \\
3 \text { Selim } \\
6 \text { Sir Peter (by High- } \\
\text { Ayer) }\end{array}$ & 1846 & $\begin{array}{l}\text { Bay Middleton } \\
\text { 4 Sir Peter } \\
\text { 4. Arethusa }\end{array}$ & $\begin{array}{l}\text { Barbelle } \\
5 \text { Eclipse } \\
5 \text { Hightyer }\end{array}$ \\
\hline 18 & $\begin{array}{l}\text { Gallinule } \\
3 \text { Stockivell } \\
5 \text { Touchstone } \\
\text { (by Camel) } \\
6 \text { Birdcatcher }\end{array}$ & 1884 & $\begin{array}{l}\text { Isonomy } \\
3 \text { Birdcatcher (Grand- } \\
\text { sire of Stockwell) }\end{array}$ & $\begin{array}{l}\text { Moorhen } \\
7 \text { Selim (Grand-sire } \\
\text { of Camel) } \\
\text { Grand-sire: } 4 \text { Camel }\end{array}$ \\
\hline 19 & $\begin{array}{l}\text { Galtee More } \\
3 \text { Thomanby } \\
4 \text { Stockmell }\end{array}$ & 1894 & $\begin{array}{l}\text { Tiendal } \\
+ \text { Birdcatcher (Grand- } \\
\text { sire of Stockwell) } \\
5 \text { Pantaloon }\end{array}$ & $\begin{array}{l}\text { Morganette } \\
5 \text { Pantaloon (Grand- } \\
\text { sire of Thormanby) }\end{array}$ \\
\hline 20 & $\begin{array}{l}\text { Glaucus } \\
3 \text { Sir Peter } \\
\text { (by Hightlyer) } \\
5 \text { Eclipse }\end{array}$ & 1830 & $\begin{array}{l}\text { Partisan } \\
2 \text { Hightyer } \\
3 \text { Eclipse } \\
4 \text { Snap (Grand-sire of } \\
\text { Sir Peter) }\end{array}$ & $\begin{array}{l}\text { Nanine } \\
4 \text { Hishflyer } \\
5 \text { Eclipse }\end{array}$ \\
\hline 21 & $\begin{array}{l}\text { Gunnersbury } \\
3 \text { Touchstone } \\
\text { (by Canel) } \\
5 \text { Sultan (by Selim) }\end{array}$ & 1876 & $\begin{array}{l}\text { Hernit } \\
4 \text { Camel } \\
6 \text { Selin }\end{array}$ & $\begin{array}{l}\text { Hippia } \\
3 \text { Sultan }\end{array}$ \\
\hline 22 & $\begin{array}{l}\text { I!ackler } \\
3 \text { Orlando } \\
5 \text { Melbourne }\end{array}$ & 1887 & $\begin{array}{l}\text { Petrarch } \\
2 \text { Touchstone }\end{array}$ & $\begin{array}{l}\text { Hacliness } \\
3 \text { Orlando }\end{array}$ \\
\hline 3 & $\begin{array}{l}\text { Ion } \\
3 \text { Evelina (by High- } \\
\text { flyer) } \\
5 \text { Sir Peter (by High- } \\
\text { flyer) }\end{array}$ & 1835 & $\begin{array}{l}\text { Cain } \\
3 \text { Hightlyer }\end{array}$ & $\begin{array}{l}\text { Margaret } \\
5 \text { Higlnyer }\end{array}$ \\
\hline
\end{tabular}


4. Inbreediner.

\begin{tabular}{|c|c|c|c|c|}
\hline No. & Names of Stallions & Born & Sire & Dam \\
\hline 24 & $\begin{array}{l}\text { Isonomy } \\
3 \text { Birdcatcher }\end{array}$ & 1975 & $\begin{array}{l}\text { Sterling } \\
5 \text { IIhalebone (Sire of } \\
\text { Sir Hercules) }\end{array}$ & 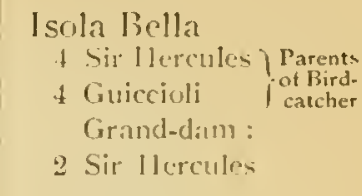 \\
\hline 25 & $\begin{array}{l}\text { King . Ilfonso } \\
3 \text { Glencre } \\
5 \text { Muley (by Orville) }\end{array}$ & $\mid \begin{array}{c}1872 \\
\text { in } \\
\text { inerica }\end{array}$ & $\begin{array}{l}\text { Phaeton } \\
6 \text { Orville }\end{array}$ & $\begin{array}{l}\text { Capitola } \\
5 \text { Orville }\end{array}$ \\
\hline $24 i$ & $\begin{array}{l}\text { Lexington } \\
3 \text { Sir . Irchy } \\
\text { (by Diomed) } \\
6 \text { Saltram (by Eclipse) }\end{array}$ & $\left|\begin{array}{c}18 ; 0 \\
\text { in } \\
\text { Anerich }\end{array}\right|$ & $\begin{array}{l}\text { Boston } \\
\text { 2 Dioned (by } \\
\text { Florizel) } \\
\text { 5 Eclipse } \\
6 \text { Marske }\end{array}$ & $\begin{array}{l}\text { Mlice Carneal } \\
6 \text { Higrhflyer (by } \\
\text { Herod, Sire of } \\
\text { Florized) }\end{array}$ \\
\hline 27 & $\begin{array}{l}\text { Lottery } \\
3 \text { Eclipse } \\
4 \text { lloodpecker } \\
\text { (by llerod) } \\
4 \text { Trentham } \\
5 \text { Herod }\end{array}$ & $18: 20$ & $\begin{array}{l}\text { Tramp } \\
3 \text { Eclipse } \\
+ \text { Herod }\end{array}$ & $\begin{array}{l}\text { Mandane } \\
+4 \text { Cade } \\
+\left\{\begin{array}{l}\text { Regulus } \\
\text { Sister to Regulu- }\end{array}\right.\end{array}$ \\
\hline 28 & $\begin{array}{l}\text { Melton } \\
3 \text { Stockwell } \\
5 \text { Touchstone } \\
6 \text { bee's } 11 \text { ingr }\end{array}$ & 185:2 & $\begin{array}{l}\text { Master Kildare } \\
+ \text { Birdcatcher }\end{array}$ & $\begin{array}{l}\text { Violet Melrose } \\
3 \text { Tuuchstone } \\
\text { } 4 \text { Pantaloon } \\
\text { Grand-dam : } \\
3 \text { Touchstone }\end{array}$ \\
\hline$\simeq 9$ & $\begin{array}{l}\text { Muncaster } \\
3 \text { Birdcatcher } \\
6 \text { Banter }\end{array}$ & 1877 & $\begin{array}{l}\text { Doncaster } \\
6 \text { Vilacklock } \\
7 \text { Whalebone }\end{array}$ & $\begin{array}{l}\text { II indermere } \\
7 \text { Orville } \\
7 \text { Bumard }\end{array}$ \\
\hline 311 & $\begin{array}{l}\text { Orlando } \\
3 \text { Selim (by luzzard) } \\
5 \text { Alexander } \\
\text { (by Felipse) } \\
6 \text { Sir Peter } \\
\text { (by Higlinger) }\end{array}$ & $18+1$ & $\begin{array}{l}\text { Touchstone } \\
4 \text {. Hexander } \\
5 \text { Buzuard } \\
\text { is Eclipse } \\
\text { is Sir Peter } \\
6 \text { Hishflyer }\end{array}$ & $\begin{array}{l}\text { Pulture } \\
\begin{array}{l}3 \text { Buzzard } \\
3 \text { Mare Paremi } \\
\text { by Mexand. I Selim } \\
+ \text { Sir Pecer }\end{array}\end{array}$ \\
\hline 31 & $\begin{array}{l}\text { Paynator } \\
3 \text { Snap (by Snip) } \\
5 \text { Godol. Mrabian }\end{array}$ & 1791 & $\begin{array}{l}\text { Trumpator } \\
+ \text { Ciulol. . Irabian } \\
+ \text { l'artner }\end{array}$ & $\begin{array}{l}\text { Mare by Mark } \\
\text { Inthong } \\
3 \text { Codol. Mabian } \\
4 \text { Mare by Eacto } \\
\text { (dam ut Sinip) }\end{array}$ \\
\hline 32 & $\begin{array}{l}\text { Pero Comez } \\
3 \text { Lady Moore Carew } \\
\text { (hy Tramp) } \\
\text { 5 Camel }\end{array}$ & $1866^{\circ}$ & $\begin{array}{l}\text { Beadsman } \\
3 \text { Tramp } \\
5 \text { Orville }\end{array}$ & $\begin{array}{l}\text { Salamanca } \\
\qquad 1 \text { Velocipede. }\end{array}$ \\
\hline 33 & $\begin{array}{l}\text { Privateer } \\
3 \text { Touchstone } \\
6 \text { Orville }\end{array}$ & $187 \mathrm{~s}$ & $\begin{array}{l}\text { Adrenturer } \\
+ \text { Orville }\end{array}$ & $\begin{array}{l}\text { La Favorita } \\
3 \text { Touchstone } \\
\text { fi Orville }\end{array}$ \\
\hline
\end{tabular}


Heredity.

\begin{tabular}{|c|c|c|c|c|}
\hline No. & Names of Stallions & Born & Sire & Dam \\
\hline$: 31$ & $\begin{array}{l}\text { Rosebery } \\
3 \text { Touchistone } \\
6 \text { Orville (by Bening- } \\
\text { brough) }\end{array}$ & 1872 & $\begin{array}{l}\text { Speculum } \\
6 \text { Orville }\end{array}$ & $\begin{array}{l}\text { Ladylike } \\
5 \text { Orville } \\
6 \text { Beningbrough }\end{array}$ \\
\hline$\therefore$ & $\begin{array}{l}\text { St. Blaise } \\
3 \text { Tuuchstone } \\
\text { (by Camel) } \\
6 \text { II halebone }\end{array}$ & 1881 & $\begin{array}{l}\text { Iermit } \\
\text { + Camel (by Whale- } \\
\text { bone) } \\
6 \text { Selim }\end{array}$ & $\begin{array}{l}\text { Fusee } \\
5 \text { Whalebone } \\
5 \text { IVaxy } \\
5 \text { Penelope }\end{array}$ \\
\hline 36 & $\begin{array}{l}\text { St. Gatien } \\
3 \text { Newminster } \\
5 \text { Birdcatcher }\end{array}$ & 1881 & $\begin{array}{l}\text { Rotherhill } \\
\text { (or The Rover) } \\
2 \text { Touchstone }\end{array}$ & $\begin{array}{l}\text { St. Editha } \\
3 \text { Birdcatcher }\end{array}$ \\
\hline 37 & $\begin{array}{l}\text { Le Sancy } \\
3 \text { Windhound } \\
3 \text {. Vlice Hawthorn } \\
5 \text { Touchstone }\end{array}$ & $188 t$ & $\begin{array}{l}\text { Atlantic } \\
7 \text { Orville } \\
7 \text { Buzzard } \\
7 \text {.1are by Nlexander }\end{array}$ & $\begin{array}{l}\text { Gem of Gems } \\
4 \text { Touchstone (Grand- } \\
\text { sire of Windhound) } \\
\text { Grand-sire: } \\
2 \text { Touchstone } \\
\text { Grand-dam : } \\
2 \text { Pantaloon (Sire of } \\
\text { IVindhound) }\end{array}$ \\
\hline :34 & $\begin{array}{l}\text { Selin } \\
3 \text { Herod } \\
5 \text { Natchem (by Cade) } \\
6 \text { Resulus (by Godol. } \\
\text {. Irabian) } \\
\text { fown brother to } \\
\text { Castrel and Rubens| }\end{array}$ & 1802 & $\begin{array}{l}\text { Buzzard } \\
3 \text { Cade (by Godol. } \\
\text { Irabian) } \\
5 \text { Partner (Grand-sire } \\
\text { of 1Herod) }\end{array}$ & $\begin{array}{l}\text { Mare by Alexander } \\
5 \text { Regulus (by Godol. } \\
\text { Arabian) } \\
6 \text { Godol. Arabian }\end{array}$ \\
\hline $39 !$ & $\begin{array}{l}\text { Silvio } \\
3 \text { Birdcatcher } \\
5 \text { Partisan }\end{array}$ & 1874 & $\begin{array}{l}\text { Blair Athol } \\
6 \text { IThalebone } \\
7 \text { Orvilie }\end{array}$ & $\begin{array}{l}\text { Silverhair } \\
7 \text { Pot8os } \\
7 \text { Orville }\end{array}$ \\
\hline 411 & $\begin{array}{l}\text { Sir Archy } \\
\text { \& llerod } \\
5 \text { Blank (by Godol. } \\
\text {. Irabian) } \\
\text { \% Childers }\end{array}$ & $\begin{array}{c}1805 \\
\text { in } \\
\text { ineric: }\end{array}$ & $\begin{array}{l}\text { Diomed } \\
+ \text { Crab } \\
+ \text { Partner (Grand-sire } \\
\text { of llerod) } \\
+ \text { Godol. Arabian } \\
5 \text { Childers }\end{array}$ & $\begin{array}{l}\text { Castianira } \\
6 \text { Godol. Arabian } \\
6 \text { Regulus }\end{array}$ \\
\hline 41 & $\begin{array}{l}\text { Sir Peter } \\
3 \text { Regulus } \\
4 \text { Godol. Arabian } \\
5 \text { Flying Childers } \\
\text { j Fox }\end{array}$ & 1784 & $\begin{array}{l}\text { Highflyer } \\
{ }_{4}\left\{\begin{array}{l}\text { Fi. Childers } \\
\text { Bart. Childers }\end{array}\right. \\
5 \text { Sister to Mixbury }\end{array}$ & $\begin{array}{l}\text { Papillon } \\
\begin{array}{l}3\left\{\begin{array}{l}\text { Fl. Childers } \\
\text { Bart. Childers }\end{array}\right. \\
+ \text { Bay Bolton }\end{array}\end{array}$ \\
\hline $4: 2$ & $\begin{array}{l}\text { Tramp } \\
3 \text { Eclipse } \\
+ \text { Herod }\end{array}$ & 1810 & $\begin{array}{l}\text { Dick Andrews } \\
4 \text { Blank } \\
5 \text { Regulus }\end{array}$ & $\begin{array}{l}\text { Mare by Gohanna } \\
3 \text { Eclipse } \\
3 \text { Herod }\end{array}$ \\
\hline
\end{tabular}




\begin{tabular}{|c|c|c|c|c|}
\hline No. & Nimes of Stallions & Born & Sire & D):Im \\
\hline 43 & $\begin{array}{l}\text { Tibthorpe } \\
3 \text { Mulatto } \\
4 \text { Blaclislocl: }\end{array}$ & 1864 & $\begin{array}{l}\text { Voltigeur } \\
\text { j Himbletonian } \\
\text { (Crand-sire of } \\
\text { Blacklock) }\end{array}$ & $\begin{array}{l}\text { Little. Irnes } \\
7 \text { Orville (Grand-sire } \\
\text { of Mulatlo) }\end{array}$ \\
\hline$H$ & $\begin{array}{l}\text { Vedette } \\
3 \text { Blacklock } \\
6 \text { Walton (by Sir } \\
\text { Peter) }\end{array}$ & 1854 & $\begin{array}{l}\text { Voltigeur } \\
\text { J llambletonian } \\
\text { (Grand-sire of } \\
\text { Blaclilock) } \\
6 \text { Sir Peter }\end{array}$ & $\begin{array}{c}\text { Mrs. Ridgway } \\
\text { 6 Jorsus }\end{array}$ \\
\hline 45 & $\begin{array}{l}\text { Vertugadin } \\
3 \text { Partisin } \\
6 \text { Whalebont } \\
7 \text { lieningbrough }\end{array}$ & 1862 & $\begin{array}{l}\text { Fitz Gladiator } \\
\text { j Sir Peter (Grand- } \\
\text { sire of Partisan) } \\
\text { j Buzzard } \\
\text { j Mare by . Nesander } \\
\text { j Golmanna }\end{array}$ & $\begin{array}{l}\text { Vermeille } \\
4 \text { llhalebone } \\
5 \text { Orville (by bening- } \\
\text { brough) }\end{array}$ \\
\hline 46 & $\begin{array}{l}\text { Walton } \\
3 \text { Snap } \\
3 \text { Herod } \\
4 \text { Regulus } \\
6 \text { Partner }\end{array}$ & 1799 & $\begin{array}{l}\text { Sir Peter } \\
3 \text { Rexulus } \\
\text { 5 Fox } \\
\text { j lilying Childers }\end{array}$ & $\begin{array}{l}\text { Arethusa } \\
3 \text { Regulus } \\
\text { j) J'ar(ner (Grand-sire } \\
\text { of Herod) }\end{array}$ \\
\hline 47 & $\begin{array}{l}\text { WTeatherbit } \\
3 \text { Orvilic } \\
5 \text { Y. Giantess } \\
6 \text { Woodpecker } \\
\text { (by Herod) }\end{array}$ & $184^{2} 2$ & $\begin{array}{l}\text { Sheet Anchor } \\
5 \text { Woodpecker } \\
6 \text { llightler } \\
6 \text { Nercury }\end{array}$ & $\begin{array}{l}\text { Miss lectly } \\
\text { l Orville } \\
6 \text { Hero! }\end{array}$ \\
\hline 48 & $\begin{array}{l}\text { IVhalebone } \\
3 \text { llerod } \\
4 \text { Snat) } \\
6 \text { Cade (by Godol } \\
\text { Arabian) }\end{array}$ & 1807 & $\begin{array}{l}\text { Wasy } \\
5 \text { Godul. Irabian } \\
6 \text { Pirtner (Grand-sire } \\
\text { of llerod) }\end{array}$ & 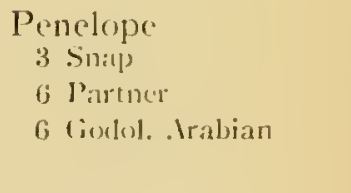 \\
\hline 49 & $\begin{array}{l}\text { Whitelock } \\
3 \text { Ilerod (by Tartar) } \\
3 \text { liclipse } \\
3 \text { Matelen }\end{array}$ & 1803 & $\begin{array}{l}\text { Hambletonian } \\
4 \text { Tiutar (by Partiner) } \\
5 \text { lienulus } \\
5 \text { Crab } \\
6 \text { Ciudol. Irabian }\end{array}$ & $\begin{array}{l}\text { Rosalind } \\
4 \text { P'artnes } \\
\text { Cirind-diun: } \\
\text { 3 l'irturer }\end{array}$ \\
\hline 5() & $\begin{array}{l}\text { IV hislier } \\
3 \text { llerod } \\
4 \text { Suap } \\
6 \text { ('inde (by Ciodol. } \\
\text { Irabian) }\end{array}$ & 1812 & $\begin{array}{l}\text { Maxy } \\
\text { j rindol. Irabian } \\
\text { 6 Partner (Grant-sire } \\
\text { of llerod) }\end{array}$ & 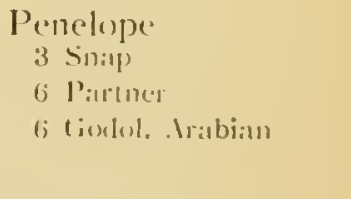 \\
\hline 51 & $\begin{array}{l}\text { Mindhound } \\
3 \text { Peruvian } \\
5 \text { Mare by .llexander } \\
\text { (by liclipe) } \\
5 \text { Bumard }\end{array}$ & $18+7$ & $\begin{array}{l}\text { Pantaloun } \\
\text { \& liclipe } \\
+ \text { llightlyce (Grimd- } \\
\text { sire of Perutian) } \\
\text { i llerod }\end{array}$ & $\begin{array}{l}\text { Phrione } \\
4 \text { il:in! }\end{array}$ \\
\hline
\end{tabular}


Stallions with + Free Generations.

\begin{tabular}{|c|c|c|c|c|}
\hline No. & Names of stallions & Born & Sire & Dam \\
\hline 1 & $\begin{array}{l}\text { Abercorn } \\
4 \text { Parasuay } \\
\text { (by Sir Hercules) }\end{array}$ & $\begin{array}{c}18 S 1 \\
\text { in } \\
\text { Austral. }\end{array}$ & $\begin{array}{l}\text { Chester } \\
5 \text { Sir Hercules } \\
5 \text { Enilius }\end{array}$ & $\begin{array}{l}\text { Cinnamon } \\
4 \text { Sir Hercules }\end{array}$ \\
\hline 2 & $\begin{array}{l}\text { Adventurer } \\
4 \text { Orrille (by Bening- } \\
\text { brough) } \\
6 \text { Stamford (by Sir } \\
\text { Peter) }\end{array}$ & 1859 & $\begin{array}{l}\text { Newminster } \\
\text {; Beningbrough } \\
\text { j Trumpator }\end{array}$ & $\begin{array}{l}\text { Palma } \\
1 \text { Orville } \\
4 \text { Sir Peter }\end{array}$ \\
\hline 3 & $\begin{array}{l}\text { Arbitrator } \\
4 \text { Touch-tone } \\
8 \text { Orville }\end{array}$ & 1874 & $\begin{array}{l}\text { Solon } \\
\text { j IIhalebone (Grand- } \\
\text { sire of Touchstone) } \\
\text { j Comus }\end{array}$ & $\begin{array}{l}\text { True Heart } \\
5 \text { Orville }\end{array}$ \\
\hline 4 & $\begin{array}{l}\text { The Bard } \\
4 \text { Melbournc } \\
7 \text { Defence (by Whale- } \\
\text { bune, Girand-sire of } \\
\text { Touchstone) }\end{array}$ & 1883 & $\begin{array}{l}\text { Petrarch } \\
2 \text { Touchstone } \\
\text { i Selim }\end{array}$ & $\begin{array}{l}\text { Magdalene } \\
6 \text { Comus (Grand-sire } \\
\text { of Nelbourne) } \\
6 \text { Sultan (by Selim) } \\
6 \text { Whutebone }\end{array}$ \\
\hline 5 & $\begin{array}{l}\text { The Barom } \\
4 \text { llisy (by Potsos) } \\
4 \text { Penelope }\end{array}$ & $18+2$ & $\begin{array}{l}\text { Birdcatcher } \\
5 \text { Iloodpecker } \\
\text { 6 Iiclipse } \\
6 \text { Highflyer } \\
6 \text { Herod }\end{array}$ & $\begin{array}{l}\text { Echidna } \\
5 \text { Potsos } \\
6 \text { Phoenunenon } \\
\text { (by Herod) } \\
6 \text { Highflyer } \\
\text { (by Herod) }\end{array}$ \\
\hline 6 & $\begin{array}{l}\text { Bay Middleton } \\
4 \text { Sir l'cter (by High- } \\
\text { flycr) } \\
4 \text {. Vrethusa } \\
7 \text { Eclip-e }\end{array}$ & 1833 & $\begin{array}{l}\text { Sultan } \\
4 \text { Highliger } \\
4 \text { llerod (Grand-sire } \\
\text { uf Diomed) } \\
4 \text { Eclipse }\end{array}$ & $\begin{array}{l}\text { Cobweb } \\
3 \text { Y. Giantess } \\
\text { (by Diomed) } \\
5 \text { Highflyer } \\
6 \text { Eclipse } \\
6 \text { IJerod }\end{array}$ \\
\hline 7 & $\begin{array}{l}\text { Bill of Portland } \\
4 \text { Pocalontas } \\
6 \text { Birdcatcher }\end{array}$ & 1890 & $\begin{array}{l}\text { St. Simon } \\
6 \text { Sultan (Grand-sire } \\
\text { of Pocahontas) }\end{array}$ & $\begin{array}{l}\text { Electric Light } \\
3 \text { Birdcatcher } \\
4 \text { Touchstone }\end{array}$ \\
\hline$s$ & $\begin{array}{l}\text { Bonavista } \\
4 \text { Pocihontas } \\
5 \text { Mindhound } \\
5 \text {. Vlice Hawthorn } \\
7 \text { Touchnone }\end{array}$ & 1889 & $\begin{array}{l}\text { Bend Or } \\
6 \text { Tinchstone (Grand- } \\
\text {-ire of Windhound) }\end{array}$ & $\begin{array}{l}\text { Vista } \\
4 \text { Banter (Dam of } \\
\text { Touchstone) } \\
4 \text { Pantaloon (Sire of } \\
\text { Windhound) }\end{array}$ \\
\hline$\because$ & $\begin{array}{l}\text { Cambuscan } \\
\text { \& Whalubone } \\
5 \text { Orville (by Bening- } \\
\text { brough) } \\
6 \text { Beninghrough }\end{array}$ & 1861 & $\begin{array}{l}\text { Newminster } \\
5 \text { Beningbrough } \\
5 \text { Trumpator }\end{array}$ & $\begin{array}{l}\text { The Arrow } \\
4 \text { Beningbrough }\end{array}$ \\
\hline
\end{tabular}




\begin{tabular}{|c|c|c|c|c|}
\hline No. & Names of Stallions & Born & Sire & Dam \\
\hline 10 & $\begin{array}{l}\text { Cannel } \\
4 \text { Highlyer } \\
5 \text { Herod } \\
5 \text { Eclipse } \\
6 \text { Snap }\end{array}$ & 19222 & $\begin{array}{l}\text { Whalebone } \\
3 \text { llerod } \\
4 \text { Snap }\end{array}$ & $\begin{array}{l}\text { Mare by Selim } \\
3 \text { Higlifyer } \\
4 \text { Herod } \\
5 \text { Eclipse }\end{array}$ \\
\hline 11 & $\begin{array}{l}\text { Catton } \\
4 \text { Herud } \\
4 \text { Eclipse } \\
5 \text { Matchem (by Cade) }\end{array}$ & 1809 & $\begin{array}{l}\text { Golumpus } \\
2 \text { Ilerod } \\
5 \text { Cade (by Godol. } \\
\text { Arabian) }\end{array}$ & $\begin{array}{l}\text { Lucy Gray } \\
3 \text { Herod } \\
6 \text { Godol. Arabian } \\
6 \text { Blanls (by Godol. } \\
\text { Arabian) } \\
6 \text { Regulus (Grand- } \\
\text { sire of Eclipse) }\end{array}$ \\
\hline 12 & $\begin{array}{l}\text { Childwick } \\
4 \text { Pocahontas } \\
5 \text { Voltigeur }\end{array}$ & $1 S 90$ & $\begin{array}{l}\text { St. Simon } \\
6 \text { Sultan (Grand-sire } \\
\text { of Pocahontas) } \\
6 \text { Velocipede's dam }\end{array}$ & $\begin{array}{l}\text { Plaisanterie } \\
6 \text { Langal } \\
\text { Grandsire: } \\
1 \text { Pocalontas }\end{array}$ \\
\hline 13 & $\begin{array}{l}\text { Count Schomberg } \\
4 \text { Stocliwell } \\
5 \text { Birdcatcher } \\
5 \text { Orlando } \\
\text { i Touchstone }\end{array}$ & 1892 & $\begin{array}{l}\text { Aughrim } \\
4 \text { Birdcatcher } \\
5 \text { Sir Hercules } \\
\text { 5 Touchstone }\end{array}$ & $\begin{array}{l}\text { Clonavarn } \\
4 \text { Touchstone } \\
5 \text { Birdcitcher }\end{array}$ \\
\hline 14 & $\begin{array}{l}\text { Cyllene } \\
4 \text { Stockwel! } \\
5 \text { lewminster } \\
\text { (by Turustone) }\end{array}$ & 1895 & $\begin{array}{l}\text { Bonavisla } \\
4 \text { Pocahontas } \\
5 \text { llindhound } \\
5 \text { Alice Hawthorn } \\
5 \text { Pantaloon } \\
\text { 7 Touclastone }\end{array}$ & $\begin{array}{l}\text { Arcadia } \\
6 \text { Touchstone }\end{array}$ \\
\hline 15 & $\begin{array}{l}\text { Le Destrier } \\
+ \text { Gladiator } \\
\text { (by Partisan) }\end{array}$ & $18 \pi i$ & $\begin{array}{l}\text { Flacreolet } \\
j \text { Partisan }\end{array}$ & $\begin{array}{l}\text { La Dheume } \\
5 \text { Partisan }\end{array}$ \\
\hline 16 & $\begin{array}{l}\text { Dick Indrews } \\
4 \text { Blank (by Godol. } \\
\text {. Irabian) } \\
5 \text { Resulus } \\
6 \text { Suip (by Fl. } \\
\text { Childers) }\end{array}$ & 1797 & $\begin{array}{l}\text { Joe Indrews } \\
+ \text { Godol. Irabian } \\
5 \text { Bart. Childers }\end{array}$ & $\begin{array}{l}\text { Mare by Highflyer } \\
3 \text { Blank } \\
6 \text { Flying Childers }\end{array}$ \\
\hline 17 & $\begin{array}{l}\text { Diomed } \\
4 \text { Crab } \\
4 \text { Codol. .Irabian } \\
4 \text { Partner } \\
5 \text { Flyiug Childers } \\
\text { (by Darl. Arabian) }\end{array}$ & 1777 & $\begin{array}{l}\text { Fiorizel } \\
+4 \text { lilying Childers }\end{array}$ & $\begin{array}{c}\text { Mare by Spectator } \\
5 \text { Ditrley" Irabian }\end{array}$ \\
\hline
\end{tabular}




\begin{tabular}{|c|c|c|c|c|}
\hline No. & Names of Stallions & Born & Sire & Dam \\
\hline 18 & $\begin{array}{l}\text { Dollar } \\
+ \text { Catton } \\
6 \text { Beningbrough } \\
6 \text { Evclina } \\
7 \text { Sir Peter } \\
7 \text { Sorcerer }\end{array}$ & $180 \%$ & $\begin{array}{l}\text { The Flying } \\
\text { Dutchman } \\
3 \text { Sclim } \\
6 \text { Sir Peter }\end{array}$ & $\begin{array}{l}\text { Payment } \\
5 \text { Sorcerer } \\
6 \text { Beningbrough }\end{array}$ \\
\hline 19 & $\begin{array}{l}\text { Economist } \\
4 \text { Herod } \\
4 \text { Eclipse } \\
5 \text { Hinhlyer }\end{array}$ & 1825 & $\begin{array}{l}\text { Whisker } \\
3 \text { Herod } \\
4 \text { Snap }\end{array}$ & $\begin{array}{l}\text { Floranthe } \\
3 \text { Herod } \\
3 \text { Eclipse }\end{array}$ \\
\hline ?) & $\begin{array}{l}\text { Energy } \\
4 \text { Sir Hercules } \\
4 \text { Birdcatcher } \\
5 \text { Touchstone } \\
5 \text { Melboune } \\
6 \text { bay Middleton } \\
\text { (by Sultan) }\end{array}$ & 1880 & $\begin{array}{l}\text { Sterling } \\
5 \text { W\%hlebone (Sire of } \\
\text { Sir Hercules) }\end{array}$ & $\begin{array}{l}\text { Cheri Ducluess } \\
4 \text { Sir Hercules (Sire } \\
\text { of Birdcatcher) } \\
5 \text { Touchstone } \\
6 \text { Sultan }\end{array}$ \\
\hline 21 & $\begin{array}{l}\text { Fisherman } \\
4 \text { Orville } \\
5 \text { Dick . Indrews }\end{array}$ & 1853 & $\begin{array}{l}\text { Herom } \\
6 \text { Eclipse (Grand-sire } \\
\text { of Dicle Indrews) } \\
7 \text { Highflyer (Grand- } \\
\text { sire of Orville) }\end{array}$ & $\begin{array}{l}\text { Mainbrace } \\
3 \text { Mandane } \\
\text { (by Potsos) }\end{array}$ \\
\hline 2 & $\begin{array}{l}\text { Flatcatcher } \\
4 \text { Wlaxy } \\
5 \text { Sir Peter } \\
6 \text { Eclipse }\end{array}$ & 1845 & $\begin{array}{l}\text { Touchstone } \\
4 \text {. Mlexander } \\
\text { (by Eclipse) } \\
\text { j Eclipse } \\
\text { 5 Sir Peter }\end{array}$ & $\begin{array}{l}\text { Decon } \\
2 \text { Sii l'eter } \\
5 \text { Eclipse }\end{array}$ \\
\hline 23 & $\begin{array}{l}\text { Florizel } \\
\text { + Flying childers } \\
\text { + Byerly Turls }\end{array}$ & 1768 & $\begin{array}{l}\text { Herud } \\
7 \text { Hautboy } \\
7 \text { Spanker } \\
7 \text { Leedes Arabian }\end{array}$ & $\begin{array}{l}\text { Mare by Cygnet } \\
3 \text { lining Clilders } \\
5 \text { Basto (by Byerly } \\
\text { Turk) }\end{array}$ \\
\hline 24 & $\begin{array}{l}\text { Galliard } \\
4 \text { Birdcatcher } \\
5 \text { Voltaire } \\
\text { (by Blacklock) }\end{array}$ & 1880 & $\begin{array}{l}\text { Galopin } \\
2 \text { Voltaire }\end{array}$ & $\begin{array}{l}\text { Maris } \\
6 \text { Blacklock } \\
\text { Grand-dam : } \\
4 \text { Blacklock }\end{array}$ \\
\hline 25 & $\begin{array}{l}\text { Gladiator } \\
4 \text { Pruncla (by High- } \\
\text { flyer) } \\
4 \text { Potsos (by Eclipse) }\end{array}$ & 1833 & $\begin{array}{l}\text { Partisan } \\
2 \text { Hicrlnfyer } \\
3 \text { Eclipse } \\
4 \text { Snap }\end{array}$ & $\begin{array}{l}\text { Pauline } \\
4 \text { Trumpator } \\
5 \text { Pot8os } \\
6 \text { Eclipse } \\
6 . \text { Highfler }\end{array}$ \\
\hline 26 & $\begin{array}{l}\text { Gouverneur } \\
4 \text { Touchstone } \\
7 \text { Whalebonc (by } \\
\text { Waxy and Penelope) }\end{array}$ & 1888 & $\begin{array}{l}\text { Energy } \\
4 \text { Sir Hercules } \\
4 \text { Birdcatcher } \\
5 \text { Touchstone } \\
5 \text { Melbourne }\end{array}$ & $\begin{array}{l}\text { Gladia } \\
5 \text { Smolenslis } \\
6 \text { Waxy } \\
6 \text { Penelope }\end{array}$ \\
\hline
\end{tabular}




\begin{tabular}{|c|c|c|c|c|}
\hline No. & Names of Stallions & Born & Sire & $1):(1,1$ \\
\hline 27 & $\begin{array}{l}\text { Hambletonian } \\
4 \text { Tartar (by Partner) } \\
5 \text { Resulus } \\
5 \text { Crab } \\
6 \text { Codul. Mrabian }\end{array}$ & 1792 & $\begin{array}{l}\text { Wing Fergus } \\
5\left\{\begin{array}{l}\text { billt. Childers } \\
\text { lil. Childers }\end{array}\right.\end{array}$ & $\begin{array}{l}\text { Mare by Highflyer } \\
\text { + Gudil. Irabian } \\
+ \text { l'artner }\end{array}$ \\
\hline 28 & $\begin{array}{l}\text { Hambures } \\
4 \text { Lexinirtur } \\
\text { 6 dileneoe }\end{array}$ & $\begin{array}{c}189.5 \\
\text { in } \\
\text { Awerlen }\end{array}$ & $\begin{array}{l}\text { Hannover } \\
2 \text { landal } \\
\text { (by Glencue) }\end{array}$ & $\begin{array}{l}\text { Lady Reel } \\
3 \text { l.exington } \\
5 \text { Giencoe } \\
\text { Cirind-dinn : } \\
2 \text { l.exington }\end{array}$ \\
\hline ניבי & $\begin{array}{l}\text { Hannibal } \\
\text { + Touchstone } \\
+ \text { Stockwell }\end{array}$ & 1891 & $\begin{array}{l}\text { Trachenbers } \\
7 \text { Bustard }\end{array}$ & $\begin{array}{l}\text { Zatma } \\
3 \text { Touchstume } \\
5 \text { Camed }\end{array}$ \\
\hline 30 & $\begin{array}{l}\text { Haphazard } \\
4 \text { Regulus (by Godol. } \\
\text { Arabian) } \\
6\left\{\begin{array}{l}\text { Bart. ('hilders } \\
\text { Fl. Childers }\end{array}\right.\end{array}$ & 1797 & $\begin{array}{l}\text { Sir Peter } \\
\text { :3 Regulus } \\
4 \text { Codol. Arabian } \\
\text { 5 Fl. Childers } \\
\text { 5 Fox }\end{array}$ & $\begin{array}{l}\text { Miss Hervey } \\
4 \text { Codol. . Mribian } \\
4 \text { bart. Chiklers }\end{array}$ \\
\hline 31 & $\begin{array}{l}\text { Hermit } \\
4 \text { Camel } \\
6 \text { Paynator } \\
\text { (by "Trumpator) } \\
6 \text { Selim } \\
7 \text { Orville (by Bening- } \\
\text { brough) }\end{array}$ & 1864 & $\begin{array}{l}\text { Newminster } \\
\text { j Frumpator } \\
\text { j Beningbroush }\end{array}$ & $\begin{array}{l}\text { Seclusion } \\
\text { 3 Sulan (by Selim) } \\
\text { i Orville }\end{array}$ \\
\hline 32 & $\begin{array}{l}\text { Joe . Indrews } \\
4 \text { Gudol. Irabian } \\
5 \text { Bart. Childers (by } \\
\text { D. Irmbian and } \\
\text { Bett! I.eedes) }\end{array}$ & 1788 & $\begin{array}{l}\text { Eclipse } \\
3 \text { Sister w Old Coun- } \\
\text { twy llench } \\
+ \text { Snilie }\end{array}$ & $\begin{array}{l}\text { Amaranda } \\
3 \text { Ciodol. Irabian } \\
4 \text { Partner } \\
6 \text { Darl. Irabian } \\
6 \text { Betty leedes }\end{array}$ \\
\hline 33 & $\begin{array}{l}\text { Iiendal } \\
4 \text { Birdcaticher } \\
5 \text { Pantaloon }\end{array}$ & 1583 & $\begin{array}{l}\text { Bend } \mathrm{Or}_{\mathrm{r}} \\
6 \text { Tinchstune } \\
6 \text { Muley }\end{array}$ & $\begin{array}{l}\text { Windermere } \\
7 \text { Bumard (Grand- } \\
\text { sire of Pantaloon) } \\
7 \text { Orville }\end{array}$ \\
\hline 34 & $\begin{array}{l}\text { Lingston } \\
+ \text { Smolenslis } \\
\text { is Sir I'eter (by High- } \\
\text { fler) } \\
\text { - Eclipse }\end{array}$ & 1849 & $\begin{array}{l}\text { Venison } \\
\text {.j Eclipse } \\
6 \text { Herod }\end{array}$ & $\begin{array}{l}\text { Queen inne } \\
6 \text { Hishiger }\end{array}$ \\
\hline $3 j$ & $\begin{array}{l}\text { Kingston } \\
4 \text { Melboune } \\
5 \text { Glencoe } \\
\text { 5 Touretistone }\end{array}$ & $\begin{array}{c}189 ! \\
\text { int } \\
\text { Arbertica }\end{array}$ & $\begin{array}{l}\text { Spendthrift } \\
\text { 5 limilius } \\
\text { fo Selim (Grand-sire } \\
\text { of Glencoe) }\end{array}$ & $\begin{array}{l}\text { Kapangal } \\
6 \text { Blacislocli } \\
7 \text { Whislebone (Grand- } \\
\text { sire of Touchstone) }\end{array}$ \\
\hline
\end{tabular}


Heredity.

\begin{tabular}{|c|c|c|c|c|}
\hline No. & Names of Stallions & Born & Sire & 1)an \\
\hline 36 & $\begin{array}{l}\text { Lambton } \\
4 \text { Orville (by Bening- } \\
\text { brough) } \\
6 \text { Whisliey } \\
7 \text { Sir Peter }\end{array}$ & 1850 & $\begin{array}{l}\text { The Cure } \\
6 \text { Sir Peter }\end{array}$ & $\begin{array}{l}\text { Elphine } \\
2 \text { Beningbrough } \\
5 \text { Sir Peter }\end{array}$ \\
\hline 37 & $\begin{array}{l}\text { Lanercost } \\
4 \text { Gohanna } \\
5 \text { Woodpeclier } \\
\text { (by Herod) } \\
5 \text { Highfler } \\
\text { (by Herod) } \\
5 \text { Trumpator } \\
6 \text { Eclipse }\end{array}$ & 1835 & $\begin{array}{l}\text { Liverpool } \\
4 \text { Eclipse (Grand-sire } \\
\text { of Gohanna) } \\
5 \text { Woodpecler } \\
5 \text { Highflyer } \\
5 \text { Trentham } \\
6 \text { Herod }\end{array}$ & $\begin{array}{l}\text { Otis } \\
3 \text { Woodpeclier } \\
4 \text { Herod } \\
\text { Grand-dam : } \\
2 \text { Herod } \\
3 \text { Eclipse }\end{array}$ \\
\hline 38 & $\begin{array}{l}\text { Liverpool } \\
4 \text { Eclipse } \\
5 \text { Highllyer } \\
\text { (by llernd) } \\
5 \text { IInodpecler } \\
\text { (by Herod) } \\
5 \text { Trentham } \\
6 \text { Herod }\end{array}$ & 1828 & $\begin{array}{l}\text { Tramp } \\
3 \text { Eclipse } \\
4 \text { Herod }\end{array}$ & $\begin{array}{l}\text { Mare by Whisker } \\
1 \text { Potsos (by Eclipse) } \\
4 \text { Herod }\end{array}$ \\
\hline 39 & $\begin{array}{l}\text { Longbow } \\
4 \text { Orville } \\
7 \text { llightlyer } \\
7 \text { lloodpecker }\end{array}$ & $18+9$ & $\begin{array}{l}\text { Ithuriel } \\
5 \text { Buz\%ard (by Wood- } \\
\text { peclier) } \\
5 \text { Mare by Alexander }\end{array}$ & $\begin{array}{l}\text { Miss Bowe } \\
5 \text { High flyer } \\
6 \text { Herod } \\
6 \text { Eclipse }\end{array}$ \\
\hline 40 & $\begin{array}{l}\text { Marsyas } \\
4 \text { Waxy } \\
4 \text { Penelope }\end{array}$ & 18.51 & $\begin{array}{l}\text { Orlando } \\
3 \text { Selim } \\
5 . \text { Hexander }\end{array}$ & $\begin{array}{l}\text { Malibran } \\
5 \text { Herod }\end{array}$ \\
\hline 41 & $\begin{array}{l}\text { Master Kildare } \\
4 \text { Birdcatcher } \\
8 \text { Blaclincli }\end{array}$ & 1875 & $\begin{array}{l}\text { Lord Ronald } \\
\text { f Whalebone (Grand- } \\
\text { sire of Birdcatcher) } \\
7 \text { Blacliocli }\end{array}$ & $\begin{array}{l}\text { Silk } \\
\quad 6 \text { Blaclilock }\end{array}$ \\
\hline 42 & $\begin{array}{l}\text { Meddler } \\
4 \text { Newninster }\end{array}$ & 1890 & $\begin{array}{l}\text { St. Gatien } \\
3 \text { Newminster } \\
5 \text { Birdcatcher } \\
\text { Grand-sire: } \\
3 \text { Melbourne }\end{array}$ & $\begin{array}{l}\text { Busybody } \\
5 \text { Venison } \\
\text { Grand-sire: } \\
2 \text { Touchstone }\end{array}$ \\
\hline 43 & $\begin{array}{l}\text { Melbourne } \\
4 \text { Termagant } \\
5 \text { Highfiger } \\
\text { (by llerod) } \\
5 \text { Trumpator } \\
6 \text { Eclipse }\end{array}$ & 1834 & $\begin{array}{l}\text { Humphrey Clinker } \\
2 \text { Sir Peter (by High- } \\
\text { Inyer) } \\
4 \text { Timpator } \\
7 \text { Eclipse }\end{array}$ & $\begin{array}{l}\text { Mare by Cervantes } \\
4 \text { Eclipse } \\
5 \text { Herod }\end{array}$ \\
\hline
\end{tabular}




\begin{tabular}{|c|c|c|c|c|}
\hline No. & Names of Stallions & Born & Sire & Dam \\
\hline$H$ & $\begin{array}{l}\text { Mercury } \\
\text { \& Godol. .Irabian }\end{array}$ & 1778 & $\begin{array}{l}\text { Eclipse } \\
\text { 3 sister to Old Coun- } \\
\text { try IIench } \\
4 \text { Sinatie }\end{array}$ & $\begin{array}{l}\text { Mare by Tartar } \\
5 \text { Curwens Bay Barb }\end{array}$ \\
\hline 45 & $\begin{array}{l}\text { Mortemer } \\
+ \text { Partisan } \\
\text { (by lbalton) } \\
6 \text { Orville }\end{array}$ & 1865 & $\begin{array}{l}\text { Compiegne } \\
6 \text { Whalebone } \\
7 \text { Buzzard } \\
7 \text { Nare by Alexander } \\
\text { i Sil l'eter (Sire of } \\
\text { Wniton) }\end{array}$ & $\begin{array}{l}\text { Comtesse } \\
1 \text { Emilius (by Orville) } \\
6 \text { Sir Peter }\end{array}$ \\
\hline 40 & $\begin{array}{l}\text { Muley } \\
\text { \& Eclipse } \\
\text { \& Herod } \\
\text { i Matchem }\end{array}$ & 1810 & $\begin{array}{l}\text { Orville } \\
2 \text { llerod }\end{array}$ & $\begin{array}{l}\text { Eleanor } \\
3 \text { Herod } \\
3 \text { Matchem }\end{array}$ \\
\hline 47 & $\begin{array}{l}\text { Mtisket } \\
+ \text { Touchnone } \\
\text { (by (amel) } \\
+ \text { Camel }\end{array}$ & 1867 & $\begin{array}{l}\text { Toxuphilite } \\
7 \text { Sir Peter } \\
\text {; } 11 \text { inolpeclier }\end{array}$ & $\begin{array}{l}\text { Mare by IVest- } \\
\text { Australian } \\
2 \text { Camel }\end{array}$ \\
\hline is & $\begin{array}{l}\text { The Nabob } \\
\text { \& Selim } \\
\text { \& Orville } \\
\text { \& J'enelope }\end{array}$ & 1819 & 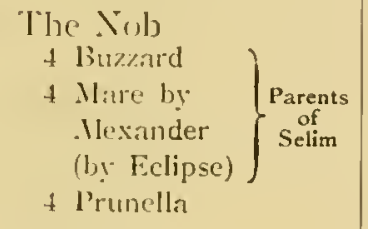 & $\begin{array}{l}\text { Hester } \\
5 \text { Wondpecker (Sire } \\
\text { of Buzzard) } \\
6 \text { Eclijse }\end{array}$ \\
\hline 49 & $\begin{array}{l}\text { The Nob } \\
4 \text { Buwzard } \\
4 \text { Mare by Alexander } \\
4 \text { Prunella (by High- } \\
\text { Myer) } \\
\text { 6 Sir l'eter }\end{array}$ & 1839 & $\begin{array}{l}\text { Glaticus } \\
3 \text { Yir loter (by High- } \\
\text { Ilyer) } \\
\text { 5 liclipse }\end{array}$ & $\begin{array}{l}\text { Octave } \\
4 \text { Hightlyer }\end{array}$ \\
\hline 50 & $\begin{array}{l}\text { Nordenfeldt } \\
4 \text { Melbourne } \\
\text { is Touchstone }\end{array}$ & 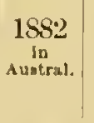 & $\begin{array}{l}\text { Musliet } \\
\text { \& Touchistone } \\
\text { \& Camel }\end{array}$ & $\begin{array}{l}\text { Onyx } \\
\text { i) Touchstone }\end{array}$ \\
\hline$\tilde{j} 1$ & $\begin{array}{l}\text { Pantaloon } \\
4 \text { Hishtlyer } \\
\text { (by Ilerod) } \\
\text { 4 Eclipse } \\
\text { 5 IIerod }\end{array}$ & 1824 & $\begin{array}{l}\text { Castrel } \\
3 \text { llerod } \\
5 \text { Matchem } \\
6 \text { lesrulus }\end{array}$ & $\begin{array}{l}\text { Idalia } \\
2 \text { Highliver } \\
3 \text { Eelipse } \\
6 \text { Recrulus }\end{array}$ \\
\hline 32 & $\begin{array}{l}\text { Phantom } \\
+ \text { Herod } \\
4 \text { Eclipse } \\
\text { i Snap }\end{array}$ & 1808 & $\begin{array}{l}\text { IV alton } \\
3 \text { ilerod } \\
3 \text { Snap } \\
4 \text { Regulus }\end{array}$ & $\begin{array}{l}\text { Julia } \\
3 \text { Herod } \\
3 \text { Matchen }\end{array}$ \\
\hline
\end{tabular}




\begin{tabular}{|c|c|c|c|c|}
\hline No. & Names of Stallions & Born & Sire & 1) $\mathrm{am}$ \\
\hline 53 & $\begin{array}{l}\text { Phönomenon } \\
4 \text { Blaze }\end{array}$ & 1780 & $\begin{array}{l}\text { Herod } \\
7 \text { Leedes Lrabian } \\
7 \text { Hautboy } \\
7 \text { Spanker }\end{array}$ & $\begin{array}{l}\text { Frenzy } \\
4 \text { (iodol. Arabian }\end{array}$ \\
\hline 54 & $\begin{array}{l}\text { Plenipotentiary } \\
4 \text { Sir Peter (by High- } \\
\text { flyer) } \\
5 \text { Highnyer } \\
7 \text { Eclipse }\end{array}$ & 1831 & $\begin{array}{l}\text { Emilius } \\
3 \text { Hlishtiyer } \\
4 \text { Eclipse }\end{array}$ & $\begin{array}{l}\text { Harriet } \\
4 \text { Highllyer } \\
6 \text { Eclipse }\end{array}$ \\
\hline 5.5 & $\begin{array}{l}\text { Plutus } \\
4 \text { limilius (by Orville) } \\
6 \text { Selim }\end{array}$ & $1866^{\circ}$ & $\begin{array}{l}\text { Prumpeter } \\
4 \text { Selim } \\
5 \text { Orville } \\
5 \text { Penelope }\end{array}$ & $\begin{array}{l}\text { Mare by Planet } \\
3 \text { Sultan (by Selim) }\end{array}$ \\
\hline 56 & $\begin{array}{l}\text { Pot8os } \\
4 \text { Crodol. Irabian }\end{array}$ & 1773 & $\begin{array}{l}\text { Eclipse } \\
3 \text { Sister to Old Coun- } \\
\text { try llench } \\
+ \text { Sinalie }\end{array}$ & $\begin{array}{l}\text { Sportsmistress } \\
5 \text {.Ancaster Turk }\end{array}$ \\
\hline 57 & $\begin{array}{l}\text { Rayon d'Or } \\
4 \text { Touchstone }\end{array}$ & 1876 & $\begin{array}{l}\text { Flaceolet } \\
5 \text { Partisan }\end{array}$ & $\begin{array}{l}\text { Araucaria } \\
5 \text { Orville } \\
5 \text { Selim }\end{array}$ \\
\hline ss & $\begin{array}{l}\text { Rosicrucian } \\
4 \text { Priam } \\
5 \text { Whilebone } \\
7 \text { Selim } \\
\text { [own broller w The } \\
\text { Palmer] }\end{array}$ & 1865 & $\begin{array}{l}\text { Beadsman } \\
\text {; Tramp } \\
\text {; Orville (Grand-sire } \\
\text { "f Priam) } \\
\text { - Bu\%ard }\end{array}$ & $\begin{array}{l}\text { Mad. Eglentine } \\
+ \text { Phanion } \\
6 \text { Buz\%ald }\end{array}$ \\
\hline 59 & $\begin{array}{l}\text { Royal Hampton } \\
4 \text { Pocahontas } \\
6 \text { Pouchstone }\end{array}$ & 188:2 & $\begin{array}{l}\text { Hampton } \\
7 \text { IVhalebone (Grand- } \\
\text { sire of Touclistone) }\end{array}$ & $\begin{array}{l}\text { Princess } \\
4 \text { Sultim (Grand-sire } \\
\text { of Pucihontas) } \\
\text { 5 IVhislier }\end{array}$ \\
\hline 60 & $\begin{array}{l}\text { Saphir } \\
4 \text { Pocahontas } \\
6 \text { Touchstone } \\
6 \text { Gladiator } \\
7 \text { Emilius }\end{array}$ & 1888 & $\begin{array}{l}\text { Channant } \\
5 \text { Enilius } \\
\text { 6 Orville }\end{array}$ & $\begin{array}{l}\text { Sappho } \\
6 \text { Touchstone } \\
\text { Grand-sire : } \\
2 \text { Pocajontas } \\
\text { Grand-dam : } \\
4 \text { Touchstone }\end{array}$ \\
\hline 61 & $\begin{array}{l}\text { Sir Hercules } \\
4 \text { Eclipse } \\
5 \text { Herod } \\
5 \text { Himhllyer } \\
7 \text { Snap }\end{array}$ & $18: 20$ & $\begin{array}{l}\text { 11 halebone } \\
3 \text { Herod } \\
4 \text { Sina }\end{array}$ & $\begin{array}{l}\text { Peri } \\
3 \text { Eclipse } \\
5 \text { llerod }\end{array}$ \\
\hline 62 & $\begin{array}{l}\text { Sir Hercules } \\
4 \text { Waxy (by Potsos) } \\
4 \text { Penelope }\end{array}$ & $\begin{array}{c}1813 \\
\text { in } \\
\text { Anstrul. }\end{array}$ & $\begin{array}{l}\text { Cap à Pie } \\
211: x y\end{array}$ & $\begin{array}{l}\text { Paraguay } \\
4 \text { l'otsos }\end{array}$ \\
\hline
\end{tabular}


4. Inbreeding.

\begin{tabular}{|c|c|c|c|c|}
\hline No. & Names of Stallions & Born & Sire & Dam \\
\hline 63 & $\begin{array}{l}\text { Sir Patul } \\
+ \text { Resulus (by Godol. } \\
\text {. Mrabian) } \\
\text { J Suip (by l\%l. } \\
\text { Childers) }\end{array}$ & $180: 2$ & $\begin{array}{l}\text { Sir Peter } \\
3 \text { liegulus } \\
1 \text { Codol. Irabian } \\
5 \text { lil. Childers } \\
\text { ifox }\end{array}$ & $\begin{array}{l}\text { Prwett } \\
3 \text { Regulus } \\
4 \text { Godol. Irabian } \\
6 \text { kl. Clailders } \\
\text { Grand-dam : } \\
3 \text { Cindol. Irabian }\end{array}$ \\
\hline 64 & $\begin{array}{l}\text { Smolenslio } \\
4 \text { Herod } \\
5 \text { Snap } \\
6 \text { Cade (by Godol. } \\
\text {. Mrabian) }\end{array}$ & 1810 & $\begin{array}{l}\text { Surcerer } \\
\because \text { Mlatchem (by cade) }\end{array}$ & $\begin{array}{l}\text { Mouski } \\
1 \text { Herod } \\
3 \text { Snap } \\
5 \text { Giodol. Irabian }\end{array}$ \\
\hline (ij) & $\begin{array}{l}\text { Sultan } \\
4 \text { Herod } \\
1 \text { Himhbyer } \\
\text { (by Herod) } \\
1 \text { Eiclipse }\end{array}$ & 1816 & $\begin{array}{l}\text { Selim } \\
3 \text { 1lerod } \\
5 \text { Matelsem }\end{array}$ & $\begin{array}{l}\text { Bacchante } \\
3 \text { Herod } \\
3 \text { Eclipse }\end{array}$ \\
\hline 60 & $\begin{array}{l}\text { Sweetmeat } \\
4 \text { llitum (by Sir } \\
\text { leter) } \\
6 \text { Potsos } \\
\text { - Iligluyger }\end{array}$ & 1842 & $\begin{array}{l}\text { Gladiator } \\
+ \text { l'otsos } \\
\text { + Prunella (by High- } \\
\text { fyer) }\end{array}$ & $\begin{array}{l}\text { Lollypop } \\
0 \text { Bilacklock } \\
\text { 6. Sir Peter (by High- } \\
\text { tlyer) }\end{array}$ \\
\hline 67 & $\begin{array}{l}\text { Touchstone } \\
\text {-: !rexander } \\
\text { (by Eclipse) } \\
5 \text { Eclipse } \\
\text { 5 Sir Peter (by High- } \\
\text { flyer) } \\
\text { 5 Buzzard } \\
6 \text { IIishflyer }\end{array}$ & 1831 & $\begin{array}{l}\text { Camel } \\
4 \text { Hichlyer } \\
\text { j Eclipse } \\
\text { 5 Herod }\end{array}$ & $\begin{array}{l}\text { Banter } \\
\quad+\text { Eclipse }\end{array}$ \\
\hline $6 t$ & $\begin{array}{l}\text { Tranby } \\
4 \text { ling lergus } \\
4 \text { Highnler } \\
5 \text { Herod }\end{array}$ & 1826 & $\begin{array}{l}\text { Blacklock } \\
\text { \& Hightyer } \\
\text { + Eclipse } \\
\text { + Herod }\end{array}$ & $\begin{array}{l}\text { Mare by Orville } \\
3 \text { Herol } \\
4 \text { Eelipse }\end{array}$ \\
\hline $6: 1$ & $\begin{array}{l}\text { Trappist } \\
+1 \text { Camel }\end{array}$ & 187.2 & $\begin{array}{l}\text { Ifermit } \\
\text { t Camel } \\
\text { 6. Selim (Cirand-sire } \\
\text { of Camel) }\end{array}$ & $\begin{array}{l}\text { Bunsh } \\
\text { I Lottery }\end{array}$ \\
\hline 71 & $\begin{array}{l}\text { Trumpator } \\
\text { + Partner } \\
\text { \& rodol. Irabian } \\
\text {; Brown Farewel] }\end{array}$ & 178:2 & $\begin{array}{l}\text { Condtictor } \\
3 \text { Partner }\end{array}$ & $\begin{array}{l}\text { Brinette } \\
\text { I Partner }\end{array}$ \\
\hline
\end{tabular}




\begin{tabular}{|c|c|c|c|c|c|}
\hline No. & Names of Stallions & Born & Sire & \multicolumn{2}{|l|}{ Dam } \\
\hline 71 & $\begin{array}{l}\text { Trumpeter } \\
4 \text { Selim } \\
5 \text { Orville } \\
5 \text { Penelope }\end{array}$ & 1856 & $\begin{array}{l}\text { Orlando } \\
3 \text { Selim } \\
5 \text { Alexander }\end{array}$ & 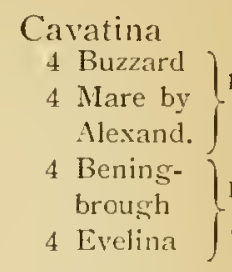 & $\begin{array}{l}\text { Parents } \\
\text { of } \\
\text { Selim } \\
\text { Parents } \\
\text { of } \\
\text { Orville }\end{array}$ \\
\hline 72 & $\begin{array}{l}\text { Vermouth } \\
4 \text { Emilius (by Orville) } \\
4 \text { Partisan } \\
5 \text { Whalebone }\end{array}$ & 1861 & $\begin{array}{l}\text { The Nabob } \\
4 \text { Orville } \\
4 \text { Penelope (Dam of } \\
\text { IIhalebone) } \\
4 \text { Selim } \\
4 \text { Prunella }\end{array}$ & $\begin{array}{l}\text { Vermeille } \\
4 \text { Whalebone } \\
5 \text { Orville }\end{array}$ & \\
\hline 73 & $\begin{array}{l}\text { Virgil } \\
4 \text { Tramp } \\
6 \text { Orville } \\
6 \text { Blacklock's dam }\end{array}$ & $\begin{array}{c}1864 \\
\text { in } \\
\text { Aweriont }\end{array}$ & $\begin{array}{l}\text { Vandal } \\
7 \text { Pot8os (Grand-sire } \\
\text { of Blaclilocle's dam) }\end{array}$ & Hymenia & \\
\hline 74 & $\begin{array}{l}\text { Yirgilius } \\
4 \text { Orville } \\
6 \text { Waxy } \\
7 \text { Sir Peter } \\
7 \text { Highflyer }\end{array}$ & 185 & $\begin{array}{l}\text { Toltigeur } \\
j \text { Hambletonian } \\
6 \text { Sir Peter (by High- } \\
\text { Ayrer. Grand-sire of } \\
\text { Orville and Hamble- } \\
\text { tonian) } \\
7 \text { Highfyer }\end{array}$ & $\begin{array}{l}\text { Eclogue } \\
5 \text { Sir Peter } \\
5 \text { Highflyer }\end{array}$ & \\
\hline 75 & $\begin{array}{l}\text { Voltaire } \\
4 \text { King Fergus } \\
5 \text { Highflyer }\end{array}$ & 1826 & $\begin{array}{l}\text { Blacklock } \\
3 \text { Highflyer } \\
4 \text { Herod } \\
4 \text { Eclipse }\end{array}$ & $\begin{array}{l}\text { Mare by Pha } \\
4 \text { Higlifiyer } \\
5 \text { Eclipse }\end{array}$ & antom \\
\hline 76 & $\begin{array}{l}\text { Wrar Dance } \\
4 \text { The Baron } \\
\text { (by Birdcatcher) } \\
7 \text { Banter }\end{array}$ & 1857 & $\begin{array}{l}\text { Galliard } \\
4 \text { Birdcatcher } \\
5 \text { Voltaire }\end{array}$ & $\begin{array}{l}\text { War Paint } \\
4 \text { Touchstone } \\
\text { (out of Bat }\end{array}$ & inter) \\
\hline 77 & $\begin{array}{l}\text { Wild Dayrell } \\
4 \text { Selim } \\
7 \text { Sir Peter }\end{array}$ & 1852 & $\begin{array}{l}\text { Ion } \\
3 \text { Evelina } \\
\text { ¿ Sir Peter }\end{array}$ & $\begin{array}{l}\text { Ellen Middle } \\
6 \text { Sir Peter }\end{array}$ & eton \\
\hline 78 & $\begin{array}{l}\text { Yattendon } \\
4 \text { Partisan } \\
6 \text { IVaxy }\end{array}$ & $\begin{array}{c}1861 \\
\text { in } \\
\text { Austral. }\end{array}$ & $\begin{array}{l}\text { Sir Hercules } \\
\text { (by Cap à Pie by } \\
\text { Colonel) } \\
4 \text { Waxy } \\
4 \text { Penelope }\end{array}$ & $\begin{array}{l}\text { Cassandra } \\
4 \text { Whiskey }\end{array}$ & \\
\hline
\end{tabular}


4. Inbreeding.

Stallions with 5 liree Generations.

\begin{tabular}{|c|c|c|c|c|}
\hline No. & Names of Stallions & born & Sire & 1) \\
\hline 1 & $\begin{array}{l}\text { Alarm } \\
\text { j Prunella (by High- } \\
\text { \#yer) } \\
\text { j Potsos (by Eclipse) } \\
6 \text { Maria (by Herod) }\end{array}$ & 1812 & $\begin{array}{l}\text { Ienison } \\
5 \text { Eclipse } \\
\text { 6 Herod }\end{array}$ & 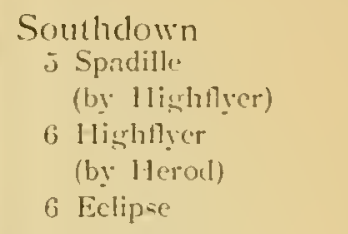 \\
\hline 2 & $\begin{array}{l}\text { Alarm } \\
\text { j Sultan (by Selim) } \\
6 \text { Sustard (by } \\
\text { Buzzard) }\end{array}$ & 1869 & $\begin{array}{l}\text { Imp. Eclipse } \\
4 \text { Selim (by Buzzard) }\end{array}$ & $\begin{array}{l}\text { Imp. Maude } \\
5 \text { Blacklock } \\
5 \text { Tramp } \\
7 \text { Buzzard }\end{array}$ \\
\hline 3 & $\begin{array}{l}\text { Aurum II. } \\
5 \text { Finherman } \\
7 \text { Touchstone }\end{array}$ & $\begin{array}{c}1594 \\
\text { in } \\
\text { Autral. }\end{array}$ & $\begin{array}{l}\text { Trenton } \\
6 \text { Touchstone } \\
6 \text { Camel }\end{array}$ & $\begin{array}{l}\text { Iurá } \\
6 \text {.larpessa }\end{array}$ \\
\hline 4 & $\begin{array}{l}\text { Avrshire } \\
5 \text { Touchstone } \\
5 \text { Bee's Wing } \\
6 \text { The Baron } \\
\text { (by Birdcatcher) } \\
6 \text { Voltaire }\end{array}$ & 1855 & $\begin{array}{l}\text { Hampton } \\
i \text { llhalebone (Grand- } \\
\text { sire of Touchstone } \\
\text { and Birlcatcher) }\end{array}$ & $\begin{array}{l}\text { Italanta } \\
\text { 5 Birdcatcher } \\
\text { Frand-sire : } \\
2 \text { Vultaire } \\
\text { Grand-dan : } \\
3 \text { Touchstone }\end{array}$ \\
\hline j & $\begin{array}{l}\text { Birdcatcher } \\
\text { J Wloodpecler } \\
\text { (by Herod) } \\
6 \text { Eclipse } \\
6 \text { llerod } \\
6 \text { Highflyer } \\
\text { (by Herod) }\end{array}$ & 1833 & $\begin{array}{l}\text { Sir Hercules } \\
4 \text { Eclipse } \\
5 \text { Herod } \\
5 \text { Hlighflyer }\end{array}$ & $\begin{array}{l}\text { Guiccioli } \\
2 \text { Bagot (by Herod) } \\
\text { Grand-sire: } \\
2 \text { Herod } \\
\text { Grand-dan : } \\
3 \text { Herod }\end{array}$ \\
\hline 6 & $\begin{array}{l}\text { Buccaneer } \\
\text { j Touchutone }\end{array}$ & 1885 & $\begin{array}{l}\text { Privateer } \\
3 \text { Touchstone }\end{array}$ & $\begin{array}{l}\text { Primula } \\
3 \text { Pocahontas } \\
4 \text { Redshank } \\
5 \text { Touchstone }\end{array}$ \\
\hline 7 & $\begin{array}{l}\text { Chamant } \\
\text { 5 Enilius (by Orville) } \\
\text { 6 Orvill. }\end{array}$ & 1874 & $\begin{array}{l}\text { Mortemer } \\
4 \text { Partisan } \\
6 \text { Orville } \\
\text { Grand-dam : } \\
1 \text { Emilius }\end{array}$ & $\begin{array}{l}\text { Araucaria } \\
5 \text { Orville } \\
5 \text { Selim } \\
\quad \text { Cirand-sire: } \\
4 \text { Orville }\end{array}$ \\
\hline$s$ & $\begin{array}{l}\text { Chanticleer } \\
\text { j Godol. Arabian }\end{array}$ & 1787 & $\begin{array}{l}\text { Moodpeclier } \\
\text { 6 Darley Arabian }\end{array}$ & $\begin{array}{l}\text { Mare by Eclipse } \\
2 \text { Regulus (by Godol. } \\
\text {. Irabian) }\end{array}$ \\
\hline 9 & $\begin{array}{l}\text { Charibert } \\
5 \text { Touchstone }\end{array}$ & 1576 & $\begin{array}{l}\text { Thormanby } \\
6 \text { Orville }\end{array}$ & $\begin{array}{l}\text { Gertrude } \\
4 \text { Sultan } \\
6 \text { Whalebone (Grand- } \\
\text { sire of Touchstone) }\end{array}$ \\
\hline
\end{tabular}




\begin{tabular}{|c|c|c|c|c|}
\hline In. & Names of Stallions & Born & Sire & Dam \\
\hline 10 & $\begin{array}{l}\text { Chester } \\
5 \text { Sir Hercules } \\
5 \text { Emilius (by Orville) } \\
6 \text { Sultan } \\
6 \text { Whisker (by II } 11 \times x \text { ) }\end{array}$ & $\begin{array}{c}1874 \\
\text { in } \\
\text { Anstrit. }\end{array}$ & $\begin{array}{l}\text { Yattendon } \\
4 \text { Partisan } \\
6 \text { llixy } \\
6 \text { Buzzard (Grand-sire } \\
\text { of Sultan) }\end{array}$ & $\begin{array}{l}\text { Lady Chester } \\
3 \text { Economist } \\
\text { (by Whisker) } \\
5 \text { Orville }\end{array}$ \\
\hline 11 & $\begin{array}{l}\text { Comus } \\
5 \text { llerud (by Tartar) } \\
5 \text { Snap } \\
6 \text { Cade (by Godol. } \\
\text { Irabian) }\end{array}$ & 1809 & $\begin{array}{l}\text { Sorcerer } \\
2 \text {. Natchem (by Cade) } \\
{\left[\begin{array}{ll}6 & \text { Partner } \\
6 & \text { Godol. Arabian }\end{array}\right]}\end{array}$ & $\begin{array}{l}\text { Houghton Lass } \\
5 \text { Blank (by Godol. } \\
\text { Arabian) } \\
5 \text { Tartar (by Partner) } \\
5 \text { Regulus (by Godol. } \\
\text {. Irabian) }\end{array}$ \\
\hline 12 & $\begin{array}{l}\text { Cowl } \\
5 \text { IIhiskey } \\
5 \mathrm{~V} \text { Giantess } \\
7 \text { Sir Peter } \\
\text { (by Higlonger) }\end{array}$ & 1842 & $\begin{array}{l}\text { Bay Middleton } \\
4 \text { Sir Peter } \\
4 \text { Arethusa } \\
7 \text { Eclipse }\end{array}$ & $\begin{array}{l}\text { Crucifix } \\
6 \text { Hightyer } \\
6 \text { Herod (Grand-sire } \\
\text { of IIhiskey) } \\
6 \text { Eclipse (Grand-sire } \\
\text { of Whiskey) }\end{array}$ \\
\hline 13 & $\begin{array}{l}\text { Defence } \\
5 \text { Herod } \\
5 \text { Higluflerer } \\
\text { (by llerod) } \\
5 \text { Eclipse } \\
5\left\{\begin{array}{l}\text {. Ilfred } \\
\text { Conductor }\end{array}\right.\end{array}$ & $1 S 24$ & $\begin{array}{l}\text { Whalebone } \\
3 \text { Herod } \\
4 \text { Snap }\end{array}$ & $\begin{array}{l}\text { Defiance } \\
4 \text { Herod } \\
4 \text { Hishflyer } \\
4 \text { Eclipse } \\
5 \text {. Nfred }\end{array}$ \\
\hline 11 & $\begin{array}{l}\text { Desmond } \\
\text { s Voltigeur } \\
\text { (by Voltaise) }\end{array}$ & 1996 & $\begin{array}{l}\text { St. Simon } \\
6 \text { Sultan } \\
6 \text { Velocipede's dan } \\
\text { Grand-sire : } \\
2 \text { Voltaire }\end{array}$ & $\begin{array}{l}\text { L'Abbesse de } \\
\text { Jouarre } \\
4 \text { Touchstone } \\
5 \text { Banter } \\
\text { Crand-dam. } \\
4 \text { Voltaire }\end{array}$ \\
\hline 15 & $\begin{array}{l}\text { Despair } \\
5 \text { Defence } \\
6 \text { Whalebone } \\
\text { (by Waxy) } \\
6 \text { Touchstone }\end{array}$ & 1881 & $\begin{array}{c}\text { See Saw } \\
7 \text { Waxy }\end{array}$ & $\begin{array}{l}\text { Peine de Coeur } \\
5 \text { Partisan } \\
6 \text { Whalebone }\end{array}$ \\
\hline 16 & $\begin{array}{l}\text { Dr. Syntax } \\
5 \text { Xlatchem (by Cade) } \\
6 \text { Snip } \\
6 \text { Cade } \\
7 \text { Crab }\end{array}$ & 1811 & $\begin{array}{l}\text { Paynator } \\
3 \text { Snap (by Snip) } \\
5 \text { Crodol. Srabian }\end{array}$ & $\begin{array}{l}\text { Mare by } \\
\text { Beningbrough } \\
5 \text { Cade (by Godol. } \\
\text {. Irabian) } \\
5 \text { Regulus (by Godol. } \\
\text {.rabian) } \\
7 \text { Mare by Basto } \\
\text { (Dam of Snip and } \\
\text { Crab) }\end{array}$ \\
\hline
\end{tabular}




\begin{tabular}{|c|c|c|c|c|}
\hline No. & Names of Stallions & Born & Sire & Dam \\
\hline 17 & $\begin{array}{l}\text { Donovan } \\
5 \text { Bay Iliddleton (by } \\
\text { Sultan-Pliantom) } \\
5 \text { Birdcatcher }\end{array}$ & 1886 & $\begin{array}{l}\text { Galopin } \\
2 \text { Voltaire (out of } \\
\text { Phantom Mare) }\end{array}$ & $\begin{array}{l}\text { Mowerina } \\
3 \text { Touchstone } \\
5 \text { Nuley } \\
6 \text { Sultan }\end{array}$ \\
\hline is & $\begin{array}{l}\text { Faugh-a-Ballagh } \\
5 \text { Woodpecker } \\
\text { (by Herod) } \\
6 \text { Eclipse } \\
6 \text { Herod } \\
6 \text { Highnyer } \\
\text { (by Herod) }\end{array}$ & 1841 & $\begin{array}{l}\text { Sir Hercules } \\
+ \text { Eclipse } \\
5 \text { Herod } \\
5 \text { Highflyer }\end{array}$ & $\begin{array}{l}\text { Guiccioli } \\
2 \text { Basrot (by Herod) } \\
\text { Girand-sire: } 2 \text { Herod } \\
\text { Grand-dam : } 3 \text { Hernil }\end{array}$ \\
\hline $1 !$ & $\begin{array}{l}\text { Fitz Gladiator } \\
\text { j Buzzard } \\
\text { j) Mare by . lexander } \\
\text { (by Eclipse) } \\
\text { j Sir Peter } \\
\text { (by Hishflyer) } \\
\text { j Gohanna }\end{array}$ & 18.50 & $\begin{array}{l}\text { Gladiator } \\
+ \text { Put8os (by Eclipse) } \\
4 \text { Prunella (by High- } \\
\text { fyer) }\end{array}$ & $\begin{array}{l}\text { Zarali } \\
4 \text { Sir l'eter } \\
6 \text { Eclipse } \\
6 \text { Ilerod }\end{array}$ \\
\hline 20 & $\begin{array}{l}\text { Flacreolet } \\
5 \text { Partican } \\
\text { (by llalton) } \\
\text { 7 Whalebone }\end{array}$ & 1570 & $\begin{array}{l}\text { Plutus } \\
+ \text { Emilius }\end{array}$ & $\begin{array}{l}\text { L.a Favorite } \\
\text { 5 Whalebone } \\
\text { Granci-daun : } 3 \text { IValton }\end{array}$ \\
\hline$\geq 1$ & $\begin{array}{l}\text { Flibustier } \\
5 \text { Tramp } \\
6 \text { Sultan } \\
7 \text { Orville [own brother } \\
\text { of Waisenknabel }\end{array}$ & 1867 & $\begin{array}{l}\text { Buccaneer } \\
3 \text { Edmund (by Orville) } \\
\text {; Paynator }\end{array}$ & $\begin{array}{l}\text { Sweet Katie } \\
5 \text { Tramp } \\
6 \text { Orville }\end{array}$ \\
\hline 22 & $\begin{array}{l}\text { Fulmen } \\
5 \text { Birdcatcher (by } \\
\text { Sir Hercules) }\end{array}$ & $\mathrm{J} 8 \mathrm{8} 0$ & $\begin{array}{l}\text { Galopin } \\
2 \text { Voltaire }\end{array}$ & $\begin{array}{l}\text { Lightning } \\
5 \text { Sir llercules }\end{array}$ \\
\hline 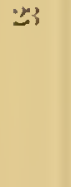 & $\begin{array}{l}\text { Glencoe } \\
5 \text {.lercury } \\
6 \text { Eclipse } \\
6 \text { Herod } \\
6 \text { Highnger }\end{array}$ & 1831 & $\begin{array}{l}\text { Sultan } \\
+ \text { Herod } \\
+ \text { Eclipse } \\
+ \text { Ilighflyer }\end{array}$ & $\begin{array}{l}\text { Trampoline } \\
4 \text { Highflyer } \\
4 \text { Eclipse } \\
\text { ¿ Herod }\end{array}$ \\
\hline 24 & $\begin{array}{l}\text { Harkaway } \\
5 \text { Potsos (by Eclipse) } \\
6 \text { Highnyer } \\
7 \text { Ilerod }\end{array}$ & 1831 & $\begin{array}{l}\text { Economist } \\
+4 \text { Felipse } \\
+ \text { llerod } \\
5 \text { llighnlyer }\end{array}$ & $\begin{array}{l}\text { Mare by Tabocklish } \\
+ \text { Highnyer } \\
\text { (by Herod) } \\
\text { Grand-dam : } \\
2 \text { Ilighnyer } \\
\text { Creat Grand-dam : } \\
2 \text { llerod }\end{array}$ \\
\hline
\end{tabular}




\begin{tabular}{|c|c|c|c|c|}
\hline No. & Names of Stallions & Born & Sire & Dan \\
\hline 25 & $\begin{array}{l}\text { Highflyer } \\
5 \text { Sister to Mixbury } \\
6 \text { Darley's Arabian }\end{array}$ & 1774 & $\begin{array}{l}\text { Herod } \\
7 \text { Leedes Arabian } \\
7 \text { Hautboy } \\
7 \text { Spanker }\end{array}$ & $\begin{array}{l}\text { Rachel } \\
1 \text { Godol. Arabian } \\
6 \text { St. Victor's Barb } \\
6 \text { Grey Whynot }\end{array}$ \\
\hline 26 & $\begin{array}{l}\text { Jerry } \\
5 \text { Herod } \\
6 \text { Matchem (by Cade) }\end{array}$ & 1821 & $\begin{array}{l}\text { Smolensko } \\
4 \text { Herod } \\
5 \text { Snap } \\
6 \text { Cade }\end{array}$ & $\begin{array}{l}\text { Louisá } \\
3 \text { Highflyer } \\
5 \text { Herod } \\
5 \text { Matchem }\end{array}$ \\
\hline 27 & $\begin{array}{l}\text { Isinglass } \\
5 \text { Birdcatcher } \\
5 \text { The Baron } \\
\text { (by Birdcatcher) } \\
5 \text { Pocahontas } \\
6 \text { Touchstone }\end{array}$ & 1890 & $\begin{array}{l}\text { Isonomy } \\
3 \text { Birdcatcher }\end{array}$ & $\begin{array}{l}\text { Dead Lock } \\
4 \text { Touchstone }\end{array}$ \\
\hline 28 & $\begin{array}{l}\text { Ithuriel } \\
5 \text { Buzzard } \\
5 \text { Mare by Alexander } \\
\text { (by Eclipse) }\end{array}$ & 1841 & $\begin{array}{l}\text { Touchstone } \\
4 \text { Alexander } \\
5 \text { Eclipse } \\
5 \text { Buzzard } \\
5 \text { Sir Peter }\end{array}$ & $\begin{array}{l}\text { Verbena } \\
4 \text { Sir Peter } \\
5 \text { Highflyer }\end{array}$ \\
\hline 29 & $\begin{array}{l}\text { King Tom } \\
5 \text { Waxy (by Pot8os) } \\
5 \text { Penelope }\end{array}$ & 1851 & $\begin{array}{l}\text { Harkaway } \\
5 \text { Pot8os (by Eclipse) } \\
6 \text { Highflyer }\end{array}$ & $\begin{array}{l}\text { Pocahontas } \\
5 \text { Gohanna } \\
\text { (by Mercury) } \\
6 \text { Mercury } \\
\text { (by Eclipse) }\end{array}$ \\
\hline 80 & $\begin{array}{l}\text { Ladas } \\
5 \text { Touchstone } \\
5 \text { Queen Mary } \\
5 \text { Melbourne }\end{array}$ & 1891 & $\begin{array}{l}\text { Hampton } \\
7 \text { Whalebone (Granci- } \\
\text { sire of Touchstone) }\end{array}$ & $\begin{array}{l}\text { Illuminata } \\
4 \text { Touchstone }\end{array}$ \\
\hline 31 & $\begin{array}{l}\text { Lamplighter } \\
5 \text { Glencoe (by Sultan) } \\
6 \text { Emilius (by Orville) } \\
6 \text { Touchstone } \\
6 \text { Sultan (by Selim) }\end{array}$ & $\begin{array}{c}1859 \\
\text { in } \\
\text { A merica }\end{array}$ & $\begin{array}{l}\text { Spendthrift } \\
5 \text { Enilius } \\
6 \text { Selin ? }\end{array}$ & $\begin{array}{l}\text { Torchlight } \\
4 \text { Touchstont } \\
7 \text { Orville } \\
\text { Grand-dam : } 3 \text { Sultan }\end{array}$ \\
\hline 32 & $\begin{array}{l}\text { Marske } \\
5 \text { Hautboy } \\
5 \text { Lister Turk } \\
7 \text { Leedes Arabian } \\
7 \text { Mare by Spanker }\end{array}$ & 1750 & $\begin{array}{l}\text { Squirt } \\
\text { ? } \\
\text { Grand-dam : } \\
1 \text { Iautboy }\end{array}$ & $\begin{array}{l}\text { Ruby Mare } \\
3 \text { Coneyskins } \\
\text { (by Lister Turk) } \\
4 \text { Hautboy }\end{array}$ \\
\hline 33 & $\begin{array}{l}\text { Matchem } \\
5 \text { Mare by Spanker }\end{array}$ & 1818 & Cade? & $\begin{array}{l}\text { Mare by Partner } \\
5 \text { Darcy's Yellow } \\
\text { Turk (Sire of } \\
\text { Spanker) }\end{array}$ \\
\hline
\end{tabular}


4. Inbreeding.

\begin{tabular}{|c|c|c|c|c|}
\hline No. & Names of Stallions & Born & Sire & D)am \\
\hline 31 & $\begin{array}{l}\text { Minting } \\
\text { 5 Birdcatcher } \\
\text { i) Youchstonc } \\
\text { 5) Cilencoe } \\
\text { - Selim } \\
\text { Cistrel }\end{array}$ & 1883 & $\begin{array}{l}\text { Lord Lyom } \\
6 \text { Selim } \\
6 \text { Whalebune (Grind- } \\
\text { sire of birdcatcher } \\
\text { and Touchstone) }\end{array}$ & $\begin{array}{l}\text { Mint Sauce } \\
\text { i Castrel } \\
7 \text { comus } \\
\text { Cirind-dam: } \\
\text { : Touchstone }\end{array}$ \\
\hline 35 & $\begin{array}{l}\text { Morion } \\
\text { 5 jouchstone } \\
\text {; Whalebone }\end{array}$ & 1897 & $\begin{array}{l}\text { Barcaldine } \\
\text { 1 1):urling"s dam } \\
5 \text { Touchstone }\end{array}$ & 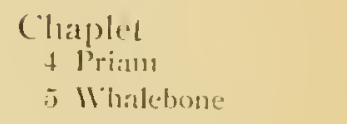 \\
\hline 3 & $\begin{array}{l}\text { Mlulatin } \\
5 \text { Florizel (by Herod) } \\
5 \text { llighl?yer } \\
\text { (by Herod) } \\
6 \text { Herod } \\
\text { 6 liclipse (by Marnlic) }\end{array}$ & $182 ; 3$ & $\begin{array}{l}\text { Calton } \\
+4 \text { llerod } \\
4 \text { liclipse } \\
5 \text { Matchem }\end{array}$ & $\begin{array}{l}\text { Desdemona } \\
2 \text { Hislifyer } \\
\text {; Virulite }\end{array}$ \\
\hline 37 & $\begin{array}{l}\text { Dewminster } \\
\text { 5) lieningbrougl (by } \\
\text { King Fergus lby } \\
\text { liclipse] and at } \\
\text { Ilerod-Mare) } \\
\text { 5 "Trumpator }\end{array}$ & lisis & $\begin{array}{l}\text { Pouchstune } \\
4 \text {. Mesander } \\
5 \text { Eclipse } \\
5 \text { Sir Peter } \\
\text { (: Ilighflyer } \\
\text { (by Herod) }\end{array}$ & $\begin{array}{l}\text { Boces lling } \\
6 \text { liclipse } \\
6 \text { llerod }\end{array}$ \\
\hline '3 & $\begin{array}{l}\text { Norfolk } \\
\text { is Sir . Irchy }\end{array}$ & 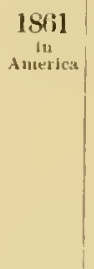 & $\begin{array}{l}\text { Lexington } \\
3 \text { Sir Archy } \\
6 \text { Saltram (by Eclipse, } \\
\text { Cirnd-sire of Wasy) }\end{array}$ & $\begin{array}{l}\text { Novice } \\
5 \text { Buzzard } \\
6 \text { Waxy } \\
\text { Grand-dam : } \\
2 \text { Sir Archy } \\
\text { Great Grand-dam: } \\
1 \text { Sir . Irchy }\end{array}$ \\
\hline :3) & $\begin{array}{l}\text { Orme } \\
5 \text { l'ocahontas } \\
\text { (by (ilencue) } \\
6 \text { Birdcatcler }\end{array}$ & 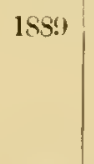 & $\begin{array}{l}\text { Ormonde } \\
\text {; Birdcatcher } \\
\text { ¡s Pantaloon }\end{array}$ & $\begin{array}{l}\text { Increlica } \\
6 \text { Sultan (Sire of } \\
\text { Glencoe) } \\
6 \text { Velocipede's dam }\end{array}$ \\
\hline III & $\begin{array}{l}\text { Ormonde } \\
5 \text { Birdcatcher } \\
5 \text { l'antaloon } \\
\text { (by Castrel) } \\
7 \text { Banter }\end{array}$ & lsis: & $\begin{array}{l}\text { Bend Or } \\
6 \text { Touchstone } \\
\text { Cirand-sire : } \\
\text { (; Blacklock }\end{array}$ & $\begin{array}{l}\text { Lily Ignes } \\
6 \text { Blacklock } \\
6\left\{\begin{array}{l}\text { Selim } \\
\text { Ciastrel }\end{array}\right.\end{array}$ \\
\hline 41 & $\begin{array}{l}\text { Persimmon } \\
5 \text { Voltigeur } \\
\text { (by Voltaire) } \\
6 \text { Pocahontas }\end{array}$ & $1 S: 13$ & $\begin{array}{l}\text { St. Simon } \\
6 \text { Sultan (Grand-sire } \\
\text { of l'ocahontas) } \\
6 \text { Velocipede's dan } \\
7 \text { Blacklock (Sire of } \\
\text { Voltaire) } \\
\text { Grand-sire: } \\
2 \text { Voltaire }\end{array}$ & $\begin{array}{l}\text { Perditta II. } \\
3 \text {. Melbourne } \\
6 \text { Voltaire } \\
6 \text {. Mirtha I,ynn } \\
6 \text { Vocahontas }\end{array}$ \\
\hline
\end{tabular}


Heredity.

\begin{tabular}{|c|c|c|c|c|}
\hline No. & Names of Stallioms & Born & Sire & Dam \\
\hline 12 & $\begin{array}{l}\text { Perth } \\
5 \text { Newininster } \\
\text { j Stockwell } \\
\text { (by The Baron) }\end{array}$ & 1846 & $\begin{array}{l}\text { IVar Dance } \\
+4 \text { The Baron } \\
7 \text { Banter }\end{array}$ & $\begin{array}{l}\text { Primrose Dame } \\
4 \text { Newminster } \\
4 \text { Stockwell } \\
5 \text { Touchstone } \\
6 \text { Birdcatcher } \\
\text { Grand-sire: } \\
2 \text { Touchstnne } \\
2 \text { Bee's Wing }\end{array}$ \\
\hline 43 & $\begin{array}{l}\text { Pyrhus the First } \\
5 \text { Buzzard } \\
\text { (by Woodpecker) } \\
5 \text { Nare by Nexander } \\
\text { (by Eclipse) }\end{array}$ & 1843 & $\begin{array}{l}\text { Epirus } \\
3 \text { Sir Peter } \\
4 \text { Diomed } \\
5 \text { Herod } \\
5 \text { Eclipse }\end{array}$ & $\begin{array}{l}\text { Fortress } \\
1 \text { Whalebone } \\
4 \text { Ilighland Fling } \\
\text { j Buzzard } \\
6 \text { Woodpecker } \\
\text { (by Herod) }\end{array}$ \\
\hline$H$ & $\begin{array}{l}\text { Rataplan } \\
5 \text { Orville } \\
6 \text { lWaxy } \\
6 \text { Penelope }\end{array}$ & 1850 & $\begin{array}{l}\text { The Baron } \\
\text { \& Waxy } \\
\text { 4 Penelope }\end{array}$ & $\begin{array}{l}\text { Pocahontas } \\
5 \text { Gohanna } \\
6 \text { Nercury } \\
7 \text { Highflyer (Cirand- } \\
\text { sire of Orville) }\end{array}$ \\
\hline ti & $\begin{array}{l}\text { Sheet Anchor } \\
5 \text { Wondpeclier } \\
\text { (by Herod) } \\
6 \text { Eclipse } \\
6 \text { Mercury } \\
6 \text { Mare by Herod }\end{array}$ & 1832 & $\begin{array}{l}\text { Lottery } \\
3 \text { Eclipse } \\
4 \text { Trentham } \\
4 \text { lloodpecher } \\
5 \text { Hernd }\end{array}$ & $\begin{array}{l}\text { Morgiana } \\
2 \mathrm{~V} \text {. Giantess } \\
6 \text { Herod } \\
6 \text { Eclipse }\end{array}$ \\
\hline 16 & $\begin{array}{l}\text { Sir Visto } \\
5 \text { Pocahontas } \\
5 \text { Newminsler } \\
6 \text { Banter (Dam of } \\
\text { Touchstone) }\end{array}$ & 1892 & $\begin{array}{l}\text { Barcaldine } \\
\text { 1 Dar'ing's dam } \\
\text { (by Birdcatcher) } \\
5 \text { Touchstone }\end{array}$ & $\begin{array}{l}\text { Vista } \\
+ \text { Banter } \\
+ \text { Pantaloo: }\end{array}$ \\
\hline 47 & $\begin{array}{l}\text { St. Florian } \\
5 \text { lon } \\
6 \text { Bay Niddleton } \\
\text { (by Sultan) }\end{array}$ & 1891 & $\begin{array}{l}\text { St. Simon } \\
\text { f Sultan }\end{array}$ & $\begin{array}{l}\text { PaInflower } \\
5 \text { Bay Niddleion } \\
5 \text { Touchistone }\end{array}$ \\
\hline 48 & $\begin{array}{l}\text { Saunterer } \\
5 \text { Waxy } \\
5 \text { Penelope }\end{array}$ & 185.4 & $\begin{array}{l}\text { Birdcatcher } \\
\text { j Woodpeclier } \\
6 \text { Eclipse (Grand-sire } \\
\text { of Waxy) }\end{array}$ & $\begin{array}{l}\text { Ennui } \\
5 \text { Waxy } \\
\text { \& Penelope } \\
6 \text { Whisley } \\
6 \text { Sorcerer }\end{array}$ \\
\hline 49 & $\begin{array}{l}\text { Scottish Chief } \\
5 \text { Orville (by Bening- } \\
\text { brough) } \\
6 \text { Sclim } \\
7 \text { Buzzard (by IYoor- } \\
\text { peclier) }\end{array}$ & 1861 & $\begin{array}{l}\text { Lord of the lsles } \\
5 \text { Buzzard } \\
5 \text { Mare by Alexander } \\
\text { (by Eclipse) } \\
6 \text { Beningbrough }\end{array}$ & $\begin{array}{l}\text { Miss Ann } \\
7 \text { Woodpecker } \\
7 \text { Mercury } \\
\text { (by Eclipse) }\end{array}$ \\
\hline
\end{tabular}




\begin{tabular}{|c|c|c|c|c|}
\hline Nu. & Namms of Stallions & Born & Sire & 1)am \\
\hline 5) & $\begin{array}{l}\text { Solon } \\
5 \text { Whalebone } \\
\text { (by Waxy) } \\
5 \text { Comus } \\
6 \text { Waxy } \\
6 \text { Penelope } \\
\text { (by Trumpator) }\end{array}$ & 1861 & $\begin{array}{l}\text { West Australian } \\
6 \text { Trumpator (Cirand- } \\
\text { sire of Comus) }\end{array}$ & $\begin{array}{l}\text { 1)arling's Dam } \\
\text { i llasy } \\
6 \text { Potsos } \\
\text { i Trumpator }\end{array}$ \\
\hline$\pi 1$ & $\begin{array}{l}\text { Spendthrift } \\
5 \text { Emilius } \\
6 \text { Selim } \\
7 \text { Waxy } \\
6 \text { Penelope }\} \text { Parents of }\end{array}$ & $\begin{array}{c}1576 \\
\text { in } \\
\text { Awerlas }\end{array}$ & $\begin{array}{l}\text { Imp. Iustralian } \\
3 \text { Whisker } \\
6 \text { Selim } \\
6 \text { Evelina (Grand-dam } \\
\text { of Emilius) } \\
7 \text { Stamford (Grand- } \\
\text { sire of Emilius) }\end{array}$ & $\begin{array}{c}\text { Aerolite } \\
?\end{array}$ \\
\hline. \pm 2 & $\begin{array}{l}\text { Springfield } \\
5 \text { Sultan (by Selim) } \\
6 \text { Camel } \\
\text { (by W'halebone) } \\
6 \text { Banter }\end{array}$ & 1873 & $\begin{array}{l}\text { St. Nlbans } \\
6 \text { Whalebone }\end{array}$ & $\begin{array}{l}\text { Viridis } \\
4 \text { Catmel } \\
4 \text { langar (by Selim) } \\
5 \text { Selim }\end{array}$ \\
\hline 53 & $\begin{array}{l}\text { Sterling } \\
5 \text { II"halebone }\end{array}$ & 1868 & $\begin{array}{l}\text { Oxford } \\
7 \text { IVaxy } \\
7 \text { Penelope }\} \text { Parents of } \\
\text { Whatehone }\end{array}$ & $\begin{array}{l}\text { Whisper } \\
5 \text { Whalebone } \\
6 \text { Waxy }\end{array}$ \\
\hline ist & $\begin{array}{l}\text { Stockwell } \\
\text { j Orville } \\
6 \text { II axy } \\
6 \text { Penelope }\end{array}$ & 1819 & $\begin{array}{l}\text { The Baron } \\
4 \text { IVaxy } \\
4 \text { Penelope }\end{array}$ & $\begin{array}{l}\text { Pocahontas } \\
5 \text { Gohanni } \\
6 \text { Mercury } \\
7 \text { Highnyer (Girind. } \\
\text { sire of Orville) }\end{array}$ \\
\hline$\therefore$ & $\begin{array}{l}\text { Timoleon } \\
5 \text { Trentham } \\
7 \text { Blank (by Godol. } \\
\text { Arabian) }\end{array}$ & 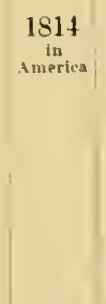 & 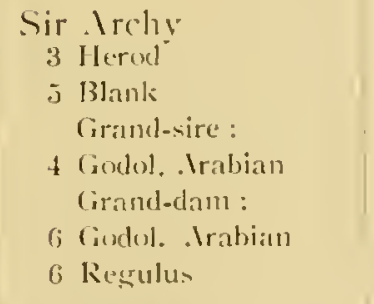 & $\begin{array}{l}\text { Mare by Saliram } \\
4 \text { Regulus (by Godol. } \\
\text {. Irabian) } \\
\text { Cirand-sire: } \\
2 \text { Regulus } \\
\text { Cirand-dan: } \\
4 \text { Recrulus }\end{array}$ \\
\hline . Ai & $\begin{array}{l}\text { Van Tromp } \\
\text { i Buzard } \\
\text { (by lloodpecler) } \\
6 \text { Pot8os (by Folipse) } \\
\text { 6 Gohanna }\end{array}$ & 1841 & 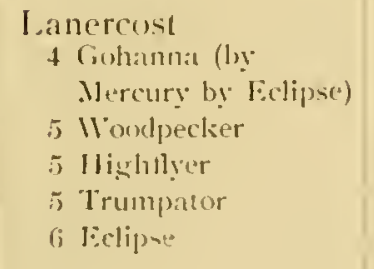 & $\begin{array}{l}\text { Barbelle } \\
6 \text { lloodpectien } \\
6 \text { liclipe } \\
6 \text { Ilerod }\end{array}$ \\
\hline
\end{tabular}


Heredity.

\begin{tabular}{|c|c|c|c|c|}
\hline No. & Names of Stallions & Born & Sire & Dam \\
\hline 87 & $\begin{array}{l}\text { Velocipede } \\
5 \text { Highfyer } \\
\text { (by Herod) } \\
5 \text { Potgos (by Eclipse) } \\
\text { 7 Matchem }\end{array}$ & 1805 & $\begin{array}{l}\text { Blacklock } \\
3 \text { Hinhfiver } \\
4 \text { Eclipse } \\
4 \text { Herod }\end{array}$ & $\begin{array}{l}\text { Mare by Juniper } \\
\text { J Eclipse } \\
\text { is Herod } \\
6 \text { Matchem }\end{array}$ \\
\hline 58 & $\begin{array}{l}\text { Venison } \\
5 \text { Eclipse } \\
6 \text { Herod } \\
7 \text { Snap }\end{array}$ & 1833 & $\begin{array}{l}\text { Partisan } \\
2 \text { Hlighfyer } \\
\text { (by Herod) } \\
3 \text { Eclipse } \\
4 \text { Snap }\end{array}$ & $\begin{array}{l}\text { Fatwn } \\
+1 \text { lerol }\end{array}$ \\
\hline (3) & $\begin{array}{l}\text { I'oltigeur } \\
\text { j Himbletonian } \\
\text { (by King liergus) } \\
6 \text { Coriander } \\
\text { 6 Sir Peter (by lligh- } \\
\text { flyer) } \\
\text { i Highflyer }\end{array}$ & 1847 & $\begin{array}{l}\text { Voltaire } \\
+ \text { King Fergus } \\
5 \text { Highfyer (Granrl- } \\
\text { sire of Hamble- } \\
\text { tonian) }\end{array}$ & $\begin{array}{l}\text { Martha Lynn } \\
4 \text { Sir Peter } \\
\text { o King Fergus }\end{array}$ \\
\hline (i) & 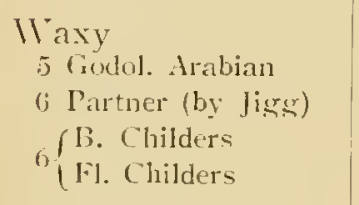 & 1796 & $\begin{array}{l}\text { Potsos } \\
4 \text { Godol. Arabian }\end{array}$ & $\begin{array}{l}\text { Maria } \\
+ \text { Flying Childers } \\
+ \text { Fox } \\
6 \text { Byerly Turk (Sire } \\
\text { of Jigg) }\end{array}$ \\
\hline 11 & $\begin{array}{l}\text { Jenophon } \\
\text { s Whalebone } \\
\text { (by llaxy) }\end{array}$ & 1872 & $\begin{array}{l}\text { Canary } \\
3 \text { Camel } \\
\text { (by Whalebone) } \\
\text { + Selim } \\
\text { 5 Orville }\end{array}$ & $\begin{array}{l}\text { Mare by Birdcatcher } \\
j \text { Waxy }\end{array}$ \\
\hline
\end{tabular}

Stallions with 6 Free Generations.

1 Beauclerc

6 W'ialebone
Bend'Or

6 Touchstone

6 .luley (by Orville)

- Selim (by Burzard)

\author{
1875 Rosicrucian \\ 4 Priam \\ j Whalebone
}

1877 Duncaster

6 Blacklock

i Whalebone

7 Selim

\section{Bonny Bell \\ 5 Walton \\ Grand-dam : \\ 3 Ithalebone}
Rouge Kose
6 Orville
6 Buzzard
6 Mare by Alexanter
7 Waxy




\begin{tabular}{|c|c|c|c|c|}
\hline Ne. & Names of Stallions & born & Sire & Dim \\
\hline$: ;$ & $\begin{array}{l}\text { Blair Athol } \\
6 \text { Whalebone } \\
\text {; Orville } \\
\text { T Evelinis }\end{array}$ & 1861 & 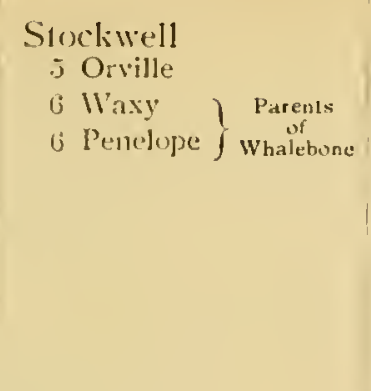 & $\begin{array}{l}\text { Blink Bonny } \\
\text { 6 Evelina (Dam of } \\
\text { Orville) } \\
6 \text { Sir Jeter } \\
\text { i lrumpator (Grand- } \\
\text { sire of Whalebone) } \\
7 \text { Iighlyer (Grand- } \\
\text { sire of Orville) } \\
\text { Grand-dan : } \\
3 \text { Whalebone }\end{array}$ \\
\hline$t$ & $\begin{array}{l}\text { Compiegne } \\
6 \text { llyalebone } \\
7 \text { Mare by . Vexander } \\
\text { 7 Buzmard } \\
\text { i Sir Peter } \\
\text { i Beningbrough } \\
7 \text { Gohanna }\end{array}$ & 18 is & $\begin{array}{l}\text { Fitz Gladiator } \\
5 \text { lizzard } \\
5 \text { Mare by Nexander } \\
\text { j Sir Peter } \\
5 \text { Guhanna }\end{array}$ & $\begin{array}{l}\text { Maid of Hart } \\
6 \text { Sir Peter } \\
6 \text { Beningbrough } \\
6 \text { W'axy } \\
\text { 15 Bvelina }\end{array}$ \\
\hline 5 & $\begin{array}{l}\text { Cremorne } \\
6 \text { Tramp } \\
\text { - Selim } \\
\text { \& Waxy } \\
8 \text { Penelupe } \\
\text { 8 Potsos }\end{array}$ & 1869 & $\begin{array}{l}\text { Parmesan } \\
6 \text { Whaxy } \\
6 \text { Penelope } \\
\text { 6 Potsos } \\
\text { is Prunella }\end{array}$ & $\begin{array}{l}\text { Rigolboche } \\
\text { s Trump } \\
\text { s. Sclim }\end{array}$ \\
\hline li & $\begin{array}{l}\text { Doncaster } \\
6 \text { Blaclilock } \\
\text { T Whalebone } \\
\text { i Selim }\end{array}$ & 1570 & $\begin{array}{l}\text { Stockwell } \\
\text { 5 Orville } \\
6 \text { Waxy } \\
\text { o P'enelope }\} \begin{array}{c}\text { Parents } \\
\text { of } \\
\text { Whalebone }\end{array}\end{array}$ & $\begin{array}{l}\text { Marigold } \\
\text { + Humphrey Clinker } \\
\text { if Selim }\end{array}$ \\
\hline 7 & $\begin{array}{l}\text { Favonius } \\
6 \text { Ithislier }\end{array}$ & 1 ses & 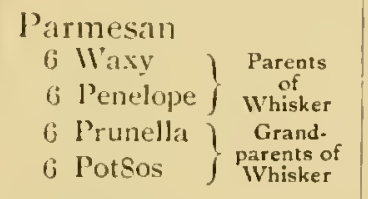 & $\begin{array}{l}\text { Zepher } \\
\text {-j Ithislier }\end{array}$ \\
\hline s & $\begin{array}{l}\text { Grand Flaneur } \\
6 \text { Sir Hercules } \\
\text { (by llhalebone) }\end{array}$ & 1877 & $\begin{array}{l}\text { Yattendon } \\
4 \text { l'artisan } \\
6 \text { llaxy (Sire of } \\
\text { ll'halcbone) }\end{array}$ & $\begin{array}{l}\text { First Lady } \\
\text { 5 Pantaloon } \\
\text { j Camel } \\
\text { (by Whalebone) }\end{array}$ \\
\hline 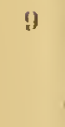 & $\begin{array}{l}\text { Hindoo } \\
6 \text { Enilius (by Orville) } \\
7 \text { Tramp }\end{array}$ & $\begin{array}{l}1575 \\
\text { in } \\
\text { Amersen }\end{array}$ & $\begin{array}{l}\text { Virgil } \\
+4 \text { Trimup } \\
6 \text { Orville }\end{array}$ & $\begin{array}{l}\text { FInrence } \\
\text { j Emilius } \\
6 \text { Orville }\end{array}$ \\
\hline 111 & $\begin{array}{l}\text { King Fercus } \\
6 \text { Bay Bolton }\end{array}$ & 177.5 & $\begin{array}{l}\text { Pelipse } \\
\text { \& Sisiler to Old Coun- } \\
\text { try liench } \\
\text { + Snake } \\
\text { (; Haulboy }\end{array}$ & $\begin{array}{l}\text { Polly } \\
6 \text { Biverly Turk }\end{array}$ \\
\hline
\end{tabular}


Heredity.

\begin{tabular}{|c|c|c|c|c|}
\hline No. & Names of Stallions & Born & Sire & Dam \\
\hline 11 & $\begin{array}{l}\text { Kisber } \\
6 \text { Sultan } \\
7 \text { Orville }\end{array}$ & 1873 & $\begin{array}{l}\text { Buccaneer } \\
3 \text { Edmund } \\
\text { (by Orville) } \\
5 \text { Paynator }\end{array}$ & $\begin{array}{l}\text { Mineral } \\
1 \text { Birdcatcher } \\
7 \text { Orville }\end{array}$ \\
\hline 12 & $\begin{array}{l}\text { Leamington } \\
6 \text { Woodpeclier } \\
6 \text { Alexander } \\
\text { (by Eclipse) }\end{array}$ & 1853 & $\begin{array}{l}\text { Faugh-a-Ballagh } \\
5 \text { Woodpecker } \\
6 \text { Eclipse }\end{array}$ & $\begin{array}{l}\text { Mare by Pantaloon } \\
4 \text { Buzzard } \\
\text { (by IVoodpecker) } \\
4 \text { Mare by Alexander }\end{array}$ \\
\hline 13 & $\begin{array}{l}\text { Lord Clifden } \\
6 \text { Paynator } \\
\text { (by Trumpator) } \\
7 \text { Orville } \\
\text { (by Beningbrough) }\end{array}$ & 1860 & $\begin{array}{l}\text { Newminster } \\
5 \text { Trumpator } \\
5 \text { Beningbrough }\end{array}$ & $\begin{array}{l}\text { The Slave } \\
5 \text { Golumpus }\end{array}$ \\
\hline 14 & $\begin{array}{l}\text { Lord Lyon } \\
6 \text { II'halebone } \\
6 \text { Selim } \\
7 \text { Orville }\end{array}$ & 1863 & 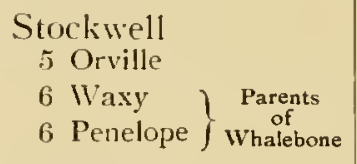 & $\begin{array}{l}\text { Paradigm } \\
5 \text { Selim } \\
5 \text { Waxy } \\
6 \text { Orville }\end{array}$ \\
\hline 15 & $\begin{array}{l}\text { Lord Ronald } \\
6 \text { Whalebone } \\
7 \text { Selim } \\
7 \text { Orville }\end{array}$ & 1862 & 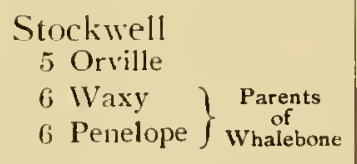 & $\begin{array}{l}\text { Edith } \\
5 \text { Whalebone } \\
6 \text { Selim }\end{array}$ \\
\hline 16 & $\begin{array}{l}\text { Macaroni } \\
6 \text { Sir Peter (by High- } \\
\text { flyer) } \\
6 \text { Orville (Grand-son } \\
\text { of Highfyer) } \\
6 \text { Buzzard } \\
6 \text { Mare by Nexander }\end{array}$ & 1860 & $\begin{array}{l}\text { Sweetmeat } \\
4 \text { Walton } \\
\text { (by Sir Peter) } \\
6 \text { Potsos (by Eclipse) }\end{array}$ & $\begin{array}{l}\text { Jocose } \\
3 \text {.lexander } \\
\text { (by Eclipse) } \\
4 \text { Highflyer } \\
4 \text { Buzzard } \\
5 \text { Sir Peter (by High- } \\
\text { flyer) }\end{array}$ \\
\hline 17 & $\begin{array}{l}\text { Matchbox } \\
6 \text { Bay Middleton } \\
\text { (by Sultan) }\end{array}$ & 1891 & $\begin{array}{l}\text { St. Simon } \\
6 \text { Sultan }\end{array}$ & $\begin{array}{l}\text { Match Girl } \\
3 \text { Orlando }\end{array}$ \\
\hline 18 & $\begin{array}{l}\text { Parmesan } \\
6 \text { Waxy (by Potsos) } \\
6 \text { Whalebone } \\
6 \text { Penelope } \\
6 \text { Potsos } \\
6 \text { Prunella (Dam of } \\
\text { Penelope) }\end{array}$ & 18.57 & $\begin{array}{l}\text { Sweetmeat } \\
4 \text { Walton } \\
6 \text { Potson }\end{array}$ & $\begin{array}{l}\text { Gruyere } \\
3 \text { Waxy } \\
3 \text { Penclupe }\end{array}$ \\
\hline 19 & $\begin{array}{l}\text { Phaeton } \\
6 \text { Orville } \\
7 \text { Waxy } \\
7 \text { Penelope } \\
6 \text { Castrel } \\
\text { Selim }\end{array}$ & $\begin{array}{c}1865 \\
\text { in } \\
\text { America }\end{array}$ & $\begin{array}{l}\text { IYing Tom } \\
5 \text { W'axy } \\
5 \text { Penelope }\end{array}$ & $\begin{array}{l}\text { Merry Sunshine } \\
1 \text { Touchstone } \\
5 \text { Orville } \\
5 \text { Castrel }\end{array}$ \\
\hline
\end{tabular}




\begin{tabular}{|c|c|c|c|c|}
\hline No. & Names of Stallions & Born & Sire & Dim \\
\hline 20 & $\begin{array}{l}\text { Rustic } \\
6 \text { Whalebone }\end{array}$ & 1863 & 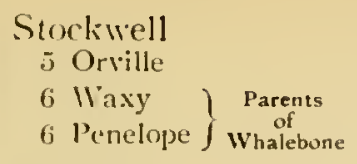 & $\begin{array}{l}\text { Village Lass } \\
5 \text { Whalcbone }\end{array}$ \\
\hline$\stackrel{21}{1}$ & $\begin{array}{l}\text { St. Nlbans } \\
6 \text { IVhalebone } \\
\text { [own brother to } \\
\text { Sivernalie] }\end{array}$ & 1857 & 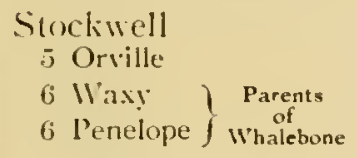 & $\begin{array}{l}\text { Bribery } \\
6 \text { Sir leter } \\
6 \text { Stamford } \\
6 \text { Whodpecler }\end{array}$ \\
\hline-2.0 & $\begin{array}{l}\text { St. Frusquin } \\
6 \text { Bay Mlidlleton } \\
\text { (by Sultan) }\end{array}$ & $181 \% 3$ & $\begin{array}{l}\text { St. Simon } \\
\text { 6 Sultan }\end{array}$ & $\begin{array}{l}\text { Isabel } \\
6 \text { Fouchstone } \\
\text { 6 Emma }\end{array}$ \\
\hline 23 & $\begin{array}{l}\text { St. Simon } \\
6 \text { Sultan (by Selinu) } \\
6 \text { Velocipede's dam } \\
7 \text { Blaclilock }\end{array}$ & 1881 & $\begin{array}{l}\text { Calopin } \\
2 \text { Voltaire } \\
\text { (b! Blacklock) }\end{array}$ & $\begin{array}{l}\text { St. Angela } \\
\text { C Selim }\end{array}$ \\
\hline 24 & $\begin{array}{l}\text { Salvator } \\
6 \text { Selin } \\
6 \text { Orville }\end{array}$ & 1872 & $\begin{array}{l}\text { Dollar } \\
4 \text { Catton } \\
\text { 6) Bening- | Parents } \\
\text { brough of } \\
\text { 6 Evelina Oorvilie } \\
\text { (irand-sire: } 3 \text { Selim }\end{array}$ & $\begin{array}{l}\text { Satragine } \\
4 \text { Selim } \\
\text { Grand-dim : } 3 \text { Stelim }\end{array}$ \\
\hline - & $\begin{array}{l}\text { Speculum } \\
6 \text { Orville }\end{array}$ & 1865 & $\begin{array}{l}\text { Vedette } \\
\text { :3 Blaclilock }\end{array}$ & $\begin{array}{l}\text { Doralice } \\
4 \text { Orville }\end{array}$ \\
\hline 20 & $\begin{array}{l}\text { Teddington } \\
6 \text { Stamford } \\
\text { (by Sir l'eter) } \\
7 \text { Trumpater } \\
7 \text { Sir Peter }\end{array}$ & 1818 & $\begin{array}{l}\text { Orlando } \\
3 \text { Selim } \\
5 \text {. Mexiander } \\
\text { (by Eclipse, (irand- } \\
\text { sire of Stamford) } \\
\text { (5 Sir l'eter }\end{array}$ & $\begin{array}{l}\text { Miss Twickenhan } \\
5 \text { Sir Peter } \\
\text { Grand-sire: } \\
3 \text { Trumpatur }\end{array}$ \\
\hline 27 & $\begin{array}{l}\text { Thormanby } \\
6 \text { Orville } \\
7 \text { Wonelpecker } \\
\text { (by Herod) }\end{array}$ & 18.7 & $\begin{array}{l}\text { Mindhound } \\
3 \text { Peruvian } \\
\text { 5 Buzard } \\
\text { (by Woodpecler) } \\
\text {; Mare by . Nexander }\end{array}$ & $\begin{array}{l}\text { Hice Haw thorn } \\
3 \text { Dick . Indrew: } \\
4 \text { Beningbrough } \\
1 \text { livelina } \\
\text { (by llighlyer) }\end{array}$ \\
\hline 24 & $\begin{array}{l}\text { Trenton } \\
6 \text { 'Tnuchstone } \\
6 \text { Camel } \\
7 \text { IIhalehonc }\end{array}$ & $\begin{array}{c}1681 \\
\text { in } \\
\text { Auntral. }\end{array}$ & $\begin{array}{l}\text { Mtusket } \\
\text { | Tuuch-tone } \\
\text { | Camel } \\
\text { Cirand-dam: } \\
\text { 2 Came] }\end{array}$ & $\begin{array}{l}\text { Fraile } \\
\text { is Sir Hercules } \\
\text { fi Whalebone }\end{array}$ \\
\hline 象! & $\begin{array}{l}\text { Tristan } \\
6 \text { Sultan (by Sclim) } \\
7 \text { Whatebure } \\
\text { \& Orville }\end{array}$ & Isis & $\begin{array}{l}\text { Hermit } \\
\text { \& Cimmel } \\
\text { (by llhalebone) } \\
\text { 6 Selim } \\
\text { 6 I'ilynator } \\
\text { F Orville }\end{array}$ & $\begin{array}{l}\text { Trieft } \\
\text { is Tramp } \\
\text { 6 } 11 \text { halebone } \\
\text { o } 11 \text { hisiser } \\
\text { f Orvilke }\end{array}$ \\
\hline
\end{tabular}




\begin{tabular}{|c|c|c|c|c|}
\hline No & Names of Stallions & Born & Sire & Dam \\
\hline 30 & $\begin{array}{l}\text { Wenlock } \\
6 \text { Whalebone }\end{array}$ & 1869 & $\begin{array}{l}\text { Lord Clifden } \\
6 \text { Paynator } \\
7 \text { Orville }\end{array}$ & $\begin{array}{l}\text { Mineral } \\
\text { I Birdcatcher } \\
\text { † Orville }\end{array}$ \\
\hline 31 & $\begin{array}{l}\text { West Australian } \\
6 \text { Evelina } \\
\text { (by Highflyer) } \\
6 \text { Trumpator } \\
7 \text { Eclipse } \\
7 \text { Sir Peter } \\
\text { (by Highflyer) }\end{array}$ & 1850 & $\begin{array}{l}\text { Melbourne } \\
4 \text { Termagant } \\
5 \text { Trumpator } \\
5 \text { Highflyer } \\
6 \text { Eclipse }\end{array}$ & $\begin{array}{l}\text { Mowerina } \\
3 \text { Waxy } \\
3 \text { Penelope } \\
\text { (by Trumpator) } \\
6 \text { Sir Peter } \\
6 \text { Eclipse }\end{array}$ \\
\hline 32 & $\begin{array}{l}\text { Woodpecker } \\
6 \text { Darley Arabian }\end{array}$ & 1773 & $\begin{array}{l}\text { Herod } \\
\text { I Leedes Mrabian } \\
7 \text { Hautboy } \\
7 \text { Spanker }\end{array}$ & $\begin{array}{c}\text { Miss Ramsden } \\
\text { ? }\end{array}$ \\
\hline 33 & $\begin{array}{l}Y . \text { Melbourne } \\
6 \text { Sir Peter } \\
\text { (by Highnyer) }\end{array}$ & 185.5 & $\begin{array}{l}\text { Melbourne } \\
4 \text { Termagant } \\
5 \text { Trumpator } \\
5 \text { Highfier } \\
6 \text { Eclipse } \\
\text { Grand-sire: } \\
2 \text { Sir Peter }\end{array}$ & $\begin{array}{l}\text { Clarissa } \\
4 \text { Buzzard } \\
4 \text { Nare by Alexander } \\
5 \text { Sir Peter }\end{array}$ \\
\hline
\end{tabular}

Stallions with 7 fires Generations.

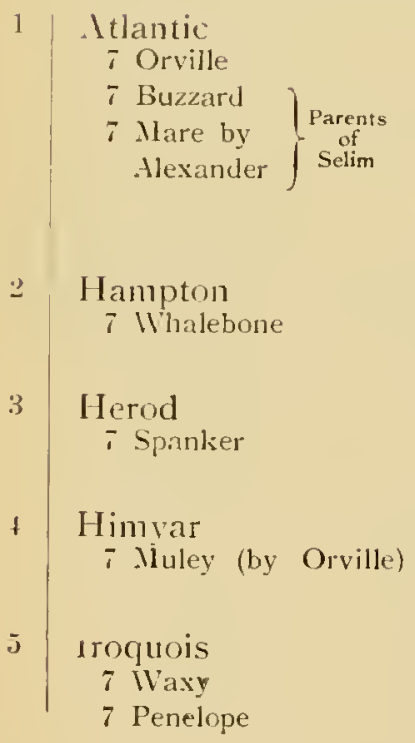

\begin{tabular}{|c|c|}
\hline 187 & $\begin{array}{l}\text { Thormanby } \\
6 \text { Orville } \\
7 \text { Woodpecker (Sire } \\
\text { of Buzzard) } \\
7 \text { Sir Peter } \\
\text { Grand-sire : \& Evelina } \\
\text { (Dam of Orville) }\end{array}$ \\
\hline 1872 & $\begin{array}{l}\text { Lord Clifden } \\
6 \text { Paynator }\end{array}$ \\
\hline 1758 & $\begin{array}{l}\text { Tartar } \\
6 \text { Old per (Dam of } \\
\text { Spanker) }\end{array}$ \\
\hline & $\begin{array}{l}\text { Ilarm } \\
5 \text { Sultan }\end{array}$ \\
\hline & $\begin{array}{l}\text { Leamington } \\
6 \text { Woodpeclier } \\
6 \text {. Mexander }\end{array}$ \\
\hline
\end{tabular}

\author{
Hurricane \\ 3 Sultan (by Selım! \\ Grand-sire : 4 Selim \\ Lady Langden \\ 3 Liverpool \\ 7 Whalebone

\section{Cypron} \\ 3 Darley's Arabian \\ Hira \\ 3 Emilitrs (by Orville) \\ 5 Sir Archy \\ Maggie
}




\begin{tabular}{|c|c|c|c|c|}
\hline No. & Names of Stallions & Born & Sire & Dam \\
\hline 6 & $\begin{array}{l}\text { Lingisher } \\
\text { \% Orville } \\
\text { \% Sir Peter } \\
\text { \& Walton }\end{array}$ & $\begin{array}{c}1867 \\
1867 \\
\text { Anericat }\end{array}$ & $\begin{array}{l}\text { 1.xington } \\
3 \text { Sir. Irehy }\end{array}$ & $\begin{array}{l}\text { Filliam I.ass } \\
\text { (j ll:ilton } \\
\text {; Orville }\end{array}$ \\
\hline 7 & $\begin{array}{l}\text { Oxford } \\
\text {; llaxy } \\
\text {; l'nelope }\end{array}$ & $15 \% 7$ & 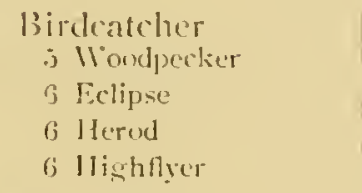 & $\begin{array}{l}\text { Homeydear } \\
3 \text { Orville } \\
4 \text { Selim }\end{array}$ \\
\hline$s$ & $\begin{array}{l}\text { Soe Sall } \\
7 \text { Orville } \\
\text { - ll:ixy } \\
\text { \& Trumpatm }\end{array}$ & $18(3.5)$ & $\begin{array}{l}\text { Buccaneer } \\
\text { 3 Filnumel } \\
\text { (by Orville) } \\
\text { j Paynator } \\
\text { (by Trumpator) }\end{array}$ & $\begin{array}{l}\text { Marcary Daw } \\
\text { j Viay } \\
\text { j Penelope } \\
\text { (hy Trumpator) } \\
\text { (j Orville }\end{array}$ \\
\hline 9 & 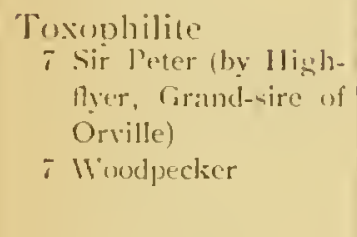 & $18.5)$ & $\begin{array}{l}\text { l.unghow } \\
1 \text { Orville } \\
7 \text { IIoodpecker }\end{array}$ & $\begin{array}{l}\text { 1.'cerdemain } \\
2 \text { Peruvian } \\
\text { (by Sir Peter) } \\
\text { j } 11 \text { oondpecker } \\
\text { Crand-dam : } \\
\text { 2 Sir Jeter }\end{array}$ \\
\hline 10 & $\begin{array}{l}\text { Trachenbers } \\
\text { ' Bustard } \\
\text { \& Orville }\end{array}$ & 1879 & $\begin{array}{l}\text { Flibustier } \\
\text { j Tramp } \\
\text { is Sultan } \\
7 \text { Orvil]e }\end{array}$ & $\begin{array}{l}\text { Dirt Cheap } \\
3 \text { Camed } \\
\text { (j Buzard (Crand-sire } \\
\text { of Bustard and } \\
\text { Sultan) }\end{array}$ \\
\hline 11 & 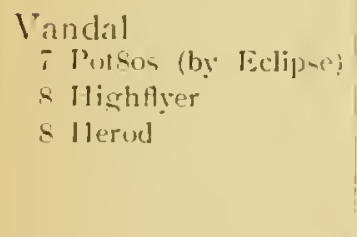 & $\begin{array}{c}18, x) \\
\text { int } \\
\text { Antricn }\end{array}$ & $\begin{array}{l}\text { Glencoe } \\
\text { 5 . Nercury } \\
\text { is Forlipse } \\
6 \text { Ileror } \\
\text { 6 Hishflyer } \\
\text { c Woodpecker }\end{array}$ & $\begin{array}{l}\text { Mara by Tranbv } \\
\text {; Highflyer }\end{array}$ \\
\hline $1: 2$ & $\begin{array}{l}\text { II ar Dance } \\
\text { i Dick Indrews } \\
\text { - Orville }\end{array}$ & $\begin{array}{c}18.5 ! \\
\text { in! } \\
\text { Avertich }\end{array}$ & $\begin{array}{l}\text { 1. (exington } \\
\text { 3 Sir Irchy } \\
\text { 6) Saltram (by Eclip-c. } \\
\text { Cirand-sire of Dicls } \\
\text {. Mulrews and } \\
\text { (m)anna) }\end{array}$ & $\begin{array}{l}\text { Reel } \\
\text { J Gohanna }\end{array}$ \\
\hline
\end{tabular}

Stallions with 8 frees Generations.

1
Missel Trusl
\& Glencoe
8 Banter (Dam of
Touchstone)
8 Bay Middleton

\author{
Throsile \\ - Tuuchstone \\ lirand-sire: \\ 2 Touchstone \\ Cirand-dam : \\ + Bisy . Widdleton
}


From these examples may be drawn the following conclusions:--

1. The greatest number of approved sires are to be found amongst those with 3,4 and 5 free generations. Also the sires and dams of the above-mentioned 270 stallions with 1 to 8 free generations, have, as a simple calculation will show, on an average, 4 (exactly 4.13$)$ free generations. This is a very remarkable and interesting result, and may be taken as a proof of the example. We here again see it confirmed that 4 free generations represent the most favourable inbreeding for successful breeding material.

2. In former times we find more successful stallions with close inbreeding than in modern times. Of stallions with 1 free generation in modern times, only Barcaldine and Flying Fox can be considered really successful. It seems to me to be an open question, in any case it has yet to be proved, whether either of them will be the founder of such a successful line as Paulowitz, born 1793. Among the stallions with 2 free generations in the past, the following may be mentioned as especially prominent : Brutandorf, Gohanna, Golumpus, Humphrey Clinker. Orville, Partisan, Sorcerer, etc. As equal to these in modern times we can only mention Galopin, Wisdom, and Sainfoin. In the case of 3 and 4 free generations, ancient and modern times are about equal. In the case of 5 and more free generations in former times are specially to be mentioned: Herod, Highflyer, King Fergus, Marske, Matchem. Waxy, and Woodpecker. The great majority of the horses mentioned in these lists are the very best stallions of the more and most recent times.

3. Is regards the repetition of closer inbreedings, it can be seen that the sires of stallions with 1 free generation had at least $t$ (on an average $4 \frac{1}{2}$ ) free generations, i.e., a little more than the remaining stallions with further removed inbreeding. Wre can therefore come to the conclusion that a repetition of such close inbreeding has not been successful. Also amongst the many examples with only 2 free generations, Golumpus is the only one that can be mentioned in which the site also had only 2 free generations, but the further removed inbreeding on the basis of Godol. Arabian is strongly supported on the dam's and sire's side.

1. In the case of the most successful stallions with a close inbreeding (1 and if free generations), a further remured inbreeding of the same is supported in nearly every case by a corresponding inbreeding of the parents, for example, with Paulowitz (1 Highfyer and 1 Termagant) the more removed inbreeding of the same is most strongly supported, viz., 6 Regulus, as well as by the sire Sir Paul (4 Regulus) as by the dam Evelina ( $t$ Regulus). Also in the case of filying Fox (1 Galopin), the more removed inbreeding of same, i.e., 6 Stockwell and 7 Pocalontas, is strongly supported by the sire Orme (j Pocahontas and 6 Birdeatcher) and a little by the dam Vampire (2) Vedette and 6 Birdcatcher). In the case of Friponnier (1 Orlando, 7 Orville, $7 \mathrm{~W}$ altom) the more removed inbreeding is strongly supported by the sire (5 Orville) and by the dam (5) Walton). In the rase of the Derby 
and 2,000 Guineas winner cadland (1 Surcerer, I Highllyer [by Herod], I IIoodpeclier [by Herod]) also, the more remored inbreading is supported by the sire Indrew (5 llered) and by the dam Soreery (.j lleresl). Among the stallions with of free arenerations, in the case of most, and those the very best. the support of more remored inbreeding is visible by a correspending inbreacling of the parents, as, for example, with l'artisan the more removed inbrecling to Snap and Regulus, with Hanower the more removed inbreeding to Emilius, with Petrarch the more remosed inbreding to Selim, with Priam (o) llerod, with The Saddler to Ilighfleer, with Wisdom to Orville, with Janissary to Touchstone, wte.

5. Inbreedings of the best stallions are supported by the inbreedings of sire or dam, of both, on the same or related basis. If the inbreeding is a close one', i.e. under f ur if free generations, it is adrantageous not to sup)port this close, hut more remoxed inbreedings, if such exist, as is explained in No. 4. It is therefore always advantageous to have several inbreedings, eate on a different basis. Such stalliuns are also easier (o) get at for mating.

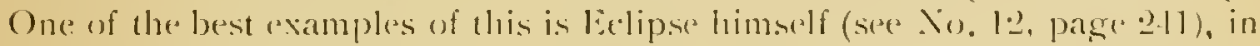

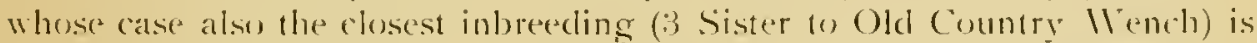
not directly supported by the inbreedings of the parents. The following more removed inbredings: 4 Snake, (j Hauboy, and 7 Coneyskins, are supported by the inbreedings of both parents and both grandmothers. This support of the inbreeding of prominent stallions by the inbreeding of their parents is alse often strikingly visible in the case of stallions with 7 and more free generations, as in the case of Atlantic, etc. The Derby winner Irepuesis, himself a prominent racer, but not quite as sucessiful at the stud. dexes not show the support of his inbreeding which is required above, as is

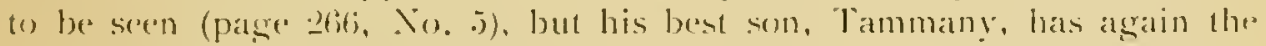
requisite support of his inbreding by (j Glenere, and his dam, I Glenere.

In the following table of the most important male bleod lines the above rule of inbreeding support is distinctly recognisable. Stallions in the catses of which these inbreding supports are especially visible, outlast in their progeny intermediatel! one crencration without this support, as in the casce of

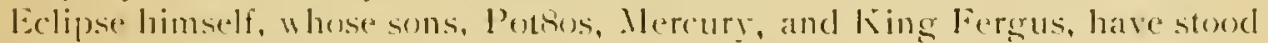
well one gentertion without this inbreding support. Their sons, Waxy, Gohanna, Beningrbrough, and llambletonian, shou already distinctly the desired supports. Joe Indrews is the only sou of ledipse who answers himself the above demands. In contrast to liclipse, the other two foundation sires, llerod and llatchem, as well as their sons (with the exreption of liforizel, sire of the lirst Derby winner, Diomed), show a much smaller, and in some cases no inbreeding support at all. This is probably the catse of the liclipse progent, in spite of the many goud racehorses which llighfler has also produced, exectling most uthers as regarels breoding value. lickipse left behind him four important make lines. Highlleer amel Moodpecker only one each. Here followed two eonserutive gremerations (IJerod and his two 
sons, Higlnfyer and Wroodpecker) without the requisite inbreeding supports. Nevertheless, Herod and his son Highflyer have proved themselves to be a very good basis of every inbreeding. The l Terod blood only commences to produce more sires for Thoroughbred breeding with the much better bred Sir Peter, born 1784 by Jighfyer, and Buzzard, born 1787 by Woodpecker. The Matchem blood has undergone a similar experience, as his son, Conductor, did not porsess sufficient inbreeding supports. Trumpator, born 1782 by Conductor, as well as his two sons, Sorcerer and Paynator, were the first to succeed in influencing Thoroughbred breeding to a greater extent, as only in their pedigree the requisite inbreeding supports became significant.

From the following list of male blood lines we can further see that the Herod and Matchem lines pussess Eclipse, or Eclipse progeny, more rarely as the basis of their inbreedings than the Eclipse line itself. In the Herod line, for example, the first Eclipse inbreeding, even yet little supported, occurs in the American bred Boston. The Sultan-Glencose line, well supported by liclipse inbreeding, has also had its chief importance in .Imerica. Europe only retained Glencue's celebrated daugliter Pocahontas. In the Bay Middletm-lFl. Dutchman line, which remained here, the Jighflyer inbreeding prevails, and in the Highflyer line, which also remained here. there is no Eclipse inbreeding at all (page 282). Only the Pantaloon line (page 28.5) shows some Eclipse inbreedings, but generally inferior to the Herod connections. The Walton line (page 283 ) has been more fortunate. The latter and the Pantaloon line have also produced the best representatives of the Herod bloud in Sweetmeat, Chamant, and Thormanby.

A similar picture is found in the Matchem line (page 286). The Smolensko-Jerry line, as well as the Paynator and Dr. Syntax line, does not show any Eclipse inbreeding at all. The Sorcerer-Comus line, beginning modestly with II mphrey Clinker, showed the first somewhat effectivcly supported Eclipse inbreedings in the case of Melbourne and West Australian. This is very probably the reason that Melbourne is the only remaining scion of the Matchem line, whose hest son, West Iustralian, was early sold to France. Fortunately, West Australian left behind him in England a stallion well strengthened by many Whalebone, Waxy, Pot8os (all Eclipse's progeny) inbreedings, $i . e .$, Solon, sire of Barcaldine. The efficacy of inbrceding supports can be most instructively seen in the long male line from Highflyer down to Fels (page 282). Only in the case of IVild Dayrell and Buccaneer is this support somewhat deficient, and, in consequence, one had to wait for a son of Buccaneer endowed with a more distinct support of inbrecdings in order to effectively continue this line. Kisher was not such an one, but Flibustier (and his own brother Waisenknabe), with his double connection over Tramp and Orville. See Saw was perhaps sufficient regarding the building of the pedigree, but of too little class. Kisber was the third member of a male line built up with insufficiently supported inbreeding. On this account his heredity did not come up to expectations which were founded on 
his great racing form. In England, lilibustier, and perhaps also Waisenknabe, with Krisber's chances, might have been a great success. Buccaneer with his inbreeding ( 3 Edmund and 5) Paynator) was certainly difficult to handle so as to obtain the required inbreeding support. Also Fenek (6 Tramp, 7 Sultan, dam 5 Cervantes), Good Hope (6 Tramp, 6 Sultan, 7 Orville, dam 7 Orville), and Pirat (5 Cain, 6 Sorcerer, dam 3 Comus, 5 Sir Peter), had not sufficient inbreeding supports in the building up of their pedigrees. Nil Desperandum was bred a little more farourably, but not sufficiently so: I Bay Middleton, fi Orville, 7 Paynator, clan 2 Touchstone, i) IThalebone. 
1. 1'u180s by Eclipse

\section{Eclipse Line.}

1733. 4 Godol. Mrabian.

2. Waxy 5 Godol. Irabian.

1790. 6 l'artner (by Jigg)

Dam 4 Fl. Childers.

+ Fox.

6 Byerly Turk (Sire of Jigg).

3. Whalebone 3 Herod.

1807. 4 Snap.

6 Cade (by God. Mrabian).

4. Camel 4 Highflyer. 5 Eclipse. 1882. 5 Herod. 6 Snap.

Dam 3 Snap.

6 Partner (Grand-sire of Herod).

6 Godol. Irabian.

1)am 3 Highflyer.

4 Herod.

5. Tunclaslunte 4 -Vexander (by Eclipse).

1831.5 Eclipse.

j Buzzard.

5. Sir Peter.

6 Highflyer.

15. Jewminster 5 beningbrough (by ling Fer1848. 5 Trumpator. [gus-Herod).

Dam 6 Eclipse (Sire of King Fergus). 6 llerod.

b. Hermil \& Camel.

1864. 6 Paynator (by Trumpator).

6 Selim.

7 Orville (by Beningbrough).

S. Trislan 6 Sultan (by Selim).

1878. 7 Whalebone.

Dam 4 Eclipse.

Dam 3 Sultan (by Selim. 5 Beningbrougl.

Dam 5 Tramp. 6 Whalebone.
6 IVhislier.

6 Orville.
4. Sir llerenles by Ithalebone

1826. 4 kclipse. 5 Hightyer.

5 Herod. 7 Snap.

j. Birdeateler 5 lloodpecker (by Herod). 1833. 6 Eclipse.

6 llerod.

(i) Hightlyer.

6. The Barou 4 llaxy (by Potsos). 1842. 4 Penelope.

S. Stutkwell 5 Orville.

1819. 6 IVaxy.

6 Penelope.

Q. Hail Atlol 6 Whalebone (by WVaxy and 1861.
Penelope).

7 Orville. 1)ann 3 Eclipse.

5 Herod.

Dam 2 Bagot (by Herod).

Dam 6 Pot8os.

6 Phonomenon.

6 Highflyer (Grand-sire of Penelope).

Dam 5 Gohanna.

6 Mercury.

7 Highflyer (Grand-sire of Orville and Penelope).

1)am 6 Evelina (Dam of Orviller.

6 Sir Peter.

7 Trumpator (Grand-sire of Whalebone).

7 Highflyer (Grand-sire of Orville). 
6. Ithuriel by Touchstonc

1841. ذँ Buzzard.

5 Mare by . llexander.

7. Longhow 4 Orville (2 Herod).

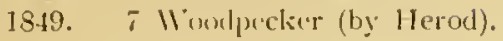

\& Toxophilile 7 Sir Peter (by Highflyer, 1855. Grand-sire of Orville).

7 Woulpeclier (by Herod).

9. Inskel + Touchistone.

186r. \& Camel.

16i. Sordenfeldt + Mellumine.
1882.
5 Touchstone.

1)am 4 Sir Peter.

5 Highflyer.

Dam 5 llighllycer.

6 llerod.

6 Eclipse.

Dam 2 l'eruvian (by Sir Peter).

5 IVoodpeclicr.

Grand-dam 2 Sir l'eter.

Dam 2 Camel.

Dam 3 Touchntone

Dam j Sir llercules.

6 Whalebone.

1881. 6 Touchstune. - Whalebone.

6 Camel.

10. Carbinc by Musket

1885. 3 Brown bess (by Camel).

5 Touchstone.
Dan 3 Touclsstone
+ Camel.

Dam 3 Stuckwell. jorlando.

3 Y. Melbourne.

\section{Melhourne. (by- Touch.).}

6. Orlando by Touchatone

14t1. 3 Selim. 6) Sir P'eter.

i) Ilexinder.

7. Trumputer + Selim.

13.56. 5 Orville.

$\therefore$ Plu(us + Emilius (by Orville). 1262. 6 Selim.

9. l'laneroled j Partisan (by Walton). 1870. : Wialebone.

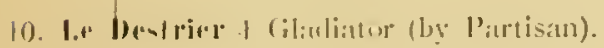
$15 \%$.
Dam 3 Buzzard Parents of
3 Mare by . Mexander j Selim.

+ Sir Peter.

1)am + Buzzard P'arents of

4 Mare by . Mexander j Selim.

4 Beningbrough / Parents of

+ Evelina $f$ Orville.

1)an is Selim.

Dam 5 Whalebonc.

Cirand-dam 3 llalton.

Dallu j Partisan. 
6. Fewminster by Touchstone

1848. 5 Beningbrough (by King Fergus [by Eclipse] and HerodMare).

5 Trumpator.

7. Loril Cliftell 6 Paynator (by Trumpator). 1860. 7 Orville (by Beningbrough).

S. Haunton 7 Whalebone. 1872.

9. Ladas 5 Touchstone. 1891. 6 The Baron.

$$
6 \text { Pocahontas. }
$$

10. Trumtheck 6 Stockiell.

1903. 7 Touchstone.
Dam 6 Eclipse.

6 Herod.

Dam 5 Golumpus.

Dan 3 Liverpool

7 Whalebone.

Dam 4 Touchstone.

Dam 3 Thormanby.

4 Stocliwell.

6 Birdcatcher.

7 Banter.

8. Petrareh by Lord Clifden

18i3. 2 Touchstone.

7 Selim.

Dan 4 Whalebone (Grand-sire of Touchstone).

5 Selim.

9. Tlie Barl 4 Melbourne.

1883. T Defence (by Whalebone).

Dam 6 Comus (Grand-sire of Melbourne).

6 Whalebone.

6 Sultan (by Selim).

7. Cambusam by Newminster

Dan 4 Beningbrough.

1861. + Whalebone.

5 Orville (by Beningbrough).

6 Beningbrough.

7. Alventurer by Newminster

1859. 4 Orville.

6 Stanford (by Sir Peter).
Dam 1 Orville. 4 Sir l'eter.
7. Eelipie by Orlando

Amer. 1855. + Selim (by Buzzard).

S. Alarm 5 Sultan (by Selim). 1869. 6 Bustard (by Buzzard).

9. Ilimyar i Nuley (by Orville) 187.5.

[2 Herod].

10. Domino 3 Lexington. 1891.

11. Commando j Lexington. 1898.
Dam 2 Phantom.

Dam 5 Blacklock.

5 Tranup.

7 Buzzard.

Dam 3 Emilius (by Orville).

5 Sir . Irclyy (3 Herod).

Dam 2 Lexinston. 
6. Oxford by Birdeatcher

1857. T Waxy.

T Penelope.

7. Stcrling $511 \%$ halebone (by Waxy and $186 \Omega$.

Penclope).

8. Isonmm! 3 Birdcatcher (by Sir Hercules and 1875.

9. Iviuntatm 5 Firdcatcher. j Pocahontas. 1890. 5 The Baron. 6 Touchstone.
Dim 3 Orsille.

+ Sclim.

Dan 5 IIhalebone.

6 llaxy.

Dam + Sir Hercules.

+ Guiccioli.

Dan \& Touchstone.
9. Callinule by lsonumy:

18\&4. 3 Stockwell. 6 Birdcatcher.

i) Touchstone (by: Camel).

10. Slicre (ialliun 6 Stockwell. T Touchstone. 1906. Tlocahontas. T Voltigeur.
Dam 7 Selim (Grand-sire of Camel). Grand-ire 4 Camel.

Danı 2 Gallopin (Grand-son of Volt.). 7 Pocahontils. $;$ Touchstone.

9. Jani-sally by 1sonomy

Dam 3 Touchstone.

1887. 2 Stockiwell.

j Touchntone.

5 Melbourne.

10. Jedilali 6 Touchstone.

Dans j Banter (Dan of Touchstone). $159 j$.

8. Enerwy by Sterling

1850. \& Sir Hercules.

4 Birdcatcher.

5 Touchstone.

9. Gourerneur $\&$ Touchstone.

18se. $i$ Whalebone (by Waxy and Penclope).
Dam 4 Sir llercules.

5 Touclistone.

Dam j Snolensio.

6 llaxy.

6 Penclope.

5. Faumla-i-Ballagh by Sir Hercules

1541. 5 lloodpeclice (by Herod).

6 Eclipse.

6. Leamington of Hoodpecker.

1853. 6 .llcxander (by Liplipse).

Dam \& Buzzard (by Woodpeclier).

4 Mare by llexander.

†. Oumblana - Buzzard (by Woodpeclier). 1879. 7 Mare by .llexander.

8. Locolutehe 3 lexington.

Dam உ Bagrot (by llerod).

18ง9. 6 Glencoe.

9. Caillan j Stockwell.

Dan 7 Dick Indrews.

Grand-dam 2 Tramp.

4 Buzzard.

Dam \& Pocahontia (by Glencoe).

1596.

Dam உ Stockinell. 
8. Hunca-ler by Siuclivell 1870. 6 B Blacklock.

7 IIhalebone.

; Selin.

9. Bend 010 Tumchstume.

187\%. 6i Muley.

† Selim (by Buzzard).

10. Orumule 5 Birdcatcher.

1883. 5 Pantaloon (by Castrel).

- Banter (1)am of Touchstone).

11. Omme 5 Pocahontas (by Glencoe). 1889. 6 Birdcatcher.

12. Flyng Fux 1 Galopin (by Vedette). 1896.

6 Stocliwell.

7 locahontas.

13. Ijax 6 living Dutchman. 1901 .
Dam 4 Humphrey Clinker. 6 Selim.

Dam 6 Orville.

6 Buzzard.

Dam 6 Blacklock.

$6\left\{\begin{array}{l}\text { Selim. } \\ \text { Castrel. }\end{array}\right.$

Dam 6 Sultan (Sire of Glencoe).

6 Velocipede's dan.

Dam 2 Vedette.

6 Birdcatcher.

Dam 5 Flying Dutchnan.

6 Pocahontas.
8. St. Alhans by Stockwell

185\%. 6 Whalebone.

9. Springliuld fo Sultan (by Selin).

1873. 6 Camel (by Whalebone).

6 Banter.

10. Sainfuin ¿ Stockwell. 1887. 6 Touchstone.
Dam 6 Sir Peter.

6 Stamford.

6 IVoodpecker.

Dam 4 Camel.

4 Langar (by Selim).

5 Selim.

Dam 3 The Baron ) Parents of

3 Pocahontas f Stocliwell.

४. Lard lyou by Stuckwell

1863. 6 Selim.

6 Whalebone (by Maxy).

T Orville.

9. Minling 5 Birdcatcher. 7 Selim. Dam 7 Castrel.

1883. 5 Touchstone. 7 / Castrel.

5 Glencoe.

S. Lord Ronald by Stoclswell

1862 .

6 IThalebone.

7 Selim.

7 Orville.

9. Yaster Kildare 4 Birdcatcher.

1875.

10. Melfon 3 Stockwell.

1882. 5 "Touchstone.
Dam 5 Selin.

5 IVaxy.

6 Orville.
Dam 5 IVhalebone. 6 Selim.

Dam 6 Blacklock.

Dam 3 Touchstone

4 Pantaloon. 
10. Kendial by Bend Or

1883. \& Birdcatcher. 5 Pantaloon.

11. Galtec Yore 3 Thormanby.

1894. \& Stockwell.

\author{
Dam 7 Buzzard \\ (Grand-aire of l'antaloon).
}

Dam 5 Pantaloun

(Grand-sire of Thormanlyy).
10. Bonarista by Bend $\mathrm{Or}$

1889. 4 Pocahontas. J .llice Hawthorn.

5 Windhound. F Touchstone. (by Pantaloon).

11. Cyllene 4 Stockwell.

1895. 5 Newminster (by Touchstone).
Dan 4 Banter.

4 Pantialoon.

Dant 6 Touchistone.
3. Whisker by IVaxy

1812. 3 Herod.

4 Snap.

6 Cade (by Godol. Irabian).

4. The Colonel 3 Hightlyer.

1825. + Herod (by Tartar).

+ Eclipre:

7 Blank.

5. Cap-il-1'ie 2 II"axy.

1837.

6. Sir Herenles +11 axy (by lot8os).

1843. 4 l'enejope.

18cil. 611 axy.

7. Yattendont 4 partion.

8. Cirand bituene 6 sir bereules 187\%. (by Whalebone).

9. Jallon 4 Stockwell. 1890. G Melbourne.
Danı 3 Sinily.

6 Partner (lirand-cire of 11 erod).

6 Gudol. . Mrabian.

Danı j Tartar.

5 Blank.

6 Regulus (rirand-sire uf

Eclipse).

Dan 5 Ficlipse (liranl-nire of Wasy).

Dan + P'otsuk.

Dan 4 Ithiskey.

Dam \& Pantaloun.

5 Camel (by IIhathbone).

Dan 2 Fisherman.

4 Melbutrne.

4 Bay . Vidcletem.

5 darpesina (lir:md-dann of Stockiveli).

9. Jerman by Grand Jlancur

1892. 5 poeahuntas.

Dam 5 bay Middletom (by sultan, Granel-sire of pecaluntas).

j Sir Herculén.

8. Cliester by billenchon 1874. 5 Sir Hereules.

6 Sultan.

5 limilius (by Orille). 6 IVhislier.

9. Ahereorn + l'arisual (by Sir Hercules). 1884.

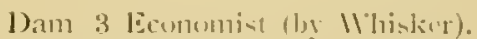

.) Orville.

Dam 4 Sir Ilereules. 
3. Whisker by II axy

1812. 3 Herod.

4 Snap.

6 Cade (by Godol. Arabian).

4. Economist 4 Herod.

1825. + Eclipse.

j Highflyer.

5 IIarlianay 5 Potsos (by- Eclipse).

1834. 6 Hightlyer.

7 Herod.

6. King Tom 5 Maxy (by PotSos).

1851. 5 Penelope.

7. Phacton 6 Orville.

1865. 7 Waxy.

7 Penelope.

$6\left\{\begin{array}{l}\text { Castrel. } \\ \text { Selim. }\end{array}\right.$

8. King Alfonso 3 Gilencoe.

1872. 5 Muley (by Orville).

9. Foxhall 3 Vandal (by Gilencoe). 1878.
Dam 3 Snap.

6 Partner (Grand-sire of Herod).

6 Godol. Arabian.

Dam 3 Herod.

4 Eclipse.

Dam 4 Highflyer (by Herod).

Dam 5 Gohanna (by Mercury).

6 Mercury (by Eclipse).

Dam 1 Touchstone.
5 Orville.
5 Castrel.

Dam 5 Orville.

Dam 7 Orville.

1. Mercury by Eclipse

17is. \& Godol. Mrabian.

‥ Golianua 2 Tartar (by Partner).

1790. \& Squirt.

5 Mosul (by Godul. Mrabian).

6 Godol. Irabian.

Dam 3 Partner.

$6\left\{\begin{array}{l}\text { Bartl. Childers. } \\ \text { Flyinar Childers. }\end{array}\right.$

3. (tolumpus 2 Herod (by Tartar).

1802. 5 Cade (by Godol. Irabian).

${ }_{4}\left\{\begin{array}{l}\text { Bartl. Childers. } \\ \text { Flying Childers. }\end{array}\right.$

4. Catton 4 Herod.

1809. 4 Eclipse.

5 Matchem (by. Cade).

5. Hulatto 5 Florizel (by llerod).

1823. 5 Hiyfffyer.

6 Herod.

6 Eclipse.

Dam 4 Godol. Irabian.

5 Partner.

Dam 3 Herod.

6 Godol. Arabian.

6 Blank.

6 Regrulus.

Dam 2 Highfyer.

5 Marslie. 
1. King Ferens by Eaclipse

\section{5.}

$$
\begin{aligned}
& 5\left\{\begin{array}{l}
\text { bintl. Childers. } \\
\text { liyiner Childers. }
\end{array}\right. \\
& 6 \text { Biy Bolton. }
\end{aligned}
$$

2. Mruinghrunsh a T:urtar (by Partner). 1 1.91.

+ Mirs slamerkin.

$6\left\{\begin{array}{l}\text { liantl. Childers. } \\ \text { lolying Childers. }\end{array}\right.$

o cindol. . Irabian.

3. Orville 2 Ilered (by Tirtar).

1799. 6 Regrulu (by (indol, . Mrabian).

4. Emilins 3 ljighnfer (by Jerod).

1820. \& Eclipse.

6 Blank (by Gudul, Irabian).

5. P’rian 5 IIhiskey.

182\%. 5 Herod.

5 Eclipse.
Dam 3 Partner.

5 Fl. Clikders.

5 Confederate Filly.
Dam 4 Regulus.

4 Blaze (by Childers).

4 Godol. Irabian.

Dam 3 Eclipse.

4 Herod.

+ Blank.

Dam 3 Herod.

4 Matchem.

2. Hambletunian by king licrous

1792. 4 Tartar. 5 Regulus.

6 Goll. Mrabian. j Crab.

3. Whitelock 3 Hered (by lantar).

1 $503 . \quad 3$ liclipse.

3 Mitchem.

4. Blathlock 3 Hlighnlyer.

Isit. 4 llerot.

4 Eclipare.

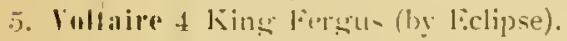

15.26. j Hightlyer.

Dan + Godol. Arabian.

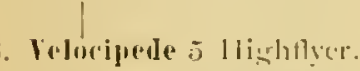

182.5. 5 l'uteus.

- Matchem.

Dam 4 Partner (Sire of Tartir).

Dam 1 Pot8os (by Eclipse).

2 Herod.

Dam 4 Jlighllyer.

5 Eclipse.

Dam 5 Eclipse.

6 Herod.

6 Matchem.

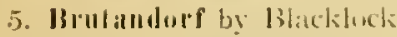

Dan 4 Cade.

18:1. 2 Puscos.

j Herod.

6. Ifelman Platudt + Poticus (by liclipse).

3837. I (imiander (by PotSos).

5 lietipase.

7. The losuack J li. liantesn.

1814. J Surcerer.

j) Stamford (by sir l'eter).

7 liclipse.

Dam 2 Sir l'uter.

5 Eclipase.

Dam 4 J. Giantess.

6 Sir l'eter.

7 Eclipse. 
6. Volligenr by Voltaire

1847. 5 Hambletonian (by ling Fergus).

6 Sir Peter (by lJighflyer).

- Highfyer.

6 Coriander.

7. Vedette 3 Blacklock.

1854. $6 \mathrm{MValton}$ (by Sir Peter).

8. (Halopin 2 Voltaire (by Blacklock). 1872.

9. St. Simm 6 Sultan (by Selim).

18S1. 6 Velocipede $\mathrm{s}$ dam.

Blacklock.

10. St. Florian 5 Jon.

1891. 6 Bay Middleton (by Sultan).

11. Ard Patrick j Cowl (bỹ Bay Middleton). 1899 .

6 Jocalontas.
Dan 4 Sir Peter.

6 Jing Fergus.

Dam 6 Potsos.

Dam \& Phantom (by Walton).

6 Potsos.

Dam 6 Selim.

Dam 5 Bay Middleton.

5 Touchstone.

Dam 5 Pantaloon.

6 Sultan (Grand-sire of

Pocahontas).
10. Matchbox by St. Simon 1891

6 Bay Middleton (by Sultan).

10. Persinmm by St. Simon

1893. 5 Voltigeur (by Voltaire and Martha Linnu).

6 Pocihontas.
Dam 3 Orlando

(3 Selim).
Dam 3 Nelbourne.

6 Voltairc.

6 Martha Lx"mn.
10. St. Frusquin by St. Simon 1893.

6 Bay Middleton (by Sultan).
Dam 6 Touchitone.

6 Enmma.
9. Donovan by Galopin

1886. 5 Bay Middleton (by Sultan).

5 Birdcatcher.

10. Velanduez j Pocahontas. 1894. 5 Touchstont.
Dam 3 Touchstone.

5 Mluley.

6 Sultan.

Dam 4 Banter (1)am of Touchstone).

8. Speculum by Vedette

Datis \& Orville.

1865. 6 Orville.

Dam „ Orville.

9. Roswery 3 "Jouchstone.

1872. 6 Orville (by Beningbrough).

6 Beningbruugh. 
1. Jue Indren- by Eclipse
1758.
4 Godol. Arabian.

5 Bartl. Childers.

2. IIick Indrew- 4 Blank (by Godol. Arabian). 179 .

5 Regrulus.

() Snip (by IPlying Childers).

Dam 3 Blank.

6 lilying Childers.

3. 'Irauı 3 Eclipse.

1810. \& Herod.

Dam 3 Eclipsc.

3 Herod.

4. Lircrpoul \& Eclipse.

1828. 5 lliwhlyer (by Herol).

5 Woodpeclier.

Dann 1 Pot8os (by Eclipse).

5 Trentham.

6 Herod.

5. Lanerenol \& Gohanna.

1835. 5 lloodpeclier.

5 Highflyer (by Ilerod).

5 "lrumpator.

6 Liclipse.

6. Ian Trump 5 Buyard (by IVoodpecker).

1844. 5 l'utsos (by liclipse).

Dam 6 Woodpecker.

6 Eclipse.

6 Herod.

Dam 3 Woodpecker.

4 Herod.

4. Lothery by Tramp

1820. 3 biclipse.

+ Trentham.

+ llvodpecker.

j Herod.

5. Shert Andhor 5 Woodpecker (by Herod).

1832.

6 Eclipse.

6 . Ncrcury.

6 Mlare by Ilcrod.

6. Weitherbit jOrville.

1842. $5 \mathrm{~K}$. Giantess.

Dam 4 Cade.

$4 \begin{aligned} & \text { Sister to legulus. } \\ & \text { Resrulus. }\end{aligned}$

Dam 6 Ilerud.

6 Eclipse.

Dam 1 Orville.

(j IVoodpecker (by Ilerod).

7. Beidsman 3 l'rimp.

1855. j Orville.

6 Herod.

Dam 6 Buzzard.

7 Buxard (by Hinodpecker).

\section{Rosicrurian}

1865. + l'riam (Cirand-son of Orville).

Dim 4 P'hatitom.

jollitebone.

7 Selim.

9. IBranchere is Whalebone. 1875.

10. Chisleshurst f l'artisan (by Walton).

1850. 7 Whalebune.

8 Orville.

Ditn 5 Willon.

Dam 5 Orville.

6 Waxy f larents of

6 Penclipe fllhalebonc. 


\section{Herod Line.}

1. Higlıllyer by Ilerod

1774. 5 Sister to Mixbury.

$4\left\{\begin{array}{l}\text { Bartl. Childers. } \\ \text { lilying Childers. }\end{array}\right.$

2. Sir Peter 3 Regulus.

1784. 4 Godol. Arabian.

5 lilying Childers.

3. Sir Paul + Regulus (by Godol. Arabian). 1802. 5 Snip (by Flying Childers).

4. Paulowitz 1 Highflyer.

1813. 1 Jermasant (by God. Arabian).

6 Regulus.

5. Caill 3 Highflyer.

1822. 6 Godol. Irabian.

6. Ion 3 Evelina (by Highther).

1835. 5 Sir l'eter (by Hightlyer).

7. Wild Wayrell 4 Selim.

18.52. 7 Sir l'eter.

8. Buccancer" 3 Edmund (by Orville).

185\%. 5 Paysator.

9. Flibusiev $\overline{5}$ Tramp.

1867. 6 Sultail.

7 Orville.

10. Trachembere 7 Bustard.

1879. S Orville.

\& Whalebone.

8 Selin (by Buzzard).

11. Inanibal 4 Touchstone.

1891. 4 Stockwell.

61011.

12. Fels 3 Hermit.

1903. 7 lon.

7 Jocaliontas.
Dam 1 Godol. Irabian.

Dam $3\left\{\begin{array}{l}\text { Bartl. Childers. } \\ \text { Flying Childers. }\end{array}\right.$

1)am 3 Regulus.

4 Godol. Irabian.

5 Flying Childers.

Dam 4 Regulus.

4 Godol. Irabian.

Dam 5 Highflyer.

1)am 5 Highflyer.

1)an 6 Sir Peter.

Dam 4 Beningbrough (Sire of Orville).

1)สแ 5 Tramp.

6 Orville.

Dam 3 Camel (by Whaleb.).

6 Buzzard (Grand-sire of Bustard and Sultan).

6 Selin.

Dam 3 Touchstone

j. Camel.

7 Sultan.

Grand-dam 4 Camel.

4 Touchstone.

Dam 5 Voltigeur.

6 Ion.

Grand-dan 4 Touchstone. 
3. IIaltou by Sir Peter

1799. 3 Snap. + Resulus.

3 llerod. 6 I'illincis.

4. Parlisau 2 llighflyer (by Herod).

1811. 3 Eclipse.

4 Snap.

6 liegulus.

5. Maldiatur + Prunella (by Highlyer). 1833. + Potsos (by Felipse).

6. Sueplunal 411 iallun (by Sir Peter). 1842. 6 potsos. 7 Highliver.

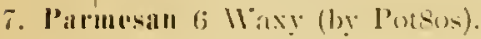

1857. 6 l'enelope.

8. Faroniu- 6 IIhislier (hy 11 axy and 1868 . l'enelope). 1)am 3 keecrulus.

5 l'artiner.

Damx 5 Kerrulus.

6 Godol. Irabian.

Dam 4 Trumpator.

5 l'otsos.

6 Eclipse.

6 Hishnyer.

Dam 0 Blatclilock.

6 Sir I'ter (by Highflyer).

Dam 3 IVaxy.

3 Penelope.

Dam 5 Ithisker.
7. Macammi by Sweetmeat 1860
6 Sir Peter.
(j Orville (Cirand-non of High- 17ver).
6 Buzzard.
6 . Mare ly Mteximder.
Dam 3 . Itexinder.
4 13tumird.
4 Hiç一yllyer.
j Sir Peter (1) Iligrhfler).

6. Fit\% (ilanlialn l, Gladiator

1850 . j lillzard.

5 Mire by . Mexilnder (by Eclipse).

5 Sir l'eter (by llishflyer).

j Guhanmí.

7. Compinanu if 14 halebone.

1858.

i Mare by . Mexinder.

- Sir l'eter.

7 beningbrough.

\% Cimatnma.

7 Putsos.

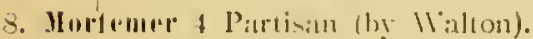
186.5. 6 Orville.

9. (')amand jomilius (by Orville). 1874. 6 Orville.

10. Saphir + P'ucahontas. 1888. 6 risuchusune.

6 Gladiatur.

7 Fmilius.

11. W(wil ; Pocahontas. 1901. 5 Strulivell.
Dam 4 Sir Peter.

6 Eclipse.

6 Ilerud.

Dam 6 Sir Peter.

6 Beninghbumgh.

li II:III (by l'otsos).

1) 1 momilius (by Orville).

6) Sir l'eter (Sire of 11 illton).

I)am -5 Orville.

5 Selim.

Cirancl-sire \& Orville.

ban 6 Touthotone.

Cirand-rire $\rightleftharpoons$ l'ocahontas.

firand-elimn \& Yuch-tune.

Dam 3 Stuelindl. 
1. Florizel by Herod

1768. 4 Fiving Childers.

$\tau$ Byerly Turk.

2. Diomed 4 Partncr.

177\%. 4 Godol. Irabian.

4 Crab.

5 Flying Childers (by Darley

Arabian).

3. Sir Arehy 3 Herod.

1805. 5 Blank (by Godol. Arabian).

$\tau$ Childers.

4. Timoleon 5 Trenthan.

1814. $T$ Blank (by Godol. Arabian).

7 Squirt.

5. Boston 2 Diumed.

1833. 5 Eclipse.

6 Marsle (by Squirt).

6. Lexiugtou 3 Sir Irclỵ (by Diomed). 1850. 6 Saltram (by Eclipse).

7. Norfolk j Sir Irchy:

1861. 5 Sumpter.
Dam 3 Flying Childers.

5 Basto (by Byerly Turlk).

Dam 5 Darley Arabian.

Dam 6 Godol. Mrabian.

6 Regulus.

Dan \& Regulus (by Godol, Arabian).

Dam J Marske.

Dam 6 Hightlyer (by Herod). Grand-sire of Diomed).

Dan 5 Buzzard.

6 VIaxy.

Grand-dan 2 Sir Archy.
2. Buzzard by Woodpeclier

178i. 3 Cade (by Godol. Mrabian).

5 Partner.

6 Flying Childers.

T Bald (ialloway.

3. Selim 3 Herod.

1802. 5 Matchem (by Cade).

6 Regulus (by Codol. . Irabian).

4. Sultan 4 llerod.

1816. + Hightiger

4 Eclipse.

5. Glencoe 5 Mercury (hy Eclipse).

1831. 6 Eclipse.

6 Hlerod.

6 Highflyer.

6. Vandal $\tau$ Pot8os (by Eclipse).

1850. \& Hightiyer.

8 Herod.

7. Virgil 4 Tramp.

1864. 6 Orville.

8. llindog 6 Emilius (by Orville).

1878. 7 Tramp.

9. Hannorer 2 Vandal (by Glencoe).

1884. 7 Enilius (5. Orville).

10. II amburn + Lexington.

1895. 6 Glencoe.
Dan 4 Codol. Arabian.

6 Bald Galloway.

Dan1 5 Regrulus.

6 Godol. .Mrabian.

Dam 3 Herod.

3 Eclipse.

Dan 4 Eclipse.

4 Highnyer.

5 Herod.

Dam 7 Hirhtlyer.

Dam ?

Dam 5 Emilius.

6 Orville.

Dam 7 Orville.

Dam 3 Lcxington.

5 Glencoe. 
3. Castiel by Buzzard

Is01. 3 llerod.

5 Matchen.

6 Regulus (by Goctol. Arabian).

4. ['anlaloon + Hight]yer.

1824. + Eclipse.

5 Herod.

5. Windhum 3 Peruvian (ly Sir Peter).

1847. 5 Mare by - Mexander (by

Eclipse).

i) Buzzard.

6. Thormanhy 6 Orville (ly Beningbrough). 1857. 7 Woodpecker (by Herod).

7. Atlantic 7 Orville.

1871. 7 Buzzard (by 1 oodp.)) Parents of

7 Mare by .llexander / Selim.

8. Le Saney 3 llindhound.

1884. 3 . vice Hawthorn.

5 Touchstone.

9. Le Sagillaire 2 Strathconan.

1892.6 Jelbourne.

10. Mainlenou 5 Newminster.

1903. 5 Stockwell.

7 Melbourne.
Dam 5 Regulus.

6 Godol. Irabian.
Dam 2 Hishlyer.

3 Eclipse.

1)am + IVaxy.

Dam 3 Dick . Indrews.

+ Beningbrough. \parents of

(by Highlyer). \ Orville.

Dam 3 Sultan (by Selim).

Dam + Touchstone (Grand-sire of IVindhound).

Grand-sire 2 Touchstone.

Grand-dam 2 Pantaloon (Sire of llindhound).

1)am 5 Touchstone.

;) Nelbourne.

1)am 6 Touchstone.

\section{Dam 3 Gladiator.}

5.) Touchstone.

9. La Justieier by Le Sancy 1892.

6 Touchstone.
Dom 3 l. Giantess.
5 Highflyer.
(i) Eclipse.

+ Sir Peter (by Highflyer).

4 . Methusa.

7 liclipse.

6. The flying Hulduman :3 Selino 1816.

6 Sir l'eter

1)ant j Hightlyer.

j Ecclipse.

-. Mollar 4 Catton.

1860. 6 Beningbrough.

(by. Hightlyer).

Dim j Sorcerer.

6 Beninglbrough.

6) Evelina.

- Sir Peter.

7 Sorcerer. 


\section{Matchem Line.}

1. Trumpitor by Concluctor (by Natchem)

1782. 4 Partner.

4 Godul. Irabian.

2. Sorcerer 2 Natchem.

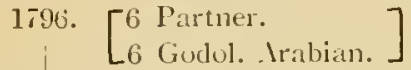

3. Comu- j Herod (by Tartar).

1809. j Snap.

6 Cade (by Godol. Irabian).

6 Eclipre.

4. Humplurey ("linlier 2 Sir Peter (by Highf.).

1829.

4 Trumpator.

7 Eclipse.

5. Mchourne \& Termagant.

1834. 5 Hightiver (by Herod).

j Trumpator.

6 Eclipse.

6. West Anstralian 6 Trumpator.

18.50.

6 Evelina (by Hightfyer).

- Eclipse.

7 Sir Peter (by Highflyer).

7. Solou 5 Whalebone (by Waxy).

1861. 5 Comus (Grand-son of Trumpator).

6 Waxy.

6 Penelope (by Trumpator).

8. Barealdine 1 Darlings's dam (by Bird1878. 5 Touchstone.

9. Yorion 5 'Touchstone. 1887. ; Whalebone.

[catcher).
Dam 4 Partner.

Dam 5 Godol. Arabian) Grand-sire of 5 Partner f Matchem.

Dam 5 Blank (by Godol. Arabian).

5 Tartar (by Partner).

5 Regulus (by Godol. Arabian).

Dan is Regulus.

6 Snip.

Dam 4 Eclipse.

5 Herod.

Dam 3 Waxy.

3 Penelope (by Trumpator).

6 Sir Peter.

6 Eclipse.

Dam 5 Waxy.

6 Pot8os.

7 Trumpator.

Dam 3 Birdcatcher.

Dam 4 Prian.

5 Whalebone.

Dan 4 Banter.

4 Pantaloon.

3. Smolen-kis by Sorcerer

1810. \& llerod.

5 Snap.

6 Cade.

4. Jerry 5 Herocl.
1892. 5 Newminster.
j Pocaliontas.
b Banter.
9. Sir Visto by Barcaldine

1821. 6 Natchem (by ('ade).

2. Paynator by Trumpator 3 Snap (by Snip). 1791. 5 Godol. Arabian.

3. Dr. Syntax 5 Matchem (by Cade). 1811. 6 Snip.

6 Cade (by Godol. Arabian).

7 Crab.
Dam 1 Herod.

3 Snap.

Dam 5 Herod.

5 Matchem.
Dam 3 Godol. Irabian.

4 Marc by Basto (Dam of Srip).

Dan 5 Cade.

5 Regulus (by Godol. Arabian).

7 Nare by Basto (Dam of Snip and Crab). 


\section{A few of the chief lines in American Trotters.}

1. Messenger x born Engl. 1780 by Mambrino xx - "lurf xx.

3 Cade (by Godol. Arabian), Jam : 3 Godol. Irabian, 5 lity bolton.

2. Mambrino xx? born Amer. 1806 by Messenger xi - Soucrout xix.

5 Cade (by Gudol. Irabian), Bam: 3 Gudol. Arabian, Grand-dam: 3 Godol. Arabian.

3. Abdallah 1 born Amer. 1823 by Mambrinu xx? and Imazmia. 1 Messenger $x x$, Dam: ?.

4. Hambleqonian (10) 1819 by Ibdallab I. and Charles lient Mare.

3 Messenger x.x, Dam : ?, Grand-dam: 0 Messenger xx.

j. George Wilkes (j19) 18.56 by Hambletonian (10) and Dolly Spanker.

Rek. 2,22 (insufficient pedigree of the Damu).

6. Patchen Wilkes (3500) 1852 by George WVilkes and Kitty Patchen.

Rels, 2,291. 5 .Mambrino xx? Dam:-1 Mambrino Patchen (Great Grand-non of Mambrino. Firand-dam: 0 .Mambrino Chief (Sire of Mambrino Pattchen).

7. Joe Patchen (30239) 1889 by Patchen Millies and Josephine.

Rek. 2,011 p. (insufficient pedigree of the I)am).

8. Dan Patch (37323) 1896 by Joe Patchen and Zelica.

Rek. 1,56ł p. 3 George Wilkes (by Hambletonian [10]). Dam : 4 J Jambletonian (10).

1. Hambletonian (10) 1849 by Abdallah I. and Charles Kent Mare.

3 .lesucnger xx, Dan: ?, Grand-dam: 0 .leswenger xx.

2. Electimeer (1.5) 1868 by Hambletonian (10) and Green . Yountain Maid. 3 . Ibdallah 1, Dam: ?

3. Menducino (20607) 1859 by Electioneer and Mano.

3 Hambletonian (10). Dim: 4 Hambletunian (10).

4. Idolita 1896 by Mendocino and Edith.

Rek. 2,091. 2 Hambletonian (10), Diun : 5 . Iambrino (Grand-sire by Jambletonian).

1. Hambletonian (10) 1849 by . Ibdallah I. and Charles Kient Mare.

3 . Hessenger xx, Dam: ?, Grand-dam : 0 . Messenger xx.

$\because$ Harold (413) 1864 by Hambletonian (10) and Enchantress.

(Sire of llaud s. born 1874 . Rek, 2,083) 0 Abdallah I, 2 Bellfoundez xx? Dam: ?

3. I'asco (10996) 1862 by Harold and V'assar.

2 Hambletonian (10) (by Abdallah I). Dam: ?.

1. Baron Wilkes (1758) 188.2 by George IVilles and Belle Patchen.

Rek. 2,18. 5 . Mambrino xx? (Crand-sire by Mambr. Chief), 1);m: 1 Mambr. Chief.

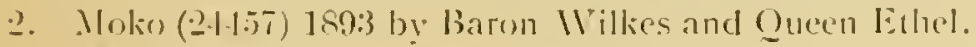

2 Hambletonian (10), Dam: I llambletonian (10).

3. Franko (33991) 1899 by . Moloo and Fraulet.

f Jambletonian (10), 6 Mambrino Chicf, Dam: : L1ambletonian (10), 5 . Iambrim Chicf. 
1. Hambletonian (10) 1849 by Abdallah I. and Charles Kent Mare. 3 Messenger :x, Dam: ?, Grand-dam : 0 Messenger xx.

2. Abdallah (15) 1852 by Hambletonian (10) and Katy Darling. 5 Mambrino xx?, Dam:?

3. Major-Edsall (211) 1859 by Abdallah (15) - Hambletonian (2).

Rek. 2,29. 5 IIessenger xx, Dam: ? (0 Messenger x.s).

4. Robert McGregor (647) 1871 by Major-Edsall (211) and Nancy Whitmann.

Rek. 2,18, 6 Messenger xx, Dam: 6 Messenger xx.

5. Crescens (26217) 1894 by Robert McGregor (647) and Mabel.

Rek, 2,021. 4 Abdallah (15), Dam : 2 Nambrino Chief (11), Grand-sire: (Allie lVest), 1 Mambrino Chief (11).

\section{A few examples of Russian Trotters.}

Polkan 111. 1817 by Lofki I., his best son Duschak 1825. 2 Bars 1 (born 1784), 3 Bars I.

Suriosni 1. 1806 by Lubesni I., his best son Ladin 1821. 0 Bars I.

2 Bars 1

Scharodei 111. 1862 by Polkan, his best son Lofki 1874.

4 Bars (born 1835). 3 Barsil (by Bars). Dam : 3 Bars.

The question of inbreeding in the case of mares is less east to handle than in the case of stallions, because there are so many of them, and secondly, because a dan can only produce a limited number of foals.

I have only found the four following examples for a coarse inbreeding, i.e., incest breeding, with -1 free generation :-

1. Bay Peg born about 1690 by Leedes Arabian and Y. Bald Peg by Leedes Arabian, was the dam of the two celebrated sires, Basto 1702 by Byerly Turk, and Fox 1714 by Clumsey.

2. A mare born about 1690 by Spanker and Old Peg (Spanker's dam), produced five celebrated foals, amongst them Jigg by Bverly Turk, and Cream Cheeks, grandmother of the two Childers (see Family 6).

3. Old Lady born about 1702 by Pulleine's Chesnut Arabian Pulleine's Chesnut Arabian, foundation mare of the Fam. 24 (Gohanna, Camel, The Baron).

4. A mare born about 1730 by Heneage's Jigg and the dam of Heneage's Jigg by Jigg, produced one filly of which there are no further reports, and six colts which became good racehorses, amongst them Hunt's Jigg (page 234), who also was bred with -1 free generation.

Of the many mares with 0 and 1 free generation which have been successful at the stud or have been prominent performers on the racecourse, the following may be mentioned :- 
4. Inbreceling.

Mares with 0 Free Generation.

\begin{tabular}{|c|c|c|c|c|c|}
\hline$\dot{\bar{\xi}}$ & \multicolumn{2}{|c|}{$\begin{array}{c}\text { Nimes of Mares and } \\
\text { their Families }\end{array}$} & liorn & Sire & Dam \\
\hline 1 & $\begin{array}{l}\text { Bess } \\
0 \text { Potsos } \\
\text { ij Ciodul. Mrabian } \\
\text { is Partner }\end{array}$ & Fam. 3 a & IsNoi & 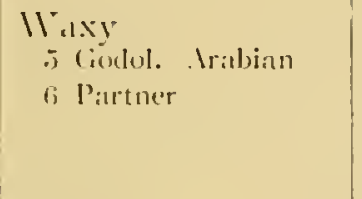 & $\begin{array}{l}\text { Vixen } \\
3 \text { Kegulus (by Godol. } \\
\text { Irabian) } \\
\text { 5 Bart. Childers } \\
6 \text { l'artner }\end{array}$ \\
\hline 2 & $\begin{array}{l}\text { Brown Russet } \\
0 \text { Hiwhtler } \\
0 \text { l'ipillon }\end{array}$ & Fam. 3 & 1802 & $\begin{array}{l}\text { Sir l'eter } \\
3 \text { leeculus } \\
4 \text { Codlul. .rabian } \\
5 \text { Flying Childers } \\
5 \text { liox }\end{array}$ & $\begin{array}{l}\text { Brown Bess } \\
3 \text { Regulus } \\
4 \text { Codol. .rabian } \\
\text { 5 Flying Childers } \\
5 \text { Fox }\end{array}$ \\
\hline 3 & $\begin{array}{l}\text { Butterfly } \\
0 \text { batrot } \\
\text { j Ciule (by Ciodul. } \\
\text { 6 Cindul. Irabian }\end{array}$ & $\begin{array}{l}\text { Fam. } 4 \\
\text { Irabian) }\end{array}$ & 18014 & $\begin{array}{l}\text { Master Bagot } \\
\text { j Codol. Trabian }\end{array}$ & $\begin{array}{l}\text { Mare by Bagot } \\
3 \text { Cade } \\
4 \text { Partner }\end{array}$ \\
\hline 1 & $\begin{array}{l}\text { Chesnut Skin } \\
0 \text { Herod } \\
6 \text { bay Bolion }\end{array}$ & Fam. & 1794 & $\begin{array}{l}\text { 11 oodpecker } \\
\text { (; Darley . Irabian }\end{array}$ & $\begin{array}{l}\text { Mare by I Ierod } \\
4 \text { Fying Childers } \\
5 \text { Sister to Mixbury }\end{array}$ \\
\hline ; & $\begin{array}{l}\text { Goldenlocks } \\
0 \text { Crab }\end{array}$ & Fan. 38 & 17.58 & $\begin{array}{r}\text { Oroonoko } \\
?\end{array}$ & Ware by Crab \\
\hline (; & $\begin{array}{l}\text { Gres Skim } \\
0 \text { llerod }\end{array}$ & Fam. $5 \mathrm{~b}$ & 1793 & $\begin{array}{l}\text { 110odpecker } \\
6 \text { Darley Irabian }\end{array}$ & $\begin{array}{c}\text { Mare by I Ierod } \\
\text { ? }\end{array}$ \\
\hline$\tau$ & $\begin{array}{l}\text { Marlot } \\
0 \text { lleroel } \\
5 \text { Ciodol. Irabian }\end{array}$ & Fam. 45 & 1783 & $\begin{array}{l}\text { IJighflyer } \\
4 \text { Dirley Irabian } \\
\text { + liety 1.eeder }\end{array}$ & $\begin{array}{l}\text { Mare b. Herod } \\
3 \text { Pirtner (Grind-sire } \\
\text { of llerod) }\end{array}$ \\
\hline s & $\begin{array}{l}\text { Juliana } \\
0 \text { Mercury } \\
\text { 2 Herod (by T:arb }\end{array}$ & Fam. j b & 1810 & $\begin{array}{l}\text { Gohanna } \\
\text { 2 Tartar } \\
\text { 5 Mogrul } \\
\text { 6 Circhl. Irabian }\end{array}$ & $\begin{array}{l}\text { Platina } \mathrm{O} . \\
2 \text { Tirtar }\end{array}$ \\
\hline 9 & 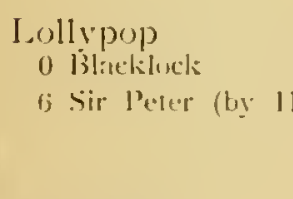 & $\begin{array}{l}\text { lamm. 21 } \\
\text { lighthyer) }\end{array}$ & $18: 36$ & $\begin{array}{l}\text { (Starch) or Voltaire } \\
+ \text { King liermus } \\
\text { 5 llimhflyer }\end{array}$ & $\begin{array}{l}\text { Belinda } \\
\text { 5 ling lergus } \\
\text { 5 Hirhllyer } \\
6 \text { Eclipse } \\
6 \text { Herol }\end{array}$ \\
\hline 11) & 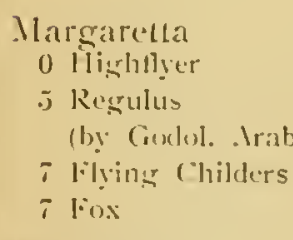 & l’aแm. $2 \mathrm{e}$ & 1802 & 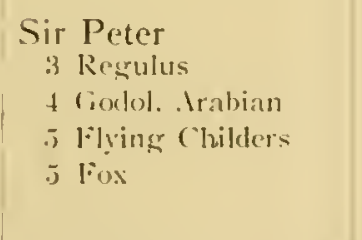 & $\begin{array}{l}\text { Mare by Highfler } \\
4 \text { foclol. Iribian } \\
4 \text { Partuer }\end{array}$ \\
\hline 11 & $\begin{array}{l}\text { Miss Elliot } \\
\text { o Parmer }\end{array}$ & Fаנแ 23 & 1756 & $\begin{array}{c}\text { Grisewond's Partner } \\
\text { ? }\end{array}$ & Cailia \\
\hline
\end{tabular}


Heredity.

\begin{tabular}{|c|c|c|c|c|}
\hline$\dot{0}$ & $\begin{array}{c}\text { Names of Mares and } \\
\text { their Families }\end{array}$ & Born & Sire & Dam \\
\hline 12 & $\begin{array}{ll}\text { Old Lady } & \text { Fam. } 17 \\
0 \text { Bald Galloway } & \end{array}$ & Alout & $\begin{array}{c}\text { Bald Galloway } \\
\text { ? }\end{array}$ & The Wharton Mare \\
\hline 13 & $\begin{array}{l}\text { Mare Fam. } 15 \\
0 \text { Little Hartley Mare }\end{array}$ & $176: 3$ & $\begin{array}{c}\text { Shakspeare } \\
?\end{array}$ & Miss Meredith \\
\hline 14 & $\begin{array}{l}\text { Valentine lam. } 12 \text { a } \\
0 \text {.lare by Phantom } \\
\text { (Dam by Voltaire) } \\
6 \text { Potsos (by Eclipse) } \\
7 \text { Highflyer }\end{array}$ & 1833 & $\begin{array}{l}\text { Voltaire } \\
4 \text { King Fergus } \\
\text { (by Eclipse) } \\
5 \text { Hightlyer }\end{array}$ & $\begin{array}{l}\text { Fisher Lass } \\
+ \text { Sir Peter (by High- } \\
\text { flyer) } \\
4 \text { Y. Giantess } \\
6 \text { Eclipse }\end{array}$ \\
\hline 15 & $\begin{array}{l}\text { Marc } \\
0 \text { Herod } \\
3 \text { Cade }\end{array}$ & 1798 & $\begin{array}{l}\text { 11 oodpeclier } \\
\text { ij Darley . Irabian }\end{array}$ & $\begin{array}{l}\text { Mare by Herod } \\
3 \text { Partner } \\
4\left\{\begin{array}{l}\text { Bartl. Childers } \\
\text { Flying Childers }\end{array}\right.\end{array}$ \\
\hline & Mares & with & 1 Free Generation. & \\
\hline 1 & $\begin{array}{l}\text { Bellissima O. Fam. } 3 \\
1 \text { Herod } \\
5 \text { Regulus } \\
\text { (by Godol. Arabian) } \\
6 \text { Fox }\end{array}$ & 1795 & $\begin{array}{l}\text { Ploenomenon } \\
\text { T Darley Arabian } \\
\text { i Fox }\end{array}$ & $\begin{array}{l}\text { Mren } \\
4 \text { Godol. Arabian }\end{array}$ \\
\hline 2 & $\begin{array}{l}\text { B]ood Red } \\
1 \text { Ellen Horne } \\
5 \text { Touchstone } \\
7 \text { Castrel } \\
7 \text { Selim }\end{array}$ & 1875 & $\begin{array}{l}\text { Lurd Lyon } \\
6 \text { Whalebone } \\
6 \text { Selin? }\end{array}$ & $\begin{array}{l}\text { Rouge Rose } \\
6 \text { Orville } \\
4\left\{\begin{array}{l}\text { Castrel } \\
\text { Selim }\end{array}\right.\end{array}$ \\
\hline 3 & $\begin{array}{l}\text { Charmer } \\
1 \text { Herod } \\
5 \text { Regulus } \\
\text { (by Gudol. .Irabian) }\end{array}$ & 1790 & $\begin{array}{l}\text { Ploenomenon } \\
\text { - Darley Arabian } \\
7 \text { Fox (Grand-sire of } \\
\text { Herod) }\end{array}$ & $\begin{array}{l}\text { Mare by Fitz Herod } \\
3 \text {. Miss Partner } \\
3 \text { Cade (by Godol. } \\
\text {. Irabian) } \\
\text { 4 Partner }\end{array}$ \\
\hline 4 & $\begin{array}{l}\text { Comtesse } \\
1 \text { Enilius } \\
6 \text { Sir Peter (by Highflyer) } \\
6 \text { Delpini }\end{array}$ & 1555 & $\begin{array}{l}\text { (The Baron) or } \\
\text { Nuncio } \\
5 \text { Sir leter } \\
5 \text { Deipini } \\
6 \text { King Fergus } \\
6 \text { Hightiger }\end{array}$ & $\begin{array}{l}\text { Euscbia } \\
\text { j Highflyer } \\
\text { j King Ferous }\end{array}$ \\
\hline 5 & $\begin{array}{l}\text { Mare Fan. } 2 \\
1 \text { Potsos } \\
2 \text { Herod } \\
5 \text { Snap } \\
6 \text { Regulus } \\
\text { (by Godol. Arabian) }\end{array}$ & 1799 & $\begin{array}{l}\text { Coriander } \\
4 \text { Cade (by Godol. } \\
\text {. Irabian) } \\
6 \text { Partner (Grandsire } \\
\text { of Herod) }\end{array}$ & $\begin{array}{l}\text { Wildgose } \\
4 \text { Blank (by Godol. } \\
\text { - Trabian) } \\
5 \text { Godol. Arabian } \\
\text { 5 Little Hartley Matre } \\
5 \text { Regulus }\end{array}$ \\
\hline
\end{tabular}




\begin{tabular}{|c|c|c|c|c|c|}
\hline$\dot{8}$ & $\begin{array}{c}\text { Names of Mares } \\
\text { their Familic }\end{array}$ & $\begin{array}{l}s \text { and } \\
\text { cs }\end{array}$ & Born & Sire & Dam \\
\hline 6 & $\begin{array}{l}\text { Flora } \\
\qquad 1 \text { Godol. Irabian }\end{array}$ & Fam. 31 & 1768 & Lofty & $\begin{array}{c}\text { Riot } \\
?\end{array}$ \\
\hline 7 & $\begin{array}{l}\text { Fortress } \\
1 \text { IIhalebone } \\
4 \text { Highland } \\
5 \text { Buzzard } \\
6 \text { Moodpecler }\end{array}$ & & 1830 & $\begin{array}{l}\text { Defence } \\
5 \text { Hcrod } \\
5 \text { Hishtlyer } \\
\text { 5 Eclipsc }\end{array}$ & $\begin{array}{l}\text { Jewess } \\
5 \text { Mare by llerod } \\
5 \text { Mercury } \\
\text { (by liclipse) } \\
5 \text { lluolpeclier } \\
6 \text { Highllyer } \\
6 \text { Eclipae }\end{array}$ \\
\hline s & $\begin{array}{l}\text { The Jewed } \\
\text { I Birdenteher } \\
\text { J Mluley (by Orvill } \\
8 \text { Gohannal }\end{array}$ & $\begin{array}{l}\text { Fann. } 28 \\
\text { le) }\end{array}$ & $186 t$ & $\begin{array}{l}\text { Stockwell } \\
\text { j Orville } \\
6 \text { Waxy } \\
6 \text { Penelope } \\
\text { (by Trumpator) }\end{array}$ & $\begin{array}{l}\text { Julv } \\
5 \text { IVaxy } \\
6 \text { Potsus } \\
6 \text { Prunella } \\
6 \text { Gohannat } \\
7 \text { Trumpator }\end{array}$ \\
\hline 9 & $\begin{array}{l}\text { Lizzic G. } \\
\text { 1 Reel (by Glencoe } \\
2 \text { Boston }\end{array}$ & $\begin{array}{l}\text { Fam?. } 23 \mathrm{a} \\
\text { e) }\end{array}$ & $\left|\begin{array}{c}1855 \\
\text { in } \\
\text { Anerick }\end{array}\right|$ & $\begin{array}{l}\text { Mar Dance } \\
+ \text { The Baron } \\
\text { i Banter }\end{array}$ & $\begin{array}{l}\text { Mare by Lecompte } \\
2 \text { Giencise }\end{array}$ \\
\hline 10 & $\begin{array}{l}\text { Mandragora } \\
\text { I Birdcatcher } \\
7 \text { Orville }\end{array}$ & Fam. $4 \mathrm{~b}$ & 1860 & $\begin{array}{l}\text { Rataplan } \\
5 \text { Orville } \\
6 \text { Waxy } \\
6 \text { Penelupe }\end{array}$ & $\begin{array}{l}\text { Manganese } \\
7 \text { Sir Peeter } \\
\text { i prumpator }\end{array}$ \\
\hline 11 & $\begin{array}{l}\text { Maria } \\
1 \text { Herod } \\
3 \text { legulus } \\
+ \text { Godol. Irabian } \\
7 \text { Bart. Childers (b) } \\
\text { 1)artey. Irabiau) }\end{array}$ & Fam. 23 & 1791 & $\begin{array}{l}\text { Jighflyer } \\
5 \text { Sister to. Vixbury } \\
6 \text { Darley. Irabian }\end{array}$ & $\begin{array}{l}\text { Maria } \\
+ \text { riodul. Mrabian } \\
4 \text { Starling } \\
5 \text { Crab } \\
6 \text { Flyiner Childers }\end{array}$ \\
\hline 12. & $\begin{array}{l}\text { Milliner } \\
\text { I Birlcatcher } \\
\text { i Orville }\end{array}$ & Fan, $4 b$ & 1869 & $\begin{array}{l}\text { Ralaplan } \\
\text { j Orville } \\
6 \text { Miaxy } \\
6 \text { Penclope }\end{array}$ & $\begin{array}{l}\text { Manganese } \\
\text { i Sir l'eter } \\
\text { 7 Trumpator }\end{array}$ \\
\hline 13 & $\begin{array}{l}\text { Minaret } \\
\text { I Birdcalcher } \\
\text { i Orville }\end{array}$ & Famm, $4 \mathrm{~b}$ & 1866 & $\begin{array}{l}\text { Rataplan } \\
5 \text { Orville } \\
\text { G llaxy } \\
6 \text { Penelupe }\end{array}$ & $\begin{array}{l}\text { Manganese } \\
\text { T Sir Peler } \\
\text { 7 Trumpator }\end{array}$ \\
\hline It & $\begin{array}{l}\text { Mlineral } \\
\text { I lirdcateher } \\
\text { † Orville }\end{array}$ & Fam. 4 & $186: 3$ & $\begin{array}{l}\text { Rataplan } \\
5 \text { Orville } \\
6 \text { II:axy } \\
6 \text { Penclope }\end{array}$ & $\begin{array}{l}\text { 'Ianganese } \\
7 \text { Sir Peter } \\
7 \text { Trumpator }\end{array}$ \\
\hline 15 & $\begin{array}{l}\text { Miss Letty O. } \\
\text { ] Orville } \\
6 \text { IIerod } \\
6 \text { Eclipse }\end{array}$ & Fam. 12 & 1831 & $\begin{array}{l}\text { Priam } \\
2 \text { IIhiskey } \\
5 \text { Herod } \\
5 \text { Eclijse }\end{array}$ & $\begin{array}{l}\text { Mare by Orville } \\
4 \text { Herod } \\
\text { f Eclipse } \\
4 \text { l'roserpine }\end{array}$ \\
\hline
\end{tabular}




\begin{tabular}{|c|c|c|c|c|c|}
\hline$\dot{\gamma}$ & \multicolumn{2}{|c|}{$\begin{array}{c}\text { Names of Mares and } \\
\text { their Pamilies }\end{array}$} & Born & Sire & Dam \\
\hline 16 & $\begin{array}{l}\text { Mlare } \\
\text { I Camel } \\
+ \text { Selin }\end{array}$ & Fam. 3 & 1853 & $\begin{array}{l}\text { Orlando } \\
3 \text { Selim } \\
5 . \text {.lexander }\end{array}$ & $\begin{array}{l}\text { Brown Bess } \\
+ \text { Potsos }\end{array}$ \\
\hline 17 & $\begin{array}{l}\text { Palma } \\
1 \text { Orville } \\
4 \text { Sir Peter (by His } \\
6 \text { Eclipse }\end{array}$ & $\begin{array}{l}\text { Fan. } 12 \\
\text { ighflyer) }\end{array}$ & 1840 & $\begin{array}{l}\text { Emilius } \\
3 \text { Highflyer } \\
+ \text { Eclipse }\end{array}$ & $\begin{array}{l}\text { Francesca } \\
4 \text { Hightlyer } \\
5 \text { Eclipse }\end{array}$ \\
\hline is & $\begin{array}{l}\text { Pazmanita } \\
1 \text { Newminster }\end{array}$ & Fam. $1 \mathrm{a}$ & 1875 & $\begin{array}{l}\text { 1 Hermit } \\
4 \text { Camel }\end{array}$ & $\begin{array}{l}\text { Nyl Gau } \\
2 \text { Touchstone }\end{array}$ \\
\hline 19 & $\begin{array}{l}\text { Mare } \\
1 \text { Herod } \\
5 \text { Squirt } \\
7 \text { Codol. Arabian }\end{array}$ & Fam. 24 & 1785 & $\begin{array}{l}\text { Ploenomenon } \\
7 \text { Darley Arabian } \\
\text { (Grand-sire of } \\
\text { Squirt) } \\
7 \text { Fox }\end{array}$ & $\begin{array}{l}\text { Matron } \\
4 \text { Partner } \\
4 \text { Godol. Arabian }\end{array}$ \\
\hline 30 & $\begin{array}{l}\text { Rachel } \\
\quad 1 \text { Godol. Irabian }\end{array}$ & Fam. 13 & 1763 & $\begin{array}{l}\text { Blank } \\
\text { ? }\end{array}$ & $\begin{array}{l}\text { Mare by Regulus } \\
\text { ? }\end{array}$ \\
\hline 21 & $\begin{array}{l}\text { Rantipole } \\
1 \text { Godol. Mrabian }\end{array}$ & Fam. 32 & 1769 & $\begin{array}{c}\text { Blank } \\
\text { ? }\end{array}$ & $\begin{array}{l}\text { Joan } \\
?\end{array}$ \\
\hline 202 & $\begin{array}{l}\text { Red Flag } \\
\text { I Ellen Horne } \\
5 \text { Touchstone } \\
7 \text { Castrel } \\
7 \text { Selin }\end{array}$ & Fam. I c & 1871 & $\begin{array}{l}\text { Lord Lyon } \\
6 \text { Whalebone } \\
6 \text { Selim } \\
7 \text { Orville }\end{array}$ & $\begin{array}{l}\text { Rouge Rose } \\
6 \text { Orville } \\
4 \text { Castrel } \\
\text { Selim }\end{array}$ \\
\hline 23 & $\begin{array}{l}\text { Red Rag } \\
1 \text { Ellen Horne } \\
5 \text { Touchstone } \\
\text { f Castrel } \\
7 \text { Selim }\end{array}$ & lant. I c & 1870 & $\begin{array}{l}\text { Lord Lyon } \\
6 \text { Whalebone } \\
6 \text { Selim }\end{array}$ & $\begin{array}{l}\text { Rouge Rose } \\
6 \text { Orville } \\
{ }_{4}\left\{\begin{array}{l}\text { Castrel } \\
\text { Selim }\end{array}\right.\end{array}$ \\
\hline 21 & $\begin{array}{l}\text { Ruth } \\
\text { l Godol. Irabian }\end{array}$ & Fam. 13 & 1761 & $\begin{array}{l}\text { Blank } \\
\text { ? }\end{array}$ & $\begin{array}{c}\text { Mare by Regulus } \\
\text { ? }\end{array}$ \\
\hline 25 & $\begin{array}{l}\text { Rutilia } \\
1 \text { Godolphin Arabia }\end{array}$ & Fan. 13 & 1769 & $\begin{array}{c}\text { Blank } \\
?\end{array}$ & $\begin{array}{l}\text { Mare by Regulus } \\
\text { ? }\end{array}$ \\
\hline 26 & $\begin{array}{l}\text { Sister to Old } \\
\text { Country Wench } \\
1 \text { Hautboy }\end{array}$ & Fam. 11 & $\begin{array}{l}\text { A bout } \\
1717\end{array}$ & $\underset{?}{\text { Snake }}$ & $\begin{array}{l}\text { Grey Wilkes } \\
\text { ? }\end{array}$ \\
\hline 27 & $\begin{array}{l}\text { Mare } \\
1 \text { Potsos } \\
4 \text { Herod }\end{array}$ & Fam. 11 & 1822 & $\begin{array}{l}\text { Whisker } \\
3 \text { Herod } \\
+ \text { Snap } \\
6 \text { Cade }\end{array}$ & $\begin{array}{l}\text { Mandane } \\
4 \text { Cade } \\
6 \text { Godol. Arabian }\end{array}$ \\
\hline 28 & $\begin{array}{l}\text { Mowstii } \\
1 \text { Herod } \\
3 \text { Snap } \\
5 \text { rodol. Irabian }\end{array}$ & Fam. 18 & 1777 & $\begin{array}{l}\text { Mentor } \\
5 \text { rodol. Arabian }\end{array}$ & $\begin{array}{l}\text { Maria } \\
3 \text { Godol. Arabian }\end{array}$ \\
\hline
\end{tabular}


For the purpose of judging of the more removed inbreedings of approved brood mares, I have arranged 60 of the best according to the deegree of their inbreeding, so as to avoid any confusion by giving ton many examples. The choosing of these 60 mares is not only a difficult task, but one about which one may have very different opinions. I have only gone into the determining of their inbreedings after having selected the mares. The result was as follows :-

\begin{tabular}{|c|c|c|c|c|c|}
\hline it & $\underline{\mathrm{f}}$ & ro & Ch. & 90 & ares \\
\hline ," & 3 & ,. & ., & 15 & , , \\
\hline , & 4 & , & , & 16 & ," \\
\hline ", & 5 & , & . & 15 & ., \\
\hline ," & 6 & ". & . & 6 & , \\
\hline
\end{tabular}

As a great number of the best brood mares are already given in the tables of stallions, there will only be given the 36 best of the chosen 60 broud mares in the following tables of mares. According to the degree of their inbreeding they are arranged nearly the same as above, viz. :-

With 2 free generations 6 mares.

$$
\begin{aligned}
& \text {, } 3 \text {,. ., } 9 \text {.. } \\
& \text {, } 4 \text {, , , } 11 \text {, }
\end{aligned}
$$

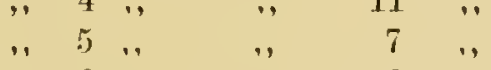

$$
\begin{aligned}
& \text { " } 6 \text {, ". } 3 \text {., } \\
& \text { Total } 36 \text { mares. }
\end{aligned}
$$

\begin{tabular}{|c|c|c|c|c|}
\hline$\dot{z}$ & $\begin{array}{l}\text { Names of Mares and } \\
\text { their Families }\end{array}$ & Born & Sire & $D_{i 1111}$ \\
\hline 1 & $\begin{array}{l}\text { Annette } \\
2 \text { Orville } \\
5 \text { Sir Peter (by Hightlyer) } \\
5 \text { King lergun (by Eelipse) } \\
6 \text { Eelipse }\end{array}$ & 1835 & $\begin{array}{l}\text { Priam } \\
211 \text { hisliey } \\
5 \text { Eclipse } \\
\text { 5 Ilerod }\end{array}$ & 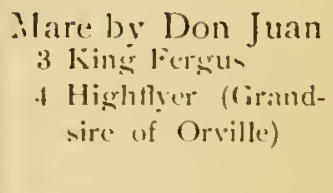 \\
\hline 2 & $\begin{array}{l}\text { Mare } \quad \text { Fam. } 3 \\
2 \text { lloudpecker (by Herod) } \\
2 \text { Mercury (by Eelipse) }\end{array}$ & 1812 & $\begin{array}{l}\text { Cancpus } \\
2 \text { llerod } \\
5 \text { Ciade (by Godel. } \\
\text { Irabian) }\end{array}$ & 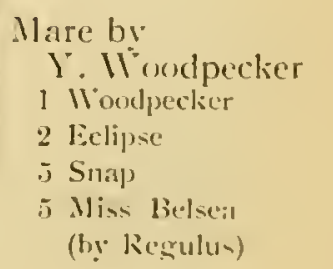 \\
\hline
\end{tabular}

Mares with : Free Generations. 


\begin{tabular}{|c|c|c|c|c|}
\hline$\ddot{z}$ & $\begin{array}{c}\text { Names of Mares and } \\
\text { their Fanilies }\end{array}$ & Born & Sire & Dam \\
\hline 3 & $\begin{array}{l}\text { Decoy Fam. } 3 \\
2 \text { Sir Peter (by Highflyer) } \\
5 \text { Eclipse }\end{array}$ & 1830 & $\begin{array}{l}\text { Filho da Puta } \\
3 \text { Eclipse } \\
4 \text { Herod } \\
5 \text { Snap }\end{array}$ & $\begin{array}{l}\text { Finesse } \\
2 \text { Highflyer } \\
4 \text { Eclipse } \\
5 \text { Snap }\end{array}$ \\
\hline 1 & $\begin{array}{l}\text { Filagree } \\
2 \text { Trumpator } \\
4 \text { Highflyer } \\
5 \text { Herod } \\
6 \text { Matchem (by Cade) }\end{array}$ & 1815 & $\begin{array}{l}\text { Soothsayer } \\
5 \text { Herod } \\
6 \text { Snap }\end{array}$ & $\begin{array}{l}\text { Web } \\
3 \text { Herod } \\
4 \text { Snap } \\
6 \text { Cade }\end{array}$ \\
\hline .j & $\begin{array}{l}\text { Prunella Fam. } 1 \\
2 \text { Blanls (Grand-son of } \\
\text { Bartl. Childers) } \\
4 \text { Partner } \\
5 \text { fox (Grand-son of Haut- } \\
\text { boy) } \\
5 \text { Flying Childers }\end{array}$ & 1788 & $\begin{array}{l}\text { Ilighflyer } \\
5 \text { Sister to Mixbury } \\
\text { (Dam of Partner) } \\
6 \text { Darley Arabian } \\
6 \text { Betty Leedes }\end{array}$ & 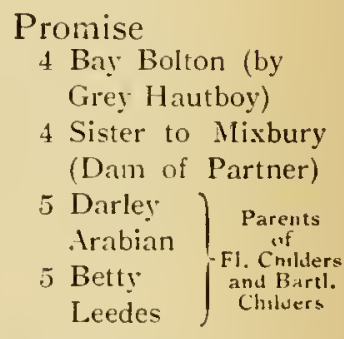 \\
\hline 6 & $\begin{array}{l}\text { V'iolet Fam. } 15 \\
2 \text { Squirt } \\
\text { (by- Bartl. Childers) } \\
6 \text { Bay Bolton }\end{array}$ & 1787 & $\begin{array}{l}\text { Shark } \\
5 \text { 1)arley Arabian } \\
5 \text { Betty Leedes } \\
5 \text { Bay Bolton }\end{array}$ & $\begin{array}{l}\text { Mare by Syphon } \\
3 \text { Barti. Childers } \\
\text { (by Darley . Irabian) } \\
3 \text { Crab }\end{array}$ \\
\hline
\end{tabular}

Mares with 3 Free Generations.

1 Mlice Hawthorn (iсp. D)p. $2 \times$.

3 Dick Indrews

+ Beningbrough

4 Evelina (by Highflyer)

6 Eclipse

2 ircot Lass

3 Volunteer (by Eclipse)

¡Herod

3 Arethusa

3 Kegulus

(by Godol. Irabian)

5 Partner
Fan, 7

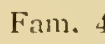

Fam.

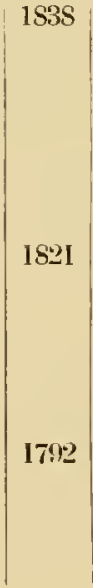

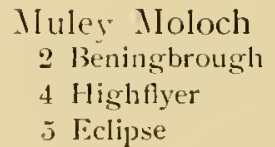

Ardrossan

2 Eclipse

3 Herod

5 Snap

Dungannon

4 Godol. Irabian

5 Bartl. Childers
Rebecca

4 Eclipse

5 Herod

5 Highflyer

Mare by

Cramlington

4 Highflyer

4 Eclipse

5 Herod

Mare by Prophet

1 Regulus 


\begin{tabular}{|c|c|c|c|c|}
\hline$\dot{8}$ & $\begin{array}{l}\text { Names of Mares and } \\
\text { their limilies }\end{array}$ & Born & Sire & Паแม \\
\hline 4 & $\begin{array}{l}\text { Cobweb 1. O. Fan. } 1 \\
3 \text { L. Giantes (by 1)iomed) } \\
5 \text { lliwhllyce } \\
6 \text { liclipsc (by Marlie) } \\
6 \text { Herud }\end{array}$ & $18: 1$ & $\begin{array}{l}\text { Phantom } \\
+4 \text { lirago } \\
4 \text { Herod } \\
4 \text { liclipse } \\
\text { i) Snap }\end{array}$ & $\begin{array}{l}\text { Filagree } \\
2 \text { Prumpator } \\
4 \text { lligrnfyer } \\
5 \text { Herod } \\
6 \text { Marslie }\end{array}$ \\
\hline 5 & $\begin{array}{l}\text { Mowerina Fam. } \\
3 \text { Tumchone } \\
5 \text { Muley (by Orville) } \\
6 \text { Sultin (by Selim) }\end{array}$ & 1576 & $\begin{array}{l}\text { Scollish Chief } \\
\text {; Orville } \\
\text { is Selim } \\
\text { i Buzzard }\end{array}$ & $\begin{array}{l}\text { Slockings } \\
5 \text { Whisker } \\
6 \text { Whalebone (Grand- } \\
\text { sire of Touchstone) }\end{array}$ \\
\hline 6) & $\begin{array}{l}\text { Penelope Fam. } 1 \\
3 \text { Snap (by Snip) } \\
6 \text { Codol. Irabian } \\
6 \text { Partner } \\
6 \text { South 's dimm }\end{array}$ & 1798 & $\begin{array}{l}\text { Trumpator } \\
4 \text { liodul. Arabian } \\
4 \text { Partner } \\
\text {; Brown Farewell } \\
\text { (by Malseless, } \\
\text { lirand-sire of } \\
\text { South's dam) }\end{array}$ & $\begin{array}{l}\text { Prunella } \\
2 \text { lilank (by Godol. } \\
\text { Arabian) } \\
4 \text { Partner } \\
5 \text { Flying (hilders } \\
\text { (Sire of Snip) } \\
5 \text { Fox }\end{array}$ \\
\hline$i$ & 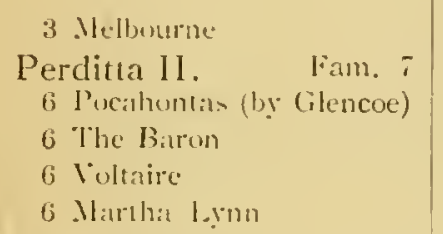 & ISSI & $\begin{array}{l}\text { Hampton } \\
\text { i Whalebone }\end{array}$ & $\begin{array}{l}\text { Hermione } \\
4 \text { Pantaloon } \\
5 \text { Glencoe (by Sultan) } \\
6 \text { Sultan }\end{array}$ \\
\hline 8 & 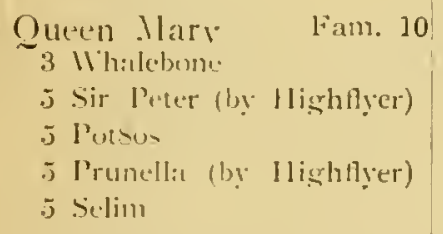 & 1813 & $\begin{array}{l}\text { Cladiator } \\
\text { \& I'runella } \\
\text { \& lutsos }\end{array}$ & $\begin{array}{l}\text { Mare by } \\
\text { Plenipotentiary } \\
\text { 5 Sir Petes } \\
\text { 6 IIighforer }\end{array}$ \\
\hline 9 & 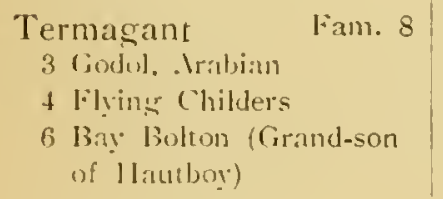 & 1772 & $\begin{array}{l}\text { Tantrum } \\
\text { 3 Flyins childers }\end{array}$ & $\begin{array}{l}\text { Canlatrice } \\
\text { o Grey Willes } \\
\text { (by Hatutboy) } \\
\text { (5 Snake (Cirand-son } \\
\text { of Hauthoy) }\end{array}$ \\
\hline
\end{tabular}

Mares with 4 Free (ienerations.

1 Banter

4 Eclipse

$6\left\{\begin{array}{l}\text { Old lingland } \\ \text { Blank }\end{array}\right.$

6 Matchem (by Cade)
Fan. 14 18:0| Master Henry

3 Ilighflier

4 Eiclipse

-j Ilerod

(j) Blank (by Gorkol. Irabian)
Boadicea

j Godol. Irabian

6 Bartl. Chilkers

(Crand-sire of Old

lingland and lilank) 
Heredity.

\begin{tabular}{|c|c|c|c|c|}
\hline$\because$ & $\begin{array}{c}\text { Names of Mares and } \\
\text { their Families }\end{array}$ & Born & Sire & Dam \\
\hline 2 & $\begin{array}{c}\text { Cinizelli Fam. } 2 \\
4 \text {.lexander (by Eclipse) } \\
5 \text { Nuzard (by Woodpeclser) } \\
5 \text { Nare by - Vlexander } \\
6 \text {. Maria (by Herod) } \\
6 \text { Sir Peter }\end{array}$ & 1812 & $\begin{array}{l}\text { Touchstone } \\
4 \text {. Mexander } \\
5 \text { Buzzard } \\
5 \text { Eclipse } \\
5 \text { Sir Peter } \\
6 \text { Highflyer }\end{array}$ & $\begin{array}{l}\text { B rocade } \\
3 \text { Alexander } \\
4 \text { Sir Peter } \\
5 \text { 110odpeclier } \\
6 \text { Herod }\end{array}$ \\
\hline 3 & $\begin{array}{cc}\text { Electress } & \text { Fam. } 2 \\
4 \text { Eclipse } & \\
5 \text { Herod } & \\
5 \text { Natchen (by Cade) } \\
6 \text { Cade (by Godol. Arabian) }\end{array}$ & 1819 & $\begin{array}{l}\text { Eilection } \\
2 \text { Herod } \\
5 \text { Cade }\end{array}$ & $\begin{array}{l}\text { Mare by Stamford } \\
4 \text { Snap } \\
5 \text { Blank (by Godol. } \\
\text { Arabian) } \\
6 \text { Godol. Mrabian }\end{array}$ \\
\hline 4 & $\begin{array}{l}\text { Emma } \\
4 \text { Eclipse } \\
4 \text { Brunette } \\
5 \text { Herod } \\
5 \text { Conductor (by Matchem) } \\
5 \text { Hightlyer (by Merod) } \\
6 \text { Matchem }\end{array}$ & 1824 & $\begin{array}{l}\text { Whislier } \\
3 \text { Herod } \\
4 \text { Snap } \\
6 \text { Cade (by Matchem) }\end{array}$ & $\begin{array}{l}\text { Gibside Fairy } \\
5 \text { Herod } \\
6 \text { Cade }\end{array}$ \\
\hline 5 & $\begin{array}{ll}\text { Mandane } & \text { Fam. } 11 \\
4 \text { Cade (by Godol. Mrabian) } \\
6 \text { Grey Robinson (by Bald } \\
\text { Gilloway) }\end{array}$ & 18110 & $\begin{array}{l}\text { Potsos } \\
+ \text { Godol. Irabian } \\
\text { () Bahl Galloway }\end{array}$ & $\begin{array}{l}\text { Y. Camilla } \\
4 \text { Godol. .Irabian } \\
6 \text { Bald Cialloway }\end{array}$ \\
\hline 6 & $\begin{array}{c}\text { Martha Lynn Fam. } 2 \\
4 \text { Sir Peter (by Highflyer) } \\
6 \text { king Fergus (by Eclipse) } \\
6 \text { Wondpecker (by Herod) }\end{array}$ & 1837 & $\begin{array}{l}\text { Mulatto } \\
5 \text { Hiwhlyer } \\
5 \text { Flurizel (by Herod) } \\
6 \text { Eclipse } \\
6 \text { Herod }\end{array}$ & $\begin{array}{l}\text { Leda } \\
5 \text { Hiyhtyer } \\
5 \text { Eclipse } \\
5 \text { Jolsos }\end{array}$ \\
\hline 7 & $\begin{array}{l}\text { Papillon } \\
4 \text { Bay Bolton } \\
5 \text { Darley Mrabian } \\
5 \text { Betty Lecdes }\end{array}$ & $176 !$ & $\begin{array}{l}\text { Snap } \\
3 \text { Bay Peg (by Leedes } \\
\text { Arabian, Grand-sire } \\
\text { of Betty Leedes) } \\
5 \text { Byerly Turk }\end{array}$ & $\begin{array}{l}\text { Miss Cleveland } \\
6 \text { Hautboy (Grand- } \\
\text { sire of Bay Bolton) }\end{array}$ \\
\hline 8 & $\begin{array}{l}\text { Mare } \\
4 \text { Eclipse } \\
4 \text { Herod } \\
5 \text { Curiosity (bỹ Snap) } \\
6 \text { Snap }\end{array}$ & 1819 & $\begin{array}{l}\text { Rubens } \\
3 \text { Herod } \\
5 \text { Matchem } \\
6 \text { Cade } \\
6 \text { Snap }\end{array}$ & $\begin{array}{l}\text { Tippitywichet } \\
3 \text { Herud } \\
5 \text { Squirt (Grand-sire } \\
\text { of Eclipse) } \\
5 \text { Snap) } \\
6 \text { Cade }\end{array}$ \\
\hline 9 & $\begin{array}{l}\text { Thistle Fam. } 4 \\
4 \text { Bay Middleton } \\
\text { (by Sultan) } \\
5 \text { Nuley (by Orville) }\end{array}$ & 187.5 & $\begin{array}{l}\text { Scottish Chief } \\
5 \text { Orville } \\
6 \text { Selim (Sire of } \\
\text { Sultan) }\end{array}$ & $\begin{array}{l}\text { Thr Flower Safety } \\
6 \text { Orville }\end{array}$ \\
\hline
\end{tabular}




\begin{tabular}{|c|c|c|c|c|c|}
\hline$\dot{\bar{z}}$ & \multicolumn{2}{|c|}{$\begin{array}{l}\text { Nimes of Mares and } \\
\text { their tomilies }\end{array}$} & liorn & Sire & Dam \\
\hline 10 & $\begin{array}{l}\text { Vermeille } \\
4 \text { llhalebone } \\
5 \text { Orville }\end{array}$ & Fam. 3 & $18 \dddot{3} 3$ & $\begin{array}{l}\text { The baron } \\
\text { \& Waxy Parents } \\
\text { \& Penclepe o whalebone }\end{array}$ & $\begin{array}{l}\text { Fair IJelen } \\
5 \text { llishtlyer (Grand- } \\
\text { sire of Orville) } \\
\text { 6 liclipse }\end{array}$ \\
\hline 11 & $\begin{array}{l}\text { Vista } \\
+ \text { banter } \\
4 \text { Pintalon }\end{array}$ & Fam. 4 & 1579 & $\begin{array}{l}\text { Macaromi } \\
6 \text { Sir Pene (Great } \\
\text { firand-sire of } \\
\text { l'antuloon) } \\
6 \text { Orville (Grand-sire } \\
\text { of Banter) }\end{array}$ & $\begin{array}{l}\text { Verdure } \\
\text { j Mluley (by Orville) }\end{array}$ \\
\hline
\end{tabular}

Mares with is liree Cienerations.

1 . Iraucaria

5 Orville

5 Selim

2. Barbelle

j Eclipse

5 Hishtlyer

6 Herod

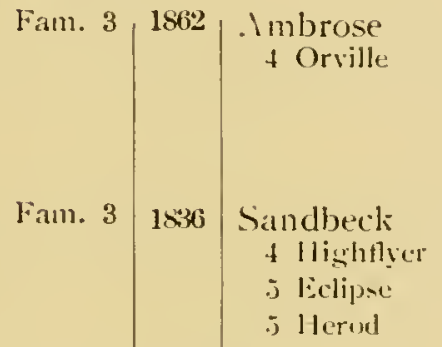

3 Morganette

j Pantialoun

6 Turchstone

6 Sultan (by Selim)

1 Paradigm
5 Selim
5 Waxy
6 Orville

5 Pocahontas Fam. 3

5 tiolsonna (hy Mercury)

6 Mercury (by Eclipse)

- Ilighnyer (by Herod)

is Sweet liatie

j Tramp

6 Orville

6 IIhisker liam. jb

$1,4(1)$
1 sst Springticld

is Sultan

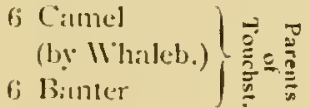

fo Binter

$185:$

Paragone

5 Orville

(;) Sir Jeter

(bỵ Hiwhtlyer)

1837

Gilencose

j Mercury

6) Eclipace

6 Hightlyer

(i) Ilerud

Suckewell

jorville.

(j) II:ixy | Paremts

li l'enclope i whisker
Pocahontas

5 Gohannat

6 Nercury

T High nyer (Grandsire of Orville)

Darioletta

3 Eclipse

4 Grecian P'rincess

5 Highhyer

5 Herod

Lady Morgan $5\{$ Selim

5 (Castrel

7 Whalebence

i Orville

\section{Ellen Horne}

4 Selim

5 Beningbrough
5 Evelinal

6 llightlyor (Frand. sire of Orville)

Marpessia

2 Whiskes

4 )ioned

6 lisclipse

6 llerud

Gathleen

6 Gohatunat (Grandsire of Tramp)

6 Potsos 


\begin{tabular}{|c|c|c|c|c|c|}
\hline$\dot{0}$ & $\begin{array}{l}\text { Names of Mares } \\
\text { their Familie }\end{array}$ & and & Born & Sire & Dam \\
\hline 7 & $\begin{array}{l}\text { Y. Giantess } \\
\dot{5} \text { Godol. Irabian } \\
5 \text { Partner }\end{array}$ & Fam. & 1790 & $\begin{array}{l}\text { Diomed } \\
+ \text { Crab } \\
+ \text { Godol. Irabian } \\
+ \text { Partner } \\
\text { \& Flying Childers }\end{array}$ & $\begin{array}{l}\text { Giantess } \\
2 \text { Godol. Arabian } \\
3 \text { Partner } \\
5 \text { Bald Galloway }\end{array}$ \\
\hline
\end{tabular}

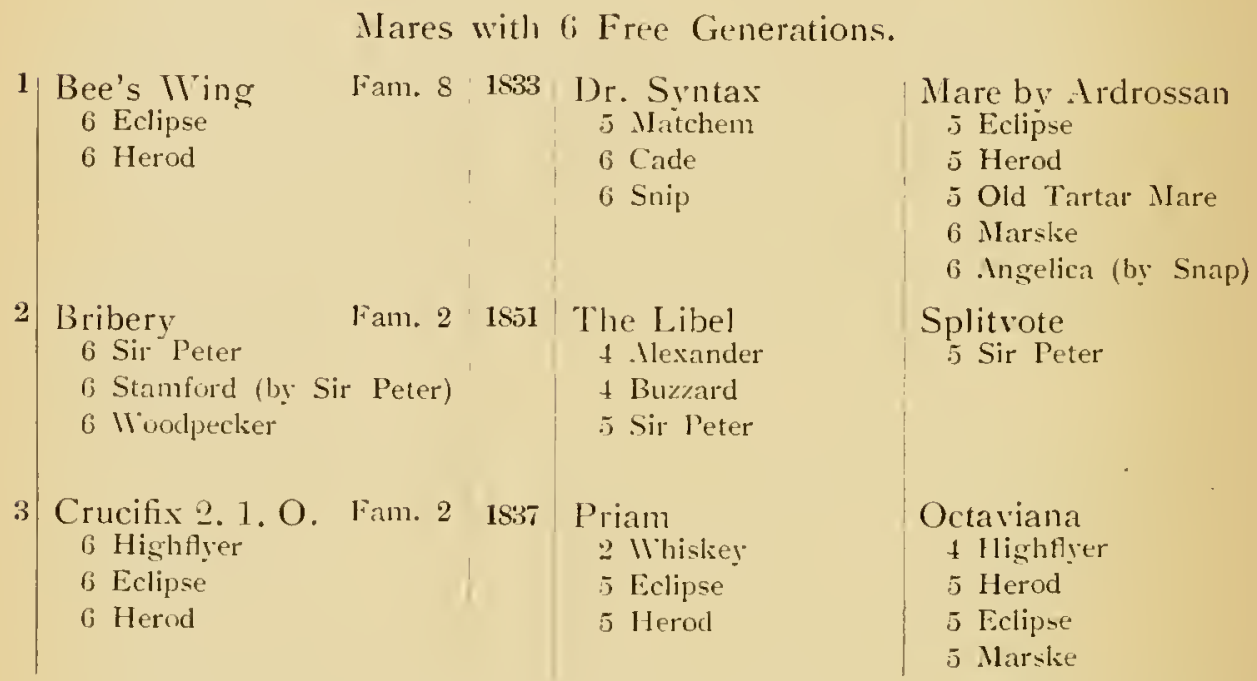

It can be seen from these examples that the same rules as regards inbreeding, and especially inbreeding supports, apply to the most approved mares just as well as to the stallions. Among the approved brood mares with more than 6 free generations I only know, unless I have made a mistake, the following nine with 7 free generations:

1. Jamaica (dam of Foxhall) - 7 Orville.

2. Manganese (dam of The Miner) 7 Sir Peter and 7 Trumpator.

3. Ilint Sauce (dam of Minting and The Lambkin) 7 Castrel and 7 Comus.

4. Miss Inn (dam of Scottish (hief) 7 Mercury and 7 Wnodpecker.

5. Mloorhen (dam of Gallinule) 7 Selim.

6. Silverhair (dam of Silvio) 7 Potsos and 7 Orville.

7. Nlare by Tranby (dam of Vandal) 7 Highflyer.

8. Suicide (dam of Imphion) 7 Whalebone.

9. Windermere (dam of Muncaster and Kendal) 7 Orville.

This number is, in comparison with the above-mentioned twelve approved stallions with 7 free generations, a very low one. With more than 7 free 
generations I only lnow Hỵenia, dam of Virgil, and Ierolite, dam of Spendthrift. Perhaps the further remowed inbreedings have heen less surressful with mares than with sires. The rery close inbrecdings, consequently, seem to be less harmful with brood mares than with sires. I do not know of one single important sire with o free generation. On page 2:30 are named the four stallions with 0 free generation of which I linum. These four sires have not played any important part in Thoroughbred breeding. Amongst mares with 0 free generation, however, besides several good foundation mares, we lind a few with exrellent breeding performances, amongst them the following:-

1. Butterfly, dam of Vabockisislı.

$\therefore$ Chesnut Slim, dam of Election D).

3. Juliana, dam of Matilda 1. .

1. Lollypop, dam of Sweetmeat.

5. Margaretta, dam of Waverley.

6. Niss Elliot, dam of Grincrack.

7. Valentine, dam of War Eagle Dcp. and 2nd in the Derb!.

8. Manourre, dam of Lioness Cs.

9. Nare 1763 by Shakespeare, dam of Sweetbriar and Mentor.

As the number of brood mares used for breeding is much larger than that of stallions, the absolute figures alone, of course, cannot be taken as equal measures of comparison. Conspicuous, howerer, is the fact that the number of sires with 1 free generation which hase done well in Thoroughbred breeding is, as abowe shown, only very small (really only Paulowitz, Barcaldine. and perhaps Flying Fox), whilst several prominent stallions have been born of mares with onl. 1 free generation, amongst which are the following :1 .Idventurer. 2. Ipology, 1. O. I... 3 Blacklock, 4 Cardinal York, 5 Drome, (i Foxhunter, 7 General Peel, 20 Dep., s Goldfinder, 9 Grey Robinson. 10 Hannibal D., 11 Highflyer, 1.2 Kisber D. GP., 13 Knnwsleg,

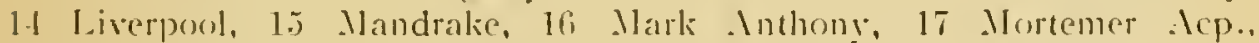
18 Padischah S.O., 19 Phaeton, 20 Przedwit OD., 21 Pyrchus I. D.. .2. Ruler L.. 23 Schwindler L. N1).. 24 Smolenski 2. D., .5 Squirt,

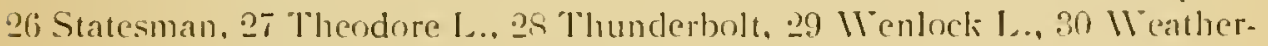
bit, 31 F1. Childers, 32 Bart. Childers.

In order w judge the inbreeding question with Half-breds, I have mentioned in the following list fil Half-bred stallions which were born in Trakehnen, with their own and their parents" inbreeding. I have only left sut a few stallions, as they had pratically no remarkable infuence in the Trakehnen breedings. Lifortunately, I have also had to leave out the following a stallions because their basis of inbreeding could not be determined, partly on account of their insuliirient pedigree: Edeling, E:lfenbein,

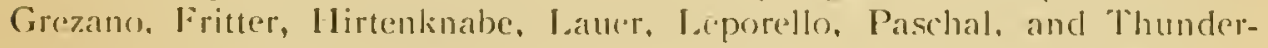
clap. The remaining 61 Half-bred stallions are divided as follows: 


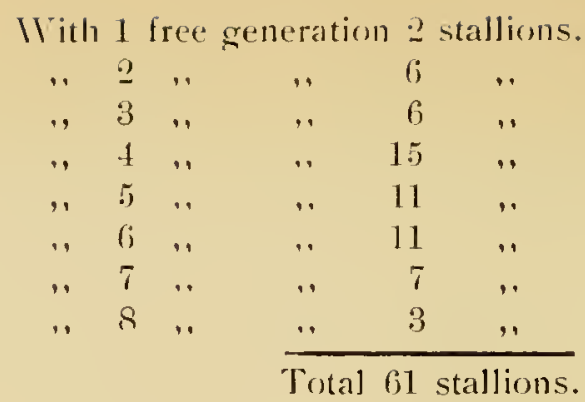

Unfortunately, in the "Trakchnen Stud Bonk we find many failures with still closer inbreeding than 1 free generation. A weakly constitution, light bones, tenderness, and finally sterility, have been also in the breeding of Trakehnen Half-breds the consequences of 100 close inbreedings. $11 \mathrm{so}$ in the Thoroughbred breeding we find the most robust and the strongest individuals among those with more remored inbreedings. Imong the close inbreedings we find many light boned and small horses. ITith the natural breeds, as, for example, the breeds of the Steppes, endowed with a more robust constitution, a close inbreeding with 1 or still less free generations may be carried on longer without bad consequences than with the modern improved breeds. Fien whin the modern improved breeds themselves, the more hardened anongst them, as for example, the Thoroughbred, seem, as above shown, to stand a closer inhreeding better than most IIalf-breds, especially the pampered ones.

Although the dangers of carrying inbreeding tou far have been finown for a long time, yet we find breeders (also in Half-bred breeding) who ignore the experiences of their predecessors, till they themselves, but generally too late, find out from their own breeding that their great, imagined cleverness has cost them very dear owing to the mistakes they have made. The evil experiences which Trakehnen suffered in the first half of the last century on account of exaggerated inbreeding should be a lesson to all who care to learn from the experience of others and who do not wish only to appear themselves clever. In the first volume of the 'lrakehnen Stud Book we find many' examples showing distinctly the deterioration and final destruction of prominent breeding material caused by exaggerated inbreeding. Thunderclap, born 1840 by .likle Fell and Toise, was perhaps the best Half-bred stallion ever bred in Trakehnen. He was used for 21 years as Royal Stud stallion, but by an exaggerated inbreeding much less has remained of him than one might have expected after 21 years' use. Thunderclap should perhaps have been the first sire of Half-breds of whom one might have been able to say that every Half-bred in East Prussia has his blood in him, even if not quite to the same extent as it has that of Eclipse. The list below gives a summary of the results of exaggerated inbreeding in Trakehnen. 
In the first volume of the Trakelhnen Stud Book, unless 1 have made a mistake in counting, there are is cases mentioned in which the stallion has covered his daughter, i.c., the breeding has been tried with --1 free generation. In the following list only the stallions are given who have covered their daughters.

\section{Simes of Stallions

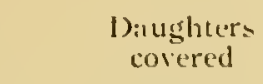

Thunderclap . . . . 24 times.

Oronocco 1. . . . . 14 ..

Caril . . . . . 6 .

Scrapall xх . . . . 4 .

Snydersxis . . . . . 1 ,

Meteor $x$. . . . . . 1 .

Culblanc 1. . . . . 1 .

Ormond . . . . . 1 ..

Oromedon . . . . 2 ..

Total it times ++

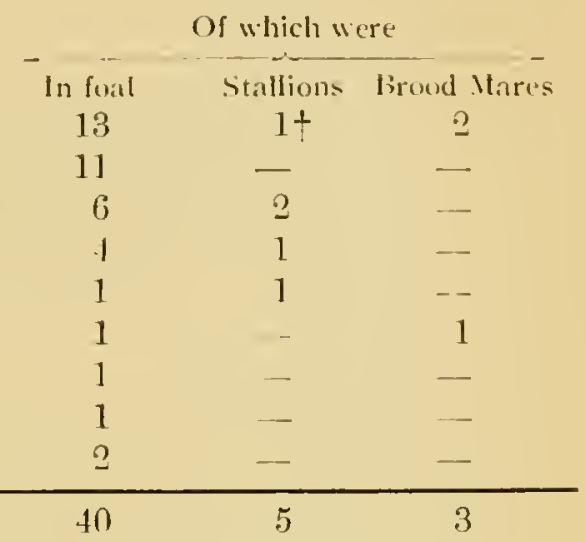

Of which were 
mares came to Gradiz: Mary 1815 by Oronocco II., and Prima 1850 by Oromedon, and died there without progeny. Two mares came to Neustadt : Aurata 18.54 by Ibarra, and Campelli 18.57 by Ibarra. The first died there without progeny, and the latter died finally in Beberbeck, with her granddaughter Clara, without any special performances.

Stallions with 1 Free Generation.

\begin{tabular}{|c|c|c|c|c|}
\hline Xo. & Nimes of Stallions & Born & Sire & $\mathrm{Dam}$ \\
\hline 1 & $\begin{array}{l}\text { Hipparch } \\
1 \text { Blackamoor } \mathrm{xx} \\
1 \text { Cyane } \\
\text { (by Oronocco I.) }\end{array}$ & $18+2$ & $\begin{array}{c}\text { Transparent } \\
?\end{array}$ & $\begin{array}{l}\text { Mercedone } \\
3 \text { Oronocco } 1 .\end{array}$ \\
\hline$\stackrel{2}{2}$ & $\begin{array}{l}\text { Leo } \\
\qquad 1 \text { Reprobate }\end{array}$ & 1867 & $\begin{array}{l}\text { Duplicat } \\
3 \text { Waterman xx } \\
5 \text { Rodrich }\end{array}$ & Lewa \\
\hline
\end{tabular}

Stallions with 2 Free Generations.

1 Ackermann

2. Aumbos

$=\quad$ Dorimont
2 Blackamoor x.

4 Oronocco I.

$: \quad$ Eurvdamus

2 Oronocco 1 .

+ Heniochus

2 Sorcerer $\mathrm{xx}$ (by Trumpator xx)

5 Journey

2 Leporello

+ Blaclsamoor xx

+ Pretender

(j) Molorchus

2 Sorcerer $x$

\begin{tabular}{|c|c|}
\hline 1899 & $\begin{array}{c}\text { Hirtenknabe } \\
?\end{array}$ \\
\hline 1818 & $\begin{array}{l}\text { Eurvdamus } \\
2 \text { Oronocco } 1 .\end{array}$ \\
\hline 1833 & Practor ? \\
\hline 1831 & $\begin{array}{l}\text { J. Whalebone } \mathrm{xx} \\
2 \text { Trumpator } \mathrm{xx}\end{array}$ \\
\hline $186^{\circ}$ & $\begin{array}{l}\text { Promoter } \\
+ \text { Oronocco 1. } \\
\text { (Grand-sire of } \\
\text { Leporello) }\end{array}$ \\
\hline 1836 & $\begin{array}{l}\text { The Cryer xx } \\
5 \text { Matchem xx } \\
\text { (Great Grand-sire of } \\
\text { Sorcerer xx) } \\
5 \text { Herod }\end{array}$ \\
\hline
\end{tabular}

Iccurate

5 Eurydanus (Great Grand-sire of Ambos)

Dairymaid

4 Sorcerer $\mathrm{x}$. (Grand-sire of Blacliamoor $\mathrm{xx}$ )

4 Oronocco 1.

Gabrielle

Pupille

Jessonda

4 Oronocco 1.

5 Sorcerer xx

(Grand-sire of

Blacliamoor $\mathrm{xx}$ )

Egisthe

3 Sorcerer $\mathrm{xx}$ 
Stallions with : Free Generations.

\begin{tabular}{|c|c|c|c|c|}
\hline$\therefore$. & Sames of Stallions & Born & Sire & Dam \\
\hline I & $\begin{array}{l}\text { Ipis } \\
3 \text { Stockwell } x x \\
6 \text { The Cryer xx }\end{array}$ & 1881 & $\begin{array}{l}\text { Paladin } \\
6 \text { Touchstone } \mathrm{x}\end{array}$ & $\begin{array}{l}\text { Ipanage } \\
7\left\{\begin{array}{l}\text { Whalebone } x x \\
\text { Whisker } x\end{array}\right.\end{array}$ \\
\hline 2 & $\begin{array}{l}\text { Djalma } \\
3 \text { Trafalgar }(75 \% \quad x x) \\
\text { j Ormond }\end{array}$ & $1 \sin 3$ & $\begin{array}{l}\text { Oromedon } \\
4 \text {... Farthing Ture } \\
\text { xx? (Grand-sire of } \\
\text { Trafalgar) } \\
\text { 5 Sir Peter sx (prob- } \\
\text { abiy Grand-sirc of } \\
\text { Trafalgar) }\end{array}$ & $\begin{array}{l}\text { Deducta } \\
8 \text { Eclipse } \mathrm{xx}\end{array}$ \\
\hline ; & $\begin{array}{l}\text { Duplicat } \\
3 \text { Waterman x. } \\
5 \text { Rodrich }\end{array}$ & 1859 & $\begin{array}{l}\text { Djalma } \\
3 \text { Trafalgar } \\
\text { j Ormond }\end{array}$ & $\begin{array}{l}\text { Daja } \\
\text { i Eclipse xx }\end{array}$ \\
\hline 4 & $\begin{array}{l}\text { Eckstein } \\
3 \text { Eurydamus } \\
6 \text { Blacliamoor } \mathrm{xx} \\
\text { (Grand-sire of } \\
\text { Eurydamus) }\end{array}$ & 1870 & $\begin{array}{l}\text { Venerato } \\
\text { J Oronocco I. } \\
\text { Grand-sire: } \\
\text { 2 Blacisamoor xx }\end{array}$ & $\begin{array}{l}\text { Echo } \\
2 \text { Eurydamus } \\
5 \text { Blackamoor xx } \\
5 \mathrm{l} \text {. Whalebone } \mathrm{xx}\end{array}$ \\
\hline$j$ & $\begin{array}{l}\text { Elwin } \\
3 \text { lorwärts }\end{array}$ & 1887 & $\begin{array}{l}\text { Passian } \\
5 \text { Syyders xx }\end{array}$ & Emilia \\
\hline is & $\begin{array}{l}\text { Hydriot } \\
\dot{3} \text { Fritter } \\
\text { j Eurydamus }\end{array}$ & 1895 & $\begin{array}{l}\text { Fiirstenberg } \\
6 \text { Ringes } x\end{array}$ & $\begin{array}{l}\text { Ifydra } \\
3 \text { Eurydamus (Grand- } \\
\text { sire of Fritter) } \\
\text { is Y. Whalebone } x x\end{array}$ \\
\hline
\end{tabular}

Stallions with + Free (ienerations.

$1 \quad .1$ al

4 Pocahuntas xx

j) Stiton $x x$

2 Intenor

4 Sorcerer $\mathrm{xx}$

4 Oronocio 1.

:3

Calcas

4 Trumpator $2 x$

j. Nexinder $x$

(by Eclipsc xx)

1 Epaminondas

41 . IIhalebone xx

4 Tirranes
185"? "runnel

6 Camel ix

Isti Leporello

1929 Scrapall x.

j) Viclipse $x$

157-2 lnspector $x$

6 Sirvererer xis (Grandsire of ligranes $x$. y. Whalebone $\mathrm{xx}$, and Blackamoor x.x) Firand-sire :

+ Surcerer $x$
Allbekannte

T Whislser xx

8 Trump xx

Ingitia

Moira

lipocca

3 Blackamoor $x x$

6 Sorcerer $x x$ 
Heredity.

\begin{tabular}{|c|c|c|c|c|}
\hline No. & Names of Stallions & Bor 11 & Sire & Dam \\
\hline$i$ & $\begin{array}{l}\text { Flügel } \\
4 \text { Vega } \\
6 \text { Catton } \mathrm{xx} \\
7 \text { Orville xx } \\
7 \text { Thunderbolt } \mathrm{xx} \\
\text { (by Sorcerer } \mathrm{xx} \text { ) } \\
8 \text { Sorcerer } \mathrm{xx}\end{array}$ & 1869 & $\begin{array}{l}\text { Vorwärts } \\
6 \text { Orville } x x \\
6 \text { Sorcerer } x x\end{array}$ & $\begin{array}{l}\text { Flasche } \\
\text { Grand-dam: } \\
5 \text { Sorcerer } x x\end{array}$ \\
\hline ii & $\begin{array}{l}\text { Hanno } \\
4 \text { Emma xx } \\
\text { (by Whisler xx) }\end{array}$ & 1861 & $\begin{array}{l}\text { Danseur xx } \\
+\left\{\begin{array}{l}\text { Whisker } x x \\
\text { Whalebone xx }\end{array}\right.\end{array}$ & Harriet \\
\hline 7 & $\begin{array}{l}\text { Ibarra } \\
4 \text { Caril } \\
7 \text { Trumpator } \mathrm{xx}\end{array}$ & $18+8$ & $\begin{array}{c}\text { Thunderclap } \\
\text { ? }\end{array}$ & Jupine \\
\hline$s$ & $\begin{array}{l}\text { Jemim } \\
\begin{array}{l}4 \text { Ganges } x \\
5 \text { Leporello } \\
\text { (by Tigranes } x)\end{array}\end{array}$ & 1876 & $\begin{array}{l}\text { Pless } \\
\text { i Thunderbolt } x x \\
7 \text { Whalebone } x x\end{array}$ & $\begin{array}{l}\text { Jessica } \\
5 \text { Tigranes x }\end{array}$ \\
\hline 9 & $\begin{array}{l}\text { Jenissei } \\
4 \text { Vecordia }\end{array}$ & 1888 & $\begin{array}{l}\text { Tenezuela } \\
6 \text { Emilius xx }\end{array}$ & $\begin{array}{l}\text { Jemba } \\
3 \text { Antenor } \\
4 \text { Ganges x }\end{array}$ \\
\hline 10 & $\begin{array}{l}\text { Massa } \\
4 \text { Vega } \\
7 \text { Thunderbolt xx } \\
\text { (by Sorcerer xx) }\end{array}$ & 1873 & $\begin{array}{l}\text { Vorwärts } \\
6 \text { Sorcerer } x x \\
6 \text { Orville } x x\end{array}$ & $\begin{array}{l}\text { Materna } \\
9 \text { Sorcerer xx } \\
\text { Grand-sire: } \\
6 \text { Sorcerer xx }\end{array}$ \\
\hline 11 & $\begin{array}{l}\text { Merlin } \\
4 \text { Whalebone xx } \\
6 \text { Sorcerer xx } \\
\text { (by Trumpator xx) }\end{array}$ & 1860 & $\begin{array}{l}\text { Sahama xx } \\
5 \text { Buzzard } \mathrm{xx} \\
5 \text { Evelina xx } \\
6 \text { Trumpator } \mathrm{xx} \\
\text { (Grand-sire of } \\
\text { Whalebone } \mathrm{xx} \text { ) }\end{array}$ & $\begin{array}{l}\text { Morea } \\
\text { 1. Y. Whalebone } \mathrm{xx} \\
\text { Grand-sire: } \\
2 \text { Sorcerer } \mathrm{xx}\end{array}$ \\
\hline 12 & $\begin{array}{l}\text { Morgenstrahl } \\
4 \text { Pocahontas } \mathrm{xx} \\
4 \text { Teddington } \mathrm{xx} \\
5 \text { Orlando } \mathrm{xx} \\
7 \text { Camel } \mathrm{xx} \\
\text { (by Whalebone } \mathrm{xx} \text { ) }\end{array}$ & 1896 & $\begin{array}{l}\text { Blue Blood xx } \\
7 \text { Selim xx (Great } \\
\text { Cirand-sire of } \\
\text { Pocahontas xx) } \\
6 \text { flhalebone xx } \\
6 \text { Whislier xx }\end{array}$ & $\begin{array}{l}\text { Moba } \\
6 \text { Camel xx (Grand- } \\
\text { sire of Orlando xx } \\
\text { and Great Grand- } \\
\text { sire of Teddington } \\
\text { xx) } \\
\text { Grand-dam: } \\
5 \text { Camel xx }\end{array}$ \\
\hline 13 & $\begin{array}{l}\text { Oromedon } \\
4 \text { Mr. Farthings } \\
\text { Turc xx? } \\
5 \text { Sir Peter xx } \\
\text { ? }\end{array}$ & 1836 & $\begin{array}{l}\text { J. Driver } \\
6 \text { Herod } \mathrm{xx}\end{array}$ & Trulla ? \\
\hline
\end{tabular}




\begin{tabular}{|c|c|c|c|c|}
\hline No. & Xames of Stallions & Burn & Sire & D:am \\
\hline 14 & $\begin{array}{l}\text { Pompejus } \\
\text { \& Pesta xx } \\
\text {; Whalebone xx }\end{array}$ & 1871 & $\begin{array}{l}\text { Inspector } x \\
\text { (i Sorcer xx }\end{array}$ & $\begin{array}{l}\text { Pometle } \\
4 \text { Limmin xx } \\
\text { (by llhislete ix) }\end{array}$ \\
\hline 1.; & $\begin{array}{l}\text { Promoter } \\
4 \text { Oronocen } 1 . \\
\text { fi Surener ix }\end{array}$ & 18.52 & $\begin{array}{l}\text { Durydamus } \\
2 \text { Oronocen } 1 .\end{array}$ & Promise ? \\
\hline
\end{tabular}

Stallions with 5 Free Cienerations.

I liberharel

jonviders $x x$

jo Vex:i

jo Give

- Impürer j Pocahontas $x$

:3

Fiscluerlinabe

5) Pucaliontas x.

1 Granicus

j. Vlellourne $\mathrm{xx}$

5
Juli
jolindianor x

ii

Malteser

j) Emma x. (by llhisker xis)

7 Ihialebone xx

7

\section{Matador}

5 11 :

5 Penculape xx

(wive brother fo)

Marteces de Sulu-)

s $\quad$ Nisos

if $l^{\circ}$. Whalebone $x$

(by lloulebone xis)

!)

Passivan

j. Snyelers $x$

(i) Limma xim

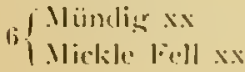

\begin{tabular}{|c|c|}
\hline 1577 & $\begin{array}{l}\text { Pliigel } \\
4 \text { Veg:t } \\
\text { 6 Catton x. }\end{array}$ \\
\hline 1890! & $\begin{array}{l}\text { 1.chnshers } \\
\text { fi Touchstone xis }\end{array}$ \\
\hline $1: 01$ & $\begin{array}{l}\text { Obelisk } \\
7 \text { llumphrey (linker xx }\end{array}$ \\
\hline 1.887 & 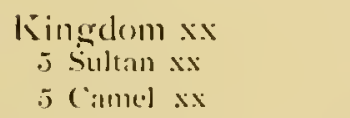 \\
\hline IS81 & $\begin{array}{l}\text { Pripounier } x \\
\text { 1 Orlando } x x\end{array}$ \\
\hline 1872 & 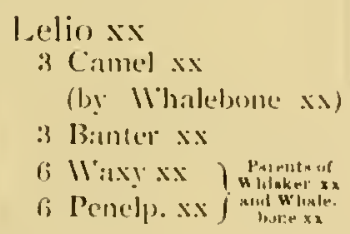 \\
\hline 184.7 & $\begin{array}{c}\text { Thunderclap } \\
\text { ? }\end{array}$ \\
\hline 1874 & $\begin{array}{l}\text { Veneralo } \\
\text { jOronoce } 1 .\end{array}$ \\
\hline IRS1 & 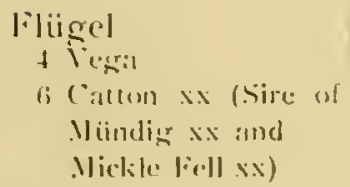 \\
\hline
\end{tabular}

E.lba

Emigrantion

5 Siluana $x$

Fischerin

.3 Poxaluntan x.

Gradlitz

i Whatebone xx

7 Biserdadly ox

Jacke

Malagamba

4 1: Whalebone x.

(j) Tiurranes $x$

Mula

Nixe

(i) Camel x:

(by 11 hallebone an

(imand-are:

a Whillebune $x \mathrm{x}$

Palme.

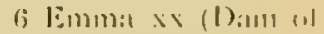

Mündig $x x$ and

Mickle linll ix) 
Heredity.

\begin{tabular}{|c|c|c|c|c|}
\hline No. & Names of Stallions & Born & Sire & Dim \\
\hline 10 & $\begin{array}{l}\text { Poët } \\
\text { j Vorw:irts } \\
\text { 6 Pocahontas xx } \\
6 \text { Stockwell xx } \\
\text { \& Orlando } x x\end{array}$ & 1904 & $\begin{array}{l}\text { Morgenstrahl } \\
4 \text { Pocahontak xx } \\
4 \text { Teddington xx } \\
\text { 5 Orlando xx } \\
\text { 7 Canel xx }\end{array}$ & $\begin{array}{l}\text { Polynesia } \\
8 \text { Touchstone xx } \\
\text { Grand-dam : } \\
3 \text { Stoclswell xx } \\
\text { Grand-sire: } \\
6 \text { Camel xx }\end{array}$ \\
\hline 11 & $\begin{array}{l}\text { Venerato } \\
\text { j Oronoceo } 1 . \\
7 \text { Sorcerer xx (Girand- } \\
\text { sire of blackamour } \\
\text { xis) }\end{array}$ & 18.52 & $\begin{array}{l}\text { Dorimont } \\
2 \text { Blackamour } \mathrm{xx} \\
4 \text { Oronocco } 1 . \\
\text { Grand-daun : } \\
4 \text { Sorcerer xx }\end{array}$ & $\begin{array}{l}\text { I'ecordia } \\
\text { ? }\end{array}$ \\
\hline
\end{tabular}

Stallions with i Free Generations.

I Discant

6 Camel x

$2 \quad$ Fürstenberg

6 Gances $\mathrm{x}$

7 Whalebone $x . x$

3 Orcus

6 Camel x.

(by Whalebone xx)

4. Paladin

6 Touchstone $\mathrm{xx}$

5 Pardo

6 Orvil]e $\mathrm{xx}$

$5\left\{\begin{array}{l}\text { Rubens xx } \\ \text { Selim xx }\end{array}\right.$

i) Polarsturm

6 Pocahontas $\mathrm{xx}$

$\succsim \quad$ Principal

6 Whalebone xx

S Prinz Optimus

6 Pocahontas $\mathrm{xx}$

6 Mclbourne xix (by Humphrey

Clinker $\mathrm{xx}$ )

\begin{tabular}{|c|c|}
\hline 1874 & $\begin{array}{l}\text { Fliigel } \\
+ \text { Fegral }\end{array}$ \\
\hline 1878 & $\begin{array}{l}\text { Ambos } \\
3 \text { Eurydamus } \\
+1 \text {. Whalebonc xx }\end{array}$ \\
\hline 1883 & $\begin{array}{l}\text { Friponnier xx } \\
1 \text { Orlando } x x \text { (Grand- } \\
\text { son of Camed) } \\
7 \text { Whalebone }\end{array}$ \\
\hline $187 t$ & $\begin{array}{l}\text { Adonis } x x \\
5 \text { Sultan xx }\end{array}$ \\
\hline $188: 3$ & $\begin{array}{l}\text { Vindex xx } \\
3 \text { Selim xx } \\
5 \text { Orville xx } \\
4 \text { Waxy xx } \\
4 \text { Penelope } x\end{array}$ \\
\hline 1900 & $\begin{array}{l}\text { Optimus } \\
\tau \text { Humphrey Clinker } x .\end{array}$ \\
\hline 1868 & $\begin{array}{l}\text { Vorwärts } \\
6 \text { Sorcerer xx } \\
6 \text { Orville xx }\end{array}$ \\
\hline 1898 & $\begin{array}{l}\text { Optimus } \\
\text { T Humphrey Clinlier } x \mathrm{x}\end{array}$ \\
\hline
\end{tabular}

Diana

Fulda $x$

5 Sir Hercules $x x$ (by Whalebone $\mathrm{xx}$ )

\section{Orelia}

8 fllhalebone $x$

8 IIThislser $x x$

Grand-sire :

4 Whalebone $\mathrm{xx}$

Palme

6 Sorcerer $\mathrm{xx}$

Perilla

5 Sorcerer $x x$

Porona

8 Touchstone xx Grand-sire :

3 Pocahontas xx

Principessa

3 Y. Whalebone $\mathrm{xx}$

4 Tigranes $x$

Prinzess

3 Sahama xx

4 Stilton $x x$ 


\begin{tabular}{|c|c|c|c|}
\hline Io. & Nomes of Stallions & Born & Sire \\
\hline$!$ & $\begin{array}{l}\text { Tunnel } \\
6 \text { ('amel xx } \\
\text { (by. Whalelone xx) } \\
\text {; Whilebone xx }\end{array}$ & $187 t$ & $\begin{array}{l}\text { The Duke of } \\
\text { Edinbourgh x. } \\
6 \text { Whalebone xx }\end{array}$ \\
\hline 111 & $\begin{array}{l}\text { Vinerzuela } \\
6 \text { Emilius xx }\end{array}$ & 1878 & $\begin{array}{l}\text { Ifectur xix } \\
6 \text { Blacklock }\end{array}$ \\
\hline 11 & $\begin{array}{l}\text { Vurwärts } \\
\text { 6 Surcer xx } \\
\text { (by Frumpator xx) } \\
\text { t) Orville } x \text {. }\end{array}$ & $185 !)$ & 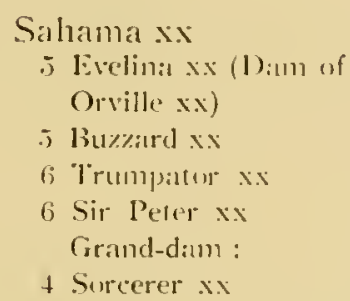 \\
\hline
\end{tabular}

Stallions with i Free Generations.

1 Ner Herr

i Pocahontals $\mathrm{xx}$

- Melbourne xis (by

llumphrey Clinker (x)

2 . Intsrorsteher

T Truchatone xx

3 Duduck

- II:Axy xx (sire of Ifoful xx)

4

Eiton

- Camel xx

;)

Justizminister

T Touchstone $\mathrm{xx}$

(;

Panzer

- Pocahontas xx

(Dam of Stockwell xx)

\section{Optimus \\ 7 Humphrey Clinker xix}

1899 Fanfarro

8 Mhiskin ws

1863

l.ightning x.

4 Sorcerer xx

(by Trumpater xx)

Grand-dam :

3 llaxy xx Parente

$\left.\begin{array}{l}3 \text { Penci. xx } \\ \text { (by Trump.) }\end{array}\right] \begin{gathered}\text { Woful } \\ \text { sx }\end{gathered}$

1sss The Duke of

Edinbourgh xx

6 Whalebone $\mathrm{xx}$

; Selim $x$

7 Buzzard xx

- Mare by .llexander $x$

lsos Boulevard x.

4 Birclenteher $\mathrm{xx}$

15)2 Greif $x$

j. Stockwell xx

\section{"utti}

6 (camed ...

Frand-nire:

3 Whatehome $x$

Viereck

(i l. Il"hilubene $\mathrm{xx}$

recordia

Grand-rire:

2 Orville

\section{LImuda}

7 Birdentcher

7 liurdamus

i Sahama xx

\section{Inisette}

4 Pocahontas xx

\section{Daisv}

311 itcrman x.x

(by lloful xx)
Eipis

6 Cimlei ax |ustitia

- Tumbahstone $x x$

Panzerliette

4 lecordin

4 liritter 


\begin{tabular}{|c|c|c|c|c|}
\hline Xio. & Names of Stallions & Born' & Sire & Dam \\
\hline 7 & $\begin{array}{l}\text { Pless } \\
7 \text { Thunderbolt xx } \\
\text { (by Surcerer xx) } \\
7 \text { Whatebone } x x\end{array}$ & 1870 & $\begin{array}{l}\text { Vorwärts } \\
6 \text { Sorcerer } x \\
6 \text { Orville xx }\end{array}$ & $\begin{array}{l}\text { Petze } \\
4 \text { Tigrimes } x\end{array}$ \\
\hline
\end{tabular}

Stallions with 8 liree Cienerations.

1 lilock

$\checkmark$ Orville $\mathrm{xx}$

2 Popilius

8 Maxy $x$

$\&$ Penelope xix

3 Thebaner

\& llihatebone xi

\begin{tabular}{|c|c|}
\hline 187.5 & $\begin{array}{l}\text { Ructic sx } \\
\text { 6 Orville } \mathrm{xx}\end{array}$ \\
\hline 3דיאו & $\begin{array}{l}\text { Iahire xx } \\
3 \text { Whalebone xx } \\
\text { Cirand-dam : } \\
\text { I Whatebone xx }\end{array}$ \\
\hline 1876 & $\begin{array}{l}\text { Pless } \\
7 \text { Whallebone } \mathrm{xx}\end{array}$ \\
\hline
\end{tabular}

Flamme

4 Vega

6 Catton $\mathrm{xx}$

7 Orville $x x$

T Thunderbolt xx

Poclie

3 Defuge (by llaterman xis, Grandecun of Waxy $x x)$

Theresia

7 Whisker xx Grand-sire:

4 Whalebone $\mathrm{xx}$

From these 6 l examples one may draw the following conclusions:-

1. Mhat the greatest number of Royal Stud stallions (just as is the case in Thorumghored breeding) are amongst those with 4 free generations. Yet the must favourable limit for Half-bred breeding seems to me to be wider than with Thoroughbreds, as not 3,4 and 5, but 4,5 and 6 free generations show the best results. Besides, the merits of Half-bred stallions with 5 , and especially with 6, free generations are much superior to those of the other stallions. Amongst the many stallions with f free generations there are only three good ones, namely, Flugel, Ilorgenstrahl, and Oromedon. Of these three Morgenstrahl (94\%) is very near to Thoroughbred, and rery likely the other two would be also very" near to Thoroughbred if one could only. deternine their pedigree more accurately. The two stallions with 1 free generation were very inferior. Amongst those with 2 free generations only Dorimont and Journey may be classified as important, but both became Royal Stud stallions only on account of their black colour. The final conclusion to be drawn is that the most favourable inbreeding for llalf-breds is 1 to 2 free generations more removed than is the case with Thoroughbreds, and that perhaps only very noble llalf-breds prefer a closer inbreeding up to I or 3 free generations, just like Thoroughbreds.

2. The inbreeding supports seem to be just as useful in Half-bred breeding as in Thoroughbred breeding. In many cases, unfortunately, the 
pedigres in so incomplete that many inbreedings cannot be ascertained. Precisely the very best stallions, as for example, Fiirstenberg, Malteser, Moreenstrahl, () reus, l'assian, Tunnel, lenerate, Vorwarts, ete., shou the same inbreeding supports either on the part of their dam or of their sire, or of buh, as is shown in the case of the best Thoroughbred stallions. Several of the most important mixed Thoroughbred stallions which are not mentioned here show these inbreeding supports very clearly, as for example, the best of them. Inspector $x$ with 6 Soreerer $x$ and the sire: 4 Sorcerer $x x$, further, Ganges $x$ with a Surerer sx and sire: 4 Sorcerer xx and Xobleman $x$ with 2 Tigranes $x, 6 \mathbb{W a x} \times x$ and 6 Penelope $x x$ (by Trumpator), sire: + Sorcerer xix (by Trumpatur and grand-sire of Tigranes) dam : 6 Trumpator x.x. Finally, to give an example from the racecourse, the at present best Half-bred steepler, Ready, born Hanover 1899 by Lorbeer (Beberbecker) and Rosamunde, with 7 . Welbourne xx, sire: 4 West . Iustralian xi (by . Melbrurne), dam: :3 Y. Melbourne xx.

Is an example for an unsatisfactory heredity of an otherwise first-class lalf-bred stallion, on account of insulficient inbreeding supports, I may here mention . Ipis (page :30:3. . $\mathrm{n}$. 1), just as I mentioned Imomis for Thoroughbred breeding.

Half-bred stallions, excellent in their conformation but with insulficicont inbreeding support, as for example. Optimus (7 II umphrey Clinker by. Comus], sire s Comus, dam 7 Whalebone and 10 Sorcerer [sire of Comus]). are often very difficult to mate to get the best results. Nelbourne (by Humplirey (Clinkier) and Pocahontas are the two cardinal puints in (Optimus' pedigree. Probably, therefore, one would obtain the best results with mares inbred to. Melbrume or Pocahontas' blond.

3. When mating the parents, of course, it is to be understexcl that only prominent ancestors common to both should be chusen as basis of inbreeding, as we have already pointed out when dealing with Thoroughhred breeding. As in the pedigree of Half-breds, Thoroughbreds are generally the best ancestors, it is recommendable in Half-bred breeding to direct thr inbreecling, as far an pussible, to a Thoroughbred ancestor. Half-bred is, lomever, in this resper often very difticult to handle. Yet the statistics of alsose of Half-bred stallions show that in 19 ases Thoroughbred (inclusive of mixed and questiunable Thoroughbred) has served as a basis of inbreeding, and Half-bred in 1.2 rases only. . Ill the 11 stallions with of tree senerations have Thurughhared as basis of their inbreding. (O) all botter stallions, mle two are inbred to Haff-bred as a basis, and these ano were lore black stallions Journey and Veneratu, which on account of their colour could claim a more indulgent criticism.

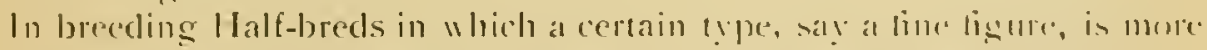
important than capabilities, as for example, with coath horses (Oldenlume. Holstein, and part of Hanover), an inbreeding to typical IIalf-brel ancesturs will often be advisable. When breeding purely for conformation it is very 
"ssential to linow the great influence which the exterior of the basis of inbreeding often exercises. High legs in the heredity of a stallion (for example (reheimrat) are, according to experiments which I have here made, more surely remoxed by a close inbreeding to a short-legged ancestor than by mating with a short-legged mare (for "xample .loba), without thereby attaining the close inbreding to a basis characterised by short legs.

In all breedings for military and riding horses, eapabilities are the most important thing, and breeding must, of course, be directed to that end. Besides, in riding and military horse breeds, the type to be obtained is in no way opposed to that of the Thorrughbred, but so nearly related to it that by the increased and well-chosen addition of Thorouglibred blond the Halfbred type an only be improved. Neither Thoroughbred breeders nor Half-bred breeders ought to be frightened away from their main purpose, i.e. capabilities, by fear of a change of type. Capabilities will automatically: form the most suitable type in Thornughbred as well as in Half-bred, if only the breeder will mate and rear his animals chiefly with respect w capabilities. The strength of the Half-bred, which is so desirable, and rightly so very much esteemed, wruld be diminished hy the early training usual with Thoroughbreds. In extended grazing, lasting up to the third vear, increases that strength, which gives type and value to the Half-breds.

From the list given below of classical male and female winners, it follows that the inbreding has been a useful factor in the production of gend stock in use, i.e., in this ease of successful racehorses. It is surprising that 4 free generations seem to be the most favourable limit of inbreeding with the best racehorse's just as well as with the best breeding horses. The idea of producing breeding material "ith special individual prepotency b! a rery - Hose inbreeding, as for example, in the case of Flying lox (I Galupio), or by a close and repeated inbreeding with distinct inbreeding support, as for example, in the rase of Hlackleck (3 Highfleer, I Herod, Sire: 3 Herod, dam : - Herod), is based on a lack of reflection. Should a stallion even be inbred as much and as often to Herod as a basis, he can never become ultimately more Iferod than Herod himself was, and therefore cannot transmit more Herod peculiarities than Herod hinself dicl. The individual prepotency has nothing to do with close inbreeding. The most stallions who have shown a special individual prepoteney had a proportionately far removed inbreeding, as for instance, Birdcatcher. Buccaneer, Chamant, Flibustier, Newminster, Perth, Rataplan, Stockwell, Volligeur, and many. others with of free generations ('ach, and Bend Or, Doncaster, Lord Clifden, St. Simon, Thomanby West Iustralian, and many others 1 ith 6 free seneratims each. In Half-bred breeding, Optimus can be mentioned as a stallion endowed with unusual individual prepotency, and he also had a very lar remored inbreeding, i.c., 7 llumphrey Clinker. 
The mate and female winners of the Derby, St. leger, and Oaks, arranged acording to the number of free generations:-

Cotsis

0) Free Generation.

1. ("edric D). líel by Phantom.

FII.I.IFS

I Free Generation.

1. Ninety-Thee L. 1790 by Jilorizcl. I. Trifle 0.1782 by Justice.

-. Islaton I. Isoce by Walnut.

$\therefore$ Bellissina O. 179.; by Phoeno-

3. Cadland D. 18.5 by . Indrew. menon.

1. Knight of St. (ieorge 1. 18.) by birdcatcher.

万. liging liox 1).I. 1896 by Orme.

3. liphemeral (). 1797 by Wrodpecker.

1. Misis Laty O. 18:34 by Priam.

- Free (ienerations.

1. Saltram D). ITisu by Eclipse

2. Noble 1). 17is: by Highfluer

3. Colt 1). $179+$ bx lidget

4. Cocktighter L. 1796 by () (Oertom

5. Quiz l.. 179s by Buzzard

6. Orvill. 1. 1799 be Beninghough

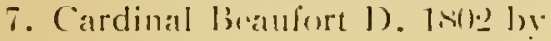
Cisllanna

8. Election 1). firot by Golannat

9. Petrunius 1.. 180.) by Sir l'e(1.0

10. Pan D. lastis be Se. Geerese

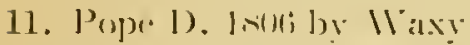

1.2. II illiam L. Isil b. (sovernor

13. Ebar 1.. 1sit by Orville

14. Sailor 1). listo be Seud

15. "Thendere L. Isig by lioful

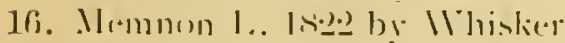

17. Mammaluk 1). 18-1 Ix Partisan

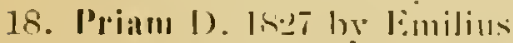

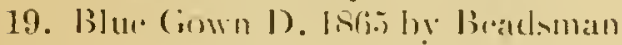

20. Haw husenden l. lavit by land ('lifden

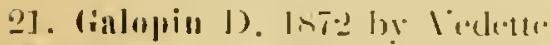

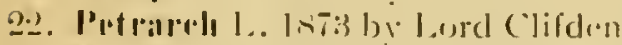

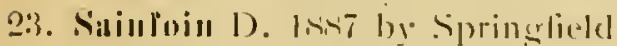

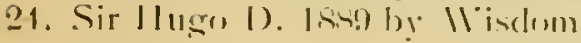

1. Stella 0.1781 by Plunder

?. Innette O. $1781 \mathrm{by}$ liclipse

3. Ilippoly 0.1787 by .Mercury

1. Portia O. 17.9s by lolunteer

5. Ilermione (O. 17! by Sir Peter

di. Platina (). 179:2 by . Merour.

7. Parisot 0.1793 by Sir P'eter

$\therefore$ Sotia O. 1799 by Dedpini

9. Pelisse O. Inil by Whiskey

10. Malluella O. 18199 b D)ick indrens:

11. Mtisidmat 1. 1810 by Dicki Indrews

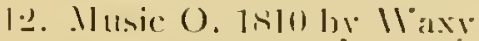

13. Minmet O. 1slen by Maxy

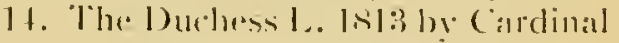
lintis

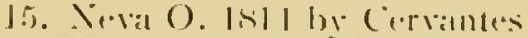

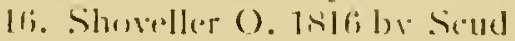

17. Galata O. freg by Sutan

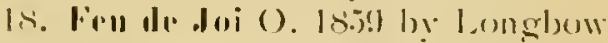

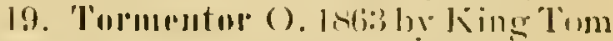

20. (iomos (). 186i- by Sunterer

-1. Thebais 0.187 by II.rmit

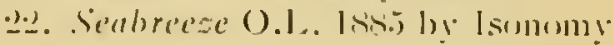


3 Free Generations.

1. Bourbon L. 1774 br Le Sang

2. Tommy L. 1776 by IVildair

3. Ruler L.. 1777 by Young Marske

4. Sir Peter D. 1784 by Highflyer

5. Beniuglorough I. 1791 by King Ferçus

6. Spread Eagle D. 1792 b! Volunterer

7. Sir Harry D. 1795 by Sir Peter

‥ Symmetry 1. 1795 by Delpini

9. Irrhduke D. 1796 by Sir Peter

10. Ditto D. 1,00 by Sir Peter

11. Staveler 1. 180.) by Shuttle

12. Paris D). 180.3 by Sir Peter

13. Whaldhom D. 1807 by $11 \mathrm{axy}$

11. Octarian L. 1807 by Stripling

15. Blücher 1). Is11 by 11 axy

16. Whisker D. 181. by Waxy

17. Filho da Puta L. 1812 by I laphazatd

18. (\%or D. 181+ by Selim

19. Sam D. 1si.j by Scud

20. Reveller L. Ixly by Comus

21. Tiresias 1). 1816 by Soothsaper

22. Antonio I. 1816 by Octavian

23. St. Patrick L. 1817 by 11 alton

24. Jack Spigot L. 181S by (.Irdrossan) or Marmion

25. Fullis D. 1 20 by Orville

26. Tarrase L. TS2:3 by Cattun

27. The Colonel L. 1825 by Whisker

28. Frederick D. 1826 b! Little John

29. Rockingham 1.. 1830 by I I momphrev Clinker

30. Elis L. 1833 by Langar

31. Phosphorus 1). 18:34 by I amplighter

32. Don John L. 1835 by (Tramp) or Maverlev

33. Satirist 1. 1838 by Pantaloon

34. Cotherstone D. 1840 by Touchstone
1. Hollandaise 1. 1775 br Matchem

?. Tetotum O. 1777 br Matchem

3. Imberatrix L. 1779 by . Iffed

4. Mail "I thw (lakis O. 1780 by Herod

5. Cowslip L. 178. by llighflyer

6. Prwett L. 1780 by Tandem

7. Tag O. 1780 by Trentham

8. Volante O. 17in by Highflyer

9. Cailia O. 1790 by Iolunteer

10. Eleamor D.( ). 179t by Whisliey

11. Theophania O. 1800 by Delpini

12. Bromzr O. 18013 by Buzzard

13. Briseris 0.1804 by Beningbrough

14. Мolm O. 180j by Surcerer

15. Oriana (). 1807 by Beningbrough

16. Laudseape O. 181:3 by Rubens

17. Corinne O. 181.j by ilaxy

18. Pastill. O. K18 by libbens

19. Zinc O. Jiso by lisoful

20. ("ullwil O. 18:1 by Phantom

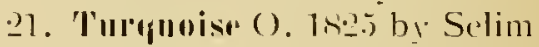

2.2. Cyprian O. 183:3 by Partisan

23. Rofuation O. 184. by Glaucus

24. ('yula O. Is l.j by Melburne

2.5. Butlerfly O. lo.j by Turnus

-6. Hippia O. lingt by King Tom

27. Marie Ntuat O.I. IsTo by Seottish (hief

28. Camelia $\frac{1}{2}$ O. 187:3 by Macaroni

29. Jamettr O.1. 187.5 by Lord Clifden

30. Wherl of Futhute O. 1876 by Adrenturer

31. Shotoeer D. 1nt!) by I Iermit

32. Mimi O. 18se by Barcaldine

33. Our Lassic O. 1900 by . Iyrshire

34. Cherry Lass O. 1902 by Isinglass

35. Glass Doll O. 1901 by Isinglass 


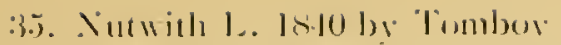

:36. Mrlauda D. 1811 by Tunchstene

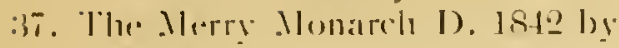
slane.

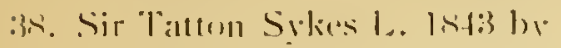
Melbourne:

3!). Tlu, Flyiug Mutelıuna 1).1. 1840 J) lia . Middletom

I1). Mardsmail D. 1s.j.j by Weatherbit

11. Pretender 1). Istili by . Idienturer

f.). Pero Come\% 1.. Isfili by Buadsman

1:3. Nilviu D.I.. 1sit by Blanir . Ithol

1.1. St. Blatise D. 18sol b) Ilermit

(Jarvester ? 1). 18S by Sterling

4.). St. (iation $\frac{1}{2}$ D. 1 Sis by Rotherhill or The Rover

16. The Lambkin 1. 1NS1 by Camballı,

47. Moltom D.I. Introg by Master Kildare

4R. Merry Hampun D. Isid by Hampton

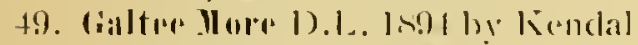

.) Vour Majesty l. 190.) by l’ersimmon

\section{Free Generations.}

1. Hiomen D). 1775 by lelerized

2. Y. Felipse D. 1778 by liclipse

3. Issassin D. 1779 by Sweetbriar

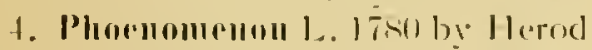

;. Serjeant 1). 1781 by Eelipse

(i. Limw(d) 1). 175.) by Mark Intony

7. Parasen 1.. 175:3 by Pasmaster

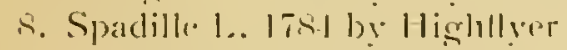

9. Sir "lu(umak 1). 178.j by Pontac

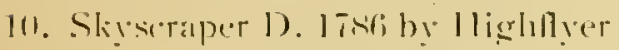

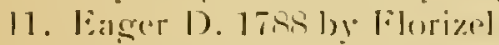

1:?. Tartar I. . 17is? bi lianrizel

1:). Hamlintumian 1.. I79.2 by King Fergus

1. Imbrosin 1. 179:3 by Sir leder

1.). 1.monger 1. . 1791 by 1)rome

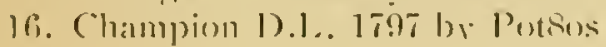

1. Bridget O. 177tiby Hered

¿. Faith O. 17To by Hered

3. Serina L. ITtis by (ioldender

4. Ceres (). 1779 by Sweet llilliam

5. Omphale 1. I

6. Nightshade 0.1785 by Potinos

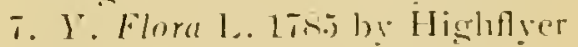

s. Yikr O. 179f by . Vixilnder

9. Bullina O. 1796 be Rockingham

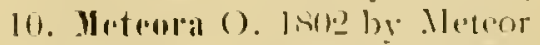

11. Pauliua I.. 1801 hy Sir Peder

1.2. Maid of Orleans $\dot{O}$. Jumi by Surreper

13. Sumery O. 180 by Surener

14. Mrdora (). 181 l by Selim

1.). ('anliur (). 1817 by 11 haldounce

16. Ingusta (). 1sis by II"oful 
17. Tyrant D. 1799 by Potsos

18. Remembrancer I. $1800 \mathrm{by}$ Pipator

19. Sancho 1. 1801 hy Don Quixote

20. Phautum 1). 1808 by IValton

21. Octavius D. 1809 by Orville

2.). Otterington L. 1809 by Golumpus

23. Smolrnsko 1). 1810 by Sorcerer

24. Gustarus D. 1818 bv Elaction

25. Iloses 1). 1819 by lithalebone or Sevmlon:

26. Barefont L. $18: 20$ b. Tramp

27. Middleton D. 182-2 by Plantom

29. Birminglom 1. 1827 by Filho da Puta

29. Chorister 1. 1N-2, by Lottery

30. Nargrave 1. . 16.29 hy Muley

31. Ploniputuntiary 1). 1831 by Emilius

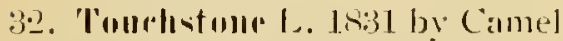

33. Miundig 1). 18:3.2 by (atton

31. Bay Hilllotun 1). is 33 b. Sultan

3.5. Imato D. 14:35 by I lelocipede

36. Little 11 onder 13. 18:37 by Muley

37. Launcelot 1. $18: 37$ by Camel

3i. Coronation D. 1 s:3s bir Hercules

39. 'The Barou 1. . 1942 by Birdeatcher

40. Surplice D.I. 1S4.5 hy Touchstome

41. Mild Dayrell 1). Tr.o by lon

-2. Hromit D. lintit by Neviminster

43. Sefton 1). 187.) by Speculum

41. Sir Bevis D. Isto by Favonius

45. Rayon d ()1 1. Inte by Flageolet

46. Rock Sand D.I. 1900) Sainfoin

47. St. Imand 10. 1901 hy. St. Frusquin

48. Challacombe 1. 1902 by St. Serf

19. Spearmint D. 1903 by Carbine

50. Orby D. 1901 by Ormo
17. Lilias $0.182: 3$ by Interpreter

18. Oxyzen O. 1828 by Emilius

19. (ilumzre O. 1838 by Pantaloon

20. B/ue Bomut L. 1839 by Touchstone

21. Poison O. 18-10 by Plenipotentiars

22. 'The Jrincess O. $18+1$ by Slane

2). Miami O. 1814 by Venison

24. Iris O. 1848 by ithuriel

2.). ('athruine Hayus O. $18: 50$ by l,anercost

296. Nummerside O. 18.56 by Mest Iustralian

-27. Reils O. 1869 by Monarque

28. Spinaway O. 187: by Macaroni

29. Placida O. 187 $+b_{y}$ Lord Lyon

30. Bonny Jean O. 1880 by . Wacaroni

31. Rêve d'Or O. Jist by Hampton

32. I'Alhosse do Jouarer O. 1886 by Trappist

33. 'I'luostle L. 1891 by Petrarch

31. Limasol O. $189+$ by Poulet

3.5. Iirs and (Amates O 189.5 by lyrshire

36. Keystone 11. O. 190:3 by Persimmon 
5 Free Generations.

1. Radamanthus D. 1787 by Justice

2. Imbidexter L. 1787 by Phoenomenon

3. Y. Traveller L. 1788 by King Fergus

1. Waxy D. 1790 by Potsos

¿. Diidalos 1) 1791 by Justice

fi. Didelot D. 179:3 by "Trumpator

i. Soothsaver 1., 1808 by Sorcerer

$\therefore$ Prince l.eopold D. 181; by Hedley

9. Jorry L. 1s:2 by Smolensko

10. Lapdog D. 1823 by Whalebone

11. Rowton L. 18:06 by Oiseatu

19. Spanice D. 18.2s by Whalebone

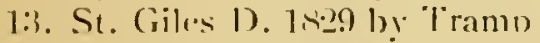

14. Dangerous 1) 18:30 by Tramp

1.). Mango L. 183t be Emilius

16. Charles XII. L. 18:36 by Voltaire

17. Attila D. 1839 by Colwick

18. Faugh-a-Ballangli 1. 1811 by Sir I Iercules

19. Pyrlutus 1. 1). 1,8t3 by Epirus

20. The Cossack 1). 181t by Hetman Platoff

21. Van Tromp L. LA1I by Lanercost

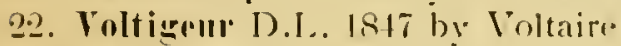

23. Yowminster 1.. 1818 by "Touchstone

24. Stoekwell L. 1sig by The Baron

2.5. Induver D. 18.1 by Bay Widdleton

206. Saucebox L. 18.5: by St. Lawrence

27. Musjid D. 1rist by . Newminster

28. Rettledrum 1). 185, by Rataplan

29. (Caractacus D) 1859 by Kingston

30. The Marquis 1. 18.59 by. Stockwell

31. Gladiateur D.I. 1862 by Ilonarque

32. Kingeraft D. 1867 by King Tom
1. Mabaculia 1.. 1773 by Sampson

‥ Matilda L. 182-1 by Comus

3. (Auluare O. 18:3 by Smolensko

4. Areell II antle O. 18206 by Sultan

5. Variation 0.1827 by Bustard

6. Vespa O. 18:30 by Muley

7. Pussy O. 18831 by Jollio

s. Qqueno "I the Trumus O.L. 18:32 by Vilocipede

9. Industry O. 18:35 by Priam

11. Laty Evalyu O. Tista by Don John

11. Rhedycinu O. 1817 by Wintonian

1.. Noungstersi O. 18t9 by Birdcatcher

13. Marrhioness O. 18.5.2 by Melbeurne

14. Mincepie (). 18.53 by Sil eetmeat

1.). Imprerieuse 1.. 19.5t by Orlando

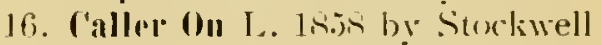

17. Queren Bretha (). 18ibu by Kingston

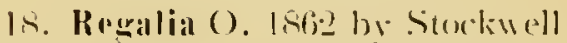

19. Brigantine $O$. INirib lisucaneer

20. Hannah ().1. 186en by King Tom

-1. Ipologey O.1. 1s71 by

Idventurer

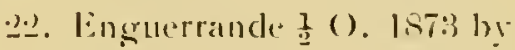
Vermouth

2:3. Nenuy Howlet O. 1,it by The Palmer

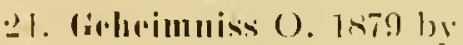
Rosicrurian

..5. Dutch Oven 1. . 185.9 b! Dutch Skater

2019. Busylumly O. 1,ist he Petrarch

27. Mis. Butterwiek O. 1890 by st. Simon

노. Imiable (). 1s:91 hy St. Simon

29. La Silgesse O. I89:- be 11 isdom

30. Canlerhury l'ilgrim (). 189:3 by

Tristin 
33. Citorge Frederick D. 1871 by Marsyas

34. Robert the Devil L. 1877 by Bertram

31. Cap and Bells II. O. 1898 by Domino

3:- Pretty Polly O.L. 1901 by Gallinule

35. Ormomle D.L. LA83 br Bend Or 33. Signorineta D.O. 1905 by

36. Ayrshire D. 18s.; by Hampton

37. Donovan D.I. 1886 by Galopin Chaleureux

38. Common D.L. 1RS8 by Isonomy

39. Isinglass D.L. 1890 by Isonomy

40. Ladas D. $189 \mathrm{~L}$ by Hampton

41. Sir Visto D. I. 1892 by Barcaldine

42. Persimmoll D.L. 1893 br St.

Simon

43. Jeddah D. 189.j by Janissary

44. Wildfowler I.. 189.5 by Gallinule

45. Diamend Jubilee D.i. 1897 by

St. Simon

46. Volodyorski D. 1898 by

Florizel II.

47. Doricles 1. 1.898 by Filorizel 11.

48. Ard Patrick D. 1899 by St.

Florian

49. Wool Winder L. 1904 by

Mlartagon

6 Free Generations.

1. John Bull D. 1789 by Fortitude

2. Hannibal D. 1801 by Driver

3. 'Teddington D, 1848 by Orlando

4 . Daniel O'Rourlie D. 1849 by Birdcatcher

5. West Iustralian D.L. $18.50 \mathrm{by}$ Melbourne

6. Ellington D. JA.si by Flying Dutchman

7. Gamester 1. 18.56 by The Cossack

8. Thormanlyy D. 18.5 by (1leebourne) or "IIndhound

9. St. Nlbaus 1.. 18.5 by Stockwell

10. Macaromi D. 1860 by Swectmeat

11. Iard clifilen L. 1860 by Xeaminster
1. The Yellow Filly O. 1783 by Tandem

2. Wings 0.1820 by The Flyer

3. Deception O. 1836 by Defence

4. (nucifix O. 1837 by Priam

.). Our Nell O. 1839 by Bran

fi. Mendirant O. 184:3 by Touchstone

7. Mincement O. 1851 by Sweetmeat

ฉ. Blink Bomny D.O. 1R.jt by Melbourne

9. Governess O. 185.) by Chatham

10. Sunhram 1.. 185.5 by (hanticleer

11. Fille de Lidir O. 1861 by Faugha-Ballagh 
12. Bair Ithol D.L. I861 by Stock- 12. Achievement L. Is6t by Stockwell

1:3. Lond Lyon D.J. 1863 by Stock- 13. Formosa O.L. 186ioby Buccancer well

14. Farmins D) 1868 be Parmesan

1.). ('remorur D. 1869 by Parmesan

11. Wenlock L., 1\$69 br Jord Clifden

17. Joncister D. 1870 by Stockwell

1s. Craig Nillar L. 1872 by Blair lthol

19. Kisher D. 167:3 by Buccaneer

20. BomI 0r D. 1877 by Doneaster

-21. Ossian L. 1880 by Salrator

2.2. Kilwarlin L. 1884 by Arbitrator

20:3. Cicero D. 1902 by Cyllene

-1. Troutbeck L. 190:3 by Jadas

11. Lumely (). 18s? by Hermu

1.). Musa O. 1896 by Martagon

16. La Roche O. 1897 by St. Simon

17. Scêptre O.I. 1899 by Persimmon

7 Free Generations.

1. Blomsbury D. 1836 by Mulatto

-. Warlock 1.. 1853 by Birdcatcher

3. Iroumois D.I. 1878 by I.eamington
1. Brown Duchess $O .18 .58$ by [i]ving Dutchman

‥ Miss Jummy O. 1883 by Petrarch

3. Memoir O.J. 1898 by St. Simon

t. Ia Flectar O.I.. 1889 by St. Simon

The names of stallions and mares printed in heavy type in above list distinguish those successful in breeding. As this classification is especially difficult with mares, a number of same are further distinguished by printing in italic type ats doubtfully successful. There is still to be noticed that the stallions and mares born after 189 . have not come into consideration, as the time of their activity is still too short to rightly classify them. 
Number of male and female winners of Derby, St. Leger and Oalks arranged according to free generations.

\begin{tabular}{|c|c|c|c|c|c|c|c|c|c|}
\hline \multirow{2}{*}{ 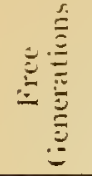 } & \multicolumn{3}{|c|}{ lionn before and in 1905 . } & \multicolumn{3}{|c|}{ Lurn before and in 1849 . } & \multicolumn{3}{|c|}{$\begin{array}{c}\text { Born between } 1850 \text { and } \\
1905 \text { inclusive. }\end{array}$} \\
\hline & Colts. & Fillies. & Total. & Colts. & Fillies. & Total. & Colts. & Fillies. & lotal. \\
\hline 11 & $\mathbf{J}$ & 一 & 1 & 1 & - & 1 & - & - & - \\
\hline 1 & (1) & + & $\begin{array}{r}9 \\
111\end{array}$ & 3 & 4 & 7 & $\stackrel{2}{(1)}$ & - & $\stackrel{\ddot{2}}{(1)}$ \\
\hline 2 & 24 & $\frac{22}{(1)}$ & $\begin{array}{l}46 \\
\text { (1) }\end{array}$ & 18 & 17 & 3.5 & 6 & $\begin{array}{r}5 \\
5 \\
\text { (1) }\end{array}$ & $\begin{array}{l}11 \\
11)\end{array}$ \\
\hline 3 & $\begin{array}{l}50 \\
(4)\end{array}$ & $\begin{array}{l}34 \frac{1}{2} \\
(3)^{2}\end{array}$ & $\frac{84 \frac{1}{2}}{(7)^{2}}$ & $\begin{array}{l}3 !) \\
\text { (11 }\end{array}$ & $\begin{array}{l}24 \\
\text { (1) }\end{array}$ & $\begin{array}{l}63 \\
\text { (2) }\end{array}$ & $\begin{array}{l}11 \\
\text { (3) }\end{array}$ & $\begin{array}{l}101 \\
(2)^{2}\end{array}$ & $\frac{21 \frac{1}{2}}{(i)}$ \\
\hline 4 & $\begin{array}{l}50 \\
\langle 31\end{array}$ & 36 & $\begin{array}{l}86 \\
131\end{array}$ & $\begin{array}{l}40 \\
\left(\begin{array}{l}(2) \\
(2)\end{array}\right.\end{array}$ & 24 & $\begin{array}{l}\text { (6) } \\
\text { (2) }\end{array}$ & $\begin{array}{l}10 \\
\text { (1) }\end{array}$ & 12 & $\frac{20}{111}$ \\
\hline.$j$ & $\begin{array}{l}49 \\
(9)\end{array}$ & $32 \frac{1}{2}$ & $\begin{array}{l}81 \frac{1}{2} \\
(14)\end{array}$ & 24 & (1) & $\begin{array}{l}36 \\
(2)\end{array}$ & ( & $20 \frac{1}{3}$ & $\begin{array}{l}45 \frac{1}{12} \\
(8)^{3}\end{array}$ \\
\hline 6 & 24 & 17 & $\begin{array}{l}+1 \\
(6)\end{array}$ & 4 & 6 & 10 & $\frac{20}{13)}$ & $\begin{array}{l}11 \\
\text { (3) }\end{array}$ & 31 \\
\hline 7 & 3 & $\begin{array}{c}+1 \\
\text { (2) }\end{array}$ & $\begin{array}{r}7 \\
(2)\end{array}$ & 1 & - & 1 & 2 & $\begin{array}{r}4 \\
(2)\end{array}$ & $\begin{array}{r}6 \\
(2)\end{array}$ \\
\hline I'utal & $\begin{array}{l}206 \\
(20)\end{array}$ & $\begin{array}{l}1501 \\
(14)\end{array}$ & $\begin{array}{l}: 356 \\
(34)\end{array}$ & $\begin{array}{l}130 \\
(4)\end{array}$ & $\begin{array}{l}87 \\
(2)\end{array}$ & $\begin{array}{l}217 \\
(6)\end{array}$ & $\begin{array}{c}76 \\
(16)\end{array}$ & $\begin{array}{l}63 \\
\text { (s) }\end{array}$ & $\begin{array}{l}139 \\
(24)\end{array}$ \\
\hline
\end{tabular}

The figures in brackets indicate the number of winners in two classical races. Each of the winners in deacl-heat races is counted with $\frac{1}{2}$. From the above unfortunately small statistics of the above table one can draw the tollowing conclusions :

1. On the whole the most of the classical winners are found among the 4 free generations, next to which follow 3 and 5 free generations.

$\because$. Lp to the middle of the past century the 4 free generations predominate also. The 3 free generations, however, excel remarkably the more removed inbreedings with $j$ free generations, as also the 2 free generations do the 6 free senerations.

3. From the middle of the past century up to now the whole picture has been changed in favour of a more removed inbreeding. Here the 5 free generations appear to be the most favourable only, after which follow the more removed inbreedings with 6 free generations, and finally that with 7 free generations.

4. Lastly, it is worthy of note that the winners of two classical races have been more numerously represented with the more removed inbreedings.

The same change in farour of the more removed inbreeding is shown in the following table, in which the percentage of stallions and mares most successful in breeding is reckoned from the above list. Accordingly, the 
pereentage of stallions most successful in breeding increases with the number of free generations up to 6 free generations, in the case of mares exen up te. Tree generations. Mlthough the statistics are somenhat small for these conclusions, and the right assessment of the stallions, and especially of the mares, "ith regard to their successful activity at the stud, is often vere dificult, the regular increase is still worthy of note. \%luere is ret to be mentioned that every doubtfully successful mare has berat inunted in the following table only with $\frac{1}{2}$.

The male and female winners of Derby, St. Leger and ()islis born until the vear 1895 inclusive.

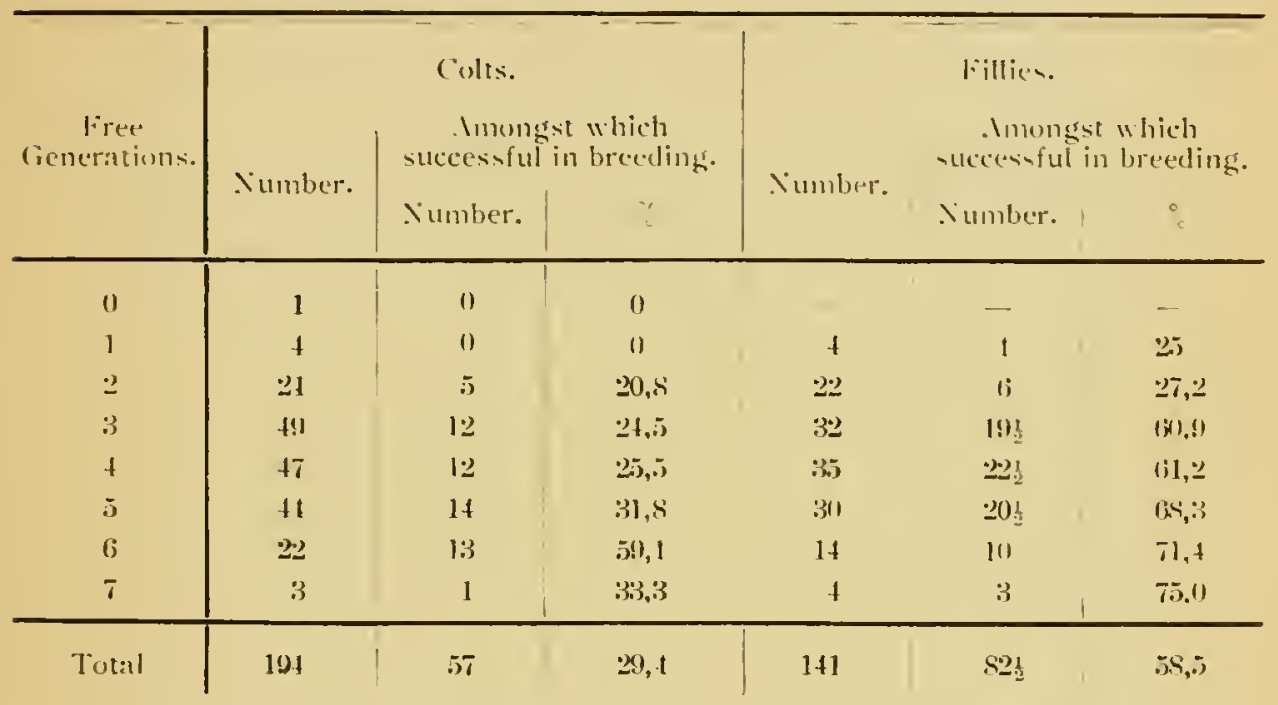

In the above list of classical winners also proved in breeding is omitted Flying Fox, as he was only born in 1890 . Te was bought by Mr. Blanc for a million francs at the auction. His successes on the racecourse were remarliable, and at the stud, at least at the commencement, he was strikingly successful. His expensive purchase further proved to be a goud business. Many breders have been induced by this example w risk such cluse inbreeding (1 free seneration) in the hope of obtaining similar suceesses. In exate study of the Thoroughbred breeding, and an impartial consideration of thi case, would have been able to provelice many seruples. Folving fou was a grandson of the marvellows horse Ormonde. and his father, Orme, who, as is well known, nearly died of poisoning wholly hefore the l) why, was just as remarliable a horse. Bred in England's best stud, traincel by the pistmaster, Porter, on the hilly training-ground of Kingsclere, and set apart as stud stallion in France's best stud, Flying Fox has heen bred to as many first-class mares as perhaps any other sire in lingland ur France. His wh- 
springs were reared and trained regardless of cost, and finally ridden in an excellent manner by the best jockey we had in Europe since the death of Ircher. Nll these things are powerful forces, which ought to increase much the chances of the offsprings of Flying Fox and repair much that exaggerated inbreeding had damaged. If filying fox should once-which I doubt-leave behind in his progeny imperishable pillars of "Thuroughbred breeding, he would be, by the side of manr miscarried attempts, the third -uccessful one of this group (with 1 free generation). Paulowit\%, born 1813 by- Sir Paul, foundation sire of Buccaneer, was the first, but even his success commenced first with his grandson, lon, when the latter produced the Derby winner Wild Dayrell. The second is Barcaldine, born 1878 by Solon, and sire of Whitefield, Morion, Cuxdfellow (sire of (halereux). Espoir, Wolf's Crag, Sir Visto, and Marco. If Flying Fox became at any time the third in this group, perhaps the natural power of the virgin soil or the excellent horse pastukes of Argentine, where two of his sons now breed. will cause this. Where and what are, however, the four own brothers and two own sisters of Flying Fox? Exempla docent! 


\section{CHAPTER V.}

\section{Hereditary Faults.}

First of all the question to be answred is, What is transmitted? lncredible small details and shades in the build, the walk, and temperament are transmitted. .lbove all, the proportions of the individual parts of the body are transmitted, as will as the length and smoothness of hair, small hair-curls, courage and timidity, maliguity and confidences, bad or good f.rtility, even immunity against certain illnesses: longevity, habits, acquired facultes; in short, nearly everthing. So-called hereditary faults, however, as blindness, spatrin, biting, broken windedness, roaring, ete. are more soldom transmitted, sometimes not at all.

In spite of the intentional use for many years of breeding material which suffered from perindic ophthalmia (moon blindness), we did not lind in Trakehnen any examples of the transmission of blindness, or of the disposition thereto, arising from periodic ophthalmia. In many low-lying distriets (in Tralielonen, the Kalpalsin Farm), and esperially by clower hay from undrained fields, horses are often aftlicted in damp years with periodic ophthalmia, which frequently leads to blindness. Aroreling to experiences at Trakelonen, foals of blind mares are no more suloject to the periodic "phthalmia than others, perhaps eren less so. Acording to the latest researches into the nature of spavin and periodic ophthalmia, scientific men (in the first rank Professon Bierkerhoff in a lecture in 1901 on the subject of hereditary faults in breeding horses) have promounced anganst their inheritance.

In the following list, the names of .5 brond mares in Traliehnen are siven, which, in consecpuenere of periodic ophthalmia became blind of one "re. This blindness usually oceurs with brond mares before their sixth year, wften aren in their first rear. In some alses the brond mares mentioned also berame, in the course of time, blind of the othereve. These brood mares hawe up to the summer of 1907 , produced 1,81 living foals, of which up to now (. Iugust, 1907) only a have suffered from periedic ophthalmia, i.e., firstle, the colt Jörn ('hl by Optimus out of Jesi ( $\mathrm{X}(\mathrm{o} .10$ of the list). born 
List of Brood Mares at Trakehnen which became Blind in consequence of Periodic Ophthalmia (Moon Blindness).

\begin{tabular}{|c|c|c|c|c|c|c|}
\hline \multirow[b]{2}{*}{ No. } & \multirow{2}{*}{$\begin{array}{c}\text { Names of Brood } \\
\text { Mares }\end{array}$} & \multirow[b]{2}{*}{ Born } & \multirow{2}{*}{$\begin{array}{c}\text { Sires of Brood } \\
\text { Nares }\end{array}$} & \multicolumn{3}{|c|}{ Of which were } \\
\hline & & & & $\begin{array}{l}\text { Living } \\
\text { Foals }\end{array}$ & $\begin{array}{l}\text { Country } \\
\text { Stallions }\end{array}$ & $\begin{array}{l}\text { Brood } \\
\text { Mares }\end{array}$ \\
\hline 1 & Eclie & 1871 & Venerato & 16 & 3 & 3 \\
\hline 2 & Ebbe & 1876 & Venerato & 8 & 2 & 1 \\
\hline 3 & Instanz & 1879 & Journey & 16 & 4 & $t$ \\
\hline+ & Hydra & 1881 & Journey & 13 & 4 & 7 \\
\hline 5 & Trojanerin & 1881 & Hector xx & 16 & 4 & ij \\
\hline 6 & Prag & 1882 & Tunnel & 13 & 4 & 2 \\
\hline 7 & Pereskie & 1884 & Fürstenberg & 7 & 2 & $\underline{2}$ \\
\hline 8 & .1lda & 1885 & Tunnel & 12 & 1 & 3 \\
\hline 9 & Veglia & 1891 & Barometer & 5 & - & 1 \\
\hline 10 & Jesi & 1893 & Hirtenknabe & 8 & 2 & 2 \\
\hline 11 & Pyrotechnil: & 1893 & Euphony xx & 6 & 2 & - \\
\hline 12 & Ibiza & 1894 & Mirmidone & 8 & 2 & 1 \\
\hline 13 & Jugrendliebe & 1894 & Leporello & 6 & - & 2 \\
\hline 14 & Learose & 1894 & Leporello & 5 & 1 & 1 \\
\hline 15 & Daniela & 1895 & Orcus & 5 & -- & 1 \\
\hline 16 & Handarbeit & 1896 & Euphony xx & 7 & 1 & 1 \\
\hline 17 & Livonia & 1896 & Mephisto $\mathrm{xx}$ & 6 & - & - \\
\hline 18 & Jedwede & 1897 & Euphony xx & 5 & 1 & - \\
\hline 19 & Indacht & 1898 & Fanfarro & 3 & $\ldots$ & - \\
\hline 20 & Gunst & 1898 & Le Borda $x x$ & 4 & - & - . \\
\hline 21 & Irche & 1899 & 1,arifari & 4 & - & 1 \\
\hline 22 & Marterbanl & 1899 & Moeros xx & 1 & & \\
\hline 23 & Irende & 1900 & Larifari & 3 & Still too & \\
\hline 24 & Irtistin & 1900 & Piper & 2 & young & \\
\hline \multirow[t]{2}{*}{25} & Paarung & 1900 & Piper & 2 & & \\
\hline & & & Total & 181 & $\begin{array}{l}33 \\
\text { Including } 3 \\
\text { Royal Stud } \\
\text { Stallions }\end{array}$ & 38 \\
\hline
\end{tabular}

29th of March, 1903. The first attack occurred on the Sth of April, 1903, and the second, 8th of October, 1904. In both instances it was in the left eye (the dam was $i l l$ and became blind also in the left eve). These two attacks were completely cured. As a three-year-old, Jörn [ $\mathrm{i}$ hl was found to be sound of eye, and was sent as a country stallion to Gudwallen, where he stands still to-day. The second is a case of a colt, Thronerbe by Optimus out of Trojanerin (No. 5 of the list), born 16th November, 1903. Thronerbe was the 16 th foal of Trojanerin (23-year-old), who first became blind in the left eye, and later on blind of both eyes, caused by periodic ophthalmia. Ite caught periodic ophthalmia on the 12th of November, 1903, and was cured. the disease leaving a small cataract behind. In damp rears, periodic 
ophthalmia is farly frequent in Trakehnen. From the Ist of Ipril. 1902:, to the 1st of liebruary, 190\%, for instance, to horses (chiefly weaning foals, yearlings and two-rear-olds) caught the periodic ophthalmia. Of the th ill ones, 17 were cured without leaving behind any abnormal changes in the eye. Nlso in the case of brood mares which suffered from periodic ophthalmia, but which did not become blind, and were cured of same without leaving behind some abnormal changes, there was not observed any transmission of a disposition to this disease in the progeny. On the contrary, as in the case of the 25 blind mares, there secms to occur the transmission of a certain immunity against the disease mentioned.

I know several cases in East Prussia, which go to show that the breeding use of stallions which have become blind, through periodic uphthalmia, is harmless. I await with special interest the results of two stallioms (Elfenfe]s 1901 by Obelisk out of Elisenau, and Musensolun 1901 by Optimus out of $1 /$ umme), each of whom became blind of one eyc in Trakehnen. through periodic ophthalmia, and who have been covering since $190 \mathrm{j}$ up to the present time in the Livland Stud, Torgel. I also still await the result of Royal Stud stallion Ingrimm, who has been covering since 1907 in Trakehnen, and who became blind of his right eye in 1904, through periodic ophthalmia.

The scientific researches into the nature and causes of spavin and ringbone have so far succeeded that neither of them may be taken as a uniform disease. The researches and opinions on sparin of horses published by Professor Eberlein (Berjin, 1897), have been verified by many celebrated scientists. One may easily accept Ebcrlein's views as to the nature and origin of sparin, also for ringbone, just as 1 myself do hereafter, without wishing to give even an approximately exhaustive explanation of the many variations and differences of sparin and ringbone.

Sparin as well as ringbone are caused either by contusion of the joint bones or by a stretching of the ligaments which hold the joint bones together, or, in other words, always by a traumatic cause. Spavin or ringbone in consequence of a rachitic disease happens rery seldom in our modern improved breeds. I, at least, have never come across such a case. In the case of spavin or ringbone caused by contusion, there ensues, in consequence of a strong and sudden pressure on the bone substance, an inflammation of the bone, at first becoming loose and then tight (ostitis rare facicns ct condensans). In the case of spavin this inflammation of the bone will principally affect the cuneiform bones (os centrale and os tarsale 111): in the case of ringbone it will affect the long pastern bone and the short pastern bone. In consequence of the inflammation of the bones, the nourishment of the articular cartilage suffers; there ensues an inflammation of articular carrilage (chronditis), and thus are caused the exudations and proliferations of bone originating in the articular surface, assuming very different dimensions, attacking the neighbouring periost, and afterwards developing into bone 
substance (formation of Osteophytes). Thus arises the real spavin or the articular ringbone.

In the case of spatin caused by stretching (periarthritis), or ringbone (periurlicular), the exudations of the bone begin from the appendage pieces of the torn ligaments, and not from the joint surfaces. They may then spread round the joint, and in serious cases also attack the joint surfaces, which they destroy, just as in the case of the articular form of the disease. Spavin commencing at the joint ligaments and the periost is very rare. This may be explained by the faet that, in the case of the so-called small ankle-joints, the joints are very tight (as opposed to the coronet joint), admitting of no, or at least rery slight, sideway movements. If the spavin originates at the appendage of the side ligaments or the periost, a push or a blow has generally been the cause of it. Perhaps the somealled break (in German Absats), which is so frequently found in the case of covering stallions, belongs very often to this periarthritic form of spavin. Iceording to Professor Eberlein, the proportion of the frequency of sparin caused by pressure to that caused by tearing, is as 9.; to \%, while in the case of ringbone (according to Ldrisky) the frequency of articular to periarticular is as 60 to 10.

Professor Lberlein writes about the transmission of sparin as follows :"The question, Mlay horses attacked with spavin disease be used for horse breeding or not?' is a very important one for horse breeding. I gainst the previously held riews, Dickerhoff rightly emphasises that every genuine case of spavin deserves a special judgment in this respect. Is I have shown, when discussing Aetiology, the primary Ostitis rare faciens of the small tarsus bones owes its origin to a mechanical contusion of the small tarsus bones, and may arise even in the best formed hocks and thighs from outside causes. Therefore spavin is in itself not hereditary. Scientifically it is proved, however, that a deficient formation of the hocks and a faulty position of the thighs, which predispose a horse to this disease (causa interna) are transmitted. Thus it follows that with regard to spavin, only horses with defective hocks or a faulty position of the thighs on the one hand, and badly constructed bones on the other hand, must be excluded from breeding."

I fully subseribe to the riew of Professor Eberlein, and extend the same also to ringbone, which, in my opinion, in itself is just as little transmittable as spavin. With regard to ringhone, I should like to mention yet that horses with short and upright pasterns are more liable to be attacked by the articular ringhone arising from contusion, while horses with long and sloping pasterns are nore exposed to the periarticular ringbone arising from tearing.

The certainty with which scientists and laymen have beliered in the undoubted transmission of spavin, ringbone, and moon blindness, shows very clearly the low standing of horse breeding as a science. I do not know of any recognised practical example as a proof of the transmission of sparin, neither have 1 ever met anyone who could point out to me such an 
(xample. Is far as I know, the following horses had the opportunity to transmit spavin :-

1. Percival, born l'rak. 1867, by I alhire xx and Peranga hy ()remedun, was for four years country stalliom in Gudwallan, and was stabled alt Röseningken. In 1872 he was set aside berause he distinetly shoued spaviu, and was used rery much afterwards as stallion at Georgenburg up to lexis.) at that time the private stud of llerr ron Simpsent. The two hoxks of Percival are preserved in the "Traliehnen hippelogical muscum, and show that he suffered from sparin, catused by entusion. Imong his very numerous foals (about 200 military remounts, jo brond mares, and e.je country stallions), I have heard of mene beinge suspeeted of spavin. Imomg the sons of Percival, (atpitain, born 18xl, later stallion in Georerenburs, must be sperialle mentioned, berause lo was used vers extensively for five pears in Georgenburer. Was then sold as stalliom to .lliumich, and was the sire of

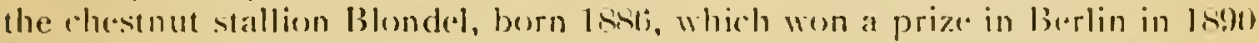
at the great horse show, and was afterwards sont to beberbeck as Roval Stud stallion. Imeng the whole of his progenge I never herard of one suspected uf sparim.

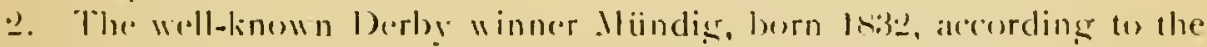
description of the prepared left hexk griven by the sted insperter. Kiutshath (published in a hand-bexk for herse breeders by ('ount l.ehnderff. page 14:3), undoubtedly suffered from spavin raused by contusion, just as Percival

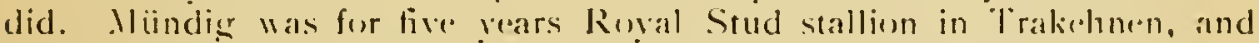
afterwards fur four years in the private stud of Julianfelde, in last l'russia,

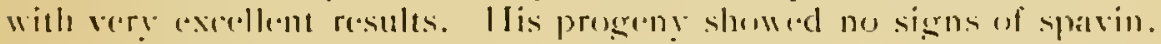

3. ()ptimus, born Ixis), by Odoardo and Optima by The (iolumel, had spavin on the near hind, as can be distincely seen from the skeleten, preserverel at the hippolegieal museum at Trakelinon. Inong his vers numerous

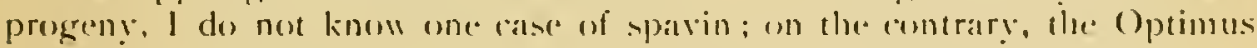

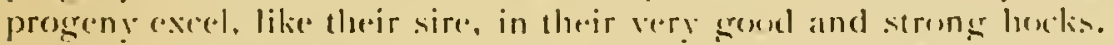

4. The Thoreughbred sire. The General, bern lis.e. by ("hilderic and Coturnix, suffered already from spavin when Royal siud stallion at

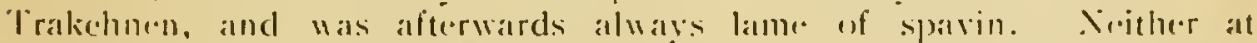
"rakelumen, mer during his sisteron years atetivity at Gudwallan, has he, as

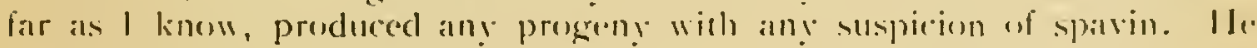
was for many lears the: lohoreushbred sire in the (itudwallan dintriet, which has produced most military remounts (abust :300)). Mloreover. he has pro-

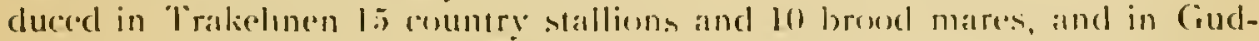

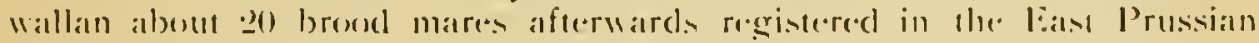
Stud besil:

․ The Derby winner llermit, hern latil, had spatin an the mear hind, as his slicletem, which is preserved in the london Veterinary Silumele dis-

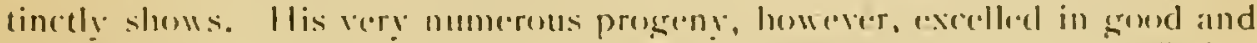

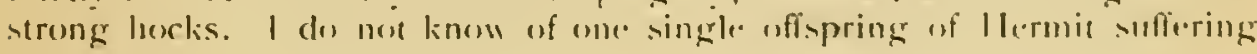
frum spatvin. 
6. The Trakehnen brood mares, Aqua, Emina, Ina, and Juniata, suffered from spavin (the hocks are in the Trakehnen Museum), and of their very numerous progeny none was suspected of spavin.

The Thoroughbred stallions, Blue Blood born 1876 by lining Tom and Marigold, and Elsass born 1870 by Napoleon and Esther, suffered from ringbone (Periarticular). The respective preparations of the two stallions are in the Trakehnen Museum. Blue Blood has not transmitted this disease in Trakehmen, and I am assured the same thing about the progeny of Elsass. I belitre, however, that the disposition to articular ringbone is often transmitted, as the upright pasterns, bulging out somewhat to the front, lend themselves easily to transmission. In some breeds of horses there are whole families which are addicted, more or less, to articular ringbone, whilst I do not know a similar case of periarticular ringbone or spavin. It is difficult to work horses lame from spavin. Their development without work is unfarourable. This is the only reason that horses which are lame from spavin when roung are little suitable for breeding purposes.

The crib-champing, gasping, tongue-sucking bad haloits, to which for the most part only very tough and durable horses are addicted, are, according to experiences in lrakehnen, transmitted by the sire to about 5 per cent, of his progeny. I presume that brood mares also transmit these faults to about the same extent. I do not know of any actual examples as, in spite of using for many years five brood mares which were crib-champers, not one of their foals has inherited it. I must here remark that gasping and tonguesucking are transmitted just as seldom as erib-champing. I have known some cases, on the other hand, in which nearly 50 per cent. of the foals of a brond mare, who herself was not a crib-champer, became already as foals rrib-champers, without their parents or grandparents having been addicted thus. It is an old rule that stallions which are crib-champers do not transmit it if burnt on account of a breali-down. This is right in those cases only in which the stallion has acquired this habit through ennui after a breakdown, during the repose which follows the burning and which lasts for many wecks. This crib-champing is not transmitted as easily as that which foals acquire whilst grazing, without any reason. Finally, I consider that the chances of transmitting erih-champing, or the disposition thereto, is so very small that the breeder need not to take it into consideration. If the lameness caused by sparin, or blindness, or hroken wind, interferes with the grmmastic development of the body, and if crib-champing often causes attacks of colic, I would not, of course, recommend such animals for breeding material.

The question on the transmission of roaring is more difficult. In the first place, roaring seems to be more general in certain breeding districts. The damp and fogg! climate of England produces more roarers than France, but in Imerical and . Iustralia they are the exception. The early born foals are in England said to be more inclined to roaring than the late born ones. Roaring is a consequence, principally, of the break-down of the hindmost, 
mostly left, aspergillum muscle (erico-arytaenoideus) or its nerve. which affects the movement of the aspergillum cartilage necessary for easy breathing. If this break-down has been caused by severe glanders, influen\%a, or by over-exertion from racing, only at the end of the third or fourth year, as in the case of Ormonde, a transmission of roaring is not to be expected. I do not know of iny roarer in the progeny of the roarer Ormonde. Prince Charlie has not transmitted this roaring in America. Pocaltontas and Chamant were roarers. The former did not transmit it at all, and the latter very seldom. The well-known I Iumply rey Clinker of former times did not transmit his roaring at all, and the most popular stallion of modern times, Gallinule, does not seen to transmit his roaring either. If the roaring. however, commences without any sperial exertions in racing, and especially so soon as in the second vear, one mat reasonably assume that the general weakness of the horse is the cause. "Weakness in horse breeding is the only real hereditary fault which is transmitted with rertainty, and it should be eradicated by the most severe measures from the breeding material. I once knew a verg much pampered stallion which apparently was not a roarer, at least not when gralloping the pace he could well perform, but of his progeny about in per cent. were roarers when they were trained for a few montlus ats two or three-year-olds. The more tenderly the borses of a breed are reared, the more roaress will appear. For this reason the Drafters, which are reared under the pressure of unreasonable shows, mostly become roarers. Ifter these come the different l Ialf-breds, according to the tenderness or severity of the method of rearing, and the fewest roarers are found amongst stoppe horses and Thoroughbreds. The only difference is that, in consequence of public races of Thoroughbreds, erery roarer is recongnised and becomes known, or that many become roarers on aceount of the tom great exertion, which is not expeeted from other breeds of horses.

The same thing applies to horses whow sinews have broken down through werexertion in training or raeing. Horses which break down alread" as two-year-olds in racing, or even in training before raw suspected of weakness. The worst, hemerer, are those which are so bad that they cannot break down because they lack the energy and the stride, without which they are not subjeet to those shocks which produce the brealidowns. Of surh herses the linglishman says, "lhey (an nerer gu quite fast enough to trouble themselves."

IIl dispositions to disease and constitutional weaknesses, such as biliousmess, bony exereserences, soft and deformed hoofs, as well ats all other diseases connected with inflammathry swelling (läinsehuss) are transmitted. Broken-nindedness belengs also to constitutional weakness. and is atsily transmitted by stallions which suffered from it in their early years. In consequence of illness, and other unknown causes, the transmission by stallions differs in erertain vears. For example, after influenzal, at mure unfasmrahle transmission has been of fen noticed. Horses which have run much transmit 
their characters sometimes worse in their early years. Ormonde, however, produced in his first covering year his most important son. Orme, and the latter again, in his lirst covering year, four winners, amongst them the tough Ameer, and in the second covering year Flying Fox, who in his turn produced in his tirst covering year l ljax and Gouvernant. Wild Dayrell produced in his first covering year as a four-year-old his best son, Buccaneer, and Florizel, who ran up to the age of seven rears, produced in his first covering year the first Derby winner, Diomed, his best son. Old stallions are said to produce more stayers, and young nnes more flyers.

Just as weakness may be considered the only real hereditary fault, in like manner hardiness is to be looked upon as the most important hereditary advantage. With hardiness is combined robust health, the most important quality of all breeding animals. I woid weakness, improve hardiness, and the . Mpha and Omega of horse breeding is obtained for producing capable horses, and not horses simply fit for shows, or horses which during their whole existence are always only sold, hut rally neser made use of : and there are many such!

Is, of course, no stud can possess fatultless breding material, one must try to ectualise faults in the conformation of brood mares by mating them with stallions which are especially good in these respective parts. Then it will be found that high-leggedness, narrowness, and bad temperament, are faults which are most diffieult to remere by mating, and therefore belong to the most serious faults. Nares which feed hadly are little suitable as brood mares, because in all probability they will also feed their foals badly. Mares which are inclined to corpulency, also, feed as a rule badly, and are not to be recommended. The same faults are to be blamed in the case of stallions, but they are less dangerous. 


\section{CIITPTER V'I.}

\section{The Transmission of the Coat Colour.}

WHAT concerns the heredity of the coat colour? There is, I suppose, a yet unknown originating force which catuses a distinct deviation from atl other laws of heredity. The sex, tex, appears 10 play a particular part, and the atavism has perhaps no influence at all. Whereas, it is remarkable that a distinct atavism is often evident in the transmission of white marks and particular spots. Is in the Roral Stud of Trakehnen, for over a hundred years, in three special studs. blacks (in Gurdszen, so to tol brood mares), browns (in Danzkehmen, 70 to 80 brend mares), and ehestnuts (in Jonasthat, 50 to fol broed mares) hawe been bred, and in twe studs (in Trakelmen so tes 100 brood mares. and in Bajohrerallen to to io brood mares) all colours are represented, and mixed with each other, we have plenty of material at hand from which to construct laws at th the transmission of coat colour.

There exists a distinct regularity with grays, chestnuts, and blates as regards transmission. This regularity is as follows: grays and chestnuts mated only to their oun colour, produce either chestnuts or grats, and black with black about in per eent. chestnuts (often dark (hestnuts), the rest always hlacks, never black-brown or dark brown. Here we must mention that rubiean horses (alsu rubican chestnuts) atso produce at times grates, as for example. Proserpine (gray) 1930:3 hy ("hittaheb) (chestnut with rubicau) and (evele (ehestnut, but I cannut sale if with rubican).

In the General stud Bowk, Volume .X... I have found two catses, and in Volume 111. three rases, in which brown foals have been proclueded by (hentinut parents:- -

1. Ofiertore 11. (brown) Jang by Kirkham out of Geld Ware.

?. II avemore (brown) 190:2 by Ocean 11 alde out of Malke More.

3. Captain Candid (brown) lsibl by Cerberus out of Mandane.

1. Ealla (brown) 1sis by Stripling out of Maniale.

j. Barnest (brown) lon:i by Buzard out of Mandane.

keferring to the first case. it must be mentioned that colded Wate is by

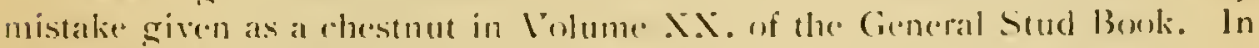

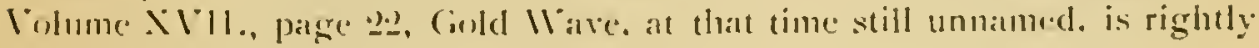
put down as a brown. 
Referring to the second case, the colour of Wave More, who never trod on a racecourse, is either wrongly given, or the dam. Make More (chestnut). has been confused with her own sister, born 1891, who was brown.

In the third case, I suspect that Orville (brown), who during the two years previously covered Mandane, also covered her again in 1812 as well as Cerberus, and that the owner of Cerberus did not mention this covering. which, it seems, was formerly, for different reasons, often omitted. Captain Candid ran second in the St. Leger.

Referring to the fourth case, it must be mentioned that Elba is by mistake given as a brown in Vol. $11 \mathrm{I}$., page 139. In Vol. III., page 67, and in the Racing Calendar 1.918 , pages .5 and 147 , Elba is rightly put down as a chestnut.

In the fifth case. I suspect that Whiskey (brown), who covered Mandane in 1803 and 180.5 , also covered her in 1.804, and that the owner did not mention this covering.

Many cases in the private studs of East Prussia, in which brown foals are said to be the offspring of chestnut parents, have proved, on closer examination, that their respective dams had been also covered by another stallion who was of a brown colour. Mso that this second stallion, either by mistake or for certain reasons, was not mentioned in the certificate of serving (only the covering for one stallion, and that the dearer one, is paid for).

In the appended pedigrees of the best linown Thoroughbreds, I have given the colours where possible (only in the (ierman edition). I have not come across any case in which chestnut parents had no chestnut fouls. The remarkably certain transmission of the chestnut colour is even completely independent of the colour of the ancestors. There are also cases in which all four grand-parents were browns and ret the two chestnut parents always produced chestnut foals, as for example, with Kinesem, The Biddy (granddam of Regalia), and many others. With Maintenon (a chestnut) it must be pointed out as a remarliable circumstance that of the four grand-parents two were brown and two grays, and with Rouge Rose. also a chestnut, three grand-parents were brown and one black. The certain transmission of the chestnut coat colour can still be more clearly recognised with the following chestnuts:-Diomed, Gladiator, Favonius, Hermit, Trampoline, Cambuscan, Pilgrimage, and Merry Sunshine, where not only the two parents, but also the four grand-parents, were brown. In the three last-mentioned cases, of eight great grand-parents, only one of each was a chestnut. The case of the chestnut Le Sagittaire is remarkable, whose sire, Le Sancy, was a gray, and the dam, La Dauphin, was a roan (not a gray as given by mistake in the French Stud Book). Niso Dryad (chestnut, and great grand-dam of Delphos), whose two parents, four grand-parents, and eight great grand-parents, were either brown or gray. In the case of Old Heroine, born 1775 (in Birdcatcher's pedigree), we may well take for granted that one of the grays was a roan, or that Old Hernine was a rubican. 
When both parents are brown, foals may be of any colour, also gray if one parent is rubican. The majority of foals, however, will likewise be brown. If the parents are of different colours, in most cases the lighter colours are more easily transmitted than the darker ones. Grays transmit their colour the most frequently and blacks the least frequently. Chestnut and black produce most often browns, chestnuts less frequently, and least seldom of all, blacks. Brown and black produce more browns and dark browns or brownish blacks than blacks. Chestnuts and dark browns produce more chestnuts. Chestnuts and light browns produce more brown, and often a dirty chestnut colour. Chestnut colour is the most suitable to get rid of the gray colour. Some of the foals will be brown.

In consequence of the very sure transmission of the chestnut colour, the chestnuts are easiest to renew in Jonasthal. They need not give anything away from their production, and are even increased by additions from the black and brown stud. The Royal Stud of Trakehnen in East Prussia is about 11,200 acres large, and is composed of 12 stud farms, among which, Gurdszen, with $90-100$ black mares: Danzkehmen, with $70-80$ bay or brown mares; Jonasthal, with $50-60$ chestnut mares: Bajohrgallen, with 60-70 mares of different colours; and Trakehnen itself, with 80-100 mares of all different colours, also gray, roan, and piebald-total 3.50-110 brood mares; with all foals -riding, carriage, working horses-altogether 1500-1700 horses.

Remarkable to say, these statements are in opposition to the fact that the chestnuts do not increase amongst Thoroughbreds, and are always in the minority, although the most celebrated foundation sire, Eclipse (of two brown parents), and his four sons, Potsos, King Fergus, Mercury, and llexander, were chestnuts; just as the famous stallions which appeared later, i.e., Birdcatcher, The Baron, Stockwell, Blair Athol, Doncaster, Bend Or, St. Ilbans, Sainfoin, Thormanby, Hermit, and many others, were also chestnuts. In the three principal Jinglish races, the coat colour is distributed as follows to the winners and seconds, up to and inclusive of 1907 :-

$$
\begin{aligned}
& \text { Derby Winners: } 94 \text { Browns, } \\
& 32 \text { Chestnuts, } \\
& 1 \text { Black, } \\
& 1 \text { Gray, }
\end{aligned}
$$

lotal 128 horses as winners

Oaks Winners : 95 Browns,

31 Chestnuts,

1 Black,

1 Gray,
Seconds : 90 Browns

30 Chestnuts

3 Blacks

5) Grays

and 128 horses as seconds.

Seconds: 8.j Browns

37 Chestnuts

5 Blacks

2 Gray's

Total 129 horses as winners

and

129 horses as seconds. 

St. Leger Winners: 102 Browns, 27 Chestnuts, 1 Black, Seconds : 98 Browns 2 Grays. 31 Chestnuts
1 Black Grays

Total 132 horses ats winners and 132 horses as seconds.

This uniform distribution of the coat colour seems, especially when we consider the certion transmission of the chestnut colour, to point to a special racing capability in conncetion with the brown colour. A classification according to decades shows a regular diminution of the chestnuts and an increase of the browns ameng the winners and the seconds. It is remarkable that the predominant colour of the Yorth-. Ifrican horses was bay or brown, and that, especially in recent years, the most important and best influence on the building up of the Thoroughbreds is ascribed to the North-ilfrican horses. It is furthermore remarliable that chestnuts mated to each other produce more colts, and black horses more fillies. 


\section{CH.MTIR VII.}

\section{The Art of Mating.}

TIIE most important thing in practioal mating at a stud is for the breeder to fatour the brood mares more than the eorering stallions, i.e., in the case of each brood mare he must consider with which of the avaitable sires she has the best chances of producing a grood foal, and not zice-iersu. People are very much inclined to give to the farourite sires the best mares, although the formere are still untried, whilst the latler have already shown their breeding capability. One very often forgets the natural demand that before mating, "specially in Half-bred breding, the special breeding result to be attained in tach single case must be 'learly pointed out, instead of which people often think that according to the result of the production one can fix its mission in every case afterwards. The uncertainty thereby caused in the judging of the special facturs of mating prevents a clear consideration of all necessary points of riew. One must endeavour to rqualise the faults of the brosed mares by corresponding merits of the covering stallions. "These faults which have to be equalised do not only consist in a faulty conformation, as for example, misplaced fore-legs, weak bocks, a too long middle part, high-leggedness, narrowness, atc., but also in defects of constitution (hard and tender), of temperament (hasty, idle, good-natured, malign, couratgonts and cowatrdy), of walk (wide all the knees, narrow at the liness, slow, and full of action), of performance (staver and flyer, action in gatloping, trotting. walking), of the use of food, and, finalle in lack of quality, beauty and vigour. Statistics serm to recommend the mating of wh sires with young mares, and icice-iersa. In order not to lose sighte of all these points of view, it is mot only neressary to have an exatet detailed linowledge of the brond mares and their previous foals, of the avaitahte sires and their progeny, but also of the aneesters. The rexersions to grand-parents and great grand-parents (.Itavism, as it is athed), are sol) frequent that at hreeder who does not duly consider them will have many bitter experiences. Furthermore, a knowledge of the ancestors is required as far as to rightly 
judge of the ancestors oceurring on both sides-representing, so to speak, a barb-i.c., to rightly estimate the basis of inbreeding when intending inbreeding with 3 to 6 free generations, which is most favourable for Thoroughbreds as well as for Half-breds. The more prominent, and the more suitable in any special case for a desired quality, the common ancestors are as a basis, the closer the inbreeding may be. It is specially farourable, if, as shown in the chapter on inbreeding, several inbreedings with different basis are passibie in one mating. Furthermore, eertain blood mixings, which experience has already proved to be good, must be preferred. This last point of view is especially important in breeding of Thoroughbreds, unless one breeds Thoroughbreds not for racing, but for riding and hunting. When mating Half-breds, one must first of all consider which breeding result, even which shade within the given breeding lines, seems to be most probable and most attainable in the case of each single brood mare. The object must not be too far removed from the type which the mare herself possesses. I very noble, wiry mare does not recommend herself for the production of a Carossier, and iicc-iersa, unless the foal is considered as the final product, and is not to be used for further breeding.

When mating, it is advisable to look at first only for the most suitable sires aceording to the pedigree of the brood mare and of the available stallions. For Thoroughbred breeding especially, the compilation of the pedigrees of the mares and of the sires, as well as of the basis of all their inbreedings, in lueid tables, is recommendable, so that one can easily see the possible and the best approved blood mixings, and that the possible and desirable basis of the inbreedings attained by mating may be found. When doing this, one will mostly find several sires which appear suitable. They must then be arranged according to the applicability which is recognised as the most suitable in exclusive consideration of the blood mixing. Then weigh the chances of same according to the other above-named points of view (deficiencies of conformation, constitution, etc.). Now begins the most difficult part of mating, the part which is considered an art, and for which no other advice can be given than to weigh exactly the advantages and disadvantages of each case of mating, and not to sacrifice in one case any more important thing than may be gained in the other. If the result of a mating decided on for good reasons does not turn out well, it is advisable not to proceed at once to another mating, but to try the same mating at least once more. Even with the best mating and the best breeding material, a small percentage will always go wrong. The fertilisation of brood mares is also surer if the covering stallion is not changed. $\mathrm{IT}^{\top} \mathrm{e}$ find the longest series of foals for the most part where the covering stallion has been changed very seldom, or not at all. Even old Fugger is of the opinion that mares conceive easier from that stallion they have been already bred to than by another. I consider the doctrine of so-called saturation wrong, for if it were right, for example, in the case of Thoroughbred breeding, many more half- 
sisters and brothers should have won classical races than own sisters and brothers, especially as the number of half-sisters and brothers is larger than that of own sisters and brothers. The following table shows that this is not the case :-

1. Derby I'inners.

(i) Own brothers and sisters.

1. Radamanthus

1787 i

2. Dädalus

3. Archduke

1. Paris

1791. by Justice and Flyer.

$1803\}$ by Sir Peter and Horatio.

5. I'halebone

6. Whisker

$1807)$

7. Lap Dog

S. Spaniel

1812 by Waxy and Penelope.

9. Persimmon

$1823\}$ by Whalebone-Canopus.

10. Diamond Jubilee 1893 )

1897 by St. Simon and Perditta.

(b) Ilalf-bruthers and Sisters.

1. Spread Eagle 1792 by Volunteer

2. Didelot

1793 by Triumpator - Highflyer.

3. Ditto

4. Pan 1800 by Sir Peter

5. St. Giles 1805 by St. George and Arethusa.

6. Bloomsbury 1836 by Mulatto $j$ and Arcot Lass.

7. Galtee More 1894 by Kendal I and Morganette.
8. Ird Patrick 1899 bi St. Florian

1. Spadille

2. I*. Flora

3. Touchstone

4. Launcelot

j. Lord Lyon

6. Achievement

7. Memoir

8. La Flèche

9. Persimmon

10. Diamond Jubilec
2. St. Leger IVinners.

(a) Own liruthers and sisters.

$178+1$

1785 by Highflyer and Jilora.

1831 ) by. Camel and Banter.

$1863)$

1864 by Stockwell and Paradigme.

1887 .

1888 ! by St. Simon and Quiver.

1893 \} by St. Simon and Perditta.

1. Van Tromp 1844 by Lanercost

?. The Flying Dutchman 1816 by Bay Middleton

3. Common

4. Throstle

1888 by Isonomy

1891 by Petrarch

and Barbelle.

and Thistle. 


\section{Oaks Winners.}

(a) Own Sisters.

1. Musis 1810)

2. Minuet 1812 by Waxy and Wroodbine.

3. Memoir 1887)

4. La Flèche 1888, by St. Simon and Quiver.

(b) Half-sisters.

1. Rhedycina 1847 by Wintonian

๑. Governess 1855 by Chatham $j$ - Laurel.

3. Spinaway

4. Wheel of Fortune 1876 by Adventurer $\}$ and Queen Bertha. 


\section{III.}

The Practical Part of Horsebreeding. 



\section{CHAPTER I.}

\section{Judging and Treating Breeding Material.}

\section{(a) Judging Covering Stallions.}

From each breeding horse, be it stallion or mare, one must demand, first and foremost, certain distinct merits, i.c., prominent parts. Only a breeding horse possessing specially prominent characters is justificd in having a few faults, and the less faults it has the greater they may be. The only unpardonable fault of a breeding animal is bad health and weakness connected with it. A too great fear of other faults, which cannot really always be avoided, is the surest way to inferiority. Of course, one will estimate the faults of breeding material differently, according to the object of the breeding in question; further, in consideration of the characters of the other brecding material: and finally according to sex.

In Half-bred breeding, a Thoroughbred sire which has little quality, and which was a distinct cypher on the course, is just as little to be recommended as a Half-bred stallion which is too light in the bone and not of sufficiently correct build. The former ought to transmit to his progeny principally capabilities and quality, and the latter strength and correctness. I myself prefer an untried Thoroughbred to a proved cypher. With the former there is always a chance that he perhaps might have done something of note on the course. Such an untried Thornughbred stallion which has produced prominently in East Prussian half-breedings was, for cxample, Cicero born 1882 by Chamant out of Liane, and perhaps also Kerl born 185:5 by Lord Colney out of Oceana. In Thoroughbred breeding only a few similar examples are known. At this moment only Golumpus and Y. Melbourne occur to me. With Thoroughbred as well as Ilalf-bred stallions, one great fault is less injurious than several small ones, especially if there are great merits together with the great fault. The best known example of former times for this is Soothsayer, born 1808, by Sorcerer nut of Golden 
Locks, who had a coarse club foot (Lymphangitis chronica). Only Bay Middleton, born 1883, whose dam, Cobweb, was a grand-daughter of Soothsayer, produced a few foals which also acquired a club foot. Further, the following are to be considered as great faults to be passed over:-A strong, misplaced forefoot, as in the case of Stockwell; a bad hock, as in the case of Birdcatcher and Saxifrage: roaring, as in the case of Chamant, Gallinule, Hawkstone (Euphony's sire), Torwärts (Ilalf-bred in Trakehnen), crooked fore-legs, as in the case of The Wizard (grand-sire of Optimus) and Hannibal; crib-champing, or gasping, as in the case of Euphony, Möros, and Mirmidone: strong wind-gall, as in the case of Marsworth; bad temperament, as in the case of Barcaldine, Friponnier, and Flugel (Halfbred in Trakehnen), etc. The progeny of the above stallions, in Thoroughbred and Half-bred breeding, has shown that even great faults may be combated with success, and that the blood streams of first-class stallions have been thus rightly preserved for breeding purposes. A great fault in walking-for example, a much misplaced fore-foot-is easier to eradicate by correct mating than an undecided walk without a great fault, as in the case of the Derby winner Ladas, who seems to walk sometimes close at the knee and sometimes wide at the linee, and who, almost without exception, transmits a bad walk. The fear of faults, especially distinct faults which any fool sees at once and criticises, has just as stagnating an effect in breeding aselsewhere in life, political or scientific.

Thoroughbred as well as Jalf-bred stallions must show the breeder distinctly for which parts he mar expect a prominent transmission, also specially suited for improvement, and for which parts he must be particular about when mating. One may obtain success in one direction, but seldom in several directions at the same time. A pronounced sire with a distinctly sharp build, a free movement from the shoulders, a courageous eye and good health, together with a thin mane, a thin, well-carried tail, shows at once a good covering stallion. Without going into the well-known demands for exterior, 1 would like to briefly mention that 1 prefer a too short neck to a too long one, and pasterns which are too long and soft rather than those which are too short and upright. Further, straight hind legs rather than those which are too curved, and a knee which is a little protruding to a knee which is too tapered.

From the table on page 185, it can be seen that the height of 15.3 lands to 16.1 hands is apparently the most farourable for Thoroughbreds. The same heights are also the best for Half-breds, if the object of the breeding is particularly aiming at capabilities. Greater heights are often demanded in many studs in order to satisfy customers, but, unfortunately, at the cost of capabilities. Here we again come across the old fight between appearance and the real thing. One may here see the danger; how that vanity, folly and want of taste may drive breeders into the production of 
appearances by producing exaggerated heiglts, which all modern improved breeds nolens iolcus aim at.

In Ialf-bred breeding as well as Thoroughbred breeding, I prefer, all things being equal, the flyer to the promounced stayer. For Hhoroughbred breeding it is certatinly more important that the stallion can travel quickly over short distances than that he can stay for long distances. The best stayers of recent times were: Gladiateur, Thurio, Fuxhall and Sheen, who, although they had good, and even the best chances, unly transmitted moderately. The phenomenal stayer, Fisherman, has not left one first-class sire behind him in Australia. Pronounced flyers over one mile who have transmitted well are, for example, Buccancer, lsonomy, Gallinule, Energie and Hannibal, etc. On account of their high class, flyers have alsn frequently won longer races, as for example, Isomony as a four-year-old. Also Chamant, who has equally well transmitted in Thoroughbred and llalf-bred breeding, was a distinct flyer, and proved himself as stuch, especially when winning the 2,000 Guineas, against the subsequent Derby winner, Silvio. Even the great Ormonde won his races gemerally by the tactics of a fler.

The best selection of chief covering stallions for llalf-bred breeding is especially difficult, because the objects to be attained are much more manifold than in the breeding of Thoroughbreds, which only are wanted for winning races. Of course, these difierent whects in Hali-hred breeding can easily be simplified by taking as the final aim of breeding a satisfactory supply of the country studs with comntry stallions, and not the sufficient capabilities and endurance of cavily remounts. The latter, however, is the chief and tinal purpose of the most important part of Prussian Half-bred breeding. Here again, however, as so often in life, especially in official life, the question arises: Shall the affairs be so conducted that people are first contented and cause no unpleasantness, or shall they be so arringed that one expects to gain the greatest advantage for the public welfaro to man's best linowledge and belief? The first method is the one usual in America, where men laugh at an idealist who desires to further the rasse, and, as they say, wants to male man happier than he can comprehend.

The less lhoroughbred breeding produces good and strong fundaments the more burning becomes the question to the llalf-bred breeder as to in which way he may do justice to this most important requirement in llalfbreds. The history of Half-bred breeds in (iermany, as well as in other countries, shous how bad-almost ruined many suds have becume by refinement and deterioration of the fundaments. That is the heel of achilles in our Half-breds, for one can scarcely werestimate the importance of a sood fundament for a soldier's horse. The worle of the breoder in this respect is the most important, as well as the most dificult one.

Unfortunately, one has tried almost everyhere cross-breeding with Draft breeds. The favourable results which have often in the first genera- 
tion been attained by this coarse crossing, have induced many breeders, even some Irish Hunter breeders, to continue breeding on this line. Even lrishmen, however, on their admirable breeding grounds, soon found out that the progeny of these coarse cross-breds was a failure, because it is just the coarseness and the wealiness of the Drafter, and the light fundament of the Thoroughbred, which most surely are transmitted. lrishmen have, in good time, got together the remains of their ancient old Half-bred, called draft horse (not cold blood), the best of which are found in County Roscommon. In more recent times they are again trying, with the help of the remains of their former native Haif-bred horse, to produce the same good and strong fundament which their horses formerly possessed to a greater extent, but which had been spoiled by mediocre Thoroughbreds and coarse Drafters.

In this strengthening of breeding material, it is at first necessary, in lreland as well as in Germany, to improve the fundament of brood mares - a very difficult, unpopular and unpoetical work indeed; because, if one strengthens, there is also brought into the breeding many ugly, unaroidable traits. One must not forget, however, that the ennobling and beautifying is afterwards the easiest work in Half-bred breeding. The most difficult work is to find or breed Half-bred stallions which may be used for the abovementioned purpose. Iccording to the actual constitution of our Half-breds and Thoroughbreds, the use of Thoroughbred stallions alone is not sufticient for making good progress. I further do not believe that one will be able to breed such a strong Half-bred stallion with the desired fundament direct from a Thoroughbred stallion. In my opinion two generations will be necessary to obtain by right mating, feeding and exercise, what is wanted. If the mating has succeeded and the soil fumishes the necessary bone-making food, one will be only able to obtain a good and strong fundament, such as we have in the Steppe horses, to perfection, by grazing lasting as long as possible, by exercise in the summer, and by a systematic daily long exercise in the winter.

If the breeder has succeeded in producing such a Half-bred stallion with a strong fundament, he must also have the courage to make good use of lim, in spite of some faults which he may undoubtedly have, and which the merest dilettante can easily recognise. These faults which must be passed wer will be mainly lack of beauty, harmony and quality, also. unfortunately often, a bad walk. The swinging and correct walk will most rertainly be bred in later, at the same time as the breed is ennobled by Thoroughbreds. Such a bony Half-bred stallion which has become big and strong in work will always, especially in its younger years, look angular and ugly. Such a stallion will rarely gain the affection of young breeders. These latter will for the most part try to attain agreeable ideals, such as are embodied in the noblest Thoroughbred, and will trouble themselves less with the wearying cares just mentioned. 


\section{(b) Judging Brood Mares.}

That I have said about stallions may also be said about the conformation of brood mares: ret in the case of the latter a greater length of trunk is allowed. eren if the middle part should suffer thereby. The feminine character alwills linds distinct expression with good brood mares. Vampire, the dam of liting Fox, with her conspicuous stallion neck and unfeminine coatrseness, helongs to the exceptions, and besides the 1899 Derby winner has not produced anything useful. Mares with pronounced hooked teeth are nearly always bad dams, and very often barren. Statistics further teach us that small, wiry mares are preferable to large, so-called show mares. If the dams of elasical winners are divided according to their conformation into the three military classes-Cuirassiers, Chlans and Hussars-the most approsed brood mares will be found in the last lot. Good brood mares of the Cuirassier class are few. Tergissmeinnicht and Pulcherrima, perhaps the best approved brood mares in Graditz. were only Hussar types, just as Sweet Fatie (dam of Flibustier, Amalie von Edel reich and VTaisenknabe), La Traviata (dam of Bauenfanger and Hochstapler), La Fille du Regiment (dam of Grimston and Primas). Cantata (dam of Hymenaus), Gorse (dam of Goura and Cuochope), Dirt Cheap (dam of Trachenbers), B Flat (dam of f'atul and Fiaterer). Of English mares may here be mentioned P'erditla (dam of two Derby winners), also Medora (dam of Zinfandel). Of Cuirassier upes which have prudued a Derby winner or similar, I only know-in lingland. St. Catien's dam, St. Lditha; in lreland, Mlorganette (dam of Cillte. More and . Ird Palrick): in France, Val D'Or's dam, Wandora. and lardy's dam, lirs and Graces; and in Germany, Zama (Hannibal's dam). Hippia, herself an Oalis winner, is also a big-framed Cuirassier horse, and yed sler rould not produce anything better than Cunnersbury by such a good sire as Hermit. I have made the same observations in the Half-bred breeding at Trakehnen. The so-called light riding horses in Tralielmen supply the best cowering stallions. The strongest brood mares in Gurdszen supply fewer groud covering stallions, and more seldom still chief stud stallions. The dams of the chief stud statlions Polarstum. Prince Optimus and Mlter Herr, lad scarcely the calibre to be

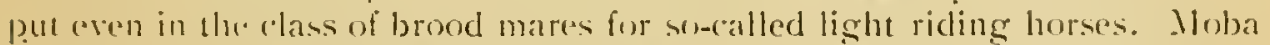
atso, the dam of Morgenstraht, belongs to the Hussar type, and the heights of some celeltratted hrond mares griven below support this therry. Linfortunately, I knum only the height of a few of them, and eren hese few, when compared with the leeights of prominent stattions given on page 18.;. show that the most favourable size for hrood mares is about 15.5 to $160 \mathrm{~cm}$. (15) hands 1 inch to 15 hands i) inches), therefore at least 5 $\mathrm{cm}$. less than the most farourable height of stallions. 
Heights of Famous Thoroughbred Mares.

\begin{tabular}{|c|c|c|c|c|c|c|}
\hline So. & Names of Mares & Born & Sire & Hands.' & $\begin{array}{l}\text { Size in } \\
\text { Inches. }\end{array}$ & cm. \\
\hline 1 & Queen Mab & 1785 & Eclipse & 1.4 & 3 & 149,9 \\
\hline 2 & M̄aniac & 1801 & Shuttle & 15 & $\ddot{\prime}$ & 157,5 \\
\hline 3 & Velocipede's dam & 1817 & Juniper & 15 & - & $152, t$ \\
\hline+ & Emma & $18 \pm 4$ & IIhisker & 15 & $1 \frac{1}{2}$ & 156,2 \\
\hline j & Bee's Wing & 18333 & Dr. Syntax & 15 & 2 & $15 \overline{1,5}$ \\
\hline ti & Crucifix & 1837 & Priami & 15 & 3 & 162,11 \\
\hline 7 & Pocahontas & 1837 & Glencoe & 14 & 3 & 149,9 \\
\hline 8 & Ghuznee & 1838 & Pantaloon & 14 & $: 3$ & 149,9 \\
\hline 9 & Miami & $18 H$ & V'enison & 1:) & 1 & 154,4 \\
\hline 10 & Lady Evelyn & $15+6$ & Don John & 15 & $1 \frac{1}{2}$ & $15 x i, 2$ \\
\hline 11 & Blink Bonny & 1854 & Melbourne & 15 & $2 \frac{1}{2}$ & 158,3 \\
\hline 12 & Impericuse & 18it & Orlando & 1.) & 3 & 160,0 \\
\hline 13 & Pulcherrima & 1873 & Beadsman & 15 & $\frac{1}{3}$ & 103,7 \\
\hline 14 & Pearlina & 1875 & Brown bread & 1.5 & 1 & $1.4,9$ \\
\hline 15 & Perditta II. & 18kil & Hampton & 15 & $\underline{-}$ & $15 \overline{5}, 5$ \\
\hline 16 & Haselnus & 1850 & Flibustier & $1 . ;$ & $1 \frac{1}{2}$ & $15 x i, 2$ \\
\hline 17 & Morganette & $185 t$ & Springfield & 16 & 1 & 165,1 \\
\hline 18 & Vitarba & 1854 & Rosicrucian & 15 & 1 & 154,9 \\
\hline 19 & Sappho & $18+1 ;$ & Wisdom & 1.) & 11 & $1.5,5$ \\
\hline 23) & Vision & 1856 & Plibustier & 1.5 & 11 & 155,5 \\
\hline 21 & La Filêche & $168 !$ & St. Simon & $16 i$ & - & 110,6 \\
\hline w & Medora & $18: 0$ & Bend $\mathrm{Or}$ & 15 & 2 & $1: 5,5$ \\
\hline 23 & Festa & 1893 & St. Simon & 15 & $\stackrel{2}{2}$ & $15 \%, 5$ \\
\hline 24 & Heln & $18 !: 3$ & Morion & 15 & $3_{i}^{3}$ & $10^{\circ}, 0$ \\
\hline 25 & Victoria & 19014 & St. Simon & 15 & 21 & $1,8,3$ \\
\hline
\end{tabular}

When purchasing or judging Thoroughbred brood mares, one must tabulate their pedigree in the female line for about four to five generations in order to easily and rightly estimate them. Is a contrast to Goos' tables, not only the classical winners, but all products of the female blood stream which have won races are noted, showing how often they have run and won. Of course, the classical winners are specially mentioned. Thereby one will often be surprised to find how few of the many offsprings in the respective female blood strean remain in the case of most mares. I give as an example of this the estimating table for the mare Thite Nun, formerly thus constructed. For want of space I have only gone back two generations. The type in bold letters means also ran or won as two-year-olds. Everything which had not won up to the sale of T'hite Nun has been left out, except where the progeny of a non-winning mare has won; for example, Wingrave Lass, as dam of Windthorpe. The sires of White Num, Lady Blanche, etc., stand above the respective mares. 
Hermit Thunderbolt

White Nun. 23. 4. Aady Blanche 27. 12.

1882

1868

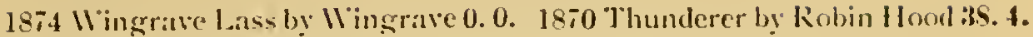

1883 Windthorpe by Tibtorpe 31. 万. 1886 Goneril by Thunderbolt 0.0.

1880 Spectre by Speculum 9. 1.

1883 Whitefriar by thermit 1S. 6 .

(Gratw. St. Gondw.)

18s:3 1)uchess of . Ilbainy 14.3. lengeance

(urdelial 15. 1.

1862

\section{(c) Age and Treatment of Covering Stallions.}

I caunot recommend the use of stallions for covering purposes before their fouth vear; firstly, because the stallions generally suffer in their development by reason of this early corering: and secondly, because also in Half-bred breeding I have never yet seen good foals produced by threeyear-old stallions. In Thoroughbred breeding also, I do not know a case if a successful use of a three-vear-old stallion. Only in . Imerican Trotter breeding I must mention the remarkable example of Hanbletonian (10), born 18.9, who produced as a two-rear-old in 18.5. Ibdallah (15). Abdallah (15) became the foundation sire of the celebrated Cresiens, born 1894, with a record of $2.02 \frac{21}{1}$. The question, up to what age the stallion may be used. depends mly how long he can cover and still fertilise. I have never yet observed at "Trakehuen a diminution in the quality of the foals due to the great age of the stallion as long as he remained healthy and was not used too much. In Thoroughbred breeding, however, the power of transmission seems to have decreased lately from the 19 th to the oth year, as may be seen from a previously given table (page s7), especially if one compares them with the performances of 19-year-olds, which are not here mentioned, but which are very favourable. In llalf-bred breeding statistics are so incomplete that one cannot arrive at any real conclusions. Many breeders in England contend that old stallions produe more stayers; further, old stallions and stallions which are much used produce generally more colts. When stallions are very much used, as sometimes happens in country studs, I have now and then observed that the foals hecome somewhat lighter, as for example, in Gudwallen with Jalm and Farnisch, and in Trakchnen with Optimus. Fertility decreases easily if stallions are too mueh used in their routh, otherwise old stallions fertilise as long as they are healthreven often better than roung stallions (as already. Iristotle and Fugger have taught us). The often observed worse transmission, as well ats worse fertilisittion, on the part of young stallions, especially those who have run much and for long, is, in my opinion, caused by the fact that the transition from the course to the stud is too sudden, and therefore injurious to health. The sensible and good treatment of young covering stallions (especially at liaton Hall, the Dule of Westminster's stud), has led in the following cases to good results in the first covering year :- 
1. Doncaster--Bend Or-Ormonde and Kendal.

2. Ormonde - Orme, l, lantony and Goldfinch.

3. Fying Fox-Ajax and Gouvernant.

4. Galtee More-Irish Lad.

5. St, Simon-Memoir, Semolina and St. Serf.

6. Persimmon-Sceptre and Cheers.

7. Flageolet-Rayon D'or.

8. Wild Dayrell-Buccancer.

9. Orlandn-Teddington.

10. Florizel-Diomed.

11. Highfler-Rockingham.

The health of the corering stallions is, therefore, one of the most important problems of the breeder. Firstly, there must be a sensible transition from the condition of training to that of covering. Stallions which have been accustomed to rugs must be slowly broken off the habit, of course. in the warm season, and always with the proviso that the stable temperature in winter does not sink below $5^{\circ} \mathrm{C}$., and is not generally less than $8^{\circ} \mathrm{C}$. Furthermore, a borse in training is used to much worls. The sudden stoppage of all work is therefore injurious to health. When, of course, quick work may be left out without injury, the roung covering stallion ought to do sufficient stow work, to which also short canters belong. To sum up, the young corering stallions must do daily fully 2012 hours' exercise. As long as Old Chamant was in Beberbeck he did his canters daily up to his soth year: but, of course. I do not wish to imply that every stallion should do cantering work up to that age. Ilere again the individualising breeder's eve must decide how much work would be beneficial to any single stallion. If, for example, such a full-blooded and phlegrmatic stallion as Minting, when he commenced curering, had done his cantering work for the $2 \frac{1}{2}$ hours necessary daily, he would not have got founder, and he would very likely not have failed in his lirst year as a covering stallion, and would very probably. have transmitted better according to his magnificent form as a racehorse and to his perfect exterior. Lingland sins most in this respect, by often taking things too easily, I believe, and many excellent Thoroughbred stallions have perished early in comsequence of having had ton little exercise. Old Wollstein already speals energetically against the insulficient exercise of covering stallions, and says linally: "If you malse doctrines, make them so that you yourselves are not ashamed of them, and that eou do not injure the animals. Ask the beast itself, and from it ynu will learn." If cantering dres not seem any more necessary for the cosering stallion, the best thing to recommend is to lead him in ermpany with another stallion, and in summer to put him in a paddock with an adjoining box. It is very uselul for the corering stallions to be able to see, in the box as well as in the paddock, their neighbouring stallions. Nothing is oo (onducive to malie covering stallions vicious than complete iscration. For llali-bred stallions 1 consider it very useful to con- 
tinue the training of four and five-year-nlds for about four months after the covering season, in order to present premature ageing and fattening, to which the best and healthiest Ialf-bred stallions are mostly prone.

The quantity of food, as well as exercise, must be regulated to suit individual cases. During the covering season 1 recommend daily 10 to at the most $15 \mathrm{lbs}$. of oats $(1 \mathrm{lb}$. of oats=albout 1 litre) in four rations, and about 10 to $15 \mathrm{lbs}$. of meadow hay (not clower haly): twice weclily, wheat bran, about 2 litres, as a mash, with oats mixed for the erening foud. Early in the spring, for about four weeks whilst casting their coats, daily abuut $\frac{1}{2}$ th. linseed ground or roasted (about a handful to each feed of oats). After the covering season in summer, about fi to $10 \mathrm{lbs}$. of oats, and als long as it is arailable, green lencerne, or green clower with Timothee. In the case of green food special attention must be paid to its being chopped twice daily and eaten fresh at once. During green food season leave out the wheat bran. Young Half-bred stallions which are still kept in training set, of course, more oats, just the same as horses in training. They must, however, get the green food just the same as the others, unless racing is intended. In the autumn, when green food is no longer available, give them, for about four weeks daily, about 6 to 8 litres fresh carrots finely chopped. Of course, a change from one ration to another must be sradual.

\section{(d) The Stallions whilst Covering.}

In my opinion, it is most advantageous that one attendant should tead the stallion when covering. If two attendants, one on each side, guide the stallion with a cavesson rein, they must both be well trained, sensible and attentive in order to avoid awkward or too sudden pulling or tightening of one or both cavessons when the stallion has w be taken or kept bick. Not only is it easy for accidents in happen "ith two attendants, but a stallion gets resed much easier, as the most important personal and friendly relation and agreement is only possible with one attendant. It is experially much better to have only one attendant leading when teaching young stallims. Besides the leading attendant and the man who holds the mare, there is only another attendant required to stand at the risht side of the mare and give the necessary assistance. He must pull the mare's tail to one side before the stallion mounts, and when necessary help on the right thigh the stallion whilst he covers, whilst the leading attendant helps him on the left thigh. To guide the penis of the stallion into the vagina is the duty of the leading attendant, but only as far as is reguired, and with the view of gradually educating the stallion to do it finally himself. The old precept of leading the sallion once before the coscring in a circle round the mare is rery sensible. This action has the effect of quietening and educating the stallion, the mare does not become so easily frightened, and the corering is done guietly and in the place desired and prepared. Very likely this nde precept "iss originally 
based on the idea that the good-looking stallion would influence the mare in producing a tine foal. It is, of course, essential that there should be the greatest quietness during the covering, and that there should not be many people, and no dogs, etc., about. I would like to mention one thing more, riz., that mounting is facilitated if the mare stands with her fore-feet on higher ground. Of the about 200 stallions which I have seen taught to cover according to the above rules, I have never yet found one which did not learn it in one or two days, with one exception, when it took eight days. Finally, in all these things, old stud attendants have more experience, and in difficult cases always more practical remedies than 1 am in general able to propose. In order to prevent the possible transmission of infectious matter of aburtion by the penis of the stallion, I recommend that same should be thoroughly washed immediately after covering with wadding soaked in a solution of chinosol 1 per 1,000, for which purpose the penis must be drawn out long with the hand. Of course, during this manipulation one cannot expect two much patience from the stallion at first, and must accustom him to it by degrees. I never yet found a staflion which did not get used to it in a very short time. To merely rinse the penis with an irrigator is not sufficient disinfection.

The best time for covering is about half an hour after the stallion has been exercised, viz., in East Prussia, in winter about 9 o'clock, and in summer about 7 oclock in the morning, and if twice covered, in the afternoon at 3 or 5 oclocli. Stallions must not cover immediately after having had their wats; there must be at least an interval of one lour. When stallions first begin covering it is advisable to let then cover only three or four times per week. Later on, in the case of most stallions, it is most advantageous for most stallions to cover once daily without missing. A socalled rest day, i.e., when no covering is done, results in most cases in the stallion making more elfort than is necessary the day after, which does hin more harm than the rest day has done him good. A rest day might be useful on the day following that in which the stallion has covered twice. Fouryear-old stallions should never be expected to cover more than once a day in their first year of covering. Older stallions ought not to mount twice a day for more than two weelis together, as after that period it would do them harm. . Ifter several days with one mount per day, two mounts daily can be repeated for two consecutive weeks. Here again, however, individuality must be the deciding factor. In the cases of especially valuable chief covering stallions I would recommend never to let them mount twice daily for more than three to four days conserutively, and then for the same number of days once daily. The over-exertion of stallions by corering twice daily for several months together, which is frequently unavoiclable in many country studs, not only causes an early infertility of the stallions, but, as already mentioned, their progeny becomes lighter. From their 20 th year the productive capacity of nost stallions gradually decreases, and for that reason valuable stallions which 
are 20 years or older must only in exceptional cases (two to three times monthly) be allowed to cover twice in one day. Slallions are most liable to refuse to cover dark colotured mares, especially black mares. It is therefore advisable to have a light coloured mare, for preference a grey mare, to get the stallion ready, and at the last moment turn him on to the black mare; a trick which in most cases is successful.

Performances of Iambletonian (10) at the Stud.

\begin{tabular}{|c|c|c|c|c|c|}
\hline $\begin{array}{l}\ln \\
\text { the } \\
\text { letir }\end{array}$ & $\begin{array}{l}\operatorname{lgge} \\
\text { in } \\
\text { lears }\end{array}$ & $\begin{array}{l}\text { Number } \\
\text { of } \\
\text { Covered } \\
\text { Mares }\end{array}$ & $\begin{array}{l}\text { Fusts } \\
\text { lorn } \\
\text { Slive }\end{array}$ & $\left|\begin{array}{c}\text { ler- } \\
\text { centilge } \\
\text { of } \\
\text { lonls }\end{array}\right|$ & $\begin{array}{l}\text { Imonsst which are the following Celebrated } \\
\text { Sons. }\end{array}$ \\
\hline 1501 & 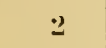 & 4 & 3 & 7.5 & . Ibdallah (15), Billy Denton (6j) : \\
\hline 1552 & 3 & 17 & 13 & 76 & \\
\hline $18 x^{2} 3$ & 4 & 101 & 78 & 7 & Volunteer (jJ) \\
\hline 154 & $\pi$ & 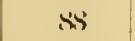 & 6i2 & 71) & lidnard liverett (\$1) \\
\hline 185.5 & fi & $(4)$ & 61 & 72 & George llillies $(\bar{s} 19)$ \\
\hline $18 \pi 3$ & $\tau$ & 87 & 64 & it & Dexter Record: 2. 17!! \\
\hline 1857 & $s$ & 87 & $(63)$ & 72 & \\
\hline $1 \sin$ & 9 & 72 & 54 & 7.5 & \\
\hline 1859 & 111 & 95 & 66 & 69 & Kobert Bonner (270) \\
\hline 1860 & 11 & 108 & 72 & is & Brumo \\
\hline 1861 & 12 & $9 s$ & lis & 69 & \\
\hline $180 \div$ & 13 & 128 & 111 & 70 & $\begin{array}{l}\text {. Ulministrator (357), Dictator (113), Happy } \\
\text { Medium (400), Harold (113), Chester, } \\
\text { Sentinel (280) }\end{array}$ \\
\hline 1503 & 11 & $1 \%$ & 92 & (i1 & $\begin{array}{l}\text { Jay Gould (197), Prosper (907), Idol (44), } \\
\text { I: Bruno }\end{array}$ \\
\hline 1864 & 15 & 217 & 148 & $6 s^{\circ}$ & $\begin{array}{l}\text { Kiniclierbucker (200), Messencer, Duroc (106), } \\
\text { Rÿadik (6.58) }\end{array}$ \\
\hline 166.5 & 16 & $1 ! x$ & $1 \geq x$ & (iii & $\begin{array}{l}\text {. Berdeen (25), General Stanton (2545), Socrates } \\
\text { (28i), Strathmore (408), Small Hopes }\end{array}$ \\
\hline 1866 & 17 & 105 & 75 & 71 & $\begin{array}{l}\text { Banker (4114), Bismarck (67), Blackstone }(72) \text {, } \\
\text { Dencation (889), loloricla (482), Bolton }(76) \text {, } \\
\text { Sweepralies (298) }\end{array}$ \\
\hline 1.407 & IR & 72 & 12 & jot & $\begin{array}{l}\text { Cuyler }(100) \text {, Elechionecr (125). Enfield (128). } \\
\text { IIasteriode (595), Drift (522). IValtham }(685)\end{array}$ \\
\hline 1 sat & 19 & $n u t$ & given & & \\
\hline 1.869 & 24) & 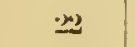 & is & 82 & \\
\hline 1.870 & 21 & 2 & 16 & 73 & . Iuditor $(7 / 3)$ \\
\hline 1871 & 些 & 30 & 26 & $s \bar{t}$ & \\
\hline 1872 & 23 & ;) & 21 & (⿻⿱⿱一口⺕亅⿱丿丶刂 & Irthurton (\$65), Irtillery ( 50 ). Lisber \\
\hline 157.3 & 21 & 31 & ध) & $6 ;$ & Meredith (136i) \\
\hline 1874 & 25 & $: 2$ & 21 & 75 & E.splere (1136) \\
\hline 1875 & 26 & 24 & 2 & 8 & \\
\hline otal & & 19130 & $1: 3 \times 3$ & 6!) & \\
\hline
\end{tabular}


As example of the capabilities of a stallion in the case of reasonable management - as is found oftener in America than in England and Irelandthe abore-mentioned Trotter. Hambletonian (10), born 5th Nay, 1849, by Abdallah I., is worthy of note. It may be especially noticed in the table on the preceding page that the percentage of fertilisation and the quality of transmission has not decreased after the 20 th year in spite of very great use (15 years old 217 mares). I Iambletonian died in Mlarch, 1876 , when 27 years old, and left behind 150 sires, with 1,490 descendants, which had a record of 2.30 and better, as well as 80 brood mares, with 110 descendants, which had also a record of 2.30 and better.

Furthermore, the Thoroughbred stallion, Red Prince II., by Kendal, has, for example, covered as a 19-year-old in four and a half months in Trakehnen 74 mares in 88 servings, and fertilised 66 of same, i.e., 89.2 per cent. Hereby it must be mentioned that Red Prince came to Trakehnen from lreland only on the Sth January, during a temperature of $30^{\circ} \mathrm{C}$., and the covering season finishes at the end of May.

\section{(e) Age of Brood Mares.}

The best age at which to cover mares is three years, except in cases where three-year-old Thoroughbred mares are still in training and have to run races, then it is better to put off the covering for another year. The same applies to Half-bred mares if they as three-year-olds are still subject to regular training, or as riding or carriage horses do so much work that they do not get fat and come too often in heat, in spite of good food. The best results as regards the number of foals and perhaps also as regards their quality are, as previous tables (pages 56-63) show, found on an average with mares which are covered and were in foal as three-year-olds. This applies to Thoroughbred as well as to Half-bred breeding. I only know one mare in Germany which has dropped 20 living foals, namely, B Flat, which was covered with success as a three-year-old. According to experiences at Trakehnen, mares which produce the first foal in their fifth year, or later, remain in the following year barren much oftener than mares which have been successfully covered as three-year-olds. Besides, the latter are, almost without exception, better nurses. Wollstein thinks that mares which bear their first foal as four-year-olds have the following advantages when compared with mares which have been covered later in life:-

1. The births are easier.

2. Mares are more fertile and have morc milk.

3. The foals are bigger and stronger.

The most prominent products of mares, on an average, are produced between their eighth and thirteenth year, as can be casily seen for Thoroughbred breeding from the Goos' tables. Goos says in the preface of his celebrated tables that the winners of the five classical races in England are distributed as follows :- 


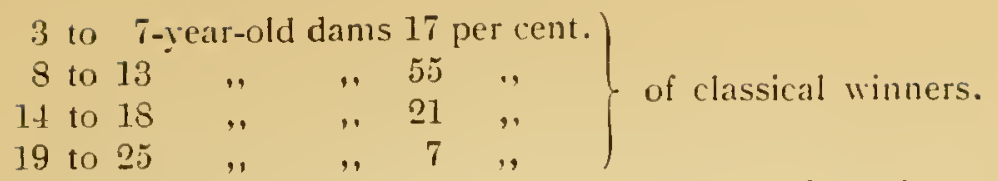

As experience at Trakehnen shows, 8 to 13 years of age is also the best and most fertile time for Half-breds. In Half-breds, however, the brood mares, up to their 7 th year inclusive-as opposed to Thoroughbreds-seem to be much more fertile than between the age of 14 and 18 . 20-year-old brood mares have up to now produced three Royal Stud stallions only, those older than 20 none at all.

Miscarriage or barrenness of mares in the first three to five years of their being made brood mares is less dangerous than if occurring in later years. Of course, this is always bad. Brood mares at the age in which, as above said, the best products are expected from them, i.e.. 10-year-old and over, have more seldom the power to overcome these unfavourable derangements of their breeding career than younger mares. Prominent products of Half-bred mares over 20 years of age are not linown to me, whilst, as shown in the previous tables (pages 65-78), in the breeding of Thorouglibreds also, 24rear-old and older brood mares have produced classical winners or other prominent progeny.

\section{(f) Twins.}

Mares which have produced twins, dead or living, are in most cases, however undesirable any single case may be, considered specially valuable brood mares, from which prominent progeny may be expected. Unfortunately, win births frequently recur, as for example, with the Oals winner Bronce, four times; with Legend born 18.5 by Merlin, and granddaughter of the celebrated Prunella, three times consecutively; and with Fairy Ring born 1878 by Macaroni, also three times consecutively. This quality is often hereditary, and there are many examples of it in "Thoroughbred breeding as well as in llalf-bred breeding.

In the last twenty-five years, from 1874 to 1898 inclusive, 33 Royal Stud stallions have been born in Trakehnen, of which the following 11 stallions are derived from dams which also had twins:-

1. Tuanel, born 187t by The Dulie of lidinburgh and Tutti.

-. Paladin, born 187t by Adonis and Palme.

3. Discant, born 1877 by Filigel and Diana.

4. Venezuela, horn 1878 by Hectur and Vierech.

5. Elfenbein, born 1879 by Marsworth and Ellis.

(i. Passvan, born 1881 by Flügel and Palme.

¡. Granicus, born 1889 by Ǩingdon and Gradlitz.

8. Jenissei, born 1888 by Venezucla and Jemba.

9. Hydriot, born 1895 by lïüstenberg and Hydra.

10. Morgenstrahl, born 1896 by Blue Blood and Moba.

11. Justizminister, born 1898 by Boulevard and Justicia. 
There can as yet be no final judgment about the later born stallions, as their dams are still alive and are too young. Of the 1,297 brood mares mentioned in the Second Volume of the Trakelnen Stud Book, only 136 have up to now produced twins; nine mares twice, and two mares three times. In the First Volume of the Beberbeck Stud Book, 342 brood mares are mentioned, of which only 13 bore twins: amongst these 13 being the celebrated Optima, dam of the best stallion which Beberbeck has produced, namely, Optimus, who was for eight years up to his death used as a Royal Stud stallion in Trakehnen. Perhaps the best son of Optimus, born in Beberbeck, was Cardinal, born 189.) by Optimus and Cedar, who commenced with twins. Only one brood mare (Lucca) has produced twins in Beberbects twice. In one case both lived, of which one (Lollo) became a brood mare and also produced twins. A jas, a twin born in Beberbeck 1800 by Dreadnought and Augusta, became stallion in Neustadt. As already mentioned, only one mare in Germany has produced -0 living foals, amongst them the Derby winner Paul, and that was the Thornughbred mare B Flat (born 1864 by Orlando and Torment), who herself was a twin.

The number of twin births, according to observations made at Trakehnen in the last twelve years, varies very much-from $\frac{1}{3}$ per cent. to 3 per cent., average about $1 \frac{1}{2}$ per cent. of the mares in foal, and is higher in those years in which fertilisation has been favourable. It is worthy of note that in human twins also the average is just over 1 per cent.

In the General Stud Book, in the first sixteen volumes, 1,05.j mares are mentioned which hase produced twins. Of these 413 mares are mentioned in Goos' tables (3rd edition), a sure sign that they are amongst the most prominent for breeding purposes: an exceedingly good state of affairs. More than 80 prominent foundation mares in the Gous' tables are dams which have produced twins. From the following list of mares which have produced twins and also performed well in breeding, it can be seen that in England 13 Derby winners, 1j Oaks winners, 9 St. Leger winners, 10 Two Thousand Guineas winners, and 10 One Thousand Guineas winners are offsprings of mares which have produced wins.

A Few Thoroughbred Brood Mares in England which have Produced Twins.

\begin{tabular}{|c|c|c|c|}
\hline No. & Name and Pedigree & Born & Family and Progeny \\
\hline 1 & $\begin{array}{l}\text { Horatia } \\
\text { by Blank - Flying Childers }\end{array}$ & 1758 & $\begin{array}{l}\text { Famous foundation mare of Fam. } \\
\text { Grand-dam of Diomed D. and } \\
\text { Y. Eclipse D. }\end{array}$ \\
\hline 2 & $\begin{array}{l}\text { Letitia } \\
\text { by Highflyer - Natchem }\end{array}$ & 1783 & Foundation mare of Fam. 46 \\
\hline 3 & $\begin{array}{l}\text { Nimble } \\
\text { by Florizel and Bantipole }\end{array}$ & 1781 & $\begin{array}{l}17 \text { foals } \\
\text { Nilié O. } \\
\text { Foundation mare of Fam. } 32\end{array}$ \\
\hline
\end{tabular}




\begin{tabular}{|c|c|}
\hline No. & Name and l'edigree \\
\hline 4 & $\begin{array}{l}\text { Maid of Ely } \\
\text { by Tandemi - Ilerod }\end{array}$ \\
\hline j) & $\begin{array}{l}\text { Palmflower } \\
\text { by Weazle and columba } \\
\text { Twice twins }\end{array}$ \\
\hline 6 & $\begin{array}{l}\text { Mare } \\
\text { by Highflyer - Golufunder }\end{array}$ \\
\hline$i$ & $\begin{array}{l}\text { Mare } \\
\text { by Trentham and Citherea }\end{array}$ \\
\hline$s$ & $\begin{array}{l}\text { I. Giantess } \\
\text { by Diomed and Giantess }\end{array}$ \\
\hline 9 & $\begin{array}{l}\text { Rally } \\
\text { by "Trumpato" and leancy. }\end{array}$ \\
\hline 10 & $\begin{array}{l}\text { Whodbine } \\
\text { by Woudpeclier and l'uzzle (lived } \\
\text { to } 32 \text { years old) } \\
\text { lwice twins } \\
\text { lIalf-cister to Homby lans }\end{array}$ \\
\hline 11 & $\begin{array}{l}\text { Mare } \\
\text { by Potson and Edithat }\end{array}$ \\
\hline 12 & $\begin{array}{l}\text { Mare } \\
\text { by l'recipitate and I.ad! IIarriet }\end{array}$ \\
\hline 13 & $\begin{array}{l}\text { IIormby I,alss } \\
\text { by Buzzard and Puzzle (lived to } \\
32 \text { years old) } \\
\text { 11alf-sistor to Wombine }\end{array}$ \\
\hline 14 & $\begin{array}{l}\text { I.ady Jane } \\
\text { by Sir Peder and Panulina } \\
\text { Own siver to } 1 \text { lormione } O\end{array}$ \\
\hline 1.) & $\begin{array}{l}\text { Mare } \\
\text { by L'recipitate anel I: Tilfany } \\
\text { Twice twins }\end{array}$ \\
\hline
\end{tabular}

\author{
178:) 13 foals \\ Foundation mare of liam. 45 \\ 1787 Cockfighter $1 .$. \\ lian. 2 \\ $1789 \quad 12$ foals \\ l'ensioneer 1795 by Dunganon \\ Innes 1805 by Shuttle \\ Fam. 26 \\ 1789 Pantina (dam of Blïcher D).) \\ lam 4 \\ 1791 Sorcerer \\ Eleanor D. O. \\ Julia (dam of Phantom D).) \\ Cressicla (dam of . Intar 2. and \\ l'rian D.) \\ Mare by Walton (dam of Nicolo 2. \\ and Langar) \\ Finm, 6
}

17(m) Fimous foundation mare of Fam. 6a

17 .1 Music $\mathrm{O}$.

Iinuet $O$.

Fant. 1 a

$1794+12$ foals

Famous foumdation mare of Fam. $3 \mathrm{e}$

1795

IVizard 2.

Finm. 12 a

1796

12 foals

Morel $O$.

loim. 1 a

Timi Briscis O.

Mise Pooler (grand-elam of Harkinw:iy $f(0) .2 x)$

คำเท. ?

1797 loubon 1811 by Surcerer

Mare by Sincerer flam of Jack Spigot I..)

liam. 5 it 


\begin{tabular}{|c|c|c|c|}
\hline No. & Name and Pedigree & Born & Family and Progeny \\
\hline 16 & $\begin{array}{l}\text { Violante } \\
\text { by John Bull - Highfyer }\end{array}$ & 1802 & $\begin{array}{l}\text { Famous foundation mare of Fam. } 3 \text { a } \\
\text { Grand-dam by Decoy }\end{array}$ \\
\hline 17 & $\begin{array}{l}\text { Bronce O. } \\
\text { by Buzzard - Nexander } \\
\text { 'Twice twins } \\
\text { Own sister to Castrel, Selim and } \\
\text { Rubens }\end{array}$ & 1803 & $\begin{array}{l}\text { Mulatto } 1811 \text { by Sorcerer } \\
\text { Busto } 1812 \text { by Clinker } \\
\text { and } 2 \text { good foundation mares in } \\
\text { Fan. } 2 \text { d }\end{array}$ \\
\hline is & $\begin{array}{l}\text { Henrietta } \\
\text { by Sir Solomon - Woodpecker }\end{array}$ & 1807 & $\begin{array}{l}\text { Tarrare L. } \\
\text { Fair Charlote } \\
\text { Fan?. } 6\end{array}$ \\
\hline 19 & $\begin{array}{l}\text { Lady Grey } \\
\text { by Stamford - Bordeaux }\end{array}$ & 1806 & $\begin{array}{l}\text { Gustavus D. } \\
\text { Fam. } 7\end{array}$ \\
\hline 20 & $\begin{array}{l}\text { Mare } \\
\text { by Walton and Y. Giantess, who } \\
\text { also had twins }\end{array}$ & $181) 8$ & $\begin{array}{l}\text { Nicolo } 2 \text {. (twins) } \\
\text { Langar } \\
\text { Fan. } 6\end{array}$ \\
\hline 21 & $\begin{array}{l}\text { Mare } \\
\text { by Shuttle - Drone }\end{array}$ & 1809 & $\begin{array}{l}13 \text { foals. Famous foundation mare } \\
\text { in Fam. } 13\end{array}$ \\
\hline $2 \cdot 2$ & $\begin{array}{l}\text { Scheherazade } \\
\text { by Selim and (21-year-old) Gipsy }\end{array}$ & 1810 & Foundation mare in Fam. $10 \mathrm{~b}$ \\
\hline 3 & $\begin{array}{l}\text { Selima } \\
\text { by Selim and a Nare by Pot8os, } \\
\text { who also had twins }\end{array}$ & 1810 & Famous foundation mare in Fan. $3 \mathrm{e}$ \\
\hline 24 & $\begin{array}{l}\text { Miss Craigie } \\
\text { by Orville and Marchioness } \\
\text { Twice twins }\end{array}$ & 1811 & $\begin{array}{l}\text { Birmingham L. } \\
\text { Foundation mare of Fam. } 34\end{array}$ \\
\hline 25 & $\begin{array}{l}\text { Sunflower } \\
\text { by Castrel - Alexander }\end{array}$ & 1813 & Foundation mare in Fam. 4 a \\
\hline 26 & $\begin{array}{l}\text { Mare } \\
\text { by Clinker and Bronce O., who } \\
\text { herself liad twins four times }\end{array}$ & 1816 & Famous foundation marc in Fam. $2 \mathrm{~d}$ \\
\hline 27 & $\begin{array}{l}\text { Mare } \\
\text { by Catton and Altisidora L. }\end{array}$ & $18 \div 3$ & $\begin{array}{l}\text { Ralph. 2. Cn. Acp. } \\
\text { Fam. } 11\end{array}$ \\
\hline 28 & $\begin{array}{l}\text { Sylph } \\
\text { by Spectre and Fanny Legh }\end{array}$ & $15: 4$ & $\begin{array}{l}\text { Lugwardine } \\
\text { Newcourt } \\
\text { Lady Lift (dam of Consul FD., } \\
\text { Mazarin, Le Marechal) } \\
\text { Fam. } 35\end{array}$ \\
\hline 29 & $\begin{array}{l}\text { Turquoise } O \text {. } \\
\text { by Selim and Pope Joan }\end{array}$ & 182.5 & $\begin{array}{l}\text { Jericho } \\
\text { Fam. } 1 \mathrm{~b}\end{array}$ \\
\hline $3 n$ & $\begin{array}{l}\text { Variation } O \text {. } \\
\text { by bustard and Johanna Soutin- } \\
\text { cote }\end{array}$ & 1827 & $\begin{array}{l}\text { Pompey } \\
\text { Elphine, famous foundation mate in } \\
\text { Fam. } 9 \mathrm{~b} \text { and dam of Warlocli } 1 . ., \\
\text { Phantom, ctc. }\end{array}$ \\
\hline
\end{tabular}




\begin{tabular}{|c|c|c|c|}
\hline Xo. & Vame and Pedigree & Burn & Family and Progeny \\
\hline 31 & $\begin{array}{l}\text { Zarina } \\
\text { by Norisco and Ina }\end{array}$ & $18: 27$ & $\begin{array}{l}\text { The Prime Warden } \\
\text { The Cur C's. } \\
\text { Fam. } 17\end{array}$ \\
\hline 32 & $\begin{array}{l}\text { Progress } \\
\text { by"Langar — Blacklock }\end{array}$ & 1833 & $\begin{array}{l}\text { Ittila 1). } \\
\text { Fann. 15 }\end{array}$ \\
\hline 33 & $\begin{array}{l}\text { Miss Fitty Cockle } \\
\text { ly Cadland and Maid of Mansfield } \\
\text { Twins twice }\end{array}$ & 1834 & $\begin{array}{l}\text { Truth Cm. } \\
\text { Fann. } 11 \mathrm{~b}\end{array}$ \\
\hline 34 & $\begin{array}{l}\text { Virginia } \\
\text { bọ Rowton and Pucelle }\end{array}$ & 1835 & $\begin{array}{l}\text { Virago 1. Gep. Dep. and Sacrifice, } \\
\text { grand-dam of Devotion, famlus } \\
\text { foundation mare in Fanl. tal }\end{array}$ \\
\hline 35 & $\begin{array}{l}\text { Black Bess } \\
\text { by Camel - Seud }\end{array}$ & 1837 & $\begin{array}{l}\text { Hernandez } 2 . \\
\text { Bataglia. good foundation mare in } \\
\text { Fam. } 6 \text { il }\end{array}$ \\
\hline 36 & $\begin{array}{l}\text { Crucifix } 2.1 . \text { O. } \\
\text { by: Priam and Octaviana }\end{array}$ & 1837 & $\begin{array}{l}\text { Surplice D. L. } \\
\text { Cow] } \\
\text { Chalice (grand-dam of I'lacida O.) } \\
\text { Fant. 2c }\end{array}$ \\
\hline 37 & $\begin{array}{l}\text { Monstrosity } \\
\text { by Plenipotentiary and Puce }\end{array}$ & $18: 38$ & $\begin{array}{l}\text { The Lgly Bucls } 2 . \\
\text { Foundation mare in Fam. \& a }\end{array}$ \\
\hline 35 & $\begin{array}{l}\text { Moonbeam } \\
\text { by Tomboy and Lunatic }\end{array}$ & 1835 & 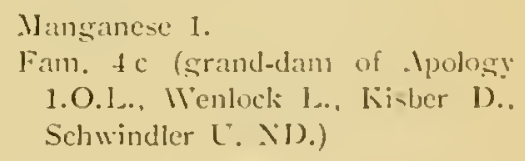 \\
\hline 39) & $\begin{array}{l}\text { Pesgy } \\
\text { by IIfuley . Joloch and Fanny }\end{array}$ & 1810 & $\begin{array}{l}15 \text { fuals, amongst others .Musjid D. } \\
\text { Fann. } 6\end{array}$ \\
\hline 40 & $\begin{array}{l}\text { Emerald } \\
\text { by Defence and Emiliana }\end{array}$ & $18+1$ & $\begin{array}{l}\text { Mentmore Lass 1. } \\
\text { Fans. } 3 \text { b (dam of Hammah 1. O. L. } \\
\text { and grand-dam of Favonius D.) } \\
\text { Fing of Diamonds }\end{array}$ \\
\hline 41 & $\begin{array}{l}\text { The Landerravine } \\
\text { by. Elis and The Nargravine }\end{array}$ & 18.11 & $\begin{array}{l}\text { Landgrave } \mathrm{Cm} \text {. } \\
\text { Sittingbourne } \\
\text { Maid of lient } \\
\text { Fam. } 5 \mathrm{~d}\end{array}$ \\
\hline 42 & $\begin{array}{l}\text { The Princess O. } \\
\text { by Slane - I'hantom }\end{array}$ & 1811 & $\begin{array}{l}\text { The Great [nknown } \\
\text { Fam. } 1 \text { c }\end{array}$ \\
\hline 43 & $\begin{array}{l}\text { Bridle } \\
\text { by 'The Saddler and Monoeda }\end{array}$ & 1844 & $\begin{array}{l}\text { Habena } 1 . \\
\text { Jady Sophic. famous foundation } \\
\text { mare in Fan. } 9\end{array}$ \\
\hline 4 & $\begin{array}{l}\text { Ellen Horne } \\
\text { bỵ Redshank and Delhi }\end{array}$ & 184 & 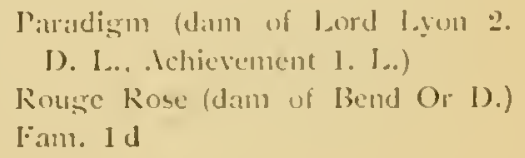 \\
\hline
\end{tabular}




\begin{tabular}{|c|c|c|c|}
\hline No. & Name and Pedigree & Born & Family and Progeny \\
\hline 15 & $\begin{array}{l}\text { Gaiety } \\
\text { by "uchotune and Cast-steel }\end{array}$ & 184 & $\begin{array}{l}\text { Gamester L. } \\
\text { Gadabout (dam of Scamander) } \\
\text { Doncaster } \\
\text { Fan. } 19\end{array}$ \\
\hline 16 & $\begin{array}{l}\text { Mare } \\
\text { by Hampton - Muley Moloch }\end{array}$ & 1844 & $\begin{array}{l}\text { Gaspard } \\
\text { Odd Trick } \mathrm{Cm} . \\
\text { Mad. Stodare } \\
\text { Fam. } 2 \text { a }\end{array}$ \\
\hline 47 & $\begin{array}{l}\text { Mainbrace } \\
\text { by Sheet Anchor - Bạ. Middleton }\end{array}$ & 1814 & $\begin{array}{l}\text { Fisherman Aep. } 2 \times \\
\text { The Peer } \\
\text { Fam. } 11\end{array}$ \\
\hline is & $\begin{array}{l}\text { Prairie Bird } \\
\text { by. Touchstone and Zillah }\end{array}$ & 184 & $\begin{array}{l}\text { Famous foundation mare in Fan. } 1 \text { b } \\
\text { England's Beauty } \\
\text { Bonny Blink } \\
\text { Vitula }\end{array}$ \\
\hline 49 & $\begin{array}{l}\text { IIיbla } \\
\text { by The Prowert and Otisina }\end{array}$ & 1846 & $\begin{array}{l}\text { Mincemeat } \mathrm{O} \text {. } \\
\text { Kiettledrum } \mathrm{D} \text {. } \\
\text { Fam. 3 d }\end{array}$ \\
\hline$\therefore 0$ & $\begin{array}{l}\text { C.egerdemain Cs. } \\
\text { by Pantaloon and Decos } \\
\text { Own sister to Sleight of Hand. } \\
\text { Van Amburg and Phryne }\end{array}$ & 1846 & $\begin{array}{l}\text { Toxophilite } \\
\text { Sagitta } 1 . \\
\text { Fair Star } \\
\text { Fam. } 3 \text { a }\end{array}$ \\
\hline 5l & $\begin{array}{l}\text { Mrs. Hobson } \\
\text { by Bay Midlleton and Vitula }\end{array}$ & 1816 & $\begin{array}{l}\text { Orestes } \\
\text { Pylades } \\
\text { Janus } \\
\text { Fan. 2] }\end{array}$ \\
\hline 52 & $\begin{array}{l}\text { Bassishaw } \\
\text { by Prime Warden and Miss } \\
\text { Whinnie, whose srand-dam by } \\
\text { Shuttle had also twins }\end{array}$ & $18+7$ & $\begin{array}{l}\text { Ben Webster } \\
\text { Isoline Gep. (dam of 1sola Bella, } \\
\text { who also had twins, } \\
\text { St. Cristophe GG., Braconnier) } \\
\text { Fam. } 19 \text { a }\end{array}$ \\
\hline 53 & $\begin{array}{l}\text { Figtree } \\
\text { by Envor and Izora }\end{array}$ & 1848 & $\begin{array}{l}\text { Palm (dam of Vauban 2. Gep. and } \\
\text { Dulie of Parma Cs.) } \\
\text { Siberia 1. (dam of Cecilia 1., who } \\
\text { also had twins) } \\
\text { Westminster Cm. } \\
\text { Fam. } 2\end{array}$ \\
\hline 54 & $\begin{array}{l}\text { Fipolic } \\
\text { by Touchstone }- \text { The Saddler }\end{array}$ & $15+8$ & $\begin{array}{l}\text { Frolicsome (dam of Frontin Ff). } \\
\text { GP.) } \\
\text { Lady Sophia } \\
\text { Fam. } 24\end{array}$ \\
\hline
\end{tabular}




\begin{tabular}{|c|c|c|c|}
\hline .Nu. & Nime and Pedigree & Burn & Family and Procreny \\
\hline 55 & $\begin{array}{l}\text { Indiana } \\
\text { by Muley . Iloloch and Pocahontas }\end{array}$ & 1848 & $\begin{array}{l}\text { lientucky } \\
\text { llumming Bird } \\
\text { Fam. 3c }\end{array}$ \\
\hline 30 & $\begin{array}{l}\text { Mare } \\
\text { by The Cure and Elphine }\end{array}$ & 1819 & $\begin{array}{l}\text { The } 11 \text { izard } 2 . \\
\text { Fam. } 9 \mathrm{~b}\end{array}$ \\
\hline $5 \bar{t}$ & $\begin{array}{l}\text { Mentmore Lass } 1 \\
\text { by Melbume and Emerald }\end{array}$ & $1551)$ & $\begin{array}{l}\text { Hannah 1. O. L. } \\
\text { Zephyr (dam of Favonius 1).) } \\
\text { Fim. } 3 \mathrm{~b}\end{array}$ \\
\hline 58 & $\begin{array}{l}\text { Torment } \\
\text { by Dlarm-Glencne }\end{array}$ & 1850 & $\begin{array}{l}\text { Tormentor O. } \\
\text { B. Flat (twins and had } 20 \text { foals) } \\
\text { I.iura (Sire Petrarch 1..) } \\
\text { liam. } 10 \mathrm{~b}\end{array}$ \\
\hline 59 & $\begin{array}{l}\text { Village Lass } \\
\text { by Pyrrhus 1. and Maid of Hart }\end{array}$ & 1851 & $\begin{array}{l}\text { Rustic } \\
\text { Countryman } \\
\text { Bittern } \\
\text { Fam. 2e }\end{array}$ \\
\hline 60 & $\begin{array}{l}\text { Chalice } \\
\text { by Orlando and Crucilix 2. 1. O., } \\
\text { who also had twins }\end{array}$ & 1852 & $\begin{array}{l}\text { Pietas (dam of Placida O.) } \\
\text { Fam. 2c }\end{array}$ \\
\hline 61 & $\begin{array}{l}\text { Homily } \\
\text { by: Suiplice and Blue Devils }\end{array}$ & $18 ; 2$ & Food foundation mare in Fam. 27 \\
\hline 62 & $\begin{array}{l}\text { Rambling Katie } \\
\text { by . Melboune and Phrone }\end{array}$ & 1852 & $\begin{array}{l}\text { Queen of the Gypies (dam of l'aul } \\
\text { Jones) } \\
\text { Chambermaid (dam of Brocken N1. } \\
\text { Hr. BB.) } \\
\text { Fam. } 3 \text { a }\end{array}$ \\
\hline $6: 3$ & $\begin{array}{l}\text { The Heiress (firstling) } \\
\text { by Birdcatcher and Inheritress } \\
\text { (Firstling. ran and won } \\
\text { up to its loth year) }\end{array}$ & $18 \% 3$ & $\begin{array}{l}\text { Vanessa (dan of Das Veilchen, } \\
\text { Wagehals, ctc.) } \\
\text { Jeannic (dam of Whim 11\%.) } \\
\text { Fam. } 4\end{array}$ \\
\hline 64 & $\begin{array}{l}\text { Verona } \\
\text { by. Orlando and Jodine }\end{array}$ & 18.31 & $\begin{array}{l}\text { Thurio Gl'. } \\
\text { l.ucetta Cm. } \\
\text { lillafranca } \\
\text { J:am. } 2\end{array}$ \\
\hline 6is & $\begin{array}{l}\text { Sourenir } \\
\text { by Chanticleer and Birthlay }\end{array}$ & $185 ;$ & $\begin{array}{l}\text { Strathconat } \\
\text { Fair Lnkinown (who also had twins) } \\
\text { Fam. } 11\end{array}$ \\
\hline 66 & $\begin{array}{l}\text { Heroine of L Lcknow } \\
\text { hy dutuith and Pocahontas }\end{array}$ & 18.30 & Famous foundiation mare in Fam. 30 \\
\hline
\end{tabular}




\begin{tabular}{|c|c|c|c|}
\hline No. & Name and Pedigree & Born & Family and Progeny \\
\hline 67 & $\begin{array}{l}\text { Diana } \\
\text { by Hartneitstein and Iris }\end{array}$ & 1858 & $\begin{array}{l}\text { Niraflora Hn. } \\
\text { Neudan (dam of Tartar OD. U. } \\
\text { ND.) } \\
\text { Wild Huntsman } \\
\text { Fam. } 8 \mathrm{c}\end{array}$ \\
\hline 68 & $\begin{array}{l}\text { Feu de Joie O. } \\
\text { by Longbow and Jeu d'Esprit }\end{array}$ & 1859 & $\begin{array}{l}\text { Numette } \\
\text { Hollandaise (dam of Solange FO.) } \\
\text { Fam. ia }\end{array}$ \\
\hline 69 & $\begin{array}{l}\text { Lady Alice Hawthorn } \\
\text { by Newminster and Lady. Haw- } \\
\text { thorn }\end{array}$ & 1859 & Famous foundation mare in Fam. $4 \mathrm{~d}$ \\
\hline 70 & $\begin{array}{l}\text { Mrs. Wood } \\
\text { by Y. Melbourne and Physalis }\end{array}$ & 18.59 & $\begin{array}{l}\text { Little Sister (dam of Thunderstorm, } \\
\text { Kiralsatoa FL. Cd., Fousi Yama } \\
\text { Cd.) } \\
\text { Fam. Sc }\end{array}$ \\
\hline 71 & $\begin{array}{l}\text { Pompadour } \\
\text { by Stockwell and Marchioness O. }\end{array}$ & 1859 & $\begin{array}{l}\text { Idrance (dam of Nlexander SD.. } \\
\text { Cäsar ND., Gallus) } \\
\text { Fan. } 21\end{array}$ \\
\hline$\gamma \geq$ & $\begin{array}{l}\text { Oueen Bertha O. } \\
\text { by lingston and Flax }\end{array}$ & 1860 & $\begin{array}{l}\text { Spinaway 1. O. } \\
\text { W"heel of Fortune 1. } 0 . \\
\text { Grandnaster } \\
\text { Queen's Messenger } \\
\text { Gertrude } \\
\text { Fam. I c }\end{array}$ \\
\hline 73 & $\begin{array}{l}\text { Baschettina } \\
\text { by Pelion and C!mba } O .\end{array}$ & 1860 & $\begin{array}{l}\text { The .lbbot } 1877 \text { by Hermit } \\
\text { Marden } 1879 \text { by IJemit } \\
\text { Canoe } 18,81 \text { by Hermit } \\
\text { Fam. } 2\end{array}$ \\
\hline$\pi 4$ & $\begin{array}{l}\text { Battaglia } \\
\text { by Kataplan and Espoir }\end{array}$ & 1861 & $\begin{array}{l}\text { Lord Gougly (the best son of } \\
\text { Gladiateur) } \\
\text { Fanı. } 12\end{array}$ \\
\hline 75 & $\begin{array}{l}\text { Breeze } \\
\text { by King Tom and Mentmore Lass } \\
\text { 1, who also had twins }\end{array}$ & 1861 & Foundation mare in Fam. 3 b \\
\hline 76 & $\begin{array}{l}\text { No Name } \\
\text { by- Teddington and Queen of } \\
\text { Beauty. }\end{array}$ & 1861 & $\begin{array}{l}\text { Nameless (dam of Geheimniss (0.) } \\
\text { Fam. } 14\end{array}$ \\
\hline 77 & $\begin{array}{l}\text { Rigolboche } \\
\text { by Rataplan-Gardham }\end{array}$ & 1861 & $\begin{array}{l}\text { Cremorne D. GI. Acp. } \\
\text { Earl of Dartrey } \\
\text { Mabille } \\
\text { Fam. } 2 \mathrm{~d}\end{array}$ \\
\hline
\end{tabular}




\begin{tabular}{|c|c|c|c|}
\hline No. & Name and Pedigree & Born & Faumily and Progeny \\
\hline is & $\begin{array}{l}\text { Mare } \\
\text { by Y. Mulbourne and biown } \\
\text { Bess }\end{array}$ & 1801 & $\begin{array}{l}\text { Suiver (dam of Memoir O. L. and } \\
\text { La Jileche 1. O. L.) } \\
\text { Fam. } 3\end{array}$ \\
\hline 79 & $\begin{array}{l}\text { Wild Ignes } \\
\text { by Wikl Dayrell and litule .Ignes }\end{array}$ & 1862 & $\begin{array}{l}\text { Little Ignes IFO. FiL. } \\
\text { Brown Ignes } \\
\text { liair Ignes } \\
\text { lliid Tommy } \\
\text { Fant. } 16\end{array}$ \\
\hline 80 & $\begin{array}{l}\text { Bounceaway } \\
\text { by Zuyder Zee and Press Forward }\end{array}$ & 1864 & $\begin{array}{l}\text { New IIolland Gep. } \\
\text { Tripaway } \\
\text { Fam. } 22 \text { a }\end{array}$ \\
\hline 81 & $\begin{array}{l}\text { Parma } \\
\text { bỵ Parmesan and Ireheress }\end{array}$ & 1864 & $\begin{array}{l}\text { Isabel (dam of St. Frusquin 2. Ec.) } \\
\text { Biserta (dam of Goletta) } \\
\text { Fam. } 22 \text { a }\end{array}$ \\
\hline 82 & $\begin{array}{l}\text { Lady Corentry } \\
\text { by Thormanby and Lady Roden }\end{array}$ & 1865 & $\begin{array}{l}\text { Yorkshire Bride } \\
\text { Farnese } \\
\text { Lady Golightly } \\
\text { Lady of Mercia (grand-dam of } \\
\text { Riggotsliy lil). GP.) } \\
\text { Fam. } 27\end{array}$ \\
\hline 83 & $\begin{array}{l}\text { Pearlfeather } \\
\text { by Newminster and liess lyon }\end{array}$ & $186 ;$ & $\begin{array}{l}\text { Paraibl (đam of St. Wolfgang, } \\
\text { Prado Tr. . N7., Lilinckalani OO.) } \\
\text { Fam. } 4 \mathrm{~b}\end{array}$ \\
\hline 84 & $\begin{array}{l}\text { Thrift } \\
\text { by Stociswell and Braxey }\end{array}$ & 1805 & $\begin{array}{l}\text { Tristan Iep. 11rd. } 3 \times \\
\text { Fam. } 10\end{array}$ \\
\hline $8 j$ & $\begin{array}{l}\text { Adelaide } \\
\text { by Y. Melbourne - Teddington }\end{array}$ & 1860 & $\begin{array}{l}\text { Peregrine } 2 . \\
\text { Quecen . Mdelaide } \\
\text { St. Wwere } \\
\text { St. Wwere } \\
\text { St. Mary (dam of I.n Sagesse O.) } \\
\text { Fam. } 9\end{array}$ \\
\hline so & $\begin{array}{l}\text { Poinsettia } \\
\text { by l: Melbourne and Lad! Ifaw- } \\
\text { thoon }\end{array}$ & J 860 & Famous foundation mare in liam. 4 d \\
\hline 87 & $\begin{array}{l}\text { Wheatear } \\
\text { by I. Melbourne and Swallow }\end{array}$ & $13(j i$ & $\begin{array}{l}\text { Harvester D. } \\
\text { Slivlauli } \\
\text { Fam. 15 }\end{array}$ \\
\hline 88 & $\begin{array}{l}\text { Isola Bella } \\
\text { by Stockwell and isoline }\end{array}$ & Is6s & $\begin{array}{l}\text { Isonomy Cm. Acp. } 2 \times \text { Cicp. Dep. } \\
\text { lernandez } \\
\text { liam. } 19 \text { a }\end{array}$ \\
\hline
\end{tabular}




\begin{tabular}{|c|c|c|c|}
\hline No. & Name and Pedigree & Jorn & Family and Progeny \\
\hline 89 & $\begin{array}{l}\text { Cicely Hacket } \\
\text { by Le Marechal and Meg O'Mar- } \\
\text { ley, who had also twins as well } \\
\text { as her dam }\end{array}$ & 1869 & $\begin{array}{l}\text { Hacliness Cm. } \\
\text { Fam. } 7\end{array}$ \\
\hline 90 & $\begin{array}{l}\text { Light Drum } \\
\text { by Rataplan and Trinlset }\end{array}$ & 1870 & $\begin{array}{l}\text { Little Duck FD. GP. } \\
\text { Lapin Cd. } \\
\text { Fam. } 13\end{array}$ \\
\hline 91 & $\begin{array}{l}\text { Enigma } \\
\text { by Tlue Rake and The Sphynx }\end{array}$ & $187: 2$ & $\begin{array}{l}\text { Florence BB. Cm. } \\
\text { Tact (dam of Amiable 1. O. and } \\
\text { Janners) } \\
\text { Gravity (dam of William III.) } \\
\text { Fam. } 2 \text { a }\end{array}$ \\
\hline 92 & $\begin{array}{l}\text { Spinaway 1. O. } \\
\text { by Nacaroni and Qucen Bertha } \\
\text { O., who had also twins }\end{array}$ & 1872 & $\begin{array}{l}\text { Busybody } 1.0 \\
\text { Arcadian } \\
\text { WValtz } \\
\text { Fam. 1c }\end{array}$ \\
\hline 93 & $\begin{array}{l}\text { Bonnie Ignes } \\
\text { by Blair Athol and little Isnes }\end{array}$ & 1875 & $\begin{array}{l}\text { Bonmy Jean } O . \\
\text { Agnostic } \\
\text { lam. } 16\end{array}$ \\
\hline 94 & $\begin{array}{l}\text { Hermione } \\
\text { by Y. Melbourne and La Belle } \\
\text { Helene }\end{array}$ & 1875 & $\begin{array}{l}\text { Perditta II. (dam of Florizel } 11 . \\
\text { Gcp., Persinmon D. L., Diamond } \\
\text { Jubilee D. L.) } \\
\text { Bride of Netherby } \\
\text { Fam. - }\end{array}$ \\
\hline 95 & $\begin{array}{l}\text { Thistle } \\
\text { by Scottish Chief and The Flower } \\
\text { Safety }\end{array}$ & 1875 & $\begin{array}{l}\text { Common 2. D. L. } \\
\text { Goldfinch } \\
\text { Throstle L. } \\
\text { Fam. 4 e }\end{array}$ \\
\hline 96 & $\begin{array}{l}\text { Lucetta Cm. } \\
\text { by Tibthorpe and Verona, who } \\
\text { also had twins }\end{array}$ & 1876 & $\begin{array}{l}\text { Silver } \\
\text { Gold Acp. } \\
\text { Speed } \\
\text { Luciana } \\
\text { Fam. } 2\end{array}$ \\
\hline 97 & $\begin{array}{l}\text { Wheel of Fortune } 1.0 \text {. } \\
\text { by . Idventurer and Queen Bertha } \\
\text { Three times twins }\end{array}$ & 1876 & $\begin{array}{l}\text { Oberon } \\
\text { Fam. } 1 \mathrm{c}\end{array}$ \\
\hline $9 \mathrm{~S}$ & $\begin{array}{l}\text { Atalanta } \\
\text { by Galopin and Feronia }\end{array}$ & 1878 & $\begin{array}{l}\text { Ayrshire 2. D. Ec. } \\
\text { Melanion } \\
\text { Troon } \\
\text { Fan. Sa }\end{array}$ \\
\hline
\end{tabular}




\begin{tabular}{|c|c|c|c|}
\hline No. & Yame and Pedigree & Burn & Family and l'rogeny \\
\hline 99 & $\begin{array}{l}\text { Sanda } \\
\text { by Winlock and Sindin] }\end{array}$ & 1575 & $\begin{array}{l}\text { Sininfoin D. } \\
\text { Golden Giarter } \\
\text { Sierral, lilaclis Sand Cs. } \\
\text { laum. 2 b }\end{array}$ \\
\hline$l(x)$ & $\begin{array}{l}\text { St. Marguerite } 1 . \\
\text { by Mermit and Devotion }\end{array}$ & 1879 & $\begin{array}{l}\text { Seabrecze O. L. Lc. } \\
\text { Villaturis } \\
\text { l.e lat l'llst. } \\
\text { Ruquebrune (dim of Rock Sand a. } \\
\text { D.1..) } \\
\text { liam. ta }\end{array}$ \\
\hline 1111 & $\begin{array}{l}\text { Matilda } \\
\text { by leauclerc - Cathedral }\end{array}$ & $18+11$ & $\begin{array}{l}\text { Xumblorpe Kis. } \\
\text { Oucen's Birthday Dcp. } \\
\text { Fam. } 11 \text { a }\end{array}$ \\
\hline 102 & $\begin{array}{l}\text { Match Girl } \\
\text { by l'lebcian and liusee }\end{array}$ & $185 \%$ & $\begin{array}{l}\text { Matchbox } \\
\text { Matchunalier } \\
\text { Fam. } 22 \text { a }\end{array}$ \\
\hline 103 & $\begin{array}{l}\text { Virginia Shore } \\
\text { by̆ John Davis and 1)istant Shore }\end{array}$ & $18 s+$ & $\begin{array}{l}\text { Whittier F. } \\
\text { lady Whing } \\
\text { Fant. Oa }\end{array}$ \\
\hline 104 & $\begin{array}{l}\text { Lady Loverule } \\
\text { by Muncaster and Sellic }\end{array}$ & 1888 & $\begin{array}{l}\text { St. Imant D. } 2 . \\
\text { Jam. } 14 \text { a }\end{array}$ \\
\hline 1115 & $\begin{array}{l}\text { Ornament } \\
\text { by Bend Or and Lil! Iguce }\end{array}$ & 1887 & $\begin{array}{l}\text { Labrador } \\
\text { Collar Hrd. } \\
\text { Sceptre O. 1. 2. 1. Fam. } 16\end{array}$ \\
\hline 106 & $\begin{array}{l}\text { Mother Siegel } \\
\text { by leriar balsitul-(iallopin }\end{array}$ & 1897 & Yinuru 2 D. Fаm. 5 \\
\hline
\end{tabular}

\section{(g) Firstlings.}

If we look more closely into the Stud Bouks, we find amongst the hirstlings of brood mares more groul animals than was generally supposed, owing te ubservations in studs where the broud mares were rery protly fed. The lirstlings of broud mares at Takelumen formerle were seldem good when pour ford was given w the broud mares, esperially before 18т0. In Half-bred as well as Thoroughbred hreeding, it is a remarkable fact that part of the best progeng wore firstlings, hut only in those studs where the

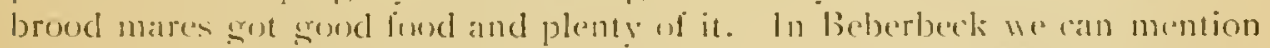

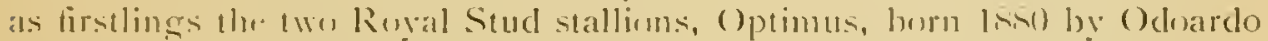
and Optima, and Jubelgreis, born lit):3 by lambers and fulic. Since the existence of beberbeck as a keyal chiof stud, we have pessibly produced no better Royal Stud stalliens than the two named bistlings. In the last fifty vears, of is Royal Stud stallions produced there, the fellewing 1.1 were lirstlings:- 
1. Norton, born 18.5t hy Stilum and Novelu.

2. Fitter, born 1856 by Nobelmann and Faucete.

3. Grezano, born 1s.5 by Stilton and Giralda.

4. Eckstein, born 1 isto by Vencrato and Echo.

r. Fiirstenbers, born 1878 by . Imbos and Fulda.

fi. Venezuela, born 1sis by IIektor and Viereck.

7. Eluin, born lisit by Passian and Emilia.

8. Elton, brom linse by Dulie of Edinburgh and Elpis.

9. Versuch, born $1 \$ 90$ br Anarch and V'estitur.

10. Ethelwold, born 1893 by Euphony and Etruskerin.

11. Intsworstcher, born 1899 by Fanfarro and Inisette.

12. Hoffnungsitrahl, born 1900 by Piper and lloffung (covered as a 3-year-s)de.

13. Fischerlinabe, born $190 \mathrm{f}$ by Obelisk and Fischerin.

11. Panzer, burn 19k2 by Greif and Panzerliette.

In the same period the dams of the following 19 Royal Stud stallions were firstlings:-1 Intenor, :- Elfenbein, 3 Ethelwold, 4 rischerknabe, 5 Flügel, fi Fürstenberg, 7 (iranicus, s Grezano, 9 Hirtenknabe, 10 Hoffnungsstrahl, 11 Insurgent. 1.2 Juli (out of a :3-vear-old covered mare), 13 Justizminister, $1+$ Laner, 1.j Malteser, 16 Nisos, 17 Orcus, 18 Pless, 19 Tunnel (out of a :3-year-eld corered mare).

In Thoroughbed bresding the following are the furstlings which are noted as classical race winners:-

\section{Derby llinners.}

1. Spread Fagle, burn 179.2 by Tolunteer - I Iighflyer.

․ Daniel O'Rourke, born 1949 by Birceatcher and Springy Jack.

3. Wild Dayrell, burn 1.5.5 by lon and Ellen Mlddleton.

1. Faronius, bern lisg by Parmesan and Kephỹr.

\section{2 . St. Leger Winners.}

1. Tommy, born 1760 by Wildair-Syphon (covered as a 3-year-old).

$\therefore$ Phönomenem, born 17 so by llerod and Frenzy.

:3. Paragun, born 17i:3 by Parmaster and Calash.

1. Ashton, born lsol by Walnut and Miss Haworth (covered as a

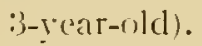

$\therefore$ Otterington, born 1809 by Conlumpus - Expectation (covered as a 3 -rear-old).

6. Filho da Puta, born 1812 by Ilaphazard and Mlirs. Barnet.

7. Touclistone, born 1831 by Camel and Banter.

$\therefore$. The Baron, born lit. by Birdcatcher and Echidna (covered as a 3-year-old).

9. Wool Winder, born 1904 by Martagon and St. Windeline. 


\subsection{Guineas II inners.}

1. The Legly buck, born lisu by linison and Monstrosity (cosered as a : $3-y($ ear-old).

2. Vedette, born 18.59 by Voltigeur and Mirs. Ridgway.

3. Surefoot, born 1 sist by Wisdom - Galopin.

\section{Oaks II inners.}

1. Bridget, born 1776 by Iforod and femima.

2. Tetotum, born $175 \mathrm{by}$.latchem (2-yeals-old) and lady Bolingbroke (10-geatr-old).

3. Sorcery, born 1808 by Sorecerer and cobbea.

4. Poison, born 18 bo by Penipotentiary and . Irsenic.

5. Nincemeat, born 18.51 by Sweetmeat and llybla.

\subsection{Guineas 11 inners.}

1. Mare, born 1812 by Sclim - Cesarin?

$\therefore$. Irab, born 18.2. by Woful and \%al.

The following are to be mentioned as lirstlings which have won other important races of which have otherw ise breme famous:-

.1. Stallions.

]. Y. Merlin

2. Cimerack

3. Paymaster

4. Trentham

5. Conductor

6. .llark . Inthony

7. Pantaloon

S. Pirthes

9. Telemachus

10. Potios

11. Joe . Indrews

1.2. D) Iungannon

13. Roxkingham

11. Fidget

15. Cires. Diomed

16. Bustard

17. Littlo. John
17.57 by Merlin and Molly Long I.egs.

1760 by Cripple and Mliss lilliot (covered an a 2 or :i-verar-(n)d).

1760 by Blank and Snapdragon.

1760 by Swopotalkes and dliss South.

176 by .latedrem - Snap.

176 by Spertater and Rachel forsered as a

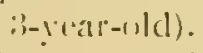

$176 \pi$ )y Matchem and Curiosity.

It67 be Sprightly Snip.

1700 by lleped - Skim.

1773 by liclipere and Sportsmistress.

17Tis by lodipse and . Muranda.

$17 \times 0$ by liclipse and Ispasia.

IRs by IIeghnyer and l'urity.

1783 by lorizal - Matehem.

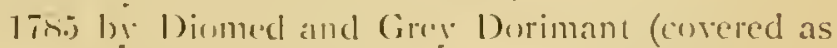

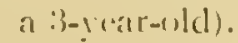

tian by Mimdperdier and Matrem (covered as a

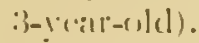

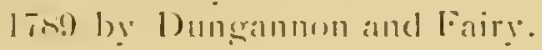


18. Oberon

19. Sorcerer

20. Catton

21. Blackamoor

22. Doctor Syntax

23. Scrapall

24. Sultan

25. Young Phantom

26. Royal Oak

27. Robin Hood

28. Sir Hercules

29. Taurus

30. Liverpool

31. Alba

32. Heron

33. Melbourne

34. Drone

35. Jon

36. The Provost

37. Robert de Gorham

38. The Cure

39. The Libel

40. The Great Lnknown

41. Elthiron

42. Newport

43. Kingston

44. Orestes

45. Arthur Wellesley

46. Fandango

47. Tournament

48. Pizarro

49. Newcastle

50. Vanquisher

51. Mandrake

52. Paul Jones

53. Hampton
$1790 \mathrm{by}$ I Iightlyer and Queen Mab.

1796 by Trumpator and Y. Giantess.

1809 by Golumpus and Lucy Gray.

1811 by Stamford -- Sorcerer.

1811 by Paynator - Beningbrough.

181ㄹ by Granicus - Young Whiskey (covered as a 3-year-old).

1816 by Selim and Bacchante (covered as a 6-yearold).

182.2 by Phantom and Emmeline.

1823 by Catton -- Smolensko.

182.t by Blacklock and Marion.

1826 by Whalebone and Peri (covered as a 3 -year-old).

1826 by Phantom or Morisco and Katherine.

1828 by Tramp - Whisker.

1831 by Nigel and Therese.

1833 by Bustard - Orville.

1834 by Humphrey Clinker - Cervantes.

183.5 by Pantaloon and Decoy.

1835 by Cain and Margaret (covered as a 3-yearold).

$1836 \mathrm{by}$ The Saddler and Rebeca.

1839 by Sir Hercules and Duvernay.

$18 \pm 1$ by Phrsician and Morsel.

1st? by Pantatoon and Pasquinade (covered as a Z-vear-old).

184.j by Voltaire and The Princess.

$18+6$ by Pantaloon and Phrone.

1846 by Epirus - Zimmermann (covered as a - -rear-old).

1849 by Venison and Qucen .Inne.

18.50 by Orlando and Mrs. Hobson.

18.5 by Melbourne and Lady Barbara.

18.2 by Barnton and Castagnette.

18.54 by Truchstone and IIappy Queen (covered as an 8-rear-old).

18.5.5 by Bloumsbury and Marina.

18.56 by Vewminster and Mary Aislabie.

18.57 by Voltigeur and Eglantine.

1864 by Weatherbit and Mlandragora.

186.5 by Buccaneer and Queen of the Gypsies.

1872 by Lord Clifden and Lady Langden (covered as a 3-year-old). 
54. Perplexe

55. Bendigo

56. Le Nord

57. Espoir

1. Tuting's Polly

‥ Virago

3. Flora

4. Frenzy

5. Y. Camilla

6. Virgin

7. Maniac

8. Katherina

9. Nermaid

10. Marpessa

11. Pocahontas

12. Monstrosity (Covered as a 2-yearold, dam of Ugly Buck 2.)

13. Pantalonade

14. Inheritress

15. Eulogy

16. Queen Mary

17. Honey Dear

18. Haricot

19. Vesuvienne

20. Little Agnes

21. Lady . Mlice J Iawthorm

22. Budagyöngye
1872 by Vermouth and Péripétie.

1880 by Ben battle and Hasty Girl.

1887 by Tristan and La Noce (covered as a 3-yearold).

1889 by Barealdine and Bel Esperanza.

B. Mares.

1756 by Black and All Black and Fanny.

$176+$ by Snap - Regulus.

1768 by Squirel and Angelica.

1774 by Eclipse - Engineer.

1787 bi lloodpecker and Camilla (covered as an s-rear-old).

1801 by Sir Peter - Potsos.

1806 by Shuttle and Anticipation (covered as a 3-lear-old).

1817 by Wiful and Landscape O. (corered as a 3-year-old).

1829 by Whalebone and Miss Emma.

1830 by Muley and Clare.

1837 by Glencoe and Marpessa.

1838 by Plenipotentiary and Puce (covered as a 3-rear-old).

1.839 by Pantaloon and fiestival (covered as a Z-rear-old).

1840 by The Saddler and Executrix (covered as a 3-vear-old).

1843 by Euclid and Martha Limn.

1843 by Gladiatur - Plenipotentiary (covered as al :-yeatr-old).

$184 t$ by Plenipuentiary and My Dear (covered as a :-rear-old).

1847 by Mangen or Lanereost and Queen Marỵ (covered as a 3 -rear-old).

1847 by Giladiator and lenus.

I8.5n by "The (ure and 1 liss I Isnes.

1859 by Newminster and Lady Hawthorn.

1882 by Buecincer and Kincsem. 


\section{(h) The Treatment of Brood Mares.}

It is, as a matter of course, essential that brood mares outsicle the grazing time should take gentle exercise for about one and a half hours daily up to the day of foaling, and about ten to fifteen days after the foaling, along with the foal, commencing with a quarter of an hour, till finally they do one hour. This is just as necessary as is in spring the gradual transition to grazing.

Like many other things in horse breeding, the food rations of the brond mares should vary accurding to the characters of the mares, and according to the soil on which the stud stands and the food grows. I now give the rations as approved of in East Prussia. After the grazing time brood mares in foal are given in three rations 6 lbs. of oats and about 1.5 lbs. of hay ; barren mares 4 lbs. of oats and about 12.2 1bs. of hay. Supposing that oats and hay are of the very best quality, and that there is an ample spread of healthy rye or summer straw, I eonsider that a larger ration, especially of oats, is only necessary in a few exceptional cases. To these exceptional calses belong, for example, mares corered as three-year-olds and hatring become pregnant, also Thoroughlored mares in foal for the first time, and some mares - mostly older and barren ones-which feed with difficulty, to whom an additional $3 \mathrm{t}$ (1) $5 \mathrm{lbs}$. of wats and the same quantity of hay may be given. Good Lucerne or Sainfoin hay is, of course, by far the best, and particularly to be recommended for Thoroughbred breeding, while for Half-bred breeding clover hay is to be recommended, together with meadow hay, in order to produce stronger bones. In many parts, as for example, in East Prussia, wheat straw, especially new, is nut suitable for brood mares, as colic and abortion are easily caused thereby. After grazing time, it is recommendable to add to the oats twice weekly 2 to 3 litres of wheat bran for the evening meal in the same manner as it is given to stallions. The last two to three weeks before foaling it is advisable to give this additional wheat bran three times weekly, or even daily, as many breeders do. In the spring, at the beginning of grazing, wheat bran is left out. To give extra food to the brood mares immediately after foating is not advisable, because the after-pains are thus unnecessarily increased. In the case of the mare foaling shortly before her feeding time, it is advisable to reduce this first feeding somewhat. In the case when the mares are not in boses, but are together with several others in lonse boses, they should be separated at once after giving birth, and remain with their foals alone for about eight to fourteen days. About eight days after birth suckling brood mares must have about 2 to $3 \mathrm{lbs}$. of oats and 5 lbs. of hay more, i.e., 8 to $9 \mathrm{lbs}$. of oats and $20 \mathrm{lbs}$. of hay. Wheat bran three times weekly, or even daily, is given up to grazing time. I recommend that young Thoroughbred brood mares and four-year-old mares should be given, after foaling, 12 lbs, of oats, but no more. The foal of a Thoroughbred mare (Noran, a very good eater) to whom I gave before foaling 12 to 16 lbs. of oats and after foaling $18 \mathrm{lbs}$. of oats, sickened soon after of bone 
discase (Osleoporosis chronica unizersalis) and died conseguently when five rears of age (Napoleon, the skeleton is in the Traliehnen .Museum). I recommend that bad feeding maresshenld ber siven an additional:3 lbs. of ground barley. In the case of the abowe-mentioned wat rations, it is talsen for granted that the suckling foals, ats stated bedrow, seet the oats extra. Salt and chalk must always be in the crib. Pregnant matres mus never be given carrots.

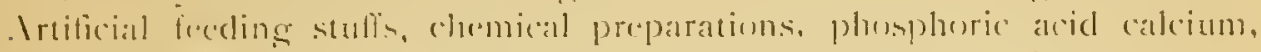
etc., are not to be recommended. Opening pills seem to be dispensable. I have mereer used them, and never knemn the need of them.

During grazing time suckling mares must geet 6 ibse of wats in two rations, batren and weancel mares 2 lbs. of oats (early in the morning). If the graxing is not rich, especially in late antumn, an additional 3 lbs. of haly in the morning, and if that is not sulizeient in the erening also, is necessiry. Fo lieep brond mares, reven in the summer when grass is plentiful and good, without wats, has proved (1) be a failure in Trakehnen. Mlso, night grazings, which were found to be gexul at Beberbeck, proved to be bad at Trakehnem. These and many other things must be managed acourding to the different experiences ubtained lexally. The more open air exercise the breeding material can be allowed without bad consequences, the more suitable is the soil for breceling. Grazing on frosty grass is to be avoided. Young clover must anly be srazed when in bloom.

\section{(i) Covering of Brood Mares.}

The question in which menth mares sheuld be corered must be answered acourdiog to keal conditions. In lingland and Imerica late born foals have excelled. West Australian and Blue Gown, for example, were born in June. Ilany contend that Linglish early born foals are specially inclined w ruaring. Prine Charlie, born in January, is a well-known example of this. John Porter sags " he never knew a roarer bern in May." The question has therefore been ratised in lingland, en calculate the age from the 1 st of 1 arch instead of from the lst of January. In East Prussia and in Courland, homever, early born foals are stronger and healthier than late burn ones. "The age of lialf-bred forls in liast Prussia is now reckoned from the 1 st of Durember. The reason is as lullows: In East Prussia the grazing continues till about the middle of October, after which follows the sol-called stabling uf the brund mares, and winter life commences with $1 \frac{1}{2}$ hours exercise on the track and -2.2 hours standiog in the stable. Dom also come the short days, so that in December mares must stand about 16 hours out of the 2.1 in a darli rum. Feeding is also less farourable, exercise less complete, air, sun and wind, with all their benefits, very limited. It can be easily undersend that the foal in the womb also suffers from these unforourable influnces, (specially als these unfavourable changes condede with the last and most impertant time of its fertal life. The shorter. therefore, this unfavourable time is from 
the stabling to the foaling, the better for the foal. The disadrantage of early horn foals in East Prussia coming to grass only when weaned may be modified by regular daily and ample exercise of the suclilings with their dams on special tracks, and is less great than keeping pregnant mares for months in the stable. Moreover, early born foals are greatly favoured by passing one of the most important and most dangerous times of their lives, namely, weaning, during the most favourable time of the year, i.e., the time of the first and most nourishing green ford, whilst late born foals only get weaned off when green food ceases or has lost already a little of its nourishing power. The early born foals, in like manner as early hatched chickens, can make more intensive and better use of all the benefits which the short East Prussian summer gives. For late born foals the summer benefits, with their light days of 16 hours' sun, offer more good things than so roung foals can take advantage of. The early born foals can get about five months fresh Lucerne or clover, and this will amply counterbalance the disadvantage of getting a smaller quantity of milk from their dams before grazing. On account of these reasons cowering begins in Trakehnen un the 15th of December and finishes at the end of May.

The most favourable day for covering foal-mares is the ninth day after foaling. Breeders of every country are agreed on that point, but views differ over any further necessary corering, especially of barren mares. It is possible that in different countries different rules have been observed with regard to mares in heat, and with regard to the readiness of mares to be served. I give my views here, based on Iong experience in East Prussia, and $I$ must add that these have been confirmed by my experiences in Beberbeck and Courland. My risits to the Thortughibred studs in England and France have, on the whole, verified my experiences in this direction. In exceptional cases 1 have had foal-mares covered eren on the serenth or eighth clay after foaling when they have showed themselves distinctly in heat already for sereral days, sometimes even beginning on the third day after the birth. Otherwise, the foral-mare is tried on the afternom of the eighth day after foaling, and if she, as often happens, is not ret well in heat, I have tried her again the following morning, and got her corered if she was well in heat. If the mare is not well in heat she is tried daily until she is. Many mares come regularly in heat only on the tenth or eleventh day. I have never had mares forcibly corered which were not in heat. Neither do I know a stud which successfully uses such a forced service, as recommended by Schwarznecker. Furthemore, it may happen that foal-mares cume distinctly in heat already on the third, fourth or fifth day after foaling, but are no longer in heat on the seventh dar, when, as abore stated, it was desirable to cover them. I consider this heat in nearly all cases a false one, without orulation (removal of a ripe egg from the nvary), especially as these mares are mostly in heat about the ninth day, and also conceive. If the foal is born more than fourteen dars too soon (i.e., the time of pregnancy is less than 319 days), the 
first heat is passed over, if the mare comes in heat as is usual the ninth day after the birth, which generally happens. The next heat is usually three or four weeks later. There are mares, however, which after premature births are always in heat at a later date, i.c., instead of on the ninth day, only on the fifteenth or wentieth day after foaling, and which, according to my experience, may be covered at ance without injurious consequences. If the too early born foal does not give the impression of not being fully developed, or if the conception of the mare is probably not attributable to the last, but to a previous covering, she may, even after premature births of less than 319 days of pregnancy, be covered the ninth day after foaling. If the mare has foaled in the night before the first of a month, she is covered, if everything is all right, on the ninth of the month, then tried nine clays later on the morning of the seventeenth, and if she is in heat is covered again. The foaling day, or the day of the first covering, is always reckoned in order to get the right ninth day. These, and the following instructions for covering, are founded on the assumption, based on long experience, that the regular heat lasts nine clars, and that if the mare has conceived in the first covering, the heat stops at latest on the ninth day after the first covering. There are numerous cases in which a mare after the first covering remains in heat even up to the eighth day, comes off on the ninth day and shows signs of pregnancy. These cases happen particularly with so-called foal-mares, i.e., mares which come in heat on the ninth day after foaling, and which have been covered at the right time. On the other hand, it is probable that the mare still in heat after the first covering up to the ninth day, then covered again, has conceived by the second and not by the first covering, which can easily be seen from the many practical examples of properly kept service registers and stud books; firstly, by comparing the number of days between the covering and the foaling with the usual time of pregnancy of the mare; and secondly, by considering the colour and form of the foal when a different stallion has covered the second time. There are, morever, many practical examples which prove that a foal-mare in heat on the ninth day after foaling could only be covered the ninth day after the first heat (as the stallion was not available), and conceived from this covering.

If the mare has not conceived after the covering, she comes, as a rule, in heat again three or four weeks later. After a strong heat the interval till the next heat is usually a little longer, and vice iersa. It often happens that mares which conceive in the first heat come again in heat three or four wceks or more later. 'This heat is, however, a false one, and can generally be recognised, as the redness, swelling and moistness of the privy parts generally to be seen with a mare really in heat is not apparent. In the wild Steppe studs of Russia, where one stallion lives in the open together with twelve to fifteen mares (called liosjaerk), one can clearly see how the stallion aroids the mares with a false heat pressing about him. He finds out the mares which are really in heat and covers them. Voung Thoroughbred stallions do not 
often understand this business in the first year, but learn it also gradually, as I myself have observed. There are, however, mares who, although they have not conceived, do not come in heat again for a few months, whilst others come in heat again in a fortnight. In the interval between the first and second heat mares should not be tried, as the many attempts often produce false heat, and it is not always possible to recognise it as such. If a mare visibly remains continually in heat after the last covering, she should not be covered again, as this heat is nearly always unnatural, and it is very improbable that the covering would lead to conception. Several cases have happened in Trakehnen where a mare, not conceiving easily, has been covered two or three times during the period of one heat, has continued to be in heat uninterruptedly for two or three weeks after the last covering, and yet proved to be pregnant without being covered again. If such mares are covered again they will very likely slip a small embryo, which in most cases is not noticed, and the mare is then wrongly considercd as barren.

In the case of suckling mares which have not conceived during the period of the first heat, or which could not be covered, it is very uncertain whether they will come in heat again after three or four weeks. The returning of heat can only be expected after weaning, or in the case of sudden warm weather. When the foal-mares are in heat for the second time, it is advisable to serve them two or three times, the second covering on the third day, or if the stallion be not available, the fourth or fifth day. In most cases this period of second heat does not continue as long as the first, and it is therefore advisable to have her covered for the second time earlier, and if the heat continues to have the third covering on the eighth or ninth day, according to experiences of previous years. The covering of barren mares must be arranged in the same manner as that of foal-mares in the second heat, unless previous experiences point otherwise. Covering of young mares must, however, be arranged as for foal-mares in the first heat, i.e., the first and ninth day. In the interval there must be no trial. When covering barren or young mares onc must pay special attention that they are well in heat, and if that be not the case, the covering nust be deferred until they are. With foalmares heat appears on the ninth day after foaling, in most cases very suddenly, and decreases generally in intensity after a few days. Therefore, the covering of these mares is most effective at the beginning of their heat. The heat of barren and young mares, however, develops very slowly, and increases in intensity in the first two or three days. The covering of same, therefore, is not the most effective on the first day, but only when the heat has reached its culminating point. The second or third covering in this heat on the third, eighth or ninth day is reckoned from the day on which the mare has reached the culminating point of her heat. The days before the culminating point are not reckoned as heat days.

There are, of course, many exceptions to the above-mentioned rules. There are mares which are only in heat one, two and three days. The stud- 
master must talie note of such mares, but, of eourse, he must not wait till the culminating point is reached, but must get them crivered as soon as they come in heat, and again on the following day. lirom my own experience I should recommend, in clifticult cases, mares to be covered in the afternoon and again on the following morning. In the case of mares which will not conceive, I would advise them to be corered with two stallions, one immediately after the other, and take the desired stallion for the second covering, as I have observed that in this ease the second stallion is ustally the fertilising one, if it succeeds at all. I recommend, moreover, that mares which conceive with difficulty should be given a good trot about half an hour before the covering, in order that they may set warm and heated, or that their mode of living should be changed; i.e., mares which run about loose in the stud should be put to hard work, and, on the other hand, mares which have been at work should be let loose. Sometimes change of place also helps. l.can mares conceive easier, but come in heat more dilficulty. There are mares which conceive with more difficuly, or even not at all, from certain stallions. In llalf-bred breeding 1 would, therefore, recommend that the stallion should be changed in the third heat, a thing which is often difficult in Thoroughbred breding. Mares which are ridden or driven usually conceive with more difficulty. I would recommend that mares which do not come in heat should be put in another stable, if possible, together with mares in heal, or in warm weather and sunshine should be let loose with several others in a paddock, but should not be driven. They then should be finally allowed to be present when other mares are covered. During the first covering of young mares everything should be avoided which might frighten them. The mounting of a trial stallion is only advisable in exceptional cases with very tromblesome and bad-tempered mares and specially valuable stallions.

Finally, I recommend breeders to try and get the mares pregnant from one covering; at any rate from as few coverings as possible. There certainly exists the danger of exciting the sexuality of the mares by too frecutent covering, and by tox many and too intensive trials, and of producing false heat without ovulation. I have come across hysterical mares often in heat, and frequently without orulation, mostly in studs in which the stallions had not enough to do, and in which the mares were consequently cosered too fften. Young mares especially are spoiled by too frequent covering. In the case of suddenly occurring heat of foal-nares, usually on the ninth day after foaling, the commencement of the heat very probably coincides with ovulation, whilst in the gradually arising heat in the case of barren mares, orulation probably begins later, perhaps only when the heat is at its culminating point. As a fertilisation without ovulation is, of course, inspossible, the above recommended manner of covering of mares also complies with this point of riew. 


\section{(k) Fertilisation.}

In order to compare the results of fertilisation, I have given in the following lists the figures of the respective stud books, but not the figures of country studs of the different provinces. It is too difficult for a country stud to get reliable statements with regard to foaling, and consequently country studs are not able to furnish statistically serviceable figures. In addition to the statements of the German General Stud Book of Thoroughbreds, I must say that the foaling results in many years-perhaps in all-appear more favourable than the really were, because all those mares about whose foalings no information is given are not reckoned, but these mares may be pretty certainly considered as barren or as having slipped their foal. Moreover, the figures on the foalings have been asked for, sometimes with more, sometimes with less energy, and this explains the striking variations in the foaling results of Thoroughbreds in Germany.

The foaling results in Trakehnen have come out too farourably, because mares which were covered and sold were not counted. The greatest number of these mares sold, however, are barren, and this is generally the reason for their being sold in autumn.

Foaling results of Thoroughbred breeding in Germany.

\begin{tabular}{|c|c|c|c|c|c|c|c|c|}
\hline \multirow{2}{*}{ lear } & \multicolumn{2}{|c|}{ Foals horn } & \multirow{2}{*}{ Total } & \multirow{2}{*}{ Aborted } & \multirow{2}{*}{ Barren } & \multirow{2}{*}{$\begin{array}{l}\text { Total } \\
\text { Number } \\
\text { Covered }\end{array}$} & \multirow{2}{*}{$\begin{array}{l}\text { Preginant } \\
0\end{array}$} & \multirow{2}{*}{$\begin{array}{c}\text { Of } \\
\text { l'regnant } \\
\text { Mlares } \\
\text {.iborled } \\
\%\end{array}$} \\
\hline & Colts & Fillies & & & & & & \\
\hline 1885 & 115 & 127 & 245 & 16 & su & 341 & 76,54 & $6,1: 3$ \\
\hline 1885 & 141 & 118 & 259 & 27 & 87 & 373 & 76,69 & 9,44 \\
\hline 1KST & $l(19)$ & $1: 37$ & $2+6$ & 17 & 112 & 375 & $70,1: 3$ & $1 i, 46$ \\
\hline 1854 & 135 & 137 & 272 & 16 & $12: 3$ & 411 & 711,07 & 5,56 \\
\hline 1889 & 161) & 14.5 & 3015 & 18 & lok & $4: 31$ & 74.94 & $.5,57$ \\
\hline $18(k)$ & 140 & 149 & 24:9! & 29 & $1: 31$ & $44 !$ & $71,8: 2$ & 0,12 \\
\hline 1891 & 172 & 179 & 851 & 31 & 140 & .202 & 73.18 & 8,11 \\
\hline 1802 & 177 & 193 & 370 & 34 & 162 & $.661^{2}$ & 71,39 & 8,42 \\
\hline 1893 & 215 & 215 & 430 & 29 & 140 & 590 & 710.63 & 6,32 \\
\hline 1894 & $2: 34$ & 215 & 449 & 34 & 175 & 662 & 73,56 & 7.80 \\
\hline 1895 & 272 & $2+1$ & 513 & 39 & 170 & $7 \cdot 2 \cdot 2$ & 76,45 & 7,07 \\
\hline 1896 & $25 \pi$ & $25 ; 3$ & 510 & 41 & 188 & $7: 20$ & 74,56 & 7,44 \\
\hline 1897 & $2(i 5$ & 366 & 531 & 47 & 164 & 742 & 77,90 & 8,13 \\
\hline 1898 & 2911 & 206 & 587 & 40 & 180 & Sil 7 & 77,70 & 6,38 \\
\hline 1899 & 298 & 263 & 561 & 37 & 221 & 819 & 73,02 & 6,19 \\
\hline 1900 & 268 & 262 & 5300 & $(i)$ & 210 & 800 & 73,78 & 10,17 \\
\hline 1901 & 279 & 285 & 54 & 238 & 236 & $\therefore 8$ & 71,84 & 6,31 \\
\hline 1902 & 318 & 259 & 576 & 42 & 231 & 8.011 & 72,82 & 6,79 \\
\hline 1903 & 274 & 260 & 540 & 45 & 241 & $\left.S^{*}\right)$ & 70,98 & $s, 1 i$ \\
\hline 1904 & 266 & 253 & 519 & 54 & $2 \cdot 27$ & $\sin 0$ & $71,\left(i^{3}\right)$ & 9,42 \\
\hline Total & 4389 & 4259 & 8645 & 701 & $33320 ;$ & 1207.5 & $7: 3,70$ & 7,50 \\
\hline
\end{tabular}


Foaling results in Trakinnen.

\begin{tabular}{|c|c|c|c|c|c|c|c|c|c|c|}
\hline \multirow{2}{*}{ Yenr } & \multicolumn{2}{|c|}{ loals born } & \multirow{2}{*}{ Total } & \multirow{2}{*}{ Aburted } & \multirow{2}{*}{ barren } & \multirow{2}{*}{$\begin{array}{l}\text { Totil } \\
\text { Number } \\
\text { Covered }\end{array}$} & \multirow{2}{*}{$\begin{array}{c}\text { Pregnant } \\
\%\end{array}$} & \multirow{2}{*}{$\begin{array}{l}\text { of } \\
\text { Pregnant } \\
\text { Mares } \\
\text { Iborted }\end{array}$} & \multirow{2}{*}{\multicolumn{2}{|c|}{$\begin{array}{l}\text { Besides } \\
\text { "Twins, } \\
\text { part living, } \\
\text { part dear }\end{array}$}} \\
\hline & Cuslts & lïllies & & & & & & & & \\
\hline 1895 & 121 & 110 & $203+1$ & 24 & 85 & 316 & 71,37 & $9 ., 30$ & & Pair \\
\hline lN!Kj & $1 \ddot{2}(j$ & 112 & 2 tis & 18 & 55 & 341 & 83,87 & $(i, \ddot{2})$ & 2 & , \\
\hline $18 \div$ & 123 & I:29 & 201 & :30 & $7 s$ & 369 & $78.86 i$ & $\mathbf{J} 0,31$ & 5 & ., \\
\hline 3698 & 145 & $1: 30$ & 281 & 19 & 63 & 363 & 82,64 & 6,33 & 1 & \\
\hline $1 s\left(x_{k}\right)$ & $1: 6$ & 108 & 231 & 99 & 63 & 396 & 81,10 & 29,73 & 4 & \\
\hline $19 x)$ & 119 & 116 & 235 & 21 & 140 & 396 & 64,$0 ;$ & $8,21)$ & 3 & ., \\
\hline $1 \%) 1$ & 148 & 143 & 291 & 26 & הT & 374 & 84,76 & 8,20 & 11) & .. \\
\hline 1902 & 163 & 185 & 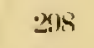 & 17 & 74 & 389 & 80,98 & 5,40 & $\therefore$ & .. \\
\hline 1963 & 152 & 148 & $: 300$ & $2 \cdot 2$ & 61 & 385 & 84,07 & 6,83 & s & ," \\
\hline 1904 & 146 & $1: 28$ & 284 & 25 & S1 & 393 & 79,36 & 8,97 & 4) &.. \\
\hline $190 ;$ & $1 H$ & 163 & 307 & 3 & 75 & 408 & 80,88 & 6,97 & $i$ & ," \\
\hline 1906 & 144 & 172 & 316 & 16 & 67 & 399 & 83,21 & 4,82 & i & .. \\
\hline $190 \bar{T}$ & 153 & 135 & 205 & 15 & 72 & 37.5 & 80,8 & $4,1,5$ & 1 & 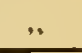 \\
\hline Total & 1813 & 1784 & 3597 & 328 & $9 \pi$ & 4932 & So, 19 & 9,105 & $60 I$ & Pair \\
\hline
\end{tabular}

Foaling results according to the East Prussian Stud Book, Iol. IV.

\begin{tabular}{|c|c|c|c|c|c|c|c|c|c|}
\hline \multirow{2}{*}{ lear } & \multicolumn{2}{|c|}{ Foals born } & \multirow{2}{*}{ rolal } & \multirow{2}{*}{. Iborted } & \multirow{2}{*}{ Barren } & \multirow{2}{*}{$\begin{array}{l}\text { Total } \\
\text { Number } \\
\text { Covered }\end{array}$} & \multirow{2}{*}{ Preginant } & \multirow{2}{*}{$\begin{array}{l}\text { Of } \\
\text { l'regnant } \\
\text { Mlares } \\
\text {. Iborted } \\
\text { o, }\end{array}$} & \multirow{2}{*}{$\begin{array}{l}\text { Besides } \\
\text { Twins, } \\
\text { part living } \\
\text { part deat }\end{array}$} \\
\hline & Colts & Fillies & & & & & & & \\
\hline 1899 & 1371 & 1.54 & : & $2 b i t i$ & 902 & 4050 & $7 \pi, 92$ & 8,35 & $\gamma$ l'ilir \\
\hline 1900$)$ & $1+71$ & 1.31 & $300 \%$ & $2 \pi$ & 1037 & $4: 300$ & $7.5,85$ & $7 ., 11$ & $8 \quad$, \\
\hline 1901 & 1.724 & 1612 & $31: 3 i$ & T5 & 1041 & 1164 & $76,6 s$ & 8,34 & 4 \\
\hline 1902 & 15202 & 115 & 3017 & 291 & $11 \%$ & 4157 & 74,019 & 8,91 & (i \\
\hline 19003 & 1.26 .5 & lints & 3153 & $3 n t$ & 107 & 1.537 & $\pi(i, 24 i$ & S.ST & $\therefore \quad$. \\
\hline lotial & 7436 & $T(x)$ & $15 \div 19$ & 1412 & $5: 1: 3$ & 21811 & 76,14 & $8, \pm !$ & 34 Pitir \\
\hline
\end{tabular}

Jualing results according to the Hanover Situd Book, Vol, 111.

\begin{tabular}{|c|c|c|c|c|c|c|c|c|c|}
\hline \multirow{2}{*}{ linis } & \multicolumn{2}{|c|}{ Fo:ils buru } & \multirow{2}{*}{ Total } & \multirow{2}{*}{ - Iborted } & \multirow{2}{*}{ liarren } & \multirow{2}{*}{$\begin{array}{l}\text { Tintal } \\
\text { Number } \\
\text { Covered }\end{array}$} & \multirow{2}{*}{$\begin{array}{c}\text { Prognant } \\
?\end{array}$} & \multirow{2}{*}{ 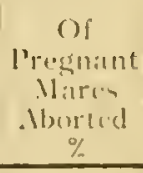 } & \multirow{2}{*}{$\begin{array}{l}\text { liceides } \\
\text { lowins, } \\
\text { partliving } \\
\text { pour dearl }\end{array}$} \\
\hline & Cislls & libllices & & & & & & & \\
\hline In' & 611 & $1: 31$ & $1: 2 \cdot 2$ & sl & :34:3 & 1736 & $77 . ! 11$ & $5,(n !)$ & 2 liair \\
\hline 1S:S(S) & (i.3.5) & $(i, 3)$ & lisini & sit & 114 & 1531 & $7 ., 7 ! !$ & $(i, 1)\}$ & $\ddot{2} \quad$, \\
\hline$i(x)$ & $6: 37$ & linis & $10(9)$ & 8. & . & 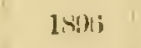 & 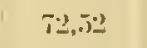 & $1 i, 1$ is & 1 \\
\hline $19 m$ & (i:2) & (iii) & 1273 & 7!) & ixti2 & 1911 & 70,64 & 5,41 & 2 \\
\hline $1: x): 2$ & (ik31 & 6.53 & 12011 & i.i & (i3i32 & $1 m ! 1$ & $64,2 x$ & $5,5 \pm 2$ & $1 \quad$, \\
\hline Tot:al & 315is & $: 3210$ & (61) & 11)1 & 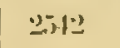 & $11: 371$ & $7: 2,87$ & 5,92 & \& l'ilir \\
\hline
\end{tabular}




\section{(i) The Time of Pregnancy of Brood Mares.}

The time of pregnaney of mares is on an average of almost exactly 11 months' duration-equal to 334 days. Mlale foals are carried un an average $\because 2$ to 3 days longer than female fuals. If the mares are well fed and their state of health is favourable, the time of pregnancy is shortened by a few days, and wice-versa. For example, in Trakehnen early covered mares which foal in November, December and January, carry on an average about 3 days less than those foaling in February, March and April, which have had for a longer time the less favourahle winter food. Mares which work hard, or suckle their foals for a long time, carry a few days more. In the modern improved studs the time of pregnancy is shorter than in the wild Stepoe studs. The so-called Kunters of Russia, Poland and Courland often carry $11_{2}^{\frac{1}{2}}$ to $12 \frac{12}{2}$ montlis. The improvement in meadows and prairies, and the better feeding of the brood mares in Trakehnen has, on an average, shortened the time of pregnancy by $5 \frac{1}{2}$ days, namely, from 335.42 days for males and 333.38 days for females in the years 1865 till 1877, to 3299.9 days for males and 327.67 days for females in the years 1903 till 1907. The regularity with which female foals are carried 2 to 3 days less can be seen more clearly from the following special list for the years 1903 to 1907 inclusive :-

The time of pregnancy necessary in the case of :

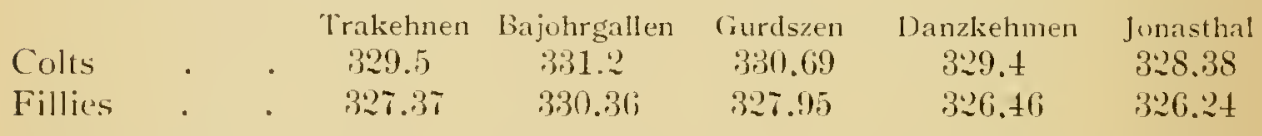

There are mares which almost alway's foal 1 to 3 weelis too early, and there are others which require the same time in addition to the 11 months, and both often transmit this character for several generations. Young mares on an average carry longer than old mares. Many Trakelnen foals, having been carried unly 315 to 320 days, have become prominent brood mares or stallions. According to experiences at Trakehnen, however, foals which have been carried more than 11 months have seldom become anything prominent, and those which have been carried more than 346 days never.

The stallion also has an influence on the duration of pregnancy corresponding to the peculiarities of his breed and family. Mares pregnant from asses carry a few days longer; twins are carried a few davs less (about 10 to 14 days), and are mostly premature births with an unripe appearance. Many firstlings are not carried their full time, and are therefore similar to twinspremature births with an unripe appearance. The dry statistics should be carefully gone into in this direction. In order to produce the first foal well developed the dam must carry it some days longer than the foals which come after, but many young brood mares are not disposed to carry their first foal longer. All prominent firstlings which 1 know, i.e., those that have become Royal Stud stallions, country stallions, brood mares, etc., were carried 5 to 
10 days longer than other prominent products. In the case of mares foaling normally the time of pregnancy often decreases a few days in the course of years.

The Royal Stud stallions which have been born during my welve years' activity at Trakelnen rank according to their merits as follows:-

\begin{tabular}{|c|c|c|c|c|c|}
\hline $\begin{array}{l}\text { Polarsturm. } \\
\text { Mlorgenstralil. }\end{array}$ & $\begin{array}{l}3326 \\
. \quad 327\end{array}$ & ay: & ry & lin & \\
\hline Fischerlinabe . & . 3333 & , & " & , & (Firstling) \\
\hline Prinz Optimus & 319 & ," & , & , & (Only 1 covering) \\
\hline Poet . . & $3: 9$ & ,. & , & ," & \\
\hline Panzer. & 334 & , & , & , , & (Firstling) \\
\hline Mlter llerr. & 32.4 & , & , & " & \\
\hline $.1 \mathrm{mts}$ orstcher & 323 & ", & ", & " & \\
\hline Ackermann & 32.4 & ," & , & ", & \\
\hline I Ioffonungsstrahl & • 339 & , , & ," & " & $\begin{array}{l}\text { (Firstling, also a } 3 \text { - } \\
\text { y.o.covered mare) }\end{array}$ \\
\hline Empörer & - $\quad 330$ & " & " & " & \\
\hline Probstein. & - $32-2$ & " & ", & " & \\
\hline Justizmunister . & - 336 & " & $"$ & " & \\
\hline Altgolel . . & - 340 & $"$ & ," & " & \\
\hline Lungerer. & 331 & " & & & \\
\hline Ethelwold . & 336 & ", & , & . & (Firstling) \\
\hline
\end{tabular}

Other Royal Stud stallions were:-

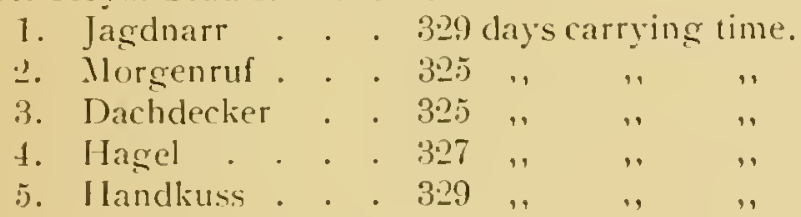

Of the above sixteen Royal Stud stallions, the first eight best were carried un an average 306.87 days, the remaining eight 33.2 .62 days, the five sent away 327 days. Brond mares (about 250) were carried on an average in Trakehnen in the last five years 329ti.7 days, and the country stallions (abuut 200, including Royal Stud stallions) for the same period 3:8.8 days.

Firm these unfortunately few figures one can see-and this 1 believe to have approximately observed also at Trakehnen-that \& to $1 . t$ days under 1.1 months of pregnancy is better than 1 to 8 days or more oxer 11 months. liurther, on looking more closely into the Trakelunen Stud books, 1 find that most mares which carried longer than 11 months were covered several times in une heat. It is rery remarkable that mares which are only covered once during one heat generally carry sonc days less than they do in other years when they are covered several times during one heat. This is owing to conception occurring immediately after covering, thus preventing a further heat, 
and causing the immediate commencement of a healthy foctal development. In the case of mares which are in heat for a longer perind, the egg-fertilisation appears to take place only several days after covering.

The climate seems also to have an influence on the carrying time. In Mesöheyes, Kisber and Babolna the average carrying time is about 3 or 4 days longer than, for example, in Graditz (colts 338.33 days, fillies 336.38 days), and in Trakehnen about 3 days less than in Graditz. Arabians in Weil and Scharnhausen are said to carry on an average about 345 days. Draft horse breecls carry about 3 to 5 days less than warm-blooded horses. According to the statements of Rudolf Endlich (Untersuchungen über physiologische Unterschiede edler und schwerer Pferde, 1895), colts of Belgian breed on the Worbzig Estate in Anhalt were carried 331.1 days, fillies 329.5 days; and the carrying time of Belgian and Rheinish mares in Mankartshof, near Neufs, was 329 days, taking the average for three rears. The average carrying time of Percherons is, according to Tessier (Goltz Handbuch der gesamten Landwirtschaft, Vol. III.), 32.2 days. Moreover, I have found from different old stud books that the carrying time of brood mates 1.50 years ago of all breeds was much longer. Is warm-blooded breeds reach a greater age than cold-blooded ones, and as the average age of all horse breeds 150 years ago was higher than it is now, we must conclude, when considering the above comparisons of carrying times, that on the whole a longer carrying time has as a consequence a greater age. The longlived Arabians are even to-day a good example in this respect.

\section{(m) Abortion and Joint-illness.}

If the milk begins to flow some time before the expected birth it is a sure sign that the foal is ill in the womb; or if the mare carries twins, that one of them is dying. Generally abortion follows. I have noticed several times, however, that the milk again ceased to flow some weeks later, and that the mare produced a healthy foal at the right time, so that one could not really see the consequences of an illness doubtless existing, but which had been fortunately surmounted by the foal. In other cases a healthy foal was born approaching the right time, and at the same time a dead twin of about the size of a cat. I also know of one case in which one dead $t w$ in was foaled in attumn and the other healthy twin in the following spring. As according to the above, the abortion may be, howerer, expected in most cases, it is better to separate the mare, at any rate during the night, so that she may not slip in the same stable where there are other pregnant mares, which might be infected by the undoubtedly contagious abortion bacteria. Late covered mares are in North Germany more inclined to slipping the foal than early covered ones. After abortion it is very important to protect the mares against colds, which they are very apt to catch in this condition. I therefore recommend that mares after abortion should be put in a warm 
stable free from dratught, and well covered up also. Noreover, it is necessary to wash the uterus twice daily with a lukewarm solution of Chinosol to 1 per 1.000 for about 6 to 9 days, i.e., till the oritice of the uterus is completely closed. I have chosen for these cleansings at Trakehnen the abore solution because it more surely kills the bacteria than the solution of $\frac{1}{2}$ per cent. LysolSolution recommended by Professor Ostertag and others. The latter has the additional disadrantage of producing in many mares strong pressure and vehement pains. As early as the beginning of 1890 , the Chief Veterinary Surgeon, Matthias, in Graditz, used such cleansings of uterus after abortion at that time by the aid of Creolin-Solution. This cleansing had such a successful effect in 1893 with the Thoroughbred mare Sappho, amongst others, that in the following rear she produced the . Iustrian Derby winner, Saphir. According to my experiences at Trakehnen, these cleansings have prevented in most, if not in cvery case, repetition of alyortion, and have led to the production, at the right time, of healthy and strong foals.

The mare should only be covered after abortion when the normal arrying time has passed. If the abortion take's place after a pregnancy of $t$ months or still less, she can be covered, after having been properly washed, when next in heat, which generally occurs ! days later, with good results. I have experienced this several times at Beberbeck as well as at Trakehnen.

The uterus of the mare should also be washed if the foals are joint-ill, or if the premature births are suspected of joint-illnest. Mares whose foals suffer from joint-illness should not be covered on the 9 th day after birth, but in the subsequent heat, i.e., 3 to f weeks later, after the above-mentioned cleansings, which should be used after birth for about 3 days, cluring the first heat for abust 6 days, and at the beginning of the second heat once or twice. It is not generally advisable to have as brond mares foals which have suffered from joint disease but which have been cured. In Trakehnen only one such mare (Trommel, 1899 by Jenissij), which as a young suckling foal suffered from a pronounced joint-illness (right hock inflamed), was made a brood mare. She has up to now without interruption produced four good and healthy foals. To prevent the transmission of abortion it is advisable:

1. To clean and disinfert the stable in which the mare has aborted.

2. To isolate the mare up to the cluse of her first heat, i.e, about Is days.

3. Tokeep the mare warm during this time, and u proteet her very carefully from cold.

1. If the abortion has taken place in the pasture, to dig up the spot and 11) discontinue the grazing of brood mare's on that part for at least 4 weeks.

IThilst nearly all seientist still denge the extolugical redation between abortion and joint-illnens of foals, all practical breeders, on the other hand, agree with the point of view hold by (count lehndorth in his handhork for horse breeders, 1A21, namely, that joint-illness is always inherited, i.c., has arisen during the pregnaner in the womb. Profesion Dieckerhoff intended 
in the next edition of his special pathology and therapy to support the view of Count Lehndorff as to the relation between abortion and joint-disease. According to the clinical observations (without microscopic investigations) which I have made at Beberbeck and 'Trakehnen, I consider Count Lehndorff's view correct, although Ostertag's researches distinctly contradict it. When making observations at Trakehnen in 1899 on the occasion of the epidemic of abortion, the Chief Veterinary Surgeon, Matthias, in his report on the abortion of mares and the joint-illness of foals, reports as follows:-

"The disease of joint-illness principally consists of an inflammation of the joint which is connected with lameness. Ilence this disease has alwars been called 'laneness' ('Lähme')."

In my opinion, we are at the present only justified in speaking of jointillness ("Lähme") if there is an inflamed swelling of the joint, together with general fever. According to Bollinger, joint-illness arises from a septic infection of the navel wound, i.e., from a navel infection after birth transmitted by the blowd. Accordingly, 1 have found in nearly 80 per cent. of all cases of joint-illness which 1 have come across, an inflammation of the navel or navel veins, with their accompanying and subsequent symptoms. Jany authorities on joint-illness consider themselves justified in diagnosing for joint-illness in the case where a foal has been born weak and dies with the symptoms of general weakness. These authorities consider a yellowish tint of the Sclera during lifetime as a pathognomonic symptom of joint-illness. If the new-born foal dies soon after with all appearance of catarth of the intestines, they consider this also as joint-illness. If at the post-mortem examination of the foal there is found a rellowish colouring of the underskin, or if the same colour is found in the peritoneal covering of the intestines, or if in the abdominal and thoracic cavity and in the pericardium there is found a quantity of yellowish fluid, and the musculature is grevish-yellow and crumbly, the diagnosis is for joint-illness. The same pathological changes may be found with products which have been slipped in consequence of epidemic abortion, or with foals still-born as a consequence of abortion, or finally, with foals which were born alive but soon died of weakness. All these things are, as 1 have been well eonvinced at Trakehnen in 1899, consequences of epidemic abortion, the cause of which has been proved by Professor Ostertag to be a distinct eoceus.

It has been asserted that "epidenic aburtion and joint-illness are caused by one and the same germ." This is contradicted by the fact of Professor Ostertag having found in cases of joint-illness a different coccus, with which he could by innoculation produce the appearances of joint-illness. He did not find this strepto-coccus in aborted foals. There is, therefore, no valid reason to consider both diseases identical. I, however, believe that I shall be safe in saying that the greater part of the so-called cases of joint-illness, which lead to death without metastatic inflammation of the joint, are really nothing but the consequences of abortus coccus. 
I have just stated that I found in at least so per extut of all cases of jointillness al navel illness. One may, therefore, consider it here proved, without further discussion, that the striptu-coceus has penetrated to the body from the navel wound after birth. The question now arises whether there also exists an intraterine origin of the joint-illness. In about on per cent. of the cases of joint-illness I have found no pathological changes of navel and navel veins. "lo take these cases as a preof of intrauterine infection serems to me too daring, for the strepto-enceus may easily have entered into the body of the young animal by some other way (feeding or breathing), or the illness of the navel may already hate gone on so far that it can no longer be proved in a rough anatumical way.

If the joint-illness had already oricrinated in the womb, then one would expect also cases of hereditary joint-illness, i.e., hereditary acute, pyamic infammations of the joint. No atuthority living at the present time has seen such a case of hereditary joint-illness. Supposing that the three cases of hereditary joint-illness (compare 11 orz. I"ber Staats-oder l.andespoferdezuchtAnstalten 17 urtten-bergs, page 10.5) extracted by Professur Solnnle from the complete literature on joint-illness have been rightly noted and interpreted, then there mas, of course, be a possibility of an intrauterine origin of jointillness. It the same time, one may, however, conclude therefrom that this sort of origrin of joint-illness by intraterine infection is very rare.

It has been observed that in the years of epidemic abortion rases of jointillness are more frequent. Of the about eno foals which are born rearly in Trakehnen, taking an average of five years, s.t die of joint-illness and 6.6 of weakness. In the abortion year 1899,10 foals died of joint-illness and 19 of weakness. The loss by joint-illness did not in this year, therefore, exced the average very much. In the case of a great number of foals suftering from joint-illness, howerer, there may have been about l.j cured of the illness. In these latter cases the illness began very late, even 6 wetes after birth, and an illness of the navel could not be proved. "The fact of joint-illness beginning so late is no profof of intratuterine infection, in spite of the non-cosisting natel illnoss.

Concresning the combating of joint-illness, good results have without doubt been ubtained by carefully attending to the navels of the new-born foals. The cleaning of the utrers of the mare and the washing of the penis of the stallion have certanly profuced satisfactory restuls in lighting epidemic abortus. Is an 'xample of this, I may say that at Trakehnen in the rpidemic year lsog, there were 93 abortions and 6 still-hirths. In the following vear there were, after this treatment, only 19 abortions and 2 still-birtlus. "he yearly average of abortions and still-births in Trakehnen is 2l. The cleansing of the uterus and washing of the penis have not led to a further decrease in cases of joint-illness in Trakehnen. "This is another prof against intratuerine infection. 
Whether there is an intrauterine existence of joint-illness or not, in any case it is recommendable, as is done in Trakehnen, to clean in the same manner as in the case of an abortion the uterus of all those mares whose foals die under symptoms of weakness, or show signs of weakness and illness shortly after birth, or suffer from joint-disease.

We have seen that the coccus of abortus does not in erery case lead to abortion, but, nevertheless, it hinders the derelopment of the foals in such a manner that they are born weakly and ill. Even if, as I suppose, these are not cases of joint-illness, nevertheless, the coccus of abortus which is in the uterus is destroyed by washing. If the orifice of the uterus is already closed when the foal shows signs of illness, the uterus must be washed at the first heat. The penis of the stallion must be washed after every covering. The most effective manner of combating joint-disease is, however, to take especial care of the navel. Complete extermination of joint-disease and of abortion will scarcely be attainable in larger studs where the greater quantity of brood mares produce such a great deal of epidemic matter, for the hygiene of confinement in stables is far from perfect, neither can the cleanliness of the stableman who acts as midwife always be relied upon. Judging from the successes of Trakehnen, however, even in large studs one may succeed in limiting sufficiently the losses caused by joint-illness and abortion.

\section{(n) The Birth.}

I normal birth occurring at the right time causes no difficulties, and seldom lasts longer than 15 minutes, reckoned from the beginning of the first visible signs of the approaching birth labour. Under normal conditions the birth itself lasts about 3 minutes. Three men are sufficient to assist. Above all, these men must keep quiet, and make their observations standing far apart, so as not to disturb the mare whilst she chooses the place which best suits her for foaling. Only when the mare, on account of the first stronger birth throes, has laid dow $n$ and stretched herself nut, should the studnaster see whether the head and the two fore-legs of the foal are in the right position. According to experiences at Trakehnen, irregular position of the foral in the womb, needing the assistance of a veterinary surgenn, is very seldom, as the studmasters are generally very capable. As soon as the head and the two fore-legs can be seen as far as the knee after the bursting of the so-called bladder, clean straw, which slould be at hand, must be put behind the mare as a bedding for the foal, and the skin of the orum must he torn far enough to enable breathing to commence. Simultaneously with the labour pains, the men must begin to pull at the fore-legs if need be with ropes round the pasterns, generally in the direction of the hocks, i.e., an ay from the anus. Then the birth is complete, at Trakehnen the navel is disinfected as follows: The navel string is cut with a pair of scissors which have heen kept in undiluted lysol, at a distance of about 3 fingers width from 
the belly of the foal. After the blond has been properly pressed out of the stump, the latter is washed with a solution of a 1 per $\downarrow, 000$ sublimat, then it is dried with wadding and painted on all sides with a 10 per cent. solution of blue pyoktanin in spirits.

Is soon as the navel has been disinfected, the foal is carried to the head of the mother, and she will then commence to lick the foal. The attendants should now leave, only one remaining in the sable, observing the mare from a short distance. As soon as the after-birth has been removed, which generally takes half an hour, the wet straw is replaced by dry.

In the twelve foaling years which I have spent at Trakehnen (from 1896 to 1907 inclusive), apart from all abortions, cut of 3.388 births, only 38 have been difficult births (including 12 breech-births), where the assistance of a veterinary surgeon was necessary or desirable. Of these 38 difficult births, 23 ended with the death of the foal alone, 2 with the death of the dam and the foal, and :- with the death of the dam alone-altogether 27 mishaps. Accordingly, 3.36(:3) foals were born without mishap, of which 1,689 were colts and 1,67t fillies. Tw in births or abortions of twins happened in these twolve years so times.

\section{(o) Treatment of the Brood Mare after the Birth.}

If the fual die's, the mare should be und, if possible, as a nurse. When this is not done, she will suffer in her health, and give less milk in the next year. If the foal at the beginning is too weak to completely empty the udder of the dam, the udder must be carefully milked at least three times daily. This happens to nearly all foals, in consequence of a slight stomach illness. when they are two or three days old. As one does not like to lift the feet of the mares up in order to round their houfs abuut two or three months before they foal, this must be done after foaling. The same thing applies to the cleaning of the stables. The wbscrvatims made at Trakehnen show that very often a few days after the cleaning of the stables abortions took place, and this seems to confirm the old dictrine that the smelling nerves of pregnant mares are very sensitive, and that evaporation of the dung and the smelf of tar cause abortions. In Throrumghbed hreeding it is, therefore, advisable to remove daily all dung. tugether with the wet straw, and to spread what fresh straw is required, as in the race stable. It is advisable to remove at once after foaling the fresh dung, io prevent the foal from eating same, a thing which they like, but which is dangerous. Foals should be weaned after $1 \frac{1}{2}$ to 6 months. A longer suckling time is seither good for the mare (even if harren) nor the foal. Ifter the frals are weaned is the best time for tinding out mares which have not conceived, and, if there is time, to get them covered again (with two criverings, evening and morning, or on the first and third dav). In my opinion, there is no benefit in omitting to cover goud mares for a year in order to spare them and improve their further 
products. According to observations I have made, this has not been successful. To cover mares every second year as is done in some countries produces very bad fertilisation results. Barren mares are easily recognisable in autumn, even if they are not in heat, by their less smooth hair, and by their worse feeding condition. After six months' pregnancy the movements of the foal can easily be felt, especially during and after drinking. In the case of younger barren mares, light work up to covering time is in most cases very useful. For mares which do not easily conceive, in most cases plentiful, but not quick work, is useful, especially if they are inclined to be fat. 


\section{CHAPTER 11.}

\section{The Judging and Treating of Foals.}

(a) Judging Suckling Foals.

IT is safest to judge the exterior of suckling foals one or two days after birth. Eight or fourteen days later one can, perhaps, judge more saf(ly as to whether they will turn out well, but in most cases the judging of the exterior has then become more difficult. Just as one can judge better the real capabilities of Thoroughbred yearlings by the autumn trials than by a few gallops as two-year-olds, so in the same way it is safer to judge the exterior immediately after birth than when the foals have been weaned, or as yearlings. To rightly judge a young suckling foal one must catch it at a moment when it stands in a natural position, and as far as possible equally on its four legs, and holding the head at a height natural and convenient to him. This moment will nearly always be brief, and the slightest changes of the centre of gravity give a wrong impression. If the centre of gravity is pushed ton far forward, i.e. if the foal stands with the fore-legs somewhat under it, it appears perhaps bow-legged, and if the head is held too high, it appears often to have a swaying back. If the fore pasterns are still very upright, it appears high-legged; if they are very sloping, it appears uver-built, etc. One must also know that suckling foals of ten grow out of some of their back characters. To these belong, first of all, the X-legged position of one or both fore-legs. There are stallions, like Chamant and several of his sons, whose progeny,

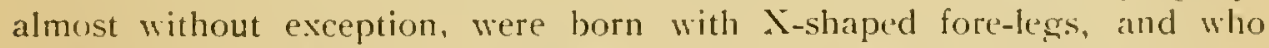
already as yearlings stood and walked perfectly straight. A son of Chamant, Panther, never transmitted this $\mathrm{X}$-shaped position of the fore-legs, but most of his progeny stood with turned-out toes and had an irregular walk. Is a rule, it seldom happens that foals born with $X$ legs walk irregularly later. Further, foals generally grow out of the following defects:-Toolong, soft pasterns, long hind-legs standing out behind, polished knees or bending back, as well as long, upright fore-pasterns, all sideway leanings, as well as all misplacements (in the womb) of the fore and particularly of the hind-legs. The bending of one or of both hind-pasterns, which occurs so frequently and often very pronounced, nearly always grows normal. The other proportions of the body do not alter. One thing remains to be noted, i.e., that foals 
always appear to be higher legged than they are when they become fully grown. The proportion of the length and the width of the body, especially of the back and crupper, strength of joints, very high or deficient withers, good or bad crest, remain the same. Short necks remain short, but are not so conspicuous in a fully-grown horse as with a suckling foal. The same thing applies to upright and tu sickle hind-legs, which change little, but are not so conspicuous in a fully-grown horse as in a young foal. Outwardly placed pasterns often become all right if this outward position only consists of a bend of the pastern joint round a horizontal axle. If, however, the outward pusition consists of a twisting of the whole fore-leg commencing at the elbow round a perpendicular axle, this fault cannot be remedied. Nost narrow-toed positions of the fore-legs consist of the twisting of the whole leg round a perpendicular axle, and therefore do not improve. Often, and usually between the second and the third year, this position becomes much worse. Only if both pasterns deviate to the same side, i.e., if the one leg stands wide-toed and the other narrow-toed, one may assume a twisting round a perpendicular axle, and therefore an improvement of both pasterns, including the inwardly placed one, is probable. Whether the crooked fore-legs wil] grow straight again can only be verified if one knows the development of foals of certain parents. For example, the progeny of Optimus born with crooked fore-legs retain same, whilst nearly all the progeny of Perfectionist were born with ("rooked fortelegs and became straight without exception. . Wl the progeny of Hazlehatch are always born with straight fore-legs, which later on, however, generally become crooked.

IJigh-leggedness, already recognisable in the suckling foal (of course, the above-mentioned upright position of pasterns must, if necessary, be taken into consideration when judging), nearly always remains. Faulty position of the ankle joint (tied in below hock) is very seldom got over so completely that traces of it cannot be perceived later, but the most distorted hocks, however, often improve remarkably, especially if the connection is broad. No improvement can be expected, however, if the upper part of the hock (calcaneus) is inclined too far forward. It is often very difficult and unsafe to rightly judge the hocks of foals born very much misplaced (in the womb). There are stallions, like Pomp and Morgenstrahl, whose progeny were born mostly with their hinder parts very much misplaced and crooked, but in the short space of two to six months they grew out of the seemingly impossible twists, almost having the appearance of curbs. One can only judge approximately correctly if one knows the manner of clevelopment of the progeny of certain stallions. Optimus' progeny, for example, which were born with hocks a little ill-shaped (which, however, seldom accurred), scarcely ever lost this fault. The same thing applies to the progeny of Hazlehatch. Generally speaking, the chance of growing out of weakly supported and improperly set hocks is least if there is no misplacement (in the womb) connected with these faults, and ice-zersa. Furthermore, small 
born fuals grow out of theil fatults lessuften than big born ones. The former have gencrally sulticient roum in the womb and, therefore, do not get misplaced.

To enrectly judge the nobility of the foal, one must not forget that this is a perint which changes very little, i.c., they will never look nobler than they (o) as young suckling foals. The young age is very apt to hide coarseness. In judging the strength of bones of foals, one must not be deceired by the long hairs on the legs. I big, dry head indicates a strong bone development, whilst the fairly small head generally indicates thin, camnon bones. Foals burn with short, smonth and shining latirs on the legs lonk lighter than they are, and must be judged farourably, as one may expect from them cuality, dryness, hatedness and health. Short and dull-looking hair is at sign that the foal has been born premature and unripe. I have never yet known a rase where stu-h premature births have developed into first-class horses. The so-calied over-built foals, which is mostly to be seen with one and tworear-olds (nearly all progeny of lolapuk), nearly always crou normally.

In judging carly the exterior, one must, of course, take into consideration the healthy development of the foal, for without such this growing out is not only not to be expected, but rather a relapse in many parts may be feared which were originally normal. The back especially gives way most easily. and becomes, wern during the streling, or immediately after the weaning, a low back, on account of disturbed development and nourishment, the rauses of which are often hidden. l.ow backs which suddenly originate after a severe illness, stch as slanders, often completely disappear, whilst those arising from no risible acute illness never get all right. The most conspicunus (xample of this which I know is the Royal Stud stallion Empörer. born 1899 by Lehnsherr (by Chamant) and Emigrantin, who as weaning foal sot a rery bad low back commencing immediately behind the withers after a rery seice attack of glanders. I le was transferred in the spring of 1900 to Guddin to the geldings. In the course of about nine momb the low back disappeared completely. In the atutum he was put to training as a tworearold, and is tomely Royal Situd stallion in Trakehnen. He has a faultess back, and his progeny also. Cienerally speaking, the foals of high-bluoded stallions, especially Thoroughbreds, develop better than those of coarser stallions. For this reason the former grow out of their faults better than the latter.

\section{(b) Treatment of Suckling Foals up to the time of Weaning.}

The lirst or sn-called Colustrum milk is especially useful to the foal on account of its somew hat opening (ffect, thus hastening the getting rid of the lirst fecal substance of the foal known as Meconium. This Colostrum milli must mot, therefore, as unfortunately often happens, be milked off. The Meconium passes away under normal conditions in the first twelve in twenty- 
four hours, and in many instances two hours after birth. If this happens too rapidly it is often not a good sign. Diarrhnea follows, frequently a sign of weakness, or beginning of joint-illness. I delay of this important removal causes meconium colics, which may very easily become dangerous. This disease generally attacks foals carried more than eleven months, and mate foals more frequently than female foals. I have never yet noticed this meconium colic in foals carried less than 320 clays. In Trakehnen in 1896 the chief reterinary surgeon, Dr. Töpper, first applied very successfully the Meconotorium, constructed by Masch, for the purpose of artificially remoring the Meconium. As this instrument can be easily handled by nonprofessionals, I here state what Dr. Töpper says about it:- "The operator sits on a chair, and the foal is held in a proper position. The instrument, and the first or second finger of the left hand, are oiled. I left finger is carefully inserted, and with it the IJecotorium (the convexity of the spoon downwards). If one presses the handle down the spoon presses into the meconium, and it is then possible to bring it out in parts as far as one can reach forwards. One $\mathrm{g}$. calomel with $50 \mathrm{~g}$. rizinus are afterwards given for the final removal, and an injection of luke-warm water made. The instrument may be had from Hauptner-Berlin." I would like to add that the spoon must be used with the greatest care, for the rectum of the foal is not capable of much resistance, and if it were pierced with the spoon death would always follow.

I slight harmless diarrhoa of the foal announces the first heat of the dam. For a continuous or malignant diarrhoa the following treatment has proved successful in Trakehnen :--

If it is a case of the dangerous grey stinking diarrhoa of foals, the intestines must be completely emptied as quickly as possible. To obtain this result, give the foal 50 to $100 \mathrm{~g}$. rizinus oil in one dose (an ordinary spoon contains about $10 \mathrm{~g}$. ). The day following give it again in one dose 10 to $20 \mathrm{~g}$. tincture of opium (a spoon contains about $1.5 \mathrm{~g}$.) with four times as much mother's milk. If necessary, this treatment with the tincture of opium, gradually increasing from 10 to $20 \mathrm{~g}$., is continued for several days. and if need be even in two doses daily. If in the course of this treatment the foal should suffer from inflated belly or colic, the treatment of opium must be abandoned.

If it is a question of the ordinary watery diarrhoa, a dose of 50 to $125 \mathrm{~g}$. of rizinus oil given at the commencement often renders further treatment superfluous.

Continuous doses of tincture of opium are essential if the diarrhoea continues for some time. As a foal soon gets into the habit of taking tincture of opium, increase the doses slowly, from 10 to $2.5 \mathrm{~g} .$, and also from once to twice daily.

Instead of the oats, which cannot be digested by the weakened gastrointestinal canal, it is advisable in cases of chronic diarrhoea to give a mixture 
of oats and linseed meal ground very fine, almost to a mash, $1 \mathrm{cut}$. of oats to about $5 \mathrm{lbs}$. of linseed. According to my experiences, a complete change of food of the dam, as regards oats and hay, as well as of bedding straw, does not have any influence on the diarrhoa of the foal.

The first nine days after birth the brood mare must be separated, along with the foal, in cases where the mares are together with several others in large stables and not in special boxes. Later on, when the foals are strong enough, it is better both for dam and foal to be able to move about freely in a large stable. Unfortunately, the arrangements in Thoroughbred studs, on account of too great anxiety, are usually not calculated to give these great advantages of freer movement, and to provide at the same time special places for oats and hay for the foals. When it is fourteen days old the suckling foal usually begins to nibble oats. It can be taken for granted that on an arerage the foals can eat as many pounds of oats daily as they are months old. Besides the oats, it is advisable to give the foals daily as much fresh hay as possible, also clover hay. I four months old suckling forl must have daily 4 to 5 lbs. of oats, and almost as much hay. If the clam gives too little milk, and it is possible to teach the foal to drink cow milk, which is often very difficult, an addition of about 3 to $\mathrm{j}$ litres of fresh undiluted cow milk (three times daily 1 to $1 \frac{1}{2}$ litres direct from the cow) is to be recommended. I have never yet found that this addition of fresh undiluted cow mills had any bad effect on suckling foals. Stunted weaning foals, as well as suckling foals, whose dams die suddenly in griving birth or somewhat later, must have daily. (f) to 9 litres fresh undiluted cow milk. One litse every two hours cluring the clay and every four hours during the night is the right quantity. It is reckoned that a good brood mare gives about 10 to 1.2 litres daily.

About four to six weeks after birth the foal's hoofs must be attended to. The foal must, however, have been already accustomed to being led for a short distance and held by the halter. This care of the hoofs, which is so important for the whole life of the horses, and which can rarely be recovered later, consists in cutting at first the foctal frog, which is provided with unhorned, soft epidernal material, with a sharp knife, holding it level, in such a manner that the hoofs get the necessary form of fros, with a soft depression in the middle and absolutely smooth surfaces. The remaining spongy substance must be removed from the sole in such a manner that it receives its proper depth. Then the wall must be cut clown on the quarters and toe as the form and position of the hoof necessitates. Frng and inferior border of the wall must lie on one level, so that the frog also helps to carry the body. The lateral lacunae of the frog must be kept smonth, and wide open below. The small cracks in the lateral lacunae of the frog are removed by so-called air-making, i.c., by a notch cut. However, under no circumstance must the strength of the bars be weakened. The most careful removal of all, even the very smallest cracks, especially in the median and the wo lateral lacunae of the frog, is very important, because it is impossible to licep 
them clean otherwise, and they therefore easily become the means of causing putridity. Every hoof must be brushed over at once, after cutting, with tar: of course, only the sole, including the frog. This must be repeated, in the case of suckling foals, every four weels before grazing time. It does not only. malie the putridity of the frog imprissible, but also assures a strong and broad derelopment of the frog and of the hoof. During grazing this work is not so often and so urgently necessary as during the winter stabling, therefore most necessary for early born foals. The wearing of the hoof caused by plentr of exercise in the pastures, and this effect of the damp earth which becomes fastened to the hoof, and which is beneficial to same, by its massaging, widening and preventing putridits, does away with, to a great extent.

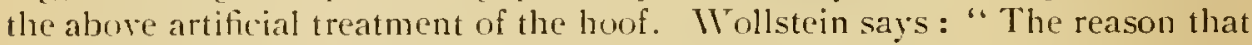
foals in natural studs suffer so little with their hoofs is because they have plenty of exercise." Even in Thoroughbred breeding the early eare of the hoof is, unfortunately, much neglected, and is the cause of so many contracted hoofs with curved walls and atrophied frogs, which again in their turn produce an early break down.

Simultaneously with the attention to the hoofs begins also the cleaning of the foals. It is not necessary to put a halter round them or to hold them. as, if it is done sensibly, they enjoy it, and it is the first means by which une can gain their confidence. The purpose of cleaning (of course, only with a dandy brush) is not only to keep them clean and prevent lice, but also for the very beneficial scrubbing of the slin, which is especially good for foals born early in the year. Later on during srazing, wind and rain do their share in this beneficial massaging of the skin.

Foals get their first exereise in the open when they, 10 to 14 days old, ge with the dam two or three times daily to drink from the trough in the yard. Very soon afterwards, at the latest at the age of 6 weeks, they must be exercised with the dam in the open, commencing with a quarter of an hour daily. Of course, these exercises must take place regularly every day, and in atl sorts of weather, being extended to at least one hour daily, taking into consideration wind and weather, so that the dams as well as foals get accustomed to fresh air, weather and wind when the grazing commences. On warm and sunny spring days it is advisable to let out at their leisure all the brood mares with their suckling foals in the yard for an hour in the afternoon, over and above the regular exercise as mentioned above. The beneficial fresh air will strengthen brood mares and foals and cause them to mix one with the other, which is very useful. Mares which have not become pregnant will often come in heat on such occasions.

Just as important as this adrice is-i.e., to have open air exercise in all kinds of weather-for our Northern climate, so is it just as unnecessary for England and France, admirable horse breeding countries, endowed with the Grace of God. My neighbours have often called the above nethod of foal rearing an exaggerated and unreasonable attempt to become hardy. Never- 
theless, I have proved that even the youngest foals can endure much more esercise, frost, wind, rain and all sorts of bad weather than I and many other people supposed. This hardening method of rearing is very useful in ever! respect for all foals, and does much to improve their health, hardness, and finally, performing capabilities. In my attempts at hardening I have not even reached the limits at which a distinct advantage to the foals ceasednot to speak of the limits at which they might begin to suffer. Of course, unreasonahle attempts at hardening which do not aroid sudden and unprepared changes would soon reach those limits, but an observing and individualising breeder will know how to act to avesid many dangers which l cannot mention here, as for example, when exercising in hard cround, foals with an inclination to upright hoofs should be suitably shod at the right times.

Ifter to to jonths suckling foals hose their foal hairs, and may then bu weaned. It is good for foals if the suckling time is extended to is or $f$ months, and this is at any rate advisable in the case of barren and late covered mares. Before weaning-it is best to begin at the age of 3 monthsfoals should be led by the halter, so that one may lead them without their dams to their own stables or to other places on the farm. The best time for weaning is towards the evening. Dam as well as foal become quict much more easily than when they are weaned in the morning. Foals which cough much, or suffer from glanders, must be weaned some weeks later, atter having wereome the illness. Suckling forals get over glanders sounce than weanced foals. Weakly weaned foals, and such as have not yet lost their coat, may be assisted for 1 or 2 months with cow milk, about 6 litres daily. To give cow milk for a longer period, especially to "Thoroughbred foals, has the disadvantage that it causes them to crow fat, and possibly somewhat soft, therefore later more difficult to train. On the other hand, one must take into consideration that weaned foals gret owr slanders more easily if they are given (u) milli during the time they have the disease. Foals which are inclined to grow fat may be given skimmed milk. In the case of brood mares which are barren, and therefore needing no protection for an embryo in the womb, it is not advisable to suclile their foals longer than 7 months at the most. In many Russian studs, where the suckling perind lasts (ven longer than lo months, I have found that this was not gond for the foals. It scems to me that they make pourer use of the oats and hay when suckling so long.

\section{(c) Treatment of Weanlings.}

With a little tact and much lore one steceeds in manipulating the weaning process, so deeply connected with the whole life of the streliling forls, in such a manuer that no interruption in their development ecors. During the lirst 21 hours at least they must, unfortunately, be liept in their now stables. In my upinion it is necessary to separate the sexes immediancly after the 
weaning, or a few weeks later. It is quite common for well fed six-month-old colts to attempt to cover, and many fillies less than one year old are already often in heat. In the stud Tammist, of Mr. B. v. Liphardt, Dorpat, in the summer of 1868, a one-year-old filly (noble Half-bred) was covered whilst grazing by a one-year-old colt (also noble Half-bred) and became pregnant. As a two-year-old she had a living, but very small colt. The Thoroughbred filly Experiment, 1812 by Bay Middleton out of Miss Craven, was covered at 11 months old by Venison, and produced when she was 1 year and 10 months old a living colt, which died after 24 hours. In the following year Experiment produced a filly by Venison. Experiment was in Lord Bentinck's stud, who had a special craze for such extravagant trials (see General Stud Book, Yol. V1., and "Post and Paddock" by The Druid, page 208).

For weaned foals a roomy, light stable, with constant fresh water and a good meadow close at hand is essential. The more foals are weaned at the same time the sonner they settle down, and when in a few weeks the next lot of weaned foals are put in the same stable, the weaning will be much more easy. The rery best picked food, always attainable fresh water, and association with horses of their own age, soon helps the foals to forget their dams. During the time of their growing the fastest, young foals require rery urgently plentiful and frequent supply of water. If that is not possible their development will be distinctly retarded. Weakly and backward foals must be separated-at least in the stable-if they are badly treated by their more robust companions.

To good grazing also should be added fresh Lucerne, Sainfoin, or green clover, with or without Timothy, ad libitum, especially in the evening, and in the hot summer weather at dinner time also. I do not recommend the giving of clover to Thoroughbreds, as experience has proved that foals fed with clover, green or dried, look very well but are much more difficult to train, suffer in the wind, i.c., have a thick wind. The best food for Thoroughbred and I Lalf-bred foals is certainly. Lucerne, and the longer foals can be fed with fresh, good, and not attacked by rust Lucerne (in East Prussia generally from the middle of May to the end of Scptember and the beginning of October) the better they will develop. When the Lucerne or the clover begins to blossom, then is the most farourable moment to commence with green food. Before the blossoming foals do not care to cat Lucerne. Foals, and particularly young ones, prefer, however, green clover to Lucerne. It is not therefore advisable to substitute often Lucerne for clover. To obtain an early second crop of Lucerne or clover, a part of it must be cut before blossoming. This early cut part will then be ready as a second cut with commencing blossom when the first part has been eaten up or become too dry. In a few words, it is only with great care and a correct division of the available land for food crops that one will succeed in obtaining an uninterrupted and regular supply of green food from spring to autumn. Those 
breeders possessing such good pastures that they do not need an addition of lucerne or clover are to be comgratulated. In Fast Prussia we have not got them.

For weaned foals 6 llos. of uats are sulticient at the commencement, and for those who desire to, or must save-for example, military horse breeders -6 lbs. are sufficient for the whole poriod, if green Lucerne or clover can be added. "Those who desire to breed breceling material or racehorses, or firstclass horses for use, must increase the oats monthly by 1 ll). per day up to at most $12 \mathrm{Jbs}$. I do not consider that generally a greater quantity is necessary. not even in Thoroughbred breding. It Trakehnen I have never given on an average more than 10 lbs. of hats. Of course, besides this arerage, an addition of about 3 llos. of oats is necessary for some backward foals, and foals which eat rery little lay. The portion of oats is split up into three meals-morning, noun and evening-for supper a little more. The foals must always be fastened up uhen the oats are giren, so that each one gets its right quantity and so that it can be seen which one has not eaten its portion. It is verv useful to give weaned foals with every feed of vats a handful of eround or ruasted linseed (about i lb. daily). When in atutumn the sreen foxd is finished and the winter ration of about 10 lbs. of hay (Luecrne or elower hay) begins, it is advisable to give them for about three to four weeks about i litres of fine chopped carrots daily, and afterwards for the whole of the winter twice woply about 2 litres wheat bran, together with wats. In those years in which the hay has grown well in consepuenere of a dry and warm summer, and has been well got in, having therefore a sreater nutritive value, we have, in Trakehnen, diminished the share of oats for "reaned foals, and given in autumn and winter at the most 9) Ibs. (6) prevent fatmess. I diminution of hay or clover would have a bad effect on the formation of bones, as the bone-forming factors are not in the grain but for the most part in the hay and sereen foud, which contains plenty of lime. Viers course clower or lacerme hay produces in roung weaned foals diarrluxa, as it irritates the intestines, and it is better to jiep same for older foals. "Thres werks" feeding with meadow hay rauses this diarrloca of "ranlings to disappear withum any other remedy. For a few years weaned

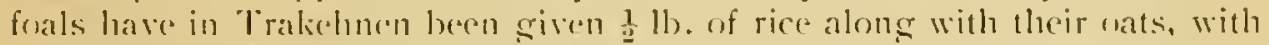
the idea that the large guantity of phosphorus comtaned in same would favourably influence the formation of bone. To sive a definite result of this is not possible get. The mese potash there is in the food of the foals, and this is particularly so in geod grazing and with green l.ucerne, the more salt is recpuired by the horses. (This ean be demonstrated by a chemical formula.) lhere ought always to be several large pieces of salt in the mangers.

Is long as erazing is possible no cliffeulty whatever is experiuneed in griving proper excecise on woanced foals. Grazing in East Prussia, which. unfortunately, only lasts about live months, must be made the best prossible 
use of, i.e., the foals must remain on the muadows as long as it is light, with a short interval for the mid-day meal, which they should receive in the stables in the form of vats, water, and if required, especially in the atutumn, an addition of green Lucerne or clover. On hot summer days this interval may at times be prolonged to about three hours, with green fond in the stable. The utmost use of the grazing time also already for weaned foals is, for the breeding of light horses, the most important and potent factor for the production of healthy and capable stock, and yet this is a thing which is very much neglected. The neglect and the final leaving off of grazing represents, in the history of many breeding studs, the turning point in the transfer from a capable breeding stud to one which is contented only with the cheaper production of showy stock. Those economical considerations which have led to the limitation or the abolition of grazing endanger our modern improved breeds in the highest degree. This applies to horses as well as to cattle. The influence of grazing, which is so very great, and which cannot be substituted by anything else, consists principally in the long, regular and slow exercise in the open air on elastic, turfy ground, and under the continuous and beneficial effect of sun, wind and rain. The tinest rereising place cannot replace these advantages of grazing, principally because the exercising place lacks the required fond-secking stimulus necessary for the said regular exercise. The healthy and useful feeding on the pasture is only of second inportance.

In winter, when there is plenty of snow, exercise mav lake place in the same pastures. In our climate it is in the spring and atutumn that the difticulty of regular exercise is experienced, as of course the pastures must be spared at these times. Horses have then to be exercised on the paved grounds, perhaps covered ner with straw, railed in and cirrular, or if it is not possible to get exercise on such places on account of the ice or because the ground is frozen, etc., horses must be exercised in half-eorered rircusformed rooms. This exercise must take place daily, about $1 \frac{7}{2}$ to 2 lours in the morning and a little less in the afternom, if possible with shepherds' horses, in urder to get the regular trotting esercise. "Whe regular exercise of weaned foals, as well as of older horses, whether on the pastures in the summer, or on the covered or uncovered exereising places in the winter, is the most important thing in the whole breeding of light horses. This point is often very much sinued against, even in Thoroughbred breeding, especially when horses are to be sold as yerlings. Ifter the finishing of grazing time, weaned Thoroughbred foals and yearlings require a sistematic, gradual and progressive course of exercise. This ensists in leading them for $1 \frac{1}{2}$ hours ii) the morning, and somewhat less in.the afternoon, at a slow pace, and galloping them daily on a railed-in track about 10 metres wide and about .500 metres long. To start with, special leading horses with a man up are useful until the foals learn later on the purpose of it. They then will gallop the distance of about 500 metres without such help. 
Whilst the weathed forats are bastened up for dinner, it is the most suitable time to lead them each day on a neighbouring track, till they are quite lit (o) be handled and are obedient for leading, as well as to place them in a proper position to judge their exterior. This first and very thankful teaching of the roung and willing foals should only commence four to six weelis after being weaned. Lnfortunately, Thuroughbred foals very seldom get this rery useful schouling, because one does not like the trouble, and, moreover, because one under-estimates the great advantages derised from this education. In I Ialf-bred breeding this would be the must opportune time to select those colts for castration which are not suitable for breeding. The carlier they are castrated the easier the fuals get wrer the operation, and the more perfectle do they develop into the desired type of gelding with thin neck and broad croup. it is still more advantageous, in order to get fine geldings, to castrate colts when they are about four weeks uld and are still with their dam, as the testictes are then sulthe ienty prominent. Foals which are early castrated srow out of many exterior faults, such as high-leggedness, narrowness, and even faulty action, often tes such a degree that when lonking at the horses when four-year-olds, one finds the reason for castrating only from the remarks written about the esterios.

\section{(d) Treatment of the Skin and Hoofs of Weanlings.}

To properly lowk after weaned fials one may rection twelve to fifteen couls to one ancindant. Of course, une man cannot clean fifteen foals properly daily, but that is not necessary, met eren in Thoroughbred breeding. I consider it sufficient if each foal is simply rubbed down each day in order to take off the worst dirt. "This is best deme in the morning whilst they eat their oats, and it requires two to three minutes for each foal. Besides this, "ach foal mus be thoroughly cleaned once a week. For this purpose every disposable altendant is given in the morning or afternoon two to three foals in the stable to clean whilst the rest are being exercised. It the same time lec lowks after the hoofs and kecps them in order by cutting them properly, rounding them and tarring them once about two or three neeks. The -mouth outting of the frog, and the air-making between the frog and the heels (opening up of the lateral lacunte of the frog), ats already stated in comnection with sucklings, are continued. In the case of contracted heels, the hech-edges must be cut sufficiently to enable one to draw the hoof wraper through the lateral lacunae of the frog from front te back with case. The stud attendants must, of course, be specially laught this work, which is nut difficult, particularly if the studmaster or manager himself understands it. If this worls, however, is given to blacksmiths. who mosty have no real interest in the work and are not always alvailable, the proper lieeping of henfs "ill never be obtained in the stables. This care of the hexts takes very. little time or trouble, but causes much allsicty, and is of the greatest 
importance, especially for Thoroughbreds. From my many rears' experience, I can confidently assert that if the hoofs are looked after in the manner stated above, of course a rotten frog is not only made impossible, but strong and resisting hoofs, with correct, broad, full and well-supporting frogs are produced to such a degree as is so much wanted for the racecourse, but which yet are so rarely found. The same order in cleaning and the same care of the hoof is to be applied later for one and two-year-old foals.

In about ten to fifteen per cent. of weaned foals it will be necessary to shoe them from time to time. In the first place, those foals which, on account of their straight pasterns, run too much on their toes, and which are therefore inclined to form upright hoofs, must be shod with half-moon shaped toe-shoes. It is generally sufficient to shoe twice every three or four weeks. Further, on account of some misplacements originating in the womb which have not been quite grown out of the I-formed position of the fore-legs belongs also to these), or in consequence of standing too wide, together with a ret too narrow chest, or again because of some crookedness in the pastern joint which may still be rectified, some foals are exposed to the clanger of wearing their hoofs off obliquely, and thus a proper development is prevented. In such cases 1 would recommend that the foals be shod according to the well-known rules for faulty standing, and in this manner until they have grown out of the supposed causes of faulty standing, and until there is no longer any fear that they will continue to wear their hoofs off obliquely. 1 mang cases shoeing for three months will be sufficient, often, however, twelve months and even more are necessary; in fact, some never grow right. A twisting round the vertical axle of the leg, originating at the carpus or wrist, the so-called "linee," or higher (not to be mistaken with the X-legged position, which is easier to handle), cannot be improved by shoeing. If these faults are not put riglut by shoeing, as unfortunately is so often the case in Thoroughbred breeding, which despises the exterior appearance, one will often find that whilst after about twelve months the formerly misplaced pastern, the narrow chest and other faults have been grown out of and become normal, but at the same time during this year an oblique hoof, i.e., contracted heel on one side, has been formed, which it will, of course, now be much more difficult to put right, especially as improvement by shoeing is very dangerous for horses in training. For twistings round a vertical axle, which cannot be put right, it is advisable, in the first years before training, to have a shoe made to suit the particular case, as by this means the early development of an oblique hoof is prevented. or at any rate the tendency to grow oblique is lessened. The following instructions are given to our local stablemen and shoeing smiths for the cutting and shoeing of hoofs in the case of faulty positions :-

Looked at sideways, faulty hoofs may be divided into pointed and blunt hoofs. pointed hoofs are those of which the long toe is conspicuously 
oblique, and the heels too low, and the blunt hoofs are those of which the short toe is conspicuously straight and the heels too high. Pointed hoofs are to be found when horses stand with their legs very much in front of the body, or if the fore-legs are knock-kneed (calf or sheep-kneed), or if the hind-legs are too angular, or if the position of the toes forms an acute angle (in most cases with long or sloping pasterns). If it is desired to favourably influence these faulty positions, the toe-wall of the hoof will have to be kept short. On the other hand, the heels are to be spared as nuch as possible. Blunt hoofs are found with horses whose fore-legs are placed too far back or are over in the knees, or if the position of the toes forms an obtuse angle (in most cases with short or upright pasterns). In this case cutting of the hoof must be limited for the most part to the ton high heels, although in many cases the keeping down of the heel does not effect a remedy. In cases where foals have a lot of exercise on hard, sharp ground, the toe sometimes becomes so worn off that a pronounced upright hoof is produced, and if not dealt with at once it cannot be remedied later, and forms a so-called pincard or ramplin foot, or otherwise ring-bone with its attendant lameness ensues. $13 y$ a timely use of a toe shoe this affliction mar be completely aroided. If it is a question of roung foals whose hoofs do not permit of a the shoe. I would recommend that the toes be hardened by repeated applications of tar, and also that small leather shoes be used. I may here mention that in some rare cases a pincard foot may arise with foals, apparently the consequence of diseased bones and sinews, and this is incurable.

Looked at from the front, the faulty hoofs may be again divided for the sake of simplicity into two groups-group 1. hoofs with wide-set toes, and group II. hoofs with narrow-set toes. Hoofs with wide-set toes are more or less pronounced in the following positions: standing wide, X-legged and wide-toed. The hoofs of the second group are found in the following cases: narrow standing. O-legged and narrow-toed.

If it is desired that the foal shall outerow these faulty pusitions, the hoofs of group I. must be cut as follows: Repeatedly cut down the outside of the toes, quarters and heels. Cut horizontally so that the sole is, if possible, in the same level with the inferior border of the wall. The sole must therefore be as little arched as possible. Do not shorten the inferior border of the wall on the inside, but from the sole so much horn must be removed, and the inner bar so far shortened (shortened, not weakened) that the sole appears much arched towards the frog. This arching of the sole of the hoof, too narrow at the inside in the case of a wide-set toe, aims at a widening of the hoof on the inside by counter-pressure of the ground.

Group 1I., hoofs of the narrow-set toes, as far as cutting is concerned. are treated in exactly the opposite manner to group 1. "The adrantages of cutting hoofs with faulty positions can best be learned when foals are exercising on soft, yielding ground. If the exercising ground is rough 
and hard the hoof soon wears to the slape necessitated by the position of the legs. In such cases the only thing to counteract this is shoeing. Shoeing should be used not before an age of six months, but usually not before they have reached the age of ten months, except in the case of uprighthoofed sucliling foals, when they should be shod earlier. It is, of course, talsen for granted that only a lirst-rate smith, perfectly acquainted with this kind of shoeing, does the work.

The shoe for the hoof of the wide-toed position must be as follows: In outer thin and an inner thick branch, the outer branch tapering towards the end, which should have the thickness of the blacle of a linife. The supporting edge of the thick branch is sloped outwards in the region of the quarters and heels. The nail holes in the inner thick branch should be made as far to the fromt as possible. The thin outside branch, the edge of which must be lept narrow, must be fitted on tight and nailed as far as the end. The inner strong branch must be kept long, and must be fitted on large. The inferion besder of the wall on its supporting surface, which slopes outwards, is inclined by each weight to slip outwards, the consequence therefrom being an early recognizable widening of the hon on the inside.

The hoof of a narrow-standing requires a shoe exactly opposite in malie. Of course, the shoe must be changed at the right time, and it is to be noted also that the faulty position cannot be remedied at once by a too strong one-sided rutting down of the inferior border of the wall, nor can it be forced by a tor great difference in the strength of the shoe-branches; both things must be done gradually, and slowly increased. It depends on how bad the faulty position is, the shape of the hoof and the results obtained, as to how long the shoeing whigt to be continued. Is soon as the animal is put to hard work it must be shod with straight shoes, but eren then it is advisable when preparing the loufs for shoeing to see that they retain the breaking ol the toe axle profuced by artificial shocing, as well as its regular form, so very laboriously acquired.

The advantages of a proper cutting and good shocing for the form of the houf and the position of the legs does not only help the foal to outgrow a faulty position well and quiokly, but does more, i.e., instead of an oblique hoof, which is always the consequence of a faulty position, a regular shaped hool is formed. For example, in the case of wide-set toes, the hoof is rery oblique, $i . e$, the inner walls incline ton much to the inside, are low and puslied under, and the whole inner half of the hoof is too narrow.

If these measures are not male use of, these evils will remain, and in most cases this houf will become later on a one-sided contracted hoof (narrowheeledness), otherwise it will not be able to overcome quitters, seams, etc., and the value of the horse is acordingly rery much reduced. But even this does not exhaust the advantages of the breaking of the toe axle. IJorses for whom nothing has been done to remedy their wide-toed position will knock themselves as soon as they are taken in use, and they are useless for 
carriage, ete., work. Foals with wide-set toes. whose toe axles by correct shoeing have acquired the desired breaking, very seldom hit their legs when put to work.

Mamy experts say, and with some justilicatiom, that the doubtless unuatural breaking of the toe axle may also cause damage. On the other hand I must state that many experiments in this direction have been made for many years, most likely only at Trakehnen, and they have demonstrated that a slow breaking of the toe axle can be endured by voung horses without injury.

If the Lithuanian peasant, or the military horse breeder, a few days before a sale prepares the hoofs in an artificial manner, this ruse has nothing to do with the real methods of improvement, which are very good and usefui for horses.

Iton sudden break of the toe axle of a three-year-old may be disastrous to young animals owing to the tearing of the ligaments and spraining of the joints, particularly so if the attempts to improve are made by shoeing. The hoof which has been shod does not so easily overcome the sudden breaking of the toe axle as an unshod one does, as the wearing off of the too high bearing part of the unshod hoof is better accomplished, and consequently no damage arises from the breaking of the the axle.

\section{(e) The Treatment of Yearlings and Older Foals.}

Treat and feed yearlings up to the beginning of grazing time in the same manner as I have already pointed out for weaned foals. When the grazing is good about + to 6 lhs. of oats are suffeient for military and other horses in use. For breeding material, abutut 6 to 8 lhs. of oats, with a simultaneous addition of green clover or l.ucerne, is required. Thoroughbred rearlings require 10 to $1.2 \mathrm{lbs}$. of oats, and in addition green l.ucerne. Half-bred vearlings which are given when grazing more than 6 to 8 lbs. of oats will eat less clover or Lucerne, and will therefore not become so strong. In some instances, as for example, where foals are growing fast, or do not feed well, a special addition of 3 to 6 lbs. of onts may be advisable.

Ifter the grazing period, Half-bred vearlings destined for use are given abuut fo lbs. of oats and 12 lbs. of hay. Horses destined for breeding stock are given about 8 lbs. of oats and $75 \mathrm{jhs}$. of cluver or lucerne. Thoroughbred yearlings should be broken in during grazing time late in the summer (about 1] hours daily), and in autumn after fonishing the best grazing they should be turned into training for racing.

In the case of two-year-olds, those bred for use receive during grazing time? lbs. of vats (early in the morning), or if grazing is groud no oats; and those required for breeding stock about $t$ to (; llos. of nats, and in both cases green Lucerne or clover in addition. If lacerne is very goud and plentiful. 1 lbs. of onts are quite sufficient: more unuld be detrimental, as it would 
cause fattening. When autumn grazing is finished, the breeding material, colts as well as fillies, should be saddled and trained. At Trakehnen we give two and three-year-old colts and fillies in training about 9 to $12 \mathrm{lbs}$. of nats. After grazing is finished $6 \mathrm{lbs}$. of oats and about $12 \mathrm{lbs}$. of hay is sufficient for two-vear-olds intended for use. In the following summer the three-year-olds at Trakehnen get during grazing time $2 \mathrm{lbs}$. of oats, but if grazing is very good none. When grazing is finished the three-year-olds are broken in, and get until the spring auction 9 lbs. of oats and about 10 to 1.2 lbs. of hay.

The above indicated food rations are only sufficient if the quality, especially of the hay, is very good. In damp years the hay is less nourishing, and it is then advisable to increase the oat ration. On the other hand, the beneficial and bone-forming qualities of hay well got in dry years may be used in more plentiful quantities at the expense of the oat ration.

A part of the oat ration can be replaced by beans or peas, say about 2 lbs. daily. $\mathrm{By}$ this means the formation of the bones is favoured, but at the same time the disagreeable gall formation appears, especially in the hocks. Beans and peas can therefore only be recommended when foals have at the same time plenty of exercise.

Every expert will know, of course, that these rations, which are ordinarily speaking used at Trakehnen, must very often be modified according to the soil of the stud and the particular requirements of individual horses. A fixed standard to suit all the different conditions - harvests, illnesses, etc.cannot be given. The eve of the breeder, especially when it works with pleasure, will itself perceive all the necessary shades in the treatment of foals. 


\section{CHAPTER 111.}

\section{Training.}

1 IITHE already mentioned in previous chapters the importance of indiviclualisation in the treatment of both breeding material and foals. It is quite evident that when training horses, whether for the purpose of racing or hunting or other performances, it is most important to indiridualise, and it is therefore impossible to give a hard and fast rule. I only intend to give general points of riew, ideas and experiences, from which each individual breeder must build up his own theory according to the particular requirements of his available material, the training track and climate, etc. The difficulty of the art of training lies in the fact that its object, the horse, like all other living creatures, is endowed with many powerful characteristics, rendering a machine-like and uniform treatment impossible. If the horse were a machine, then training would not be an art, and racing tests to the lifeless elock time would be all that would be required. Furthermore, the work of the trainer is made difficult by the task of having to obtain the highest possible degree of fitness by a ficed time, i.e., the race day. The wbject of all training consists in removing all superfluous fat and connective tissues, in strengthening the muscles and sinews, and in clearing the wind. In order to obtain this result the horses must be watehed carefully and correctly every day and properly dealt with. The trainer's eye is the cause of horses being fit or unfit.

The suitable English and Irish soil, as well as the admirable racing tracks -given by the grace of God-especially at Newmarket, has led the Englishmen also in this branch into the confortable and tenacious conservatism which is just as dangerous and hostile to all progress as was the former conservatism of artillerymen with reference to breech-loaders not invented by them. It has taken a long time before the simplest doctrines of hygiene could remove much of the eril in English training. The distinct successes of the Imericans in training and riding during the course of the last ten years have caused Englishmen to think and reform where necessary. In both training and riding, Americans, who are not bound down by tradition, 
and who are not spoiled by English pastures and galloping grounds, (bbtained great successes through their practical ideas and almost inconsiderate leaning to what is natural. Iloreover, the Americans have reason to be proud of the fact that Old England, with its long history of classical races, has had to learn such a rough lesson from them !

\section{(a) The Training Methods.}

The training methods of the eighteenth century, that is, at the time of Eclipse, born 1st April. 176t, had to adapt thenselves to the following circumstances :-

1. There were only a few attainable racecourses for each horse, and the racing season was much shorter than it is to-day, often only three months.

2. In most cases only four-year-ridd and older horses ran. Three-yearolds only since 17.56 , and two-rear-olds wnly since 1773 .

3. There were no railwars, and consequently the visiting of the different racecourses entailed long journeys on fort.

4. Most races were run for a distance of 2 to 4 English miles, with heats, the weights for six-rear-olds being 12 stone.

At that time it was very usual to keep horses in training for only three to four months, and to send them for the rest of the time to grass. Ifter the grazing, training began with weekly plyysics and bleedings. Then the horse received about two sweating gallops weekly, over distances of 2 to $f$ English miles. During the sweating gallops with wonllen rugs, some parts of the body which had ton much flesh very often-for example, the neckwere covered with extra heary rugs. In these gallops the last quarter of a mile had to be ricden a little more quick, that means at half speed. After the sweating gallop the nose and mouth were washed, then the horses were brought into the stable or in the so-called rubbing-down house, and there covered with several woollen rugs until the sweat oozed out in sufficient quantities. The sweat was then remored with a sweating linife, and the lorse rubbed dry by four persons with woollen cloths, then covered with fresh rugs and given walking exercise for half an hour. Some days a week complete rest days were usual, whilst long walking exercise, as is common to-day, was almost unknown. The usual daily canter or gallop was over 2 to 4 English niles, often without a leading horse, and in any case at a slower pace than is usual to-day. The word "canter," meaning a quiet gallop, arises from the slow manner in which the pilgrims walked to the grave of Irchbishop Thomas Becket at Canterbury, murdered 1170.

The development of training in the last century, after the coming ints. prominence of the classical races for two and three-rear-olds, proceeded as follows :-

1. Iecording to Darvill, at the beginning of the nineteenth eentury, the following sweating gallops were given. For yearlings nver a miles, for 
two-rear-olds orer 212 miles, for thece-pear-olds over 3 to 323 miles, for fouryear-olds over 4 to $4 \frac{1}{2}$ miles, and for 5 and $f$ year-olds over 5 miles.

2. The sweating gallops gradually became more scasce, and were held wer somewhat shorter distances. The sweating gallops of two-year-olds (at the beginning once weekly, over 1 English mile) gradually ceased altogether.

3. Sweating gallops at the heginning of the nineteenth century were given in adclition to the daily work, i.e., in addition to the quick work or so-called gallop. Iater on there was no quick gallop on the days of the sweating gallops.

4. Opening medicines, called physics, became more rare, and are finally limited to one or two dosss a year, especially in spring, shortly before the beginning of quicker work, $i . c$, beginning as they are transferred from the straw-bed to the racecourse.

5. The claily work consisted of $1 \frac{1}{2}$ to 2 hours in the morning, and about 1 heur in the afternoon. This afternoon work, however, does not seem to have been generally practised, and crases almost everywhere towards the end of the nineterntin century.

fi. The work in the formoun for the two-year-olds consisted of a short walk and trot, then two quiet canters of about 1.000 metres, and then a somewhat quicker canter of about 1,200 metres, the latter once or twice weckly, at full or half speed. The older horses cantered and galloped just as often, but over longer distances. The distance was gradually increased to the distance of the racecourse, i.e., extending eventually over 4 miles, equal to 6.437 metres. In lutumn the yearlings cantered two or three times daily, each time 600 to 800 metres, and in the late autumn were tried up to son metres with the assistance of an older leading horse. The afternom work consisted only in walking and a little trotting.

7. Ibout the second half of the nineteenth century the work of the rearlings and of the two and three-year-olds was limited to two canters daily, of which the second canter was somewhat longer and quicker. Once or wice weekly the sccond canter was made almost at racing pace, in accordance with the progressive condition. The three-year-old and older horses cantered generally about 1 English mile, seldom more than 2,000 metres. Derby horses, for example, galloped at least two or three times before the race $1 \frac{1}{2}$ miles, equal to 2,414 metres, at full racing speed. Gallops over longer distances than $1 \frac{1}{2}$ English miles gradually cease altogether, even in the case of horses, for example, which were trained for the Doncaster St. Leger (distance 1 mile, 6 furlongs, 132 yards, equal to 2,937 metres).

The present day views on training are characterised as follows:-

1. Sweating gallops and physics arc only applied in exceptional cases when the condition of the legs does not permit that quantity of work by which the useless fat and flesh, called in German luder, can be removed, and yet at the same time muscle can be formed. Further physics are given if a horse, in consequence of too much work, has become stale or has broken 
down, so that during the time of its enforced rest it may not put on too much flesh. If a broken down horse has to be blistered or fired, it is given a pill before and after the rest of four to six weeks. Where needed it is also given a physic about eight days before the race when some slight accident to a fit horse requires an important reduction of work.

2. The daily work consists in the morning of $1 \frac{1}{2}$ to $2 \frac{7}{4}$ hours' walking exercise, none or very little trotting, and two canters or gallops. In the afternoon $\frac{3}{4}$ to 1 hour's walking exercise, either led or with a man up. After the beginning of the fast work, it is calculated that under normal conditions about six weeks are sufficient to make the horse fit for racing up to $1 \frac{1}{4}$ English miles, equal to 2,000 metres; whilst at least two months are required if the distance is $1 \frac{1}{2}$ English miles, equal to 2.400 metres.

3. In the case of the two daily canters or gallops, the last 500 to 800 metres (or as some trainers say, the last 300 to 600 metres) should, according to the Imerican idea, be done at medium pace, and in the case of a more advanced condition at racing pace, at first only in the second canter, later on in both. The beginning of this gallop in any case must be done at such a slow pace that one can trot alongside. This slow part of the canter may be accordingly extended up to 2,000 to 3,000 metres. Once or twice weekly, in the case of more adranced condition, the quick part of the second gallop may be extended up to 1,200 to 1,600 metres, later on up to at the most 2,000 metres, when, of course, the slow part can be very much reduced, or omitted altogether. Only few trainers are of the opinion that the gallop at racing pace may be extended up to 2,400 metres.

The idea which underlies this kind of training is that the daily gallops over short distances, at a medium or at racing pace, bring the muscles which are used for quicker work, and the lungs, better and more surely into condition than the former usual longer gallops, undertaken once or twice weekly, for which the horses were not sufficiently prepared, as the other daily work was only slow cantering.

The new method of training is supposed to effect a daily, uninterrupted and gradual improvement of condition, whilst formerly, according to the doctrine of the old trainers, every two steps forward should be counteracted by one in the opposite direction. Moreover, experience has taught that gallops at racing pace for longer distances than about 2,000 metres do not improve the condition, but rather the reverse. The winner of the St. Leger, 1906. Troutbeck, has, as I have been assured by his trainer, IV. IVaugh, never during the whole of his existence galloped or cantered over a longer distance than 2,000 metres. On the other hand, the American trainer, Walker, who gets perhaps the most out of his horses, gave his Derby candidate, Fels, several gallops at racing pace over 2,400 metres, sometimes even with relay leading horses. But even this trainer is of opinion that this distance is the extreme limit, and is even sufficient, for example, for the preparation of the Grand Prix, which is run over a distance of 3,000 metres. 
In the bigger and better. Imerican racing stables, nne often finds the practical arrangement of using one part of the stablemen for riding only, whilst the greater part is emplosed in cleaning, feeding and leading the horses. I racing stable of about 30 horses not far from lew York, had, for example, only two stable boys 1 ho could ride. Besides these, the stable jockey also rode. The cleaning and feeding of the horses, as well as taking them to their daily work on the racesourse, and in the afternoon senerally to the yard, was undertaken by about eight to ten young felluws who, however, never were allowed to ride. By this arrangement the two stable lads had plenty of opportunity 10 practise galloping, as ther galloped each of their about 7 horses wice daily. It is principally owing to this very practical division of work that the .Imericans are in the position to produce so many gund jockers. Some of them even learn to acomplish fairly accurately the very dithcult task of doing a gallop whilst training at at certain defined pace (eventually I mile in about 1 min. 50 secs.). In Imerican training such tasks are rery popular. The most difficult task, however, is to ride definite distances in the shortest possible time. The partisans of racing against time do not recognise, in my upiniom, sufficiently the difficulties attached (n) same. They think that in our racing to a finish the art of the juckey in riding is more important than the capacity of riding in the shortest pessible time. If the horse were at mere marhine it would be an easy thing to get the best record by letting it gu full speed ahead from start to finish. With living horses, however, the best record would certainly not he obtained by this method.

For stecplechasing the same kind of training is also recommended, except that instead of galloping them once or twice weekly over 1,200 to 1.600 metres as recommended. they are more often given a hunting gallop, over obstacles, for 3,000 to 5,000 metres. Many trainers assume that if a horse is fit to gallop 1,600 metres on the flat, and if it has had sufficient practice in jumping. it is also fit to do 5,000 metres and more at a steeplechase pace, and that finally at the finish it can still put on the required well-prepared speed which it has not used up whilst going at a steeplechase pace.

Experience has furthermore laught that short guick gallops arce less dangeruus for the legs than longer canters, provided that the horses do not need to stretch themselves in these quick frallops. In these longer canters energy and a desire for going soun disappear, conse(puently the absence of strained nerves and muscles causes the sinews to break down easily or the legs $t$ become splints. If this long ranter is over obstacles it causes excitement, aturntion and the lesire for going, which are so useful and so necessary in training. La\%y horses, as well as filles in heat, often gallop with loose joints, and therefore uften break down very easily. A change in the galloping track is in this respect of un very liseful, as it serves in increase the attention of the horses, and removes the so dangerous weariness. One of the most important instructions giren to the stable bogs is: In your daily 
canters or gallops be rery careful to aroid pushing your horse. Sit still, so that the horse may feel and think that the rider really wishes to go a little more slowly. This soothing belief sustains the desire for going, and strengthens the nerves of the horses.

Besides a horse's galloping performances, there are several other very remarkable signs to show how far its condition has improved. To commence with, notice must be taken how long it takes the horse to snort (clear the wind) after it has been pulled up. The souner this happens the more forward is its condition, especially the breathing. If the horse, for example, requires a minute or more to snort, it is a sure sign that the horse has been asked to do too much in the gallop in question, i.e., a mistake has been made. If the horse is very fit and the gallop has been tou short, i.e., it has been easy work for it, it takes a long time to snort, or it does not snort at all. The length and pace of the gallop must fit the condition, so that if it clears the wind by snorting 10 to 30 seconds after being pulled up, it is a sure sign that everything is all right.

In the case of a horse which is fit the skin becomes thinner, the hair more shiny, and the flesh firmer. The latter, as well as the disappearance of useless fat, can best be noticed at the mane and on the ribs. A little easy sweating is by no means a sign of bad condition, on the contrary, a fit horse certainly sweats less but more readily, as its sweat is more liquid and its skin thinner and more readily penetrated. The sweat of a fit horse looks like clear water; on the other hand, that of an unfit one like lather, which can be most distinctly seen between the hind legs, and dries up less quickly.

If the horse when galloping begins to become long, or to breathe more deeply, pressing the knees of the jockey untwards, it is a sign for the jockey to pull up.

Finally, I must add that before the race, and in order to get a perfect condition, in most cases several gallops at full speed, over not more than 2,000 metres, are required, and that the last quick gallop-which often works wonders-must be undertaken two or three days before race day. Of course, even after this last gallop the horse must do its usual wo canters daily up to the day of the race. On the day of the race itself, early in the morning, give the horse 1 to $1 \frac{1}{2}$ hours' walking exercise, a short canter of 800 metres, and a so-called sprint for the same distance. If desired, you can do as the Americans like to-let the sprint follow the canter without any interval. About five hours before the race give the horse some oats with a little water. An hour before racing lead the horse out of the stable.

The following points may be briefly mentioned with reference to the important treatment of horses' legs when in training:

1. As long as the legs give no cause for anxiety bandages must not be made use of, except for steeplechasers as a orotection against external injuries when jumping, and when only this protection seems necessary. 
Bandages only then fullil their purpose when they are carefully wound and fastened round the right place. The rule is to cord somewhat loosely but und round rather firmly, and in such a manner that it is easy to put one finger between the bandage and the leg. It must be corded about two inches below the upper edge of the bandage, so that the strings of the bandage lie even and only on one place.

2. This leg which is causing ansiety is generally discovered in the wethknown afternom cleaning and revision hour, and it is then first of all necessary to find out whether a changing of the shocing is required. It will very often be found that the heels have been shortened too much, and in all probability this has been the chief cause for the beginning of the break down. In such a case new shoes with thick branches must be got at once. The straighter position of the pasterns, which often takes place when training, requires the heels to be lifted slightly.

3. Is soon as the sinews give way when working, it is necessary to begin the well-known treatment of stitched-on stockings, wet bandagas, cold douches, blistering and firing, according to the stage of the disease. Mlore effective, however, than all these remedies is supposed to be the llydrothermoregulator, Sistem Ullman (see Archiv. fur wissenschaftlich. und prakt. Tirrheilkunde, V' 1.31 , page 196). Thick sinews, and ('specially fresh breakdowns, are exposed by this apparatus for 30 to 50 hours to a temperature of 50 to 5.5 deg. C. Professor Eberlein says that by this treatment a complete reformation and thus a cure of the break down is possible.

1. Besides the break down of the sinews (apart from abumt 50 per cent. of all lameness produced by hoof discases), lameness frequently occurs through splint-exostosis. This exostosis is never or very rarely caused from external injury, such as by knocking or striking by the other leg, but it arises from the tearing of the ligaments by which the splint bones are connected to their cannon bone. This tearing of the ligaments is caused when galloping during training by the shock, which increases in proportion to the speed, when galloping. Even when doing slow work, howeror, cxostosis may arise from sudden and irregular effort of the upper parts of the splint bones, which may be caused in various ways (lazincss, heat, neglect). In the case of lameness thus arising, exostosis itself is, of course, non ret resisting, and therefore not yet to be perceived. If exustesis is apparent, the lameness has often already passed away. In order to prevent or (o) limit as much as possible an interruption of the training, it is necessary te cereroise a permanent pressure on the splint bones by careful handaging. Ifter riding, it is advisable for the remainder of the day to apply wet bandages with dry ones over them, and to change them at thres hours interval. For riding. and of course during the night, apply only dry bandages. When the place where the splint-exostosis arises is already to be recongised, place a piece of lead about the size of a two-shilling piere into the bandage, so that it exer- 
cises the necessary pressure. Perhaps also in the case of splintexostosis the application of the Hydrothermoregulator might be useful. There are good racehorses which, on account of exostosis caused whilst training and not looked to at the proper time, could never be propery trained for the whole of the season. Sperber's brother is a well-known example of this.

\section{(b) Stable Regulation and Instruction for Rubbing Down Horses.}

Nork in the stable begins in the morning about $1 \frac{1}{2}$ to 2 hours before the horses are taken out. The first thing to be done is to give the horses water and a little oats, clean the stable, and rub cach horse down, after which the men go to breakfast and put on their riding clothes. On their return from the training track, the horses are to be regularly attended to in the following manner:

1. Remove the snaffle, take off the sacldle and put same on the cleaning bench, put on the horses' halters, and if necessary fasten them up. The stable boy then commences to wipe the place where the saddle has been with a straw wisp, and with the same straw wisp removes the greater part of the dirt from the belly and legs, etc., the horses in the meantime eating the hay. or in the summer, the fresh Lucerne, which must be there ready for them $i n$ the manger. If the horses are under rugs, after the completion of the above work, cover them up again, and in such a manner that the inside of the rug comes on the top.

2. The next work is the treatment of the legs and hoofs. The bandages are taken off, and all the four legs, beginning with the near fore, are thoroughly cleaned, if required, washed or douched, and then again rubbed dry. When cleaning or drying the legs, the stable boy must kneel down and take firm hold of the leg of the horse from the front. The hoofs are now washed, rubbed dry and filled inside with damp clay. If the hoofs are not washed it is unnecrssary to put damp clay on the frog and sole, as the soil which adheres to them is sufficient protection against the stable urine and against wer-drying of the houfs. The hoofs must be washed once a week at least, but as above-mentioned, damp clay must always be smeared in the sole. The blacking of boofs is not required, and is often dangerous. If more is desired for the decoration as well as for the preservation of the hoofs, the outer part of the honf can be well brushed daily and wiped with a wool cloth in order to make them smooth and shing. When above has been done. the legs must, when required, be rubbed with fluid and bandaged. Then give the horse balf a bucket of water to drink.

3. The real process of cleaning the horse begins with the head and ears, care being taken that the horses keep their heads low. Take the rug off partly or altogether, brush first the left, and then the riglit side, always commencing with the neck and shoulders. Then massage the horse with a somewhat 
damp wisp consisting of hay and bast, then wipe with a woollen choth. Beat the dust well out of the rugs, put them on again, and put on the girth, then once more clean the tail and mane. Finally wipe the eyes, nose, mouth and backside with a soft sponge, and the horse's toilet is complete. The halter is then taken off, the horse bedded and given sufficient water. The saddleand harness are then cleaned and put away in the harmess room.

In hour after coming home the horses are given their chief feed of oats, the stable shut, and if necessiry in the summer on account of flies and heat, darkened. The horses must now have four hours' rest up to the afternoon stabling time, when the legs are first examined and the appetite controlled. They are then got ready for their afternoun's exercise of about three-quarters of an hour, after which they are again cleaned, given water and oats. This is the time when risitors may be received to inspect the stables, and the work for the next day considered. In the evening give water unce again, as well as oats and hay. In the long hot summer days, especially with us in the Forth, it is advisable to worli very early, and to arrange for five feeding times instead of four. The above-mentioned stable rest of four hrours would then be after the third feed of oats. In this case the five feeds would be about as follows :-

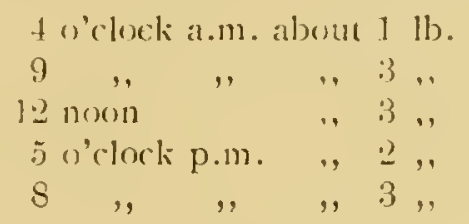

(c) Food whilst Training.

For horses in training for racing abuut 1.2 to 1.5113 .5 of oats will be, on an average, sufficient for one day. Very few horses in training eat more than 16 lbs. of oats daily for long, and I consider it inadrisable, except in rare instances, especially where horses will not eat hay, for horses to receive more than this quantity, as there might easily be a relapse, when they will scarcely ('at $10 \mathrm{lbs}$. Horses which have sufficient time and rest for eating, as for example, racehorses, as opposed to farm horses, digest their oats better "ithut chaff. If it is desired to give chaff, however, I would advise the use of Lucerne or Sanfoin hay. Part of the oats, about 2 to $4 \mathrm{lbs}$., may be replaced by the same quantity of peas or beans, especially if horses in quick work lose their alppetite. I find it more practical to give the peas unground and unswollen, as most horses prefer them like that, and as the peas or beans which have not been ground remove the tartar from the teeth, and do not get dirty so easily. An addition of about 2 litres of wheat bran twice weekly, damped a little, with oats (mash), is recommended, especially after the guicker gallops; in any case, not the day before. During the hairing season 1 recommend that there be added to this so-called 
mash a little slime of linseed, or about $\frac{1}{2} \mathrm{lb}$. of ground or roasted linseed. Formerly the portion of hay was fixed in England as 5 lbs. per day maximum, because one wished to get the racehorses more slender after the training than to-day, and they did not think it possible that the horse with more flesh, or even with a so-called hay stomach, could be fit for racing. To-day raceborses in training are given 10 to $12 \mathrm{lbs}$. of hay daily (but no clover hay), and in addition, after their morning work, a few pounds of green Lucerne as long as it is to be had. Americans have introduced a very good custom, i.e., to put a bucket of water in the stables so that the racehorses can drink whenever they like. Horses in consequence drink oftener, but in quantity less than when they are watered three to four times daily.

Half-bred horses which are not trained for racing but for the improvement of muscle formation, lung activity and health, receive, in order to produce stronger bones, less oats and more hay, especially clover hay. In Trakehnen the two and three-year-old colts in training are given on an average $10 \mathrm{lbs}$. of oats and 18 lbs. of clover or Lucerne hay, or in summer green Lucerne ad libitum, but care must be taken that it is mown twice daily and given to the horses as soon as possible after it has been cut. 


\section{CHIPTER IV.}

\section{Establishment of Studs.}

THE Medes were one of the first and most ancient nations which gained great political power in Asia, mostly on account of their good horses and their clever caralry. Only after allying Medea, which was rich in horses, with Persia, did Cyrus succeed in overoming the rich Crosus (5.49 B.C.), in destroying Babylon (5.38 B,C.), and in founding a great Persian Empire, whose kings were so fond of horses that they had their celebrated studs for their own use and for that of their cavalry even in the far distant lledea. Victor Hehn supposes that Medea was the home and starting point of the horse breeding and horsemanship for Interior Asia. Amongst the grasses which improved especially the horse breeding in Medea, the Greeks and Romans found one particularly suitable, and imported this grass about lou B.C. inte laty under the name of lledic grass, the name "Iledieago" arising later. In the fifternth century this grass was brought first to France and Belgium sia Spain, and received the name Lucerne from the little lablian town (lauserne: acording to other information from the nid Spanish word " L"serdas." Soon after the dis-

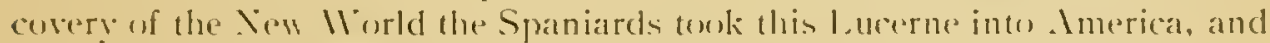
called it there by the Irabian word "Iffalfa," later on the Spanish word for elever. Lueerne las mle taken rout in Germany since the middle of the

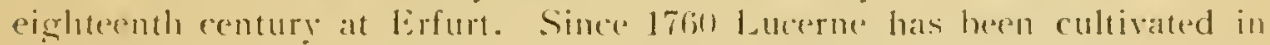
England in the frelds, and in the sunny East of England (especially in Essex and kent) very much extended. In 1892 about 6.640 acres grew Lucerne, and in rome already about $-2.2,160$ aceres.

The horse, on acount of the simpler organiom of its stomach and thes slurter intestines, has a much less caparity for digestion than cud-ehen ing animals. For this reason the horse utilises less hay and straw than cows or sheep. Of all kinds of hay, the horse utilises best Lucerne hay, and excels

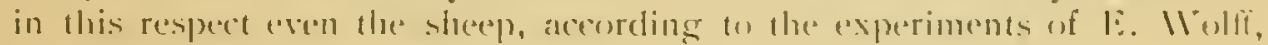


Hohenheim, especially as far as the use of the raw protein and digestible carbohydrates is concerned. Therefore, not only the history of horse breeding, and the experiences of the present time, but also scientific considerations, seem to point to the special value of Lucerne for horse breeding.

Medicago sativa, or common Lucerne-named "aspert" by the Persians (i.e., literally forage for horses)-given either green or dried, is the most nourishing and healthiest food for horses, and most suitable to produce strong and hard bones. This grass requires a chalky, strong, deep and warm soil, with deep level of subsoil water, but it also grows well in mountainous clistricts, where the rain water, coming suddenly and in great quantities, is able to run off well. In the South of France it often grows in the same spot fifteen years and longer. In the middle of Germany about six years; in Trakehnen only three to four years. It can be cut in France five to six times yearly, but in Trakehnen only three times. It thrives better in dry years than in wet years. On account of its roots, which grow 2 to 3 metres deep, it can withstand the greatest scarcity of water. Lucerne thrives best after a crop of vegetables, about $20 \mathrm{lbs}$. to one acre, sown in oats or barley, and which can be mown green, as long as the Lucerne appears strong enough to suppress weeds. After every crop it is advisable to harrow the Lucerne, and even in special cases to harrow continuously even with the usurpator, in order to destror the weed. In winter it is casily affected by strong frost, and it is therefore advisable to cut it for the last time in atumn, about two to three weeks before the first night frosts. Nanure of $3 \mathrm{cwts}$. Thomas meal per acre every autumn, as well as 6 cwts. in spring, has been proved to be sufficient at Trakebnen. Ifter Lucerne the best food for horses is Sainfoin (onobrychis sativa maxima). Sainfoin contains a little less chalk than clover, but like Lucerne, it is not injurious to the breathing organs, and is, therefore, more suitable for Thoroughbred breeding than clover. Sainfoin is less capable of resistance than Lucerne. It takes more from the soil, gives only one crop, and is more difficult to get in. Iccording to the tables of E. Wolff, there is contained in every 1,000 part :-

Wrood Sorrel . . 4.7 lime.
Sweet Meadow Hay 16.6 ",
Sainfoin Hay . . 16.8 ",
White Clnver Hay . 19.3 ",
Red Clover Hay . . 20.0 ",
lucerne Hay . . . $26.2, "$

There is clouble the quantity of lime in Lucerne as green food as in red clover, almost three times as much as in meadow grass, and five times as much as in Timothy grass.

The thriving of I-ucerne is one of the surest indications that the soil is good for horse breeding, as has been proved in its home to the old Medes 
and Persians. Where I, ueerne thrives well, it can be taken for granted that there will be found geud pasture and meadew lanel, ur that they can be laid out. Cood meadows and grazing are amongst the most important conditions for the thriving of horse breeding. Ilthough horses have a great power of resistance against heat and cold, wind and weather, yet there is no doubt that they presper bester in dry and high-lying districts than in damp and lowlying unes, because the food which grows on the former is more nutritive. Horewer, on high-lying plains the lungs of the horses develop better on acount of the thinner air, which causes the horse to breathe more often and more deep. The great superiority of that wonderful horse, ()rmonde, must partly be ascribed to the high-lying, otherwise rather deficient, training place, Kingselere. The high-lying training place, Becklampton, near Calne, in Miltshire, has proved to be just as gomd; witness the two Derby winnors, Galtere More and Ird Patrick, wained there. The best Arabs are ferund en the highest-lying plains of . Irabia, and probably the first home of the Trabs is the same as that of I,ucerne, namme, the mountainous Medea. Adniral Rous writes about the "stablishment of a stud as follows :-

"The best site for at hreeding "stablishment is undulating ground, on a light soil, wer limesteme, then wer chalk, gravel, sand and loam, well drained: and the worst situation is a rich, wet pasture on stiff clar, which is only fit fur cattle and cart horses."

A wind! climate is bealthier than one which is not windy. The massage of the skin which is effected by the wind strengthens the nerves and improves the health of men as well as of animals. The almost constant wind in East Prussia, and the good loam soil there, on which clover flourishes luxuriantly, are the elements which cause horse breeding to thrive so well in that district, in spite of many other unfarourable conditions, due to its Northern position. The possibility of foals catching cold after heary rain is much greater in districts where there is not much wind than in districts where there is much wind, as the wind, by drying the hair, is the best protection against colds. Moreover, in districts where there is not much wind it is very difficult to get hay really dried by the wind, especially lucerne and clover, without diminishing the nutritive power. To sum up, a chalky soil and a windy climate produce good and streng nerves, and they in theit turn produce health. courage and lratuly, in both men and horses!

The noble horse, it is true, arelimatises itself easily, and may be used and hred all over the world, and yet a keen observer must confess that it prospers, and is better in "very respect on a soil similar to the one just described. Although the influence of the soil is generally recugnised, and every practical breeder can see it erery year in his onn as well as in his neighbour's ease, yet breeders who are not living on favourable soil admit it very unwillingly, and often deceive themselves. I have often beard liast

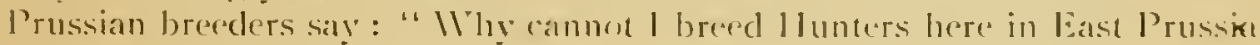
just as well as they du in Ireland?" The infleence of the soil is, 
however, much greater than even experienced breeders believe. If we were even to import into East Prussia the finest fond from Medea, yet we could never import two very important conditions for breeding good Junters - firstly, a grazing period lasting at best longer than five months: secondly, the splendid galloping country on which three-year-old Hunters carrying small apprentices gallop behind the hounds, over walls and obstacles, and even are able to gallop through the whole of the winter! If we, on the other hand, look at the difficulties which the East Prussian breeder experiences in giving his horses the required exercise for seven or eight winter months, one can well understand, from this standpoint alone, the very great importance of the soil.

The United Kingdom (England and lreland) undoubtedly possesses the best soil for breeding horses. Perhaps, as is often said, the English fogs which are so frequent, caused by the sea, are the cause of more roarers being found there than in other breeding districts. Without this fog, however, the meadows, and especially the galloping tracks, would not be so good as they are in this country and nowhere else in the world. Besides, the oceanic climate of England favours the specially high proportion of amid in the horse forage, and as according to the latest researches of Dr. IV. Völtz, amid combinations are especially useful for the building up of firm bones and muscles, consequently the oceanic climate of England seems to be specially favourable for horse breeding. In the less sunny Western part of England, the plants contain more amid, whilst in the more sunny East, as already mentioned, l, ucerne thrives better.

France is behind England in this respect, and in Imerica the ground cannot stand being galloped on, and for that reason all races are run on artificial Macadam courses. The conseguence of these advantages of the English soil is that the English reared and trained Thoroughbred is far superior to all other Thoroughbreds, by reason of the elasticity of its galloping and its high quality. This blood, whicl produces wonders, is to be had most surely in England. The other Thoroughbreds have not show as yet that they can make progress, or even remain at their height, without continuous borrowing from England. England, on the other hand, in spite of the yearly growing export of good and the best breeding material, has retained its power. The danger to this horse country, endowed with the Grace of God, consists in the increasing neglect which, by reason of all producing wonderful nature, has rooted itself so often in mankind, as Th. Buckle has shown in his "Mistory of Civilisation in England."

The best soil for the breeding of draft horses is in fertile low-lying and diluvial regions, which, on account of their plentiful and fattening forage, are suitable for producing the desired large and heary-limbed cold-blood horses. Even the home of the Suffolks in England has the trace of a marshy nature, and more su the Clyde valley, the home of the Clydesdales. It is, however, possible, and may be cxpected, that the breeding of heary horses 
for performanees may require something else from the soil. One of the best exampless l know of for the greale influence which the soil exercises on the type is the previously mentioned pure draft breeds in the Russian Strppe stud of 1)erliul. The Percherons grew like Irabs here, and the Suflolks and Clydesdales berane stunted, in spite of the splendid forage from the Steppe.

When ohoosing a ground suitable for a stud for horses. one must further take into consideration that the hay of higher lying meadous (especially mountainous and $1 / p$ meadows) is superior as far as taste and nutritive value are concerned. The hay of lower lying valley moadows, and still more that of irrigated madows and marshes, is, all things bring equal, inferior to the hay of higher lying regions, on account of less taste and greater quantity of worly libres, even if these same grasses should prevail here as well as there. Mestly on higher meadous less weed will be found, and less grasses of inferior value, or even dangerous. I myelf comsider it improbable that a continuous dreught reduces the mincral ingredients of the foud, and that a food is produced pour in line and phosphoric acid, causing diseases of the bones (as l'rofessor kedner say's in his well-known manual. "Dic Lirnährung der landwirtschaftlichen Nutzticere"). Is far as practice is concerned, in dry yars one can only complain about a smaller cjuantity, the cuality is alwais better, and the horses prefer it, and therefore develop mueh better, in spite of a smaller quantity.

Of all demestic animals, the horse is the ment sensitive to bad drinking water. I"nclean, surface or stagnant water is the most injurious. Even the vapours arising from stagnant llaters are very unhealthy for horses, especially young foals, since they cause an increase of trubblesome fies. Continually running water containing lime, which is colourless and without smell, is bent for horses. It has often been ubserved that slanders breaks wat in a milder form after the horses are griven better water from fresh springs newly bored.

The growth of beeches is generally and rightly considered a favourable sign as regards a geod soil for horse breeding. Is oaks grow best in wet, low-lying countries, or in countries whith much rain, and as such countries are not farrourable for horse breeding, the idea has arisen that horses do not prosper where oaks prosper. Oaks grow, nevertheless un high-lying ground, and thrive wedl on strong clay soil, which in also sutable for horse breeding. On the other hand, limes, especially the small-leaved ones, ats well as all kind of barberries, are undesirable in a stud, because they are the most popular carriers of rust parasies, which are apecially unfarourable for Lucerne, and also for chover, as well as other grasses. fiurthermores, wheat strall suffers mostly from rust, as in a lesser degree do also bat and barley strall.

Even the best soil reguires, in order to derive any benefit from its advantages for breeding groud, capable and sound horses, two very important facturs, i.e', padelecks and permanent pastures. "These remarlis are perhaps 
superfluos when speaking of two benefited horse breeding countries like England and Ireland. But the method of rearing in the stable without paddocks and permanent pastures, which is still so widespread in Germany for all kinds of breeds, threatens to ruin many breedings. First of all must be mentioned here the breeding of horses. Paddocks and permanent pastures serve, therefore, as a contrast to the method of rearing in the stable, to leep the breeding material as long as possible outside the stable and in conformity with nature, to nourish same. The following advantages thus arise: -

1. The influence of light. The recently well recognised heneficial influences of light consist principally in destroying many very dangerous microbes, especially tuberculusis baccili, and in increasing the energy of life by multiplying the red corpusculli and the hamoglobis. According to the observatinns of Professur Gaule in Zurich, for example, Pular travellers often suffered more or less from ancmia.

2. The influence of good air. The air rich in oxyen in the open is a primary condition of every healthy development. The continuous remaining out in the open jucreases the need of oxygen, and in order to satisfy their needs, horses must, by deep breathing, make a greater use of their lungs. Accordingly, the lungs will be extended and strengthened, and also the energy of life increased.

3. The influence of wind and weather. The constant skin massage by wind and weather strengthens the whole nervous system. Is a matter of fact, wind and weather preserves the whole animal organism in a continuous and beneficial training through frequent and sudden changes, and forces it to get accustomed to outside circumstances for the sake of self-preservation. In conjunction with the beneficial influence of light and air, wind and weather, owing to a normal and strengthening development of the nervous system, favour the health in such a good and energetic way altogether impossible if the horses are brought up in the stable.

4. The influence of excrcise. A roluntary, continunus and mostly slow exercise on the meadows is necessary when secking their fond. By this means the sinews, muscles and bones are under the influence of a farourable slow, continuous and effective training quite impussible outside the meadows. The longer grazing is possible (in East Prussia, unfortunately, five months at the most), and especially night grazing, the more distinctly is to be observed a favourable development of the formation of the body, especially of the shape of the limbs, as well as that so important correct walk. The voluntary desire of the horses to visit distinct parts of the meadow, the possibility of their moving about as they please, and so noticing all that is going on around them, the attention which is required for observing changes, the many chances to caper and play with their companions-all these strengthen the intellect and senses, and are the best and only preventives against timidity. 
5. Food grasing. The adrantages of grazing on the meadow, as against green fond in the stable. lie, hirsty, in the fact that the horses never get as much in their mouth in the meadow as in the stable, and that, therefore, sudden overloading of the stomach is avoided: secondly, many and just the best and youngest grasses lose their taste between the time of being mowed and eaten: thirdly, the useful combinations of amids are, for the most part, in the younger plants, and these are the most difficult to mow, but the horses whilst on the meadow get them easily.

For the good preservation of pastures it is very important that they should be grazed alternately, as far as possible, by horses, cows or oxen (but not by sheep). Grazing by horses only, continued through many years, seems to produce some peculiarities on the respertive pastures which are dutbtlessly injurious, but up to the present not yet investigated scientifically. These damaging characters are developed more intensively the longer grazing is able to be extended in the respective countries. The pastures in England and Ireland, for example, suffer much more fiom continuous grazing by horses alone than pastures in East Prussia, where five to seven months winter, with snow and frost, shortens the grazing season and prevents somewhat the development of these injurious peculiarities. In countries with a short winter and no snow the pastures are especially sensitive to a one-sided use. Who, however, can afford the lusury of manuring his pastures plentifully every year with composts, will suffer little, or not at all, from the above damaging characters.

When preparing the meadows or pastures, it is useful to grow a green crop of potatoes or turnips, on purpose to destroy weeds as radically as possible. The following composition of seeds, mised with vats or barler as suard-corn, have proved suitable for Traliehnen :-

\section{For Clayey Soil.}

Sow per 1 . Murgen -1 ha. 0.63 .lcre.

Red clover (Trifolium pratense) . . . . . . . . . 2 lbs.

White or Dutch clover (Trifolium repens) . . . . . . . . . . .

Cow grass (Trifolium pratense perenne) . . . . . . . . 3 ,.

Smooth-stalked meadow grass (Poa pratense) . . . . . . . 10 ..

Dogstail (Cynosurus cristatus) . . . . . . . . . . . . .

Meadow Fescue (Festuca pratense) . . . . . . . . . . 3 "

Fiorin or creeping bent grass (Igrostis alba stolonifera) . . . 2 ",

Cocksfoot grass (Dactylis glomerata) . . . . . . . . 2 "

Timothy grass (Phleum pratense) . . . . . . . . . . 2 "

lotal. . 28 lbs. 


\section{2 . For a Sandy Soil.}

Sow per 1 Morgen $=1$ ha. $=0.63$ Acre.

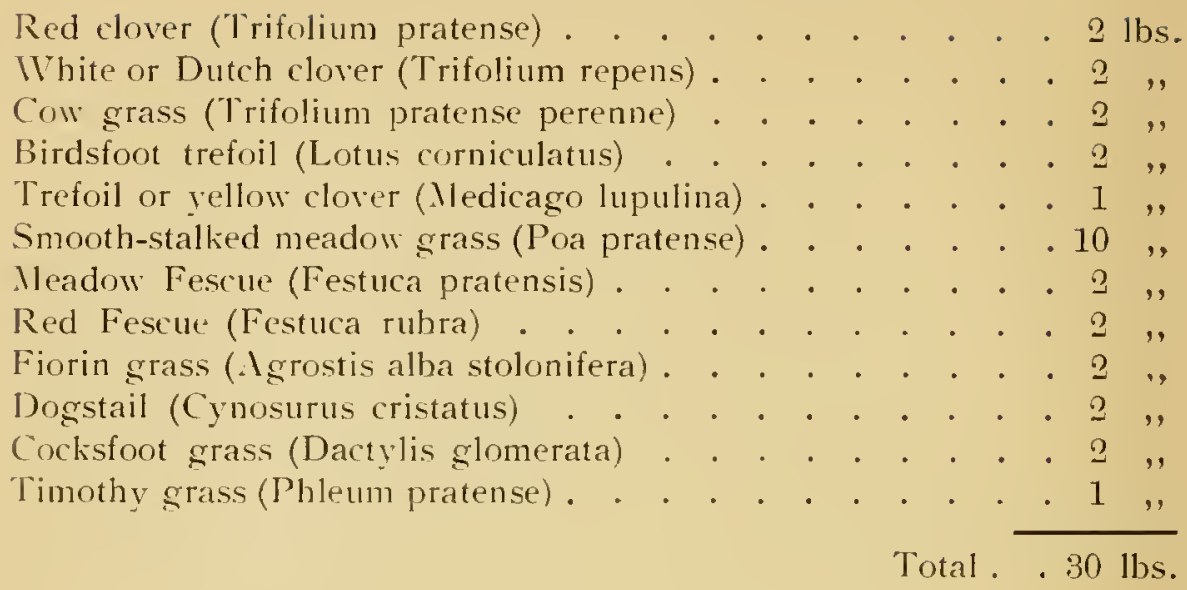

To arrange a stud it is necessary to have the stud books corresponding to the breeding aimed at. It is very much to be regretted that in the most studs uf England and Ireland these stud books are liept very irregularly and in a loose manner. The import certificates from England do not even state the most important dates, which we mark on each covering and foal certificate, namely, day of birth (not only year of birth), time of pregnancy reckoned from the coverings indicated on the service certificates, colour and marks (not colour alone). The entry of all the coverings with their dates is especially important when several stallions have been used for covering. It is also. important to know whether the horse has been a premature birth. A well organised and properly kept stud book can be the storehouse of many and different breeding and also biological problems. For the sake of reference, all coverings should be entered in the stud book, i.e., registers of coverings. and foalings, notices of the judging of foals, the whereabouts of same, etc., etc., must be lucidly arranged together to save space, and also for the sake of lucidity special signs for the judgment of foals at different times are practicable. Below I give the model of a stud book introduced at Trakehnen for the last twelve years, with the practically tried signs for the judging of fuals. These signs are intended to specially reproduce the exterior peculiarities, which are useful for the immediately following matings, as well as to judge of transmission on the part of the parents and the development of the foals themselves. If, as it appears to me, this stud book introduced at Trakehnen is sufficient for IIalf-bred breeding, nevertheless, 
for uther breeds, $i$. . for Thoroughboreds or Trotters, special modifications will be necessary. Tluese will comsist principally of a luciclly arranged classitication of the racing and breeding performances of the family in question, as I have similarly shown on page 290 . In addition, short remarks about the development, capability and peculiarities of the foals during the breaking-in, first trials and further trainings, would he very useful, to which it might be advisable we insert special columns. 


\section{Stud Book}

No.46. Isar born (after 331d.) 8/1 1897 in Bojohrgallen.

liy lilfenbein and lsmir by Malteser and Issy by I)jalma - Vindex $x x$ - Reprobate

- Y. Emilius xx. Fr. Gener.: $\begin{aligned} & 5 \text { Nobleminn } \\ & 6 \text { Antenor }\end{aligned}$

$725 \% \mathrm{XX}, 81 \%$ OX.

Colour, Mark, Size: dark brown without special mark, $169 / 158 \mathrm{~cm}$.

F. No. 181 Foundation Mare: Bonne E. H. born Engl. 1784.

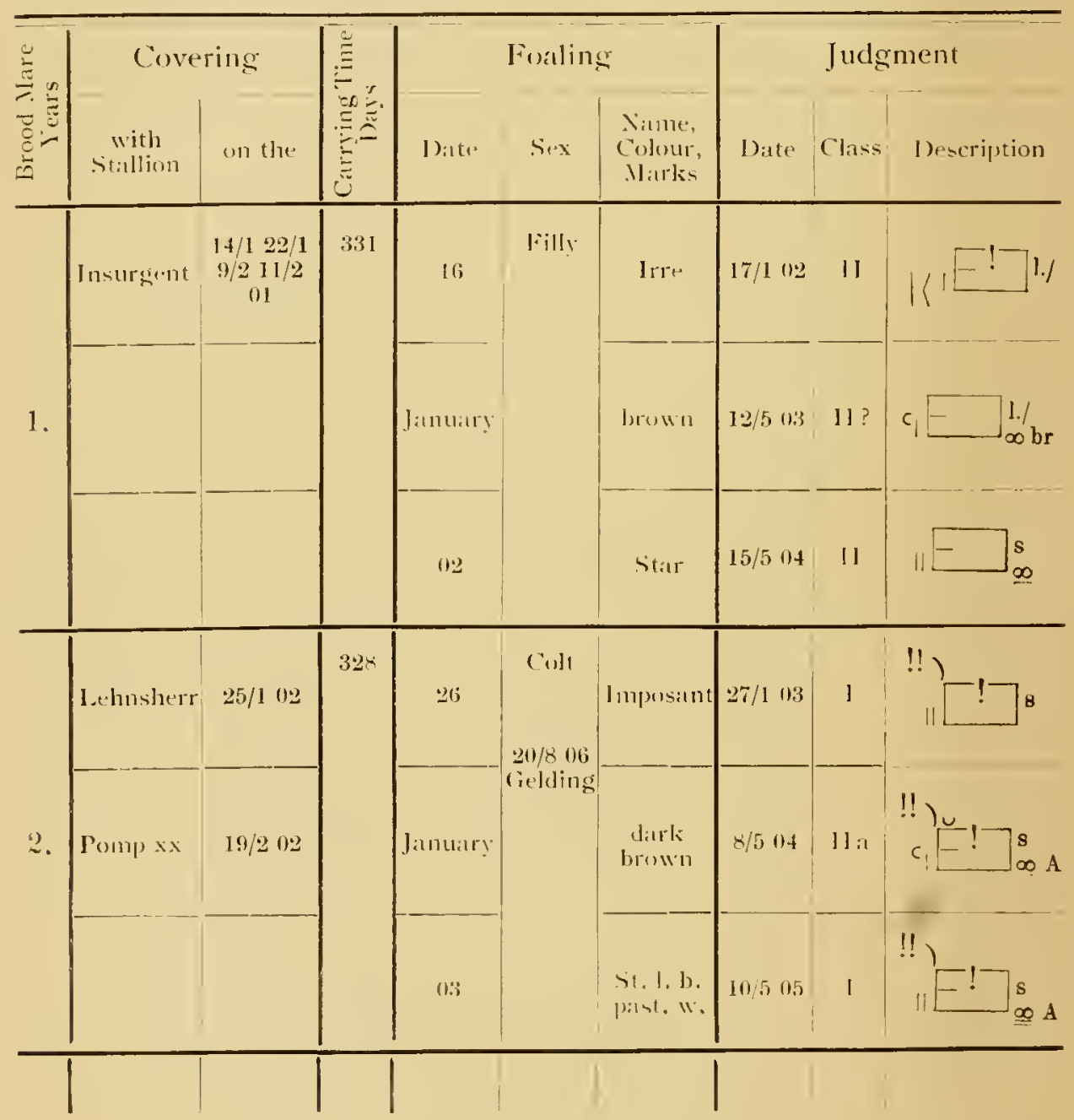




\section{Formula.}

\section{Of the Brood Hare}

\begin{tabular}{|c|c|c|c|c|}
\hline 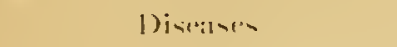 & Condition whilst Tritining & 1) weription: & : ind & Rentuirkis \\
\hline $\begin{array}{l}\text { Servere glanders when } \\
\text { neaned. }\end{array}$ & $\begin{array}{l}\text { Eyeatrold. very idleand slow } \\
\text { in the atutumn trainings. }\end{array}$ & 1 & \multirow{3}{*}{\multicolumn{2}{|c|}{ 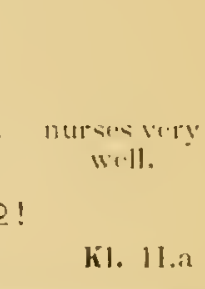 }} \\
\hline $\begin{array}{l}\text { 3/1 49) 1. periodical eye in- } \\
\text { 11:1mumiationt. } \\
\text { 4/3 !99 1. blind. }\end{array}$ & $\begin{array}{l}\text { " year old, firm hurse. } \\
\text { liscrilent temperament. }\end{array}$ & 11 & & \\
\hline $\begin{array}{l}5 / 4 \text { !n l, h, inflitm, - wallings: } \\
\text { recovered. }\end{array}$ & 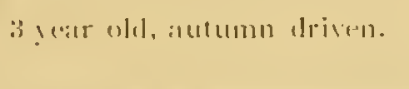 & & & \\
\hline
\end{tabular}

and Development of the Foals.

\begin{tabular}{|c|c|}
\hline 1) inearse & Conclition whilat louining \\
\hline
\end{tabular}

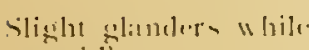
-uckling.

Often colic when weatned.

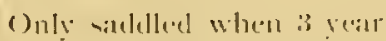
ald in the stmmer : : 1 f:arm herses, offen l:anle.

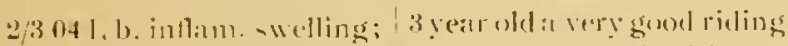
remedied. hornd, allaigs healthy. liroul Vare.

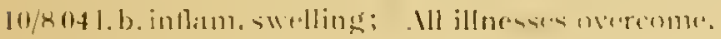
yot $2 / 1104$ recoveriel.

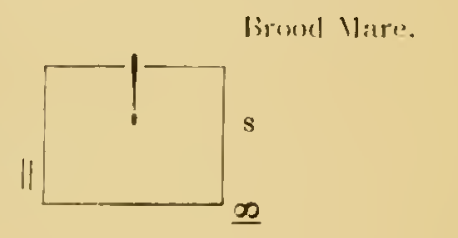

Z. Ki. 11 .

1i. Ki. 11,il

$171 / 162 \mathrm{~cm}$.

\begin{tabular}{|c|c|c|c|}
\hline $\begin{array}{l}\text { Meconium remoned with } \\
\text { instruments. }\end{array}$ & 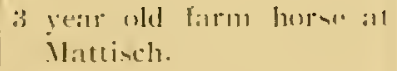 & $! !)$ & ("rib hiter. \\
\hline $\begin{array}{l}\text { Very slight slanders whon } \\
\text { weaned. }\end{array}$ & lixceflent tempuratment. & & $\begin{array}{l}15+117 \\
\text { in . Iuk!. }\end{array}$ \\
\hline 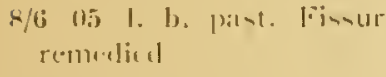 & 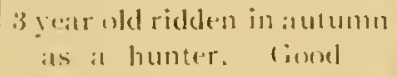 & & IX5o Vl:arli \\
\hline $11 / 805$ crib biler. & jшורוрег. & & \\
\hline I!nk; uferen colie. & & $\begin{array}{l}\text { Z. kl. } 111 . \\
\text { li. kil. I. }\end{array}$ & $17316.1 \mathrm{~cm}$. \\
\hline
\end{tabular}




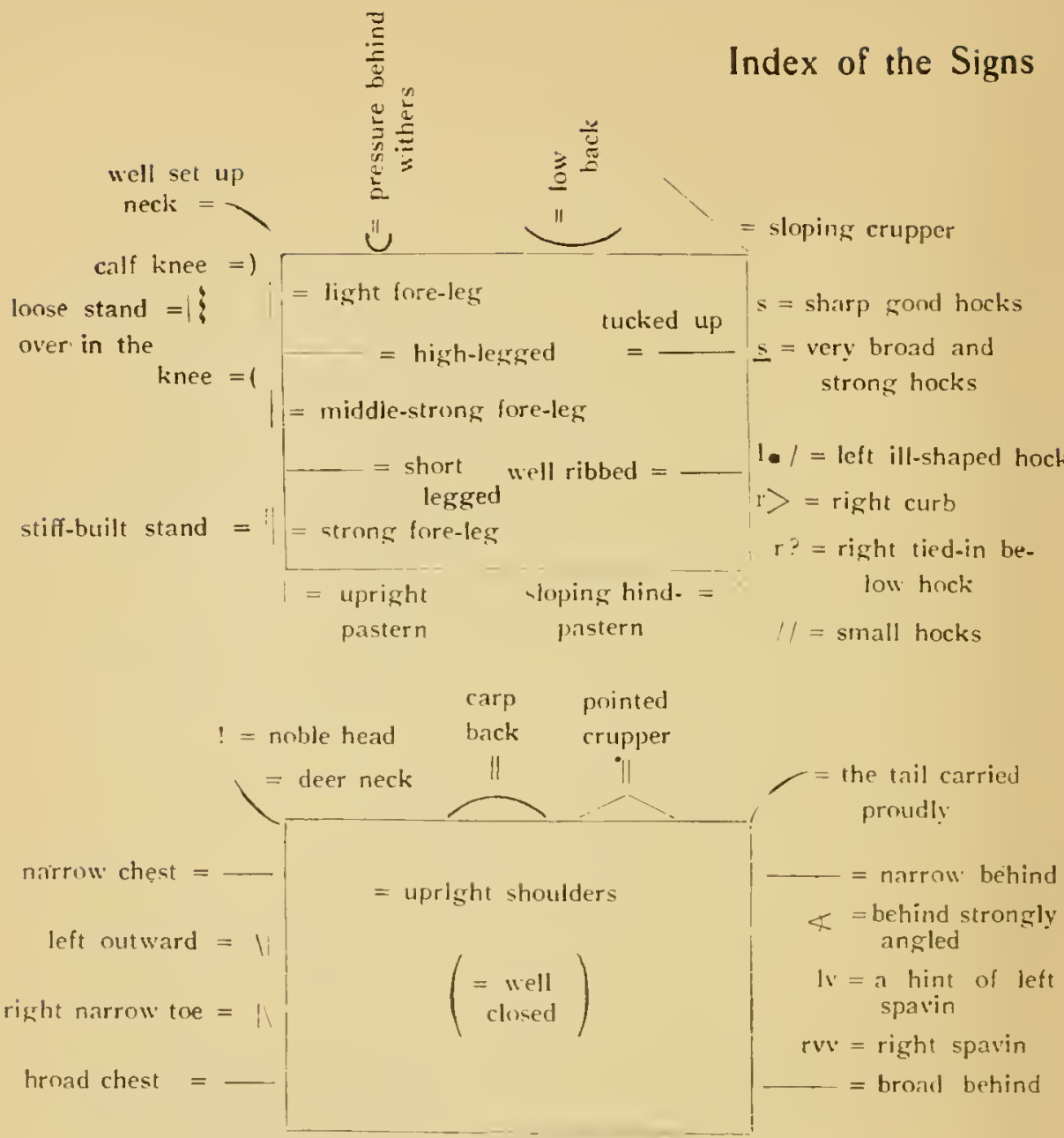

1. b. = left front upright hoof.

$$
\mathrm{KI}=\text { Class: 1.a, 1.. 11.a, 11?,111. }
$$

Z. $K \mathbf{l}=$ Class for breeding purposes

G. Kl $=$ Class for use purposes

Crib = crib biter

kr. $=\mathrm{ill}$

liz $=$ hip-shot

$k=$ small

$k=$ very small

$\mathrm{N}=$ needy being

$\mathrm{g}=$ large

$\mathbf{c}=$ correct

$\mathrm{h}=$ harmonious

$w=$ little
$\mathrm{R}=$ large frame

$\mathrm{L}=$ joint ill

$\mathrm{u}=$ unripe

sch $=$ weak

fill = gall

$!$ = noble

$! !=$ very noble

$a=$ expressively

$\operatorname{tr}=$ lean

$!=$ common

$1 !$ = very common

1. A. = left eye cataract-spor 


\section{for Judging the Exterior.}
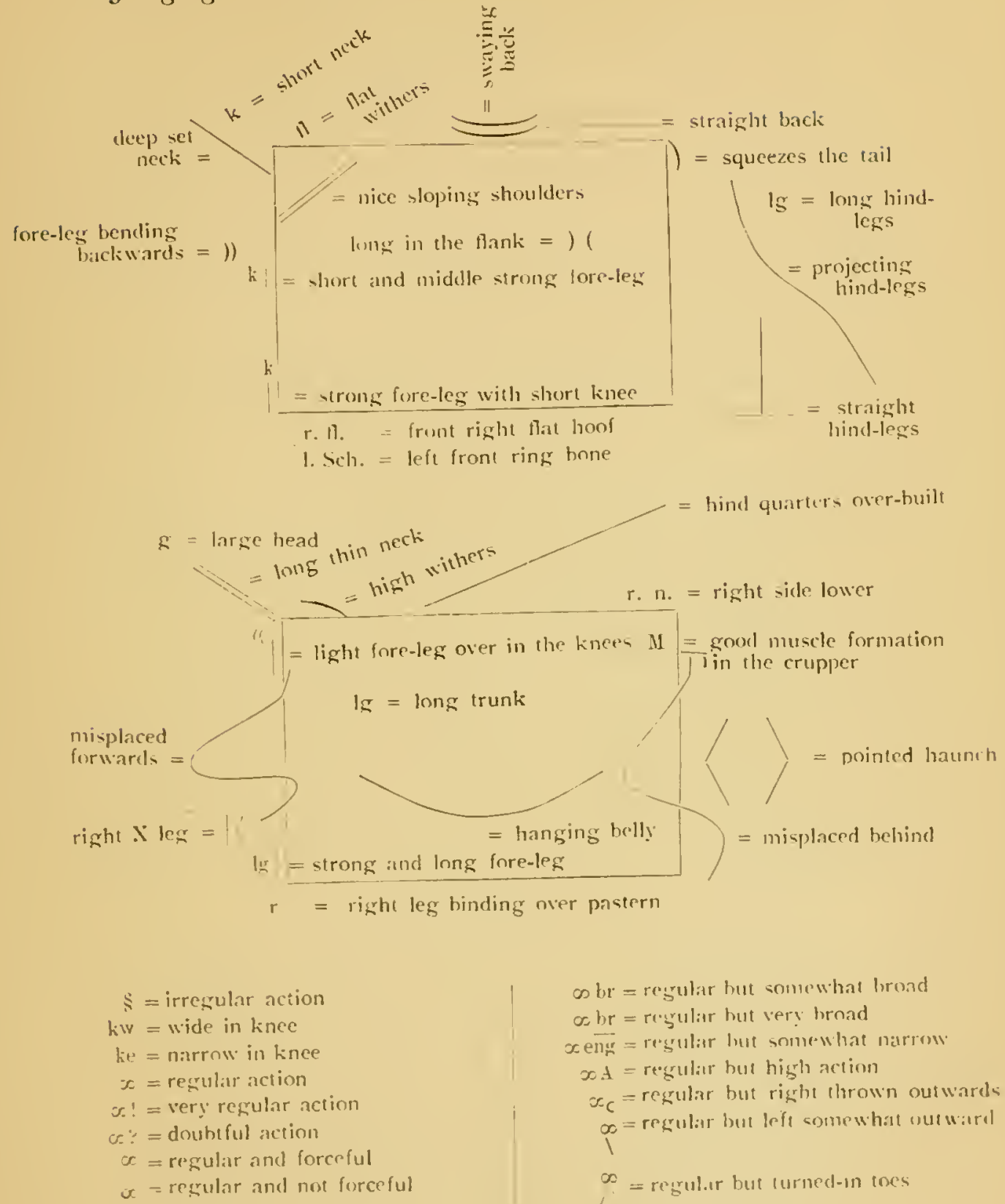

os br = regular but somewhat broad $\infty$ br $=$ regular hut very hroad $x$ entr = resular lut somewhat narrom xod = regulat bus high action $o_{c}=$ regular but right thrown outwards $\infty=$ regular but leff somewhat uutuatrol 1

$\infty=$ reguldur but lurned-in toes 


\section{CIIAPTER V.}

Tables for comparison of various Measurements.

Linear Measure.

1 Yard in lingland=3 Feet . . . = $=0,91438 \mathrm{~m}$

1 lard in America . . . . . . . = 0.91439 ,.

1 Furlong $=2: 20$ Yards . . . . . . $=201,16 \quad$,

1 Distance $=240$ Yards . . . . . . $=219,5$,,

1 .tile in England=8 Furlongs . . = 1609,31 ",

1 Mile in America . . . . . . . $=1609,33$ ",

1 Mile in Prussia . . . . . . . $=7532,5$ ",

1 Nile in Fungary . . . . . . . $=8353,6$,

1 Mile in Kurland $=7$ Versts . . . . = $=7467,5$,"

1 Faden (Saxony) in Russia . . . . = 2,13356 ,"

1 Verst $=500$ laden . . . . . . . $=1066,78$,.

1 Faden in Sweden and Finnland . . = 1,7814 ,,

1 Inch in Prussia (Rheinland) . . . = $2,6154 \mathrm{~cm}$

1 Inch in England and Russia . . . = 2.539954 ,.

1 Inch in America . . . . . . = 2,539977 ,"

8 Inches (English) . . . . . . . = 20 ",

$8 \frac{1}{2}$ Inches $\quad . \quad$. . . . . . . $=21 \frac{1}{2}$ ",

9 Inches $"$. . . . . . . $=22.8, "$

1 Foot in Prussia (Rheinl.) $=12$ Inches = 31,38535 ",

1 Foot in England and Russia $=12$ Inches $=30,47945$ ",

1 Font in . Imerica=12 Inches . . . = 30,47973 ",

1 Werschok in Russia . . . . . = = 4,445 ,"

$1 \wedge$ rschin $=16$ IVerschok . . . . . = = 71,1187 ," 
5. Tables for Comparison of Various Measurements.

Height Measurement for I Iorses.

1. In England.

I lnch $=2,3509 \mathrm{~cm}, 1$ 11:and $=4$ Inche $\mathrm{s}=10,1596 \mathrm{~cm}$.

\begin{tabular}{|c|c|c|c|c|c|c|c|c|c|c|c|}
\hline Ilands & Incl & & $\mathrm{cm}$. & 1lands & Inch & & $\mathrm{cm}$. & Hands & Inch & & $\mathrm{cm}$ \\
\hline 12 & - & $=$ & 121,9 & 14 & 3 & $=$ & 149,9 & 17 & 2 & $=$ & 177,8 \\
\hline 12 & 1 & $=$ & 124,5 & 15 & - & $=$ & 152,4 & 17 & 3 & $=$ & 180,3 \\
\hline 12 & 2 & $=$ & 127 & 15 & 1 & $=$ & 154,9 & 18 & - & $=$ & 182.9 \\
\hline 12 & 3 & $=$ & $129, \pi)$ & 15 & 2 & $=$ & $1: \pi, 5$ & 18 & 1 & $=$ & 185.4 \\
\hline 13 & - & $=$ & 132,1 & 15 & 3 & $=$ & $160)$ & 18 & 2 & $=$ & 188 \\
\hline 13 & 1 & $=$ & 134,6 & 16 & - & $=$ & $16:-6$ & 18 & 3 & - & 190,5 \\
\hline 13 & 2 & $=$ & 137,2 & 16 & 1 & $=$ & $16 \%, 1$ & 19 & - & $=$ & $19: 3$ \\
\hline 13 & 3 & $=$ & 139,7 & 16 & 2 & $=$ & $16 \mathrm{iT}, 6$ & 19 & 1 & $=$ & 195,6 \\
\hline 14 & - & $=$ & $1+1: 2,2$ & 16 & 3 & $=$ & 170,2 & 19 & 2 & $=$ & 198,1 \\
\hline 14 & 1 & $=$ & 141,8 & 17 & - & $=$ & $17 \cdot 27$ & 19 & 3 & - & 200,7 \\
\hline 14 & 2 & $=$ & 117.3 & 17 & 1 & $=$ & 175,8 & & & & \\
\hline
\end{tabular}

\section{In fierman!?.}

1 Inth $=2,6154 \mathrm{~cm}, 1$ fout $=12$ Inches $=31,38535 \mathrm{~cm}$.

Fect Inches $\mathrm{cm}$. Hands Inches Arschin Werschok

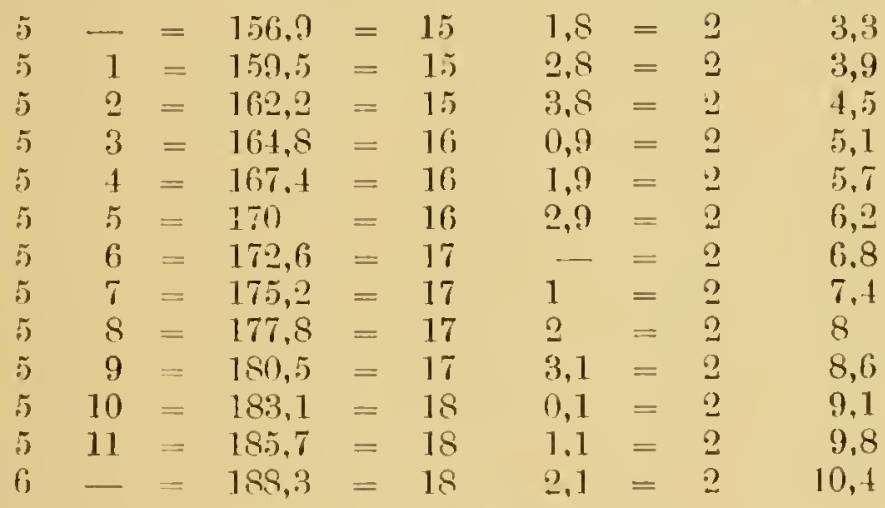

\section{In Rus-iil.}

111 erschuk $=4.4 .5 \mathrm{~cm}, 1$. Irschin $=1611$ erachok $=71,1187 \mathrm{~cm}$.

Arschin Werschok 2
2
2
2
2
2
2
2
2
2
2
2
2 $\mathrm{cm}$.

142,2

$116,7=$ $151,1=$ $155,6=$

160

164,5

$$
169
$$

173.1

177.8

182,2

186,7
Hands Inches

$\begin{array}{ll}1.4 & - \\ 1.4 & 1,8 \\ 1.4 & 3,5 \\ 15 & 1,3 \\ 15 & 3 \\ 16 & 0,8 \\ 16 & 2,5 \\ 17 & 0,3 \\ 17 & 2 \\ 17 & 3.8 \\ 18 & 1,5\end{array}$




\section{Liquid and Dry Measure.}

1 Quart in England=1,136 Liter, 1 ()uart in America=0,946 Liter.

1 Pint in England=0,568 Liter, 1 Pint in America $=0,474$ Liter.

1 Garnez in Russia=3,2797 Liter.

1 Liter $=1$ Cubicdecimeter.

1 Hektoliter $=100$ Liter.

1 Liter of Oats weighs abost a Pound= $\frac{1}{2}$ Kilogramm.

\section{Surface Measure.}

$1 \Lambda \tau=100 \mathrm{s.m.,} 1$. \сте=2.5,.53 ar.

1 - Ielitar $=100$ ar $=3,9166$. Icre.

1 Acre in England and America $=10,47$ ar $=1,59$ Acre.

1 Dessätine in Russia = 1,09 1 Jelitar =3 Lofslellen in Iiurland.

\section{Weights.}

1 Pound (ib.) in Germany, Denmark, Switrerland $=500$ st. = $\frac{1}{2}$ Kilogr. 1 lb. in England and America $=453,0 \mathrm{gr} .1 \mathrm{lb}$. in Russia $=409,5 \mathrm{kr} .1 \mathrm{lb}$. in Austria Hungary $=560,1 \mathrm{gr} .1 \mathrm{lb}$. in Sweden $=425.1 \mathrm{gr}$. I Stone in England $=14$ English lbs. $=$ $6.3 .5 \mathrm{~kg} .1$ Pud in Russiat $=40$ Ruscian $\mathrm{lbc} .=16,38 \mathrm{lsg}$.

\begin{tabular}{|c|c|c|c|c|c|c|c|c|c|c|c|c|c|}
\hline Stone & lb. & & lis. & & Pud & lb. & Stone & lb. & & $\mathrm{kg}$. & & Pud & lb. \\
\hline 5 & - & $=$ & $31 \frac{3}{4}$ & $=$ & 1 & 37,5 & 7 & - & $=$ & $44 \frac{1}{2}$ & $=$ & 2 & 28.5 \\
\hline 5 & 1 & $=$ & $32 \frac{7}{4}$ & $=$ & 1 & 38,6 & 7 & 1 & $=$ & $45^{\circ}$ & $=$ & 2 & 29,7 \\
\hline 5 & 2 & $=$ & $32 \frac{3}{4}$ & $=$ & 1 & 39,7 & 7 & 2 & $=$ & $45 \frac{1}{4}$ & $=$ & 2 & 30,8 \\
\hline 5 & 3 & $=$ & 33 & $=$ & ? & 0,9 & 7 & 3 & $=$ & $15 \frac{3}{4}$ & $=$ & 2 & 31,9 \\
\hline 5 & 4 & $=$ & $3: 3 \frac{7}{2}$ & $=$ & 2 & 2 & 7 & 4 & $=$ & $46 \frac{1}{4}$ & $=$ & 2 & $3: 3$ \\
\hline 5 & 5 & $=$ & $3 t$ & $=$ & 2 & 3 & 7 & $\tilde{5}$ & $=$ & $46 \frac{3}{4}$ & $=$ & 2 & 34.1 \\
\hline 5 & 6 & $=$ & $34 \frac{1}{2}$ & $=$ & 2 & $4, D$ & 7 & 6 & $=$ & $47 \frac{1}{4}$ & $=$ & 2 & $3.5,2$ \\
\hline 5 & 7 & $=$ & 35 & $=$ & 2 & 5,3 & 7 & 7 & $=$ & $47 \frac{3}{4}$ & $=$ & 2 & 36.3 \\
\hline 5 & 8 & $=$ & $35 \frac{1}{2}$ & $=$ & 2 & 6,4 & 7 & $B$ & $=$ & 18 & $=$ & 2 & 37,4 \\
\hline 5 & 9 & $=$ & $35 \frac{3}{4}$ & $=$ & 2 & 7,5 & 7 & 9 & $=$ & $48 \frac{1}{2}$ & $=$ & 2 & 38,5 \\
\hline 5 & 10 & $=$ & $36 \frac{1}{4}$ & $=$ & 3 & 8,6 & 7 & 10 & $=$ & 49 & $=$ & 2 & 39,6 \\
\hline 5 & 11 & $=$ & $36 \frac{3}{4}$ & $=$ & 2 & 9,7 & 7 & 11 & $=$ & $49 \frac{1}{2}$ & $=$ & 3 & 0,7 \\
\hline 5 & 12 & $=$ & $37 \frac{1}{4}$ & $=$ & 2 & 10.8 & 7 & 1.2 & $=$ & 50 & $=$ & 3 & 1,8 \\
\hline 5 & 13 & $=$ & $37 \frac{3}{4}$ & $=$ & 2 & 11,9 & 7 & 13 & $=$ & $50 \frac{1}{4}$ & $=$ & 3 & 3 \\
\hline
\end{tabular}




\begin{tabular}{|c|c|c|c|c|c|c|c|c|c|c|c|c|c|}
\hline Stome & lb. & & kis. & & Puel & lb. & Stone & lib. & & kig. & & Pud & I1). \\
\hline ii & $\ldots$ & $=$ & 38 & $=$ & !) & 13 & s & - & $=$ & $=; 0)_{1}^{3}$ & $=$ & $: 3$ & 4,1 \\
\hline i) & 1 & $=$ & 3 & $=$ & 2 & 14,2 & 8 & 1 & $=$ & 513 & $=$ & $: 3$ & 5,2 \\
\hline (; & 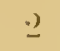 & $=$ & 39 & $=$ & 2 & 15,3 & 8 & 2 & $=$ & $51 \frac{3}{4}$ & - & 3 & 10,3 \\
\hline i & 3 & $=$ & $: 39 \frac{1}{2}$ & $=$ & 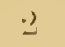 & 16,4 & 8 & $: 3$ & $=$ & 5.) $\frac{1}{-1}$ & $=$ & 3 & $7,-1$ \\
\hline (i & 4 & $=$ & 40 & - & $\because$ & 17,5 & $S$ & 1 & $=$ & 521 & - & :3 & 8.5 \\
\hline (i) & ; & $=$ & $40_{-1}^{J}$ & $=$ & $\because$ & 18,6 & 8 & j) & $=$ & $533^{-}$ & $=$ & :3 & 9,8 \\
\hline ii & i) & $=$ & $40 \frac{3}{4}$ & $=$ & .3 & 19,7 & 8 & 6 & $=$ & (5) 31 & $=$ & 3 & 10.7 \\
\hline i & 7 & $=$ & $11 \frac{1}{1}$ & - & .2 & 20,8 & 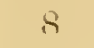 & 7 & 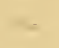 & 51 & $=$ & :3 & 11.8 \\
\hline i & 8 & $=$ & 413 & - & $\underline{-}$ & $=1,9$ & 8 & is & $=$ & i) $4 \frac{1}{2}$ & $=$ & 3 & 1.2.,!) \\
\hline ii & 9 & $=$ & 423 & $=$ & 2 & $2: 3$ & 8 & 9 & 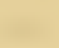 & 5.5 & - & 3 & 11 \\
\hline ii & 10 & -- & $1=3$ & $=$ & .2 & 24.1 & 8 & 10 & - & $55_{\frac{1}{4}}$ & $=$ & 3 & $1 ; \pi, 1$ \\
\hline (j) & 11 & $=$ & 43 & - & .) & $2.5,2$ & s & 11 & $=$ & $5.5 \frac{3}{4}$ & $=$ & 3 & 16,3 \\
\hline 6 & $1: 2$ & $=$ & $4: 3 !$ & - & .2 & 26,3 & $B$ & 12 & 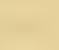 & $56 \frac{1}{1}$ & $=$ & $: 3$ & 17,1 \\
\hline 6 & $1: 3$ & $=$ & $H 4$ & 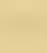 & )$. & $.27,4$ & 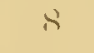 & $1: 3$ & 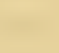 & $50 j_{1}^{3}$ & - & 3 & $1 \lambda_{,} ;$ \\
\hline$!$ & - & $=$ & $.57 !$ & - & 3 & 19,6 & 10 & 6 & $=$ & $66 \frac{1}{4}$ & $=$ & 4 & 1,7 \\
\hline ?) & 1 & 2 & $57 \frac{1}{2}$ & - & :3 & 20,7 & 10 & 7 & - & lifi & $=$ & 1 & $\because, 8$ \\
\hline !) & 2 & $=$ & 58 & 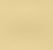 & :3 & $.21,0$ & 10 & 8 & $=$ & $(i]_{1}^{3}$ & $=$ & 4 & 3,9 \\
\hline 9 & 3 & $=$ & $58 \frac{1}{2}$ & $=$ & :3 & $2.20,9$ & 10 & 9 & $=$ & $i T_{2}^{1}$ & $=$ & 4 & i). 1 \\
\hline 9 & 1 & $=$ & 59 & $=$ & :3 & $\because 4$ & 10 & 10) & $=$ & fis & $=$ & 4 & $(i, \stackrel{2}{ }$ \\
\hline !) & $\tilde{j}$ & $=$ & $59 \frac{1}{2}$ & - & :3 & $2.5,1$ & 10) & 11 & $=$ & 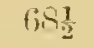 & $=$ & 4 & 7.3 \\
\hline 9) & (i) & $=$ & 593 & $=$ & :3 & 26,2 & 10 & $1: 1$ & $=$ & lig & $=$ & $t$ & 8,1 \\
\hline ?) & 7 & $=$ & $60^{\frac{1}{1}}$ & 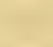 & $: 3$ & 27,3 & 10 & 13 & $=$ & (18.9) & $=$ & 1 & 9,0 \\
\hline$?$ & 8 & $=$ & $60_{4}^{3}$ & $=$ & :3 & $=-8,4$ & & & & & & & \\
\hline ?) & 9 & $=$ & $61 \frac{1}{4}$ & 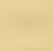 & $: 3$ & 29,5 & $\pi$ & . & $=$ & & $=$ & 1 & \\
\hline ?) & 10 & $=$ & $61 \frac{3}{4}$ & $=$ & 3 & 30,7 & 12 & - & $=$ & $T 6 i_{4}^{1}$ & $=$ & 4 & $2(1,1$ \\
\hline 9) & 11 & $=$ & $6: 23$ & 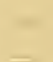 & $\begin{array}{l}3 \\
3\end{array}$ & $\begin{array}{l}31,8 \\
309\end{array}$ & $1: 3$ & - & $=$ & $(-2) \frac{1}{2}$ & $=$ & j & 1,0 \\
\hline $\begin{array}{l}9 \\
\text { !) }\end{array}$ & $\begin{array}{l}1: 3 \\
1: 3\end{array}$ & $\begin{array}{l}= \\
=\end{array}$ & $\begin{array}{l}6: 2.3 \\
1 i i 3\end{array}$ & $=$ & $\begin{array}{l}3 \\
3\end{array}$ & $\begin{array}{l}3=0.9 \\
34\end{array}$ & 14 & - & 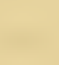 & S? & $=$ & 5 & 17,1 \\
\hline 10 & - & - & $1 ;: 3 \frac{1}{2}$ & $=$ & :3 & 35.1 & 1.i & & & $95 \frac{1}{1}$ & $=$ & $\check{\jmath}$ & 30.6 \\
\hline 10 & 1 & $=$ & $61^{\circ}$ & $=$ & $: 3$ & $: 36,2$ & $16 i$ & - & - & $1111 \frac{1}{2}$ & $=$ & 6 & 8,1 \\
\hline 10 & $\because$ & $=$ & $6+\frac{1}{2}$ & 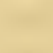 & :3 & 37,3 & 17 & $\ldots$ & $=$ & 108 & $=$ & 6 & $2: 3,1 i$ \\
\hline 10 & 3 & $=$ & 643 & $=$ & ;) & $: 3 \pi, 1$ & 14 & $=$ & - & 111 & $=$ & 6 & 89,1 \\
\hline $\begin{array}{l}10 \\
10\end{array}$ & 1 & $=$ & $\begin{array}{l}\text { fi.) } \frac{1}{2} \\
\text { (i.). } 3\end{array}$ & 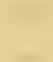 & 3 & $\begin{array}{r}39,5) \\
(1,1 \mathrm{i}\end{array}$ & 19 & & 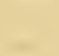 & 1203 & $=$ & 7 & 1.6 \\
\hline & & 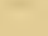 & & 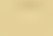 & & & & & & & & & \\
\hline
\end{tabular}


Most Important Distances at Newmarket.

The Beacon Course (B. C.)

since 18.52

since 1864

since 1865

since 1889

Round Course (R. (')

since 1819

since 185:

since 1889

Iitch in (D. J.)

since 1852

since 1865

since 1889

Ankaster Mile (1. II. since 1889

Two vears old Course (.I Y. U. C.) July St.

New Two years old Course (on the B. M.) since 1888 July St. since 1902

Two years old Course (on the flat) since 185" since 1902

Yearling Course ( $Y . C$. since 1853 (on the flat) since 185.54

Cambridgeshire Course (1839)

Old Cambr. Course since 1848

New Cambr. Course since 1901 since 1902

Cesarewitch Course (1839) since 1852

since 1889

since 1902

Across the Flat (A. F.). since 1818 since 1852 since 1887

Dewhurst Pl. Course

Criterion Course since 1865

Suffolk St. Course since 1889 since 1902
Mile Furl. Yds. Meter$$
41138=6764,6
$$$$
41173=6796,6
$$$$
4 \quad 1 \quad 157=6782
$$$$
4 \quad 1 \quad 143=6769,2
$$$$
4 \quad 1 \quad 177=6800.2
$$$$
\text { 3 } 6 \quad 93=6119,9
$$$$
34 \quad 187=5803,6
$$$$
34 \quad 139=5759,7
$$$$
34138=5758,8
$$$$
2
$$$$
2
$$

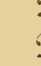$$
-
$$$$
97=3307,3
$$$$
119=3327,4
$$$$
105=3314,6
$$$$
118=3326,5
$$$$
18=1625,8
$$$$
22=1629,4
$$$$
\text { .) } \quad 136=1130.2
$$$$
5
$$$$
5
$$

$$
142=1135,6
$$$$
140=1133,8
$$$$
140=1133,8
$$$$
134=1128,3
$$$$
147=536,7
$$$$
2 \Omega=422,4
$$$$
52=449,9
$$$$
156=1953,1
$$$$
30=1828,8
$$$$
17=1826
$$$$
-=1810.5
$$$$
215=3615,4
$$$$
28=3646,5
$$$$
35=3652,9
$$$$
-=3620.9
$$$$
44=2051,9
$$$$
2 t=2033,6
$$$$
73=2078,4
$$$$
=2011,6
$$$$
\text { - }=1408,1
$$$$
182=1172,2
$$$$
-=1207
$$$$
2=2415,8
$$$$
25=2436,8
$$$$
-=2414
$$ 


\section{CHAPTER VI.}

Tables showing the Ancestors of Thoroughbreds in the Male Line. 


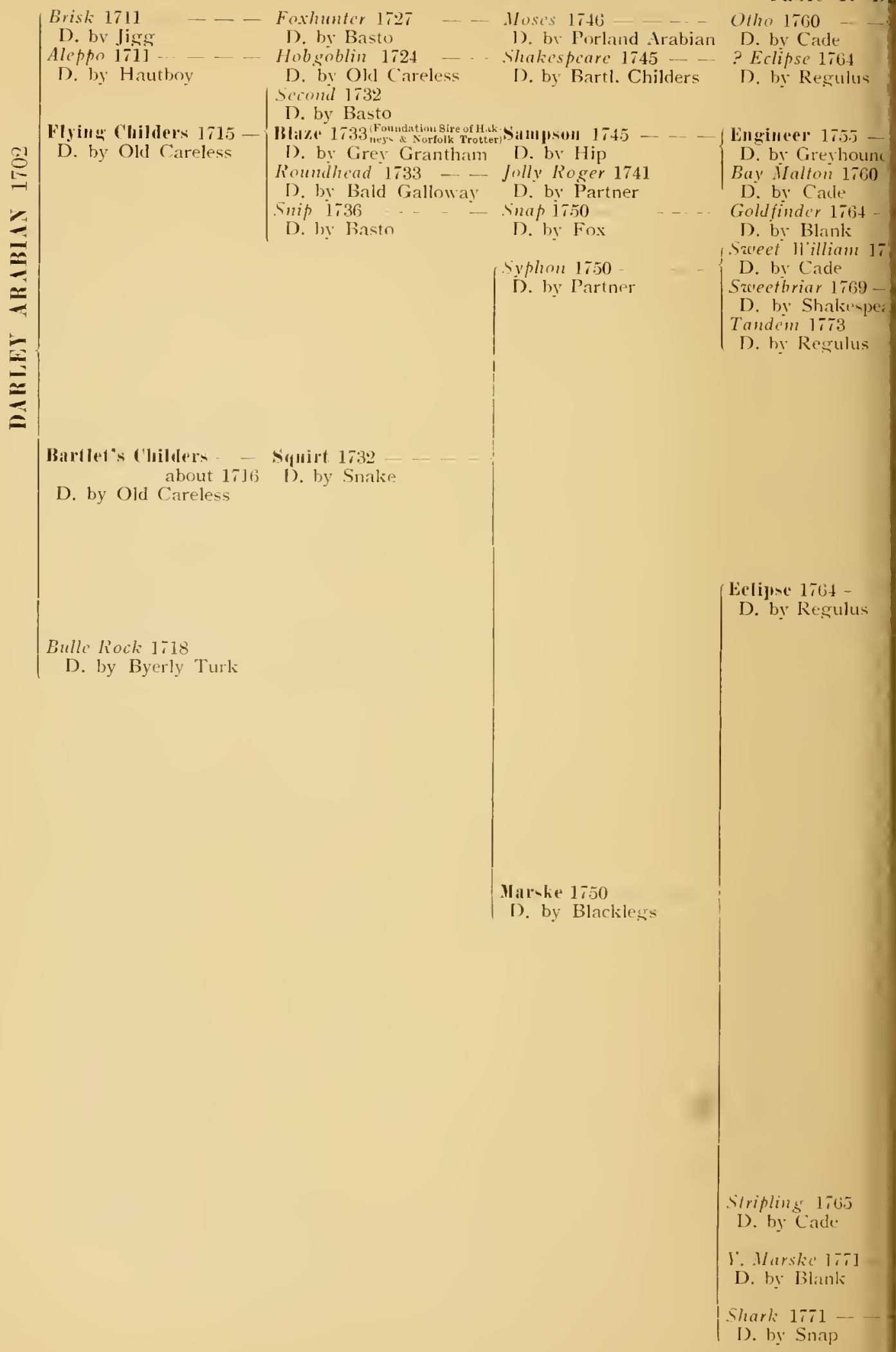


rab. Linte.

(ixionle 17il

1). by Biabraham

Darimant 17T2

1). by Babrah:m

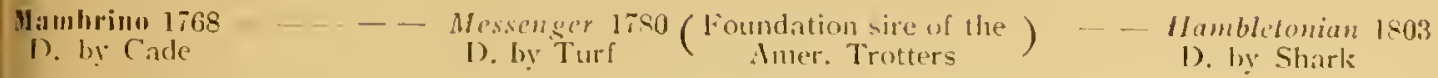

Nincler $17-76$

I). by Sedley Irabian

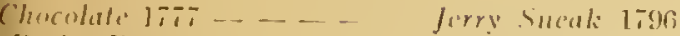

I). Iy liancly I). by Trunnion

Jarolin 1-is

D. by Trunnion

D. ly. Sipectato

D. by llerod i). by l'lumenomenon

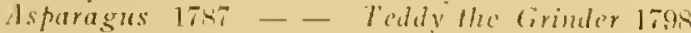

D. by Justice 1). Wx llightlve:

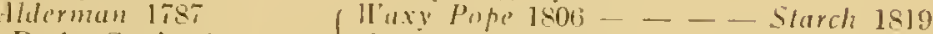

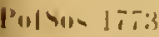

D. by Syuirrel

1). hy lishllyer

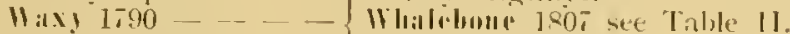

I). by IV:trens Sportsman

Jupiler $17-7$

Chamion 179\%

I). W Tartar

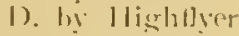

D. Is Trumpator

llioful 1.809

()inrfun 1788

1). he Herod

I). by Trumpator

Whioline JSI2 ser Table III.

Kime runum 17,5

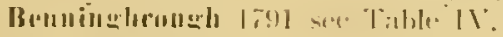

1). Ы lilack and all Black

Brmalrual $17 \%$

1). by Sinreger

D. by llerod

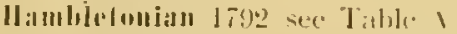

1). by llighllyer

Precipilate $178 \%$ Bublail 1-9.5

1). Iㄴ.rml

1). log liclipsc

finlaumas l 402 ser Table VI.

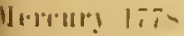

1). 140

llall's Foclipse 170

1). he Rerulus

1). Jlarud

1). he Iloculpeclier

(irberus 1,00

I). by llarod

18:2lliv 180:3

I). liy IV imaduecker

('umopus 180:3

1). h llimelpeker

Election 1 1 00

1). hy Mondpecker

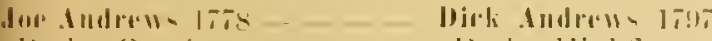

'I'iami, I>10 -ee Table 1 II.

1). 12 ()mnium

I). In llighllior

1). hi (iollommat

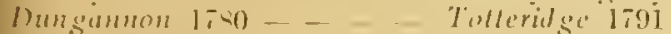

1). by llexol 1). My Mambrino

inimierer 1-N0

J). by Tirtar

1). ly llightyor

Saltrim 17-0

Pinme"tr l:01

1). In I lishtlyer

D. Џу Shuttle

1). lo Snap

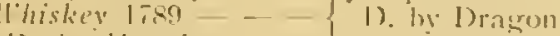

I). Is llererl

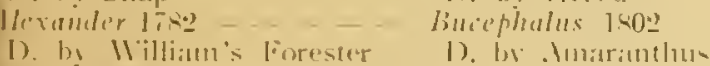

varmion levefi

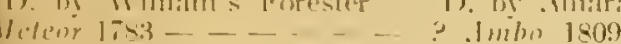

1). by Merlin

I). his sij l'eter

Cimpoteder IT-A

1). hy (Oniar

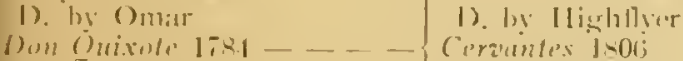

1). |x 1)ioned

1). bi William's forester

l'enasis 1784

I). by liosphorus

Sirpionil listi

1). lis Simetallai

limlen $17 \%$

I). Is: I.tifty

Shullic 170:3

1). by liauxhall Sinatp

Whark 1791

1). แ limmap

Sultion $1801 \quad$ (iumon liall 1810

Corvintes Is06;

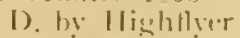

Inudis Jint

[). by vir Peleer

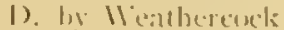

- Emigrant 1<22

D. by Buzzard 
Tab]e II. Darl. Irabian line.

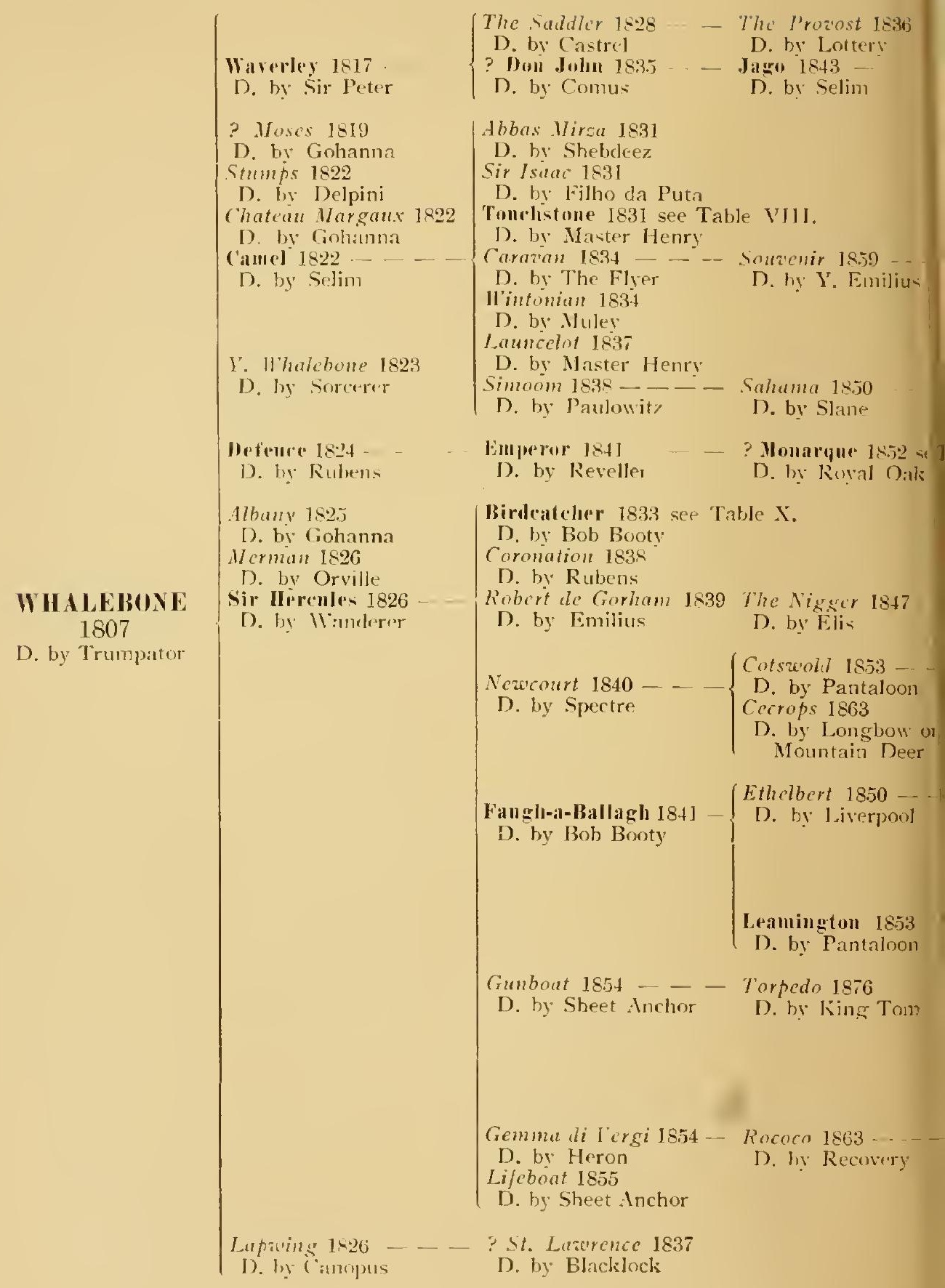


Shere 18,01

I). hy Moriseo

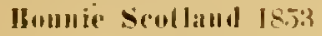

I). by Gladiator
Bomlic siotland jun. 18(j).

D. by Clay Trustie

Bramble 1875_.. _ Ben Brush 189?

1). W Australian 1). Wy M:ırm

Jallypup 1873

I). by Suclewill

lahire 1861

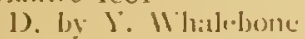

1..

lirst Lord 1866

D. by Terldinguon

\footnotetext{
liing of Kont 18j8 - - J'usumini 1865

$\begin{array}{ll}\text { 1). by Touchstune } & \text { I). by l'eppermint }\end{array}$
}

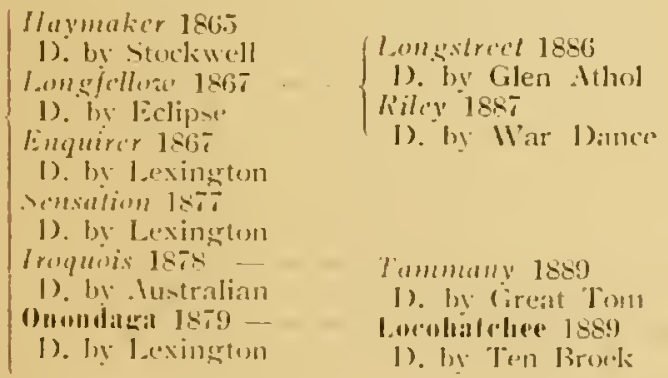

- (aiman) I-94i

1). ly ( incruleu-

(Mippendale 1876

1). hy idventurer 


\section{Tahe III. Darl. Mrabian Line.}

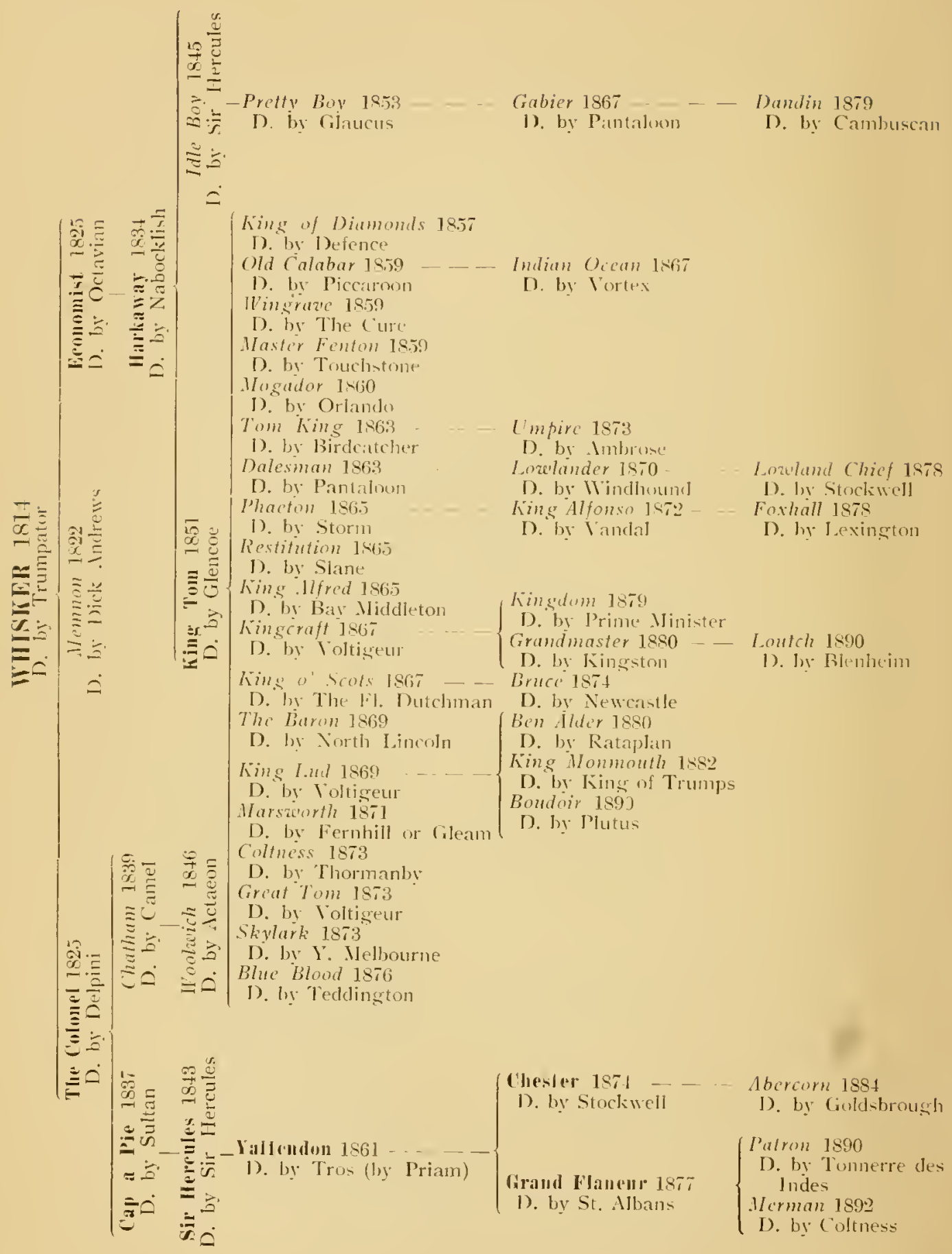


6. Tables Showing the Incestors of Thoroughbreds in the Nale Line. 433
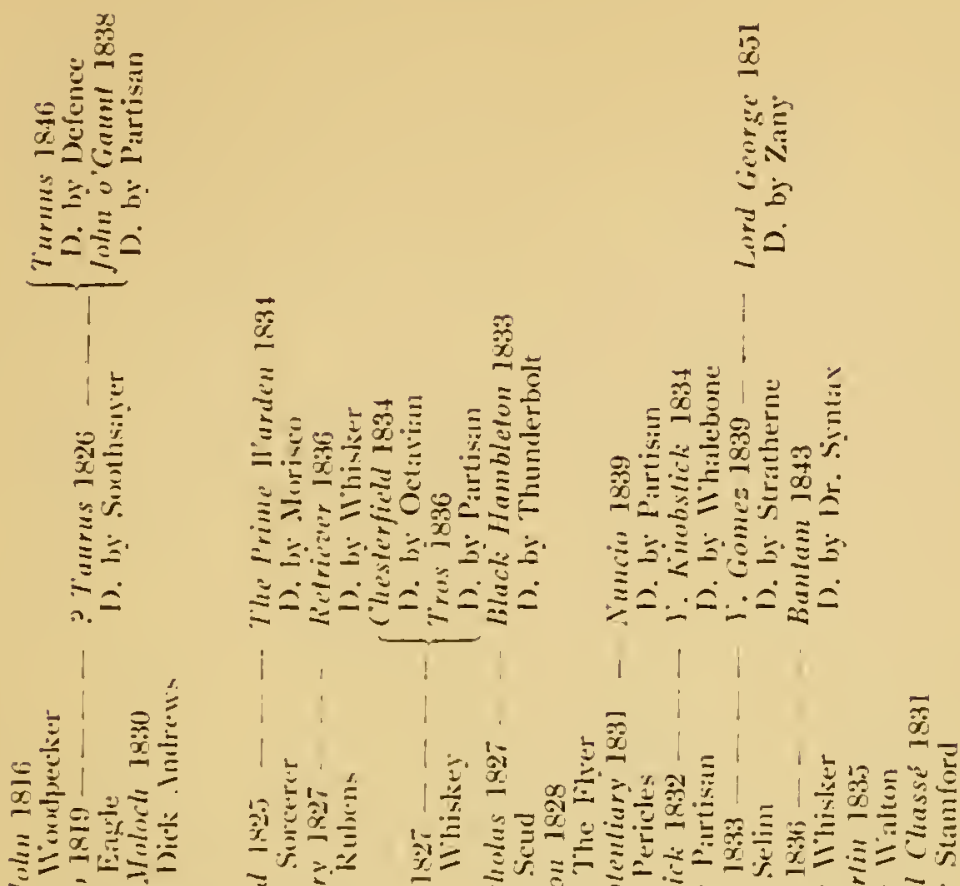

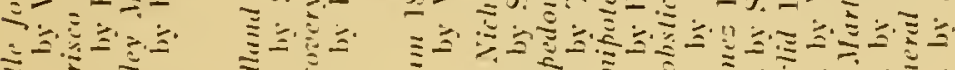

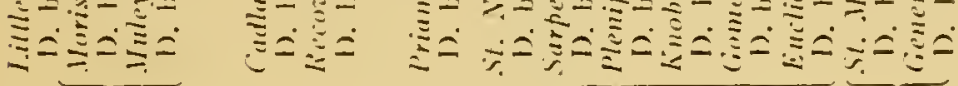

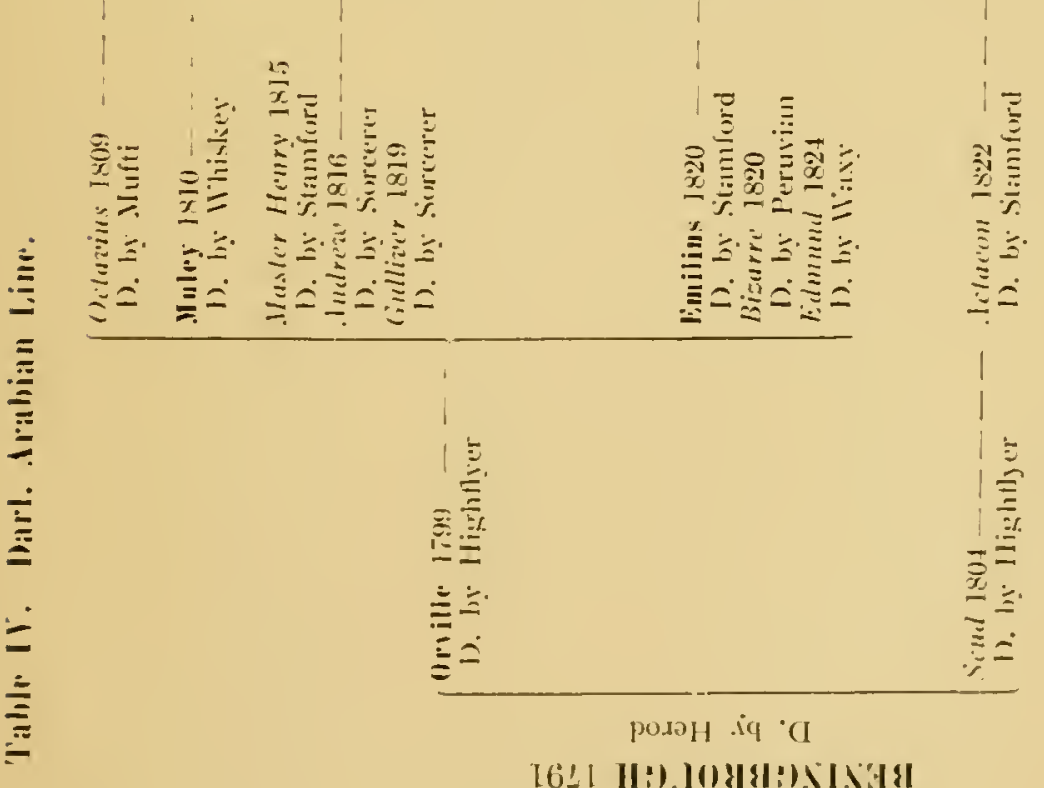




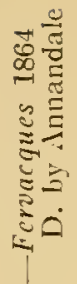

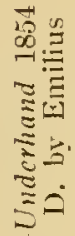

总

츠

$\approx 0$

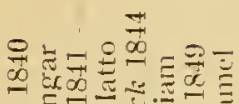

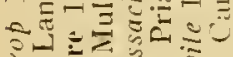

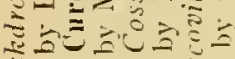

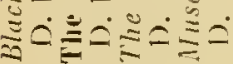

$i$

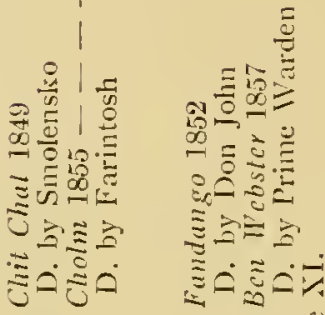

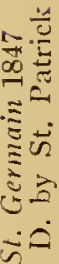

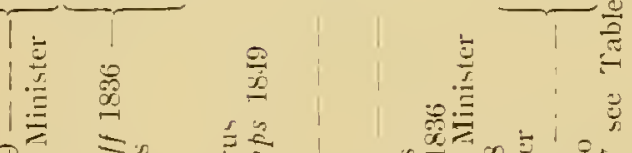

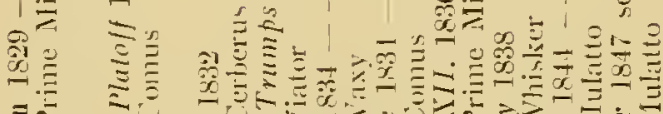

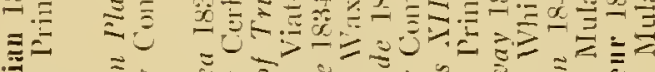

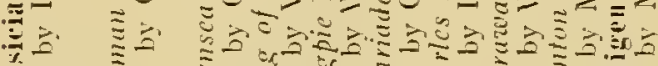

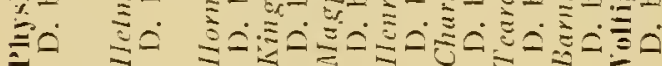

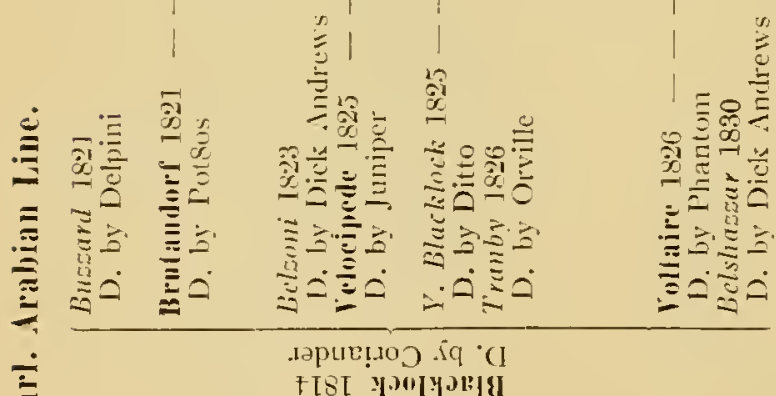

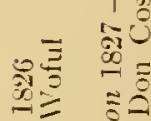

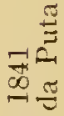


6. Tables Showing the Ancestors of Thoroughbreds in the Male Line. 435
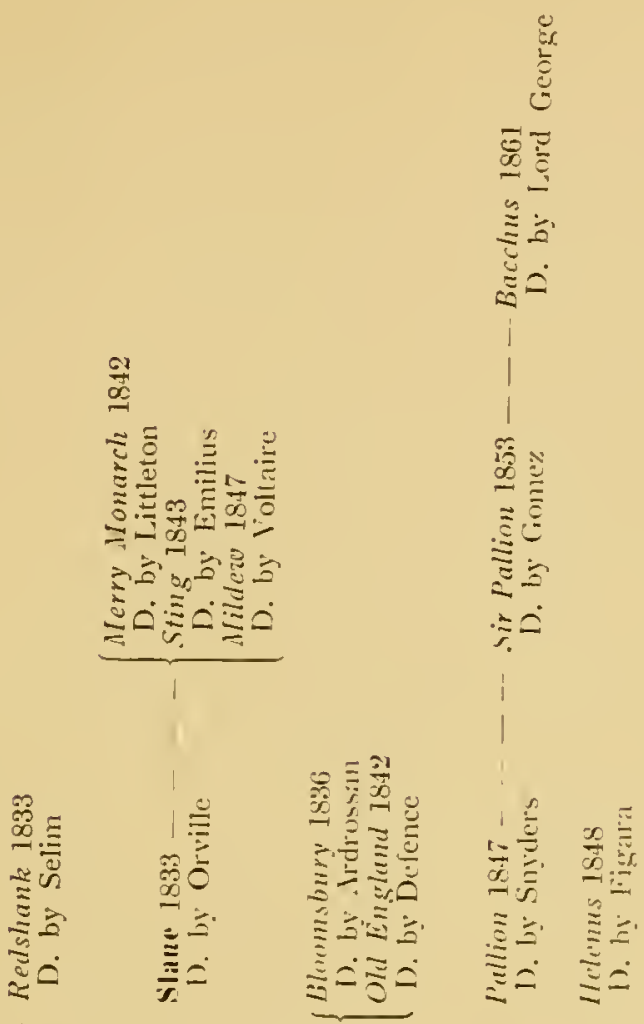

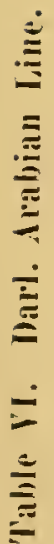

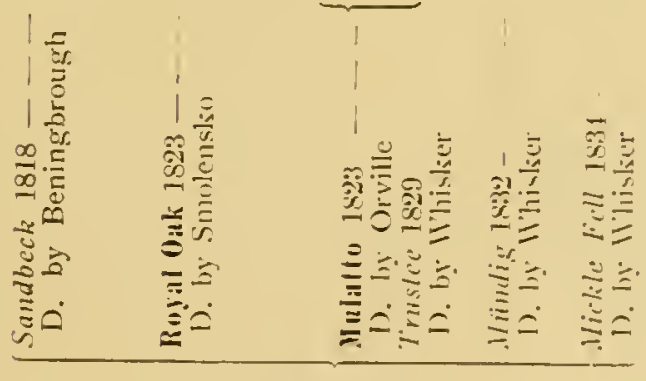

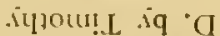

6081 I(I.I,L)

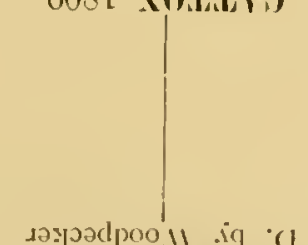

c08 S'DAIf I'I0:) 


\section{Table VII. Jarl. Arabian Line.}

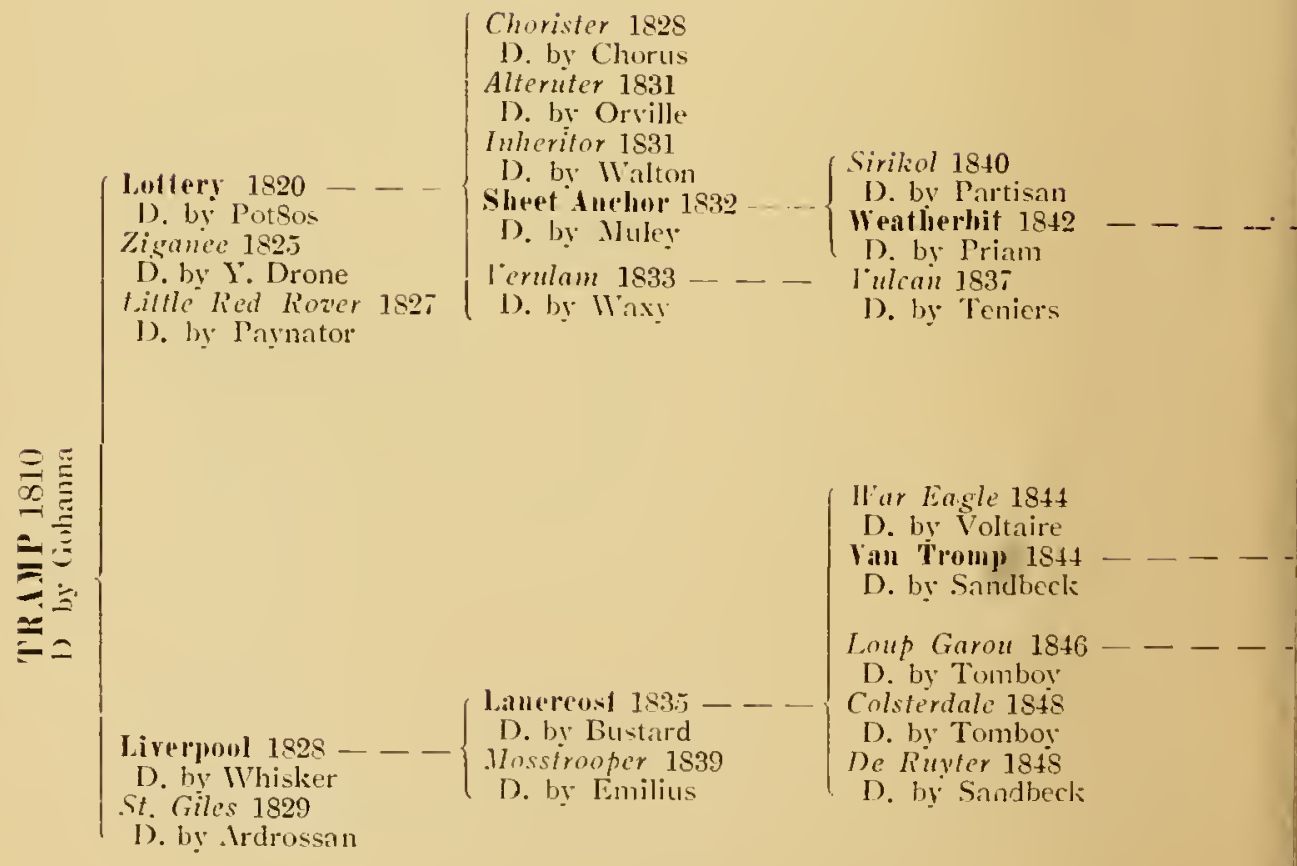




\title{
Table VIII. Darl. Arabian Line.
}

\author{
Auckland 1839 \\ D. by Champion \\ Cotherstone 1810 \\ D. by IThisker
$\{$ Danseur 1854
Danseur 1854
D. by New Light
Durclilaucht 1858
D. by Snyders
Glenmasson 1854 - - l'estminster 1866
D. by Priam
D. by Envoy \\ Orlando 1841 see Table VIJj. a \\ D. by Langar \\ Ithuriel 1841 \\ D. by Velocipede \\ Annaindale 1812 \\ D. by Lottery \\ Falstaff 1842 \\ D. by Filho da Puta \\ Paragone 1843 - - Orpheus 1860 - - . \\ D. by Tonboy \\ Flatcatcher 1815 \\ D. br Filho da Puta \\ Surplice 1815 - - - \\ D. by Priam \\ Pontifex 1817 \\ Pontifex $18 \pm 7-$
D. by Priam
Yewminter 1848 s
D. by Dr. Syntax \\ D. by Pantaloon \\ Mountain Deer 1848 \\ D. by Belshazzar \\ Harbinger 1949 \\ D. by Elis \\ Magnes 1849 \\ D. by Langar \\ rindex $1950-$ \\ 'D, by liangar \\ Lurd of the I-les 1852 \\ D. by Pantaloon \\ De: Clure 18.52 \\ D. by Catton \\ lifleman 18.52 \\ D. by The Colonel
Claret $1852--$
D. by Belshazar
Artillery 18.53 \\ D. by Birdcatcher \\ Lomglow 1849 \\ D. by Catton \\ Toxophilite 1855 \\ D. by Pantaloon \\ D. by St. Nicholas \\ Pylades 1852 - - - - \\ D. by Bay Middleton \\ Nortl Lincoln 1856 \\ D. by Redshank \\ Flasti in the Pan 1856 \\ D. by Pompey
}




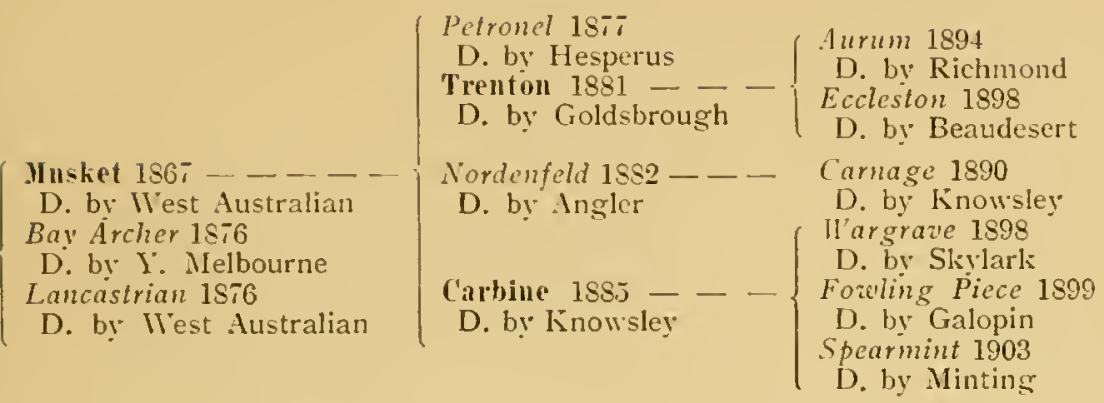

Noble Chieftuin 1885

D. by Moulsey

The General 1882

D. by Thunderbolt 
Table MHl. a. Jarl. Mrabian Linn.
Challanooga $1862--$
I). by Birdeatcher
Wellinglouia 1869 D. by limbrose
Clozer 1886 - - - - -
D. hy Prince Clarlie 


\section{Diaklia 1893}

D. bi Mortemer

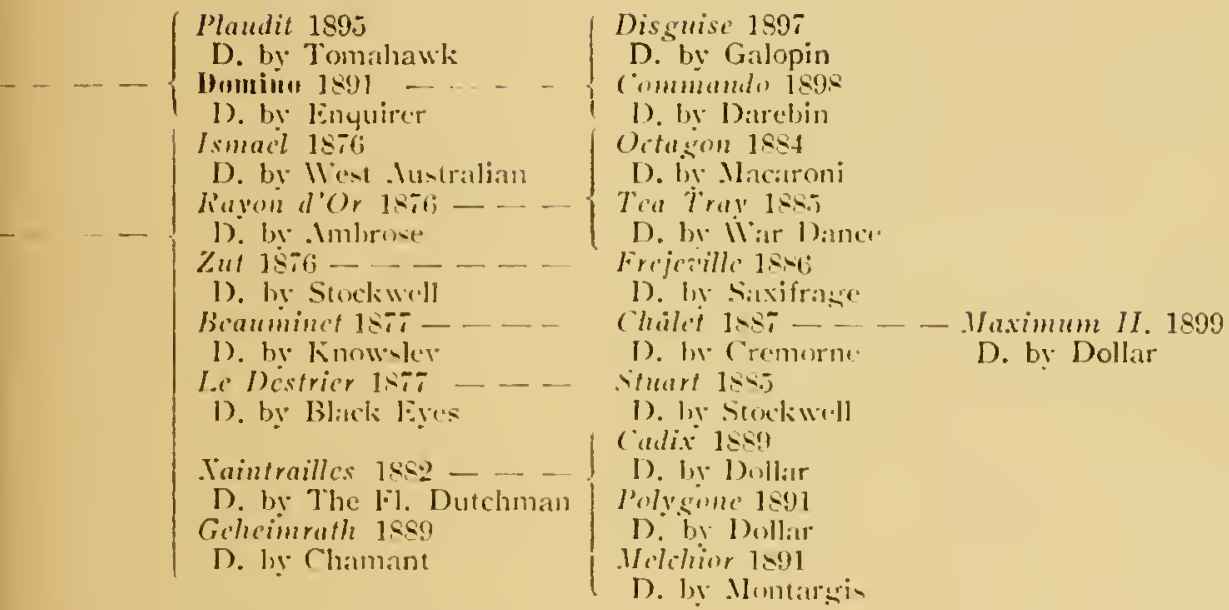

D. by Cambuscan 


\section{Table VIII. b. Darl. Arabian Line.}

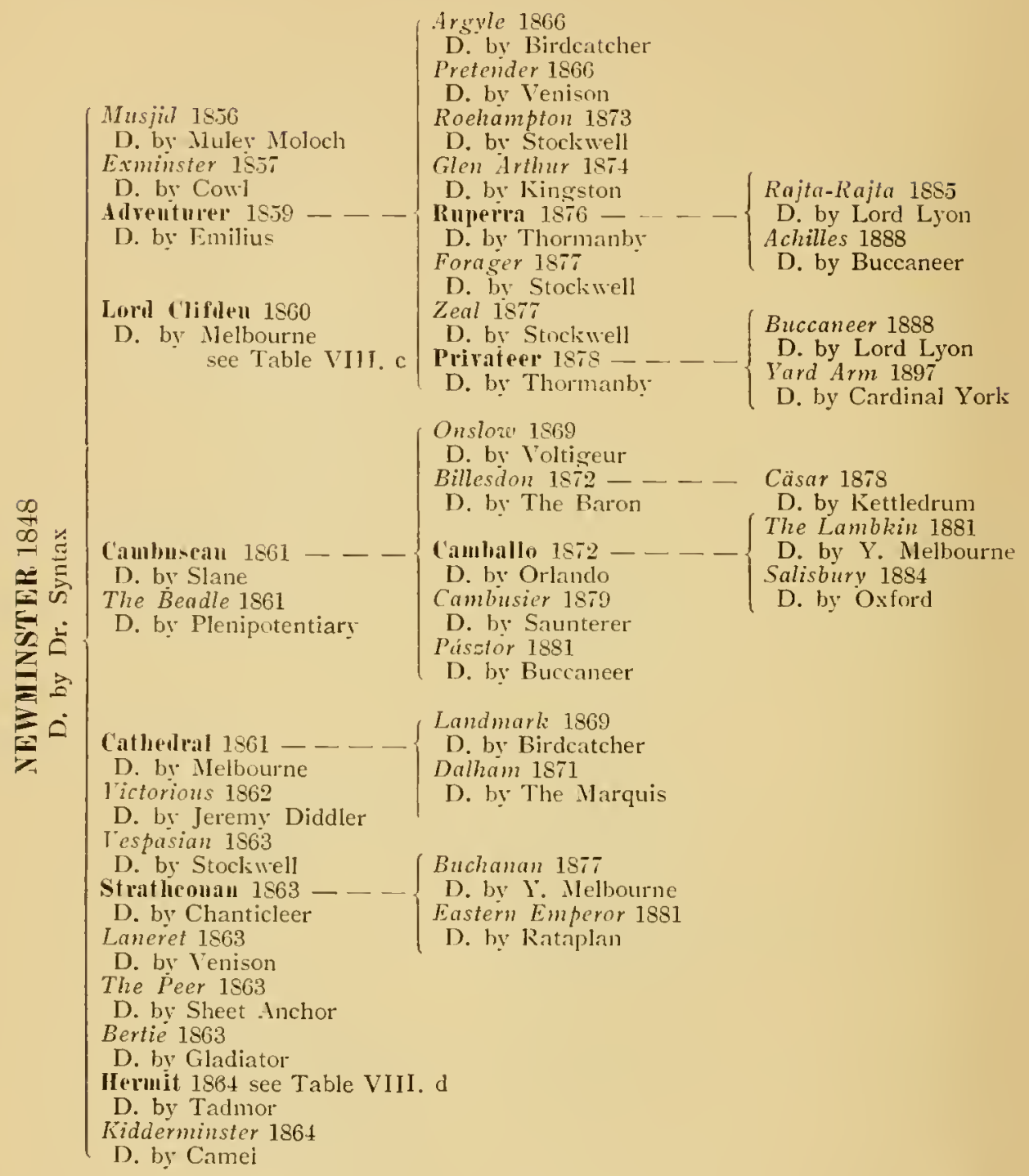


6. Tables Showing the Ancestors of Thoroughbreds in the Male Line.

Table VII. c. Darl. Arabian Iine.

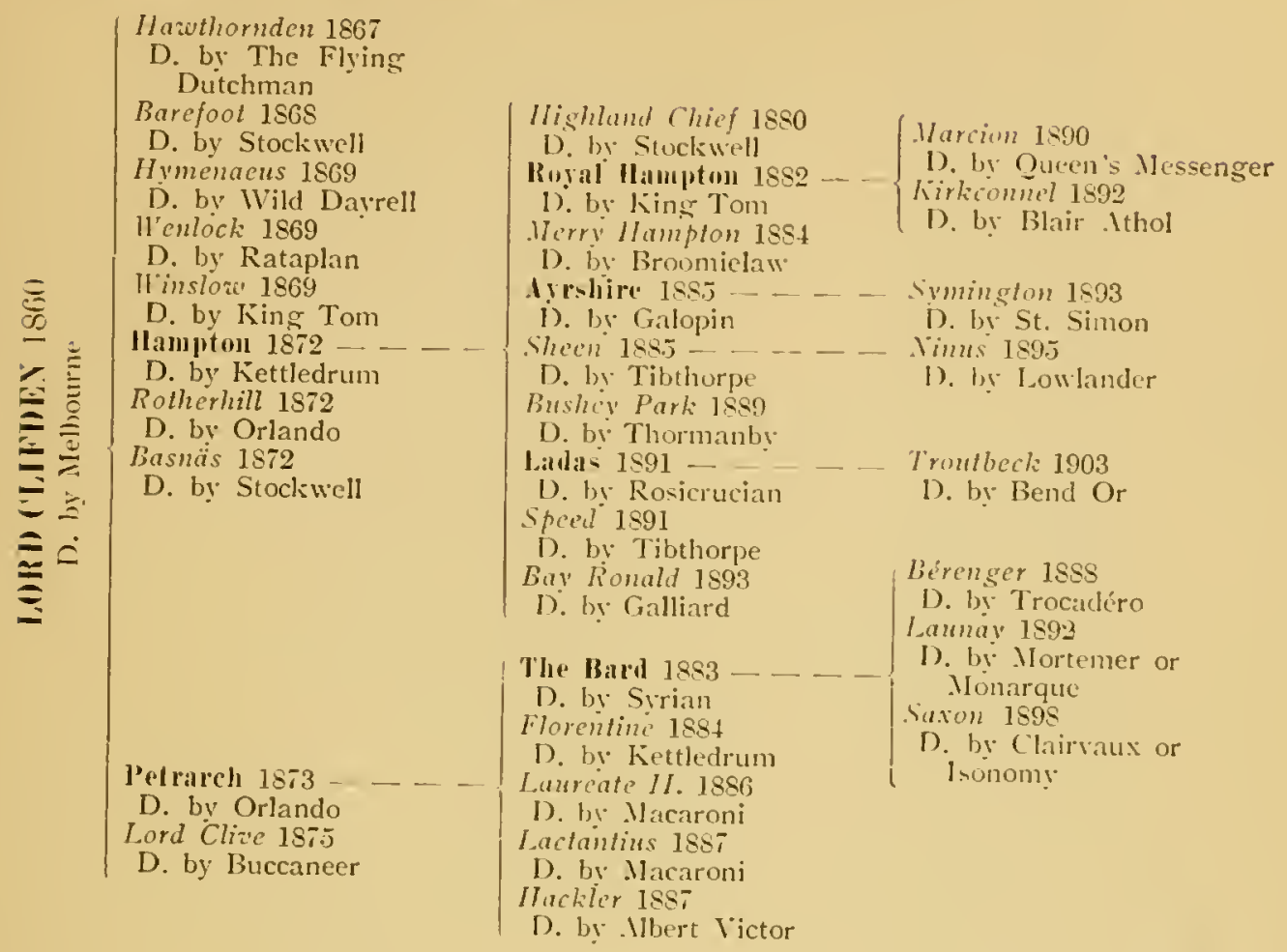




\section{'Table VIII. d. Marl. Arabian line.}

Ancelic $15-1$

1). by Mcelbourme.

Holv Friar 18\%2

1). hy "Thormanby

Recurder 15:2

1). by St. Illown

Trappist 18:2

1). lis .lluseovite.

.1mbirstis 18 8:

D. by longrow

Gumershary 180

D. be linir 'Tom

The iliser 18:-

D. b st. Ilbans

Zealot 18Ti

D. by Stacliwedl

leeter ista

D. W Frither to Stratford

The ibbot $1877_{-\ldots} \ldots$

1). by Pelion

Retreat 18:-

D. ly Rataplan

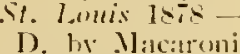

Edicard the Conjessor $18 \mathrm{~s}$

1). by Stmelewell

Jrintain Isis.

D. by Stuckwell

Warlini Is-s

D. W Tinephilite

Exilc $11.15-\mathrm{s}$

D). by l.urd l.ron

Mardin 18,!

1). be P'elion

(7airiatax 1850

1). by Stuckinell

St. Biaise 1880 -

D. by .11:arata

Torpeder Ts-o

1). he Tosuphilite

St. Hemurat 18s?

1). by Situclivell

froillingtun 1842

1). by Stuckwell

Tatilus 1850

D. W Y. Ilelkourne

Gamii 1-8 3 -

1). by tontiob chief

Harelstume 1853

1). by Stexckwell

Gay Ilomil 1se?

D. by bromicesam

Timuling 1sat

D. W Brother to Strational

Haflihatich 188.5

1). W (athedral

Friar's Balsam 188.j _.

1). Wr Jicudalbane

Astrologer 18s.j

D. by Brother to Strafford

Mclanion 1886 - - -

D. by Gitlopin

Heallite 188-

D. br Breadalbane

Misial 1491

1). by "lowmlerbolt
Koval Meath 1884

I). We Curoner

Sir Palricli 1890

1). by Herbertotown

lard liblot 1898

I). by Chippendalce

k̈önigst'in 1888

1). by Stratheonam

Gajari 188.5

1). by . Albion

Hacros 1886

1). by Rumicruetan

I rami-balyam 188fi

1). by Buccineer

Gulerimumel 1890

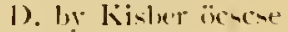

Gialifard 1896

1). Wy Gialopjin

\%oupran 18st

1). |x Blair .|tho|

Father (onfaswer 18S.5

1). Wy 'The Hadji

Indré Is!!?

D. by raslupin

l.c lliardy 1888

1). by S. Ilb:111=

l.e . Merd los

1). by IVcllingtomia

Lé Villam II. 1890

D. by Wellingtunia ity $1 \leq 91$

1). Wr Galopin

burigine $1 \leq 90$

D. hy liken . Irthur

17.trerate 1893

1). in 111-l hed

Ciesperdar 1891

1). by Trociadero

7he ()rack 1893

1). by Gialopin

Hillonium 1896

1). by Barcitdine

Bhack tand 1897

1). by llenlock

l.c Rii .rolcil l895

i). by biviad 


\section{Table IX. Jarl. drabian Line.}

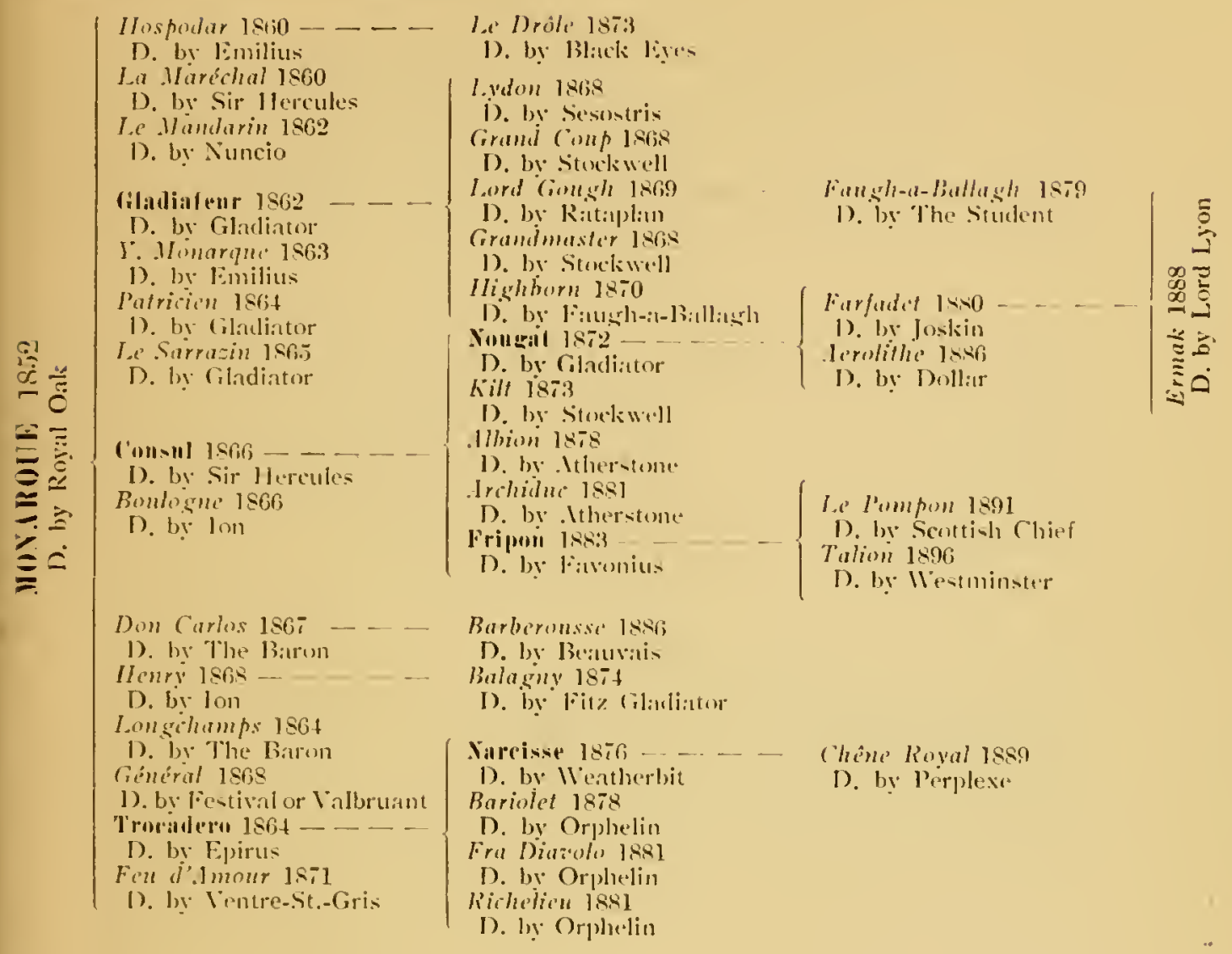




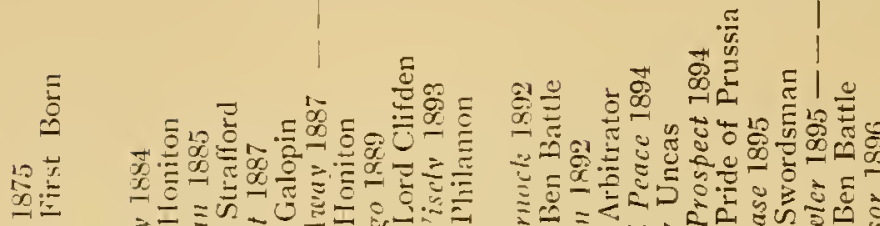

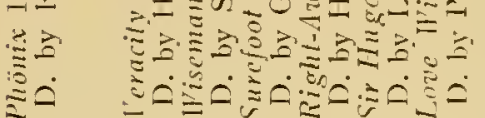

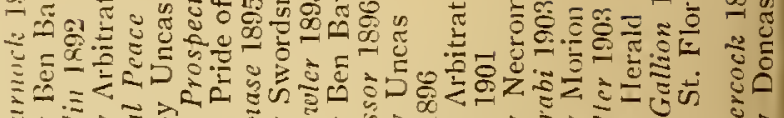

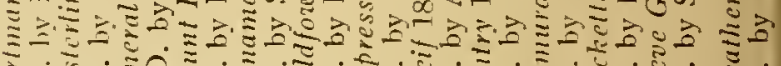

$\approx$

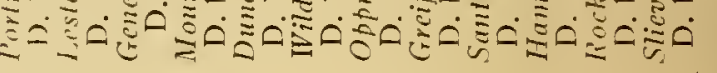

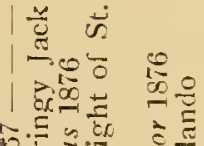

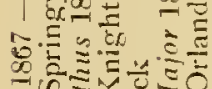

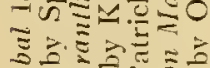

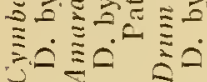

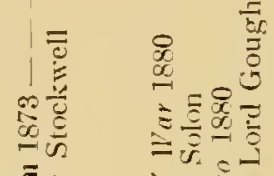

$$
\begin{aligned}
& \text { 三三 }
\end{aligned}
$$

$\stackrel{\grave{c}}{\grave{\Xi}}$

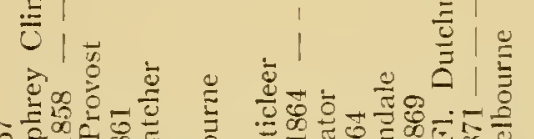

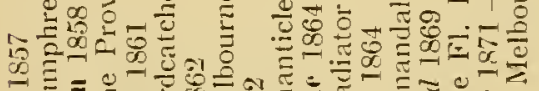
Q

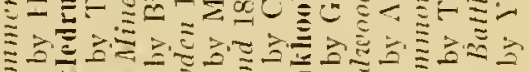

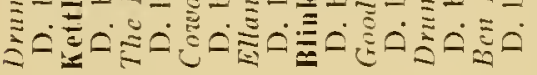

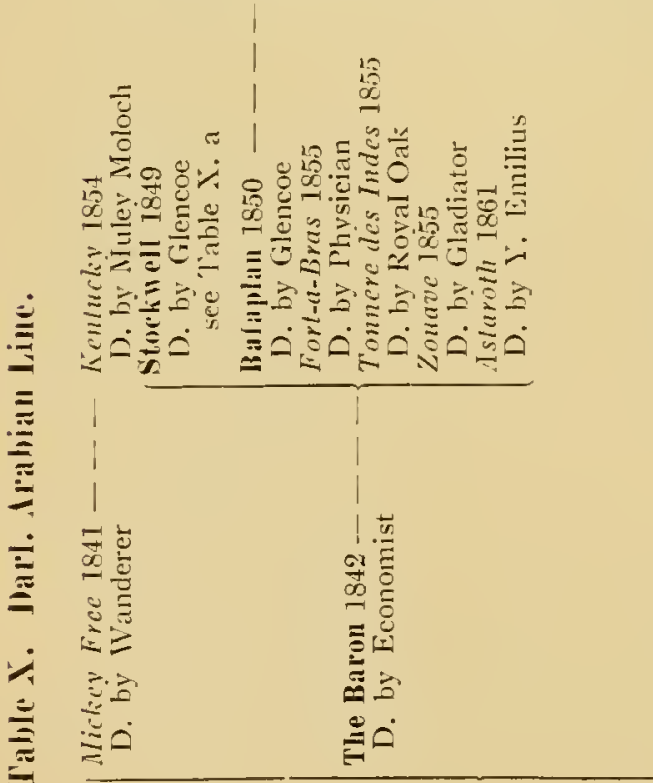

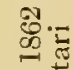
芌芯

층

in

$\stackrel{\infty}{\stackrel{\infty}{-}}$

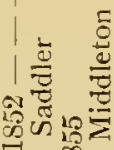
我为

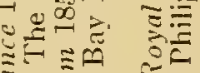

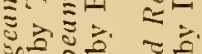

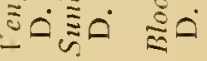

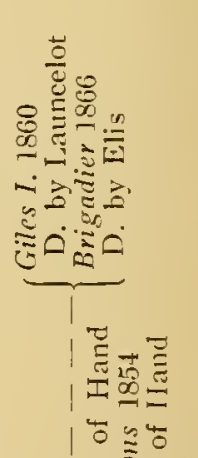
$10 \cong 0$

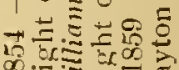
$\infty=\bar{c}=\frac{\pi}{\pi}$ ज得 $\overline{0}$

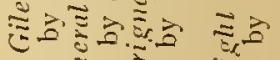

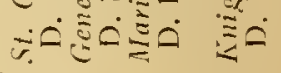

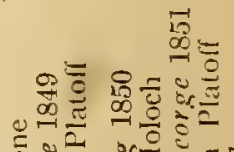

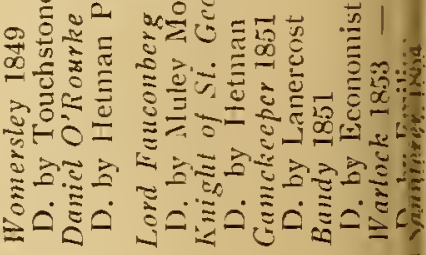


6. Tables Showing the Incestors of Thoroughbreds in the llale l.ine. 447
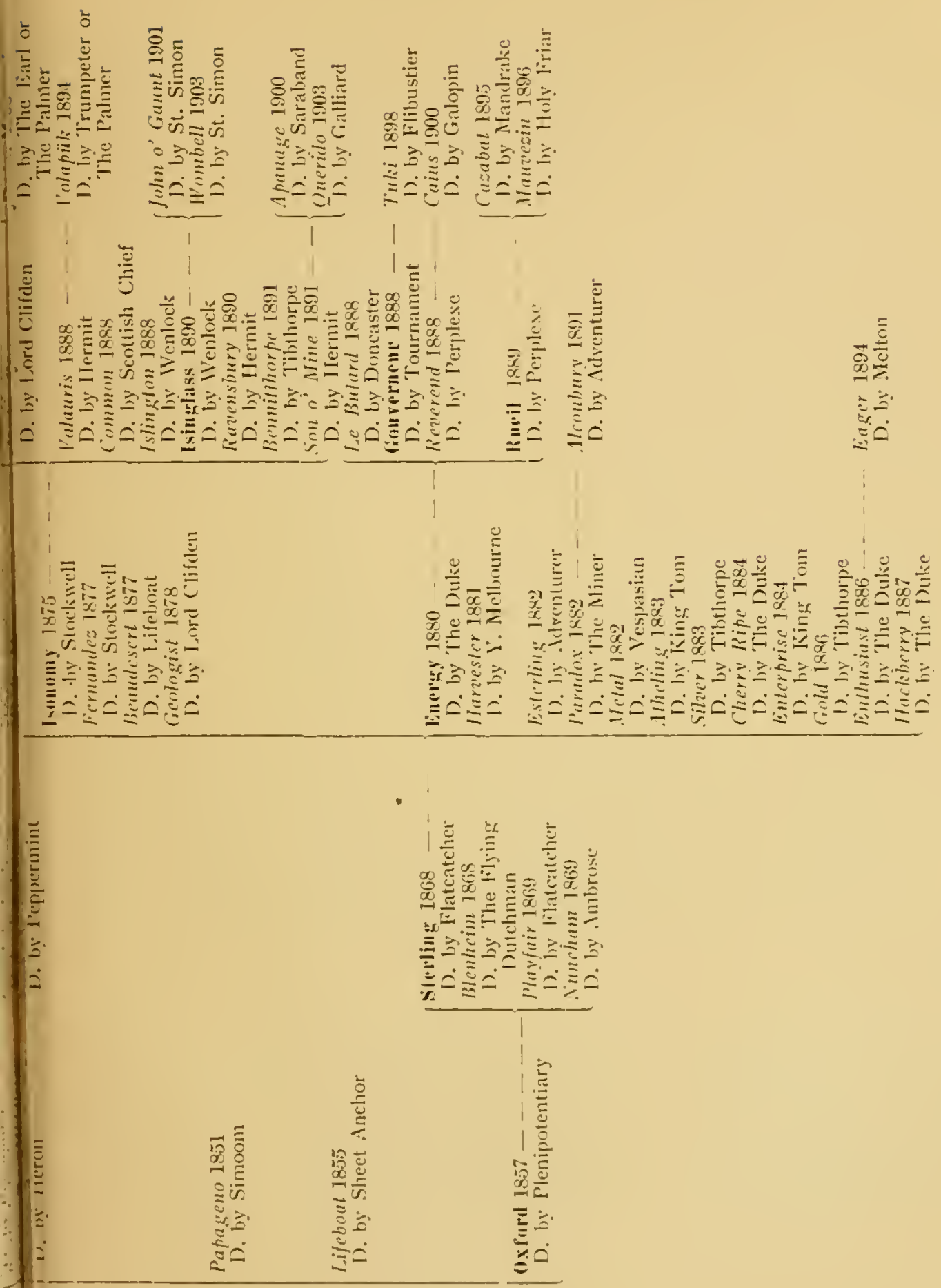


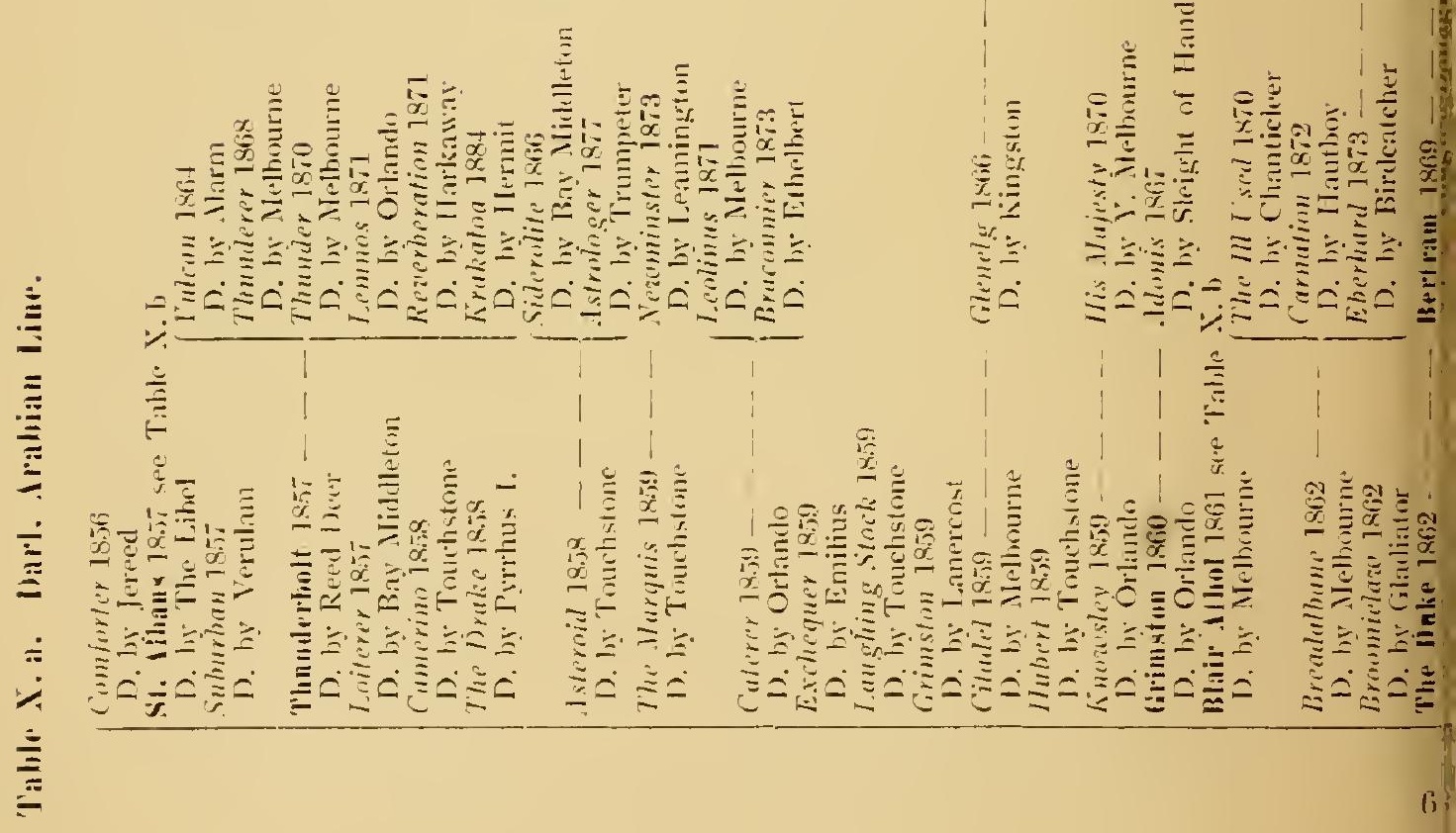


6. Tables Showing the Ancestors of Thoroughbreds in the Male Line. 449

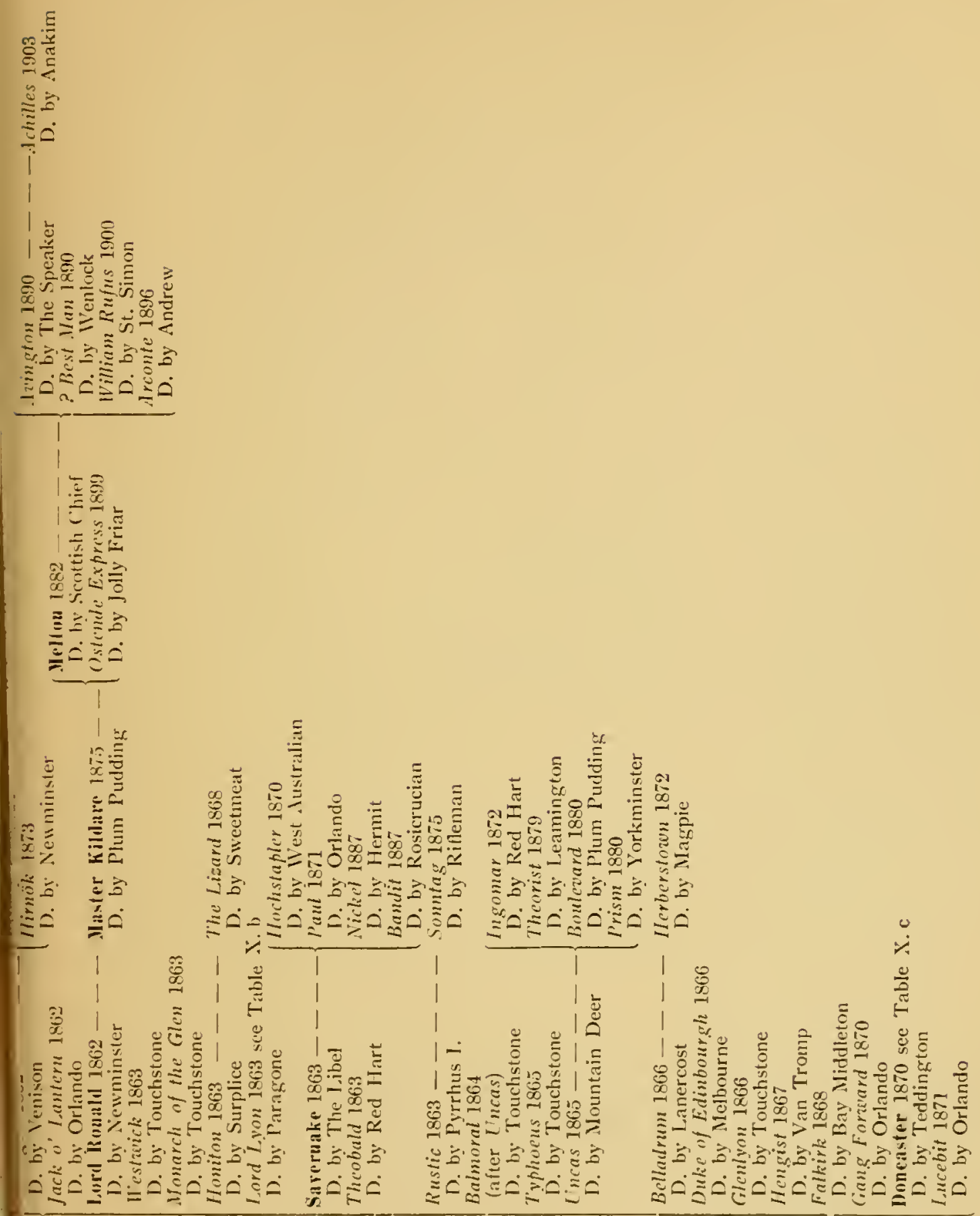


Table X. b. Darl. Arabian Line.
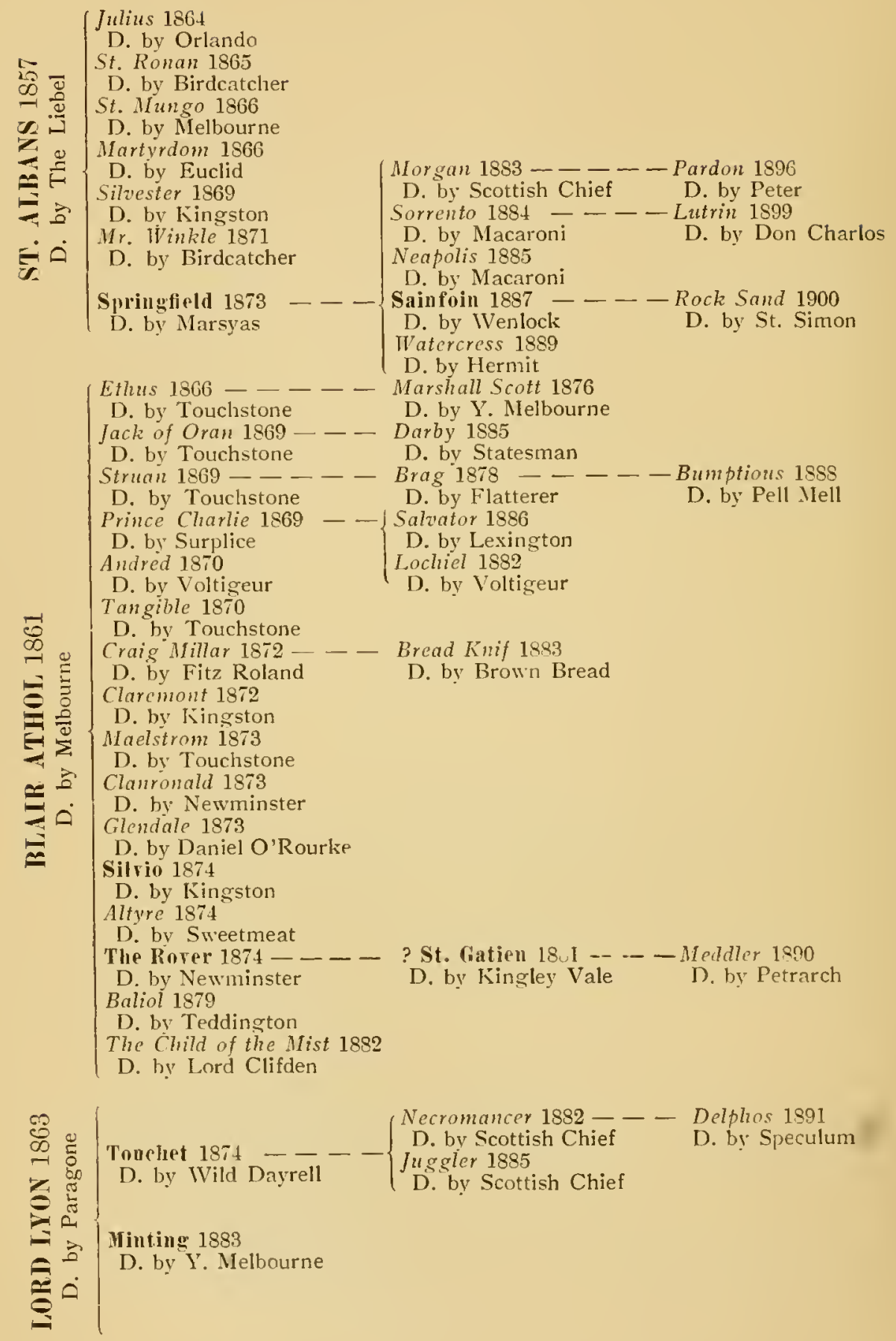
6. Tables Showing the Ancestors of Thoroughbreds in the Male Line.

451

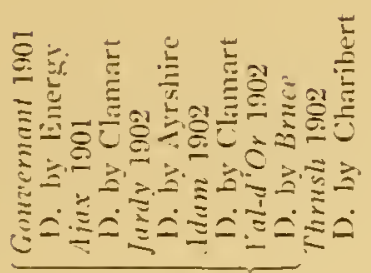

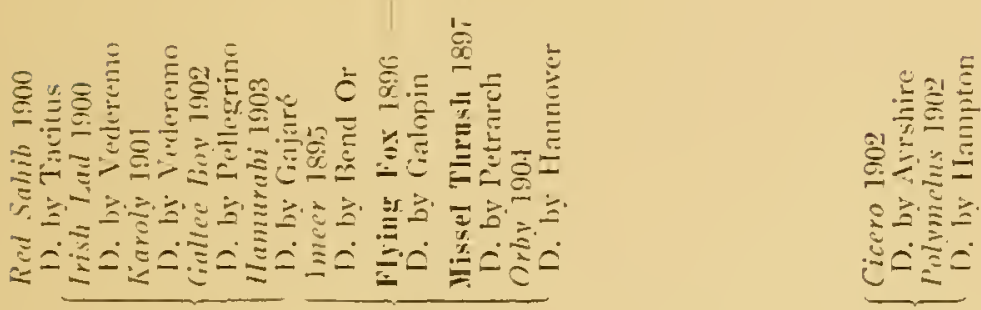

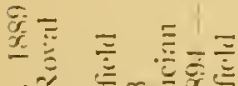

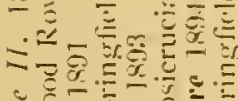

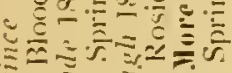

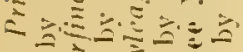

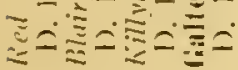
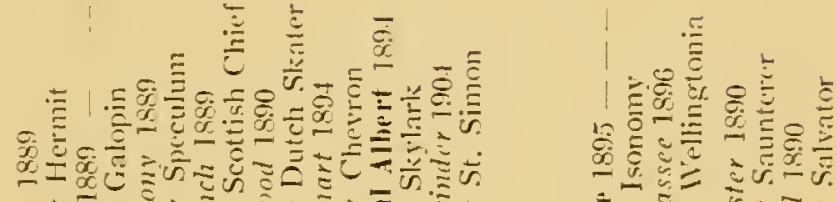

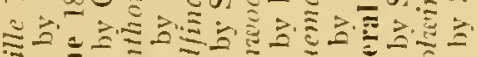

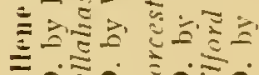

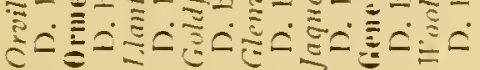
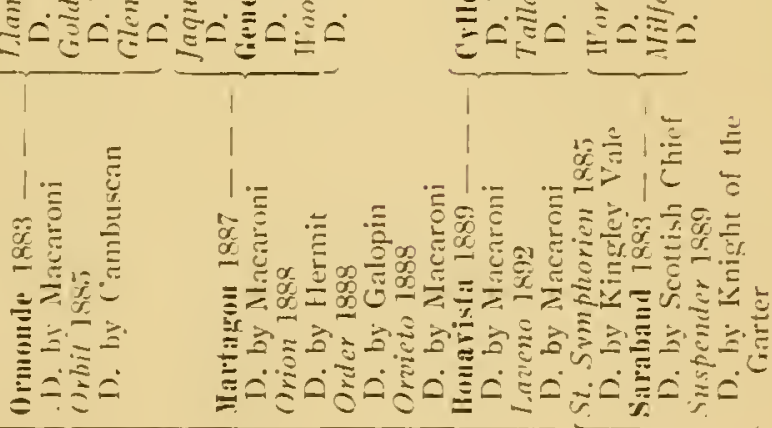

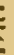

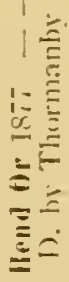

1 ह हैं

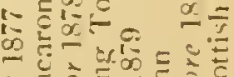

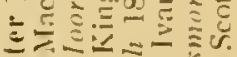

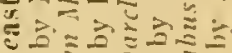

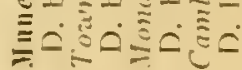




\section{Tablo XI. Darl. Arabian Line.}

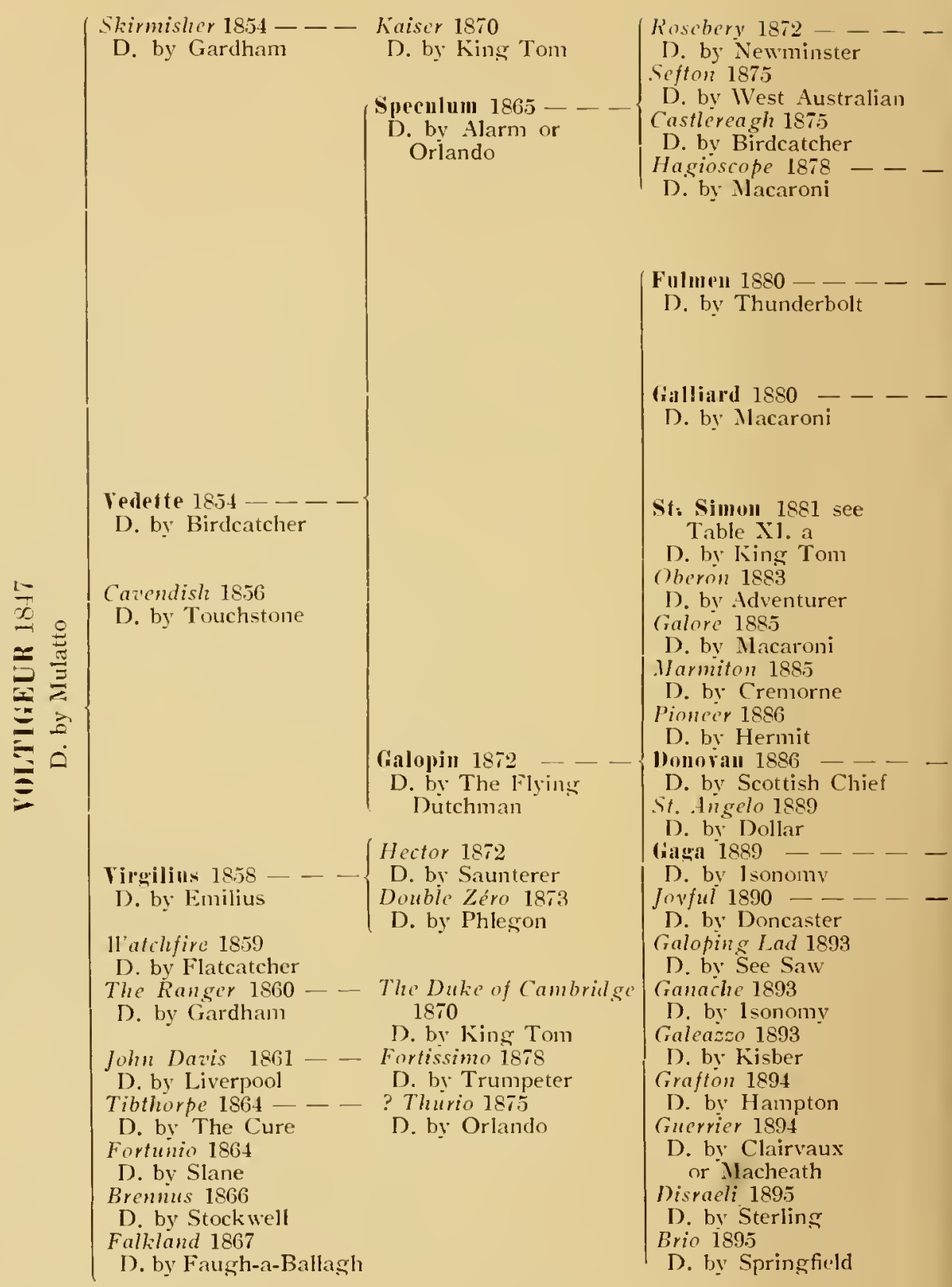




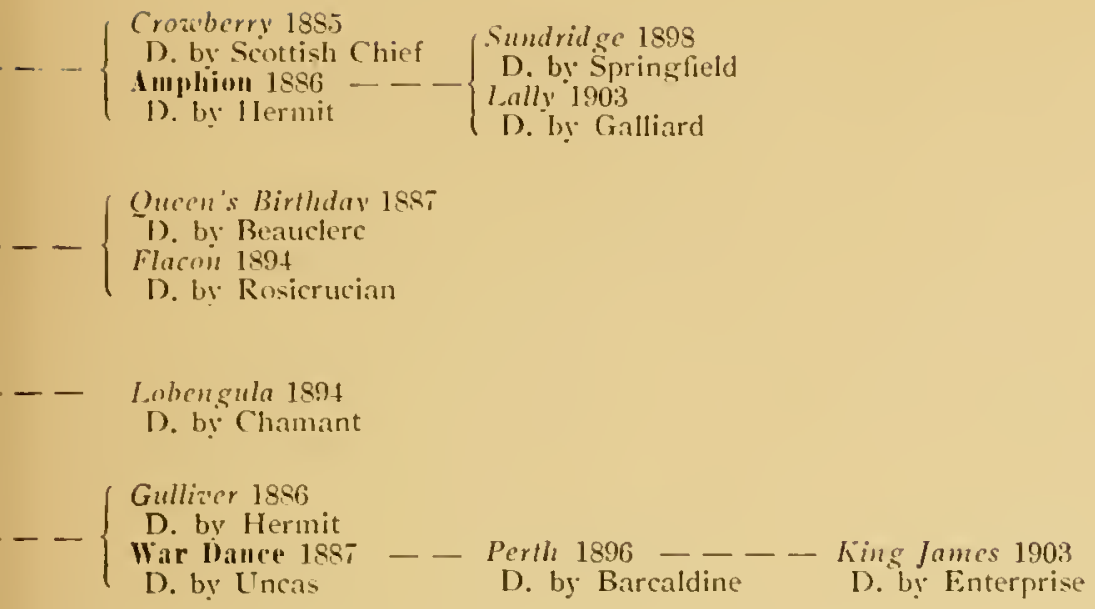

\section{Matchmaleer 1892 - Handicapper 1898}

D. by Plebeian

D. IN Beaudesert

lilasquez 1894

Adimiral Brceze 1901

D. by Macaroni

D. by Isonomy

Mindig 1895

D. by Scottish (Thief

IInlländer 1902

D. by Isonomy 

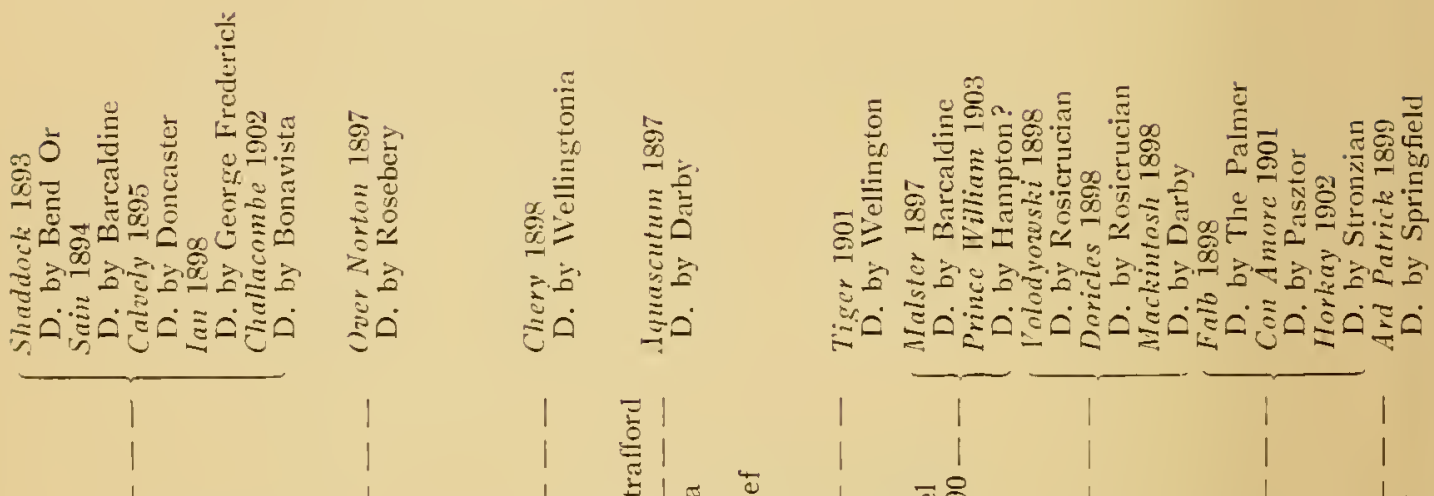

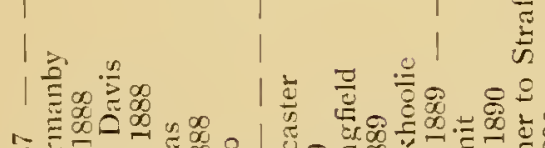
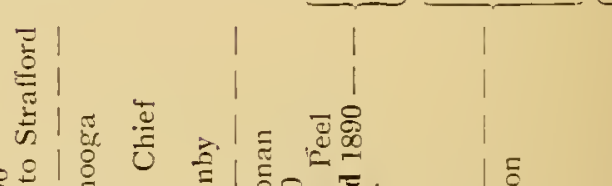

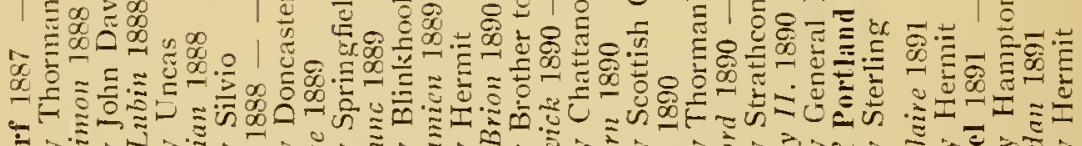

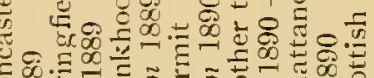

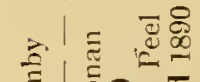

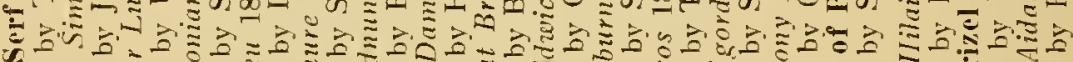

三

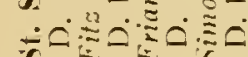
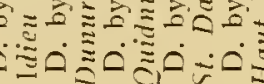
6. Tables Showing the Ancestors of Thoroughbreds in the Male Line.

455

乌

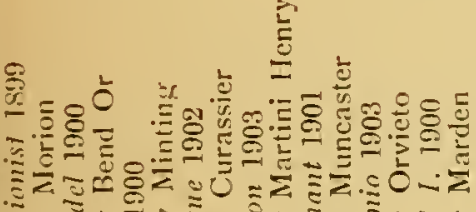

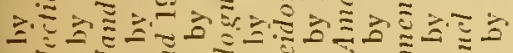

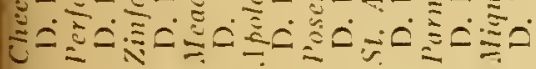

$\stackrel{8}{3}$

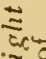

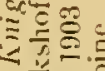

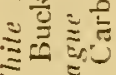

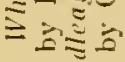

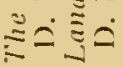

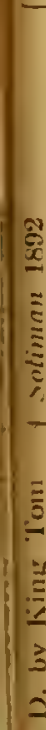

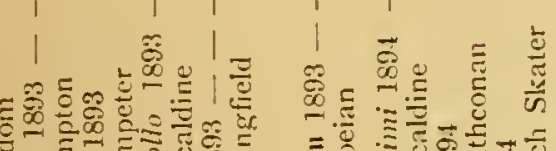

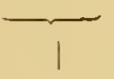

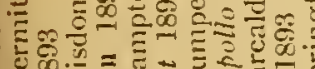

$\overline{\underline{E}}$

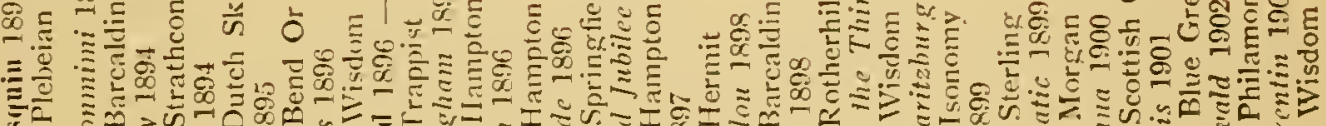

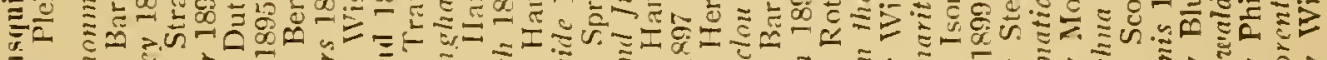

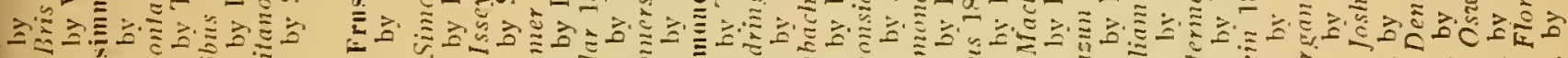

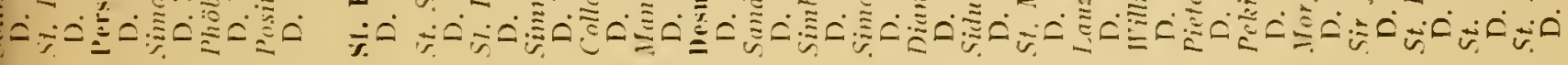




\section{'T'able I. Byerly 'T'urk Line.}

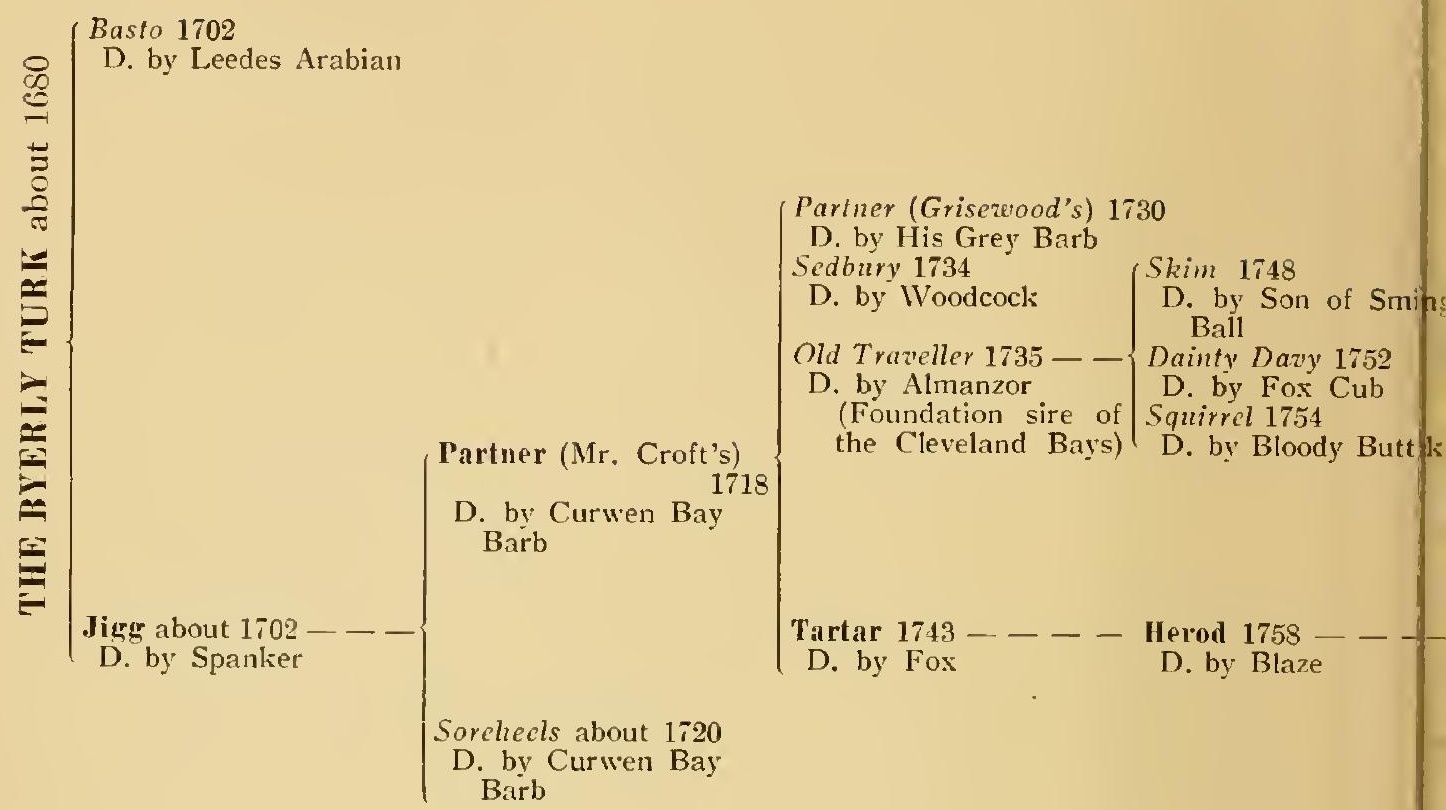

Table I. a.

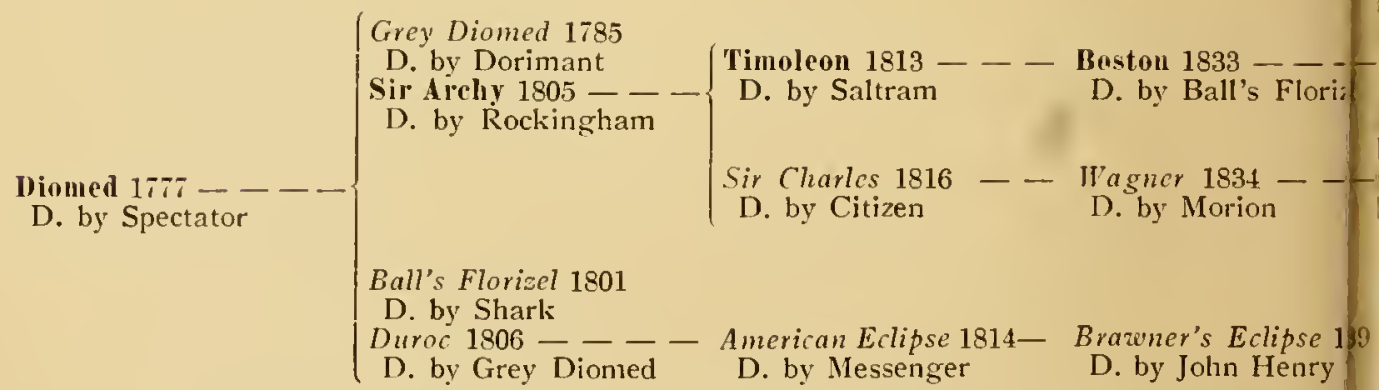




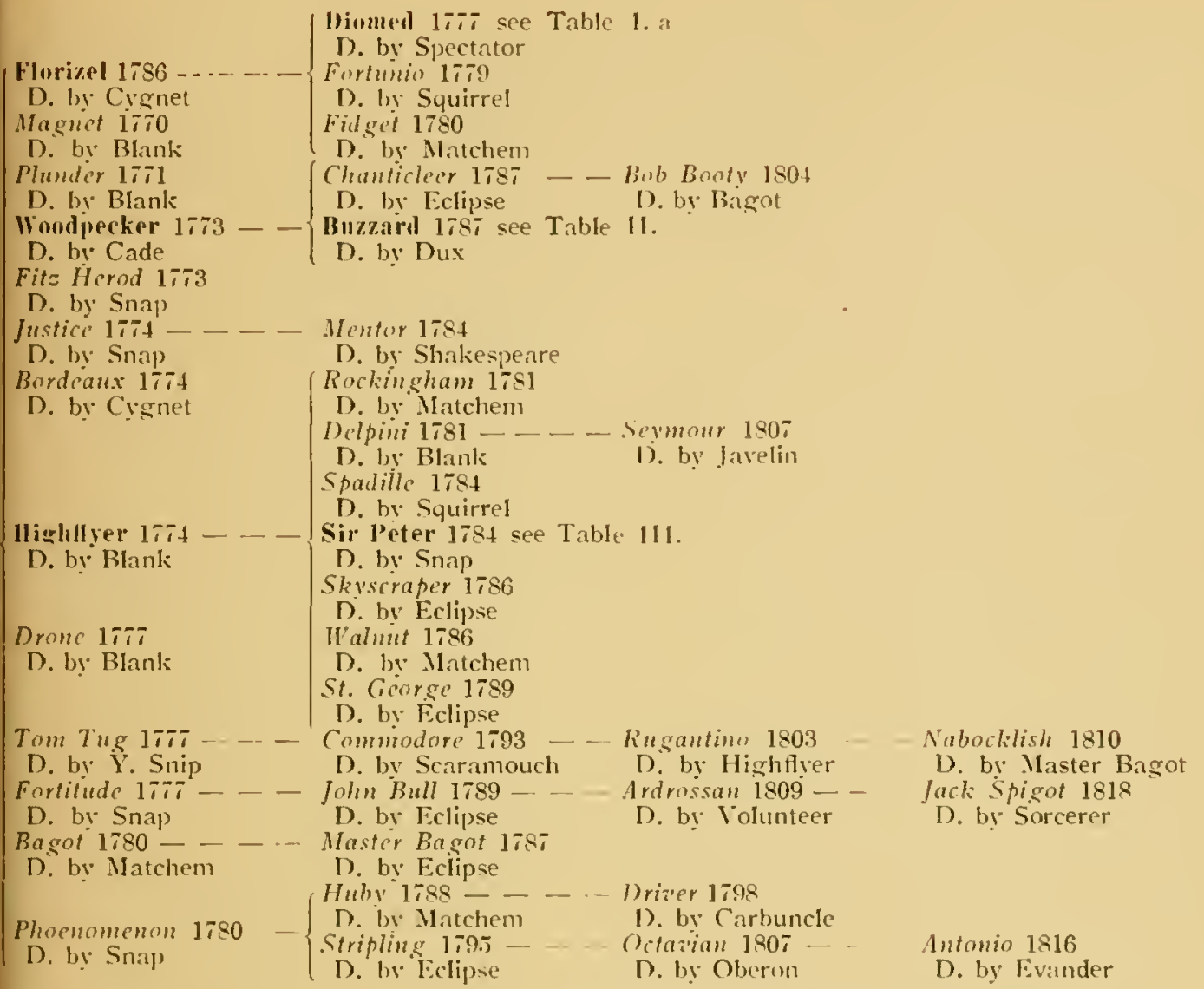


Table II. Byerly T'urk Line.

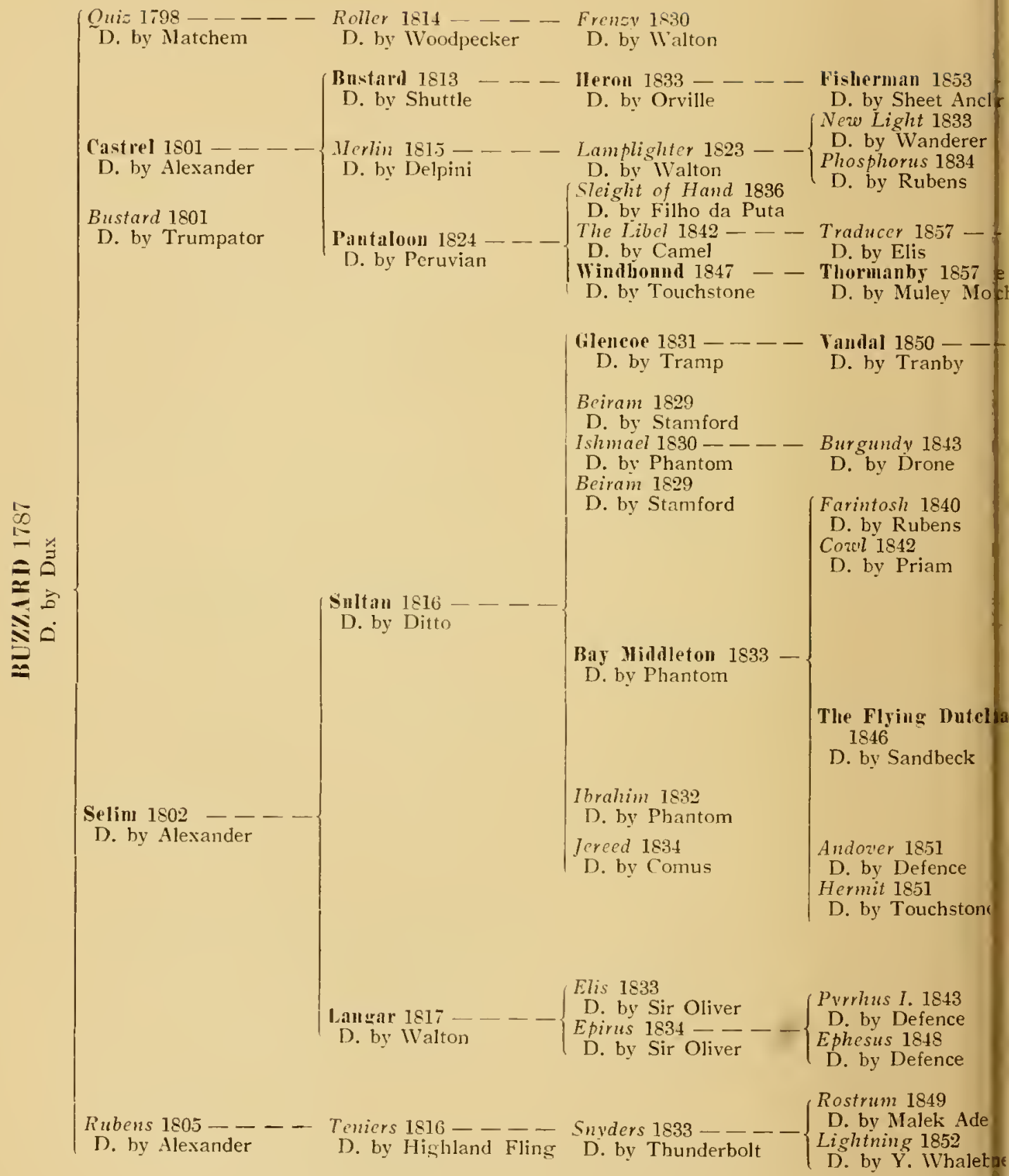


Angler 1862 - - - hobinson Crusoe 1873

D. by Melbourne D. by Siockwell

Maribyrnong 1863 - - Richmond $187^{2}$ - - The Admiral 1887 - The l'ictory 1898

1). by lil. Dutehman D. by The Premier D. Dy Countryman D. by Henchman

ir . Modred 1877

D. by Cambuscan

Table IV.

Wiruil $1864--$
D. by Yorkshire

Hindoo $1878---1$ - lannorer $1884---$
D. by lexington by Bonnie Scotland

Hamburg $1895-1-\left\{\begin{array}{l}\text { D. by Fellowcraft } \\ \text { D. Callistrate } 1890\end{array}\right.$

Ellington 1853

1). by Bonnie Scotland D. by Fellowcraft

D. by Lanercost

Amsterdam 1854

D. by Elis

Ignoramus 1854

D. by The little

known

Ainsterdam 1855

D. by Idle Boy

(by Satan)

Capi Flvatiny 18.57

D. br Melbourne

Mollar $1860-$
D. by Slane

- Sclicdam 1865

D. by Heron

Indrocles 1870 - _ - Cambyse 1884

Cambyse I8s4
D. by Plutus

D. by Light or Serious Saliaior $18 i 2$

1). by lon

St. Cyr Isiz _ _... Pastisson 1890

D. by lon

D. by Jlarlisman

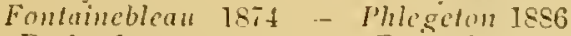

D. by lon D. by Plutus

Patriarche I874 - Iutin 1891

D. by Birdeatcher D. by Don Carlos

I'ignemale 1876

D. by The Baron

Prologue 1866

D. by Gladiateur

Sammir 1878 - _ C.. Clamart 1888

D. by Jon D. by Prince Charlie frizona 1899

The Condor 1882 Omuim II. $1892 \ldots$ D. by Melton

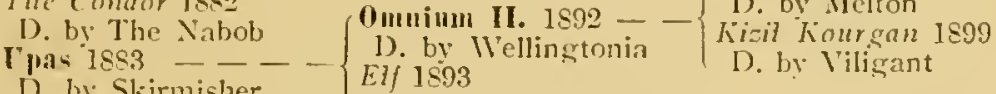

D. by Jon

D. by Skirmisher (D. by Adventurer

Bocage 1885 - _ - Roilelet $1 \$ 92$

D. by Chattannoga D. by Mars

Dauphin 1885

D. by Father Thames

Dutcly skater 1866 - Insulaire 1875

D. by Gladiator D. by Beadsman

Gardefeu 189:

D. by Bruce

D. by D'Estournel

Codoman 1897

D. by The Bard

D. by Mars 


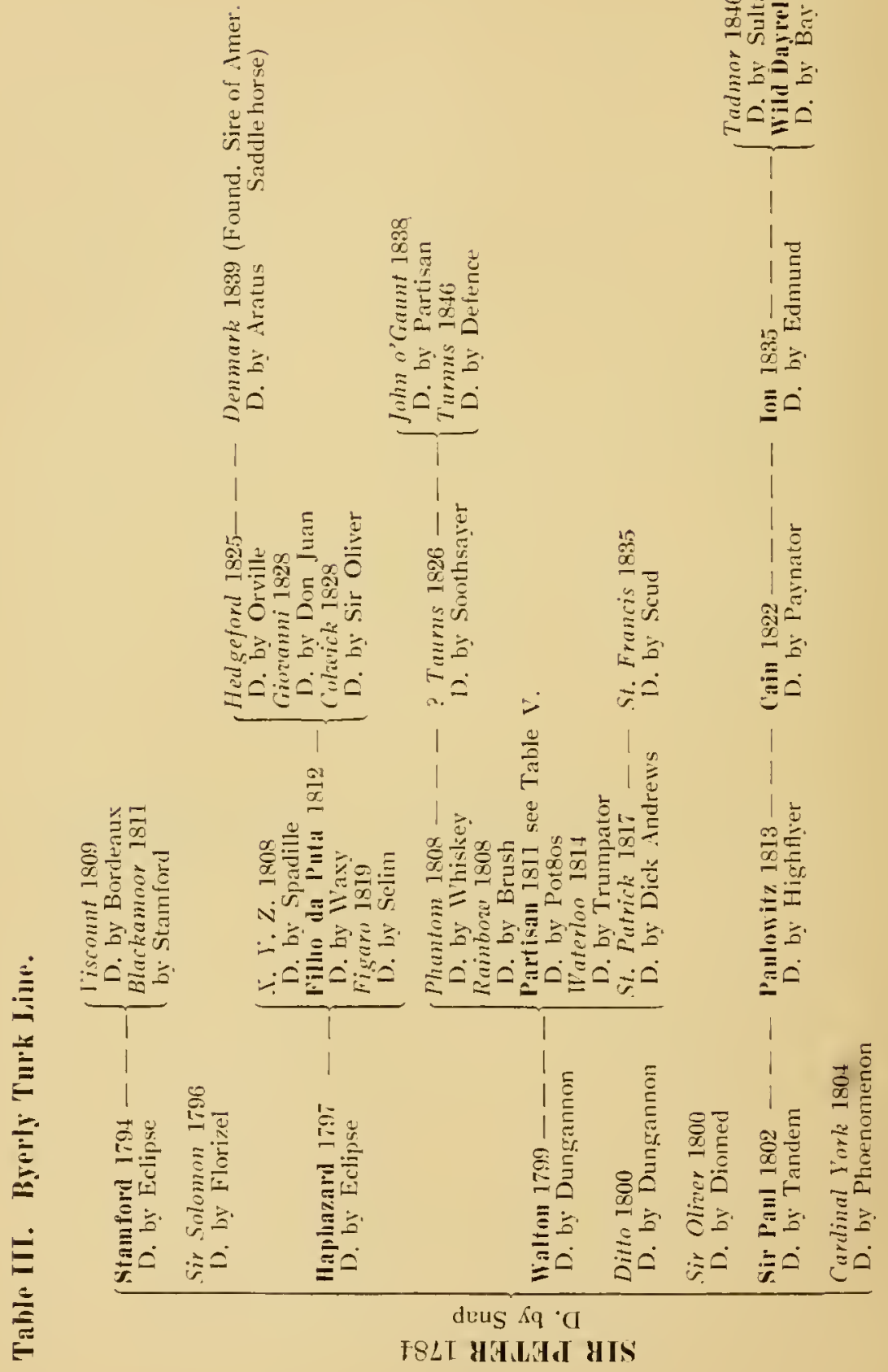


6. Tables Showing the Ancestors of Thoroughbreds in the Male Line. 461

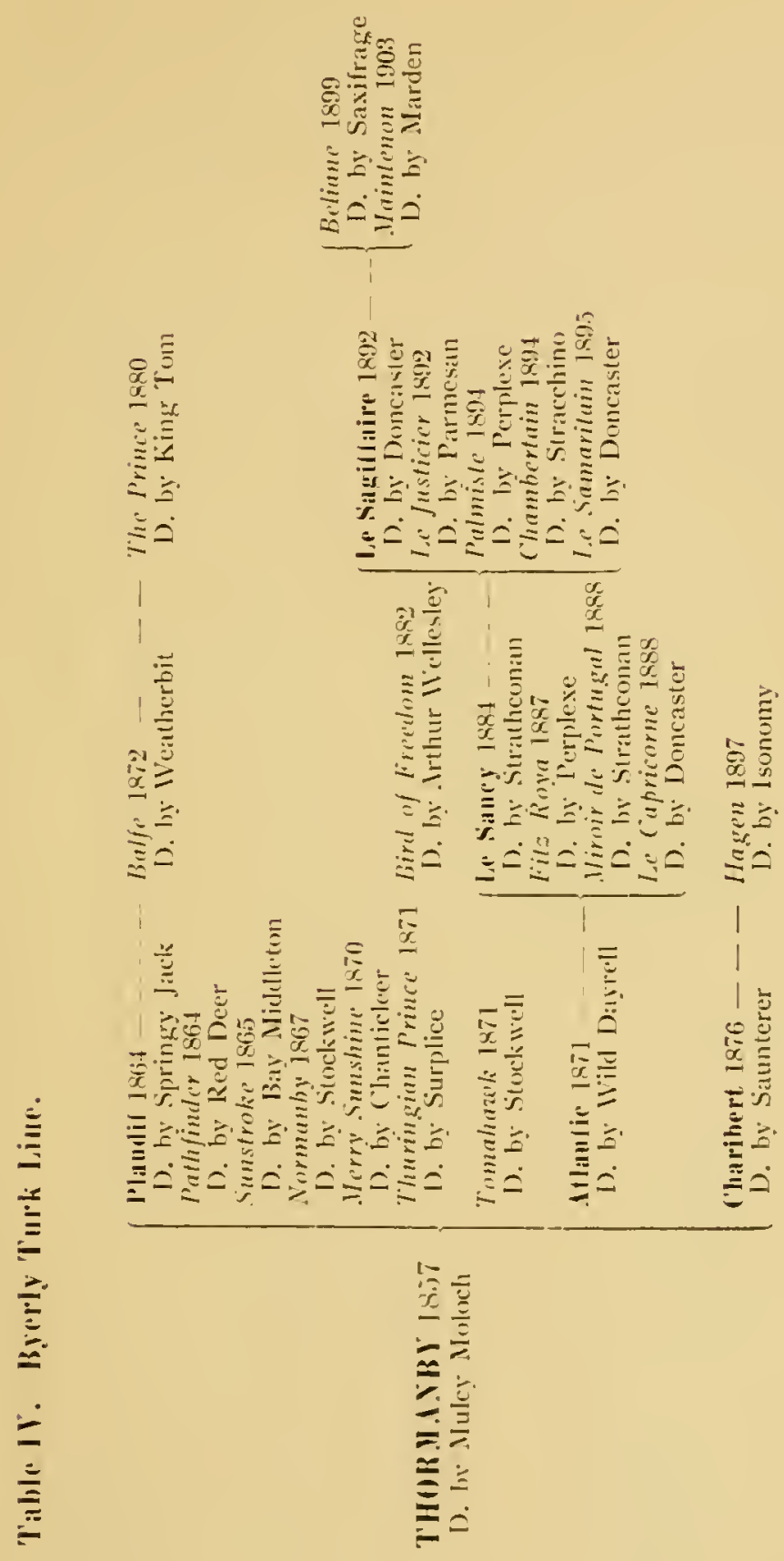




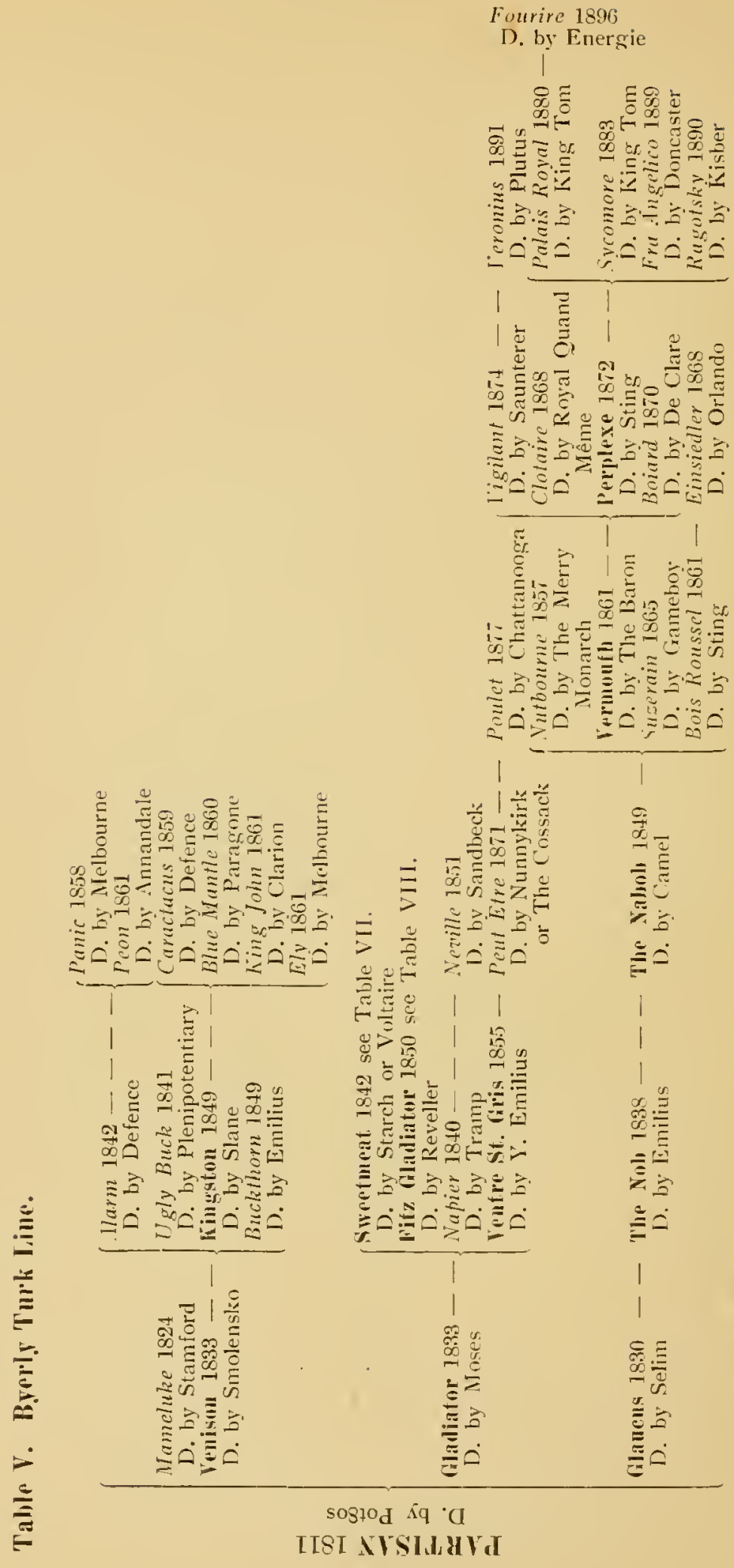




\section{'Table VI. Byerly 'Jurk I.iut.}

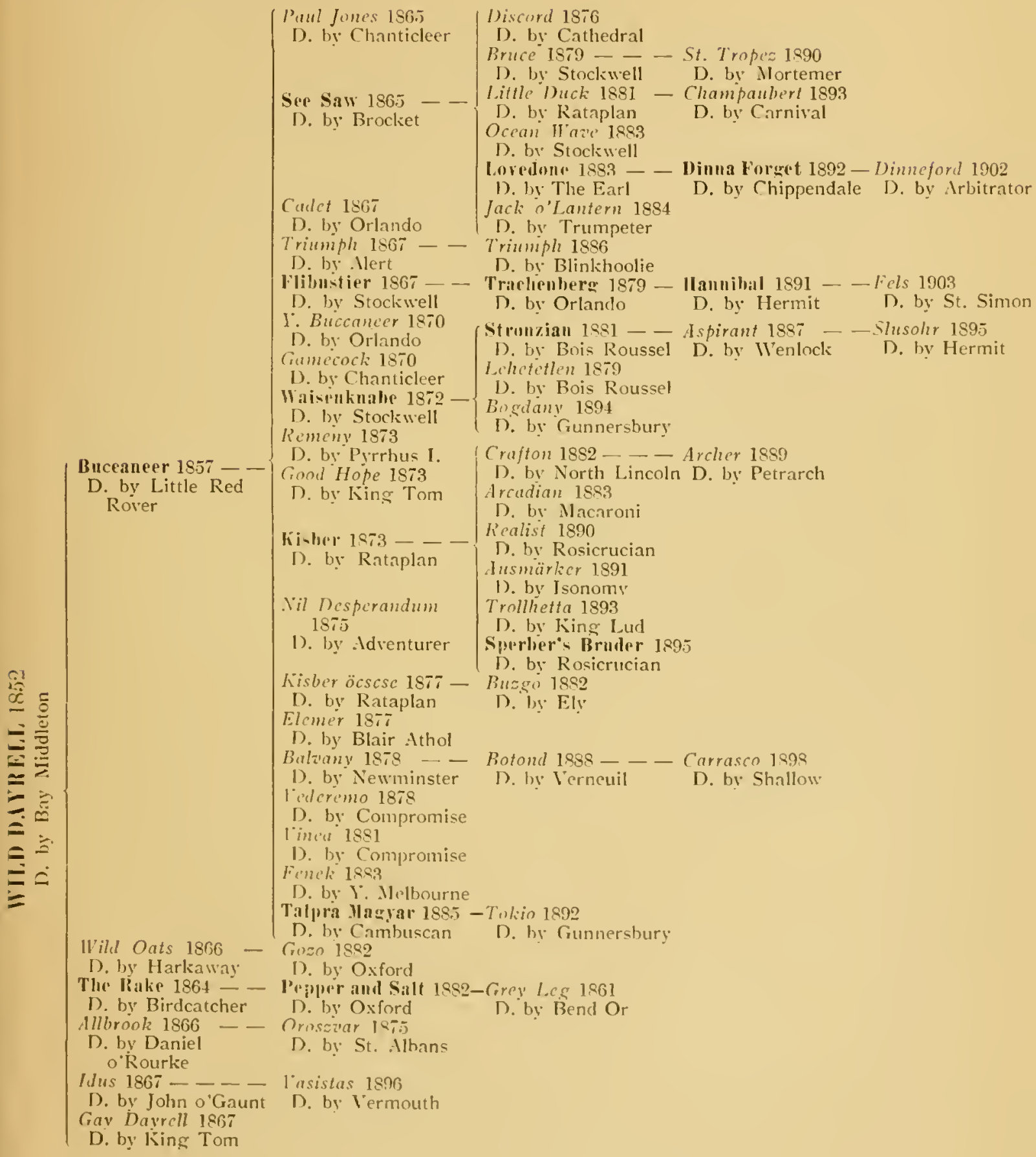




\section{Tahle VII. Byerly Turk Line.}

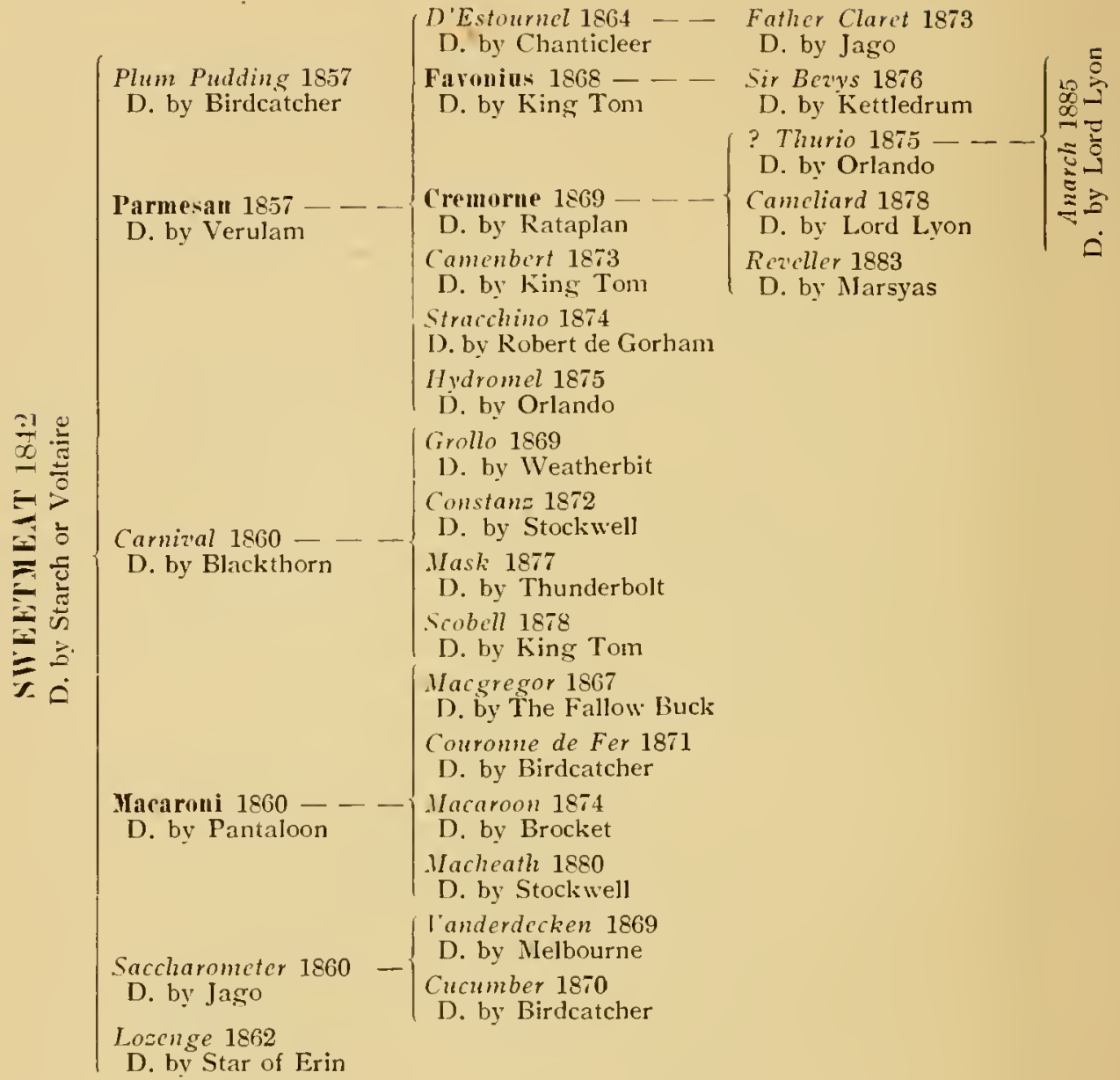



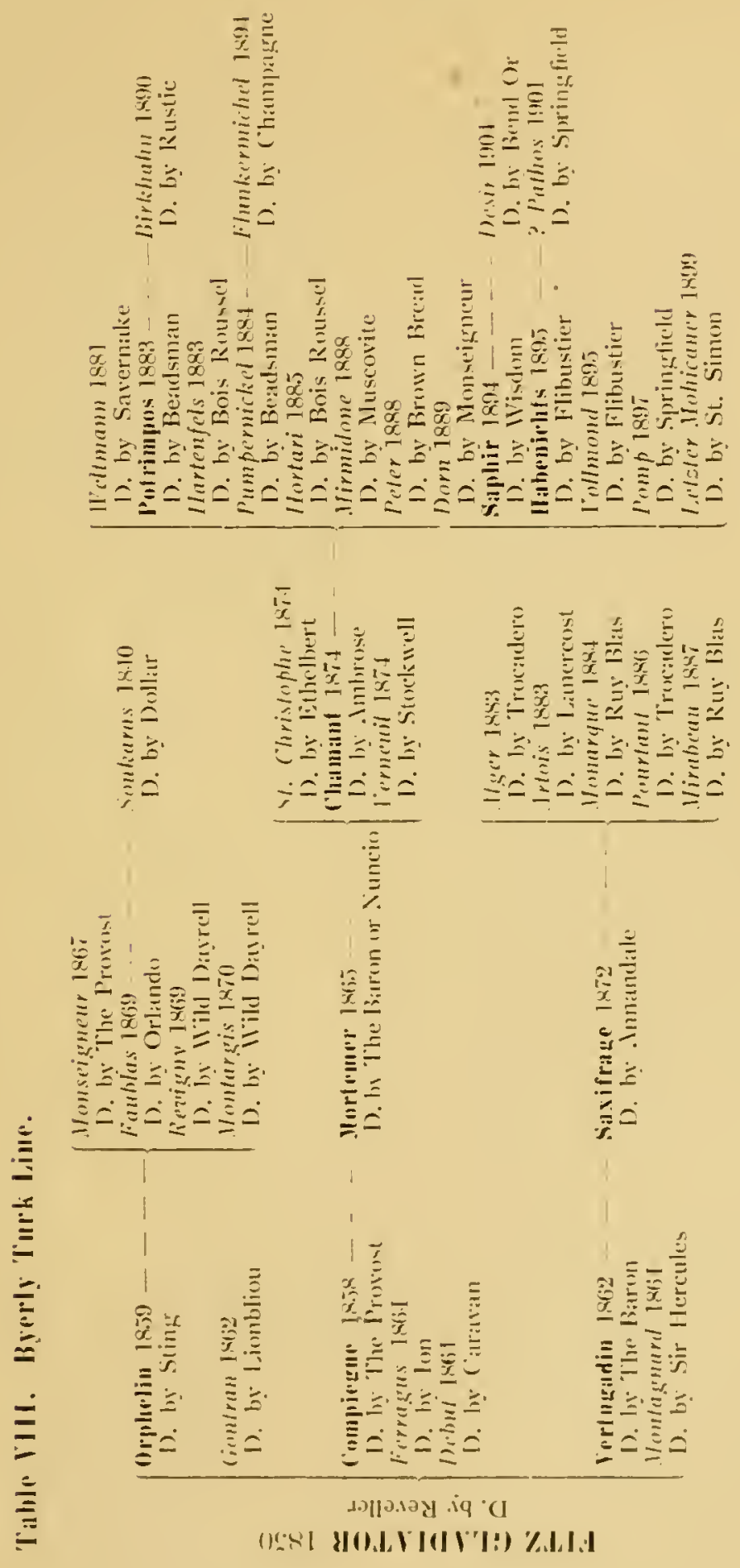


\section{Table I. Godolphin Irahian Line.}


Table II. Fomb. Arabian line.

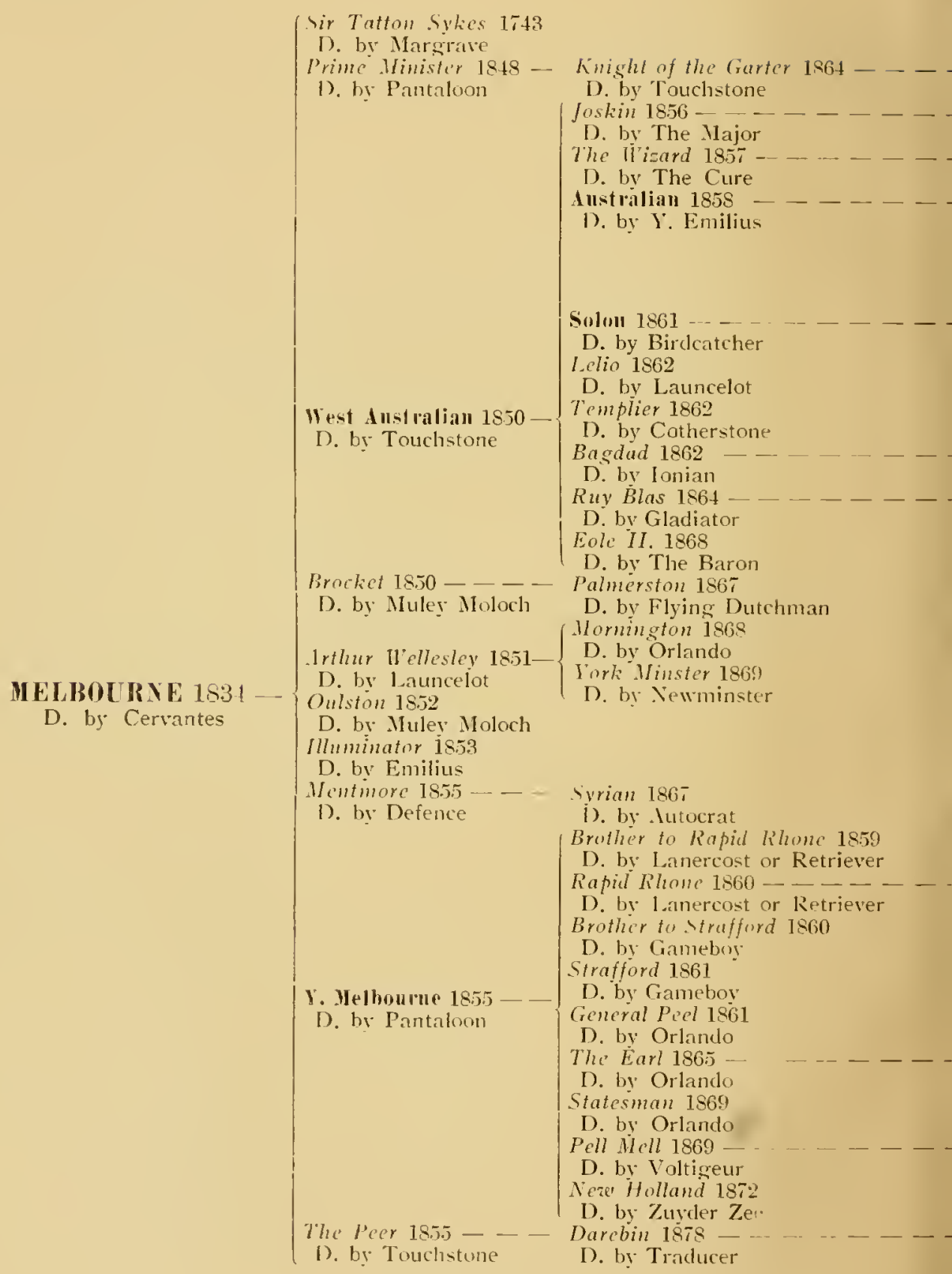


茞 


$$
\begin{aligned}
& \text { pec, } \\
& \text { taindans Gris } \\
& 636.108 \\
& \text { Q.8OE }
\end{aligned}
$$




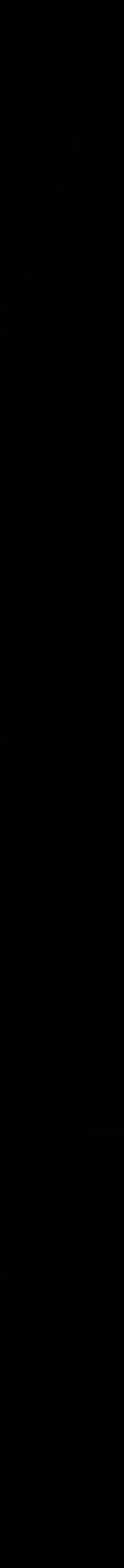

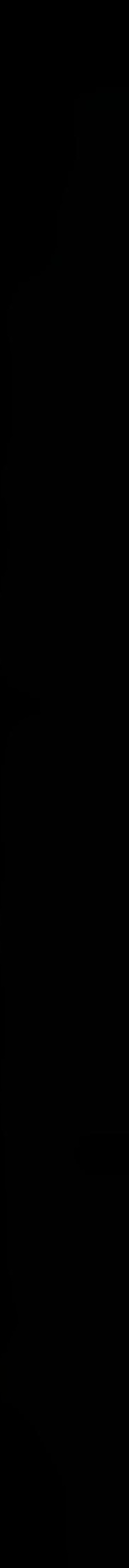



